\title{
Axiomatisches Denken und Arbeiten im Mathematikunterricht
}

\author{
VON DER FAKULTÄT FÜR MATHEMATIK, INFORMATIK UND \\ NATURWISSENSCHAFTEN DER RWTH AACHEN UNIVERSITY ZUR ERLANGUNG DES \\ AKADEMISCHEN GRADES EINES DOKTORS DER NATURWISSENSCHAFTEN \\ GENEHMIGTE DISSERTATION
}

vorgelegt von

Diplom-Gymnasiallehrer

Tobias Hock

aus

Jülich, Nordrhein-Westfalen

$\begin{array}{ll}\text { Berichter: } & \text { Prof. Dr. Johanna Heitzer } \\ & \text { Prof. Dr. Inge Schwank } \\ & \text { Prof. Dr. Hans Niels Jahnke }\end{array}$

Tag der mündlichen Prüfung: 23. April 2018

Diese Dissertation ist auf den Internetseiten der Universitätsbibliothek verfügbar. 



\section{Kurzfassung}

Aussagen, die im Rahmen mathematischer Theorien bewiesen werden, genießen ein besonders hohes $\mathrm{Maß}$ an Sicherheit und Beständigkeit. Maßgeblich verantwortlich für diesen Ruf ist die axiomatische Methode: Ausgehend von Aussagen, die über jeden Zweifel erhaben sind, lassen sich streng logisch und unter ausschließlicher Verwendung bereits bewiesener Sätze weitere Resultate herleiten. So wird ein umfassendes, strukturiertes Theoriegebäude errichtet. Seit Euklid mit seinem Werk Die Elemente im 3. Jahrhundert v. Chr. die Geometrie erstmals axiomatisch-deduktiv aufbaute, war diese Darstellungsform paradigmatisch für exakte Wissenschaft.

Im Verlauf der Jahrhunderte haben sich das Bild von Mathematik und damit auch der Blick auf die Rolle der Axiomatik grundlegend gewandelt: Zu Beginn des 20. Jahrhunderts setzte sich ein abstrakt-formalistischer Standpunkt durch, dem zufolge mathematische Begriffe als Variablen für inhaltlich nicht näher bestimmte Entitäten aufgefasst werden. Axiome beschreiben vor diesem Hintergrund die Beziehungen, die zwischen diesen bestehen, und definieren die Begriffe so implizit. Damit entfiel der klassische Wahrheitsanspruch mathematischer Aussagen, wie er sich in der angenommenen Selbstevidenz der Axiome äußerte, zugunsten des formalen Konzepts der Widerspruchsfreiheit. Dieser Paradigmenwechsel ebnete den Weg für eine Mathematik, in der inhaltlich verschiedene, aber strukturell ähnliche Themengebiete in abstrakt formulierten Theorien zusammengefasst und geordnet werden können.

Trotz ihrer grundlegenden Bedeutung für das Selbstverständnis der Mathematik als beweisende Disziplin spielt die axiomatische Methode im gegenwärtigen Mathematikunterricht kaum eine Rolle. Reformversuche, welche unter anderem eine stärkere Berücksich-

tigung axiomatischer Aspekte in den Curricula umfassten, gelten als gescheitert. Das in sämtlichen Bildungsstandards ausgewiesene Kompetenzfeld Argumentieren umfasst zwar verschiedene Strengeniveaus; axiomatische Betrachtungen werden darin jedoch konsequent ausgeklammert.

Die Hauptziele dieser Arbeit bestehen darin, dem interessierten Leser einen Gesamtüberblick über die Bedeutung der Axiomatik in der Mathematik zu geben und Lehrkräften eine 
didaktische Grundlage für eine eigene reflektierte Entscheidung zur Verfügung zu stellen, ob und in welchem Maße sie axiomatische Themen mit Schülerinnen und Schülern behandeln wollen. Schließlich sollen konkrete Möglichkeiten aufgezeigt werden, axiomatische Denk- und Arbeitsweisen zum Gegenstand von Lernumgebungen zu machen. Um diese Ziele zu erreichen, werden

- wesentliche Eigenschaften der axiomatischen Methode aus mathematikhistorischer und -philosophischer Sicht beleuchtet;

- die Argumente für und wider eine Behandlung axiomatischer Themen im Mathematikunterricht eruiert und aus heutiger didaktischer Sicht bewertet;

- übergeordnete Lernziele und didaktische Leitlinien für eine abgerundete Behandlung von Axiomatik auf Oberstufenniveau formuliert;

- gelungene Unterrichtskonzepte zu ausgewählten axiomatischen Gesichtspunkten vorgestellt;

- ein eigenes Konzept inkl. umfangreicher Materialien zur Behandlung axiomatischer Themen zur Verfügung gestellt, welches für den Einsatz in Zusatzkursen der Sekundarstufe II gedacht ist. 


\section{Abstract}

Theorems that are proven within the framework of mathematical theories enjoy an especially high degree of security and stability. This reputation is intrinsically tied to the axiomatic method: Starting with propositions whose truth is indubitable, it is possible to logically deduce further results that exclusively rely on already accepted theorems. This leads to a comprehensive theoretical structure. Since in the 3rd century BC Euclid's Elements provided an axiomatic-deductive description of geometry for the first time, this form of presentation has been paradigmatic of exact sciences.

Over the course of the centuries, the picture of mathematics and with it the view on the role of axiomatics have fundamentally changed: At the beginning of the 20th century an abstract-formalistic position prevailed, according to which mathematical terms are seen as variables for entities that are not further determined with regard to their content. Against this background, axioms describe the relations that exist between these terms, thus defining them implicitly. As a consequence, the classical claim to truth of mathematical statements, as it manifested itself in the supposed self-evidence of the axioms, was dispensed with in favour of the formal concept of consistency. This paradigm shift paved the way for a type of mathematics in which topics that differ in content but are structurally similar can be subsumed under and organized in abstract theories.

Despite its fundamental importance for the self-conception of mathematics as a discipline of proof the axiomatic method plays hardly any role in contemporary mathematics education. Reform programmes comprising among other things a greater consideration of axiomatic aspects in curricula are considered to have failed. Reasoning skills, which are included in all educational standards, may comprise different levels of rigour; however, axiomatic considerations are consequently excluded in them.

The main goals of this thesis are to give the reader a comprehensive overview of the significance of axiomatics in mathematics and to provide teachers with a didactic basis for a well-reflected decision as to whether and to what extent they wish to treat axiomatic topics with their pupils. Eventually, concrete ways of integrating axiomatic thinking and working methods into learning environments are illustrated. To achieve these goals, 
- the main characteristics of the axiomatic method are depicted from a historical and philosophical perspective;

- the arguments for and against dealing with axiomatics in mathematics education are analysed and evaluated from a contemporary didactic point of view;

- overarching learning objectives and didactic guidelines for a comprehensive treatment of axiomatics at Sekundarstufe II are set up;

- successful teaching concepts on selected axiomatic aspects are presented;

- a teaching concept of my own is provided that includes extensive learning materials for treating axiomatic topics in supplementary courses at Sekundarstufe II. 


\section{Danksagung}

Feeling gratitude and not expressing it is like wrapping a present and not giving it.

- William Arthur Ward

Mein größter Dank gilt meiner Doktormutter Frau Prof. Dr. Johanna Heitzer. Ihre unschätzbare Unterstützung und ihr steter Zuspruch - nicht nur in Bezug auf mein Promotionsvorhaben - haben mir durch so manche Frustrationsphase geholfen, mich ,auf Kurs gehalten" und die Gestalt der Arbeit entscheidend mitgeprägt.

Frau Prof. Dr. Inge Schwank danke ich für die Übernahme des Zweitgutachtens sowie für fruchtbare Treffen in Osnabrück und später Köln, die mir insbesondere im Hinblick auf den unterrichtspraktischen Teil der Arbeit sehr geholfen haben.

Ferner habe ich in hohem Maße von Herrn Prof. Dr. Hans Niels Jahnkes Expertise profitiert. Ihm möchte ich für einen anregenden Gedankenaustausch, sein Interesse an meinem Promotionsthema sowie die Übernahme des dritten Gutachtens herzlich danken.

Bei Herrn Prof. Dr. Sebastian Walcher möchte ich mich für viele hilfreichen Anregungen gerade in der Anfangsphase meiner Arbeit bedanken. Herrn Prof. Dr. Erich Grädel, Frau Prof. Dr. Franziska Jahnke und Herrn Dr. Marc Ensenbach danke ich für ihre fachlichen Anmerkungen zu meinem Kapitel über die Gödelschen Unvollständigkeitssätze.

Die mehrjährige Arbeit an einer Promotion wäre sicher um ein Vielfaches mühsamer ohne ein angenehmes Arbeitsklima. Die Zusammenarbeit mit meinen Kolleginnen und Kollegen hat mir stets großen Spaß gemacht und viele Freundschaften hervorgebracht. Vor allem möchte ich mich (in alphabetischer Reihenfolge) bei folgenden Personen bedanken: Ruth Bisterfeld, Dr. Cornelia Dieckmann, Dr. Till Dieckmann, Ivo Mei, Laura Neisius, Dr. Agnes Peters, Stefan Pohlkamp, Marvin Titz, Regine Wallraf und Tobias WiernickiKrips. Bei Georg Mierau und Ramón Janßen bedanke ich mich für die Hilfe bei diversen LATEX-bezogenen Problemen.

Mein abschließender Dank gilt meiner Familie, die mich immer und uneingeschränkt bei all meinen Entscheidungen unterstützt und gefördert hat. 



\section{Inhaltsverzeichnis}

Kurzfassung iii

Abstract $\quad$ v

Danksagung vii

1 Einleitung 1

1.1 Motivation und Zielsetzung . . . . . . . . . . . . . . . . . . 1

1.2 Kapitelübersicht und Zielgruppe . . . . . . . . . . . . . . . . . . 4

2 Axiomatik in der Mathematik $\quad 7$

2.1 Axiomatik in der Geometrie . . . . . . . . . . . . . . 8

2.1.1 Euklids Elemente . . . . . . . . . . . . . . . . . . 8

2.1.2 Nichteuklidische Geometrie . . . . . . . . . . . . . 16

2.1.3 Hilberts Grundlagen der Geometrie . . . . . . . . . . . . . . . . 29

2.2 Axiomatik im 20. Jahrhundert . . . . . . . . . . . . . . . 36

2.2.1 Strukturmathematik . . . . . . . . . . . . 36

2.2.2 Grenzen der Axiomatik: Die Gödelschen Unvollständigkeitssätze 41

2.3 Mathematikphilosophische Aspekte . . . . . . . . . . . . . . . 51

2.4 Zusammenfassung: Zur Rolle der Axiomatik in der Mathematik . . . . . 61

3 Axiomatik im Mathematikunterricht: Didaktische Perspektiven 65

3.1 Neue Mathematik: Historischer und inhaltlicher Überblick . . . . . . . . 65

3.2 Fertigprodukt versus Tätigkeit: Zwei Sichtweisen auf Axiomatik . . . . 75

3.3 Wesentliche Bedenken . . . . . . . . . . . . . . . 78

3.4 Einordnung der Axiomatik in das Kompetenzfeld Argumentieren . . . . . 83

3.5 Zusammenfassung: Zur Rolle der Axiomatik im Mathematikunterricht . . 87

4 Axiomatik im Mathematikunterricht: Unterrichtspraktische Perspektiven $\quad 93$

4.1 Übergeordnete Lernziele zur Behandlung von Axiomatik im Mathematikunterricht .......................... 93 
4.2 Unterrichtsvorschläge: Bestandsaufnahme . . . . . . . . . . . . . . . 95

4.2.1 Axiomatisierung von Abstimmungsmengen (H.-G. Steiner) . . . 96

4.2.2 Sätze aus dem Wüstensand (E. Cohors-Fresenborg et al.) . . . . . 104

4.2.3 Die Entdeckung der Axiomatik am Sechsstern (M. Wagenschein und M. Gerwig) . . . . . . . . . . . . . . . . . . 113

4.2.4 Hypothesen in der Mathematik (H. N. Jahnke) . . . . . . . . . . . 118

4.3 Zusammenfassung mit Blick auf die Lernziele . . . . . . . . . . . . . . 120

5 Axiomatik mit Oberstufenschülern: Eigene Unterrichtsmaterialien 125

5.1 Didaktische Grundsatzentscheidungen . . . . . . . . . . . . . . 126

5.1.1 Themenauswahl . . . . . . . . . . . . . 126

5.1.2 Methodische und inhaltliche Leitideen . . . . . . . . . . . . . . 128

5.2 Unterrichtsreihe 1: Geometrie . . . . . . . . . . . . . . . 131

5.2 .1 Sachanalyse . . . . . . . . . . . . . . . 131

5.2.2 Didaktische Anmerkungen . . . . . . . . . . . . . . 132

5.2.3 Beschreibung der Unterrichtsreihe . . . . . . . . . . . . . . 136

5.3 Unterrichtsreihe 2: Wahrscheinlichkeitsrechnung . . . . . . . . . 152

5.3 .1 Sachanalyse . . . . . . . . . . . . . . . 152

5.3.2 Didaktische Anmerkungen . . . . . . . . . . . . . 156

5.3.3 Beschreibung der Unterrichtsreihe . . . . . . . . . . . . . 158

5.4 Unterrichtsreihe 3: Zahlbereichserweiterungen . . . . . . . . . . . . . . 169

5.4.1 Sachanalyse . . . . . . . . . . . . . . . 169

5.4 .2 Didaktische Anmerkungen . . . . . . . . . . . . . . . 170

5.4.3 Beschreibung der Unterrichtsreihe . . . . . . . . . . . . . . 173

5.5 Erfahrungen aus der Umsetzung mit Schülergruppen . . . . . . . . . 180

5.5 .1 Pilotierung . . . . . . . . . . . . 180

5.5.2 Erprobung im Rahmen einer Workshop-Woche . . . . . . . . . . 183

5.5.3 Lernzielerreichung . . . . . . . . . . . . . . . . . . 191

5.5.4 Zusammenfassung und Konsequenzen . . . . . . . . . . . . 197

6 Fazit 199

A Unterrichtsmaterialien 203

A.1 Arbeitsblätter . . . . . . . . . . . . . . . . . . . 203

A.2 Historische Textauszüge . . . . . . . . . . . . . . . 231

A.3 Auszüge aus dem Geometrikon . . . . . . . . . . . . . . . . . . . . . . . 244

A.4 Lösungen und Kommentare . . . . . . . . . . . . . . . . . . . . 270

A.4.1 Geometrie . . . . . . . . . . . . . . . . . 270 
A.4.2 Wahrscheinlichkeitsrechnung . . . . . . . . . 278

A.4.3 Zahlbereichserweiterungen . . . . . . . . . . . . 286

A.5 Hinweise zu Bildquellen und -nutzungsrechten . . . . . . . . . . . . 291

B Schüler-Kurzaufsätze $\quad 293$

$\begin{array}{ll}\text { C Evaluationsergebnisse der Workshop-Woche } & 307\end{array}$

$\begin{array}{ll}\text { Literatur } & 311\end{array}$

$\begin{array}{ll}\text { Index } & 327\end{array}$ 



\section{Einleitung}

\subsection{Motivation und Zielsetzung}

Of the many odd and various things we believe, few are believed more confidently than the truths of simple mathematics. When asked for an example of a thoroughly dependable fact, many will turn from common sense - 'after all, they used to think humans couldn't fly'-from science - 'the sun has risen every day so far, but it might fail us tomorrow'- to the security of arithmetic - 'but 2 plus 2 is surely 4'. (Maddy 1990, S. 1)

Die amerikanische Wissenschaftsphilosophin Penelope Maddy beschreibt mit dem Alleinstellungsmerkmal security ein Charakteristikum, das für viele Menschen die Mathematik auszeichnet. Die logische Stringenz und formale Strenge, die von mathematischen Argumentationsketten gefordert werden, machen den Beweis zum unentbehrlichen Bestandteil des mathematischen Selbstverständnisses; die Mathematik ist sozusagen ,über den Beweis definiert - und genau darin liegt ihre Besonderheit und ihre Differenz zu allen anderen Wissenschaften“ (Heintz 2000, S. 216).

Beim Beweisen wird die Gültigkeit einer mathematischen Aussage gemäß zuvor vereinbarter logischer Schlussregeln auf die Gültigkeit anderer, bereits bewiesener Aussagen zurückgeführt. Dies würde zu einem ,unendlichen Regress“ führen (Lakatos 1982b), wenn nicht letztlich auf vorangestellte Axiome und Grundbegriffe zurückgegriffen würde, welche das Fundament für die innerhalb einer Theorie deduzierbaren Aussagen liefern. Diese eigentlich simple Überlegung ist der Keim, aus dem heraus sich die Mathematik zu einem Musterbeispiel für exakte Wissenschaft entwickelt hat. Gerwig (2015, S. 11) übertreibt daher nicht, wenn er schreibt: „Die Weiterentwicklung der babylonischen und ägyptischen Rechen- und Messkunst zu einer auf Axiomen und Definitionen ruhenden Mathematik stellt die Geburtsstunde der Wissenschaften dar" (Herv. im Orig.).

Die ausschließliche Berufung auf bereits bewiesene Aussagen (bzw. die Axiome und Grundbegriffe) nach den Prinzipien des logischen Schließens beim deduktiven Prozess - wie sie paradigmatisch bei Euklids Elementen vorzufinden ist - gepaart mit der postu- 
lierten Selbstevidenz der Axiome gab den Aussagen der Mathematik über Jahrhunderte den Status unumstößlicher Wahrheiten. Dies entspricht nicht der modernen Auffassung von Mathematik. Spätestens seit David Hilberts Arbeiten zu Beginn des 20. Jahrhunderts hat sich eine Sichtweise durchgesetzt, welche die Frage nach einer (wie auch immer gearteten) „Wahrheit“ von Axiomen im Rahmen der mathematischen Theorieentwicklung ausklammert. Axiome und Grundbegriffe werden als semantisch zunächst nicht interpretierte Formeln und Symbole aufgefasst, die keinen ontischen Wahrheitsgehalt beanspruchen. Im Vordergrund stehen der systemkonforme Umgang mit den durch die Axiome implizit definierten Grundbegriffen und somit das formal-deduktive Erschließungspotential eines Axiomensystems.

Courant und Robbins (2010, S. xxi) konstatieren:

Trotz der Gefahr der einseitigen Übertreibung hat die Axiomatik zu einem tieferen Verständnis der mathematischen Tatsachen und ihrer Zusammenhänge und zu einer klareren Einsicht in das Wesen mathematischer Begriffe geführt. Hieraus hat sich eine Auffassung entwickelt, welche über die Mathematik hinaus für moderne Wissenschaft typisch ist.

Angesichts ihrer hohen Relevanz für die mathematische Erkenntnissicherung erscheint eine Thematisierung axiomatischer Denk- und Arbeitsweisen im Schulunterricht erstrebenswert. De facto findet das Thema allerdings kaum Beachtung: In den aktuellen Lehrplänen und Bildungsstandards ist Mathematisches Argumentieren zwar ein zentrales Kompetenzfeld, axiomatische oder allgemeiner erkenntnistheoretische Aspekte werden aber nicht explizit erwähnt. Auch in der fachdidaktischen Literatur finden sich derzeit nur wenige Beiträge zu diesem Kernthema der Mathematik.

Diese Tatsache hängt vermutlich mit dem Scheitern der unter dem Schlagwort Neue Mathematik geführten strukturmathematischen Reformversuche in den 1960er und 1970er Jahren zusammen, im Rahmen derer auch axiomatisch-deduktive Darstellungen schulmathematischer Themengebiete angestrebt wurden. Mittlerweile dominiert eine gewisse Reserviertheit gegenüber der expliziten unterrichtlichen Auseinandersetzung mit axiomatischen Fragestellungen. Es besteht die Befürchtung, zu viel Axiomatik im Schulunterricht lasse die Mathematik als starres, fertig gegebenes Theoriegebäude erscheinen, wo doch eigentlich die Genese mathematischer Konzepte und Beweise einen verständnisorientierten Einblick in diese Disziplin erst ermögliche. Allerdings betonen Vollrath und Roth (2012, S. 26):

Angesichts der großen Bedeutung der Axiomatik in der Mathematik [...] ist es unbefriedigend, dass die Mehrzahl der Abiturientinnen und Abiturienten nie etwas von Axiomatik gehört hat. 
Die Hauptziele dieser Arbeit bestehen darin, dem interessierten Leser einen Gesamtüberblick über die Bedeutung der Axiomatik in der Mathematik zu geben und Lehrkräften eine didaktische Grundlage für eine eigene reflektierte Entscheidung zur Verfügung zu stellen, ob und in welchem Maße sie axiomatische Themen mit Schülerinnen und Schülern behandeln wollen. Schließlich sollen konkrete Möglichkeiten aufgezeigt werden, axiomatische Denk- und Arbeitsweisen zum Gegenstand von Lernumgebungen zu machen. Um diese Ziele zu erreichen, werden

- wesentliche Eigenschaften der axiomatischen Methode aus mathematikhistorischer und -philosophischer Sicht beleuchtet;

- die Argumente für und wider eine Behandlung axiomatischer Themen im Mathematikunterricht eruiert und aus heutiger didaktischer Sicht bewertet;

- übergeordnete Lernziele und didaktische Leitlinien für eine abgerundete Behandlung von Axiomatik auf Oberstufenniveau formuliert;

- gelungene Unterrichtskonzepte zu ausgewählten axiomatischen Gesichtspunkten vorgestellt;

- ein eigenes Konzept inkl. umfangreicher Materialien zur Behandlung axiomatischer Themen zur Verfügung gestellt, welches für den Einsatz in Zusatzkursen der Sekundarstufe II gedacht ist.

Die laut den Lehrplänen im Mathematikunterricht behandelten Inhalte bieten sinnvolle Anknüpfungspunkte, um sich ausgehend von den Schülerinnen und Schülern bekannten Themengebieten mit wesentlichen Elementen der Natur mathematischer Erkenntnis deutlicher als bislang üblich zu beschäftigen und insofern die Art und Weise, wie in der Mathematik Aussagen gesichert werden, explizit zu diskutieren. Jahnke und Ufer (2015, S. 351) kommen zu dem Ergebnis:

Die Tatsache, dass aus guten pädagogischen Gründen der axiomatische Aufbau der Mathematik in der Schule nicht thematisiert wird, hat [...] für viele Schülerinnen und Schüler die negative Folge, dass sie auch am Ende der Sekundarstufe II keine Vorstellung davon haben, welche Geltungsansprüche die Mathematik stellen kann, und in welchem Sinne in der Mathematik Allgemeinaussagen bewiesen werden.

Eine Thematisierung des Gebäudes Mathematik kann und sollte sowohl den Prozess des Errichtens dieses Gebäudes beleuchten als auch eine Wertschätzung für das fertige Endprodukt und dessen Stabilität vermitteln. 


\subsection{Kapitelübersicht und Zielgruppe}

Die Arbeit ist in drei große Bereiche unterteilt. Zunächst wird das Thema Axiomatik aus rein fachlicher Sicht beleuchtet (Kapitel 2). Daraufhin werden wesentliche fachdidaktische Aspekte im Hinblick auf die unterrichtliche Behandlung axiomatischer Denk- und Arbeitsweisen thematisiert (Kapitel 3). Abschließend wird eine unterrichtspraktische Perspektive eingenommen. Dabei werden zunächst bereits existierende und aus meiner Sicht gelungene Unterrichtsbeispiele (Kapitel 4) und im Anschluss drei von mir konzipierte und erprobte Unterrichtsreihen vorgestellt und didaktisch kommentiert (Kapitel 5).

Kapitel 2 beginnt mit der historischen Entwicklung der axiomatischen Methode am Beispiel der Geometrie (Abschnitt 2.1). Wesentliche Etappen sind die erstmals in Euklids Elementen vorgenommene deduktive Strukturierung eines mathematischen Themengebiets, die im Kontext der Diskussion um die Unabhängigkeit des Parallelenpostulats entdeckte nichteuklidische Geometrie sowie die Begründung der modernen formalistischen Auffassung mathematischer Aussagen. Die historische Übersicht wird durch eine Darstellung der zwei im Hinblick auf Axiomatik wesentlichen Entwicklungen des 20. Jahrhunderts abgeschlossen: der Entstehung der Strukturmathematik rund um die BourbakiGruppe und Kurt Gödels Erkenntnis, dass eine rein formalistische Absicherung der gesamten Mathematik nicht möglich ist (Abschnitt 2.2). Diese Entwicklungen werden anschließend aus mathematikphilosophischer Sicht beleuchtet; denn der Axiomen zugesprochene ontische und epistemische Status hat sich mit den Jahrhunderten gewandelt, wodurch sich auch der mit mathematischen Aussagen verbundene Wahrheitsanspruch änderte (Abschnitt 2.3).

In Kapitel 3 wird das Thema Axiomatik aus mathematikdidaktischer Perspektive beleuchtet. Da es im Rahmen der Reformbestrebungen der Neuen Mathematik in den 1960er und 1970er Jahren bereits umfassende Versuche gab, axiomatische Themen im Mathematikunterricht zu verankern, erfolgt zunächst eine Auseinandersetzung mit den damaligen Ideen und möglichen Schwächen der als gescheitert geltenden Reform (Abschnitte 3.1 bis 3.3). Darauf aufbauend werden Anknüpfungspunkte an das in heutigen Lehrplänen und Bildungsstandards ausgewiesene Kompetenzfeld Mathematisches Argumentieren aufgezeigt (Abschnitt 3.4).

Konkrete Möglichkeiten für die Behandlung axiomatischer Gesichtspunkte sind Gegenstand von Kapitel 4. Zunächst werden sechs übergeordnete Lernziele formuliert, welche die wesentlichen Aspekte der axiomatischen Methode abdecken und als Orientierung für 
die didaktische Kommentierung von vorhandenen sowie für die Konzeption von neuen Unterrichtsmaterialien dienen (Abschnitt 4.1). In Abschnitt 4.2 werden vier bereits existierende, ausgiebig erprobte und aus meiner Sicht besonders gelungene Unterrichtsideen vorgestellt und didaktisch beleuchtet. Im abschließenden Abschnitt 4.3 werden diese Unterrichtskonzepte mit den formulierten Lernzielen abgeglichen, um weiteren Handlungsbedarf aufzuzeigen und meine selbst entwickelten Unterrichtsideen genauer einzuordnen.

Im Zentrum von Kapitel 5 stehen drei von mir entwickelte Unterrichtsreihen für Zusatzkurse in der Sekundarstufe II (Abschnitte 5.2 bis 5.4), denen ein Abschnitt zu allgemeinen didaktischen Grundsatzentscheidungen vorangestellt ist (Abschnitt 5.1). Die zu den Unterrichtsreihen gehörenden Arbeitsblätter und Textauszüge einschließlich zugehöriger Lösungen und Kommentare sind in Anhang A zu finden. Erfahrungen aus der Umsetzung der Unterrichtskonzepte mit Schülerinnen und Schülern im Rahmen einer Workshop-Woche an der RWTH Aachen schließen das Kapitel ab (Abschnitt 5.5).

Zielgruppe sind in erster Linie Lehrerinnen und Lehrer sowie Referendarinnen, Referendare und Lehramtsstudierende, die sich tiefergehend und bewusst auf einer Meta-Ebene mit der axiomatischen Struktur der Mathematik beschäftigen möchten und nach Ideen suchen, dieses Thema im Oberstufenunterricht oder im Rahmen von Zusatzkursen zu behandeln. Zugleich stellt die Arbeit eine geeignete Informationsquelle für all diejenigen dar, die sich privat ein abgerundetes Bild der mathematischen Axiomatik aus heutiger Sicht aneignen möchten.

Die Lektüre setzt eine grundlegende Vertrautheit mit der formal-abstrakten Darstellung mathematischer Inhalte voraus, wie man sie in der Regel nach einigen Semestern eines Mathematikstudiums erworben hat. Ferner sind Kenntnisse aus Einführungsveranstaltungen oder entsprechenden Lehrbüchern zur ebenen Geometrie, Stochastik und zu Zahlbereichserweiterungen aus Lehrersicht notwendig für eine kompetente Nutzung der in Kapitel 5 vorgestellten Unterrichtsmaterialien, da die mathematischen Hintergründe dieser Themengebiete nicht detailliert dargelegt werden.

Aus der Arbeit am hier behandelten Thema ist 2016 eine Veröffentlichung hervorgegangen (Hock, Heitzer und Schwank 2016). Vereinzelt wurden Gedanken oder wenige Zeilen umfassende Textpassagen in diese Dissertationsschrift übernommen. Dies betrifft im Wesentlichen die Auseinandersetzung mit der Neuen Mathematik (Abschnitte 3.3 und 3.5), die Lernzielformulierung (Abschnitt 4.1) sowie die Beschreibung der Unterrichtsreihe zur Wahrscheinlichkeitsrechnung (Abschnitt 5.3). 



\section{Axiomatik in der Mathematik}

In diesem Kapitel wird die Bedeutung der Axiomatik in der Mathematik insbesondere aus historischer und philosophischer Perspektive beleuchtet. Ein axiomatischer Aufbau mathematischer Sachverhalte etablierte sich zuerst in der Geometrie. Auch die wesentlichen ontologischen und epistemologischen Umbrüche $^{1}$ in der axiomatischen Sicht auf Mathematik erfolgten allesamt im Rahmen von Auseinandersetzungen mit geometrischen Fragestellungen. Die Schilderung der Entwicklung der axiomatischen Methode beschränkt sich in dieser Arbeit daher weitestgehend auf die Geometrie. Die drei wesentlichen Etappen, die dabei zu beachten sind, werden in Abschnitt 2.1 beschrieben. Da der Fokus auf den mit der Entwicklung der axiomatischen Methode verbundenen Auffassungswechseln über die Rolle von Axiomen in der Mathematik liegt, wird in diesem Kapitel nicht ausführlich Geometrie betrieben. Wer sich für die eigentliche deduktive Darstellung der euklidischen und nichteuklidischen Geometrien inkl. zugehöriger Beweise interessiert, sei auf die einschlägige Literatur verwiesen (beispielsweise Filler 1993 oder Klotzek 2001).

Als Ergänzung der Ausführungen zur Geometrie werden in Abschnitt 2.2 zwei im Hinblick auf die Axiomatik wesentliche Entwicklungen des 20. Jahrhunderts geschildert: die Entstehung der Strukturmathematik rund um die Bourbaki-Gruppe und Kurt Gödels Erkenntnis, dass eine rein formalistische Absicherung der gesamten Mathematik nicht möglich ist. Die in den historischen Beschreibungen bereits aufleuchtenden mathematikphilosophischen Ansichten werden in Abschnitt 2.3 gebündelt und intensiver vorgestellt. Der zusammenfassende Abschnitt 2.4 ordnet abschließend die unterschiedlichen Auffassungen und Zielsetzungen axiomatischer Arbeits- und Denkweisen in einer Typologie.

\footnotetext{
${ }^{1}$ Die Ontologie (wörtlich: Lehre vom Sein) befasst sich unter anderem mit Fragen der Existenz und Realität von Objekten, während die Epistemologie (Erkenntnistheorie) sich mit den Bedingungen für Wissen sowie dessen Genese und Begründung beschäftigt.
} 


\subsection{Axiomatik in der Geometrie}

\subsubsection{Euklids Elemente}

Schönbeck (2003, S. 5) stellt in seiner Euklid-Biographie fest: „Das Leben des Euklid ist uns so verborgen wie jenes von Homer, dem ältesten epischen Dichter des Abendlandes; von beiden besitzen wir kaum mehr als ihre Werke - wenn es denn ihre Werke sind. Über ,Euklid aus Alexandria', den alle Mathematiker der Welt kennen, ist nichts uns wirklich mit Bestimmtheit bekannt". Er lebte vermutlich etwa im 3. Jahrhundert v. Chr. in Alexandria und hat in seinem Hauptwerk Die Elemente das bis dato bekannte mathematische Wissen zusammengetragen und erstmals in eine axiomatisch-deduktive Struktur gebracht. Die Elemente zählen zu den einflussreichsten Büchern der Menschheitsgeschichte und haben die Mathematik als Wissenschaft über mehr als zwei Jahrtausende geprägt.

Sie bestehen aus insgesamt 13 Büchern (Kapitel nach heutiger Auffassung), über deren Inhalte Tabelle 2.1 einen groben Überblick gibt. Die Betrachtungen in dieser Arbeit beschränken sich auf das erste Buch, da in diesem als einzigem neben Definitionen auch Axiome und Postulate aufgestellt werden. Im Folgenden werden die wesentlichen in den Elementen verwendeten meta-mathematischen Begriffe (Definitionen, Postulate, Axiome, Propositionen, u. a.) sowie der prinzipielle deduktive Aufbau der euklidischen Geometrie vorgestellt. Von den 23 Definitionen, 5 Postulaten und 9 Axiomen, die zu Beginn von Buch I angeführt werden, sei folgende Auswahl zitiert (Herv. im Orig.): ${ }^{2}$

\begin{tabular}{|c|l|}
\hline Buch & Inhalt \\
\hline \hline I & Ebene Geometrie bis zur Satzgruppe des Pythagoras \\
II & Geometrische Algebra \\
III & Kreisgeometrie (u. a. Satz des Thales) \\
IV & In- und Umkreise regelmäßiger Vielecke \\
V & Proportionenlehre \\
VI & Ähnlichkeitslehre \\
VII-IX & Arithmetik und Zahlentheorie (z. B. Euklidischer Algorithmus \\
& oder Existenz unendlich vieler Primzahlen) \\
X & Inkommensurabilität \\
XI-XIII & Raumgeometrie bis zur Konstruktion der fünf Platonischen Körper \\
& (und dem Nachweis, dass es keine weiteren gibt) \\
\hline
\end{tabular}

Tabelle 2.1: Die 13 Bücher der Elemente

\footnotetext{
${ }^{2}$ Alle Auszüge aus den Elementen sind der deutschen Übersetzung von Clemens Thaer (Euklid 2010) entnommen.
} 


\section{DEFINITIONEN:}

1. Ein Punkt ist, was keine Teile hat,

2. Eine Linie breitenlose Länge.

4. Eine gerade Linie (Strecke) ist eine solche, die zu den Punkten auf ihr gleichmäßig liegt.

10. Wenn eine gerade Linie, auf eine gerade Linie gestellt, einander gleiche Nebenwinkel bildet, dann ist jeder der beiden gleichen Winkel ein Rechter; und die stehende gerade Linie heißt senkrecht zu (Lot auf) der, auf der sie steht.

11. Stumpf ist ein Winkel, wenn er größer als ein Rechter ist,

15. Ein Kreis ist eine ebene, von einer einzigen Linie [die Umfang (Bogen) heißt] umfaßte Figur mit der Eigenschaft, daß alle von einem innerhalb der Figur gelegenen Punkte bis zur Linie [zum Umfang des Kreises] laufenden Strecken einander gleich sind;

20. Von den dreiseitigen Figuren ist ein gleichseitiges Dreieck jede mit drei gleichen Seiten,

23. Parallel sind gerade Linien, die in derselben Ebene liegen und dabei, wenn man sie nach beiden Seiten ins unendliche verlängert, auf keiner einander treffen.

Postulate:

Gefordert soll sein:

1. Daß man von jedem Punkt nach jedem Punkt die Strecke ziehen kann,

2. Daß man eine begrenzte gerade Linie zusammenhängend gerade verlängern kann,

3. Daß man mit jedem Mittelpunkt und Abstand den Kreis zeichnen kann,

4. Daß alle rechten Winkel einander gleich sind,

5. Und daß, wenn eine gerade Linie beim Schnitt mit zwei geraden Linien bewirkt, daß innen auf derselben Seite entstehende Winkel zusammen kleiner als zwei Rechte werden, dann die zwei geraden Linien bei Verlängerung ins unendliche sich treffen auf der Seite, auf der die Winkel liegen, die zusammen kleiner als zwei Rechte sind.

AXIOME:

1. Was demselben gleich ist, ist auch einander gleich.

2. Wenn Gleichem Gleiches hinzugefügt wird, sind die Ganzen gleich.

3. Wenn von Gleichem Gleiches weggenommen wird, sind die Reste gleich. 
7. Was einander deckt, ist einander gleich.

8. Das Ganze ist größer als der Teil.

Die Definitionen fallen in zwei Kategorien. Zum einen handelt es sich um die - auch modernen wissenschaftstheoretischen Maßstäben genügende - Erklärung bestimmter Begriffe mit Hilfe bereits bekannter Begriffe. Beispielsweise wird aufgrund von Definition 11 klar, was ein „stumpfer Winkel“ ist, wenn man bereits weiß, was ein „rechter Winkel“ ist und was es für einen Winkel bedeutet, ,größer“ als ein anderer zu sein. Solche Definitionen haben den Charakter von Abkürzungen, indem sie für teilweise lange sprachliche Ausdrücke kürzere festlegen (vgl. Radnitzky 1989, S. 27-28). Zum anderen befassen sich bestimmte Definitionen mit geometrischen Grundbegriffen und sind aus heutiger Sicht unbefriedigend (aus formalistischer Sicht sogar überflüssig, siehe Abschnitt 2.1.3), so zum Beispiel Euklids Charakterisierung eines Punktes als etwas, das keine Teile hat (Definition 1). Dieser Typ von Definitionen spielt für die Beweisführung in den Elementen jedoch keine Rolle (vgl. Scriba und Schreiber 2010, S. 51) und ist womöglich als Versuch zu betrachten, die Bedeutung der Grundbegriffe intuitiv zu erfassen.

Die Aufstellung von Ausgangssätzen und deren Unterteilung in Axiome und Postulate wird meist auf die methodologischen Ansichten des Aristoteles zurückgeführt, der sich vor allem in seiner Analytica posteriora umfassend zum wissenschaftlichen Beweisen äußert (vgl. Höffe 2009, S. 62-85). So beginnt er besagtes Werk mit der Feststellung: „Jede Unterweisung und jedes verständige Erwerben von Wissen entsteht aus bereits vorhandener Kenntnis“ (zit. n. Höffe 2009, S. 62). Für wissenschaftliches Beweisen bedeutet dies, dass eine zu beweisende Aussage auf bereits bekannte Aussagen zurückgeführt werden muss. Gleichzeitig ergibt sich daraus jedoch, dass gewisse Prinzipien unbewiesen an den Anfang einer Argumentation gestellt werden müssen, denn ,,sonst würde ja ein Fortschritt ins Unendliche eintreten und auch so kein Beweis stattfinden“ (zit. n. Höffe 2009, S. 241). Der wesentliche Unterschied zwischen Axiomen und Postulaten besteht nun darin, dass erstere als evidente Wahrheiten angesehen werden, die keines Beweises bedürfen, ${ }^{3}$ wohingegen die Postulate bloße Forderungen sind, die man akzeptieren kann oder auch nicht. Darüber hinaus werden bisweilen auch noch weitere Unterschiede zwischen den beiden Termini hervorgehoben, die zwar bis zu einem gewissen Grad plausibel, bei genauerer Betrachtung jedoch nicht unproblematisch sind:

1. Axiome besitzen wissenschaftsübergreifende Gültigkeit, wohingegen die Postulate theoriespezifisch, in Euklids Fall also nur auf die Geometrie bezogen sind. Eine sol-

\footnotetext{
${ }^{3}$ Man vergleiche dazu auch den Euklid-Kommentar des Proklos (5. Jh. n. Chr.): „, [A]xioms take for granted things that are immediately evident to our knowledge and easily grasped by our untaught understandings“"(Proklos 1970, S. 140).
} 
che terminologische Unterscheidung findet sich unter anderem bei Aristoteles, der als Beispiel für ein Axiom den auch bei Euklid in ähnlicher Formulierung als Axiom auftretenden Satz ,Wenn man Gleiches von Gleichem abzieht, [ist] das Übrige gleich“" nennt (zit. n. Höffe 2009, S. 71). ${ }^{4}$ Gegen diese Unterscheidung könnte man jedoch einwenden, dass Euklids siebtes Axiom („Was einander deckt, ist einander gleich.") eindeutig planimetrischer Natur und somit theoriespezifisch ist (siehe auch Szabó 1960, S. 71).

2. Axiome und Postulate unterscheiden sich darin, dass letztere die Ausführbarkeit geometrischer Konstruktionen fordern. Diese Erklärung findet man in ähnlicher Weise bei Proklos (1970, S. 140-143). Hierbei treten aber Schwierigkeiten mit Euklids Postulaten 4 und 5 auf, da diese offensichtlich keine Konstruktionen beschreiben. Während man dieses Problem im Fall von Postulat 5 noch umgehen kann, indem man dessen Aussage so deutet, dass hier die Konstruierbarkeit eines Schnittpunktes zweier Geraden unter gewissen Voraussetzungen angenommen wird (siehe auch Schreiber 2010b, S. 54-55), so scheint das vierte Postulat aus der Reihe zu fallen, weshalb Proklos auch vorschlägt, es unter die selbstevidenten Axiome zu fassen (vgl. Proklos 1970, S. 147).

Die Unterscheidung der Begriffe Axiom und Postulat ist in der nacheuklidischen Zeit immer weniger beachtet worden, so dass mitunter auch die ursprünglich als Postulate formulierten Aussagen als unzweifelhaft wahr betrachtet wurden (vgl. Kline 1972, S. 59, Scriba und Schreiber 2010, S. 55-56 und Schreiber 2010b, S. 3-5). Um den hypothetischen Status der Postulate besser nachvollziehen zu können, ist es hilfreich, sie vor dem Hintergrund ihres (oben bereits erwähnten) konstruktiven Charakters zu betrachten (siehe auch Schreiber 2010a, S. ix-x, Hartshorne 2010, S. 18-20 und Scriba und Schreiber 2010, S. 53-54): Aus moderner Sicht werden die geometrischen Ausgangssätze als Existenzaussagen aufgefasst (z. B.: Zu zwei verschiedenen Punkten existiert (genau) eine Gerade, die durch diese Punkte verläuft.); ursprünglich forderten sie jedoch die Ausführbarkeit bestimmter geometrischer Operationen (abgesehen vom vierten Postulat). Beachtet man zusätzlich, dass zu Euklids Zeiten Geometrie ausschließlich mit der Vorstellung begrenzter Geraden-

\footnotetext{
${ }^{4}$ In der Literatur wird häufig auf die Gemeinsamkeiten zwischen Euklid und Aristoteles hingewiesen, beispielsweise in Heath (2013, S. 117-124), Bedürftig und Murawski (2012, S. 36-42) oder Scriba und Schreiber (2010, S. 50-51). In der Tat lassen sich einige von Aristoteles' Ausführungen sehr gut an Euklids Elementen illustrieren. Von einer prinzipiellen terminologischen Übereinstimmung kann aber nicht die Rede sein. Aristoteles verwendet eine Vielzahl von Begriffen (Prinzipien, Festsetzung, These, Hypothese, Forderung, Postulat, Axiom, Definition usw.), deren epistemischer Status und Abgrenzung untereinander nicht immer klar werden, was einen Vergleich mit Euklid deutlich erschwert. Szabó (1978, S. 230-231) geht sogar noch weiter: „Aristotle's views about axiomatics and the terminology which goes along with them simply cannot be applied to Euclid, unless they are first subjected to an arbitrary reinterpretation".
} 
stücke betrieben wurde, wird deutlich, dass die Gültigkeit der Postulate keinesfalls trivial ist und mehr umfasst als eine reine Existenzaussage. So könnte beispielsweise durchaus angezweifelt werden, dass die im fünften Postulat beschriebene sukzessive Verlängerung der beiden Geradenstücke in einer endlichen (aber unbekannten) Anzahl von Schritten einen Schnittpunkt liefert.

Dieser konstruktive Ansatz zeigt sich auch in den Propositionen, die in den Elementen bewiesen werden. Diese sind in Lehrsätze und (Konstruktions-)Aufgaben unterteilt, wobei die Lehrsätze im Wesentlichen bestimmte Eigenschaften geometrischer Figuren behandeln (z. B. Proposition 6: „Wenn in einem Dreieck zwei Winkel einander gleich sind, müssen auch die den gleichen Winkeln gegenüberliegenden Seiten einander gleich sein“.). $\mathrm{Zu}$ den Aufgaben wird stets ein Lösungsverfahren (in modernen Worten: ein Konstruktionsalgorithmus; siehe auch Schreiber 2010b, S. 3-5 und Scriba und Schreiber 2010, S. 52-53) angegeben und bewiesen, dass das beschriebene Verfahren das Gewünschte leistet. Dies sei exemplarisch an Proposition 1 verdeutlicht (der Beweis wurde sprachlich leicht angepasst, sämtliche von Euklids Argumentationsschritten sind jedoch enthalten):

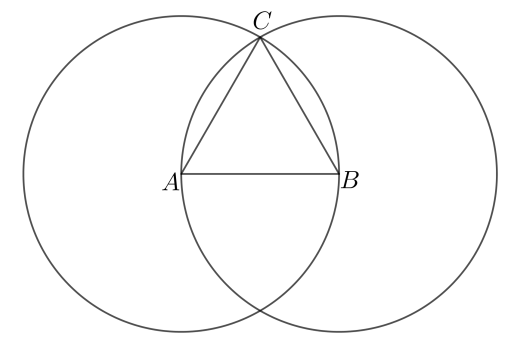

[Es ist möglich,] über einer gegebenen Strecke ein gleichseitiges Dreieck zu errichten.

Beweis: Die gegebene Strecke sei $A B$. Man zeichne nun den Kreis mit Mittelpunkt $A$ und Radius $A B$ (Post. 3), ebenso den Kreis mit Mittelpunkt $B$ und Radius $B A$ (Post. 3). Die beiden Kreise schneiden sich in einem Punkt $C$. Man ziehe nun die Strecken $C A$ und $C B$ (Post. 1). Da $C$ und $B$ auf dem Kreis mit Mittelpunkt $A$ liegen, gilt $A C=A B$ (Def. 15); da $C$ und $A$ auf dem Kreis mit Mittelpunkt $B$ liegen, gilt $B C=B A$ (Def. 15). Insgesamt ist also $C A=A B$ und $C B=A B$, woraus $C A=C B$ folgt (Ax. 1). Also sind $A B, B C$ und $C A$ alle einander gleich und das Dreieck ist somit gleichseitig und über der Strecke $A B$ errichtet.

Hier wird nicht nur die Existenz eines gleichseitigen Dreiecks - welches in Definition 20 zwar beschrieben, aber nicht als existent angenommen wird - bewiesen, sondern zugleich ein konstruktives Verfahren zu dessen Erzeugung mit Zirkel und Lineal angegeben.

An diesem Beweis wird außerdem häufig illustriert, dass es Euklid trotz aller Bemühungen, nach Aufstellung der Postulate und Axiome ohne Appell an die Intuition oder 
Anschauung auszukommen und ausschließlich logisch-deduktiv zu argumentieren, nicht gelingt, sämtliche verwendeten Annahmen zu explizieren. So ist die Tatsache, dass die beiden konstruierten Kreise (mindestens) einen Schnittpunkt besitzen, zwar grundlegend für den Beweis, sie wird aber nicht vorher bewiesen oder als Postulat aufgeführt (siehe auch Heath 2013, S. 242-243). Auch wenn diese Kritik aus moderner Sicht einleuchtend und berechtigt ist, so setzt sie doch voraus, dass die vollständige Offenlegung aller verwendeten Annahmen und Prinzipien beim mathematischen Beweisen Euklids primäres Anliegen war. Dies ist unter Historiken jedoch durchaus umstritten (vgl. Scriba und Schreiber 2010, S. 51). Es ist auch möglich, „dass Euklids Hauptabsicht war, die von den Ägyptern und Babyloniern überlieferte mathematische Kultur der Angabe von Lösungsverfahren für Aufgabentypen durch die argumentative Rechtfertigung der angegebenen Verfahren zu ergänzen, wobei er zwangsläufig erste Fragmente von deduktiven Theorien schuf“" (Schreiber 2010a, S. x).

Dennoch ist auffällig, dass Euklid in seinen Ausführungen ,weder auf die materielle Welt noch auf irgendwelche Anwendungen explizit Bezug nimmt“" (Scriba und Schreiber 2010, S. 50), wenngleich er die Geometrie nicht von ihrem anschaulichen Bezug löst. Darin spiegeln sich implizit die philosophischen Ansichten Platons, gemäß derer ,der eigentliche Zweck dieser ganzen Wissenschaft [der Geometrie, T.H.] nichts anderes als die reine Erkenntnis“ ist (Platon 2010, S. 324). Mathematische Objekte haben laut seiner Ideenlehre eine vom menschlichen Denken unabhängige Existenz außerhalb von Raum und Zeit und damit auch außerhalb der materiellen, sinnlich wahrnehmbaren Welt (vgl. Bedürftig und Murawski 2012, S. 33-35). Ähnlich fasst Euklid Objekte wie Punkte und Geraden als Idealisierungen der physischen Welt auf, mit denen geometrische Probleme nicht mehr nur approximativ, sondern exakt gelöst werden können (vgl. Hartshorne 2010, S. 9-10). Diese Loslösung von empirischen Gesichtspunkten und der Sinneswahrnehmung war der erste Schritt, mit dem sich die Geometrie zu einer theoretischen Wissenschaft entwickelte.

Um einen Eindruck davon zu vermitteln, wie elaboriert die deduktive Struktur der Elemente ist, habe ich in Abbildung 2.1 auf Seite 15 die Herleitung des Innenwinkelsatzes (Proposition 32$)^{5}$ aus den Postulaten und Axiomen analysiert und veranschaulicht. Die Abbildung zeigt Proposition 32 an oberster Stelle. Von denjenigen Propositionen (und Axiomen), die Euklid in dem zugehörigen Beweis verwendetet $\left(A_{1}, A_{2}, 13,29\right.$ und 31), zeigen Pfeile auf Proposition 32. In analoger Weise zeigen Pfeile auf diese Propositionen, die wiederum von denjenigen Propositionen ausgehen, die in deren Beweisen jeweils benötigt werden und so weiter. Insgesamt lässt sich somit Euklids vollständiges

\footnotetext{
${ }^{5}$ Proposition 32 beinhaltet neben dem Innenwinkel- auch den Außenwinkelsatz: „An jedem Dreieck ist der bei Verlängerung einer Seite entstehende Außenwinkel den beiden gegenüberliegenden Innenwinkeln zusammen gleich, und die drei Winkel innerhalb des Dreiecks sind zusammen zwei Rechten gleich“.
} 
Beweisschema visualisieren, dessen Ausgangspunkte die gelb markierten Postulate und Axiome sind. Die Anordnung der Aussagen wurde so gewählt, dass alle Pfeile möglichst gut sichtbar sind. Um die Abbildung nicht zu unübersichtlich zu machen, werden die Propositionen, in deren Beweis Postulat 1 direkt verwendet wird, unten separat aufgeführt. Ähnliche Veranschaulichungen für drei andere Sätze aus den Elementen sind bei Gerwig (2015, S. 379-381) zu finden.

Abschließend möchte ich auf die Arbeiten Árpád Szabós (Szabó 1960 und Szabó 1978) eingehen, der ausführlich die Etymologie der bei Euklid verwendeten Termini für die Definitionen, Postulate und Axiome untersucht hat. ${ }^{6}$ Seine wesentliche Erkenntnis ist, dass die von Euklid verwendeten Begriffe ursprünglich der vorplatonischen Dialektik ${ }^{7}$ entstammen, deren zentraler Grundgedanke es ist, dass sich Dialogpartner vor Beginn einer argumentativen Auseinandersetzung zunächst auf eine Definition des zu diskutierenden Themenbereichs einigen sowie gewisse Ausgangsaussagen akzeptieren müssen. Insbesondere das Wort $\alpha \xi \iota \omega \mu \alpha$ (axioma) wurde in der Dialektik bisweilen in der Bedeutung „Annahme, Forderung“ gebraucht, deren Zustimmung durch den Gesprächspartner auch in der Schwebe gelassen werden konnte (vgl. Szabó 1960, S. 69). ${ }^{8}$ Beispielsweise wurden die in den Elementen als Axiome bezeichneten Aussagen, die im Wesentlichen Eigenschaften der Gleichheitsrelation beschreiben, in der eleatischen Philosophie vor Platon vor allem durch die bekannten Paradoxien des Zenon - durchaus angezweifelt (vgl. Szabó 1960, S. 77). Die Auffassung von Axiomen als unbezweifelbaren Wahrheiten scheint sich erst seit Aristoteles durchgesetzt zu haben. ${ }^{9}$ Der dialektische Ursprung mathematischer Argumentation verdeutlicht ihren hypothetischen Charakter. Mathematische Aussagen beruhen auf Voraussetzungen bzw. Hypothesen, die man akzeptieren kann, aber nicht muss. Von Bedeutung und Interesse sind die Konsequenzen, die man aus den Voraussetzungen ziehen kann. Hält man die Ausgangshypothesen für besonders überzeugend, muss man auch an die sich aus ihnen ergebenden Folgerungen glauben. Steht hingegen die Gültigkeit der zu Beginn aufgestellten Annahmen zur Debatte, so können die Folgerungen zu deren Stärkung - z. B. im Falle einer Passung mit empirischen Messungen, Sinneswahr-

\footnotetext{
${ }^{6}$ Eine Zusammenfassung findet sich u. a. bei Jahnke (2007a, S. 10-13) und Jahnke (2010, S. 17-21).

${ }^{7}$ Kunst der Gesprächsführung; die Griechen verstanden darunter vor allem die Diskussion kontroverser Themen.

${ }^{8}$ Interessant ist der hierbei auftretende Zusammenhang zu den Postulaten der Elemente, welche bezeichnenderweise stets mit den Worten „Gefordert soll sein“ eingeleitet werden.

${ }^{9}$ Auffällig ist auch, dass der Begriff $\alpha \xi \iota \omega \mu \alpha$ in dem überlieferten Euklid-Manuskript gar nicht auftaucht. Stattdessen steht dort Kotv $\alpha \mathbf{l}$ Evvor $\alpha \mathbf{l}$ (koinai ennoiai), die ,allen Menschen gemeinsamen Vorstellungen“ (in englischen Ausgaben der Elemente findet man auch meistens den Ausdruck „common notions“ anstelle von ,,axioms“). Szabó vermutet, dass Proklos, der wiederum von Axiomen redet, womöglich ein älteres Euklid-Manuskript vorgelegen hat und dass die $\alpha \xi i \omega \mu \alpha \tau \alpha$ in späteren Versionen der Elemente durch den Ausdruck Kolv $\alpha$ l $\varepsilon v v o r \alpha$ ersetzt wurden, um den epistemischen Status der Ausgangssätze bewusst von dem einer bloßen Forderung oder Annahme abzugrenzen (vgl. Szabó 1960, S. 40-41).
} 


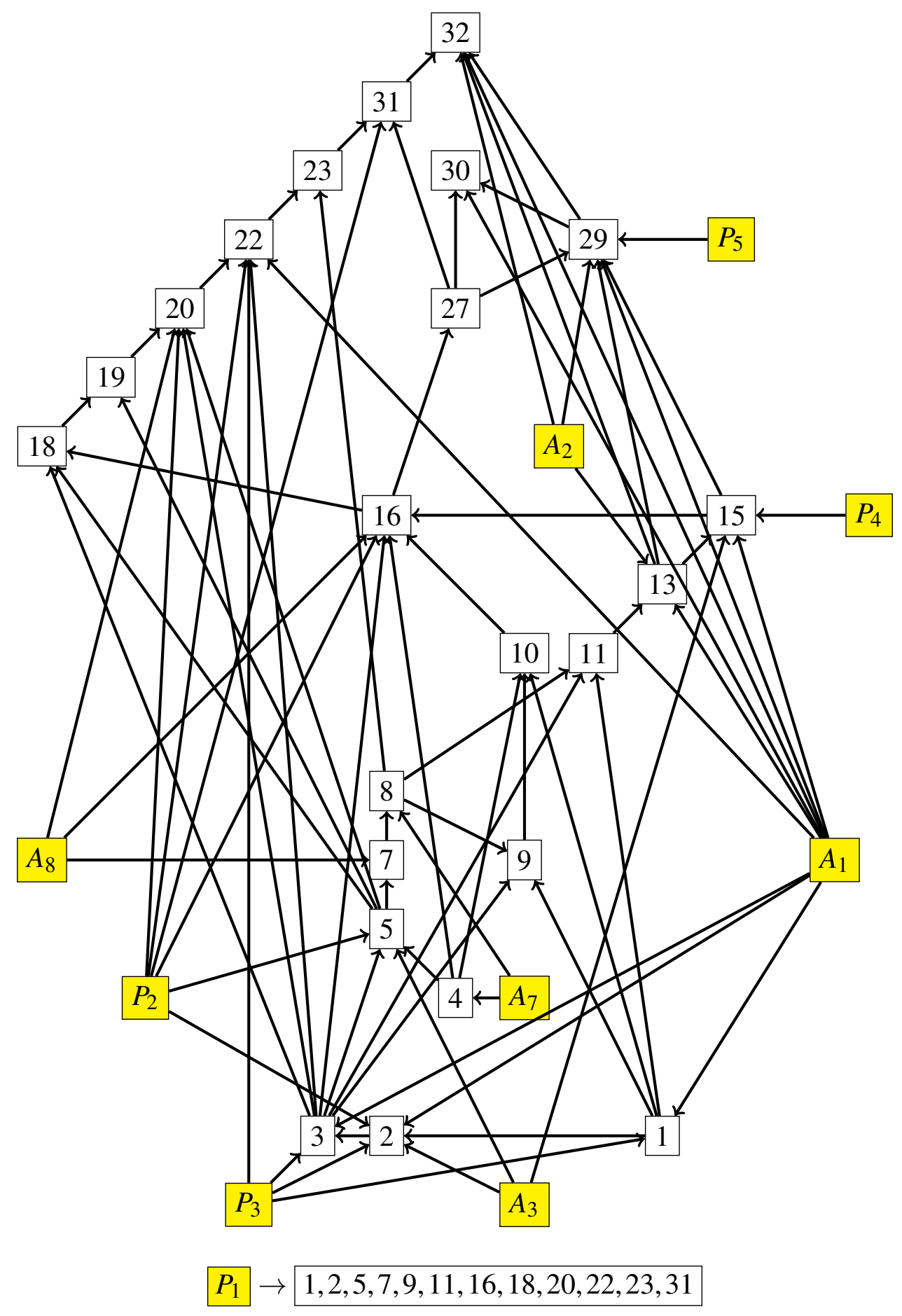

Abbildung 2.1: Deduktionsschema zum Innenwinkelsatz 
nehmungen oder sonstigen menschlichen Erfahrungen - oder auch Ablehnung führen, wenn sich beispielsweise ein logischer Widerspruch ergibt (vgl. Szabó 1960, S. 52). ${ }^{10}$

Zusammenfassend kann man sagen, dass Euklid mit seinen Elementen wie kein anderer das Außenbild und das wissenschaftliche Selbstverständnis der Mathematik geprägt hat. Die Auffassung von Mathematik als einer Disziplin, die aus evidenten Grundaussagen streng logisch absolute Wahrheiten generiert, hatte lange Zeit Bestand und wird auch heute teilweise noch vertreten. Lakatos (1982b, S. 4) fasst diese Sichtweise wie folgt zusammen:

Ich nenne ein deduktives System eine ,Euklidische Theorie ${ }^{6}$, wenn die Aussagen an der Spitze (die Axiome) aus völlig bekannten Ausdrücken (Grundausdrücken) bestehen, und wenn es hier unfehlbare Wahrheitswertsetzungen des Wahrheitswerts ,wahr' gibt, der durch die deduktiven Kanäle der Wahrheitsübertragung (Beweise) hinabfließt und das ganze System durchdringt. (Herv. im Orig.)

Szabós Ausführungen zeigen jedoch, dass der mit dem Terminus Euklidische Theorie beschriebene Standpunkt nicht nur aus einer modernen Perspektive betrachtet, sondern bereits mit Blick auf die Anfangszeit axiomatischer Theoriedarstellungen relativiert werden muss.

\subsubsection{Nichteuklidische Geometrie}

Wie im letzten Abschnitt bereits angedeutet, wurden die geometrischen Axiome ${ }^{11}$ sowie die daraus deduzierbaren Sätze in der Zeit nach Euklid als unbezweifelbare Wahrheiten betrachtet. Nicht umsonst wurden andere mathematische Gebiete lange Zeit überwiegend in geometrischer Sprache dargestellt (wie beispielsweise die Arithmetik und Zahlentheorie bei Euklid in den Büchern VI bis IX der Elemente). Durch die Eingliederung in die Geometrie beabsichtigte man eine klare Grundlegung der anderen mathematischen Gebiete und somit die Sicherung ihrer uneingeschränkten Gültigkeit (vgl. Kline 1972, S. 861862). Zudem war man davon überzeugt, dass die Aussagen der euklidischen Geometrie den real existierenden physikalischen Raum beschreiben, wenn auch in idealisierter Form. Erst im Laufe des 19. Jahrhunderts gelangte man zu der Einsicht, dass geometrische Theorien, die teilweise andere Axiome als Ausgangsbasis nehmen, nicht nur logisch konsis-

\footnotetext{
${ }^{10}$ Diese dialektische Vorgehensweise der Hypothesenüberprüfung anhand ihrer Konsequenzen ist eng verwurzelt mit der Methode der indirekten Beweisführung, bei der man aus einer für falsch gehaltenen Hypothese einen logischen Widerspruch erzeugt, um die gegenteilige Aussage zu beweisen.

${ }^{11}$ Der Einfachheit halber und im Einklang mit der nacheuklidischen Auffassung von Geometrie werden die Begriffe Axiom und Postulat im Folgenden als gleichbedeutend betrachtet und verwendet.
} 
tent sein, sondern auch zur Beschreibung von Phänomenen des uns umgebenden Raumes dienen können. Die Denknotwendigkeit des euklidischen Raumes ${ }^{12}$ und damit einhergehend die klassische Auffassung von der Wahrheit mathematischer Aussagen waren damit hinfällig. Ausgangspunkt dieser langwierigen und komplexen Entwicklung war die Auseinandersetzung mit Euklids fünftem Postulat:

Gefordert soll sein [...] daß, wenn eine gerade Linie beim Schnitt mit zwei geraden Linien bewirkt, daß innen auf derselben Seite entstehende Winkel zusammen kleiner als zwei Rechte werden, dann die zwei geraden Linien bei Verlängerung ins unendliche sich treffen auf der Seite, auf der die Winkel liegen, die zusammen kleiner als zwei Rechte sind.

Trotz der Überzeugung vom absoluten Geltungsanspruch der euklidischen Geometrie bereitete dieses Postulat den Mathematikern Kopfzerbrechen. Für die eingehende Beschäftigung mit dieser - auch Parallelenpostulat ${ }^{13}$ genannten - Aussage sind verschiedene Gründe vorstellbar (siehe auch Klotzek 2001, S. 188):

- Das Parallelenpostulat ist im Vergleich zu den übrigen Postulaten und Axiomen deutlich länger und komplizierter formuliert.

- Euklid selbst scheint bemüht, das Parallelenpostulat erst so spät wie möglich zu verwenden. Es wird zum ersten Mal beim Beweis von Proposition 29 benötigt. ${ }^{14}$

- Die Umkehrung des Parallelenpostulats wird von Euklid in Proposition 17 bewiesen: „In jedem Dreieck sind zwei Winkel, beliebig zusammengenommen, kleiner als zwei Rechte“.

Bereits Proklos merkt in seinem Kommentar zu den Elementen an: „This [das Parallelenpostulat, T.H.] ought to be struck from the postulates altogether. For it is a theorem [...] and requires for its demonstration a number of definitions as well as theorems" (Proklos 1970, S. 150). Er deduziert das Parallelenpostulat aus dem Theorem „Wenn eine gerade Linie eine von zwei Parallelen schneidet, so schneidet sie auch die andere“, allerdings unter Verwendung eines zusätzlichen Axioms (siehe Proklos 1970, S. 289-292). Die

\footnotetext{
${ }^{12}$ Man vergleiche dazu auch Kants Kritik der reinen Vernunft (1781), die in diesem Zusammenhang oft zitiert wird. Darin bezeichnet er die geometrischen Axiome als ,,synthetische Grundsätze a priori“ (Kant 1966, S. 749), d. h. der (anschaulichen und empirischen) Erfahrung vorausgehend und damit sonstige Erkenntnis überhaupt erst ermöglichend. Inwiefern die euklidische Geometrie damit aber als denknotwendig charakterisiert wird, ist in der Kant-Interpretation durchaus umstritten (siehe dazu beispielsweise Jürgen Josts Kommentar in Riemann 2013, S. 16-20).

${ }^{13}$ Diese Bezeichnung rührt daher, dass das Postulat äquivalent zur Forderung der Existenz genau einer Parallelen zu einer gegebenen Geraden ist (siehe weiter unten).

${ }^{14}$ „Beim Schnitt einer geraden Linie mit (zwei) parallelen geraden Linien werden (innere) Wechselwinkel einander gleich, jeder äußere Winkel wird dem innen gegenüberliegenden gleich, und innen auf derselben Seite entstehende Winkel werden zusammen zwei Rechten gleich“.
} 
zahlreichen Versuche, das Parallelenpostulat entweder direkt zu beweisen oder zumindest durch eine ,evidentere“ Aussage zu ersetzen, haben eine Reihe zu ihm äquivalenter Aussagen hervorgebracht. Diese waren zwar nicht allesamt neu und tauchen teilweise schon bei Euklid als Theoreme auf; insgesamt haben sie jedoch ,,viele wertvolle Erkenntnisse über die logischen Zusammenhänge zwischen Sachverhalten [geliefert], die eine zweidimensionale Geometrie als euklidisch charakterisieren“ (Schreiber 2010b, S. 6). Einige der wichtigsten Ergebnisse sind im Folgenden aufgelistet (die in Klammern angegebenen Namen bezeichnen dabei nicht unbedingt die Personen, die die jeweiligen Sätze zum ersten Mal in einem Beweisversuch des Parallelenpostulats verwendet haben, wohl aber die bekanntesten Vertreter): ${ }^{15}$

- Die Menge aller Punkte, die zu einer Geraden gleichen Abstand haben und auf der gleichen Seite derselben liegen, bildet wieder eine Gerade. (Christoph Clavius, 1537-1612)

- Zu jedem Dreieck gibt es ein ähnliches Dreieck beliebiger Größe (oder anders formuliert: Es gibt ähnliche, nicht kongruente Dreiecke). (John Wallis, 1616-1703)

- Zu einer gegebenen Geraden und einem Punkt außerhalb dieser Geraden gibt es genau eine Parallele, die durch diesen Punkt verläuft. (John Playfair, 1748-1819) ${ }^{16}$

- Die Winkelsumme in jedem Dreieck beträgt 180 . (Girolamo Saccheri, 1667-1733; Adrien-Marie Legendre, 1752-1833) ${ }^{17}$

- Durch drei beliebige nicht kollineare Punkte kann man einen Kreis ziehen. (Wolfgang von Bolyai, 1775-1856)

Insbesondere Saccheri leistete wichtige Vorarbeit auf dem Gebiet der später als nichteuklidisch bezeichneten - da auf der Verneinung des Parallelenpostulats beruhenden - Geometrie. Es gelang ihm zum einen, in seinem 1733 in lateinischer Sprache erschienenen Buch mit dem ambitionierten Titel Euclides ab omnio naevo vindicatus (,Der von jedem

\footnotetext{
${ }^{15}$ Genauere Informationen zu den zugehörigen Beweisideen (mitunter allerdings von einer vollständigeren axiomatischen Grundlage als bei Euklid ausgehend) finden sich unter anderem bei Hartshorne (2010, S. 296-326), Heath (2013, S. 204-220) und Klotzek (2001, S. 179-187).

${ }^{16}$ Diese Fassung ist die bekannteste Alternative zu Euklids Formulierung und verantwortlich dafür, dass sich die Bezeichnung Parallelenpostulat etabliert hat. Sie wird in der Regel in modernen Textbüchern zur Geometrie verwendet. Die ursprüngliche Formulierung fordert die Existenz höchstens einer Parallelen. Die Existenz mindestens einer Parallelen lässt sich unabhängig vom Parallelenpostulat beweisen, wie z. B. bei Euklid in Proposition 31: Es ist möglich, ,,[d]urch einen gegebenen Punkt eine einer gegebenen geraden Linie parallele gerade Linie zu ziehen“.

${ }^{17}$ Diese Aussage ist nur dann äquivalent zum Parallelenpostulat, wenn man zusätzlich das Archimedische Axiom voraussetzt: „Sind $A B$ und $C D$ irgendwelche Strecken, so gibt es eine Anzahl $n$ derart, daß das $n$-malige Hintereinander-Abtragen der Strecke $C D$ von $A$ aus auf den durch $B$ gehenden Halbstrahl über den Punkt $B$ hinausführt“ (Hilbert 1962, S. 30).
} 
Makel befreite Euklid"), einige (bei Euklid nicht in dieser Form vorkommende) geometrische Aussagen ohne Rückgriff auf das Parallelenpostulat zu beweisen, beispielsweise: In jedem Dreieck beträgt die Winkelsumme höchstens $180^{\circ}$. Wenn die Winkelsumme in mindestens einem Dreieck $180^{\circ}$ beträgt, so hat jedes Dreieck diese Winkelsumme. ${ }^{18}$ Zum anderen untersuchte er die Eigenschaften des nach ihm benannten Saccherischen Vierecks. Es handelt sich dabei um ein Viereck $A B C D$ mit rechten Winkeln bei $B$ und $C$ sowie $A B=C D$ (siehe Abbildung 2.2). Saccheri zeigte zunächst, dass die Winkel bei $A$ und $D$ (in der Abbildung mit $\alpha$ bezeichnet) identisch sein müssen, und untersuchte daraufhin die drei verschiedenen Hypothesen, dass $\alpha$ ein stumpfer, spitzer oder rechter Winkel ist. Letztere Annahme ist äquivalent zum Parallelenpostulat, die Annahme eines stumpfen Winkels lässt sich mit den übrigen euklidischen Axiomen nicht vereinbaren (gilt aber beispielsweise auf der Kugeloberfläche). Saccheris Versuche, die Hypothese eines spitzen Winkels ebenfalls zum Widerspruch zu führen, scheiterten jedoch. Er gelangte durch verschiedene Deduktionen aus dieser Annahme zwar letztlich zu Aussagen, die der - typischerweise durch Euklids Bild der Geometrie geprägten - Anschauung zu widersprechen schienen, einen logischen Widerspruch hingegen konnte er nicht herbeiführen. Saccheri genügte es aber scheinbar, eine seiner Meinung nach anschaulich widersinnige Schlussfolgerung deduziert zu haben, so dass er von der Gültigkeit der Hypothese des rechten Winkels überzeugt war (vgl. Kline 1972, S. 866-867).

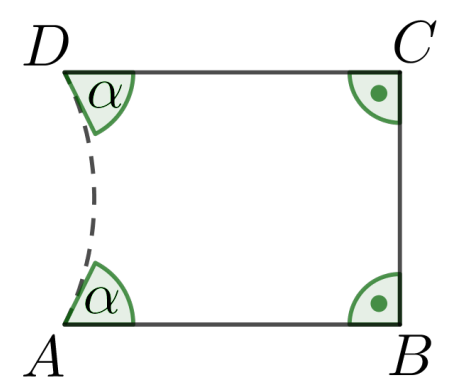

Abbildung 2.2: Saccheri-Viereck

Einen deutlichen Schritt weiter ging Johann Heinrich Lambert (1728-1777). Er untersuchte eine Vierecksfigur, die der von Saccheri ähnlich war, bei der er jedoch von drei rechten Winkeln ausging und anschließend wie Saccheri die drei Möglichkeiten für den verbleibenden Winkel untersuchte (vgl. Scriba und Schreiber 2010, S. 365-366). Er kam zu ähnlichen Ergebnissen wie Saccheri, erkannte im Gegensatz zu diesem aber, dass eine Passung mit den Gegebenheiten des physikalischen Raumes nicht länger das entscheidende Kriterium zur Validierung der Ausgangshypothese sein konnte (vgl. Schreiber 2010b, S. 7-8). Vielmehr hat jede geometrische Theorie, in der keine logischen Widersprüche

\footnotetext{
${ }^{18}$ Der Nachweis dieser Sätze wird häufig auch Legendre zugeschrieben, da sie in einem 1794 von ihm verfassten Geometrielehrbuch ebenfalls enthalten sind (vgl. Schreiber 2010b, S. 7).
} 
existieren, ihre Daseinsberechtigung. Darüber hinaus stellte Lambert fest, dass sich die trigonometrischen Formeln, die sich jeweils aus den Hypothesen eines stumpfen bzw. spitzen Winkels ergaben, nur durch ein Vorzeichen voneinander unterschieden. Da die Annahme eines stumpfen Winkels zur sphärischen Geometrie führte, stellte er die Vermutung auf, dass eine Geometrie, die auf der Annahme eines spitzen Winkels beruhte, auf einer Kugeloberfläche mit imaginärem Radius Gültigkeit besitzen könnte (siehe auch Schreiber 2010b, S. 8 oder Kline 1972, S. 868). Bemerkenswert an diesen Überlegungen ist, dass Lambert damit bereits über eine - modern formuliert - Modellvorstellung (siehe weiter unten) dieser neuen Art von Geometrie verfügte, auch wenn diese nicht anschaulich war.

Die eigentliche Entdeckung ${ }^{19}$ der nichteuklidischen Geometrie wird in der Regel drei Personen zugeschrieben: Johann von Bolyai (1802-1860), Nikolai Iwanowitsch Lobatschewski (1792-1856) und Carl Friedrich Gauß (1777-1855). Alle gelangten zu mehr oder weniger ähnlichen Ergebnissen, indem sie (unter Beibehaltung aller euklidischen Axiome bzw. Postulate mit Ausnahme des Parallelenpostulats) eine sehr umfangreiche mathematische Theorie aus der Annahme entwickelten, dass es zu einer gegebenen Geraden nicht genau eine, sondern mehrere Parallelen gibt, die durch einen vorgegebenen Punkt verlaufen. ${ }^{20}$ Während Gauß sich aber scheute, seine Ergebnisse publik zu machen, veröffentlichten Bolyai und Lobatschewski ihre Arbeiten in den 1820er und 1830er Jahren, weswegen der Name Gauß in vielen Darstellungen der Entstehungsgeschichte der nichteuklidischen Geometrie nur an dritter Stelle genannt wird. ${ }^{21}$

Der wesentliche Unterschied zu ihren Vorgängern, die ebenfalls bereits wesentliche Resultate aus der Negation des Parallelenpostulats gefolgert hatten, bestand darin, dass Gauß, Bolyai und Lobatschewski davon überzeugt waren, dass sich die Annahme mehrerer Parallelen nicht zu einem logischen Widerspruch führen lasse. Damit wäre das Parallelenpostulat tatsächlich ein Postulat, also kein aus den übrigen Postulaten folgerbarer Satz. Exemplarisch sei hierfür aus einem Brief von Gauß zitiert:

In diesem Sinne enthält die Nicht-Euklidische Geometrie durchaus nichts Widersprechendes, wenngleich diejenigen [die sie kennen lernen] viele Ergeb-

\footnotetext{
${ }^{19} \mathrm{Ob}$ man besser von Entdeckung oder Entwicklung reden sollte, ist eine Frage des philosophischen Standpunktes und wird an dieser Stelle nicht weiter diskutiert.

${ }^{20}$ Aus der Annahme, dass es keine Parallelen gibt, lässt sich die sogenannte elliptische Geometrie aufbauen, deren bekannteste Form - bei geeigneter Definition eines Punktes - die sphärische Geometrie ist. In der elliptischen Geometrie sind jedoch noch weitere euklidische Axiome nicht erfüllt.

${ }^{21}$ Kline (1972, S. 877-879) weist jedoch darauf hin, dass die Darstellung von Bolyai und Lobatschewski als Protagonisten dieser Entdeckungen den historischen Gegebenheiten nicht gerecht werde. Zum einen konnten alle drei Mathematiker auf die wertvollen Vorarbeiten von Personen wie Saccheri und Lambert zurückgreifen. Zum anderen befasste sich Gauß deutlich früher mit dem Parallelenproblem und es ist zu vermuten, dass Bolyai und Lobatschewski in nicht unerheblichem Maße von dessen Ideen profitierten.
} 
nisse derselben anfangs für paradox halten müssen, was aber für widersprechend zu halten nur eine Selbsttäuschung sein würde, hervorgebracht von der frühen Gewöhnung, die Euklidische Geometrie für streng wahr zu halten. (zit.n. Volkert 2013, S. 14)

An dieser Stelle muss betont werden, dass die Beschäftigung mit dem Parallelenproblem selbst im 19. Jahrhundert lange Zeit als kuriose und wenig sinnvolle Nebenbeschäftigung galt. Erst als diverse Briefwechsel von Gauß nach dessen Tod zu Beginn der 1860er Jahre veröffentlicht wurden und zeigten, dass der anerkannteste Mathematiker seiner Zeit sich ernsthaft mit dieser Thematik beschäftigt hatte, rückte die nichteuklidische Geometrie zunehmend ins öffentliche Interesse (vgl. Volkert 2013, S. 13-14).

Die Auseinandersetzung mit verschiedenen Geometrien warf zwangsläufig die Frage auf, welche denn nun die „richtige“ Geometrie sei im Sinne einer adäquaten Beschreibung des realen physikalischen Raumes. Philosophisch setzte sich zunehmend eine empiristische Haltung durch, die davon ausging, dass man die (realweltliche) Gültigkeit geometrischer Axiome (und ihrer Konsequenzen) letztlich nur durch möglichst präzise Messungen ermitteln könne (siehe auch Volkert 2013, S. 203-209). Beispielhaft hierfür ist Gauß' Vermessung des Dreiecks Brocken-Inselberg-Hoherhagen, mit der er die Gültigkeit des zum Parallelenpostulat äquivalenten Innenwinkelsatzes überprüfen wollte (vgl. Volkert 2013, S. 23). Als weiterer prominenter Repräsentant dieser Grundhaltung hat Hermann von Helmholtz (1821-1894) in seinem Vortrag Über den Ursprung und die Bedeutung der geometrischen Axiome (von Helmholtz 1883) ein Gedankenexperiment vorgestellt, um aufzuzeigen, wie unterschiedliche Erfahrungen unterschiedliche Geometrien hervorbringen können:

Denken wir uns - darin liegt keine logische Unmöglichkeit - verstandbegabte Wesen von nur zwei Dimensionen, die an der Oberfläche irgend eines unserer festen Körper leben und sich bewegen. Wir nehmen an, dass sie nicht die Fähigkeit haben, irgend etwas außerhalb dieser Oberfläche wahrzunehmen, wohl aber Wahrnehmungen zu machen, ähnlich den unsrigen, innerhalb der Ausdehnung der Fläche, in der sie sich bewegen. Wenn sich solche Wesen ihre Geometrie ausbilden, so würden sie ihrem Raume natürlich nur zwei Dimensionen zuschreiben. Sie würden ermitteln, dass ein Punkt, der sich bewegt, eine Linie beschreibt, und eine Linie, die sich bewegt, eine Fläche, was für sie das vollständigste Raumgebilde wäre, das sie kennen. Aber sie würden sich ebenso wenig eine Vorstellung machen können von einem weiteren räumlichen Gebilde, das entstände, wenn eine Fläche sich aus ihrem flächenhaften Raume herausbewegte, als wir es können von einem Gebilde, das durch Herausbewegung eines Körpers aus dem uns bekannten Raume entstände. Unter dem viel gemissbrauchten Ausdrucke „,sich vorstellen“ oder „,sich denken können, wie etwas geschieht" verstehe ich - und ich sehe nicht, 
wie man etwas Anderes darunter verstehen kann, ohne allen Sinn des Ausdrucks aufzugeben -, dass man sich die Reihe der sinnlichen Eindrücke ausmalen könne, die man haben würde, wenn so etwas in einem einzelnen Falle vor sich ginge. Ist nun gar kein sinnlicher Eindruck bekannt, der sich auf einen solchen nie beobachteten Vorgang bezöge, wie für uns eine Bewegung nach einer vierten, für jene Flächenwesen eine Bewegung nach der uns bekannten dritten Dimension des Raumes wäre, so ist ein solches „Vorstellen“ nicht möglich, ebenso wenig als ein von Jugend auf absolut Blinder sich wird die Farben „vorstellen“ können, auch wenn man ihm eine begriffliche Beschreibung derselben geben könnte.

Jene Flächenwesen würden ferner auch kürzeste Linien in ihrem flächenhaften Raume ziehen können. Das wären nicht nothwendig gerade Linien in unserem Sinne, sondern was wir nach geometrischer Terminologie geodätische Linien der Fläche, auf der jene leben, nennen würden, Linien, wie sie ein gespannter Faden beschreibt, den man an die Fläche anlegt, und der ungehindert an ihr gleiten kann. Ich will mir erlauben, im Folgenden dergleichen Linien als die geradesten Linien der bezeichneten Fläche (beziehlich eines gegebenen Raumes) zu bezeichnen, um dadurch ihre Analogie mit der geraden Linie in der Ebene hervorzuheben. Ich hoffe den Begriff durch diesen Ausdruck der Anschauung meiner nicht mathematischen Zuhörer näher zu rücken, ohne doch Verwechselungen zu veranlassen.

Wenn nun Wesen dieser Art auf einer unendlichen Ebene lebten, so würden sie genau dieselbe Geometrie aufstellen, welche in unserer Planimetrie enthalten ist. Sie würden behaupten, dass zwischen zwei Punkten nur eine gerade Linie möglich ist, dass durch einen dritten, außerhalb derselben liegenden Punkt nur eine Parallele mit der ersten geführt werden kann, dass übrigens gerade Linien in das Unendliche verlängert werden können, ohne dass ihre Enden sich wieder begegnen, und so weiter. Ihr Raum könnte unendlich ausgedehnt sein, aber auch, wenn sie an Grenzen ihrer Bewegung und Wahrnehmung stießen, würden sie sich eine Fortsetzung jenseits dieser Grenzen anschaulich vorstellen können. In dieser Vorstellung würde ihnen ihr Raum unendlich ausgedehnt erscheinen, gerade wie uns der unsrige, obgleich auch wir mit unserem Leibe nicht unsere Erde verlassen können, und unser Blick nur so weit reicht, als sichtbare Fixsterne vorhanden sind.

Nun könnten aber intelligente Wesen dieser Art auch an der Oberfläche einer Kugel leben. Ihre kürzeste oder geradeste Linie zwischen zwei Punkten würde dann ein Bogen des größten Kreises sein, der durch die betreffenden Punkte zu legen ist. Jeder größte Kreis, der durch zwei gegebene Punkte geht, zerfällt dabei in zwei Theile. Wenn beide ungleich lang sind, ist der kleinere Theil allerdings die einzige kürzeste Linie auf der Kugel, die zwischen diesen beiden Punkten besteht. Aber auch der andere größere Bogen desselben größten Kreises ist eine geodätische oder geradeste Linie, d. h. jedes kleinere Stück desselben ist eine kürzeste Linie zwischen seinen beiden Endpunkten. Wegen dieses Umstandes können wir den Begriff der geodätischen oder 
geradesten Linie nicht kurzweg mit dem der kürzesten Linie identifizieren. Wenn nun die beiden gegebenen Punkte Endpunkte desselben Durchmessers der Kugel sind, so schneiden alle durch diesen Durchmesser gelegten Ebenen Halbkreise aus der Kugelfläche, welche alle kürzesten Linien zwischen den beiden Endpunkten sind. In einem solchen Falle gibt es also unendlich viele unter einander gleiche kürzeste Linien zwischen den beiden gegebenen Punkten. Somit würde das Axiom, dass nur eine kürzeste Linie zwischen zwei Punkten bestehe, für die Kugelbewohner nicht ohne eine gewisse Ausnahme gültig sein.

Parallele Linien würden die Bewohner der Kugel gar nicht kennen. Sie würden behaupten, dass beliebige zwei geradeste Linien, gehörig verlängert, sich schließlich nicht nur in einem sondern in zwei Punkten schneiden müssten. Die Summe der Winkel in einem Dreieck würde immer größer sein als zwei Rechte, und umso größer, je größer die Fläche des Dreiecks. Eben deshalb würde ihnen auch der Begriff der geometrischen Ähnlichkeit der Form zwischen größeren und kleineren Figuren derselben Art fehlen. Denn ein größeres Dreieck muss nothwendig andere Winkel haben als ein kleineres. Ihr Raum würde allerdings unbegrenzt, aber endlich ausgedehnt gefunden oder mindestens vorgestellt werden müssen.

Es ist klar, dass die Wesen auf der Kugel bei denselben logischen Fähigkeiten, doch ein ganz anderes System geometrischer Axiome aufstellen müssten, als die Wesen auf der Ebene, und als wir selbst in unserem Raume von drei Dimensionen. Diese Beispiele zeigen uns schon, dass, je nach der Art des Wohnraumes, verschiedene geometrische Axiome aufgestellt werden müssten von Wesen, deren Verstandeskräfte den unsrigen ganz entsprechend sein könnten. (von Helmholtz 1883, S. 8-10)

Von Helmholtz richtet sich damit explizit gegen eine Kantianische Auffassung von Mathematik, der zufolge die geometrischen Axiome der reinen Anschauung entstammen, die transzendentaler Natur ist und vor jeder empirischen Erfahrung liegt. ${ }^{22}$

Der Status der auf der Annahme mehrerer Parallelen aufbauenden Geometrie - im Folgenden auch hyperbolische Geometrie ${ }^{23}$ genannt - wurde schließlich durch die Veröffentlichung diverser Modelle zunehmend gefestigt. Dieser Begriff wurde damals noch nicht verwendet, stattdessen sprach man beispielsweise von Versinnlichungen (vgl. Volkert 2013, S. 3). Aus moderner Sicht ist ein Modell eines Axiomensystems ,ein Bereich von Objekten, innerhalb dessen die Axiome gelten, oder etwas weniger hochgestochen, ein konkretes Beispiel“" (Deiser 2010, S. 153). So sind etwa der $\mathbb{R}^{n}$ und die Menge aller

\footnotetext{
${ }^{22}$ Gegen von Helmholtz wurde allerdings berechtigterweise eingewendet, dass seine nichteuklidischen Modelle mit euklidischen Mitteln beschrieben werden, da beispielsweise die Oberfläche einer Kugel ein in den dreidimensionalen euklidischen Raum eingebettetes Gebilde ist. Eine Widerlegung der Position Kants ist damit also keineswegs erbracht (vgl. Wille 2015, S. 15-17).

${ }^{23}$ Die Bezeichnung als hyperbolisch rührt daher, dass man diese Art der Geometrie unter anderem auf der Oberfläche einer Schale eines zweischaligen Hyperboloids visualisieren kann.
} 
Polynome vom Grad $\leq n$ mit den üblichen Definitionen der Addition und Skalarmultiplikation Modelle eines (euklidischen) Vektorraums; ebenso ist die Menge $\{-1,1\}$ mit der üblichen Multiplikation ein Modell für eine Gruppe. ${ }^{24}$

Vor allem die Modelle von Felix Klein (1849-1925) und Henri Poincaré (1854-1912) waren sehr einflussreich. Deren Grundideen sind im Folgenden kurz beschrieben. ${ }^{25}$

\section{FELIX KLEIN: Kreisscheibe}

Dieses Modell der hyperbolischen Geometrie spielt sich auf dem Inneren einer Kreisscheibe ab und wird daher auch „Bierdeckelgeometrie“ genannt. Geraden sind in diesem Modell Kreissehnen (ohne Endpunkte). Man sieht sofort, dass es in diesem Modell unendlich viele Parallelen zu einer Geraden (definiert als Geraden ohne Schnittpunkt mit der gegebenen) durch einen gegebenen Punkt gibt. Die Längen- und Winkelmessung werden durch eine nichteuklidische Metrik angepasst. Ein räumliches Analogon stellt das Innere einer Kugel dar, wobei hyperbolische Ebenen durch den Schnitt einer

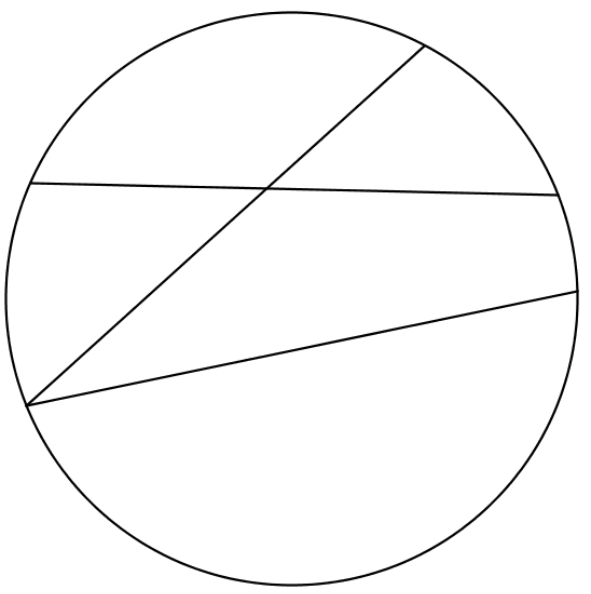

Abbildung 2.3: Klein-Modell euklidischen Ebene mit dem Kugelinneren entstehen.

\section{Henri Poincaré: Halbebene und Kreisscheibe}

Poincarés hyperbolische Ebene ist die oberhalb einer beliebigen vorgegebenen Geraden $g$ liegende Halbebene. Die hyperbolischen Geraden sind alle Halbkreise, deren Mittelpunkt auf $g$ liegt sowie alle auf $g$ senkrecht stehenden euklidischen Geraden (jeweils ohne die auf $g$ liegenden Randpunkte). Die Abstandsmessung muss wieder durch eine nichteuklidische Variante angepasst werden. Durch eine konforme Abbildung erhält man eine zweite Variante des Poincaré-Modells, welche wie das Modell von Klein aus dem Inneren einer Kreisscheibe besteht. Die hyperbolischen Geraden sind hier allerdings Kreisbögen, die die Kreisscheibe senkrecht schneiden, sowie Durchmesserlinien der Kreisscheibe. Ein Vorteil der beiden Poincaré-Modelle gegenüber dem Kleinschen Modell ist, dass die Winkelmes-

\footnotetext{
${ }^{24}$ Der Modell-Begriff ist also zu unterscheiden von dem in der heutigen Mathematikdidaktik oft gebrauchten Konzept des Modellierens, wenngleich dieses durchaus Gemeinsamkeiten zur Tätigkeit des Axiomatisierens aufweist (siehe dazu ausführlicher Hischer 2016).

${ }^{25}$ Ausführlichere Darstellungen der mathematischen Details der nichteuklidischen Geometrie sowie ihrer Modelle findet man unter anderem in Filler 1993, Klotzek 2001 und Koch 2004, S. 335-354.
} 

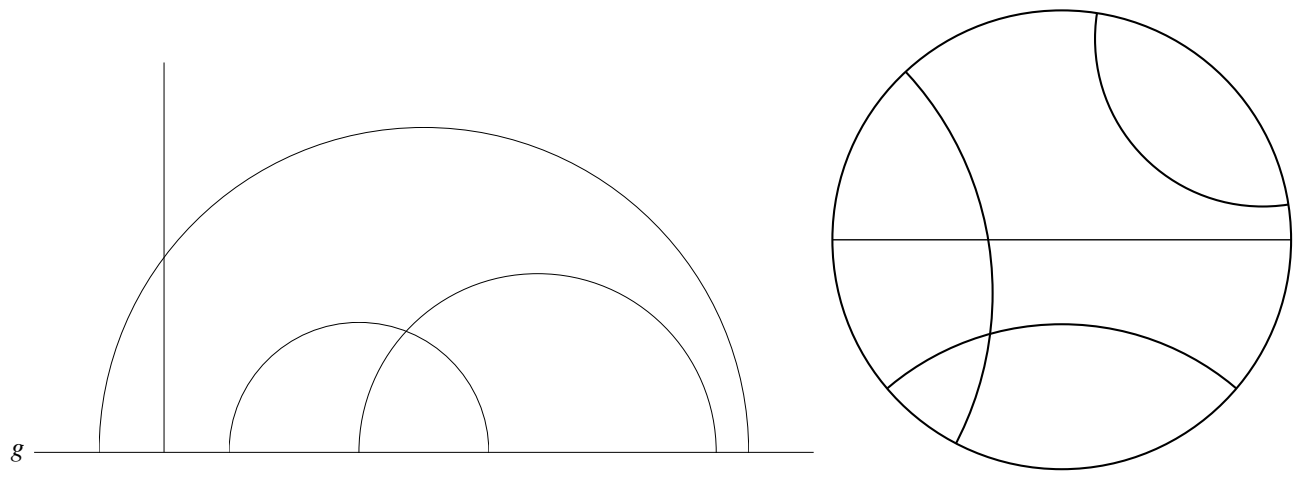

Abbildung 2.4: Poincaré-Modelle

sung euklidisch vorgenommen werden kann: Der Winkel zwischen zwei hyperbolischen Geraden (d.h. zwischen zwei Halbkreisen oder Kreisbögen), die sich in einem Punkt $P$ schneiden, ist gleich dem Winkel der euklidischen Tangenten dieser Kreislinien in $P$.

Bemerkenswert sind Poincarés eigene Kommentare zu seinen Modellen, in denen er einen Vergleich zur Tätigkeit des Übersetzens zieht:

Wir wollen eine gewisse Ebene betrachten, welche ich als Fundamental-Ebene bezeichnen will, und wir wollen eine Art von Wörterbuch herstellen, indem wir mit einer doppelten Reihe von Gliedern, welche in zwei Kolonnen aufgeschrieben sind, in derselben Art wie in den gewöhnlichen Wörterbüchern die Worte zweier Sprachen, die denselben Sinn haben, einander korrespondieren lassen:

Raum

Ebene

Gerade

Kugel

Kreis

Winkel

Gegenseitige Entfernung zweier Punkte
Teil des Raumes oberhalb der Fundamentalebene. Kugel, welche die Fundamentalebene rechtwinklig schneidet.

Kreis, welcher die Fundamentalebene rechtwinklig schneidet.

Kugel.

Kreis.

Winkel.

Logarithmus des Doppel-Verhältnisses, welches diese beiden Punkte mit zwei anderen Punkten bilden, wenn letztere als Schnittpunkte der Fundamentalebene mit einem Kreise definiert werden, der durch diese beiden Punkte hindurchgeht und die Fundamentalebene rechtwinklig schneidet.

etc. etc.

Wir nehmen jetzt die Lehrsätze von Lobatschewsky und übersetzen sie mit Hilfe dieses Wörterbuches [...]. Wir werden so Lehrsätze der gewöhnlichen [d.h. euklidischen, T.H.] Geometrie erhalten. [...] 
Auf diese Weise wird man niemals, soweit man auch die Folgerungen der Hypothesen von Lobatschewsky treibt, auf einen Widerspruch stoßen. In der Tat, wenn zwei Lehrsätze von Lobatschewsky einander widersprechen würden, so würde dasselbe der Fall sein mit den Übersetzungen dieser beiden Lehrsätze, welche mit Hilfe unseres Wörterbuches gemacht sind, aber diese Übersetzungen sind Lehrsätze der gewöhnlichen Geometrie, und niemand zweifelt daran, daß die gewöhnliche Geometrie von Widersprüchen frei ist. $[\ldots]$

Das ist nicht alles. Die Geometrie von Lobatschewsky, welche einer konkreten Veranschaulichung fähig ist, hört auf, ein leeres Spiel des Verstandes zu sein, und kann Anwendungen erhalten; [...] (Poincaré 1904, S. 42-44)

Poincaré formuliert hier zwei wesentliche Erkenntnisse, die durch die Modelle der hyperbolischen Geometrie gegen Ende des 19. Jahrhunderts hervorgebracht wurden: Zum einen wurde durch sie gezeigt, dass die zunächst rein logisch-axiomatisch entwickelte Theorie von Gauß, Bolyai und Lobatschewski einer anschaulichen Interpretation fähig ist; nach damaligen Standards war dies gleichbedeutend mit dem Nachweis der ontischen Existenz der in dieser Theorie behandelten mathematischen Objekte (vgl. Volkert 2013, S. 73). Zum anderen wird hier erstmals deutlich betont, dass Modelle die relative Widerspruchsfreiheit einer Theorie garantieren. Man spricht hier von relativer Widerspruchsfreiheit, da ein Modell die Realisierung einer „unbekannten“ Theorie in einer „bekannten“ Theorie darstellt (vgl. Volkert 2013, S. 73). Beispielsweise kann man davon ausgehen, dass die ebene euklidische Geometrie widerspruchsfrei ist, da man sie im $\mathbb{R}^{2}$ modellieren kann und die geometrischen Axiome dort erfüllt sind. Dabei setzt man aber stillschweigend die Widerspruchsfreiheit der Arithmetik der reellen Zahlen voraus. Man hat also die Widerspruchsfreiheit der euklidischen Geometrie durch Angabe des $\mathbb{R}^{2}$-Modells nur relativ zur Widerspruchsfreiheit der Arithmetik der reellen Zahlen gezeigt. Ebenso ist die hyperbolische Geometrie widerspruchsfrei, falls die euklidische Geometrie widerspruchsfrei ist, denn ein Widerspruch in der hyperbolischen Geometrie führt zwangsläufig auch zu einem Widerspruch in der euklidischen Geometrie und umgekehrt.

Damit war nebenbei die Jahrhunderte alte Frage nach dem Status des euklidischen Parallelenpostulats geklärt, denn wäre dieses ein von den übrigen Axiomen abhängiges Theorem, so müsste eine entsprechende Aussage auch in der hyperbolischen Geometrie gelten, was jedoch einen direkten Widerspruch zum hyperbolischen Axiom über die Existenz mehrerer Parallelen liefern würde.

So eröffnete sich eine völlig neue Sichtweise auf die Natur mathematischer Axiome. Bis dato hatte die Beschäftigung mit dem Parallelenpostulat - wie bereits erwähnt - stets in engem Zusammenhang mit der Frage gestanden, inwiefern mit der Festlegung auf die eu- 
klidischen Axiome bzw. Postulate eine Beschreibung des physikalischen Raumes gegeben sei. Damit einher ging die Auffassung, dass die fundamentale Bedeutsamkeit von Axiomen auch darin begründet liegt, dass mit ihnen Wahrheiten bezüglich einer (in welcher Form auch immer existierenden) Realität formuliert werden. Die euklidische und hyperbolische Geometrie widersprechen sich jedoch, können also nicht beide wahr sein (im Sinne einer Übereinstimmung mit der realen Welt). Gleichzeitig würde ein Widerspruch in der einen aber notwendigerweise auch einen Widerspruch in der anderen Geometrie produzieren. Die Entwicklung der hyperbolischen Geometrie zeigte somit, dass Fragen der Widerspruchsfreiheit und Beweisbarkeit auch ohne eine Überprüfung der Axiome hinsichtlich einer inhaltlich-anschaulichen „Wahrheit“ geklärt werden konnten bzw. mussten (vgl. Schreiber 2010b, S. 10).

Passend dazu formulierte Poincaré - geprägt durch die in obigem Zitat verdeutlichte „Wörterbuch“-Auffassung der nichteuklidischen Modelle - seine auch als konventionalistisch bezeichneten Ansichten vom Wesen geometrischer Axiome:

Die meisten Mathematiker betrachten die Lobatschewskysche Theorie nur als eine einfache logische Merkwürdigkeit; einige von ihnen sind allerdings weiter gegangen. Ist es gewiß, daß unsere [die euklidische, T.H.] Geometrie die rechte ist, denn es sind doch mehrere Geometrien möglich? Die Erfahrung lehrt uns ohne Zweifel, daß die Summe der Winkel eines Dreiecks gleich zwei Rechten ist; aber das ist nur der Fall, wenn wir mit zu kleinen Dreiecken operieren; nach Lobatschewsky ist der Unterschied von zwei Rechten der Oberfläche des Dreiecks proportional: kann diese Differenz nicht wahrnehmbar werden, wenn wir mit größeren Dreiecken operieren und wenn unsere Messungen genauer werden? Die Euklidische Geometrie würde für uns damit nur eine vorläufig richtige Geometrie sein.

Um über diese Meinung zu disputieren, müssen wir uns vor allem fragen: Welches ist die Natur der geometrischen Axiome?

Sind es synthetische Urteile a priori, wie Kant sie nennt? [...]

Wir wollen andererseits die Vorstellung von Lebewesen ohne Dicke (resp. Höhe) wieder aufnehmen [...]; wir können wohl kaum annehmen, daß sich diese Wesen, wenn sie einen Verstand gleich dem unsrigen hätten, die Euklidische Geometrie aneignen würden, welche allen ihren Erfahrungen widerspräche.

Sollen wir nun daraus schließen, daß die geometrischen Axiome erfahrungsmäßige Wahrheiten sind? Man experimentiert doch nicht mit idealen geraden Linien oder Kreisen; man kann dazu nur wirkliche Gegenstände brauchen. $[\ldots]$

Wenn die Geometrie eine Experimental-Wissenschaft wäre, so würde sie aufhören, eine exakte Wissenschaft zu sein, sie würde also einer beständigen 
Revision zu unterwerfen sein. [...]

Die geometrischen Axiome sind also weder synthetische Urteile a priori noch experimentelle Tatsachen.

Es sind auf Übereinkommen beruhende Festsetzungen; unter allen möglichen Festsetzungen wird unsere Wahl von experimentellen Tatsachen geleitet; aber sie bleibt frei und ist nur durch die Notwendigkeit begrenzt, jeden Widerspruch zu vermeiden. In dieser Weise können auch die Postulate streng richtig bleiben, selbst wenn die erfahrungsmäßigen Gesetze, welche ihre Annahme bewirkt haben, nur annähernd richtig sein sollten.

Mit anderen Worten: die geometrischen Axiome [...] sind nur verkleidete Definitionen.

Was soll man dann aber von der folgenden Frage denken: Ist die Euklidische Geometrie richtig?

Die Frage hat keinen Sinn.

Ebenso könnte man fragen, ob das metrische System richtig ist und die älteren Maß-Systeme falsch sind [...]. Eine Geometrie kann nicht richtiger sein wie eine andere; sie kann nur bequemer sein. (Poincaré 1904, S. 49-52; Herv. im Orig.)

Poincaré unterscheidet deutlicher als seine Vorgänger zwischen einer formalen Geometrie, deren Axiome den Charakter von Konventionen bzw. Definitionen haben und für die sich die Wahrheitsfrage daher nicht stellen kann, und einer inhaltlich interpretierten Geometrie, innerhalb der man die Richtigkeit einer Aussage durch empirische Messungen zumindest approximativ untermauern kann. Zwar werde die Entwicklung einer formalen Theorie durch experimentelle Erkenntnisse beeinflusst, letztlich rechtfertige aber alleine die logische Widerspruchsfreiheit ein Axiomensystem. Die euklidische Geometrie hat laut ihm eine besondere Stellung unter den mathematisch prinzipiell gleichberechtigten Theorien, weil sie für viele praktische Zwecke einfacher zu handhaben ist. Man erkennt an Poincarés Ausführungen gewisse formalistische Züge. ${ }^{26}$ An anderer Stelle schreibt er beispielsweise: „Ein mathematisches Objekt existiert, sobald nur seine Definition weder mit sich selbst noch mit den vorher schon bewiesenen Sätzen in Widerspruch steht“ (Poincaré 1904, S. 45). ${ }^{27}$

\footnotetext{
${ }^{26}$ Nota bene: Hilberts Grundlagen der Geometrie (siehe Abschnitt 2.1.3) waren zu dem Zeitpunkt, als Poincaré die oben zitierten Ausführungen veröffentlichte, bereits erschienen. Dennoch war Poincaré weit davon entfernt, ein radikaler Formalist zu sein.

${ }^{27}$ Man vergleiche dies mit Hilberts Aussage aus einem 1899 verfassten Brief an Frege: „Wenn sich die willkürlich gesetzten Axiome nicht einander widersprechen mit sämtlichen Folgen, so sind sie wahr, so existieren die durch die Axiome definirten Dinge. Das ist für mich das Criterium der Wahrheit und der Existenz“" (zit. n. Frege 1976, S. 66; siehe auch Abschnitt 2.1.3).
} 


\subsubsection{Hilberts Grundlagen der Geometrie}

Die im vorigen Abschnitt beschriebene zunehmende Loslösung der Geometrie von ontologischen Fragestellungen und der damit einhergehende „Wandel der Mathematik von einer Quasi-Naturwissenschaft zur Strukturwissenschaft“" (Scriba und Schreiber 2010, S. 497) fand ihren Höhepunkt in der erstmals 1899 anlässlich der Enthüllung des GaußWeber-Denkmals veröffentlichten Festschrift Grundlagen der Geometrie des deutschen Mathematikers David Hilbert (1862-1943). ${ }^{28}$

Darin verfolgt er das Ziel, ,für die Geometrie ein vollständiges und möglichst einfaches System von Axiomen aufzustellen und aus denselben die wichtigsten geometrischen Sätze in der Weise abzuleiten, daß dabei die Bedeutung der verschiedenen Axiomgruppen und die Tragweite der aus den einzelnen Axiomen zu ziehenden Folgerungen klar zutage tritt“ (Hilbert 1962, S. 1). Für diese Zwecke stellt er ein sehr umfangreiches Axiomensystem vor, welches in fünf Gruppen unterteilt ist: I. Axiome der Verknüpfung/Inzidenz, II. Anordnung, III. Kongruenz, IV. Parallelen und V. Stetigkeit. ${ }^{29}$ Beispielhaft seien einige Axiome der ebenen Geometrie genannt (zit. n. Hilbert 1962):

I.1 Zu zwei Punkten $A, B$ gibt es stets eine Gerade $a$, die mit jedem der beiden Punkte $A, B$ zusammengehört.

I.2 Zu zwei Punkten $A, B$ gibt es nicht mehr als eine Gerade, die mit jedem der beiden Punkte $A, B$ zusammengehört.

I.3 Auf einer Geraden gibt es stets wenigstens zwei Punkte. Es gibt wenigstens drei Punkte, die nicht auf einer Geraden liegen.

II.1 Wenn ein Punkt $B$ zwischen einem Punkt $A$ und einem Punkt $C$ liegt, so sind $A, B, C$ drei verschiedene Punkte einer Geraden, und $B$ liegt dann auch zwischen $C$ und $A$.

II.2 Zu zwei Punkten $A$ und $C$ gibt es stets wenigstens einen Punkt $B$ auf der Geraden $A C$, so daß $C$ zwischen $A$ und $B$ liegt.

III.1 Wenn $A, B$ zwei Punkte auf einer Geraden $a$ und ferner $A^{\prime}$ ein Punkt auf derselben oder einer anderen Geraden $a^{\prime}$ ist, so kann man auf einer gegebenen Seite der Geraden $a^{\prime}$ von $A^{\prime}$ stets einen Punkt $B^{\prime}$ finden, so daß

\footnotetext{
${ }^{28}$ Einflussreiche Wegbereiter waren neben Moritz Pasch (1843-1930) unter anderem Giuseppe Peano (1858-1932) und Hermann Wiener (1857-1939) (siehe auch Scriba und Schreiber 2010, S. 499-501 und Kline 1972, S. 1007-1015).

${ }^{29}$ Diese Strukturierung wurde in späteren Auflagen immer wieder geringfügig modifiziert. Die neunte Auflage, aus der in dieser Arbeit überwiegend zitiert wird (Hilbert 1962), umfasst 8 Inzidenzaxiome, 4 Anordnungsaxiome, 5 Kongruenzaxiome, das Parallelenaxiom und das Archimedische Axiom. Zudem enthält Gruppe V im Gegensatz zur Erstauflage neben dem Archimedischen Axiom noch das sogenannte Vollständigkeitsaxiom (siehe dazu auch Hilbert 2015, S. 177-180).
} 
die Strecke $A B$ der Strecke $A^{\prime} B^{\prime}$ kongruent oder gleich ist, in Zeichen:

$$
A B \equiv A^{\prime} B^{\prime}
$$

IV (Euklidisches Axiom) Es sei $a$ eine beliebige Gerade und $A$ ein Punkt außerhalb $a$ : dann gibt es in der durch $a$ und $A$ bestimmten Ebene höchstens eine Gerade, die durch $A$ läuft und $a$ nicht schneidet.

V.1 (Axiom des Messens oder Archimedisches Axiom) Sind $A B$ und $C D$ irgendwelche Strecken, so gibt es eine Anzahl $n$ derart, daß das $n$-malige Hintereinander-Abtragen der Strecke $C D$ von $A$ aus auf den durch $B$ gehenden Halbstrahl über den Punkt $B$ hinausführt.

Hilbert stellt drei Anforderungen an Axiomensysteme: Widerspruchsfreiheit, Unabhängigkeit und Vollständigkeit. Widerspruchsfreiheit bedeutet, dass aus einem Axiomensystem nicht gleichzeitig eine Aussage und deren Negation deduzierbar ist. Dies ist für Hilbert das essentielle Kriterium zur Rechtfertigung von Axiomensystemen. Die Unabhängigkeit der Axiome (d. h. es soll kein Axiom aus den anderen ableitbar und damit prinzipiell entbehrlich sein) ist eher eine pragmatische Forderung und daher nur von sekundärer Bedeutung (vgl. Tapp 2013, S. 66-67). Besondere Beachtung verdient der Begriff der Vollständigkeit. Gerade in Hilberts früheren Schriften wird er abweichend von seiner Bedeutung in der modernen Logik verwendet. Grundgedanke von Hilberts Konzeption der Vollständigkeit einer Theorie ist, dass durch eine axiomatische Darstellung eine bereits vorliegende, ,informelle“ Theorie logisch geordnet wird. Vor diesem Hintergrund nennt er ein Axiomensystem vollständig, wenn alle (bekannten) Sätze dieses bereits vorliegenden Wissensgebietes aus den Axiomen hergeleitet werden können. ${ }^{30} \mathrm{Im}$ Falle von Hilberts Grundlagen der Geometrie bezieht sich die Vollständigkeit also auf die klassischen Sätze der euklidischen Geometrie (vgl. Tapp 2013, S. 62-63).

Die (relative) Widerspruchsfreiheit des Axiomensystems sowie die Unabhängigkeit der Axiome untereinander wird bei Hilbert durch die Angabe von Modellen gezeigt. So wird die Unabhängigkeit des Parallelenaxioms von den übrigen durch eine kurze Beschreibung von Poincarés Kugelmodell der hyperbolischen Geometrie bewiesen. Für den Nachweis der Widerspruchsfreiheit definiert Hilbert den pythagoreischen Zahlkörper $\Omega$, welcher ausgehend von der Zahl 1 - durch endliche Anwendung der Rechenoperationen +, -, , , / sowie $\omega \mapsto \sqrt{1+\omega^{2}}$ (wobei $\omega$ eine bereits konstruierte Zahl von $\Omega$ ist) entsteht (vgl. Hilbert 1962, S. 34). Auf $\Omega$ lässt sich dann in gewohnter Weise eine ebene bzw. räumliche analytische Geometrie definieren, in der sämtliche von Hilberts Axiomen gültig sind (Dort werden dann Punkte als Zahlentupel bzw. -tripel aufgefasst und Geraden in der Ebene

\footnotetext{
${ }^{30}$ Etwa gegen Ende der 1920er Jahre hat Hilbert sich auch zunehmend mit der sogenannten syntaktischen Vollständigkeit beschäftigt (vgl. Tapp 2013, S. 65-66; siehe auch Abschnitt 2.2.2).
} 
durch Gleichungen des Typs $u x+v y+w=0$ mit $(x, y) \in \Omega^{2}$ und $u, v, w \in \Omega(u, v$ nicht beide gleich Null) charakterisiert.). ${ }^{31}$ Hilbert führt also die Widerspruchsfreiheit seines Axiomensystems auf die Widerspruchsfreiheit der Arithmetik der reellen Zahlen zurück, die er in den Grundlagen der Geometrie allerdings nicht weiter hinterfragt.

Das Revolutionäre an Hilberts Ansichten über Axiomatik ist die vollständige Abkehr von der klassischen, korrespondenztheoretisch geprägten Auffassung des Wahrheitsbegriffs. ${ }^{32}$ In der vielzitierten Erklärung, welche der Präsentation seines Axiomensystems vorangestellt ist, schreibt er:

Wir denken drei verschiedene Systeme von Dingen: die Dinge des ersten Systems nennen wir Punkte und bezeichnen sie mit $A, B, C, \ldots$; die Dinge des zweiten Systems nennen wir Geraden und bezeichnen sie mit $a, b, c, \ldots$; die Dinge des dritten Systems nennen wir Ebenen und bezeichnen sie mit $\alpha, \beta, \gamma, \ldots ;[\ldots]$

Wir denken die Punkte, Geraden, Ebenen in gewissen gegenseitigen Beziehungen und bezeichnen diese Beziehungen durch Worte wie „liegen“, ,zwischen“, „kongruent“, „parallel“, „stetig“; die genaue und für mathematische Zwecke vollständige Beschreibung dieser Beziehungen erfolgt durch die Axiome der Geometrie. (Hilbert 1962, S. 2; Herv. im Orig.)

Hilberts Axiomensystem ist an keinerlei inhaltliche, weltwissen-bezogene Annahmen gebunden. Die Grundbegriffe einer mathematischen Theorie werden durch Axiome in bestimmte Beziehungen zueinander gestellt und sind dadurch implizit definiert. Es stellt sich nicht mehr die Frage, was beispielsweise ein Punkt ist, sondern, wie mit einem Ding namens Punkt systemkonform umzugehen ist. Berühmt geworden ist der Hilbert zugeschriebene Ausspruch, man müsse jederzeit anstelle von Punkten, Geraden und Ebenen auch Tische, Stühle und Bierseidel sagen können (vgl. Wußing 2009, S. 174). Mathematische Theorien werden damit als formale Denksysteme charakterisiert, weshalb Hilberts Ansatz auch häufig als Formalismus bezeichnet wird.

Durch den Wegfall des Wirklichkeitsbezugs mathematischer Objekte wird natürlich auch die Frage nach der Wahrheit von Aussagen, die diese Objekte zum Gegenstand haben,

\footnotetext{
${ }^{31}$ Eine Ausnahme bildet das Vollständigkeitsaxiom V.2, welches fordert, dass zu dem System der Punkte einer Geraden keine weiteren Elemente hinzugefügt werden können, ohne mindestens eines der Axiome aus den Gruppen I-III oder das Archimedische Axiom V.1 zu verletzen. Die Menge $\Omega$ ist jedoch eine echte Teilmenge der rellen Zahlen. Erst mit der Erweiterung des Hilbertschen Modells auf die gewohnte analytische Geometrie des $\mathbb{R}^{2} / \mathbb{R}^{3}$ ist auch V.2 erfüllt (siehe Hilbert 1962, S. 36-37).

${ }^{32} \mathrm{Die}$ auf Aristoteles zurückgehende Korrespondenztheorie fasst Wahrheit als Übereinstimmung mit der (wie auch immer gearteten) Wirklichkeit auf. Empiristisch ausgerichtete Erkenntnistheorien machen eine solche Übereinstimmung beispielsweise an Erfahrungen fest; auch besteht ein enger Zusammenhang mit evidenzbasierten Argumentationen, die von dem menschlichen Intellekt unmittelbar einsichtigen und somit als wahr erscheinenden Aussagen ausgehen (vgl. Andersson 1989, S. 369-371).
} 
obsolet. ${ }^{33}$ Die in den Axiomen auftretenden Begriffe werden nur noch als Variablen für Objekte oder Eigenschaften verstanden. Die Axiome selber haben nicht mehr den Charakter von Aussagen, sondern sind vielmehr Aussageformen, die erst dann wieder den Status von Aussagen erlangen, wenn man die Variablen inhaltlich interpretiert (vgl. Steiner 1959, S. 18). Zu dieser Sichtweise passt auch der sogenannte algebraische Zugang zu axiomatischen Theorien, dem zufolge Axiomensysteme mit Gleichungssystemen vergleichbar sind:

The axioms are held to "define" their primitive terms as whatever would be needed to make those axioms true, in the same way as systems of equations "define" their unknown values as whatever would be needed to make those equations hold. [...] The primitive terms act as place holders for the many possible "solutions" to the "equations" set by the axioms (Leng 2010, S. 4748).

Viele Mathematiker sträubten sich dagegen, die ihnen vertraute Haltung zur zweifellosen Verankerung mathematischer Wahrheit in der Welt aufzugeben. Der Konflikt zwischen der „,klassischen“ und der „,modernen“ Auffassung von Mathematik wird besonders deutlich in einem Briefwechsel zwischen Hilbert und Gottlob Frege (1848-1925), der in der Literatur zur Axiomatik häufig zitiert wird. ${ }^{34}$ Für Frege ist Hilberts Konzept der impliziten Definition sehr problematisch:

\footnotetext{
${ }^{33}$ Bereits 1882 versuchte Moritz Pasch, eine modernen Ansprüchen genügende logisch-strenge Grundlegung der projektiven Geometrie zu liefern. Insbesondere seine dabei angestellten Ausführungen zu den geometrischen Grundbegriffen und ihrem Zusammenhang mit den Axiomen kommen der Hilbertschen Auffasung stellenweise schon recht nahe. Im Gegensatz zu Hilbert betont Pasch allerdings noch den Einfluss der durch wiederholte Beobachtungen gestützten Erfahrung auf die Rechtfertigung der Axiome und vertritt somit einen empiristischen Standpunkt:
}

Die Mathematik stellt Relationen zwischen den mathematischen Begriffen auf, welche den Erfahrungsthatsachen entsprechen sollen [...]. Nach Ausscheidung der auf Beweise gestützten Sätze, der Lehrsätze, bleibt eine Gruppe von Sätzen zurück, aus denen alle übrigen sich folgern lassen, die Grundsätze; diese sind unmittelbar auf Beobachtungen gegründet, freilich auf Beobachtungen, welche seit undenklichen Zeiten sich unaufhörlich wiederholt haben, welche klarer erfasst werden, als die irgend einer anderen Art, und mit denen die Menschen deshalb längst so vertraut geworden sind, dass ihr Ursprung in Vergessenheit gerathen und Gegenstand des Streites werden konnte. Die Grundsätze sollen das von der Mathematik zu verarbeitende empirische Material vollständig umfassen, so dass man nach ihrer Aufstellung auf die Sinneswahrnehmungen nicht mehr zurückzugehen braucht. (Pasch 1882, S. 16-17)

${ }^{34}$ Die Wiedergabe von isolierten Zitaten einzelner Mathematiker birgt natürlich immer die Gefahr, ihre grundlagentheoretischen Positionen verfälscht - zumindest aber vereinfacht - wiederzugeben. Die in den Briefen gemachten Aussagen Freges und Hilberts sind aber so prägnant, dass sie an dieser Stelle zur Illustration zweier stark verschiedener Ansichten über Axiome herangezogen werden. Man sollte allerdings Vorsicht walten lassen, von den zitierten Passagen auf die umfassendere philosophische Orientierung der Beteiligten zu schließen. 
Axiome und Lehrsätze können [...] nie die Bedeutung eines in ihnen vorkommenden Zeichens oder Wortes erst festsetzen wollen, die vielmehr schon feststehen muss. (zit. n. Frege 1976, S. 62-63)

Hilbert erwidert, dass er keinen wesentlichen Unterschied zwischen Axiomen und Grundbegriffsdefinitionen sieht:

Ich will nichts als bekannt voraussetzen: [...] Wenn man nach andern Definitionen für „Punkt“, etwa durch Umschreibungen wie ausdehnungslos etc. sucht, so muss ich solchem Beginnen allerdings aufs entschiedenste widersprechen; man sucht da etwas, was man nie finden kann, weil nichts da ist, und alles verliert sich und wird wirr und vage und artet in Versteckspiel aus. Wollen Sie meine Axiome lieber Merkmale der in den „Erklärungen“ gesetzten und dadurch vorhandenen Begriffe nennen, so würde ich dagegen gar nichts einzuwenden haben [...]. (zit. n. Frege 1976, S. 66)

Meine Meinung ist eben die, dass ein Begriff nur durch seine Beziehungen zu anderen Begriffen logisch festgelegt werden kann. Diese Beziehungen, in bestimmten Aussagen formulirt, nenne ich Axiome und komme so dazu, dass die Axiome (ev[tl]. mit Hinzunahme der Namensgebungen für die Begriffe) die Definitionen der Begriffe sind. (zit. n. Frege 1976, S. 79)

Noch deutlicher werden Freges und Hilberts unterschiedliche Ansichten hinsichtlich der oben bereits erwähnten Wahrheitsfrage. Laut Frege drücken Axiome Grundwahrheiten aus, die über jeden Zweifel erhaben sind:

Axiome nenne ich Sätze, die wahr sind, die aber nicht bewiesen werden, weil ihre Erkenntnis aus einer von der logischen ganz verschiedenen Erkenntnisquelle fliesst, die man Raumanschauung nennen kann. Aus der Wahrheit der Axiome folgt, dass sie einander nicht widersprechen. (zit. n. Frege 1976, S. 63)

Entsprechend vernichtend fällt sein Urteil über die nichteuklidische Geometrie aus:

Niemand kann zwei Herren dienen. Man kann nicht der Wahrheit dienen und der Unwahrheit. Wenn die euklidische Geometrie wahr ist, so ist die nichteuklidische Geometrie falsch, und wenn die nichteuklidische wahr ist, so ist die euklidische Geometrie falsch. [...] Soll nun die euklidische oder die nichteuklidische Geometrie hinausfliegen? Das ist die Frage. Wagt man es, Euklids Elemente, die mehr als 2000 Jahre ein unbestrittenes Ansehen behauptet haben, als Astrologie zu behandeln? Nur dann, wenn man es nicht wagt, kann man auch Euklids Axiome nicht als falsch oder zweifelhaft hinstellen. Dann muss die nicht-euklidische Geometrie zu den Unwissenschaften gezählt werden, die man nur noch als geschichtliche Seltsamkeiten einer geringen Beachtung wert achtet. (zit. n. Frege 1969, S. 183-184) 
Für Hilbert stellt sich der Sachverhalt genau umgekehrt dar:

Wenn sich die willkürlich gesetzten Axiome nicht einander widersprechen mit sämtlichen Folgen, so sind sie wahr, so existieren die durch die Axiome definirten Dinge. Das ist für mich das Criterium der Wahrheit und der Existenz. (zit. n. Frege 1976, S. 66)

Wahrheit und Existenz werden hier mit Widerspruchsfreiheit gleichgesetzt, d. h. sie ,werden bei Hilbert zu systemrelativen Begriffen ohne ontische Qualität“ (Heintz 2000, S. 50). Auf den ersten Blick lösen sich dadurch diejenigen erkenntnistheoretischen Probleme, die mit der Frage zusammenhängen, wie die Wahrheit von Axiomen gerechtfertigt werden kann. Aus formalistischer Sicht stellen sich diese Probleme nicht, denn die Frage nach der Bedeutung mathematischer Aussagen (und damit nach einer Wahrheit mit ontischem Bezug) ist für die Theorie an sich irrelevant. Es zählt das basale Beziehungsgefüge mit seinem Erschließungs- und Rückführungspotential und damit der hypothetisch-deduktive Charakter von Mathematik. Von Wahrheit wird nur noch in dem Sinne gesprochen, dass eine Aussage letztlich aus den Axiomen logisch ableitbar ist.

Die Frage nach einem lebensweltlichen Bezug von Axiomensystemen stellt sich allerdings wiederum bei der Anwendung von Mathematik, die einer inhaltlichen Qualifizierung von Axiomen bedarf. Auch bleibt die obige Sichtweise die Antwort auf die Frage schuldig, warum manche Axiomensysteme aufgestellt werden und sich durchsetzen, andere aber nicht. Widerspruchsfreiheit kann als rein systemimmanentes Kriterium diesen Umstand nicht erklären. Courant und Robbins schreiben hinsichtlich der in ihren Augen nicht sinnvollerweise als gegeben anzunehmenden „Willkür des Mathematikers“:

Der Lebensnerv der mathematischen Wissenschaft ist bedroht durch die Behauptung, Mathematik sei nichts anderes als ein System von Schlüssen aus Definitionen und Annahmen, die zwar in sich widerspruchsfrei sein müssen, sonst aber von der Willkür des Mathematikers geschaffen werden. Wäre das wahr, dann würde die Mathematik keinen intelligenten Menschen anziehen. Sie wäre eine Spielerei mit Definitionen, Regeln und Syllogismen ohne Ziel und Sinn. (Courant und Robbins 2010, S. xxi)

Auch Hilbert hätte dieser Überlegung wohl zugestimmt. Bei der Darstellung seiner grundlagentheoretischen Arbeiten entsteht häufig der Eindruck, Hilbert sei ein Formalist im radikalen Sinne gewesen. Die Auffassung mathematischer Aussagen als inhalts- und bedeutungslose Formeln ist jedoch vor allem im Rahmen seines beweistheoretischen Programms von Bedeutung, welches sich in zwei Schritte unterteilt:

Erstens: Alles, was bisher die eigentliche Mathematik ausmacht, wird nunmehr streng formalisiert, so daß die eigentliche Mathematik oder die Mathe- 
matik in engerem Sinne zu einem Bestande an beweisbaren Formeln wird. $[\ldots]$

Zweitens: $\mathrm{Zu}$ dieser eigentlichen Mathematik kommt eine gewissermaßen neue Mathematik, eine Metamathematik, hinzu, die zur Sicherung jener dient [...]. In dieser Metamathematik kommt - im Gegensatz zu den rein formalen Schlußweisen der eigentlichen Mathematik - das inhaltliche Schließen zur Anwendung, und zwar zum Nachweis der Widerspruchsfreiheit der Axiome. (Hilbert 1964, S. 29; Herv. im Orig.)

Die Formalisierung mathematischer Theorien (zu der schließlich auch das Ausklammern ontologischer Fragestellungen gehört) ist also lediglich ein Mittel zum - metatheoretisch motivierten - Zweck, die Widerspruchsfreiheit dieser Theorien zu beweisen (siehe auch Abschnitt 2.2.2). Interessant ist auch Hilberts Formulierung, dass die ,eigentliche Mathematik" durch ihre Formalisierung und den Nachweis ihrer Widerspruchsfreiheit gesichert werden soll. Bei der Darstellung eines mathematischen Wissensgebietes in axiomatisierter und formalisierter Form handelt es sich also um eine nachträgliche logische Systematisierung und ,Sicherung eines schon vorgängigen [d.h. insbesondere auch inhaltlich interpretierten oder sogar ontologisch verankerten, T.H.] Erkenntnisinhaltes“ (Tapp 2013, S. 59). Dahingehend kann man auch die Tatsache interpretieren, dass Hilbert seinen Grundlagen der Geometrie folgendes Kant-Zitat voranstellt: ,So fängt denn alle menschliche Erkenntnis mit Anschauungen an, geht von da zu Begriffen und endigt mit Ideen“ (zit. n. Hilbert 1962, S. 1). Hilberts Formalismus ist also vielmehr eine methodische Einstellung (siehe auch Tapp 2013, S. 116-120), die von radikalen Auffassungen wie dem sogenannten Spielformalismus ${ }^{35}$ zu unterscheiden ist. Diese Relativierung des Hilbertschen Standpunktes hat auch Auswirkungen auf die angebliche Willkürlichkeit, mit der die Axiome gewählt werden. In aller Deutlichkeit hat Hilbert selbst seine Einstellung zu dieser Willkür dargelegt:

[Mathematics] has nothing to do with arbitrariness. Mathematics is in no sense like a game, in which certain tasks are determined by arbitrarily established rules. Rather, it is a conceptual system guided by internal necessity, that can only be so, and never otherwise. (zit. n. Corry 1997, S. 116)

\footnotetext{
${ }^{35}$ Als Spielformalismus bezeichnet man eine Sichtweise, die das Wesen der Mathematik in der Manipulation bedeutungsloser Zeichen sieht. Nicht nur die mathematischen Aussagen werden dabei zu bloßen Zeichenketten, sondern auch den Regeln, nach denen man diese Zeichenketten manipuliert, wird keinerlei inhaltliche Bedeutung zugeschrieben. Das Treiben von Mathematik wird damit vergleichbar mit einem reinen Formelspiel, dessen Regeln man sich ähnlich denen eines Schachspiels unterwirft. Eine konzise, aber sehr instruktive Diskussion der verschiedenen Ausprägungen des Formalismusbegriffs sowie eine Einordnung Hilberts findet sich bei Tapp (2013, S. 115-134). Eine eher populärwissenschaftliche Darstellung liefert Shapiro (2000, S. 140-171).
} 


\subsection{Axiomatik im 20. Jahrhundert}

\subsubsection{Strukturmathematik}

Spätestens mit Hilberts Grundlagen der Geometrie hatte sich der Blick auf das Wesen mathematischer Axiome grundlegend geändert. Der formalistische Standpunkt, Axiome (zunächst) als inhaltlich uninterpretiert aufzufassen, lieferte eine wichtige Grundlage dafür, strukturelle Gemeinsamkeiten, die in unterschiedlichen mathematischen Gebieten entdeckt wurden, in einer einheitlichen abstrakten Theorie zu bündeln.

Ein typisches Beispiel hierfür ist die Gruppentheorie, welche durch einen Prozess der Abstraktion aus unterschiedlichen mathematischen Gebieten entstanden ist (Hischer 2012, S. 47; Herv. im Orig.):

- die Untersuchung der Auflösbarkeit algebraischer Gleichungen n-ten Grades in der Gleichungslehre [...] (Abel, Galois)

- Bewegungen und ihre Invarianten in der Geometrie (Klein)

- Quadratische Formen in der Zahlentheorie (Lagrange, Gauß)

Eine erste abstrakte Definition des Gruppenbegriffs stammt von Arthur Cayley (18211895); die Formulierung der im Wesentlichen auch heute noch gebräuchlichen Axiome lieferte Heinrich Weber (1842-1913) (siehe auch Alten et al. 2014, S. 533-547):

Ein System $\mathfrak{S}$ von Dingen (Elementen) irgend welcher Art in endlicher oder unendlicher Anzahl wird zur Gruppe, wenn folgende Voraussetzungen erfüllt sind.

1) Es ist eine Vorschrift gegeben, nach der aus einem ersten und einem zweiten Element des Systems ein ganz bestimmtes drittes Element desselben Systems abgeleitet wird.

Man schreibt symbolisch, wenn $A$ das erste, $B$ das zweite, $C$ das dritte Element ist

$$
A B=C, \quad C=A B,
$$

und nennt $C$ aus $A$ und $B$ componirt.

Bei dieser Composition wird im Allgemeinen [...]

2) das associative Gesetz vorausgesetzt,

d. h. wenn $A, B, C$ irgend drei Elemente aus $\mathfrak{S}$ sind, so ist

$$
(A B) C=A(B C),
$$


$[\ldots]$

3) Es wird vorausgesetzt, dass, wenn $A B=A B^{\prime}$ oder $A B=A^{\prime} B$ ist, nothwen$\operatorname{dig} B=B^{\prime}$ oder $A=A^{\prime}$ sein muss. [...]

4) Wenn von den drei Elementen $A, B, C$ zwei beliebig aus $\mathfrak{S}$ genommen werden, so kann man das dritte immer und nur auf eine Weise so bestimmen, dass

$$
A B=C
$$

ist.

(Weber 1893, S. 522; Herv. im Orig.)

Die Entstehung der abstrakt formulierten Gruppentheorie kann man als „Beginn einer grundsätzlichen Tendenz in der Mathematik bis weit in die zweite Hälfte des 20. Jahrhunderts hinein“ betrachten, die auf eine ,axiomatische Fundierung und Strukturierung nahezu aller Teilgebiete der Mathematik" hinauslief (Hischer 2012, S. 41).

Den zweiten wichtigen Baustein für eine vereinheitlichende Darstellung mathematischer Theorien lieferte die von Georg Cantor (1845-1918) begründete Mengenlehre. In seiner Abhandlung Beiträge zur Begründung der transfiniten Mengenlehre begründete er den sogenannten naiven Mengenbegriff:

Unter einer ,Menge“ verstehen wir jede Zusammenfassung $M$ von bestimmten wohlunterschiedenen Objecten $m$ unsrer Anschauung oder unseres Denkens (welche die ,Elemente “ von $M$ genannt werden) zu einem Ganzen. (Cantor 1895, S. 481)

Diese „Definition“ ist bekanntlich nicht unproblematisch, da sie beispielsweise die Bildung der Menge aller Mengen, die sich nicht selbst enthalten, erlaubt. Die Frage, ob diese Menge nun sich selbst enthält, führt - egal, ob man sie mit Ja oder Nein beantwortet - unweigerlich zu einem Widerspruch, der Russelschen Antinomie (siehe auch Hischer 2012, S. 74). Eine Lösung dieses Problems besteht in einer Axiomatisierung der Mengenlehre, welche die Konstruktion ,problematischer“ Mengen verbietet und es gleichzeitig gestattet, die ursprünglich von Cantor entwickelte Theorie darauf aufzubauen. Eine solche Fundierung wurde durch Ernst Zermelo (1871-1953) und Abraham Fraenkel (1891-1965) vorgenommen. Ich möchte an dieser Stelle nicht näher auf die Entwicklung der Mengenlehre eingehen und verweise stattdessen auf die umfangreichen Darstellungen in Deiser 2010. Die für diesen Abschnitt wesentliche Erkenntnis ist, dass mit dem Mengenbegriff ein sehr umfassendes Konzept zur abstrakten Beschreibung unterschiedlichster mathematischer Objekte vorliegt. 


\section{Strukturierte Mengen}

Das Vorhaben der Neustrukturierung sämtlicher Gebiete der Mathematik wurde in der ersten Hälfte des 20. Jahrhunderts vor allem von der Bourbaki-Gruppe vorangetrieben. ${ }^{36}$ Vor dem Hintergrund der auf den vorherigen Seiten geschilderten Entwicklungen betrachtete man die Mathematik als „Schatzkammer von abstrakten Formen“ (Bourbaki 1961b, S. 218). ${ }^{37}$ Als Grundlage für den Aufbau mathematischer Theorien dienen strukturierte Mengen. Einer Menge $M$ kann durch die Definition verschiedener Relationen und Abbildungen sowie die Auszeichnung bestimmter Teilmengen eine Struktur aufgeprägt werden: Relationen setzen gewisse Elemente einer Menge direkt in Beziehung zueinander, Abbildungen strukturieren Mengen häufig durch die Angabe von „Verknüpfungsregeln“ (d.h. die Verknüpfung zweier Elemente liefert ein wohlbestimmtes Element der gleichen Menge) und die Auszeichnung eines Teilmengensystems verbindet diejenigen Elemente einer Menge, die jeweils zu einer ausgezeichneten Teilmenge gehören (vgl. Grotemeyer 1965, S. 76-77). Je nach Art der Strukturierung werden drei Mutterstrukturen unterschieden: (1) algebraische Strukturen, (2) Ordnungsstrukturen und (3) toplogische Strukturen.

Algebraische Strukturen können mit Hilfe des Konzepts der Verknüpfung beschrieben werden. Ganz allgemein kann man eine Verknüpfung $\circ$ als eine Abbildung $\circ: A \times B \rightarrow C$ definieren. Man spricht dann von einer

- inneren Verknüpfung auf einer Menge $M$, falls $M:=A=B=C$, d. h. man erhält eine Abbildung $\circ_{1}: M \times M \rightarrow M$;

- äußeren Verknüpfung erster Art, falls nur $M:=B=C$ gefordert wird, d. h. man erhält eine Abbildung $\circ_{2}: A \times M \rightarrow M$;

- äußeren Verknüpfung zweiter Art, falls nur $M:=A=B$ gefordert wird, d. h. man erhält eine Abbildung ${ }_{3}: M \times M \rightarrow C .{ }^{38}$

Anstatt $c=\circ((a, b))$ schreibt man in der Regel einfach $c=a \circ b$.

\footnotetext{
${ }^{36}$ Nicolas Bourbaki war das Pseudonym einer Gruppe überwiegend französischer Mathematiker, die es sich zum Ziel gesetzt hatte, einen modernen Ansprüchen genügenden, systematischen Aufbau der Mathematik darzulegen. Ihr Hauptwerk war die 40-bändige Serie Éléments de mathématique. Einige Hintergrundinformationen finden sich bei Halmos (1957) und Wußing (2009, S. 483-487).

${ }^{37}$ Der Bourbaki-Artikel wurde ursprünglich 1948 in französischer Sprache veröffentlicht und erschien zwei Jahre später in englischer Übersetzung in der Zeitschrift The American Mathematical Monthly (Bourbaki 1950). Ich zitiere in dieser Arbeit aus der deutschen Übersetzung von K. Strubecker (Bourbaki 1961a, Bourbaki 1961b).

${ }^{38}$ Die Terminologie wurde von Steiner (1959, S. 33) übernommen.
} 


\section{Algebraische Struktur}

Eine Menge $M$, auf der eine oder mehrere Verknüpfungen definiert sind, heißt algebraische Struktur.

Die grundlegenden algebraischen Strukturen werden dabei zumeist durch eine Auswahl der folgenden Axiome charakterisiert:

- Assoziativgesetz: $(a \circ b) \circ c=a \circ(b \circ c)$ für alle $a, b, c \in M$

- Kommutativgesetz: $a \circ b=b \circ a$ für alle $a, b \in M$

- Existenz eines neutralen Elements $e$ mit der Eigenschaft $e \circ a=a \circ e=a$ für alle $a \in M$

- Existenz eines inversen Elements $a^{-1}$ zu jedem $a \in M$ mit der Eigenschaft $a^{-1} \circ a=$ $a \circ a^{-1}=e$

Bei algebraischen Strukturen, die durch zwei innere Verknüpfungen definiert sind (typischerweise mit + und · bezeichnet, z. B. bei Ringen und Körpern), existieren dann jeweils zwei Versionen der obigen Axiome (einmal bezogen auf die Verknüpfung + und einmal bezogen auf ·). Zudem wird in diesen Fällen häufig noch das Distributivgesetz gefordert:

- Distributivgesetz $I:(a+b) \cdot c=a \cdot c+b \cdot c$ für alle $a, b, c \in M$

- Distributivgesetz II: $a \cdot(b+c)=a \cdot b+a \cdot c$ für alle $a, b, c \in M$

Für Ordnungsstrukturen ist der Begriff der Relation von zentraler Bedeutung. Allgemein und mengentheoretisch formuliert ist eine $n$-stellige Relation $R$ zwischen den Mengen $M_{1}, \ldots, M_{n}$ eine Teilmenge des kartesischen Produkts $M_{1} \times \ldots \times M_{n}$. Man sagt, dass die Elemente $a_{i} \in M_{i}$ (für $\left.i=1, \ldots, n\right)$ in Relation zueinander stehen, wenn $\left(a_{1}, \ldots, a_{n}\right) \in R$ gilt. Von besonderem Interesse sind zweistellige Relationen $R \subseteq M_{1} \times M_{2}$ : Zwei Elemente $a \in M_{1}$ und $b \in M_{2}$ stehen in Relation zueinander, wenn $(a, b) \in R$. Verkürzt verwendet man dafür häufig die Schreibweise $a R b .^{39}$

\section{Ordnungsstruktur}

Eine Menge $M$ wird zu einer Ordnungsstruktur bzw. einer geordneten Menge, wenn auf ihr eine Ordnungsrelation $\preceq$ definiert ist, die folgende Axiome erfüllt:

\footnotetext{
${ }^{39}$ Der Abbildungsbegriff lässt sich als rechtseindeutige, zweistellige Relation auffassen, d. h. als Relation mit der zusätzlichen Eigenschaft $\left(a R b_{1} \wedge a R b_{2}\right) \Rightarrow b_{1}=b_{2}$ (vgl. Hischer 2012, S. 168), so dass man die bei algebraischen Strukturen vorkommenden Verknüpfungen formal auch als dreistellige Relationen betrachten kann.
} 


$$
\begin{aligned}
& O_{1}: \text { Für alle } x \in M \text { gilt } x \preceq x . \quad \text { (Reflexivität) } \\
& O_{2}: \text { Aus } x \preceq y \text { und } y \preceq x \text { folgt } x=y . \quad \text { (Anti-Symmetrie) } \\
& O_{3}: \text { Aus } x \preceq y \text { und } y \preceq z \text { folgt } x \preceq z . \quad \text { (Transitivität) }
\end{aligned}
$$

Zwei Beispiele für eine Ordnungsrelation sind die „Kleiner-gleich“-Beziehung (symbolisch: $\leq$ ) zwischen Elementen der , üblichen“ Zahlbereiche $(\mathbb{N}, \mathbb{Z}, \mathbb{Q}$ und $\mathbb{R})$ und die Teilmengen-Beziehung $\subseteq$, aufgefasst als Relation zwischen den Elementen der Potenzmenge einer Menge $M$. Die oben angegebene Definition einer Ordnungsrelation (häufig auch Halbordnung genannt) ist zwar sehr gebräuchlich, ${ }^{40}$ streng genommen aber keine Minimalanforderung, um von Ordnungsstrukturen sprechen zu können. So untersucht man häufig auch Relationen, die nur reflexiv und transitiv sind (Quasiordnung), oder bei denen man die Axiome $O_{1}$ und $O_{2}$ durch die Forderung der Irreflexivität (d.h. für kein $x \in M$ gilt $x \preceq x$ ) ersetzt (Striktordnung). ${ }^{41}$ Eine Totalordnung oder lineare Ordnung erhält man, wenn man den Axiomen $O_{1}$ bis $O_{3}$ noch die Forderung ,Es gilt stets entweder

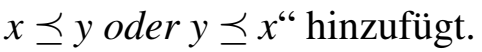

Eine topologische Struktur erhält man dadurch, dass man ein bestimmtes System von Teilmengen einer Menge $M$ durch gewisse Axiome auszeichnet. Es gibt verschiedene Möglichkeiten, topologische Strukturen axiomatisch zu definieren. ${ }^{42}$ Die folgende Charakterisierung findet sich häufig in neueren Lehrbüchern (z. B. Jänisch 2008, Ossa 2009 oder Laures und Szymik 2015).

\section{Topologische Struktur}

Ein System $\mathbb{T}$ von Teilmengen einer Menge $M$ nennt man eine Topologie auf $M$, wenn folgende Axiome erfüllt sind:

$T_{1}$ : Aus $U_{1}, U_{2} \in \mathbb{T}$ folgt $U_{1} \cap U_{2} \in \mathbb{T}$.

$T_{2}$ : Sei $I$ eine Indexmenge und $U_{i} \in \mathbb{T}$ für alle $i \in I$, dann gilt auch $\bigcup_{i \in I} U_{i} \in \mathbb{T}$.

$T_{3}$ : Es sind $\emptyset \in \mathbb{T}$ und $M \in \mathbb{T}$.

\footnotetext{
${ }^{40}$ Siehe unter anderem Bourbaki 1961b, S. 212, Meschkowski 1964, S. 119, Berghammer 2013, S. 13 sowie Schichl und Steinbauer 2009, S. 151.

${ }^{41}$ Strikte Ordnungsrelationen sind beispielsweise die Beziehung ,echt kleiner“ $(<)$ bei Zahlbereichen oder „echte Teilmenge“ $(\subset)$ bei Mengen. In der abstrakten Beschreibung verwendet man daher auch häufig anstelle von $\preceq$ das Symbol $\prec$.

${ }^{42}$ Siehe https://de.wikipedia.org/wiki/Axiomensysteme_der_Allgemeinen_Topologie (letzter Zugriff: 12.09.2017).
} 
Die drei Mutterstrukturen bzw. Mutterstruktur-Typen (denn jeder Typ umfasst je nach Auswahl der in Frage kommenden Axiome verschiedene Strukturen; vgl. Bourbaki 1961b, S. 214-215) lassen sich zu multiplen Strukturen kombinieren; so kann man beispielsweise algebraische Strukturen zusätzlich mit einer Ordnung und/oder Topologie ausstatten. Schließlich kann man die Elemente einer strukturierten Menge $M$, die bisher - abgesehen von den ihnen aufgeprägten Strukturen - nicht näher bestimmt wurden, noch konkreter inhaltlich interpretieren und gelangt so zu den „Theorien der klassischen Mathematik“ (Bourbaki 1961b, S. 215). Beispielsweise kann man die Menge der ganzen Zahlen $\mathbb{Z}$, versehen mit der Verknüpfung + und der Ordnungsrelation $\leq$, als konkrete Realisierung (d. h. als Modell) einer geordneten Gruppe auffassen.

„Die axiomatische Theorie einer so gegebenen Struktur aufstellen, läuft dann hinaus auf die Deduktion der logischen Folgerungen aus den Axiomen dieser Struktur, ohne Berücksichtigung irgendeiner weiteren Hypothese über die betrachteten Elemente oder die Natur dieser Elemente“ (Bourbaki 1961a, S. 166). Der wesentliche Vorteil dieser strukturtheoretischen Auffassung besteht darin, dass man speziellere Strukturen als Modelle abstrakterer Strukturen auffassen kann, wodurch eine „Hierarchie von Strukturen“ entsteht, die „vom Einfachen zum Komplizierten und vom Allgemeinen zum Besonderen geht“" (Bourbaki 1961b, S. 214; Herv. im Orig.). Die Aussagen, die sich aus den Axiomen einer allgemeineren Struktur deduzieren lassen, gelten automatisch auch für alle konkreteren Modelle dieser Strukturen: „Die Strukturen sind Werkzeuge für den Mathematiker; sobald er zwischen den Elementen, die er studiert, Relationen erkannt hat, die bekannten Axiomen genügen, hat er sofort das ganze Arsenal von allgemeinen Sätzen zur Verfügung, die zu den Strukturen dieses Typus gehören“; die axiomatische Darstellung einer derart aufgefassten Strukturtheorie hat eine „,beträchtliche Ökonomie des Denkens“ zur Folge (Bourbaki 1961b, S. 213).

\subsubsection{Grenzen der Axiomatik: Die Gödelschen Unvollständigkeitssätze}

[Die] Überzeugung von der Lösbarkeit eines jeden mathematischen Problems ist uns ein kräftiger Ansporn während der Arbeit; wir hören in uns den steten Zuruf: Da ist das Problem, suche die Lösung. Du kannst sie durch reines Denken finden; denn in der Mathematik giebt es kein Ignorabimus ${ }^{43}$ ! (Hilbert 1900, S. 262; Herv. im Orig.)

\footnotetext{
${ }^{43}$ Ignorabimus (lat.) = ,Wir werden nicht wissen“. Für nähere Informationen zur sogenannten Ignorabimus-Kontroverse siehe Tapp 2013, S. 12-14.
} 
Wie in Abschnitt 2.1.3 bereits angedeutet, wollte Hilbert die axiomatische Methode nutzen, um die Mathematik ein für allemal auf sichere Füße zu stellen. Seine im vorangestellten Zitat dargelegte erkenntnisoptimistische Überzeugung, dass sich jedes mathematische Problem letztlich lösen lasse (mit anderen Worten: dass sich jede Behauptung beweisen oder widerlegen lasse), äußert sich in seiner Forderung, widerspruchsfreie und vollständige Axiomensysteme aufzustellen. Das sogenannte Hilbertprogramm umfasst dabei zwei wesentliche Schritte (Hilbert 1964, S. 29; Herv. im Orig.):

(1) „Alles, was bisher die eigentliche Mathematik ausmacht, wird nunmehr streng formalisiert, so daß die eigentliche Mathematik oder die Mathematik in engerem Sinne zu einem Bestande an beweisbaren Formeln wird“.

(2) „, $\mathrm{Zu}$ dieser eigentlichen Mathematik kommt eine gewissermaßen neue Mathematik, eine Metamathematik, hinzu, die zur Sicherung jener dient [...]. In dieser Metamathematik kommt - im Gegensatz zu den rein formalen Schlußweisen der eigentlichen Mathematik - das inhaltliche Schließen zur Anwendung, und zwar zum Nachweis der Widerspruchsfreiheit der Axiome“.

Hilberts Vorhaben ist - so liest man häufig, beispielsweise bei Laugwitz (1965, S. 1517) - in Form der von Kurt Gödel (1906-1978) aufgestellten Unvollständigkeitssätze gescheitert, wodurch die Grenzen der Axiomatik klar aufgezeigt werden: Weder kann man die gesamte Mathematik in einem einzigen axiomatisch-formalen System abbilden, noch lässt sich ihre Widerspruchsfreiheit mit ausschließlich „,unverdächtigen“ logischen Mitteln sichern, die Hilbert in seiner Metamathematik nur zulassen möchte. ${ }^{44}$

„Ist das nicht eine schlagende Widerlegung des Axiomatizismus als Weltanschauung?“, könnte man nun wie Laugwitz (1965, S. 16) fragen. Eine pauschale Antwort auf diese (ebenso pauschal wie suggestiv formulierte) Frage ist schwierig, da eine oberflächliche Behandlung des Hilbertprogramms und der Gödelschen Arbeiten der Komplexität der Thematik nicht gerecht wird.

Eine nachvollziehbare Darstellung der Gödelschen Resultate wie auch des Hilbertprogramms, das den Anlass zu Gödels Untersuchungen bildete, erfordert - auch unter der Annahme eines mathematisch vorgebildeten Leserkreises - eine intensive Einführung in verschiedene Aspekte der mathematischen Logik, die weit außerhalb der Möglichkeiten dieser Arbeit liegt. Die folgenden Ausführungen sind daher als grober Einblick in die

\footnotetext{
${ }^{44}$ Unverdächtige logische Mittel sollen solche sein, die von den verschiedenen philosophischen Lagern gleichermaßen akzeptiert werden. Auf die Frage, was genau die gültigen Beweismittel für derartige Widerspruchsfreiheitsbeweise sein sollen, kann und soll an dieser Stelle nicht genauer eingegangen werden. Sie ist eng mit dem Konzept des Finitismus verknüpft, das unter anderem bei Tapp (2013, S. 135-153) genauer beschrieben wird.
} 
Grundgedanken der Gödelschen Resultate für interessierte Leserinnen und Leser gedacht. An verschiedenen Stellen wird auf weiterführende Literatur verwiesen. ${ }^{45}$

\section{Formalisierung der Mathematik}

Der erste Schritt des Hilbertprogramms betrifft die Formalisierung der Mathematik. Die Kernidee ist dabei, mathematische Aussagen als (nach bestimmten Vorgaben zusammengesetzte) Zeichenketten aufzufassen und Regeln dafür anzugeben, wie aus bereits vorhandenen, wohlgeformten Zeichenketten weitere Zeichenketten gebildet werden dürfen. Die so entstehenden formalen Systeme lassen sich grob wie folgt definieren:

Ein formales System besteht aus

1. einem Alphabet, welches die prinzipiell verwendbaren Symbolzeichen vorgibt,

2. einer entscheidbaren Menge ausgewählter Symbolketten (Terme und Formeln), die man die Sprache des Systems nennt,

3. einer entscheidbaren Menge ausgewählter Formeln, den Axiomen, und

4. einer endlichen Menge von Schlussregeln, nach denen aus bestimmten Formeln weitere Formeln gebildet werden können.

Der Begriff Entscheidbarkeit besagt dabei, dass ein Algorithmus existiert, der entscheidet, ob eine vorgegebene Zeichenkette eine Formel ist oder nicht.

Als Beispiel für ein formales System möchte ich die Peano-Arithmetik skizzieren, welche die Theorie der natürlichen Zahlen einschließlich der Addition und Multiplikation formalisiert und im Rahmen der Gödelschen Unvollständigkeitssätze eine wichtige Rolle spielt (eine ausführliche Darstellung, auf der die folgenden Ausführungen wesentlich beruhen, findet sich in Hoffmann 2013b, S. 134-146). Ihre Terme und Formeln lassen sich induktiv nach folgenden Regeln bilden:

\footnotetext{
${ }^{45}$ Als erste Orientierung sei Kahle 2007 empfohlen. Eine intensive Auseinandersetzung mit Gödels Originalarbeiten bietet Hoffmann 2013a. Eine tendenziell leichter verständliche und besser strukturierte Behandlung der Thematik stellt jedoch Hoffmann 2013b dar. Viele Ausführungen in diesem Abschnitt orientieren sich wesentlich an diesem Werk. Das Hilbertprogramm wiederum steht in Tapp $2013 \mathrm{im}$ Fokus.
} 
- $0, x_{1}, x_{2}, x_{3}, \ldots$ sind Terme.

- Sind $\sigma$ und $\tau$ Terme, so sind es auch

$$
s(\sigma),(\sigma+\tau),(\sigma \cdot \tau) .
$$

- Sind $\sigma$ und $\tau$ Terme, so ist $(\sigma=\tau)$ eine Formel.

- Sind $\varphi$ und $\psi$ Formeln, so sind es auch

$$
(\neg \varphi),(\varphi \wedge \psi),(\varphi \vee \psi),(\varphi \rightarrow \psi),(\varphi \leftrightarrow \psi),(\varphi \leftrightarrow \psi) .
$$

- Ist $\varphi$ eine Formel, dann sind auch $\forall \xi \varphi$ und $\exists \xi \varphi$ Formeln, wobei $\xi \in\left\{x_{1}, x_{2}, x_{3}, \ldots\right\}$

Die Axiome der Peano-Arithmetik sind in Theorie- und Logikaxiome aufgeteilt. Erstere sind die formalisierten Abbilder bekannter Eigenschaften der natürlichen Zahlen. Die Logikaxiome formalisieren zusammen mit den Schlussregeln die Prädikatenlogik erster Stufe und sind nötig, um neue gültige Aussagen bilden zu können. ${ }^{46}$

\section{Theorieaxiome:}

(S 1) $\sigma=\tau \rightarrow(\sigma=\rho \rightarrow \tau=\rho)$

(S 2) $\sigma=\tau \rightarrow s(\sigma)=s(\tau)$

(S 3) $\neg(0=s(\sigma))$

(S 4) $s(\sigma)=s(\tau) \rightarrow \sigma=\tau$

(S 5) $\sigma+0=\sigma$

(S 6) $\sigma+s(\tau)=s(\sigma+\tau)$

(S 7) $\sigma \cdot 0=0$

(S 8) $\sigma \cdot s(\tau)=(\sigma \cdot \tau)+\sigma$

(S 9) $\varphi(0) \rightarrow(\forall x(\varphi(x) \rightarrow \varphi(s(x))) \rightarrow \forall x \varphi(x))$

\section{Logikaxiome:}

(A 1) $\varphi \rightarrow(\psi \rightarrow \varphi)$

(A 2) $(\varphi \rightarrow(\psi \rightarrow \chi)) \rightarrow((\varphi \rightarrow \psi) \rightarrow(\varphi \rightarrow \chi))$

(A 3) $(\neg \varphi \rightarrow \neg \psi) \rightarrow(\psi \rightarrow \varphi)$

(A 4) $\forall \xi \varphi \rightarrow \varphi[\xi \leftarrow \sigma] \quad([\xi \leftarrow \sigma]$ kollisionsfrei $)$

(A 5) $\forall \xi(\varphi \rightarrow \psi) \rightarrow(\varphi \rightarrow \forall \xi \psi)(\xi \notin \varphi)$

\section{Schlussregeln:}

(MP) $\frac{\varphi, \varphi \rightarrow \psi}{\psi}$

(G) $\frac{\varphi}{\forall \xi \varphi}$

In den obigen Ausführungen sind $\sigma$ und $\tau$ Platzhalter für Terme, $\varphi, \psi$ und $\chi$ für Formeln und $\xi$ für eine Variable. Man beachte, dass erst die Substitution jedes solchen Platzhalters durch einen passenden Teilausdruck eine wohlgeformte Zeichenkette liefert. Ferner ist an dieser Stelle der Unterschied zwischen freien und gebundenen Variablen wichtig: „Eine Variable heißt gebunden, wenn sie im [...] Einflussbereich eines Quantors [ $\forall$ oder $\exists$, T.H.] steht. Sonst heißt sie frei“ (Bedürftig und Murawski 2012, S. 289; Herv. im Orig.). Nur Formeln, in denen keine freien Variablen vorkommen (also nur gebundene wie in

${ }^{46}$ Es ist für die Zwecke dieses Abschnittes nicht notwendig, alle Notationen (beispielsweise bei (A 4) und (A 5)) zu verstehen. Nähere Informationen zur Prädikatenlogik finden sich in Hoffmann 2013b, S. 103112. 
$\forall x_{1} \forall x_{2}\left(x_{1}+x_{2}=x_{2}+x_{1}\right)$ oder gar keine wie in $\left.s(0)=0\right)$, haben den Charakter von Aussagen; Formeln mit freien Variablen (z. B. $x_{1}=0$ ) haben dagegen den Charakter von Aussageformen.

Für das Arbeiten in formalen Systemen ist der Begriff der Ableitung bzw. des formalen Beweises essentiell (vgl. Hoffmann 2013b, S. 74):

Eine Ableitung bzw. ein formaler Beweis ist definiert als eine Kette von Aussagen $\varphi_{1}, \varphi_{2}, \ldots, \varphi_{n}$, wobei $\varphi_{i}$ entweder ein Axiom ist oder aus den vorangegangenen Gliedern der Beweiskette durch die Anwendung einer Schlussregel entstanden ist. Die letzte Aussage dieser Kette ist das bewiesene Theorem. Man sagt auch: $\varphi_{n}$ ist (in dem System) formal beweisbar bzw. ableitbar.

Einige in solcher Notation geführte Beweise für Theoreme der Peano-Arithmetik finden sich in Hoffmann 2013b, S. 143-146.

An dieser Stelle muss betont werden, dass wir uns bisher auf einer rein syntaktischen Ebene bewegt haben. Symbole wie $\rightarrow$ oder $\neg$ mögen an bekannte aussagenlogische Begriffe wie ,wenn, dann“ oder ,nicht“ erinnern. Ebenso liegt die arithmetische Bedeutung der Symbole $s,+$ und · nahe. In einem formalen System werden sie aber völlig ohne Bedeutung mechanisch (d.h. unter ausschließlicher Verwendung der definierten Schlussregeln) verwendet. Natürlich geht es bei der Formalisierung der Mathematik darum, formale Systeme derart aufzustellen, dass sie ein „Abbild“ der bekannten mathematischen Sätze und Schlussweisen darstellen. Damit kommt neben dem syntaktischen Aspekt eine semantische Perspektive hinzu: Man kann die verwendeten Symbole inhaltlich interpretieren, so dass wir beispielsweise im Falle der Peano-Arithmetik das (Standard-)Modell der natürlichen Zahlen erhalten, in dem $s$ für die Nachfolgerabbildung, + für die Addition und . für die Multiplikation stehen. Die Aussagen der (nicht formalisierten) inhaltlichen Mathematik lassen sich nach dem dort gültigen Prinzip der Zweiwertigkeit in wahre und falsche Aussagen unterteilen. Historisch gesehen war vor allem die Frage nach dem $\mathrm{Zu}-$ sammenhang zwischen der Wahrheit mathematischer Aussagen und ihrer (syntaktischen) Beweisbarkeit von Interesse.

\section{Die Gödelschen Resultate}

Nach dieser kursorischen Einführung in die wesentlichen Aspekte formaler Systeme am Beispiel der Peano-Arithmetik lassen sich einige Begriffe definieren, die für das Hilbertprogramm und Gödels Arbeiten grundlegend sind: 
Ein formales System heißt

- widerspruchsfrei, wenn es nicht möglich ist, in ihm eine Aussage $\varphi$ und ihre Negation $\neg \varphi$ abzuleiten.

- negationsvollständig (syntaktisch vollständig), wenn für jede Aussage $\varphi$ entweder $\varphi$ selbst oder $\neg \varphi$ abgeleitet werden kann.

- korrekt, wenn jede formal beweisbare Aussage $\varphi$ auch wahr ist.

- vollständig (semantisch vollständig), wenn jede wahre Aussage $\varphi$ auch formal beweisbar ist.

Mit diesen Begriffen lassen sich die Aussagen der beiden Gödelschen Unvollständigkeitssätze grob umreißen. Dabei ist zu beachten, dass der erste Gödelsche Unvollständigkeitssatz in der Literatur in unterschiedlichen Varianten formuliert wird, was leicht zu Missverständnissen führen kann. Ich möchte daher zwei wesentliche Varianten angeben und in Beziehung zueinander setzen (siehe auch Hoffmann 2013b, S. 202):

\section{Erster Gödelscher Unvollständigkeitssatz}

VERSION 1 (SEMANTISCH):

Jedes korrekte formale System, das die Peano-Arithmetik umfasst, ist (semantisch) unvollständig.

VERSION 2 (SYNTAKTISCH):

Jedes widerspruchsfreie formale System, das die Peano-Arithmetik umfasst, ist negationsunvollständig.

Da aus der Negationsunvollständigkeit die semantische Unvollständigkeit folgt und die Korrektheit eines formalen Systems seine Widerspruchsfreiheit impliziert (siehe dazu Hoffmann 2013b, S. 77), ergibt sich die semantische Variante des Satzes unmittelbar aus der syntaktischen. Dieser Zusammenhang wird in Abbildung 2.5 visualisiert.

Gödel selbst beweist in seinem Artikel Über formal unentscheidbare Sätze der Principia mathematica und verwandter Systeme I (Gödel 1931a) eine leicht abgeschwächte Variante der syntaktischen Version (siehe Hoffmann 2013b, S. 203). ${ }^{47}$

\footnotetext{
${ }^{47}$ Er setzt die so genannte $\omega$-Widerspruchsfreiheit des formalen Systems voraus, was eine stärkere Voraussetzung als die Widerspruchsfreiheit ist. Später konnte John Barkley Rosser (1907-1989) Gödels Beweis so modifizieren, dass sich das Unvollständigkeitsresultat auch unter der Annahme der ,normalen“ Widerspruchsfreiheit formulieren lässt (Rosser 1936; siehe auch Hoffmann 2013b, S. 225-228).
} 

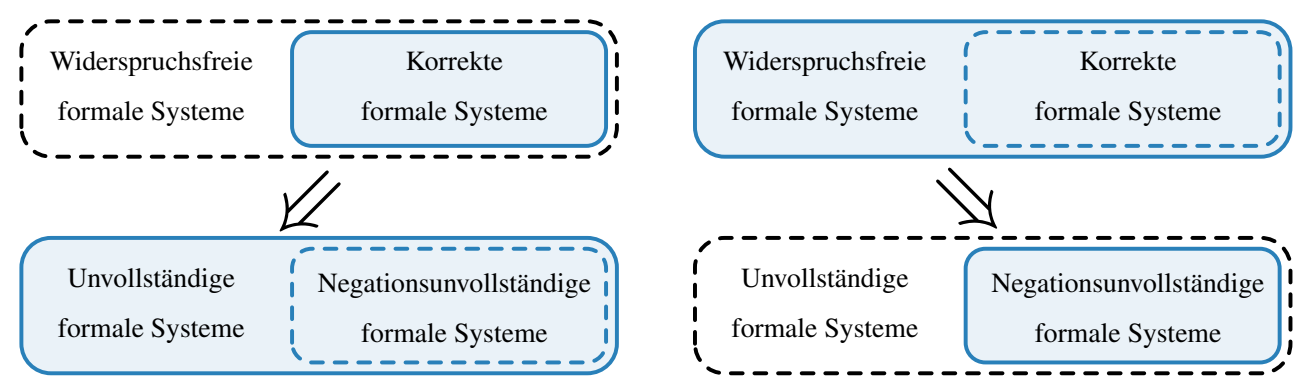

Abbildung 2.5: Erster Unvollständigkeitssatz: Semantische Variante (links) und syntaktische Variante $(\text { rechts })^{48}$

Unter anderem besagt der erste Unvollständigkeitssatz, dass die Konzepte der Ableitung bzw. (formalen) Beweisbarkeit und der Wahrheit in hinreichend ausdrucksstarken formalen Systemen ${ }^{49}$ nicht deckungsgleich sind, denn nur in korrekten, vollständigen Systemen wären diese in dem Sinne äquivalent, dass aus der formalen Beweisbarkeit einer Aussage ihre Wahrheit folgt und umgekehrt. Hilberts Vorhaben, die Mathematik so zu formalisieren, dass ihre Aussagen ,zu einem Bestande an beweisbaren Formeln“ werden (Hilbert 1964, S. 29), was der Forderung nach (semantischer) Vollständigkeit entspricht, ist also in dieser Form nicht realisierbar: Man kann nicht Widerspruchsfreiheit und Vollständigkeit zugleich erreichen. ${ }^{50}$

Da die Widerspruchsfreiheit eines formalen Systems die wichtigste aller Forderungen ist (denn aus widersprüchlichen Systemen lässt sich nach dem Prinzip ex falso quodlibet alles ableiten), könnte man noch die Hoffnung hegen, unter Aufgabe eines allzu umfassenden Vollständigkeitsanspruchs die Widerspruchsfreiheit zumindest wesentlicher Teile der Mathematik zu zeigen.

Auch dieses Vorhaben ist jedoch mit dem zweiten Gödelschen Unvollständigkeitssatz gescheitert, der hier in vereinfachter Form angegeben wird: ${ }^{51}$

\footnotetext{
${ }^{48}$ Quelle: entnommen aus Hoffmann 2013b, S. 202; leicht angepasst.

${ }^{49}$ Mit dieser Charakterisierung soll hier gemeint sein, dass das System mindestens die Peano-Arithmetik umfasst. Man kann diese Voraussetzung allerdings noch abschwächen, ohne die Gültigkeit der GödelSätze zu gefährden (vgl. Hoffmann 2013b, S. 253-254).

${ }^{50}$ Bei dieser Aussage spielt es nach dem zuvor Gesagten keine Rolle, ob hier die semantische oder syntaktische Vollständigkeit gemeint ist.

${ }^{51}$ Für ein genaueres Verständnis des zweiten Unvollständigkeitssatzes muss genauer geklärt werden, was es für ein formales System bedeutet, seine eigene Widerspruchsfreiheit zu beweisen. Gödels Grundidee bestand darin, eine Aussage Con in der Peano-Arithmetik zu formulieren, die genau dann wahr ist, wenn das zugrunde liegende formale System widerspruchsfrei ist. Wäre Con auch formal beweisbar, so würde man sagen, dass das formale System seine eigene Widerspruchsfreiheit beweisen kann. Der zweite Unvollständigkeitssatz besagt aber, dass Con in jedem widerspruchsfreien formalen System, das die Peano-Arithmetik umfasst, nicht ableitbar ist (vgl. Hoffmann 2013b, S. 242-245).
} 


\section{Zweiter Gödelscher Unvollständigkeitssatz}

Ein widerspruchsfreies formales System, das die Peano-Arithmetik umfasst, kann nicht seine eigene Widerspruchsfreiheit beweisen.

Da der Nachweis der Widerspruchsfreiheit eines hinreichend ausdrucksstarken formalen Systems nicht mit den Mitteln des Systems selber gelingen kann, ist er erst recht nicht mit noch schwächeren logischen Mitteln erreichbar, die Hilbert im Rahmen der Metamathematik ja nur zulassen wollte. Gödel selbst beschreibt die Kernaussage des zweiten Unvollständigkeitssatzes wie folgt: „,[E]in Widerspruchsfreiheitsbeweis für eines dieser Systeme $\mathfrak{S}$ kann nur mit Hilfe von Schlußweisen geführt werden, die in $\mathfrak{S}$ selbst nicht formalisiert sind. Für ein System, in dem alle finiten [...] Beweisformen formalisiert sind, wäre also ein finiter Widerspruchsfreiheitsbeweis, wie ihn die Formalisten suchen, überhaupt unmöglich“ (Gödel 1931b, S. 150-151).

\section{Häufige Missverständnisse}

Ich habe in den vorangehenden Ausführungen Wert darauf gelegt, trotz des beschränkten zur Verfügung stehenden Gesamtumfangs grobe Missverständnisse in Bezug auf den Aussagegehalt der Gödelschen Sätze zu vermeiden. Einige besonders häufig vorkommende, falsche Schlussfolgerungen möchte ich an dieser Stelle zusätzlich kurz kommentieren (siehe auch Hoffmann 2013b, S. 252-257):

- In der Mathematik gibt es wahre Sätze, die nicht beweisbar sind.

Bei der Beweisbarkeit ist zu beachten, dass es sich hierbei um einen Begriff handelt, der nur in Bezug auf ein zuvor festgelegtes formales System, bestehend aus Axiomen und Schlussregeln, definiert ist. Es gibt den Begriff unbeweisbar nicht in einem absoluten Sinne. Ist eine Aussage $\varphi$ in einem formalen System $S$ nicht beweisbar, so kann man ein neues formales System $\tilde{S}$ aufstellen, welches als zusätzliches Axiom $\varphi$ enthält, wodurch diese Aussage dann per Definition in $\tilde{S}$ beweisbar ist.

- In jedem formalen System existieren Aussagen, die nicht beweisbar sind, obwohl sie wahr sind. / Jedes formale System ist unvollständig.

Hier wird die wesentliche Voraussetzung unterschlagen, dass das System hinreichend ausdrucksstark sein muss. In der Regel ist damit gemeint, dass man zumindest über die additiven und multiplikativen Eigenschaften der natürlichen Zahlen reden können muss. Es gibt durchaus ausdrucksschwache Systeme, die widerspruchs- 
frei und vollständig sind. Ein Beispiel ist die Presburger-Arithmetik, deren TheorieAxiome den Axiomen (S 1) bis (S 6) sowie (S 9) der Peano-Arithmetik entsprechen und damit denjenigen Axiomen, die lediglich die additiven Eigenschaften der natürlichen Zahlen beschreiben (vgl. Hoffmann 2013b, S. 253-254).

- Die Widerspruchsfreiheit eines hinreichend ausdrucksstarken formalen Systems ist nicht beweisbar.

Der zweite Gödelsche Unvollständigkeitssatz besagt, dass die Widerspruchsfreiheit eines hinreichend ausdrucksstarken formalen Systems mit den Mitteln des formalen Systems selbst nicht beweisbar ist. Es ist aber möglich, die Widerspruchsfreiheit eines formalen Systems mit nicht-finiten Mitteln nachzuweisen, die in dem System selbst nicht formalisiert sind. So gelang es beispielsweise Gerhard Gentzen (19091945), die Widerspruchsfreiheit der Peano-Arithmetik unter Hinzunahme des Prinzips der transfiniten Induktion nachzuweisen, welches in der Peano-Arithmetik jedoch nicht formalisierbar ist (Gentzen 1936). Ferner muss auch noch die Möglichkeit in Betracht gezogen werden, dass es ,,auf der metamathematischen Ebene Beweismittel gibt, die sich nicht in der betrachteten Theorie formalisieren lassen, aber dennoch finit zulässig sind“ (Tapp 2013, S. 317). Hilbert hat für seine Metamathematik - jene „unverdächtigen“ logischen Mittel, mit denen formale Systeme auf ihre Widerspruchsfreiheit untersucht werden sollen - explizit keine Formalisierung gefordert. Der zweite Gödelsche Unvollständigkeitssatz macht aber nur eine Aussage über die Unmöglichkeit eines Widerspruchsfreiheitsnachweises mit den formalisierten Mitteln des betrachteten Systems.

Diese Beispiele zeigen, dass es bei einer adäquaten Einschätzung der Auswirkungen der Unvollständigkeitssätze auf das Hilbertprogramm auch auf sprachliche Präzision ankommt. Dies erklärt vielleicht auch die unterschiedlichen Positionen, die in der Sekundärtliteratur in Bezug auf den Zusammenhang zwischen Gödel und dem Hilbertprogramm (HP) auftreten (Tapp 2013, S. 312):

(GH1) Die Gödelsätze haben das HP ohne Einschränkung zum Scheitern gebracht.

(GH2) Die Gödelsätze haben das ursprüngliche HP zum Scheitern gebracht.

(GH3) Die Gödelsätze zeigen, daß im HP Beweismittel verwendet werden müssen, die über die untersuchte Theorie hinausgehen.

(GH4) Die Gödelsätze zeigen, daß im HP Beweismittel verwendet werden müssen, die über die striktest finiten Methoden hinausgehen.

(GH5) Die Gödelsätze zeigen, daß im HP der finite Standpunkt „,in einer schärferen Weise" ausgenutzt werden muß. 
Ich möchte auf die einzelnen Standpunkte nicht näher eingehen, jedoch erscheint die Schlussfolgerung einleuchtend, „daß der Mainstream der Logiker und Mathematikphilosophen das ursprüngliche HP mit den Gödelsätzen mehr oder weniger für erledigt hält"“ (Tapp 2013, S. 313; Herv. im Orig.). Zugleich wird jedoch deutlich, dass Hilberts Vorhaben immerhin in modifizierter Form nicht zum Scheitern verurteilt sein muss.

\section{Fazit: Grenzen der Axiomatik?}

Fest steht, dass die Axiomatik (bezogen auf eine formalisierte Mathematik) nicht länger als die Methode zur zweifelsfreien Sicherung der gesamten Mathematik betrachtet werden kann. Die Gödelschen Unvollständigkeitssätze zeigen klar die Grenzen der Bemühungen auf, Vorstellungen von Wahrheit umfassend durch formalistische Konzepte wie Widerspruchsfreiheit und Ableitbarkeit zu ersetzen. Als Ordnungsprinzip zur systematischen und strukturierten Darstellung von Theorien ist die Axiomatik in der Mathematik jedoch nach wie vor unverzichtbar (siehe auch Abschnitt 2.2.1). Hier hat die Idee der ontologische Unbestimmtheit mathematischer Objekte große Früchte getragen.

Die Erkenntnis, dass es eine letzte Sicherheit in der Mathematik nicht gibt, hat auf die mathematische Praxis allerdings nur geringen Einfluss. Verhulst (2010, S. 242) hält fest:

Die meisten Mathematiker sind zufrieden damit, dass sie an die Arbeit gehen können mit:

- einigen Grundbegriffen wie Punkt, Gerade und Zahl [...];

- einem geeigneten System von Axiomen als Ausgangspunkt für Argumentationen;

- dem Gebrauch von Techniken wie vollständige Induktion [...].

Eine treffende Beschreibung liefert auch Heintz (2000, S. 69), die eine von vielen Mathematikerinnen und Mathematikern in Gesprächen eingenommene Haltung wie folgt zusammenfasst:

Die Gödel-Sätze sind mir völlig egal! [...] Ich bin sehr komfortabel mit dem Gedanken, dass man nie imstande sein wird, die Widerspruchslosigkeit der Mathematik zu beweisen. Wir haben etwas geschaffen, die Mathematik. Wir können nicht beweisen, dass sie widerspruchsfrei ist. Das bedeutet: da ist etwas mächtiger als wir. Und das geht. Es könnte natürlich auch fehlgehen, einmal. Das können wir nicht sagen. Ich habe völliges Vertrauen, aber ich kann es nicht beweisen. Niemand kann es beweisen. Niemand wird je fähig dazu sein, das zu beweisen - denn ich glaube an den Beweis der Gödelschen Sätze! 


\subsection{Mathematikphilosophische Aspekte}

Wie in der Einleitung dieser Arbeit bereits angedeutet, wird den Aussagen der Mathematik ein besonders hohes Maß an Sicherheit zugeschrieben. Die Mathematik zeichnet sich dabei als Wissenschaft nach Ansicht Vieler durch folgende Charakteristika aus (siehe auch Heintz 2000, S. 17-23, Leuders 2011, S. 18 und Shapiro 2000, S. 21-24):

- Mathematisches Wissen beruht nicht auf (empirischen) Erfahrungen, sondern auf reinem Denken. In diesem Sinne ist es ein Wissen a priori.

- Die Objekte, mit denen sich die Mathematik beschäftigt, sind nicht physikalischer Natur. Ob sie dabei außerhalb von Raum und Zeit in einem abstrakten Ideenreich existieren (Platonismus) oder bloße Konstruktionen des menschlichen Geistes sind (Konstruktivismus), wird unterschiedlich gesehen.

- Die Mathematik zeichnet sich durch ein hohes Maß an kognitiver Kohärenz und sozialem Konsens aus. Obwohl soziologische Ansätze auf die Bedeutung der Gemeinschaft zur Akzeptanz mathematischer Ergebnisse hinweisen, ${ }^{52}$ scheint es innerhalb dieser Wissenschaft wenig, ,interpretative Flexibilität“ (Heintz 2000, S. 20) zu geben. Streitigkeiten sind selten und werden in der Regel schnell entschieden.

- Das Validierungsinstrument der Mathematik ist der Beweis, welcher mathematischen Resultaten ihren „zwingenden und objektiven Charakter“ (Leuders 2011, S. 18; Herv. im Orig.) verleiht: ,[A] mathematical theorem, once proved, is established once and for all“" (Hempel 1945, S. 7).

- Insbesondere ist mathematisches Wissen daher kumulativ. Es unterliegt keinem Prozess der Revision oder gar Falsifikation, wie dies in empirisch arbeitenden Wissenschaften der Fall ist: „A new mathematical theory may lead to the abandonment of an older one by making it appear uninteresting or perhaps superfluous, but never wrong“" (Corry 1989, S. 419).

$\mathrm{Zu}$ jedem dieser Punkte gibt es natürlich Gegenpositionen und nur wenige Mathematiker würden dem soeben skizzierten Bild der Mathematik uneingeschränkt zustimmen. Dennoch scheint der allgemeine Glaube an die Zuverlässigkeit mathematischer Aussagen hinreichend groß zu sein, um ihr einen Sonderstatus unter den Wissenschaften zu garantieren.

\footnotetext{
${ }^{52}$ „The compelling force of mathematical procedures does not derive from their being transcendent, but from their being accepted and used by a group of people. The procedures are not accepted because they are correct, or correspond to an ideal; they are deemed correct because they are accepted“ (Bloor 1983, S. 92).
} 
Eine naheliegende Frage ist, warum die Mathematik (scheinbar) so sicher ist. Der Versuch einer Antwort führt unweigerlich zu einer Auseinandersetzung mit den Grundlagen der Mathematik und damit einhergehenden philosophischen Themen. Aus der Mathematik stehen hier elementare Konzepte wie der Zahl-, Größen- und Mengenbegriff sowie die Unendlichkeit im Fokus. Übergreifende philosophische Fragestellungen gibt es viele. Im Folgenden ist eine Beispielliste wichtiger Fragen aufgeführt:

Was ist Mathematik? Gibt es mathematische Objekte? Wie sind sie beschaffen und wo sind sie lokalisiert? Sind sie Teil der physikalischen Welt, existieren sie bloß im Kopf der einzelnen Mathematiker oder sind sie soziale Objekte vergleichbar mit anderen kulturellen Artefakten? Aufgrund welcher Kriterien werden bestimmte Entitäten als mathematische Objekte definiert und andere nicht? Wo verläuft die Grenzlinie zwischen Mathematik und theoretischer Physik? Wie gelangen Mathematiker zu mathematischem Wissen durch eine spezifisch mathematische Intuition, durch Generalisierung empirischer Sachverhalte oder durch bloße Setzung? Aufgrund welcher Kriterien entscheiden sie, ob eine mathematische Aussage wahr ist oder falsch? Weshalb, so eine weitere Frage, erweist sich ein Wissen, das autonom und völlig losgelöst von empirischen Bezügen entwickelt wurde, immer wieder als brauchbar für die Beschreibung der empirischen Wirklichkeit? Und weshalb kommt es umgekehrt dennoch nie dazu, dass mathematische Aussagen durch empirische Fakten falsifiziert werden? (Heintz 2000, S. 36)

Die meisten Teilfragen lassen sich drei Themenkomplexen zuordnen: (1) der Existenz mathematischer Objekte (Ontologie), (2) dem Wahrheitsbegriff in der Mathematik (Epistemologie) und (3) dem Verhältnis von Mathematik und Realität. Es ist leicht zu erkennen, dass alle drei Aspekte eng mit der Rolle der Axiomatik in der Mathematik verknüpft sind. In diesem Abschnitt möchte ich einige grundlegende Positionen der Mathematikphilosphie, die für das Thema der vorliegenden Arbeit wichtig sind, genauer beleuchten, ohne jedoch den Anspruch einer erschöpfenden Darstellung zu erheben. Meine Ausführungen beruhen dabei wesentlich auf den sehr guten und übersichtlichen Schilderungen in Heintz 2000. Als Orientierung dienen folgende Leitfragen:

(1) In welchem Sinne existieren mathematische Objekte?

(2) Welcher Geltungsanspruch ist mit der Kennzeichnung einer mathematischen Aussage als wahr oder falsch verbunden?

(3) Welche Kriterien zur Rechtfertigung mathematischer Theorien gibt es?

Während ich der Existenzfrage im Folgenden einen eigenen Abschnitt widme, werden die Fragen (2) und (3) anschließend gemeinsam behandelt. Abschließend folgen einige Ausführungen über sogenannte quasi-empiristische Positionen, welche ebenfalls die Fra- 
gen nach dem Geltungsanspruch und den Rechtfertigungsmöglichkeiten mathematischer Theorien beleuchten.

\section{Existenz mathematischer Objekte}

Die philosophischen Standpunkte zur Existenz mathematischer Objekte lassen sich in die drei folgenden Ansätze gruppieren: Der Realismus spricht mathematischen Objekten eine (noch genauer zu erläuternde) Existenz zu, der Intuitionismus fasst sie als mentale Konstruktionen auf und der Nominalismus sieht in ihnen bloße sprachliche und symbolische Konventionen (vgl. Heintz 2000, S. 38). Ich werde den Platonismus als wichtigste Variante des Realismus sowie den Formalismus als bekanntesten Vertreter des Nominalismus genauer erläutern. Auf den Intuitionismus werde ich im Rahmen ontologischer Fragestellungen nicht näher eingehen. Damit ist nicht gesagt, dass konstruktive Aspekte beim Mathematiktreiben nicht wichtig ist. Als philosophische Grundposition ist der Intuitionismus aber - unter anderem aufgrund der Ablehnung des aktual Unendlichen und einiger klassischer logischer Schlussweisen - mit weitreichenden inhaltlichen Einschränkungen verbunden, die ihn für weite Teile der mathematischen Praxis unpraktikabel machen (vgl. Heintz 2000, S. 67-69). ${ }^{53}$

Der Platonismus lässt sich in fünf Thesen kompakt zusammenfassen (Heintz 2000, S. 39; siehe auch Irvine 1990, S. xix-xx):

(1) Die Objekte der Mathematik existieren unabhängig von uns und unserem Bewusstsein.

(2) Die Objekte der Mathematik sind nicht physikalischer Natur. Sie existieren außerhalb von Zeit und Raum und sind uns über unsere Sinne nicht zugänglich.

(3) Mathematische Aussagen sind entweder wahr oder falsch, und zwar unabhängig von unserer Kenntnis des jeweiligen Wahrheitswertes.

(4) Der Wahrheitswert einer mathematischen Aussage ergibt sich aus der Beschaffenheit der mathematischen Objekte, auf die sich die Aussage bezieht.

(5) Es ist uns möglich, mathematische Objekte zu erkennen.

Der Platonismus ,liefert eine praktikable Erklärung für die Erfahrung [praktizierender Mathematiker, T.H.], es mit einer eigenständigen - und manchmal auch widerständigen

\footnotetext{
${ }^{53}$ Beispielsweise lehnen Intuitionisten das Prinzip der Zweiwertigkeit ab, dem zufolge jede Aussage entweder wahr oder falsch ist. Damit werden auch sämtliche Widerspruchsbeweise grundsätzlich abgelehnt, zu denen der Intuitionismus jedoch häufig keine Alternativbeweise liefert (vgl. Verhulst 2010, S. 238).
} 
- Wirklichkeit zu tun zu haben“ (Heintz 2000, S. 40): Mathematiker entwickeln keine Theorien, sondern sie entdecken mathematische Wahrheiten.

Vom philosophischen Standpunkt aus bereitet er jedoch einige Probleme. Ein kritischer Aspekt ist die Kombination ontologischer (siehe (1) und (2)) und epistemologischer (siehe (3), (4) und (5)) Aspekte. ${ }^{54}$ Es drängt sich nämlich die Frage auf, wie wir irgendeine Art von Erkenntnis über die Beschaffenheit mathematischer Objekte - und damit über die Wahrheit oder Falschheit mathematischer Aussagen - erlangen können (dies wird durch (4) und (5) postuliert), wenn diese doch unabhängig vom menschlichen Denken existieren, raum-zeitlich nicht gebunden und damit sinnlich nicht wahrnehmbar sind (siehe (1) und (2)). Ein zweites Problem betrifft die breite Anwendbarkeit mathematischer Theorien auf die reale, empirisch erfassbare Welt: „Wie lässt sich erklären, dass Theorien, die sich auf eine nicht-physikalische Wirklichkeit beziehen und wahr sind in Hinblick auf diese, sich immer wieder als brauchbar erweisen für die Modellierung der physikalischen Wirklichkeit“ (Heintz 2000, S. 41)?

Denkt man an Platons berühmtes Höhlengleichnis (siehe Platon 2010, S. 303-307), könnte man auf diese beiden Fragen antworten, dass man immerhin in der Lage ist, in der empirisch erfahrbaren Welt die „Schatten“ der ideellen Objekte wahrzunehmen und somit einen Zugang zur mathematischen Wirklichkeit zu erlangen. Einer der prominentesten modernen Vertreter des Platonismus ist Kurt Gödel, über dessen Unvollständigkeitssätze im letzten Abschnitt bereits berichtet wurde. Er glaubt an die Existenz einer mathematischen Intuition, durch die der Mensch mathematisches Wissen erlangt:

[D]espite their remoteness from sense experience, we do have something like a perception also of the objects of set theory, as is seen from the fact that the axioms force themselves upon us as being true. I don't see any reason why we should have less confidence in this kind of perception, i.e., in mathematical intuition, than in sense perception [...]. (Gödel 1947/1983, S. 483-484)

Unabhängig davon, wie genau man nun die menschliche „Erkenntnisfähigkeit“ der platonischen Wirklichkeit beschreibt, ergibt sich zwangsläufig ein Konflikt mit These (2), welche die prinzipielle empirische Unzugänglichkeit mathematischer Objekte postuliert. ${ }^{55}$

\footnotetext{
${ }^{54}$ Gelegentlich wird auch zwischen einem ontologischen und einem epistemologischen Platonismus unterschieden (siehe beispielsweise Steiner 1973).

${ }^{55}$ Eine Lösung besteht darin, mathematischen Objekten eine empirische Existenz zuzusprechen (mit anderen Worten: These (2) explizit abzulehnen). Diesen Ansatz verfolgt der Physikalismus. Die häufig erwähnte ,unreasonable effectiveness“ der Mathematik (Wigner 1960), die für den Platonisten ein Problem darstellt, erklären Physikalisten mit dem Argument, dass die hohe Relevanz der Mathematik für physikalische Theorien eine irgendwie geartete reale Existenz mathematischer Objekte impliziert. Der ontische Status mathematischer Objekte wird dabei häufig mit dem Status theoretischer Entitäten wie Elektronen in der Physik verglichen, ,die theoretisch postuliert werden, dem Auge jedoch aus prinzipiellen Gründen nicht zugänglich sind“ (Heintz 2000, S. 42). Eine solche Sichtweise, die die Mathe-
} 
Der Formalismus löst die Probleme, mit denen sich alle Formen des Realismus konfrontiert sehen, durch eine Entontologisierung des Existenz- und Wahrheitsbegriffs:

Durch die Jahrhunderte hatten die Mathematiker ihre Objekte [...] als „Dinge an sich" betrachtet. Da diese Objekte aber den Versuchen, sie angemessen zu definieren, von jeher getrotzt haben, dämmerte es den Mathematikern des 19. Jahrhunderts allmählich, daß die Frage nach der Bedeutung dieser Objekte als „,wirkliche Dinge“ für die Mathematik keinen Sinn hat - wenn sie überhaupt einen hat. Die einzigen sinnvollen Aussagen über sie beziehen sich nicht auf die dingliche Realität; sie betreffen nur die gegenseitigen Beziehungen zwischen undefinierten Objekten und die Regeln, die die Operationen mit ihnen beherrschen. Was Punkte, Linien, Zahlen ,wirklich“ sind, kann und braucht in der mathematischen Wissenschaft nicht erörtert zu werden. Worauf es ankommt und was ,nachprüfbaren“ Tatsachen entspricht, ist Struktur und Beziehung, etwa, daß zwei Punkte eine Gerade bestimmen, daß aus Zahlen nach gewissen Regeln andere Zahlen gebildet werden, usw. Eine klare Einsicht in die Notwendigkeit, die elementaren mathematischen Begriffe ihrer Dinglichkeit zu entkleiden, ist eines der fruchtbarsten Ergebnisse der modernen Entwicklung der Axiomatik. (Courant und Robbins 2010, S. xxii)

Über den formalistischen Standpunkt, wie er häufig am Beispiel von Hilberts Grundlagen der Geometrie illustriert wird, wurde in Abschnitt 2.1.3 bereits ausführlicher berichtet. In Bezug auf den ontischen Status mathematischer Objekte lässt sich festhalten, dass für (radikale) Formalisten „Existenz im mathematischen Sinne nichts anderes bedeute[t] als Widerspruchsfreiheit. Hiermit ist gemeint, dass für die Mathematik keine philosophische Existenzfrage besteh[t]“" (Bernays 1950/1976, S. 92).

Für manche Mathematiker mag der Formalismus ein Standpunkt sein, auf den man sich „zurückziehen“ kann, wenn man mit den philosophischen Problemen anderer Sichtweisen konfrontiert wird. Ein berühmtes Zitat besagt, dass der typische Mathematiker ,an Werktagen Platonist und an Sonntagen Formalist ist“" (Davis und Hersh 1996, S. 337).

Das heißt, daß er, wenn er aktiv Mathematik betreibt, überzeugt ist, daß er es mit einer objektiven Realität zu tun hat, deren Eigenschaften er zu ergründen sucht. Wird er jedoch mit der Forderung konfrontiert, eine philosophische Darlegung dieser Realität zu geben, findet er es doch einfacher vorzugeben, daß er letztlich nicht an sie glaubt. (Davis und Hersh 1996, S. 337)

matik zumindest in die Nähe empirischer Wissenschaften rückt und damit die apriorische Auffassung mathematischen Wissens ablehnt, impliziert jedoch zugleich, dass mathematische Theorien prinzipiell falsifizierbar sind. De facto ist es aber noch nie vorgekommen, dass mathematische Resultate durch empirische Beobachtungen widerlegt wurden. Beispielsweise mag die euklidische Geometrie nicht geeignet sein, um den uns umgebenden dreidimensionalen Raum in kosmischen Maßstäben adäquat zu beschreiben; damit ist sie als mathematische Theorie aber keineswegs falsifiziert. Ferner steht der Physikalismus vor dem Problem, den Bereich der reinen Mathematik überhaupt nicht abzudecken (vgl. Heintz 2000, S. 42-47 und Maddy 1990, S. 28-31). 
Der Formalismus mag zwar philosophisch bequemer sein, die Substitution des Existenzbegriffs durch das Konzept der Widerspruchsfreiheit ist jedoch vor dem Hintergrund des zweiten Gödelschen Unvollständigkeitssatzes (siehe Abschnitt 2.2.2) problematisch. Da man die Widerspruchsfreiheit weiter Teile der Mathematik nicht zweifelsfrei nachweisen kann, ist in der formalistischen Auffassung direkt die Existenz der mathematischen Welt in Frage gestellt. ${ }^{56}$

\section{Geltungsanspruch und Rechtfertigung mathematischer Theorien}

Die Einstellung von Mathematikern hinsichtlich der Existenz mathematischer Objekte ist eng mit Fragen zum Wahrheitsbegriff in der Mathematik und dem Geltungsanspruch der dort getroffenen Aussagen verbunden.

Mathematische Aussagen gelten gemeinhin dann als gesichert, ,wenn es gelingt, sie anhand einer Reihe von Ableitungsregeln Schritt für Schritt aus einer Menge von als wahr erkannten Ausgangssätzen abzuleiten, d.h. zu beweisen“ (Heintz 2000, S. 53). Damit wird die Frage nach der Wahrheit mathematischer Aussagen aber keinesfalls gelöst, sondern auf die Frage nach der Wahrheit der Ausgangssätze, also der Axiome, verlagert: „What the rigorous proof of a theorem [...] establishes is not the truth of the proposition in question but rather a conditional insight to the effect that that proposition is certainly true provided that the postulates are true" (Hempel 1945, S. 8-9; Herv. im Orig.). Gerade an der Frage, inwiefern die Ausgangssätze einer mathematischen Theorie als wahr bezeichnet werden können, scheiden sich jedoch die Vertreter unterschiedlicher Auffassungen: „Die Sicherheit der Mathematik liegt [...] nicht in ihren Aussagen, sondern in ihren Schlüssen. [...] Die Akzeptabilität der Hypothesen, die man in der Mathematik benutzt, ist eine Sache der Bewertung“(Jahnke 2009, S. 27; Herv. im Orig.).

In der platonistischen Auffassung wird die Wahrheit der Axiome über deren Selbstevidenz in Bezug auf ein metaphysisches Ideenreich begründet. Mathematische Aussagen haben damit einen universellen Geltungsanspruch: „Beweise vermitteln uns deshalb Wissen über die mathematische Wirklichkeit, weil ihre axiomatische Basis in einem korrespondenztheoretischen Sinne wahr ist“" (Heintz 2000, S. 58). Für den Platonismus entsteht damit jedoch ein Spannungsfeld zwischen den Begriffen der Wahrheit und Beweisbarkeit. Die Idealvorstellung, dass ein Satz genau dann wahr ist, wenn er beweisbar ist, kollidiert mit der für den Platonismus charakteristischen Prämisse, dass mathematische Aussagen genau dann wahr sind, wenn sie die mathematische Wirklichkeit adäquat beschreiben.

\footnotetext{
${ }^{56}$ Streng genommen müsste hier sprachlich genauer erklärt werden, was ,Zweifelsfrei nachweisen“ bedeutet, siehe dazu Abschnitt 2.2.2.
} 
Dieses von Tait (1986) auch als Truth/Proof problem bezeichnete Dilemma ergibt sich auf den ersten Blick im Formalismus nicht. Dort findet eine „Interiorisierung der Wahrheitsinstanz" (Heintz 2000, S. 50) statt. Für radikale Formalisten stellt sich die Frage nach der Wahrheit einer mathematischen Aussage nicht bzw. sie ist identisch mit der Frage nach der Widerspruchsfreiheit des zugrunde liegenden Axiomensystems und der formalen Beweisbarkeit der Aussage. Dies ändert sich jedoch, sobald man neben syntaktischen auch semantische Gesichtspunkte betrachtet: Die Gödelschen Unvollständigkeitssätze (siehe Abschnitt 2.2.2) haben gezeigt, dass die Konzepte der Wahrheit und der (formalen) Beweisbarkeit nicht in Einklang gebracht werden können. ${ }^{57}$

Doch selbst, wenn man sich auf ein radikal formalistisches Mathematikbild einlässt, stellt sich die Frage nach der Rechtfertigung axiomatisch aufgebauter Theorien: Wenn mathematische Aussagen nichts weiter sind als nach syntaktischen Regeln zusammengesetzte Symbolketten, deren Zeichen keine inhaltliche Bedeutung haben und ontologisch unbestimmt sind, wieso setzen sich dann bestimmte Axiomensysteme bzw. Theorien durch und andere nicht? Widerspruchsfreiheit kann als rein systemimmanentes Kriterium diesen Umstand nicht erklären. Wäre Mathematik eine rein syntaktische Angelegenheit, „dann würde [sie] keinen intelligenten Menschen anziehen. Sie wäre eine Spielerei mit Definitionen, Regeln und Syllogismen ohne Ziel und Sinn“ (Courant und Robbins 2010, S. xxi). Selbst erklärte Formalisten wie Haskell B. Curry (1900-1982) betonen: „Neither mathematics nor any other science can develop in complete isolation“ (Curry 1951, S. 56). Wenn es um die Frage gehe, welche formalen Systeme für Mathematiker von Interesse sind, sei die Annehmbarkeit (acceptability) einer Theorie der wesentliche Faktor: „By acceptability [...] I mean the considerations which lead us to choose one formal system rather than another" (Curry 1951, S. 59). Solche Überlegungen erfordern jedoch immer eine inhaltliche Interpretation einer formalen Theorie und umfassen - wenn es nicht um Anwendungen auf ein anderes mathematisches Themengebiet geht - auch außermathematische Argumente wie die Passung mit empirischen Messungen (vgl. Curry 1951, S. 59-62).

Ein wichtiger Vertreter dieser Art von Rechtfertigung einer Theorie aufgrund ihrer Nützlichkeit ist Gödel. Neben Axiomen, die über die mathematische Intuition als evident betrachtet werden können, gebe es auch solche, die sich einer unmittelbaren Rechtfertigung entziehen. In diesen Fällen müsse man auf andere Weise über ihre „Wahrheit“" entscheiden:

\footnotetext{
${ }^{57}$ Eng mit den Gödelschen Resultaten verbunden ist Alfred Tarskis (1901-1983) Nachweis, dass sich der Wahrheitsbegriff in einer formalen Sprache nicht definieren lässt (siehe Tarski 1936). Ich werde darauf an dieser Stelle nicht weiter eingehen; einige Erläuterungen dieses Satzes in Bezug auf die PeanoArithmetik finden sich in Hoffmann 2013b, S. 233-238.
} 
[A] probable decision about its [a new axiom's, T.H.] truth $^{58}$ is possible also in another way, namely, inductively by studying its "success." Success here means fruitfulness in consequences, in particular in "verifiable" consequences, i. e., consequences demonstrable without the new axiom, whose proofs with the help of the new axiom, however, are considerably simpler and easier to discover, and make it possible to contract into one proof many different proofs. [...] There might exist axioms so abundant in their verifiable consequences, shedding so much light upon a whole field, and yielding such powerful methods for solving problems [...] that [...] they would have to be accepted at least in the same sense as any well-established physical theory. (Gödel 1947/1983, S. 477; Herv. d. Verf.)

Gödels erkenntnistheoretische Ansichten sind also zweistufig (vgl. Maddy 1990, S. 33): Einige Axiome sind intuitiv direkt einleuchtend und daher als wahr gerechtfertigt. Andere Axiome sind jedoch nicht offensichtlich wahr und werden daher über die aus ihnen herleitbaren Konsequenzen gerechtfertigt. Aufbauend auf Gödels Ausführungen spricht Maddy (1990) von zwei grundsätzlichen Rechtfertigungsmethoden in der Mathematik. Ein Axiom, welches als intuitiv einleuchtend erachtet wird, nennt sie intrinsisch gerechtfertigt. Ein Axiom, dessen Akzeptanz über die Nützlichkeit der aus ihm deduzierbaren Konsequenzen begründet wird, nennt sie extrinsisch gerechtfertigt, d. h. ,justified by their role in our theory, by their explanatory power, their predictive success, their fruitful interconnections with other well-confirmed theories, and so on“ (Maddy 1990, S. 32).

Maddy hat das Zusammenspiel von intrinsischen und extrinsischen Rechtfertigungsmustern eingehend am Beispiel der Mengenlehre diskutiert (Maddy 1988a,b, 1990, 2011). Gerade in der modernen Mathematik spielen extrinsische Argumente für die Auswahl der Axiome eine fundamentale Rolle. Während einige ,simple“ Axiome wie das Extensionalitätsaxiom ${ }^{59}$ noch intrinsisch gerechtfertigt würden - Maddy nennt es das ,,most obvious of axioms“" (Maddy 1988a, S. 484) -, gründe sich die Verwendung komplexerer Axiome wie des Unendlichkeitsaxioms ${ }^{60}$ in deren Fruchtbarkeit für die weitere Theorieentwicklung. Neben der Vermeidung bzw. der Beseitigung von Paradoxien stünde häufig auch die Frage nach der Beweisbarkeit bestimmter Aussagen aus einem gegebenen Axiomensystem als Kriterium für dessen Verwendung im Vordergrund. Ein Beispiel ist das Auswahl-

\footnotetext{
${ }^{58}$ Man beachte, dass Gödel Platonist war und daher sein Gebrauch des Wahrheitsbegriffs auch vor dem Hintergrund einer als existent angenommenen mathematischen Wirklichkeit zu verstehen ist.

59 „Zwei Mengen sind genau dann gleich, wenn sie die gleichen Elemente haben“ (Deiser 2010, S. 422).

${ }^{60}$ „Es existiert eine Menge $x$, die die leere Menge als Element enthält und die mit jedem ihrer Elemente $y$ auch $\{y\}$ als Element enthält“ (Deiser 2010, S. 427). Damit enthält $x$ die Elemente $\emptyset,\{\emptyset\},\{\{\emptyset\}\}$ usw., was letztlich die Existenz unendlicher Mengen garantiert.
} 
axiom $^{61}$, welches eine wichtige Rolle bei der Herleitung des Wohlordnungssatzes ${ }^{62}$ spielt (vgl. Maddy 1988a, S. 483).

\section{Quasi-Empirismus}

Das Prinzip der extrinsischen Rechtfertigung rückt die Mathematik in die Nähe der empirisch arbeitenden Naturwissenschaften, denn extrinsisch gerechtfertigte Axiome sind nicht sicher; vielmehr müssen sie als vorläufige Hypothesen betrachtet werden, die abhängig von ihren Konsequenzen auch wieder revidiert werden können. ${ }^{63}$ Ähnlich argumentieren auch Vertreter quasi-empiristischer Positionen, die ich zum Abschluss dieses Abschnitts vorstellen möchte.

Ausgangspunkt des Quasi-Empirismus ist die Feststellung, dass alle grundlagentheoretischen Versuche, die Sicherheit der Mathematik philosophisch zweifelsfrei zu begründen, bisher gescheitert sind. Kalmár (1967, S. 192-193) betont die empirischen Wurzeln der Mathematik:

- Viele Axiome können (und sollten) als Abstraktionen empirischer Fakten verstanden werden.

- Die Widerspruchsfreiheit der Mathematik kann nicht bewiesen werden, sondern beruht auf der Erfahrung, dass gravierende Widersprüche bisher nicht aufgetreten sind bzw. beseitigt werden konnten.

- Die in der Mathematik verwendeten logischen Schlussweisen verdanken ihre Anwendbarkeit der über Jahrtausende empirisch gefestigten menschlichen Denkpraxis.

Die extrinsische Rechtfertigung passt in dieses Mathematikbild, da sie die Grundlagen der Mathematik für Revisionen aufgrund außermathematischer Faktoren öffnet. Kalmár (1967, S. 193) stellt dazu die provokante Frage: „Why do we not confess that mathematics, like other sciences, is ultimately based upon, and has to be tested in, practice?“

Ein wichtiger Wegbereiter des Quasi-Empirismus war Imre Lakatos (1922-1974). Er hält der Überzeugung von der Unfehlbarkeit der Mathematik (die trotz aller Differenzen die

\footnotetext{
${ }^{61}$ „Ist $x$ eine Menge, deren Elemente [wiederum Mengen sind, welche] nichtleer und paarweise disjunkt sind, so existiert eine Menge $y$, die mit jedem Element von $x$ genau ein Element gemeinsam hat" (Deiser 2010, S. 437).

${ }^{62}$ „Sei $M$ eine Menge. Dann existiert eine Wohlordnung < auf $M$ “ (Deiser 2010, S. 238). Zur Definition einer Wohlordnung siehe Deiser 2010, S. 225.

${ }^{63}$ Hierin unterscheidet sich Gödels Platonismus von den klassischen Varianten, denen zufolge mathematische Aussagen apriorische Sicherheit genießen.
} 
gängigen philosophischen Ansätze gemeinsam haben) eine „fallibilistische Position“ entgegen (Heintz 2000, S. 89), der zufolge eine prinzipielle „Fehlbarkeit der Mathematik und damit ihre Ähnlichkeit mit der empirischen Wissenschaft" vorliegt (Lakatos 1982a, S. 40). Seiner Ansicht nach entwickelt sich mathematisches Wissen ,nicht durch die andauernde Vermehrung der Zahl unbezweifelbar begründeter Sätze [...], sondern durch die unaufhörliche Verbesserung von Vermutungen durch Spekulation und Kritik" (Lakatos 1979, S. xii). In seiner Monographie Beweise und Widerlegungen (Lakatos 1979) illustriert er anhand eines fiktiven Lehrer-Schüler-Dialogs über den Beweis der Eulerschen Polyederformel, dass grundsätzlich zu einem gegebenen Beweis Gegenbeispiele konstruierbar sind und die Gültigkeit einzelner Beweisschritte angezweifelt werden kann. Dies bedinge die Notwendigkeit, bislang verwendete mathematische Begriffe präziser zu definieren und implizit benutzte Voraussetzungen zu explizieren.

Wenn man die Mathematik wie Lakatos in Analogie zu empirischen Wissenschaften setzt, muss man jedoch die Frage nach möglichen Falsifikatoren beantworten, denn: ,[A]n empirical hypothesis is theoretically disconfirmable; i.e., it is possible to indicate what kind of evidence, if actually encountered, would disconfirm the hypothesis" (Hempel 1945/1983, S. 378). Hier tut sich der Quasi-Empirismus jedoch schwer. „Im Falle einer formalisierten Mathematik, so wie sie Hilbert im Sinn gehabt hatte, sind genau genommen nur logische Falsifikationen denkbar“" (Heintz 2000, S. 76; Herv. im Orig.), d. h. logische Widersprüche der Form $A \wedge \neg A$. Lakatos’ Ausführungen sind aufgrund der Historizität des Eulerschen Polyedersatzes nicht einfach auf die moderne Mathematik übertragbar (vgl. Heintz 2000, S. 73). So könnte man Lihm aus formalistischer Sicht ein zu informelles Beweisverständnis vorwerfen: ,There is [...] no serious sense in which [...] a proof [gemeint ist ein formaler Beweis, T.H.] is fallible. And, because the rules of proof are 'sound', no counterexample to the proof can be found" (Worrall 1979, S. 72). Heintz (2000, S. 77) ergänzt: „Nur die Axiome können Gegenstand von Kritik oder Zweifel sein. Dies braucht den Formalismus allerdings nicht weiter kümmern, da die Axiome nicht (mehr) den Status von inhaltlichen (und entsprechend anzweifelbaren) Wahrheiten haben“.

Letztlich kann keine der hier vorgestellten Positionen eine vollends zufriedenstellende Antwort auf die eingangs erwähnten Fragen der Mathematikphilosophie geben. Die unterschiedlichen Sichtweisen liefern aber trotz ihrer Grenzen wertvolle Einsichten in das Wesen der Mathematik:

- Der Platonismus spiegelt die Überzeugung eines Großteils der Mathematikerinnen und Mathematiker wider, es beim Mathematiktreiben bis zu einem gewissen Grad mit einer wie auch immer gearteten Wirklichkeit und der Entdeckung von ,wahren“ Resultaten zu tun zu haben. 
- Die im Formalismus betriebene Loslösung mathematischer Theorien von ihren realweltlichen Bezügen und die Konzentration auf die logischen Beziehungen zwischen Aussagen ist aus der modernen Mathematik nicht mehr wegzudenken und hat maßgeblich zu ihrer Weiterentwicklung beigetragen.

- Das Konzept der extrinsischen Rechtfertigung wie auch die quasi-empiristische Auffassung von Mathematik stellen pragmatische Sichtweisen dar, die der mathematischen Forschungspraxis deutlich näher kommen als die übrigen philosophischen Ansätze. Auch historisch gesehen ist eine enge Verwandschaft zwischen der Mathematik und den empirischen (Natur-)Wissenschaften einschließlich der in ihnen verwendeten Methoden der Erkenntnisgenerierung und -validierung nicht bestreitbar (siehe beispielsweise Witzke 2011).

\subsection{Zusammenfassung: Zur Rolle der Axiomatik in der Mathematik}

Betrachtet man die historische Entwicklung der axiomatischen Methode, wie sie bis hierhin beschrieben wurde, so kristallisieren sich drei unterschiedliche Typen von Axiomatik heraus, die mit jeweils unterschiedlichen Zielsetzungen in Bezug auf die Darstellung mathematischer Theorien wie auch divergierenden Auffassungen über den ontischen Status mathematischer Objekte und den epistemischen Status mathematischer Aussagen verbunden sind. Ich orientiere mich bei den folgenden Ausführungen an Freudenthal 1963 und van der Waerden 1967.

\section{Euklidische Axiomatik}

Die klassische Axiomatik, wie sie paradigmatisch in Euklids Elementen festgehalten ist, hat zwei wesentliche Merkmale:

1) „Die Gegenstände, auf die sich die klassischen Axiome beziehen, sind von vornherein bestimmt und bekannt" (van der Waerden 1967, S. 1). Man kann auch sagen, die euklidische Axiomatik ist „ontologisch verankert“ (Freudenthal 1963, S. 5), da den mathematischen Objekten eine (physikalische oder idealisierte) Existenz ${ }^{64} \mathrm{zu}$ -

\footnotetext{
${ }^{64}$ Die Frage nach der genauen Art der Existenz dieser Objekte ist für die hier vorgenommene Charakterisierung zweitrangig. Denkt man vorwiegend an die Geometrie, so würde man wohl von einer platonischen Existenz im Ideenhimmel sprechen. Man kann unter den hier beschriebenen Typ von Axiomatik aber beispielsweise auch Newtons Philosophiae Naturalis Principia Mathematica fassen, welche überwiegend von materiell existierenden Objekten handeln (vgl. van der Waerden 1967, S. 1).
} 
gesprochen wird. Die ontologische Verankerung spiegelt sich besonders stark in den Begriffsdefinitionen wider: Zwar unternimmt Euklid den Versuch, auch grundlegende Begriffe wie Punkt und Linie zu definieren; die Tatsache, dass er diese Definitionen dann in seinen Beweisen nicht verwendet, zeigt jedoch, dass die anschaulichen Vorstellungen der geometrischen Objekte noch eine entscheidende Rolle spielen.

2) Die Axiome werden als wahre, evidente Aussagen betrachtet. Insofern sind auch die aus den Axiomen deduzierten Sätze wahre Aussagen.

Ziel dieser Art von Axiomatik ist es, ,die [ideelle oder materielle, T.H.] Wirklichkeit zu ordnen, Beziehungsgefüge herzustellen und sie bis zu einem Horizont der Evidenz zu führen, der nicht genau festgelegt und recht variabel [ist]“" (Freudenthal 1963, S. 6).

\section{Hilbertsche Axiomatik}

Die neuere Sicht auf Axiomatik, wie sie sich in Hilberts Grundlagen der Geometrie widerspiegelt, ist vor allem mit einem Wegfall der ontologischen Bindung verbunden. Im Gegensatz zu Euklid, der Definitionen an den Anfang seiner Darstellungen stellt, beginnt Hilbert direkt mit den Axiomen, durch die die darin (zum ersten Mal) auftretenden Begriffe dann implizit definiert werden. Die Frage nach einer Wahrheit mathematischer Sätze stellt sich nicht mehr. Stattdessen rückt der hypothetisch-deduktive Charakter der Mathematik in den Vordergrund, d. h. die Frage, welche Folgerungen sich aus welchen Voraussetzungen rein logisch ziehen lassen. Die für die euklidische Axiomatik so wichtigen Begriffe der Existenz und Wahrheit werden durch das formale Konzept der Widerspruchsfreiheit ersetzt.

Als Ziel wird vor allem die Vollständigkeit der aufgestellten Axiomensysteme wichtig. Man möchte eine axiomatisch aufgebaute Theorie so gestalten, dass sie bis auf Isomorphie nur ein Modell besitzt, d. h. das Axiomensystem ist monomorph. Neben der Axiomatisierung der euklidischen Geometrie durch Hilbert sind die Dedekind-Peano-Axiome für die natürlichen Zahlen ein typisches Beispiel. Laugwitz (1966, S. 19) verwendet in diesem Zusammenhang auch den Begriff der charakterisierenden Axiomatik: „Man ist [...] bestrebt, den mathematischen Bereich durch ein Axiomensystem zu charakterisieren“. 


\section{Bourbakische Axiomatik / Abstrahierende Axiomatik}

Der Begriff der abstrahierenden Axiomatik wird sowohl von Freudenthal (1963) als auch von Laugwitz (1966) in expliziter Abgrenzung von der charakterisierenden, Hilbertschen Axiomatik verwendet. Mit diesem Typ ist die in Abschnitt 2.2.1 beschriebene Strukturmathematik der Bourbaki-Gruppe gemeint. Während die Hilbertsche und Bourbakische Axiomatik die Ausklammerung ontologischer Fragestellungen und damit eine formale Sichtweise auf mathematische Objekte und Aussagen gemeinsam haben, verzichtet die Strukturmathematik ganz bewusst auf die oben genannte Monomorphie von Axiomensystemen. Durch die Abstraktion struktureller Gemeinsamkeiten soll gerade bezweckt werden, dass ein Axiomensystem polymorph ist, d. h. viele nicht-isomorphe Modelle hat, die in unterschiedlichen mathematischen Gebieten anwendbar sind. Die abstrahierende Axiomatik ,dient in erster Linie nicht der Grundlagenforschung, sondern der Organisation und Vereinheitlichung der Mathematik“ (Freudenthal 1963, S. 7).

\section{Zusammenfassende Einordnung}

Die voranstehende Dreiteilung geht aus zwei sich überlappenden Dichotomisierungen der Axiomatik hervor:

Zum einen können die Sichtweisen von Axiomatik anhand der Frage unterschieden werden, inwiefern den mathematischen Objekten bzw. Aussagen eine (wie auch immer geartete) inhaltliche Bedeutung zukommt. Daraus ergibt sich die Aufteilung in inhaltliche und formale Axiomatik (beispielsweise in Hilbert und Bernays 1934). Gebräuchlich sind auch die Begriffe klassisch - modern (siehe van der Waerden 1967). Die formale Axiomatik umfasst dabei - trotz im Detail unterschiedlicher Zielsetzungen - die Hilbertsche wie auch die Bourbakische Axiomatik.

Eine zweite Möglichkeit der Dichotomisierung ergibt sich aus den unterschiedlichen Zielsetzungen. Die Begriffe der charakterisierenden und abstrahierenden Axiomatik erscheinen mir hierfür geeignet. Auch wenn die Bezeichnung als „charakterisierend“ von Laugwitz (1966) vor allem für die Axiomatik im Sinne Hilberts verwendet wird, lässt sie sich auf die euklidische Axiomatik ausweiten. Zwar geht es bei Euklid nicht um die Aufstellung eines (im modernen Sinne) vollständigen Axiomensystems, sehr wohl aber um die deduktive Beschreibung eines bestimmten mathematischen Themenfeldes. Der Prozess des Abstrahierens wird hier - abgesehen von den in der Mathematik natürlich immer vorhandenen Idealisierungen - nicht so weit getrieben, dass die entstehende Theorie auf ganz unterschiedliche Bereiche anwendbar wäre. 
Unter Verwendung der in diesem Abschnitt eingeführten Begrifflichkeiten ergibt sich somit folgende Typologisierung axiomatischer Vorgehens- und Sichtweisen:

\begin{tabular}{|c|c|c|}
\hline EUKLID & HILBERT & BOURBAKI \\
\hline \hline inhaltlich/klassisch & formal/modern \\
\hline \multicolumn{2}{|c|}{ charakterisierend } & abstrahierend \\
\hline
\end{tabular}

Prinzipiell kann man von diesen drei Auffassungen noch eine vierte Art der Axiomatik unterscheiden, die zusätzlich zur abstrakten Auffassung der Axiome die Formalisierung der mathematischen Aussagen in Form von Zeichenketten sowie die vollständige Angabe der zulässigen Schlussweisen in einem (ebenfalls axiomatisierten und formalisierten) Logikkalkül umfasst. Steiner (1966c, S. 38) nennt dies formalistische Axiomatik. Die formalistische Axiomatik ist - wie in Abschnitt 2.2.2 dargelegt - vor allen Dingen ein Mittel zur Überprüfung der Widerspruchsfreiheit der Mathematik, hat jedoch seit den GödelResultaten für die mathematische Praxis keine wesentliche Bedeutung mehr und wird in dieser Arbeit nicht näher behandelt. 


\section{Axiomatik im Mathematikunterricht: Didaktische Perspektiven}

In diesem Kapitel sollen fachdidaktische Gesichtspunkte in den Blick genommen werden, um das Potential des Themas Axiomatik für die Behandlung im Mathematikunterricht zu eruieren.

Das Vorhaben, axiomatische Aspekte zum Gegenstand des Mathematikunterrichts zu machen, ruft bei vielen Menschen Erinnerungen an die Bestrebungen zur Modernisierung des Mathematikunterrichts im Rahmen der Neuen Mathematik - international auch als New Math bezeichnet - wach. Die Integration axiomatisch-deduktiver Inhalte und Methoden in die Schulmathematik war ein wesentlicher Bestandteil dieses Reformprogramms, welches - überspitzt formuliert - mit einem Großteil seiner Ziele genauso schnell scheiterte, wie es zunächst in die Schulsysteme implementiert worden war. Vermutlich unter anderem als Folge dieser historischen Entwicklung sind die Reaktionen von Fachmathematikern, Didaktikern und Lehrkräften auf das Thema Axiomatik heutzutage oft skeptisch bis ablehnend. Es ist daher notwendig, sich eingehend mit den Zielen der Neuen Mathematik in Bezug auf axiomatische Fragestellungen zu beschäftigen und die in der Auseinandersetzung hervorgebrachten Argumente vor dem Hintergrund aktueller didaktischer Ansichten neu zu reflektieren.

\subsection{Neue Mathematik: Historischer und inhaltlicher Überblick}

Eine einheitliche Darstellung der Neuen Mathematik ist aus mehreren Gründen nicht einfach. Zum einen bezieht sich der Begriff Neue Mathematik in seiner englischen Formulierung New Math sowohl auf eine internationale Bewegung, welche durch diverse Konferenzen und Seminare vorangetrieben wurde (vgl. Kilpatrick 2012 und Moon 1986, S. 4370), als auch auf zahlreiche Reformbestrebungen in einzelnen Ländern (siehe beispiels- 
weise die Kapitel 4 bis 8 in Moon 1986). Beide Aspekte hängen natürlich zusammen, da die internationalen Entwicklungen Einfluss auf nationale Projekte hatten und umgekehrt. Zum anderen gab es unter den an den diversen Initiativen beteiligten Personen zum Teil höchst unterschiedliche Ansichten über die konkrete Ausgestaltung eines modernen Mathematikunterrichts. ${ }^{1}$ Dies erschwert eine möglichst allgemeingültige Beschreibung.

Ich möchte daher zunächst die wesentlichen Entwicklungen auf internationaler Ebene nachzeichnen und mich in den anschließenden inhaltlichen Erläuterungen auf die Ausprägungen der Neuen Mathematik in Deutschland konzentrieren.

\section{Entstehungsbedingungen auf internationaler Ebene}

Inhaltliche Triebfeder für die Reformbemühungen war die - in Abschnitt 2.2.1 genauer beschriebene - Strukturmathematik, welche sich zu Beginn der 1950er Jahre in der Entwicklung befand. Vor dem Hintergrund dieser Entwicklungen auf Seiten der Fachmathematik wuchs die Unzufriedenheit mit der Gestalt des Mathematikunterrichts an weiterführenden Schulen. Dem traditionellen Schulunterricht wurde gar eine ,fachstrukturelle Abstinenz" (Lenné 1969, S. 77) bescheinigt; die Befürchtungen, den Anschluss an die Wissenschaft Mathematik zu verlieren, waren groß. Der Ruf nach einer Modernisierung des Mathematikunterrichts wurde zudem durch eine Reihe wirtschaftlicher, politischer und gesellschaftlicher Faktoren verstärkt.

Häufig wird der Sputnikschock 1957 als das Ereignis genannt, das in den USA als Katalysator für in Ansätzen bereits vorhandene Reformvorhaben wirkte (vgl. Kilpatrick 2012, S. 563, Kline 1973, S. 16, Lenné 1969, S. 78 oder Moon 1986, S. 45). Auf europäischer Ebene erfüllte die Gründung wirtschaftlich ausgerichteter Organisationen wie der OEEC (Organisation for European Economic Co-operation, seit 1961 in OECD umbenannt) eine ähnliche Funktion (vgl. Lenné 1969, S. 78). Welchen Einfluss die anhaltenden Spannungen zwischen den Vereinigten Staaten und der ehemaligen UdSSR auch auf Fragen der europäischen Bildungspolitik hatten, verdeutlicht das folgende Zitat von Meschkowski (1965, S. 183):

Unsere politische Zukunft hängt auch davon ab, ob wir genug Fachkräfte für die Wirtschaft ausbilden. Nehmen wir an, daß es in den nächsten Jahrzehnten zu keinem weltweiten Atomkrieg kommt, daß immer wieder mindestens

\footnotetext{
${ }^{1}$ Kapur (1977, S. 260-261) weist daraufhin, welche unterschiedlichen Personengruppen an der Ausgestaltung der New Math-Programme beteiligt sein konnten: reine und angewandte Mathematiker, Hochschuldozenten, Mathematiklehrer, Psychologen, Pädagogen, (Schulbuch-)Autoren und Verleger sowie Politiker. Diese Auflistung zeigt, dass es sehr unterschiedliche Interessen, Meinungen und Anforderungen zusammenzubringen galt.
} 
im letzten Augenblick die Probleme zwischen den Weltmächten friedlich gelöst werden. Damit sind doch die politischen und ideologischen Gegensätze nicht aus der Welt geschafft, und die Entscheidungen der jungen Völker werden nicht zuletzt durch die Wissenschaftler, Ingenieure und Lehrer bestimmt sein, die die alten Großmächte ihnen stellen. Es ist also eine für die politische Zukunft höchst bedeutsame Frage, ob wir wieder den Anschluß an die Forschung finden, ob wir genug Ingenieure und Techniker ausbilden, mit denen wir den Entwicklungsländern helfen können.

Die Zeit nach dem Zweiten Weltkrieg war unter anderem geprägt durch eine ,stark wachsende Bedeutung der Technik und damit auch der Mathematik für Form und Bestand der [...] Gesellschaft" (Lenné 1969, S. 78). Die Forderung der Anschlussfähigkeit des Mathematikunterrichts an den aktuellen Stand von Forschung und Technik zielte dabei nicht nur auf die Ausbildung künftiger Eliten für die Industrie und die Naturwissenschaften, sondern hatte auch jene Auswirkungen im Blick, die der technische Fortschritt auf das alltägliche Leben der meisten Menschen hatte. ${ }^{2}$ Ein wichtiges Phänomen, das beide genannten Bereiche (Beruf und Alltag) betraf, war die rasch voranschreitende Entwicklung von Rechenmaschinen und des Computers (vgl. Lenné 1969, S. 80 und Bogensperger 2014, S. 13-15).

Auf internationaler Ebene werden zumeist drei Konferenzen bzw. die auf diesen basierenden Publikationen unter der Federführung der OEEC/OECD als wegweisend für die inhaltliche Ausformulierung der Neuen Mathematik angesehen (vgl. Lenné 1969, S. 81, Kilpatrick 2012, S. 563-564 und Moon 1986, S. 48-55): (1) New Thinking in School Mathematics (23.11.-04.12.1959 in Royaumont, Frankreich), (2) Synopses for Modern School Mathematics (26.08.-19.09.1960 in Dubrovnik, Jugoslawien) und (3) Mathematics Today: A Guide for Teachers (17.-23.11.1963 in Athen, Griechenland).

Insbesondere die Royaumont-Konferenz nimmt in der Retrospektive eine zentrale Stellung ein. In seiner Eröffnungsrede nannte der Konferenzleiter und Mathematiker Marshall Harvey Stone (1903-1989) die beiden wesentlichen Faktoren, aus denen ein dringender Modernisierungsbedarf für den Mathematikunterricht abgeleitet wurde:

There are two major factors which require us to examine with fresh eyes the mathematics we propose to teach to young people in the secondary schools and in the first years at the university. One is the extraordinary growth of pure mathematics in modern times. The other is the increasing dependence of scientific thought upon mathematical methods, coinciding in time with a more and more urgent social demand for the services of scientists of every description.

\footnotetext{
${ }^{2}$ Einige Zitate zur „Mathematisierung aller Lebensbereiche“ nennt Bogensperger (2014, S. 15-16).
} 
The forces exerted by these two factors on our educational system are quite clearly on the point of shattering the traditional framework of mathematical instruction and thus preparing the way for an overdue modernization and improvement of our teaching of mathematics. (Stone 1961, S. 15; Herv. d. Verf.)

Programmatisch für eine radikale Ausrichtung der Reformbewegung wurde der Vortrag von Jean Dieudonné (1906-1992), einem Gründungsmitglied der Bourbaki-Gruppe, in dem er unter anderem für die Abschaffung weiter Teile der euklidischen Elementargeometrie zugunsten einer allgemeineren Behandlung zwei- und dreidimensionaler reeller Vektorräume plädierte. ${ }^{3}$ Insgesamt beklagte er: ,[W] [Wen a student enters university, he will most probably never have heard such common mathematical words as set, mapping, group, vector space, etc.“ (Dieudonné 1961, S. 34). Aus Sicht der Universitäten würden dagegen folgende Anforderungen an Studienanfänger gestellt: ,[A] first-year student should, on the one hand, be familiar with a certain number of elementary techniques [...] such as elementary linear algebra, analytic geometry, trigonometry and some calculus. On the other hand, the student should already be fairly well trained in the use of logical deduction and have some idea of the axiomatic method“" (Dieudonné 1961, S. 32).

In der Folge der Royaumont-Konferenz starteten in zahlreichen OEEC/OECD-Ländern umfangreiche Projekte zur Erstellung neuer Lehrmaterialien und zur Überarbeitung der nationalen Curricula. Im Laufe der Jahre weitete sich der Fokus der Reformdiskussionen die sich anfangs überwiegend mit dem sekundären Bildungsbereich (secondary education) beschäftigten und dabei hauptsächlich künftige Mathematiker im Blick hatten - auf den Primarbereich sowie diejenigen Schülergruppen aus, die später nicht studieren und auch keinen mathematikintensiven Beruf ergreifen würden (vgl. Kilpatrick 2012, S. 565-566).

\section{Die Neue Mathematik in Deutschland}

Bestrebungen zur Modernisierung des Mathematikunterrichts hatte es in Deutschland schon seit Mitte der 1950er Jahre gegeben, jedoch wurden die Ideen der Neuen Mathematik erst ca. zehn Jahre später durch konkrete Reformvorschläge spürbar wahrgenommen (vgl. Damerow 1977, S. 53). Eine ähnlich treibende Kraft wie der Start der russischen Sputnikraketen bildeten dabei Veröffentlichungen über die angeblich drohende große Bil-

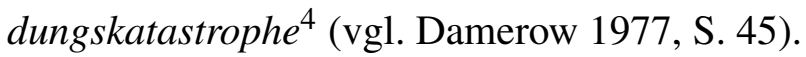

Fachliche Grundlage für sämtliche inhaltlichen Reformvorschläge der Neuen Mathematik war - wie oben bereits erwähnt - die Strukturmathematik. Darauf aufbauend entwickel-

\footnotetext{
${ }^{3}$,And if the whole program I have in mind had to be summarised in one slogan it would be: Euclid must go!“ (Dieudonné 1961, S. 35; Herv. im Orig.).

${ }^{4}$ Siehe hierzu vor allem Picht 1964.
} 
ten sich die Begriffe Menge, Struktur und Abbildung zu Leitideen curricularer Vorstellungen. ${ }^{5}$ Der Mengenbegriff war dabei „,der eigentlich fundamentale Begriff“ (Steiner 1959, S. 28), denn Strukturen existieren auf Mengen und der Abbildungsbegriff kann zum einen mengentheoretisch als Teilmenge eines kartesischen Produkts definiert und zum anderen verwendet werden, um ,strukturierte Mengen [...] miteinander zu vergleichen und in Beziehung zu setzen, insbesondere auch, um auf Mengen Strukturen zu induzieren“ (Steiner 1959, S. 35).

Einen sehr detaillierten Vorschlag zur inhaltlichen Ausgestaltung eines modernen Mathematikunterrichts stellt der Nürnberger Rahmenplan des Deutschen Vereins zur Förderung des mathematischen und naturwissenschaftlichen Unterrichts ${ }^{6}$ dar, welcher auf der 56. MNU-Hauptversammlung im April 1965 vorgestellt wurde.

Der Lehrplan wurde „,nach rein innermathematischen Erwägungen“ erstellt und hatte das Ziel, „den modernen mathematischen Gegebenheiten weitmöglichst gerecht zu werden“ (Athen 1966, S. 87). Die fachwissenschaftliche Ausrichtung im Sinne der Strukturmathematik wird bereits in der Präambel und den Vorbemerkungen zum eigentlichen Stoffplan deutlich:

Indem wir uns an der Weiterentwicklung der Wissenschaften orientierten und neue Ideen, Begriffe und Ordnungsschemata verwendeten, hoffen wir einmal dazu beizutragen, den Graben einzuebnen, der sich gerade in unseren Fächern ${ }^{7}$ zwischen Gymnasium und Hochschule aufgetan hat. (Lenné 1969, S. 314-315)

Die Mathematik beschäftigt sich mit strukturierten Gebilden, d.h. mit bestimmten Mengen von Objekten und den auf ihnen definierten Strukturen, mit der gedanklichen Konstruktion neuer Gebilde und mit Zusammenhängen zwischen Gebilden, insbesondere mit Zuordnungen (Relationen und Funktionen bzw. Abbildungen). Die Gebilde selber erscheinen dabei als Modelle für abstrakte Strukturen, die durch entsprechende Axiomensysteme beschrieben und mit logischen Hilfsmitteln begrifflich beherrscht werden. (Lenné 1969, S. 316-317)

Ebenfalls in den Vorbemerkungen aufgeführt ist eine Übersicht der wichtigsten „,zu vermittelnden strukturmathematischen Grundkategorien“ (Damerow 1977, S. 216; siehe auch Lenné 1969, S. 317-318):

\footnotetext{
${ }^{5}$ Siehe dazu exemplarisch Steiner 1959 (insb. S. 15-37), Steiner 1965 und Griesel 1965.

${ }^{6}$ Im Folgenden wird nach dem Abdruck in Lenné (1969, S. 313-325) zitiert.

${ }^{7}$ Auf der MNU-Hauptversammlung wurden Rahmenpläne für die Fächer Mathematik, Physik, Biologie und Chemie vorgestellt.
} 
- Objekte (z. B. Zahlen oder Punkte),

- Mengen (z. B. Zahlenmengen, Geraden und Ebenen aufgefasst als Punktmengen, Mengen von $n$-Tupeln),

- Strukturen (Gruppe, Ring, Körper, Vektorraum, Ordnung u. a.),

- Abbildungen (z. B. stetige Abbildungen, strukturerhaltende Abbildungen, geometrische Abbildungen wie Drehung und Spiegelung) und

- logische Begriffe und Hilfsmittel (z. B. Variable, Term, Aussage(form), aussagenlogische Verknüpfungen, Quantoren).

In der Unterstufe, in der ,,praktische Rechenfertigkeiten, die Beherrschung einfacher Anwendungsmöglichkeiten des Zahlenrechnens [...] und Vertrautheit mit den Grundkonstruktionen in der Geometrie“ (Lenné 1969, S. 318) im Vordergrund stehen, sollen die strukturmathematischen Begriffe der Lehrperson lediglich als Leitbegriffe dienen: „Der Lehrer muß deswegen stets die [...] genannten Strukturen als Leitbegriffe vor Augen haben und in der Anordnung und Darbietung des Stoffes wirksam werden lassen“ (Lenné 1969, S. 318). Damit soll die explizite Einführung der Strukturbegriffe in der Mittelstufe vorbereitet werden. In der Geometrie soll das lokale Ordnen anschaulich-geometrischer Sachverhalte die Basis für spätere axiomatische Betrachtungen liefern.

Die Mittelstufe dient zunächst der Festigung folgender Fertigkeiten: „Handhabung von algebraischen Termen, Lösen von Gleichungen, Auffassen und Darstellen von Funktionen bzw. Abbildungen, Anwendung numerischer Verfahren, anschaulich-konstruktiver Umgang mit geometrischen Figuren, Verständnis für Zusammenhänge zwischen Geometrie und Wirklichkeit, Beziehungen zwischen Geometrie und Algebra (z. B. lineare Systeme und Vektoren u.a.), Aneignung und Verständnis elementarer Methoden des Definierens und Beweisens“ (Lenné 1969, S. 319-320). Ein wesentliches Ziel ist „die saubere Definition der Funktion“ (Lenné 1969, S. 320). „Darüber hinaus sollen Einsichten in den deduktiven Aufbau der Geometrie und die Fähigkeit entwickelt werden, eine gegebene Situation zu mathematisieren; sie kann vor allem dadurch ausgebildet werden, daß durch induktives Vorgehen und stufenweise Abstraktion die Hinführung zu grundlegenden Begriffsbildungen der Strukturmathematik erfolgt, die die Grundkategorien des Mathematisierens darstellen“ (Lenné 1969, S. 320). ${ }^{8}$

\footnotetext{
${ }^{8}$ Damerow (1977, S. 219) merkt an, dass das Konzept des Mathematisierens hier ,auf einen innermathematischen Prozeß reduziert [wird], so als sei der Übergang [...] zu den Strukturbegriffen als Übergang zur wahren Mathematik zu begreifen“".
} 
Die Eigenschaften der Zahlbereiche sollen dabei ,unter allmählicher, aber ausdrücklicher Benutzung der Begriffe Menge, Relation, Verknüpfung, Gruppe, Ring, Körper, Anordnung, Isomorphie“ erarbeitet werden, wobei das Permanenzprinzip ,als strukturelles Leitprinzip“ verwendet werden soll (Lenné 1969, S. 320). In der Geometrie, die abbildungstheoretisch aufzubauen sei, soll zudem der „Übergang zu einem deduktiven Aufbau im Sinne einer voraxiomatischen Ordnung anschaulich gegebener geometrischer Tatsachen“ sowie die „Zurückführung auf ein bestimmtes System von geometrischen Grundbegriffen und Grundsätzen“ vermittelt werden (Lenné 1969, S. 320). Zudem wird die „Erreichung einwandfreier Denk- und Sprechgewohnheiten“ angestrebt, wozu unter anderem die „Formulierung von Aussagen in der, wenn - so '-Form [...], Unterscheidung von notwendigen und hinreichenden Bedingungen“ und ein ,Verständnis für den deduktiven Beweis (zugleich als Vorbereitung für das Verständnis der axiomatischen Methode)“ gehören (Lenné 1969, S. 320).

In der Oberstufe werden der Zahl- und Funktionenbegriff durch die Themen der Analysis vertieft (topologische Vollständigkeit der reellen Zahlen, Begriffe wie beispielsweise Umgebung, Grenzwert, Stetigkeit und Differenzierbarkeit). Auch Grundfragen der Wahrscheinlichkeitsrechnung und Statistik sollen behandelt werden. In der Geometrie und linearen Algebra rücken die Begriffe des Vektorraums und der Abbildungsgruppe in den Vordergrund (vgl. Lenné 1969, S. 322-323). „Darüber hinaus soll der Oberstufenunterricht zum Verständnis der axiomatischen Denkweise beitragen und auf Probleme der mathematischen Grundlagenforschung auf wenigstens einem begrenzten Teilgebiet eingehen“ (Lenné 1969, S. 323). Für eine derartige Vertiefung axiomatischer Aspekte wird am Ende des Lehrplans eine Reihe von Themen genannt, aus denen die Lehrkraft eine Auswahl treffen kann (Lenné 1969, S. 325):

- Kennzeichnung des Körpers der reellen Zahlen durch Grundeigenschaften.

- Der Körper der komplexen Zahlen.

- Gruppe, Ring, Körper.

- Axiomatischer Aufbau der Geometrie, z. B. aus dem Spiegelungsprinzip oder aus der Parallelogrammrelation (Äquipollenz).

- Inzidenzgeometrie (endliche Geometrien).

- Nicht-euklidische Geometrie.

- Boolesche Algebra und Anwendungen (z. B. Logik und logische Maschinen, Schaltalgebra u. a.).

- Wahrscheinlichkeitsrechnung (axiomatischer Aufbau). 
Als eigentliche Initiierung der Neuen Mathematik sind die Empfehlungen und Richtlinien der KMK zur Modernisierung des Mathematikunterrichts an den allgemeinbildenden Schulen zu sehen, welche am 03.10.1968 und damit dreieinhalb Jahre nach der Vorstellung des Nürnberger Rahmenplans verabschiedet wurden. Dieser KMK-Beschluss forderte - verkürzt formuliert - ,die flächendeckende Einführung der sogenannten ,Neuen Mathematik' ab dem Schuljahr 1972/73 [...] für alle Schulformen“" (Hamann 2011, S. 347).

Gegen die Reformpläne regte sich relativ unmittelbar nach ihrer Einführung massiver Widerstand in der Bevölkerung. In Deutschland standen vor allem die Auswirkungen auf den Primarbereich im öffentlichen Fokus. Die Einführung der Mengenlehre in der Grundschule wurde heftig und teilweise polemisch kritisiert. Als paradigmatisch für die erheblichen gesellschaftlichen und bildungspolitischen Unruhen kann der Spiegel-Leitartikel der Ausgabe vom 25. März 1974 angesehen werden, auf deren Titelseite provokativ die Frage Macht Mengenlehre krank? abgedruckt war.

Spätestens Anfang der 1980er Jahre mussten weite Teile der Reform vor allem im Primarbereich als zurückgenommen gelten (vgl. Hamann 2017, S. 105-106). Die Auseinandersetzungen rund um die Grundschulmathematik sollen an dieser Stelle nicht weiter verfolgt werden, da sie mit dem Thema dieser Arbeit nur bedingt zu tun haben. Der interessierte Leser sei auf Hamann 2017 und das weitgehend den Entwicklungen im Primarbereich in Deutschland gewidmete Kapitel bei Moon (1986, S. 161-184) verwiesen.

\section{Gründe für das Scheitern}

Die Gründe für das verhältnismäßig schnelle Scheitern der Neuen Mathematik sind vielfältig und können in dieser Arbeit nicht umfassend dargestellt oder analysiert werden. Insgesamt hat wohl eine „Kombination verschiedener Einflussfaktoren“ (Hamann 2013, S. 415) zur raschen Rücknahme großer Teile der Reforminhalte geführt. Einige in der Literatur dargelegte Aspekte sind:

- Die neuen Inhalte führten zu einer Überforderung der Lernenden. Insbesondere der intensive Gebrauch mengentheoretischer und aussagenlogischer Begrifflichkeiten sowie die Forderung von präzisem Sprachgebrauch schon in der Grundschule bereitete vielen Schülerinnen und Schülern Probleme und stieß in der Bevölkerung auf Befremden. Besonders pointiert wird diese Situation von Kline (1973, S. 1-3) und Feynman (1965) beschrieben.

- Durch die Lehrplanreformen wurden die Inhalte des traditionellen Mathematikunterrichts nicht ersetzt, sondern häufig mit Hilfe der modernen Begrifflichkeiten neu 
formuliert sowie durch strukturmathematische Inhalte ergänzt, was insgesamt zu einem erheblichen Stofffülle-Problem führte (vgl. Lenné 1969, S. 95-103).

- Nicht zu unterschätzen ist auch der gesellschaftliche Druck auf die Politik, welcher unter anderem durch den erheblichen Widerstand der Bevölkerung entstand. Eltern konnten ihren Kindern nicht mehr bei den Mathematikhausaufgaben helfen und mussten selber „Nachhilfe“ nehmen, beispielsweise durch Lektüre von Ratgebern wie Walter Fuchs' Eltern entdecken die neue Mathematik, die damals zu Bestsellern avancierten. Neue methodische Ansätze wie die logischen Blöcke von Zoltán Pál Dienes (siehe Abbildung 3.1) wurden mit Skepsis betrachtet, weil scheinbar „gespielt und nicht gelernt“" wurde (,Mengenlehre: 3+5=5+3“ 1974, S. 63).

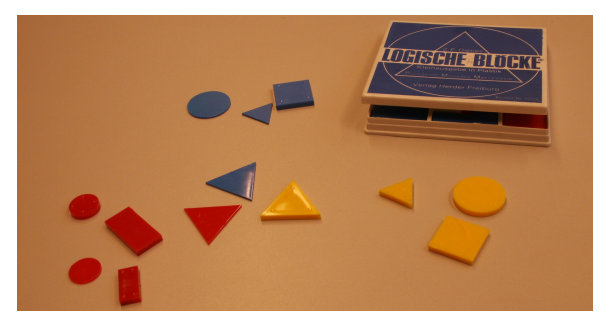

Abbildung 3.1: Logische Blöcke

- Auch die Unterstützung der Lehrerschaft fehlte bei den Vorhaben der Neuen Mathematik größtenteils. Dies lag unter anderem daran, dass viele Lehrkräfte mit den neuen Unterrichtsinhalten nicht hinreichend vertraut waren und die Fortbildungsmaßnahmen inhaltlich wie organisatorisch verbesserungsbedürftig waren (vgl. Hamann 2013, S. 413-414). Sowohl der Widerstand der Lehrerinnen und Lehrer wie auch der Eltern wird von Heymann (1996, S. 155-156) als ein wesentlicher Faktor für das Scheitern der Neuen Mathematik benannt:

Das Prinzip der kulturellen Kohärenz wurde eklatant verletzt, die Kontinuität der mathematischen Alltagskultur war durch diese Reform in Frage gestellt. Viele Eltern verstanden nicht mehr, was ihre Kinder in der Grundschule im Fach Mathematik lernen sollten, die Verständigung zwischen den Generationen gestaltete sich in einem vermeintlich basalen Wissensgebiet unerwartet schwierig. Und von einer Anwendbarkeit der neuen Inhalte in alltäglichen Situationen konnte keine Rede sein. Der Konservativismus der Eltern (und weitgehend auch der Lehrer) hatte in diesem Fall also einen rationalen Kern.

- Obwohl die Reforminitiativen explizit alle Schulformen miteinbezogen, waren die neugestalteten Lehrpläne insgesamt stark an den Belangen des Gymnasiums orientiert (vgl. Damerow 1977, S. 236-239). Es wurde kritisiert, dass „,den Rahmenplänen der KMK kein durchdachtes Konzept der Differenzierung zwischen den Lehr- 
plänen der Schularten zugrunde liegt. Die Pläne für die Realschule und die Hauptschule scheinen durch eine recht willkürliche Kürzung des reformierten Stoffplans für das Gymnasium entstanden zu sein“ (Damerow 1977, S. 238).

- Ein weiterer Faktor, der die übrigen Problemfelder wahrscheinlich erheblich mitbeeinflusst hat, ist die bildungspolitische Struktur, welche in Deutschland als Reformgrundlage vorherrschte: „Die Struktur des deutschen Erziehungswesens, das curriculare Änderungen nur im Rahmen ministerieller Bürokratien erlaubt, hat eine unabhängige und wissenschaftlich fundierte Curriculumentwicklung größeren Stils verhindert" (Tietze und Förster 2000, S. 3). Insbesondere die Kritik an den im KMK-Beschluss von 1968 vorgestellten Rahmenplänen, welche die Umsetzung der Reform in die Praxis maßgeblich geprägt haben, fällt bei Damerow (1977) vernichtend aus. Nach einer detaillierten Analyse kommt er zu dem Fazit:

Angesichts [der] Bedeutung der Rahmenpläne muß es geradezu als skandalös erscheinen, daß sie von einem Ausschuß, in dem weder Mathematiker noch Fachdidaktiker vertreten waren, in insgesamt vier Arbeitstagen zusammengeschustert worden sind. Lernpsychologische, fachdidaktische und fachwissenschaftliche Überlegungen, die in diese einflossen, waren nur aus zweiter Hand, so daß die Inkonsistenz der Darstellung nicht verwundert. (Damerow 1977, S. 235)

Derartige Rahmenbedingungen dürften dazu beigetragen haben, dass die Neue Mathematik von den meisten Betroffenen ,als von oben auferlegt und bürokratisch empfunden“ (Tietze und Förster 2000, S. 3) wurde.

Bei aller Kritik muss jedoch darauf hingewiesen werden, dass die Charakterisierung der Reform als ,gescheitert“ zu pauschal ist, denn einzelne Bestandteile haben durchaus eine langfristige Wirkung erzielt. Besonders hervorzuheben ist hier die zentrale Stellung, die der Funktionsbegriff bzw. die Idee des funktionalen Zusammenhangs in den heutigen Lehrplänen und Bildungsstandards hat. Richtig ist jedoch, dass sich die angestrebte umfassende inhaltliche Umstrukturierung des Mathematikunterrichts und insbesondere die Orientierung an Begrifflichkeiten der Mengenlehre, der (algebraischen) Strukturen sowie der Aussagenlogik nicht durchgesetzt haben. Auch von der Absicht, Schülerinnen und Schüler in Aspekte der axiomatischen Methode einzuführen, hat man mittlerweile Abstand genommen. $\mathrm{Ob}$ dafür allerdings eine prinzipielle Ungeeignetheit der betreffenden mathematischen Inhalte für den Schulunterricht ursächlich war und es nicht in viel stärkerem Ausmaß die institutionell-organisatorischen Rahmenbedingungen der Reformimplementierung waren, darf durchaus bezweifelt werden. 


\subsection{Fertigprodukt versus Tätigkeit: Zwei Sichtweisen auf Axiomatik}

Als Grundlage eigener Investitionen in die Entwicklung von Unterrichtsmaterialien (siehe Kapitel 5) sollen in diesem und dem folgenden Abschnitt die im Rahmen der Neuen Mathematik auftretenden Positionen zur Axiomatik sowie die in die Diskussion eingebrachten Argumente gegen ihre Behandlung im Mathematikunterricht thematisiert werden.

Lenné (1969, S. 84) beobachtet bei der Neuen Mathematik eine „Differenzierung in zwei Hauptrichtungen, eine rigorose und eine gemäßigte“. Während Vertreter der rigorosen Richtung rein fachliche Argumente zur Neuorganisation des Mathematikunterrichts in den Vordergrund stellen und dabei vor allem die ,Vermehrung des mathematisch-naturwissenschaftlichen Nachwuchses“ (Lenné 1969, S. 85) im Blick haben, finden bei der gemäßigten Richtung pädagogische Argumente für die Stoffauswahl stärkere Berücksichtigung. Besonders deutlich werden die unterschiedlichen Standpunkte der Befürworter einer Neuen Mathematik in der Auseinandersetzung mit axiomatischen Fragestellungen. ${ }^{9}$ Die folgende Dichotomisierung von Zielsetzungen von Kapur (1977, S. 261-262) stützt Lennés Beobachtungen (Herv. d. Verf.):

- One goal was to present mathematics as an axiomatic deductive structure and to feed the students on a ready-made definitions-axioms-theorems diet. Another goal was to encourage a discovery approach, to give freedom to learn, to let students re-invent mathematics and use the Socratic method. These were not easily compatible goals. In one, axiom systems were the starting point, in the other they could possibly be the end products.

- One goal was complete rigour. Number systems were to be deduced from Peano's axioms and geometry was to be based on Hilbert axioms. Another goal was to encourage creativity and intuition. One goal required being formal, another goal required being informal.

- One goal was global axiomatization, another was to replace it by local axiomatization and by small deductive structures. [...]

- One goal was to prepare every child to be a future research mathematician. [...] Another goal was to prepare every child for life and to give him that mathematics which would let him deal with day-to-day problems. [...]

\footnotetext{
${ }^{9}$ „Kaum ein Problem der konkreten Stoffbestimmung von Mathematik hat die mathematisch-didaktische Diskussion mehr angefacht als die Frage nach der Rolle der Axiomatik in der Schule“ (Lenné 1969, S. 233).
} 
- In one goal ready-made mathematics was emphasized, in the other, acted-out mathematics was more important. In one case, axioms are to be given by the teacher or the text-book author, in the other the student has to discover the axioms. In one goal, mathematical models (axioms) were given to students, consequences (theorems) had to be deduced, in the other goal, mathematical model making, whether inside or outside mathematics, is the main object of mathematics education.

Die rigorose Richtung betont die ,,ausführliche Behandlung von Axiomatik im Sinne von Deduktionen“ (Lenné 1969, S. 87). Kennzeichnend dafür ist unter anderem die Forderung „nach streng axiomatischer Behandlung ganzer Gebiete der Schulmathematik etwa vom 15. Lebensjahr an“(Lenné 1969, S. 88; siehe auch Dieudonné 1961).

Als Hauptvertreter der gemäßigten Richtung kann der niederländische Mathematiker und Didaktiker Hans Freudenthal (1905-1990) angesehen werden, der sich in verschiedenen Publikationen intensiv mit der Rolle der Axiomatik im Mathematikunterricht auseinandergesetzt hat. ${ }^{10}$ Er stellt zunächst fest: ,Worte wie ,Sprache“ oder ,Mathematik“ haben eine Doppelbedeutung. Sie können eine Tätigkeit bezeichnen oder auch das Resultat dieser Tätigkeit. Nach diesen zwei Gesichtspunkten kann man sie auch unterrichten, als ein Fertigprodukt oder als etwas neu zu Entdeckendes, zu Erfindendes“ (Freudenthal 1963, S. 12; Herv. d. Verf.). Freudenthal plädiert dafür, die Mathematik nicht als Fertigprodukt - d. h. als fertig geordnetes axiomatisch-deduktives System - zu lehren, sondern den Prozess, der zu einer solchen axiomatischen Ordnung führt, in den Vordergrund unterrichtlicher Bemühungen zu stellen. Mathematik bzw. Axiomatik als Fertigprodukt finde man vor allem in mathematischen Fachtexten: „Der Mathematiker ist gewöhnt zu objektivieren. Er publiziert nicht seine Gedankengänge, sondern eine objektivierende Bearbeitung: Definitionen, Satz, Beweis. Wenn er von den Überlegungen, die ihn zum Ziele führten, etwas veröffentlichte, käme er sich vor, als stände er in der Unterhose auf der Straße“ (Freudenthal 1963, S. 16). ${ }^{11}$ Eine überwiegend fachsystematisch ausgerichtete Schulmathematik gehe von der Prämisse aus, den Schülerinnen und Schülern eine fertige Mathematik beizubringen, wie sie jedoch erst nach einem langwierigen Prozess heuristischer, mathematisierender und systematisierender Aktivitäten entstehen kann (vgl. Freudenthal 1963, S. 16-17; siehe auch Kline 1966). Für einen derart gestalteten Unterricht hat Freudenthal den Begriff antididaktische Inversion geprägt: ,[D]em Schüler wird das Resultat

\footnotetext{
${ }^{10}$ Am bedeutendsten in diesem Zusammenhang ist sein Werk Mathematik als pädagogische Aufgabe (Freudenthal 1973a,b). Die wichtigsten seiner darin geschilderten Gedanken zur Axiomatik finden sich jedoch bereits in Freudenthal 1963.

${ }^{11}$ Das prominenteste Beispiel für diese Praxis stellt wohl die Arbeitsweise Carl Friedrich Gauß' dar, der seine Ideen erst dann veröffentlichte, wenn er für seine Behauptungen besonders ausgereifte und elegante Beweise gefunden hatte. Er verglich die Mathematik dabei häufig mit einem Bauwerk, dem man sein Gerüst nach der Fertigstellung nicht mehr ansehen dürfe (vgl. Meschkowski 1990, S. 113).
} 
der Analyse vorgesetzt [...]; die Gedanken, die uns zum Resultat führten, verheimlichen wir" (Freudenthal 1973a, S. 100-101). Für die Behandlung axiomatischer Aspekte im Mathematikunterricht folgert er:

Gehört die Axiomatik auf die Schule? Wenn die Frage so wie in den meisten Entwürfen der letzten Jahre beantwortet wird, sage ich: nein. Auf die Schule paßt keine präfabrizierte Axiomatik, ebensowenig wie präfabrizierte Mathematik im Allgemeinen. Aber das, was der erwachsene Mathematiker an der Axiomatik hochschätzt, gehört wohl auf die Schule: das Axiomatisieren. (Freudenthal 1963, S. 22; Herv. im Orig.)

Als Vorstufe des Axiomatisierens betont er die Tätigkeit des lokalen Ordnens, die im Folgenden für die Geometrie beschrieben wird, aber in offensichtlicher Weise auf andere mathematische Gebiete übertragbar ist:

Man analysiert die geometrischen Begriffe und Beziehungen bis zu einer recht willkürlichen Grenze, sagen wir, bis zu dem Punkte, wo man von den Begriffen mit dem bloßen Auge sieht, was sie bedeuten, und von den Sätzen, daß sie wahr sind. So räsonniert man immer in der Geometrie unseres Lebensraumes; niemals aus Axiomen, die viel zu weit weg liegen, sondern, nach einem verschwimmenden und sich verschiebenden Horizont von Sätzen hin, die jeweils als wahr angenommen werden. Das Feld wird auf kleine oder größere Strecken, aber nicht als Ganzes geordnet. (Freudenthal 1973a, S. 142)

Freudenthal deutet am Beispiel des Satzes über die Mittelsenkrechten im Dreieck an, wie lokales Ordnen auf Schulniveau aussehen kann (siehe Freudenthal 1973b, S. 423426); daneben gehören die Vorschläge zum lokalen Ordnen der Winkelsätze in der ebenen Geometrie zu den bekanntesten Umsetzungen (siehe vor allem Griesel 1963).

Neben Freudenthal betont vor allem der deutsche Mathematikdidaktiker Hans-Georg Steiner (1928-2004) die Bedeutung des lokalen Ordnens und Axiomatisierens (siehe beispielsweise seine in Abschnitt 4.2.1 vorgestellten Unterrichtsideen zur Axiomatisierung von Abstimmungsmengen). Bereits in seinen frühen Publikationen spricht er sich ausdrücklich für die Vermittlung des Prozesscharakters mathematischer Theoriebildung aus:

Es ist ein Irrtum, zu glauben, daß Mathematik heute in derselben Weise geschaffen wird, wie sie sich nach systematischer und logischer Abklärung als Endresultat in der Spezialvorlesung darstellt. Auch bei der Entstehung der modernen mathematischen Theorien spielen induktive Prozesse und vom „Konkreten“ ausgehende Abstraktionen eine ganz wesentliche Rolle. Die Axiomatisierung, die ursprünglich nichts weiter als ein besonders erfolgreiches Mittel zur logischen Beherrschung von Strukturzusammenhängen innerhalb einer Disziplin darstellt und deren primärer Sinn es ist, daß man sich genau 
Rechenschaft darüber gibt, was man als gesichert voraussetzen will und was nicht, erfolgt immer erst, nachdem eine große Menge induktiver, abstrahierender, vergleichender und stückweise deduktiver Vorarbeit geleistet worden ist. Vor der vollkommenen Axiomatisierung eines ganzen mathematischen Gebiets steht die logische Durchordnung von kleineren Gruppen zusammenhängender Teilresultate desselben. Das ist eine mathematische Tätigkeit, die in allen Stadien der Erschließung einer mathematischen Theorie und generell einer jeden mathematisch erfaßbaren Wissenschaft vorkommt. (Steiner 1959, S. 13-14; Herv. d. Verf.)

Die Prozess-Produkt-Unterscheidung ist für die Auseinandersetzung mit der didaktischen Literatur insofern bedeutsam, als dort mitunter nicht immer klar benannt wird, welcher Aspekt der Axiomatik eigentlich gemeint ist. Häufig wird pauschal von Axiomatik gesprochen, wodurch der Eindruck einer umfassenden Ablehnung einer unterrichtlichen Behandlung axiomatischer Themen entstehen kann. Dabei dürfte eine Fokussierung der Entwicklung und des Ordnens mathematischen Wissens - im Rahmen derer das Axiomatisieren eine wichtige Rolle spielt - auf weitaus weniger Widerstand stoßen als eine mit Fachbegriffen überfrachtete axiomatisch-deduktive Präsentation schulmathematischer Stoffgebiete.

\subsection{Wesentliche Bedenken}

In diesem Abschnitt sollen die wesentlichen Kritikpunkte dargestellt werden, die häufig gegen eine Behandlung axiomatischer Aspekte im Mathematikunterricht ins Feld geführt werden. Es wird sich dabei herausstellen, dass diese sich in zwei Kategorien einordnen lassen: methodische Bedenken gegen eine zu rigorose, statische Behandlung der Axiomatik im Unterricht sowie grundsätzliche Bedenken, dass eine sinnvolle Thematisierung auf Schulniveau überhaupt gelingen kann.

Der Großteil der erwähnten Literatur stammt aus der Zeit der Neuen Mathematik, da die didaktische Auseinandersetzung mit dem Thema in dieser Zeit besonders intensiv geführt wurde. In den letzten Jahrzehnten gab es hingegen kaum Publikationen zu diesem Thema. Möglicherweise betrachten viele Mathematikdidaktikerinnen und -didaktker die Frage nach dem Platz von Axiomatik im Unterricht als mit dem Scheitern der Neuen Mathematik abschließend beantwortet.

Häufig wird kritisiert, dass die starke Betonung eines abstrakten, axiomatischen Standpunkts die Mathematik als isolierte Wissenschaft erscheinen lasse, deren Anwendungsbezug nur noch zweitrangig ist. Die bekannte Spiel-Metapher (Mathematik als formales 
Spiel mit Symbolen und Regeln für deren Gebrauch) mag zwar für bestimmte Zwecke ihren Charme besitzen, lässt die Schülerinnen und Schüler aber letztlich nicht den Sinn und Zweck von Mathematik erkennen (vgl. Blank 1966, S. 15, Kline 1973, S. 74-83 und Tietze und Förster 2000, S. 5). Freudenthal (1973b, S. 419) spricht gar von einer ,pädagogischen Bankrotterklärung“, wenn Lernenden gegenüber das Thema mit dem Argument gerechtfertigt werde, man solle sich unter Axiomen Spielregeln vorstellen. Fragen nach der Herkunft und der Sinnhaftigkeit dieser Spielregeln würden so unterdrückt. Sie sind aber essentiell, wenn Mathematik gerade nicht als Fertigprodukt, sondern als Prozess erfahrbar gemacht werden soll. Deduktive Ansätze, die Axiome an den Anfang stellen, um daraus anschließend Aussagen abzuleiten, liefern nur ein unvollständiges, sogar verzerrtes Bild von der Bedeutung der Axiomatik für die Mathematik, denn Axiomensysteme stellen das Endprodukt eines langwierigen Prozesses des Systematisierens und Ordnens dar. $^{12}$

Auch aus lernpsychologischer Sicht ist eine verfrühte Konzentration auf Abstraktion und Formalisierung im Rahmen eines axiomatisch-deduktiven Unterrichtsansatzes ungeeignet. Theorien der kognitiven Entwicklung weisen axiomatische Elemente in der Regel ihren jeweils letzten Stufen zu. Ein bekanntes Beispiel ist van Hieles Modell der geometrischen Denkentwicklung, dem zufolge erst auf der letzten Stufe die Bedeutung von Deduktionen und Axiomen begriffen wird (vgl. van Hiele 1959, S. 246). Auch neuere Theorien wie David Talls „Three Worlds of Mathematics“ weisen die Axiomatik als Endstufe der kognitiven Entwicklung aus (siehe Tall 2013, S. 133-154).

Unterrichtsmethodisch gesehen ist eine strikt auf Axiomen aufbauende Beweisführung für Lernende zu unübersichtlich und mühselig: ,[D]er Weg von den Axiomen zu wichtigen Sätzen ist zu lang“ (Freudenthal 1973b, S. 426); derweil geht es um die Beschäftigung mit Deduktionen von Aussagen, die den Schülerinnen und Schülern intuitiv klar und nicht beweisbedürftig erscheinen (siehe auch Kline 1973, S. 24-33, 55-56 und Buck 1966, S. 21). ${ }^{13}$

Die bisher angebrachten Argumente können im Wesentlichen als Kritik an einer Überbetonung deduktiver Aspekte im Mathematikunterricht verstanden werden. Die im vorigen Abschnitt erläuterte Prozess-Produkt-Unterscheidung spielt dabei eine wichtige Rolle. Der Unterricht soll sich nicht an der fertigen Mathematik orientieren, sondern ,,an den natürlichen erkenntnistheoretischen Prozessen der Erschaffung und Anwendung von Mathematik“ (Wittmann 1981, S. 130). Insofern wird eine unterrichtliche Behandlung axio-

\footnotetext{
${ }^{12}$ Ähnlich argumentieren auch Kline (1966, S. 58), Blank (1966, S. 16) und Wittmann (1981, S. 144-147).

${ }^{13}$ Zur Problematik der Einsicht in die Beweisnotwendigkeit aus Schülersicht hat sich Winter (1983b) umfassend geäußert.
} 
matischer Themen dadurch nicht prinzipiell abgelehnt, die Art und Weise der Behandlung aber kritisch in den Blick genommen.

So wird unter anderem von Vollrath (1994, S. 17), Steiner (1965, S. 17) und Kline (1973, S. 98) betont, dass das Verständnis eines abstrakt vorliegenden Axiomensystems nur auf Grundlage der Betrachtung hinreichend vieler konkreter, mit der Realität verknüpfter Beispiele erfolgen könne, die den Schülerinnen und Schülern strukturelle Gemeinsamkeiten deutlich machen und die Vorteile einer gemeinsamen axiomatischen Beschreibung hervorheben. Generell darf das „Lösen der ontologischen Bindung“ mathematischer Aussagen und Begriffe von ihrem realweltlichen Bezug erst erfolgen, wenn dieser Bezug bei den Schülerinnen und Schülern ,vorhanden und kräftig“ ist (Freudenthal 1973b, S. 417). Man kann Abstraktion nur dann verstehen, wenn man weiß, wovon abstrahiert wird. Gleichzeitig muss laut Steiner (1965, S. 17) aber auch sichergestellt sein, dass die so erschlossenen axiomatischen Strukturen ,für deduktives Vorgehen auf elementarer Stufe in einem gewissen Ausmaß geeignet sind, so daß auch ein Stück der zugehörigen allgemeinen Theorie entwickelt und nutzbar gemacht werden kann“.

Abgesehen von Ansätzen, die ,,[n]eben die Mathematik als Fertigprodukt [...] gleichrangig die Mathematik als schöpferische Methode“ (Steiner 1965, S. 7) setzen wollen, gibt es allerdings auch die Meinung, Axiomatik gehöre generell nicht in den Mathematikunterricht. Es wird angeführt, die Axiomatik sei für praktizierende Mathematiker nicht so bedeutsam, dass dadurch eine unterrichtliche Behandlung gerechtfertigt werden könne. So schildert beispielsweise Kline (1973) anhand historischer Beispiele die Rolle heuristischer und intuitiver Ansätze bei der Entwicklung mathematischer Ideen und Polya (1949/1995, S. 9) betont im Vorwort seines bekannten Werkes Schule des Denkens, dass die „Mathematik im Entstehen [...] als experimentelle induktive Wissenschaft“" erscheine. In der Tat gibt es ein ,,außerordentlich gutes und systematisches Quellenmaterial für die empirische Theorieentwicklung in der Mathematik“ (Witzke 2011, S. 911). Es ist demnach auch historisch gesehen nicht abwegig, wenn beispielsweise Schoenfeld (1985) beobachtet, dass Schülerinnen und Schüler die Schulmathematik überwiegend als empirische Wissenschaft kennenlernen. Witzke (2012, S. 952) meint dazu,

dass Schulmathematik - im naturwissenschaftlichen Sinne vermittelt und aufgefasst - eine vernünftige Mathematik ist. Diese ist aber wegen der empirischen Verortung der Grundbegriffe eine andere als die moderne Hochschulmathematik. Der ,Abstraktionsschock' ist mithin eine fast logische Konsequenz, der [sic!] sich nicht vermeiden aber (z. B. historisch) erklären lässt. Versuche einer Angleichung erscheinen beim Blick auf die historische Entwicklung problematisch. 
Warum also, könnte man fragen, sollten Schülerinnen und Schüler sich überhaupt mit dem Thema Axiomatik im Schulunterricht auseinandersetzen, wenn doch für die Entdeckung und Weiterentwicklung interessanter mathematischer Resultate axiomatisches Vorgehen mitunter wenig relevant ist und ein empirisch orientiertes Mathematikbild für schulische Zwecke völlig ausreichend erscheint? Als Gegenargument kann man natürlich auf die Bedeutung der Axiomatik für die strukturierte Darstellung mathematischen Wissens verweisen wie auch auf die Rolle, die axiomatische Fragestellungen bei der Entwicklung der Mathematik von einer empirischen, der Anschauung verhafteten zu einer modernen, abstrakt-formalen Wissenschaft gespielt haben. Darauf wird wiederum eingewendet, diese Bedeutung für die Wissenschaft Mathematik sei von Schülerinnen und Schülern anhand der Schulmathematik nicht hinreichend nachvollziehbar, so dass eine Berücksichtigung dieser Thematik im Unterricht nicht sinnvoll sei. Eine derartige Argumentation findet sich beispielsweise bei Wittenberg (1963, S. 50-51):

Im Unterricht muß sich für den Schüler eine gültige Begegnung mit der Mathematik, mit deren Tragweite, mit deren Beziehungsreichtum, vollziehen; es muß ihm am Elementaren ein echtes Erlebnis dieser Wissenschaft erschlossen werden. Der Unterricht muß dem gerecht werden, was Mathematik wirklich ist. (Herv. im Orig.)

Ein solches Erlebnis werde jedoch nicht durch eine äußerliche Anpassung der Schulmathematik an moderne höhere Mathematik erreicht, welche seiner Ansicht nach durch die Reformvorhaben der Neuen Mathematik angestrebt werde (vgl. Wittenberg 1963, S. 5354). Auch Wittenberg plädiert für ein Lehren der Mathematik als Prozess, wenn er darauf hinweist, dass von einer gültigen Erfahrung ,nur in dem Maße die Rede sein [kann], wie der Unterricht nicht nur die Ergebnisse, sondern das ganze Vorgehen in überzeugender Weise innerhalb des geistigen Erfahrungsbereichs des Schülers zustandekommen läßt" (Wittenberg 1963, S. 59). Im Hinblick unter anderem auf die Axiomatik geht er aber im Gegensatz zu Steiner und Freudenthal noch einen Schritt weiter. Er ist der Überzeugung, dass

die frappanten äußerlichen Züge der modernen Mathematik - Allgemeinheit, axiomatische Begründung, formaler Aufbau, Strenge - ihr allmähliches Zustandekommen nicht einer Laune der Mathematiker, sondern organischen Notwendigkeiten verdanken, die dem begrenzten Erfahrungsbereich des Gymnasiasten notwendigerweise größtenteils fremd bleiben müssen. Aus dem historischen und sachlichen Zusammenhang dieser Notwendigkeiten gerissen, werden sie aber willkürlich und sinnlos, und damit durch und durch unmathematisch. [...]

In der höheren Mathematik werden jene Begriffe und Methoden nicht um ihrer selbst willen eingeführt, sondern weil sie mathematisch etwas leisten - 
sie dienen dazu, neuartige mathematische Erkenntnisse zu erschließen. Wäre dem nicht so, so würde sich kein schöpferischer Mathematiker dazu hergeben, auch nur einen Gedanken an sie zu verschwenden. Am Gymnasium leisten sie aber charakteristischerweise nichts. Sie bleiben Selbstzweck - und damit Unsinn [...]. (Wittenberg 1963, S. 55)

Wittenberg bezweifelt also, dass die „organischen Notwendigkeiten“ des Axiomatisierungsprozesses und das deduktive Erschließungspotential von ausgewählten Axiomensystemen im Schulunterricht „sachgemäß und sauber“ (Wittenberg 1963, S. 249) behandelt werden können. Diese Formulierung erinnert an Jerome Bruners (1915-2016) Forderung, Lehrinhalte ,in einer intellektuell ehrlichen Form“ zu vermitteln (Bruner 1973, S. 44). ${ }^{14}$

Mit einem indirekten Verweis auf die Gödelschen Resultate (siehe Abschnitt 2.2.2) hält Wittenberg fest:

Unter diesen Umständen kann man in guten Treuen geteilter Meinung darüber sein, ob es überhaupt angezeigt ist, axiomatische Gesichtspunkte ausdrücklich am Gymnasium zu behandeln; besonders heute, da die Fachleute einsehen mußten, daß, im Gegensatz zu früher gehegten Hoffnungen, auch die axiomatische Methode weder letzte Wahrheit liefert noch der letzte Schritt in der Analyse der Grundlagen der Mathematik ist. (Wittenberg 1963, S. 248)

Mit Blick auf die Geometrie räumt er allenfalls die Möglichkeit einer „rückblickenden Untersuchung“ (Wittenberg 1963, S. 250) am Ende der Schullaufbahn ein, in der - im Rahmen einer Auseinandersetzung mit einem anschaulichen Beispiel nicht-euklidischer Geometrie - auf die Fragen nach deren Wahrheit und logischen Struktur eingegangen werden kann. Im Sinne einer echten Erfahrung der Mathematik müsse dann jedoch auch „die Bedeutung des axiomatischen Vorgehens angemessen gewürdigt“" (Wittenberg 1963, S. 252) werden, indem die prinzipielle freie Wählbarkeit der Axiome, die durch sie gegebene implizite Definition der geometrischen Grundbegriffe wie auch die damit aufgeworfenen Probleme hinsichtlich der Widerspruchsfreiheit thematisiert werden (vgl. Wittenberg 1963, S. 252-253). Wittenberg bleibt schlussendlich skeptisch, ob ein solches Vorhaben gelingen kann:

Dies sind allerdings keine leichten Einsichten. Vielleicht sind sie unserem Schüler unerreichbar oder würden einen ungebührlichen Unterrichtsaufwand erfordern. Sollte dies aber der Fall sein, so gebietet es eben den Verzicht auf axiomatische Betrachtungen. [...] Keinesfalls werden wir in die Ursünde pädagogischer Selbsttäuschung zurückfallen, die hier darin bestünde, unter Berufung auf die Wichtigkeit axiomatischer Gesichtspunkte diese in unseren Unterricht einzubeziehen, unter Berufung auf deren Schwieirigkeit aber davor zu

\footnotetext{
${ }^{14}$ Im mathematikdidaktischen Bereich ist vor allem Arnold Kirsch (1922-2013) für die Verwendung dieser Formulierung bekannt (siehe beispielsweise Kirsch 1976).
} 
resignieren, vom Schüler ein wirkliches, prüfbares Verständnis zu verlangen.

(Wittenberg 1963, S. 253)

Rückblickend lassen sich die vorgebrachten Einwände in zwei Bereiche unterteilen:

(1) Methodische Bedenken: Im Vordergrund einer unterrichtlichen Behandlung sollte nicht (nur) das deduktive Arbeiten mit vorgegebenen Axiomensystemen stehen, sondern (auch) die Tätigkeit des Axiomatisierens und lokalen Ordnens. Insbesondere die vollständige axiomatische Darstellung ganzer Themengebiete wie der Geometrie ist im Mathematikunterricht weder durchführbar noch sinnvoll.

(2) Grundsätzliche Bedenken: Es ist die Frage zu klären, ob axiomatische Aspekte für eine echte Erfahrung der Mathematik überhaupt bedeutsam sind bzw. inwieweit eine intellektuell ehrliche Thematisierung der Axiomatik mit Schülerinnen und Schülern möglich ist.

Die grundsätzlichen Bedenken haben sich offenbar durchgesetzt. Jedenfalls spielen axiomatische Aspekte in den heutigen Lehrplänen und Bildungsstandards (zumindest explizit) sowie in der didaktischen Diskussion kaum eine Rolle. Andererseits ist das Thema Axiomatik eng mit dem Kompetenzfeld (Mathematisches) Argumentieren verknüpft, zu dem eine Fülle an Literatur existiert. Der nächste Abschnitt beschäftigt sich daher mit der Frage, inwieweit sich die Behandlung axiomatischer Aspekte in die heutige (didaktische) Sicht auf die Rolle des Beweisens und Argumentierens im Mathematikunterricht einbetten lässt.

\subsection{Einordnung der Axiomatik in das Kompetenzfeld Argumentieren}

(Mathematisches) Argumentieren ist in sämtlichen Bildungsstandards, Lehrplänen und Kompetenzmodellen - nicht nur in Deutschland - ein zentraler Bestandteil, wenngleich der Gebrauch der Begriffe Argumentieren, Begründen und Beweisen oftmals uneinheitlich ist (siehe Brunner 2014, insb. S. 27-53). Während manche Forscher vom Argumentieren in bewusster Abgrenzung zum Konzept des mathematischen Beweisens reden (z. B. Duval 1991), betrachten es andere eher als Sammelbegriff für eine sehr umfassende Kompetenz, in die sich das Beweisen als Spezialfall eingliedert bzw. die zumindest viele strukturelle Gemeinsamkeiten zum Beweisen aufweist (z. B. Pedemonte 2007).

Konsens besteht aber darüber, dass der Mathematikunterricht den verschiedenen Stufen der Strenge beim mathematischen Argumentieren bzw. Beweisen Rechnung tragen muss. 
Winter (1975, S. 109) fordert als ,eines der zentralen Lernziele [...] speziell des Mathematikunterrichts“, er müsse den Schülerinnen und Schülern „Möglichkeiten geben, rationale Argumentation zu üben“. Dies umfasse ,all jene [Lernsituationen], in denen eine Behauptung gerechtfertigt werden soll“. Zwar gehe es dabei primär um die „Schulung der Strenge“, gleichzeitig sei aber auch der ,Verzicht auf den Glauben, es gebe eine absolute und unveränderliche Strenge“" von grundlegender Bedeutung (Herv. im Orig.).

Für die Kompetenz Mathematisch argumentieren (Kl) wird in den KMK-Bildungsstandards betont: „Das Spektrum reicht dabei von einfachen Plausibilitätsargumenten über inhaltlich-anschauliche Begründungen bis zu formalen Beweisen“ (Kultusministerkonferenz 2015, S. 14). Im Fokus der didaktischen Auseinandersetzung mit dem Thema Beweisen steht also nicht primär die formal möglichst strenge Herleitung einer Aussage aus bereits bekannten Sätzen bis hin zur schlussendlichen Rückführung auf ein Axiomensystem. Viele aktuelle Beiträge betonen vielmehr die zentrale Rolle heuristischer, experimenteller und explorativer Elemente beim Prozess der Beweisfindung (z. B. Hanna 2000, Meyer 2007, de Villiers 2010, Arzarello et al. 2012 sowie Leuders und Philipp 2014).

Auch bei der Formulierung von Beweisen gibt es - je nach kognitivem Leistungsstand der Schülerinnen und Schüler - unterschiedliche Niveaus hinsichtlich der formalen Strenge und der Art der zulässigen Schlussweisen. Prominente Literaturbeispiele zum informellen Beweisen sind unter anderem Kirsch 1979 (prämathematische Beweise), Wittmann und Müller 1988 (inhaltlich-anschauliche Beweise) sowie Blum und Kirsch 1991 (präformale Beweise). Exemplarisch zitiere ich aus Wittmann und Müller 1988, S. 254:

Eine sinngemäße Übertragung von Beweisaktivitäten in die schulischen Rahmenbedingungen erfordert [...] eine Loslösung von formalen, deduktiv durchorganisierten Darstellungen der für die Schule relevanten elementarmathematischen Gebiete zugunsten inhaltlich-anschaulicher Darstellungen. Diese sind gekennzeichnet durch Einbettung in sinnvolle Kontexte, durch Entwicklung von Motivationen, durch ein Vorgehen gemäß heuristischen Strategien, durch die Verwendung bedeutungshaltiger präformaler Darstellungen und durch entsprechende inhaltlich-anschauliche Beweise.

Um den unterschiedlichen Beweisniveaus im Mathematikunterricht gerecht zu werden, ist der Begriff der Argumentationsbasis nützlich:

Um eine Aussage $\alpha$ durch Schließen begründen zu können, ist notwendig, daß Aussagen $\beta, \gamma, \ldots$ vorliegen, die als richtig angesehen werden und aus denen auf die Richtigkeit von $\alpha$ geschlossen werden kann. Eine Menge von Aussagen, die als richtig angesehen werden, soll zusammen mit den Schlußweisen, die als zulässig anerkannt werden, als Argumentationsbasis bezeichnet werden. (Bürger 1979, S. 106; Herv. im Orig.) 
Man beachte, dass hier keine einschränkenden Bedingungen hinsichtlich der gültigen Schlussweisen formuliert werden, die somit ausdrücklich auch nicht-deduktive Argumentationsmuster beinhalten können. Gerade im schulischen Bereich herrschen oft Argumentationsbasen „,mit einer meist geringen Explikation von Annahmen und Schlussregeln“ vor (Jahnke und Ufer 2015, S. 334). Das kann problematisch sein, insbesondere wenn die Lernenden und die Lehrkräfte unterschiedliche Argumentationsbasen zugrunde legen. In solchen Fällen kann es zu dem oft beschriebenen Phänomen kommen, dass die Schülerinnen und Schüler die Notwendigkeit eines von der Lehrperson eingeforderten Beweises nicht sehen (können), da der zu beweisende Satz für sie „einfach klar“, also bereits Teil der Argumentationsbasis ist. ${ }^{15}$ Ein Ziel des Mathematikunterrichts muss deshalb sein, den Lernenden das Vorhandensein unterschiedlicher Argumentationsbasen bewusst zu machen und ihre Argumentationsbasen schrittweise weiterzuentwickeln (vgl. Fischer und Malle 2004, S. 181-183).

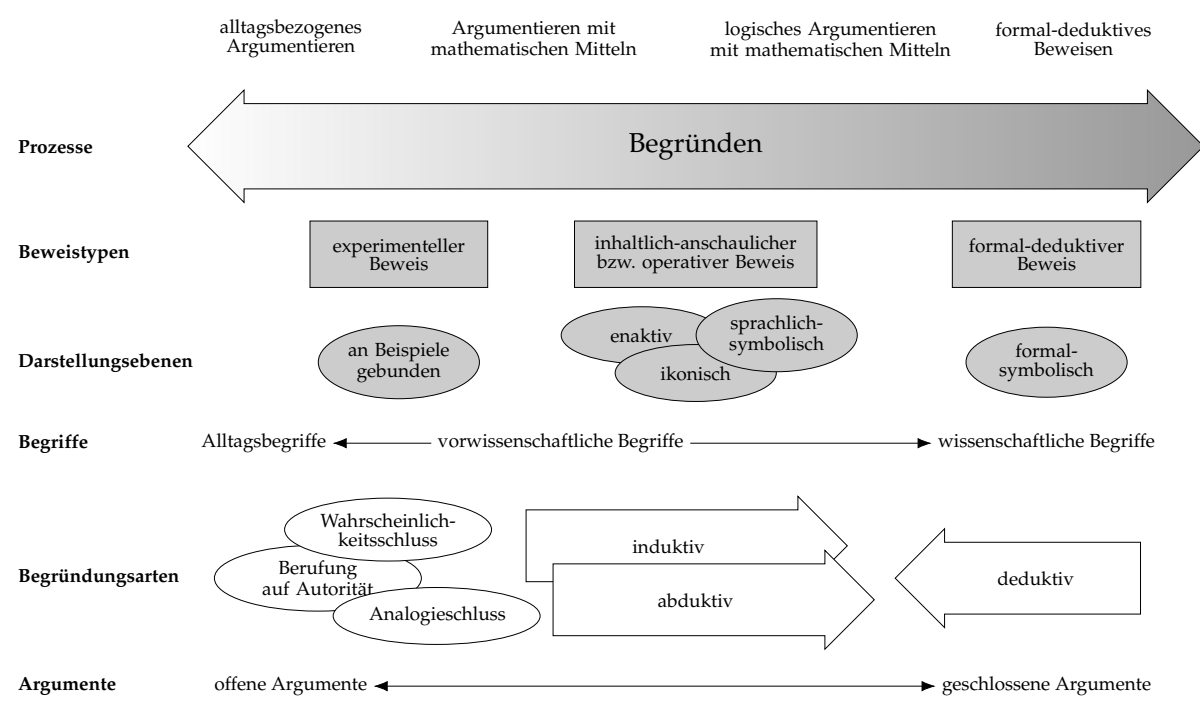

Abbildung 3.2: Verhältnis zwischen Argumentieren, Begründen und Beweisen ${ }^{16}$

Ein aus meiner Sicht nachvollziehbares und für die Mathematikdidaktik weitgehend konsensfähiges Modell für das Verhältnis zwischen Argumentieren, Begründen und Beweisen stammt aus Brunner 2014 (siehe Abbildung 3.2). Begründen wird hier als Oberbegriff für ein Kontinuum verschiedener Aktivitäten verwendet, welches beim alltagsnahen Argumentieren beginnt und unter sukzessiver Hinzunahme von mathematischen und logischen Mitteln schließlich beim formal-deduktiven Beweisen endet. Eine besondere Stärke des Modells ist, dass es viele für das Argumentieren und Beweisen grundlegende didaktische

\footnotetext{
${ }^{15}$ Fischer und Malle (2004, S. 180-181) beschreiben unterschiedliche Argumentationsbasen, die zur Begründung der Aussage „In einem gleichschenkligen Trapez sind die Diagonalen gleich lang. “ herangezogen werden können.

${ }^{16}$ Quelle: selbst erstellt nach Brunner 2014, S. 49.
} 
Konzepte integriert: Entlang des oben beschriebenen Kontinuums lassen sich unterschiedliche Beweistypen (Wittmann und Müller 1988), Darstellungsebenen (Bruner 1974; Zech 2002, S. 104-110), Begriffe, Begründungsarten (vor allem Abduktion, Induktion und Deduktion; siehe auch Meyer 2006) sowie offene und geschlossene Arten von Argumenten (Jahnke 2008) passend einordnen.

Ein weiteres Anliegen der Mathematikdidaktik ist das Herausarbeiten verschiedener Funktionen des Beweisens in der Mathematik. de Villiers (1990) unterscheidet fünf Funktionen:

1. Verifikation (Nachweis, dass eine Aussage wahr ist)

2. Erklärung (Einsicht, warum eine Aussage wahr ist)

3. Systematisierung (Organisation verschiedener Aussagen in einem logisch-deduktiven System)

4. Entdeckung neuer mathematischer Sätze

5. Kommunikation (Vermittlung mathematischen Wissens innerhalb einer sozialen Gemeinschaft)

Dabei behandeln die ersten beiden Funktionen die kognitive Rolle von Beweisen, während die dritte Funktion die epistemologische und die letzten beiden die soziale Rolle beschreiben (vgl. Brunner 2014, S. 14). Während aus rein fachlicher Sicht häufig die Verifikations- und Systematisierungsfunktion im Vordergrund stehen, wird für den Schulunterricht auch die Bedeutung der anderen Funktionen betont; insbesondere die erklärende Rolle eines Beweises wird immer wieder an verschiedenen Beispielen thematisiert: „Schulische Beweise sollen nicht in erster Linie dazu dienen zu verstehen, daß etwas so ist, sondern, warum etwas so ist" (Tietze und Förster 2000, S. 167; Herv. im Orig.). ${ }^{17}$

Insgesamt lässt sich festhalten, dass seitens der Mathematikdidaktik für eine umfassende Einbettung mathematischer Beweisaktivitäten auf verschiedenen Niveaus der Strenge in die Unterrichtskultur plädiert wird. Es muss jedoch als erstaunlich bezeichnet werden, dass axiomatische Aspekte, die im Rahmen des in Abbildung 3.2 dargestellten Kontinuums in den Bereich formal-deduktiver Beweise fallen, in der Regel nicht Gegenstand der didaktischen Diskussion sind.

Außerdem wird zunehmend gefordert, dass Schülerinnen und Schüler über ein hinreichendes Metawissen zum Thema Beweisen verfügen und sich der von ihnen verwendeten

\footnotetext{
${ }^{17}$ Ein erklärender Beweis für die Aussage, dass der Umfang eines konvexen Vierecks größer ist als die Summe der Länge der beiden Diagonalen wird von Hefendehl-Hebeker und Hußmann (2003, S. 97) thematisiert.
} 
Aussagen und Schlussweisen bewusst werden (siehe hierzu beispielsweise Hemmi 2008, Kuntze 2009 und Cabassut et al. 2012). Axiomatische Gesichtspunkte stellen vor diesem Hintergrund sicher nicht den für die Schule bedeutsamsten Aspekt dar. Sie sind jedoch ein wichtiger Bestandteil eines abgerundeten Bildes vom mathematischen Beweisen. Winter (1975, S. 110) erläutert, ,worauf es bei rationaler Argumentation allgemein ankommt“:

- Die Einsicht in die Richtigkeit einer Aussage geschieht erst innerhalb eines Systems von „zugehörigen“ Aussagen. Isolierte Einsicht ist gar keine Einsicht $[\ldots]$.

- Notgedrungen führt das logische Zurückverfolgen auf einen „Anfang“, d. h. auf Aussagen, die man nicht weiter auf andere zurückführen kann, deren Wahrheit ,angenommen“ wird.

Damit spricht Winter das zentrale Charakteristikum mathematischen Begründens und Beweisens an, wie es auch in der Definition des Begriffs Argumentationsbasis (siehe oben) zum Vorschein tritt: das Zurückführen der Gültigkeit einer Aussage auf die Gültigkeit bereits akzeptierter Aussagen und die damit einhergehende logische Notwendigkeit einer (wie auch immer gearteten) Ausgangsbasis. Dieser Aspekt ist der Kern axiomatischen Denkens, lässt aber genügend Raum, um dieses auf einem variablen Niveau der Strenge zu behandeln. Die exemplarische Einsicht in den axiomatischen Aufbau von Mathematik trägt dazu bei, diese ,als eine deduktiv geordnete Welt eigener Art“ (Winter 1995, S. 37) erfahrbar zu machen und die Fragen zu thematisieren, was einen Beweis ausmacht und welche Funktion(en) er in der Mathematik erfüllt: „Wer vom Beweisen reden will, kann letztlich nicht von Theorien schweigen“ (Jahnke und Ufer 2015, S. 351).

\subsection{Zusammenfassung: Zur Rolle der Axiomatik im Mathematikunterricht}

In diesem Abschnitt wird vor dem Hintergrund der bisher in diesem Kapitel angestellten Überlegungen mein eigener Standpunkt zur Behandlung axiomatischer Aspekte im Mathematikunterricht dargelegt, welcher die Konzeption meiner Unterrichtsmaterialien (siehe Kapitel 5) wesentlich trägt.

Zunächst möchte ich auf die grundsätzliche Frage eingehen, inwiefern die unterrichtliche Behandlung von Axiomatik überhaupt erstrebenswert ist. Die aus meiner Sicht wesentlichen Argumente, die für das Thema Axiomatik als Unterrichtsgegenstand sprechen, sind: 
- Der axiomatische Aufbau der Mathematik ist nicht nur historisch bedeutsam, sondern gehört - genauso wie ihre breite Anwendbarkeit - zu einem abgerundeten Gesamtbild dieser Wissenschaft. In diesen Kontext gehört definitiv auch eine Betrachtung des Wahrheitsanspruchs mathematischer Aussagen. Die Ansicht, dass es in der Mathematik um absolute Wahrheit gehe, ist heutzutage nicht mehr zeitgemäß und sollte zumindest gegen Ende der Schullaufbahn kritisch diskutiert werden.

- Die Frage nach dem warum beim mathematischen Argumentieren und Beweisen führt sehr schnell zu axiomatischen Fragestellungen. Die Einsicht, dass mathematische Sätze stets unter Verwendung bereits bekannter und bewiesener Sätze begründet werden, impliziert die Notwendigkeit von Axiomen.

- Der Bezug der Mathematik zur Wirklichkeit (insbesondere zu den Resultaten der Naturwissenschaften) ist ein wesentlicher Aspekt der Bildungsstandards und Lehrpläne. Er manifestiert sich unter anderem in der zentralen Kompetenz des $\mathrm{Mo}$ dellierens. Die Beschäftigung mit der axiomatischen Natur der Mathematik kann einen wichtigen Beitrag dazu leisten, die Möglichkeiten und Grenzen von Modellierungen besser einzuschätzen; denn die Prozesse, die zur Herausbildung und letztlichen Rechtfertigung von Axiomensystemen führen, sind denen beim Modellierungskreislauf häufig sehr ähnlich.

- Betrachtet man die Axiomatik aus einer philosophischen Perspektive, so bietet sie die Gelegenheit zu engeren Vernetzungen mit den Geisteswissenschaften im Mathematikunterricht, welche prinzipiell wertvoll, aber in der Regel schwierig herzustellen und unterrepräsentiert sind.

- Die häufig beschriebene und von vielen Studierenden in den ersten Semestern erlebte Diskrepanz zwischen Schul- und Hochschulmathematik ist nicht nur auf mangelnde Kenntnisse bzw. Routine der Studierenden im Sekundarstufenstoff (v. a. im Bereich elementarer Algebra) zurückzuführen, sondern beruht auch auf einem epistemologischen Auffassungswechsel von der eher empirisch-gegenständlich vermittelten Schulmathematik zur formalistischen, axiomatisch-deduktiv präsentierten Hochschulmathematik (vgl. Witzke 2013). Dieser Bruch ist für Schülerinnen und Schüler besser zu verstehen, wenn er bereits in der Schule zumindest in Ansätzen thematisiert wurde.

Die beiden vorgebrachten Gegenargumente sind, dass die Axiomatik für die praktische Anwendung der Mathematik kaum relevant sei und dass man die eigentliche Bedeutung der axiomatischen Methode auf schulischem Niveau nicht in intellektuell redlicher Weise vermitteln könne. 
Im Hinblick auf das erste Argument bin ich der Auffassung, dass die Relevanz für den praktizierenden Mathematiker kein notwendiges (und auch kein hinreichendes) Merkmal der Sinnhaftigkeit eines Unterrichtsthemas ist. Es ist zwar richtig, dass Mathematiker sich heutzutage größtenteils nicht mit grundlagentheoretischen Fragen beschäftigen und ihre Beweise auch nicht bis zu ihrer axiomatischen Basis zurückverfolgen. Dennoch arbeiten sie innerhalb etablierter Theorien und Theorienetzwerke, die letzten Endes deduktiv geordnet sind. Die Tatsache, dass die Mathematik in axiomatisch-deduktiven Theorien organisiert ist, ist und bleibt eines der fundamentalen Merkmale dieser Wissenschaft, so dass fehlende unmittelbare praktische Relevanz nicht als Argument ausreicht, um Axiomatik als mögliches Unterrichtsthema abzulehnen.

Wittenbergs Zweifel, ob eine Auseinandersetzung mit axiomatischen Aspekten, die ,sachgemäß und sauber“ (Wittenberg 1963, S. 249) ist und den Schülerinnen und Schülern somit ein echtes Erlebnis der Mathematik ermöglicht, überhaupt durchführbar sei, wiegen dagegen deutlich schwerer: Der axiomatische Aufbau der Mathematik ist ein recht theoretisches Thema. Die mathematischen und epistemologischen Prozesse, die zur Herausbildung, Modifikation und Rechtfertigung von Axiomensystemen führen, sind komplex und werden selbst im Mathematikstudium an der Universität nur selten auf einer Meta-Ebene reflektiert und diskutiert.

Es ist im Grunde nicht erläuterungsbedürftig, dass man die Rolle, welche die Axiomatik aus mathematikhistorischer und -philosophischer Sicht wie auch im Hinblick auf die systematische Darstellung und Ordnung mathematischen Wissens gespielt hat, nicht vollumfänglich im schulischen Kontext vermitteln kann. Genauso wenig kann aber beispielsweise die fachliche Tragweite des Vektorbegriffs im Mathematikunterricht aufgezeigt werden. Man muss jederzeit bei der Behandlung mathematischer Inhalte lerngruppenadäquate didaktische Reduktionen vornehmen, d. h. insbesondere bestimmte Teilaspekte vernachlässigen und komplexe Zusammenhänge elementarisieren.

Vor diesem Hintergrund darf intellektuelle Redlichkeit nicht mit der Vermittlung eines aus Fachperspektive umfassenden Verständnisses verwechselt werden. Als Beispiel möchte ich auf Wittenbergs Kommentar eingehen, dass Begriffe der modernen Strukturmathematik im Schulunterricht zwangsläufig Selbstweck blieben. Es ist richtig, dass Schülerinnen und Schüler das Potential wie auch die Entstehungsgründe beispielsweise des abstrakten Gruppenbegriffs nach einer Auseinandersetzung mit den zugehörigen Axiomen, ersten einfachen Deduktionsbeweisen sowie der Identifikation mehrerer konkreter Beispiele (d. h. Modelle) für Gruppen noch nicht einschätzen können. Sie können dadurch allerdings einen Einblick in deduktives Arbeiten gewinnen und im Ansatz erfahren, was Abstraktion bedeutet. Eine Beschränkung auf einen (ggf. kleinen) Ausschnitt axiomatischen 
Denkens und Arbeitens bedeutet nicht automatisch, dass man als Lehrkraft intellektuell unredlich handelt, solange man nichts fachlich Falsches vermittelt oder den Schülerinnen und Schülern suggeriert, das Wesen der Mathematik erschöpfe sich in der Aufstellung von Axiomensystemen und der Herleitung von Aussagen aus diesen. Eine Reduzierung auf grundlegende und aus meiner Sicht vermittelbare Aspekte der axiomatischen Methode schlage ich in Form von sechs übergeordneten Lernzielen in Abschnitt 4.1 vor.

Natürlich spielt das konkrete Vorgehen bei der Behandlung axiomatischer Themen eine wichtige Rolle für die intellektuelle Redlichkeit eines Unterrichtsvorhabens. Damit befindet man sich jedoch nicht mehr auf der Ebene der grundsätzlichen, sondern der methodischen Bedenken. Daher soll nun die Tragweite der in Abschnitt 3.3 diesbezüglich gegenüber der Neuen Mathematik geäußerten Kritikpunkte betrachtet werden, um daraus Konsequenzen für die unterrichtliche Behandlung axiomatischer Aspekte zu ziehen:

- Die im Hinblick auf axiomatische Unterrichtsinhalte geäußerten Bedenken waren gegen Reformbemühungen gerichtet, die eine umfassende Implementierung der axiomatischen Methode im Mathematikunterricht beabsichtigten, beispielsweise in Form einer deduktiven Präsentation ganzer Stoffgebiete der Schulmathematik. Es bestand die berechtigte Befürchtung, dass gerade die explorativen, kreativen Phasen bei der Entdeckung mathematischer Sätze und Zusammenhänge in den Hintergrund gedrängt und die Mathematik auf ihre fertige, deduktiv geordnete Struktur reduziert würde. Auch wenn deduktives Schließen selbst kein Automatismus ist und bei zunehmender Komplexität des theoretischen Gebäudes durchaus kreative und heuristische Kompetenzen erfordert, schließe ich mich der Kritik an, dass sich die axiomatische Methode nicht dazu eignet, im Mathematikunterricht Vertrautheit mit neuen Inhalten zu gewinnen. Auch eine vollständige axiomatische Darstellung ganzer Stoffgebiete ist weder sinnvoll noch durchführbar. Vielmehr sollten Schülerinnen und Schüler lernen, anhand ihnen bekannter Stoffgebiete mathematische Themenfelder rückblickend aus einer neuen Perspektive zu betrachten. Diese Möglichkeit hat - wie in Abschnitt 3.3 bereits erwähnt - auch Wittenberg nicht grundsätzlich abgelehnt.

- Dies impliziert zugleich, dass eine Auseinandersetzung mit axiomatischen Aspekten nicht zu früh erfolgen darf. Die Einwände gegen eine Überbetonung axiomatischer Elemente bezogen sich verstärkt auf die unteren Klassenstufen. ${ }^{18}$ Eine The-

\footnotetext{
${ }^{18}$ Siehe beispielsweise Laugwitz 1966, S. 36: „Ein Weltbild, in dem Mathematik als eine Sammlung von aus Axiomen gefolgerten Sätzen erscheint, ist nicht nur der Altersstufe nicht angepaßt, sondern sogar falsch. Man sollte insbesondere bei Lehrplänen nicht dieses Weltbild zur Orientierung verwenden. Gerade das aber geschieht, wenn man die Lehrpläne schon von Sexta an an Axiomen ausrichtet [...]“ (Herv. d. Verf.).
} 
matisierung in der Oberstufe hingegen wurde auch von Kritikern als sinnvoll bzw. möglich erachtet. Insbesondere für die Behandlung von Themen, die über die euklidische Charakterisierung von Axiomen als evidenten Tatsachen hinausgehen, erscheint eine Beschränkung auf die Sekundarstufe II angebracht. Dennoch möchte ich darauf hinweisen, dass es durchaus Unterrichtsvorschläge gibt, die axiomatische und epistemologische Fragestellungen auf einem für die Sekundarstufe I angemessenen Niveau behandeln. ${ }^{19}$ Einige dieser Vorschläge werden in Abschnitt 4.2 genauer vorgestellt.

- Die Forderung, Mathematik nicht als Fertigprodukt zu lehren, sondern als Tätigkeit, ist - nicht nur im Hinblick auf das Thema der vorliegenden Arbeit - von grundlegender Bedeutung. Strebt man die Vermittlung eines authentischen Bildes der axiomatischen Methode an, muss die Frage nach der Entstehung und Rechtfertigung von Axiomensystemen Bestandteil des unterrichtlichen Bemühens sein. Dies muss (und sollte) jedoch nicht ausschließen, Schülerinnen und Schülern auch Auszüge einer deduktiv geordneten Theorie zu präsentieren und sie mit einfachen und überschaubaren Axiomensystemen arbeiten zu lassen.

Zusammenfassend ist eine Thematisierung axiomatischer Aspekte mit Schülerinnen und Schülern aus meiner Sicht trotz der geäußerten Bedenken erstrebenswert, sofern man klar unterscheidet zwischen der (didaktisch in der Tat problematischen) Intention, bestimmte Themengebiete vollständig axiomatisch zu behandeln, und dem Bestreben, axiomatisches Denken und Arbeiten anhand exemplarischer Themengebiete zu verdeutlichen. Sehr treffend wird dies von Blank (1966, S. 16) formuliert: „Although the axiomatic method does not seem to be an appropriate vehicle for teaching new ideas, we need not use the axiomatic method of teaching to teach axiomatic method".

\footnotetext{
${ }^{19}$ Beispiele sind Griesel 1963, Cohors-Fresenborg, Kaune und Griep 1998b, Cohors-Fresenborg et al. 2003b, Jahnke 2007b, 2009 sowie Jahnke und Wambach 2013.
} 



\section{Axiomatik im Mathematikunterricht: Unterrichtspraktische Perspektiven}

Wie bereits in der Einleitung dieser Arbeit erwähnt, ist es eines meiner Haupziele, Möglichkeiten aufzuzeigen, axiomatische Denk- und Arbeitsweisen im Mathematikunterricht zu thematisieren. Zu diesem Zweck werden in diesem Kapitel auf Basis der Analysen des fachlichen und didaktischen Hintergrunds aus den vorherigen beiden Kapiteln übergeordnete Lernziele aufgestellt, die als Orientierung für die Konzeption eigener Unterrichtsmaterialien dienen sollen. Ferner werden ausgewählte, bereits existierende Unterrichtskonzepte vorgestellt und didaktisch kommentiert. Dies geschieht zum einen, um auf besonders gelungene und aufgreifenswerte Ideen hinzuweisen und zum anderen, um die Notwendigkeit der Entwicklung weiterer Unterrichtsmaterialien zu prüfen. Im abschlieBenden Abschnitt dieses Kapitels werden die vorgestellten Unterrichtskonzepte daher mit den formulierten Lernzielen abgeglichen, um weiteren Handlungsbedarf aufzuzeigen und meine selbst entwickelten Unterrichtsideen genauer einzuordnen.

\section{1 Übergeordnete Lernziele zur Behandlung von Axiomatik im Mathematikunterricht}

Die folgenden Lernziele wurden aufgrund der in den Kapiteln 2 und 3 angeführten fachmathematischen, mathematikhistorischen, -philosophischen und -didaktischen Aspekte aufgestellt. Sie tragen zum einen dem Umstand Rechnung, dass sich die epistemologische und ontologische Sicht auf Axiome im Laufe der Geschichte grundlegend verändert hat und eine unterrichtliche Behandlung diesen Auffassungswechsel thematisieren sollte. Zum anderen spiegeln sie das Bestreben wider, sowohl die fertige axiomatisch-deduktive Struktur einer mathematischen Theorie als auch den zur Auswahl geeigneter Axiome führenden Prozess der logischen Strukturierung und letztendlichen Axiomatisierung im Unterricht abzubilden. 
Die Schülerinnen und Schüler

1. erklären, dass beim mathematischen Beweisen die Gültigkeit einer Aussage auf die Gültigkeit bereits akzeptierter Aussagen zurückgeführt wird, und begründen damit die Notwendigkeit, als Ausgangspunkte mathematischer Beweisführungen Axiome zu setzen.

2. erläutern die Deutung von Axiomen als Tatsachen, die aufgrund ihrer Evidenz ohne Beweis akzeptierbar sind.

3. erläutern die Deutung von Axiomen als Aussagen, welche inhaltlich nicht interpretiert werden und die Grundbegriffe eines mathematischen Gebiets implizit definieren.

4. untersuchen die logischen Zusammenhänge ausgewählter mathematischer Aussagen sowie verschiedene Möglichkeiten, aus diesen Aussagen ein (minimales) Axiomensystem auszuwählen.

5. leiten exemplarisch aus einem gegebenen Axiomensystem deduktiv weitere Aussagen her.

6. beschreiben Kriterien zur Rechtfertigung einer auf Axiomen aufbauenden Theorie.

Die Nummerierung der Lernziele (im Folgenden auch mit LZ abgekürzt) soll keine zwingende, durchgehende Behandlungsreihenfolge suggerieren, jedoch stellt LZ 1 eine unverzichtbare Grundlage für jedwede Beschäftigung mit mathematischem Beweisen dar. Der dort benannte relative Charakter mathematischer Aussagen und die euklidische Auffassung von Axiomen als evidenten Wahrheiten (LZ2) sind dem Erfahrungsbereich von Schülerinnen und Schülern dabei am nächsten, da die Objekte der Schulmathematik in der Regel stark ontologisch verankert sind (vgl. van der Waerden 1967, S. 1). LZ 3 ergänzt die klassische Sicht um die formalistische Interpretation axiomatischer Theorien.

LZ 4 und 5 sind nicht scharf voneinander zu trennen. Grundsätzlich zielt LZ 4 auf das lokale Ordnen ab und fokussiert damit den Prozess, durch eine nachträgliche logische Systematisierung von Aussagen einen deduktiven Aufbau des zugehörigen Theoriegebäudes zu erreichen. In LZ 5 hingegen wird die Existenz eines Axiomensystems vorausgesetzt und das regelgeleitete Deduzieren in den Vordergrund gestellt. Dennoch spielen natürlich auch beim lokalen Ordnen deduktive Elemente eine Rolle, so dass es vorstellbar und sinnvoll ist, LZ 4 und LZ 5 zusammenhängend zu behandeln. 
LZ 6 schließlich adressiert die Frage, wie man mathematische Theorien (und damit die zugehörige axiomatische Basis) über das euklidische Argument der Selbstevidenz hinaus rechtfertigen kann, beispielsweise durch die Beschreibung extrinsischer Faktoren wie inner- und außermathematische Nützlichkeit. Gerade im Kontext formalistischer Auffassungen stellt es eine wichtige Ergänzung dar. Eine Diskussion der „Nützlichkeit“ kann aber auch über inhaltlich interpretierte Axiome erfolgen, wie die Entstehungsgeschichte der nichteuklidischen Geometrie zeigt.

Die Lernziele sind bewusst als Grobziele formuliert und nicht operationalisiert. Sie lassen vorerst offen, mit welchen methodischen Mitteln sie zu erreichen und in welcher Altersstufe sie zu behandeln sind oder auch in welcher (fachlichen, philosophischen) Tiefe auf die angesprochenen Aspekte einzugehen ist. In Bezug auf meine eigenen Unterrichtsmaterialien finden sich diesbezüglich nähere Angaben in Kapitel 5.

\subsection{Unterrichtsvorschläge: Bestandsaufnahme}

Aus den 1960er und 1970er Jahren gibt es in Folge der strukturmathematischen Reformbestrebungen zahlreiche Überlegungen und Vorschläge, axiomatische Gesichtspunkte zum Gegenstand des Mathematikunterrichts zu machen. Beispielhaft möchte ich einen Blick auf die Themen der Zeitschrift Der Mathematikunterricht (MU) werfen. Die Veröffentlichungen reichen von sehr theoretischen und kaum schulpraktisch orientierten Darstellungen der bei einem mathematischen Gebiet zu berücksichtigenden Aspekte (z. B. Behnke und Steiner 1956a,b) über eher didaktisch orientierte Abhandlungen (z. B. Artmann und Weller 1981) bis hin zu sehr konkret ausgearbeiteten Unterrichtsszenarien und Erfahrungsberichten (z. B. Steiner 1966a,b). Inhaltlich werden vor allem die Geometrie und algebraische Strukturen (vor allem Gruppentheorie) behandelt, vereinzelt auch andere Themen wie die Dedekind-Peano-Axiome der natürlichen Zahlen (z. B. Kirsch 1967 oder Ebbinghaus 1974). Tabelle 4.1 gibt einen Überblick über diejenigen Themenhefte, die sich entweder explizit mit der Geometrie oder algebraischen Strukturen befassen oder die Behandlung axiomatischer Aspekte im Mathematikunterricht auf grundsätzlicher Ebene diskutieren.

Eine weitergehende inhaltliche und didaktische Auseinandersetzung mit diesen Unterrichtsvorschlägen kann und soll hier nicht erfolgen. Stattdessen werden im Folgenden ausgewählte, aus meiner Sicht besonders ausgereifte und gelungene Unterrichtskonzepte vorgestellt und didaktisch kommentiert. 


\begin{tabular}{lcl} 
Jahrgang & Heft & Titel \\
\hline $1959(5)$ & 3 & Axiomatik und Geometrieunterricht \\
$1961(7)$ & 1 & Logische Probleme im Mathematikunterricht II \\
$1963(9)$ & 4 & Axiomatik und Geometrieunterricht II \\
$1965(11)$ & 1 & Mathematische Strukturen im Unterricht \\
$1965(11)$ & 4 & Moderne Bestrebungen im Mathematikunterricht aller Schularten \\
$1966(12)$ & 2 & Der Gruppenbegriff im Unterricht \\
$1966(12)$ & 3 & Axiomatische Methode im Schulunterricht \\
$1967(13)$ & 1 & Axiomatik und Geometrie III \\
$1968(14)$ & 3 & Gruppen in der Geometrie \\
$1970(16)$ & 3 & Gruppentheorie - Anwendungen \\
$1970(16)$ & 4 & Inzidenzgeometrie \\
$1975(21)$ & 3 & Nichteuklidische Geometrie \\
$1976(22)$ & 4 & Axiomatik affiner und euklidischer Ebenen \\
$1976(22)$ & 6 & Anwendung der Gruppentheorie \\
$1977(23)$ & 2 & Algebraische Strukturen \\
$1977(23)$ & 6 & Gruppen in der Geometrie
\end{tabular}

Tabelle 4.1: Ausgewählte MU-Hefte zu axiomatischen Themen

\subsubsection{Axiomatisierung von Abstimmungsmengen (H.-G. Steiner)}

Die aus meiner Sicht überzeugendste und weitreichendste Unterrichtsidee zur Integration axiomatischer Denk- und Arbeitsweisen in den Mathematikunterricht stammt von HansGeorg Steiner. Aufbauend auf der Theorie der $n$-Personen-Spiele (siehe beispielsweise von Neumann und Morgenstern 1944), entwickelte Steiner eine mathematische Theorie von sogenannten Abstimmungsmengen, wie sie häufig im Alltag anzutreffen sind. Zu den mathematischen und didaktischen Aspekten dieses Themas wie auch zu den Ergebnissen zahlreicher Unterrichtserprobungen hat er sich wiederholt und über Jahrzente hinweg geäußert (siehe unter anderem Steiner 1966b,c, 1968, 1969a,b, 1975, 1976a,b, 1988).

Laut Steiners eigenen Aussagen (siehe Steiner 1988, S. 199) wurden die im Folgenden beschriebenen Unterrichtsideen mehrfach an deutschen und amerikanischen Schulen erprobt, und zwar vorwiegend mit Schülerinnen und Schülern der Jahrgangsstufen 11 und 12. Die Unterrichtsreihe im Ganzen hat einen Umfang von ca. 12-15 Schulstunden (Schulstunde $=45$ Minuten). 


\section{Inhaltliche Beschreibung}

Ausgangspunkt der Unterrichtsreihe ist die Betrachtung von Alltagssituationen, in denen eine Gruppe von Personen Entscheidungen durch eine Abstimmung herbeizuführen hat. Beispiele hierfür sind zahlreich und können von Schülerinnen und Schülern problemlos zusammengetragen werden, etwa die Wahl des Bundespräsidenten durch die Bundesversammlung, die Beschlussfassung in einem Vereinsvorstand, eine Klassensprecherwahl oder Geschworenenurteile in Rechtssystemen wie dem der USA.

Allen Situationen ist gemein, dass eine Gruppe von Personen, genannt Abstimmungsmenge $A=\left\{a_{1}, \ldots, a_{n}\right\}$, am Prozess der Entscheidungsfindung beteiligt ist. Typischerweise erhält jedes Mitglied $a_{i}$ einer Abstimmungsmenge eine gewisse Anzahl $s_{i}$ von Stimmen; zusätzlich wird eine Entscheidungsregel in Form eines Mehrheitsquotienten $\frac{1}{2} \leq q \leq 1$ festgelegt. ${ }^{1}$ Teilmengen $K \subseteq A$ werden Koalitionen genannt. Eine Koalition heißt auch Gewinnkoalition, wenn sie eine Abstimmung für sich entscheiden kann, d. h. wenn der relative Anteil der Stimmen der in ihr zusammengefassten Mitglieder in Bezug auf die Gesamtstimmenzahl größer oder gleich $q$ ist (im Falle $q=\frac{1}{2}$ echt größer als $q$ ).

Anhand verschiedener Beispiele können die Lernenden nun Beobachtungen aufstellen und sammeln. Diese wiederum geben Anlass zu weiteren Begriffsbildungen:

\section{Beobachtung:}

(i) Wenn $K$ eine Gewinnkoalition ist, dann auch jede Obermenge von $K$. Es genügt also, „minimale“ Gewinnkoalitionen zu kennen.

(ii) Wenn $K$ eine Gewinnkoalition ist, dann ist $\bar{K}$ keine Gewinnkoalition, also eine „Verlustkoalition“.

\section{Definition:}

(1) Eine Gewinnkoalition $K$ wird $m i$ nimale Gewinnkoalition genannt, wenn $K$, aber keine echte Teilmenge von $K$, Gewinnkoalition ist.

(2) Eine Teilmenge $K$ heißt Verlustkoalition, wenn $\bar{K}$ eine Gewinnkoalition ist.

\footnotetext{
${ }^{1}$ Eine explizite Angabe einer Stimmenverteilung bzw. eines Mehrheitsquotienten muss jedoch nicht immer gegeben oder offensichtlich sein. Ein Beispiel stellt der UN-Sicherheitsrat dar: Er besteht aus fünf ständigen und zehn nichtständigen Mitgliedern. Gemäß Artikel 27(3) der UN-Charta (siehe http://www.unric.org/de/charta (letzter Zugriff: 29.09.2017)) bedürfen Beschlüsse - abgesehen von Verfahrensfragen - der Zustimmung aller ständigen Mitglieder sowie von mindestens vier der nichtständigen Mitglieder. Eine konkrete Stimmenverteilung auf die 15 Mitglieder samt Angabe eines Mehrheitsquotienten liegt hier nicht auf der Hand.
} 
(iii) Es gibt Abstimmungssituationen, in denen eine Koalition $K$ weder eine Gewinn- noch eine Verlustkoalition ist. ${ }^{2}$

(iv) Es gibt Abstimmungssituationen, in denen einzelne Mitglieder keinerlei Einfluss auf die Entscheidung haben. ${ }^{3}$
(3) Eine Teilmenge $K$ heißt Blockkoalition, wenn $\bar{K}$ (also auch $K$ ) weder eine Gewinn- noch eine Verlustkoalition ist.

(4) Eine Person $a \in A$ heißt machtlos, wenn $a$ zu keiner minimalen $\mathrm{Ge}$ winnkoalition gehört.

Die verschiedenen „Koalitionsstrukturen“ lassen sich mit HASSE-Diagrammen veranschaulichen. Abbildung 4.1 zeigt zwei mögliche Strukturen für die Abstimmungsmenge $A=\{a, b, c, d\}$, wenn jedes der vier Mitglieder eine Stimme hat. Gewinnkoalitionen sind grün markiert, Verlustkoalitionen rot und Blockkoalitionen blau. Das linke Diagramm veranschaulicht die Situation, dass für eine Entscheidung die absolute Mehrheit der Stimmen notwendig ist; das rechte Diagramm gibt die Gewinn- und Verlustkoalitionen an, wenn bei Stimmengleichheit die Stimme des Mitglieds $a$ ausschlaggebend ist.
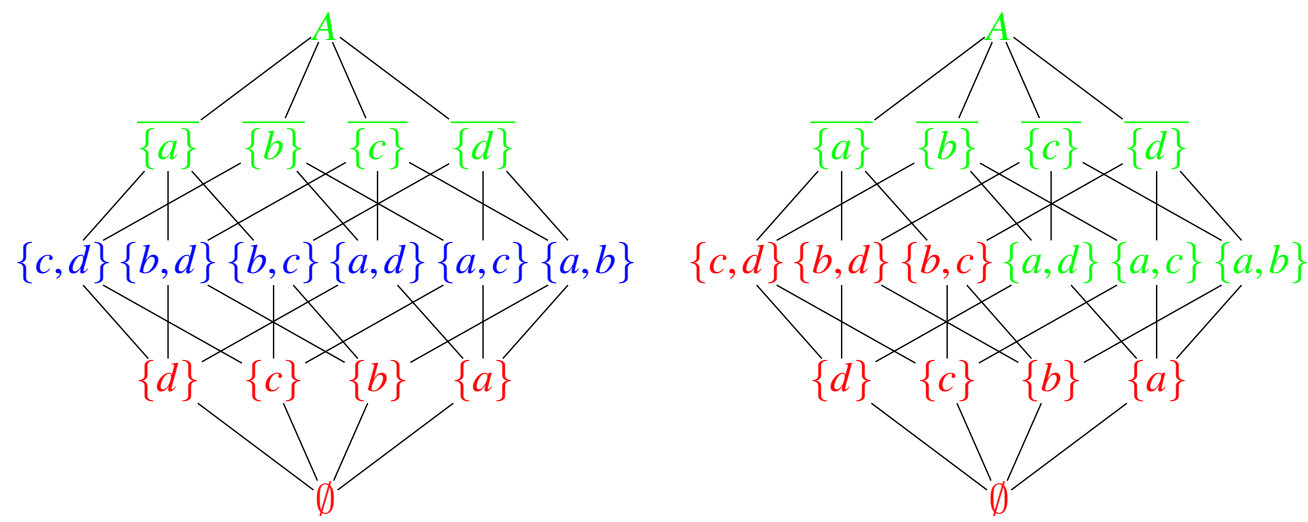

Abbildung 4.1: Mögliche Koalitionsstrukturen der Menge $A=\{a, b, c, d\}$

Eine besonders ergiebige Herausforderung für Schülerinnen und Schüler stellt die Aufgabe dar, im bisher aufgebauten theoretischen Gebäude zu definieren, was ein Diktator ist. Aufgrund der Schülervorschläge sind beispielsweise die folgenden drei Ansätze denkbar:

$D_{1}: x \in A$ heißt Diktator, wenn alle von $x$ verschiedenen Mitglieder aus $A$ machtlos sind.

$D_{2}: x \in A$ heißt Diktator, wenn $x$ zu jeder minimalen Gewinnkoalition gehört.

$D_{3}: x \in A$ heißt Diktator, wenn $\{x\}$ minimale Gewinnkoalition ist.

\footnotetext{
${ }^{2}$ Beispiel: $A=\{a, b, c, d\}$, wobei jedes Mitglied genau eine Stimme habe und $q=\frac{1}{2}$ sei. Dann ist jede zwei-elementige Teilmenge von $A$ weder eine Gewinn- noch eine Verlustkoalition.

${ }^{3}$ Beispiel: Person $d$, wenn $A=\{a, b, c, d\}$ mit der Stimmenverteilung $(3,3,2,1)$ und $q=\frac{5}{9}$.
} 
Anhand dieses Beispiels lässt sich sehr gut über den Sinn und Zweck von Definitionen diskutieren: Die Frage nach ihrer Wahrheit stellt sich nicht, vielmehr geht es darum, eine möglichst adäquate Charakterisierung des Begriffs Diktator im gegebenen theoretischen Kontext zu finden. Ein erster Schritt, um sich für eine der drei Definitionsansätze zu entscheiden, besteht in der Analyse der logischen Abhängigkeiten zwischen den darin enthaltenen Aussagen. Man kann zeigen, dass $D_{1} \Leftrightarrow D_{3}$ gilt sowie $D_{3} \Rightarrow D_{2}$, nicht jedoch $D_{2} \Rightarrow D_{3} .{ }^{4}$ Als Definition kann man sich also beispielsweise auf $D_{3}$ einigen; die Äquivalenz zu $D_{1}$ und die Implikation von $D_{2}$ sind in diesem theoretischen Rahmen dann Sätze. Als Beweis sei exemplarisch die Implikation $D_{3} \Rightarrow D_{1}$ vorgeführt (für weitere Deduktionen siehe Steiner 1966b, S. 72-74):

Eine von $\{x\}$ verschiedene minimale Gewinnkoalition $G \subseteq A$ kann nicht $x$ enthalten, da $\{x\}$ bereits minimale Gewinnkoalition ist. Also muss $G \subseteq \overline{\{x\}}$ gelten. Die Menge $\overline{\{x\}}$ ist als Komplement einer Gewinnkoalition jedoch eine Verlustkoalition. Damit ist auch $G$ als Teilmenge eine Verlustkoalition, was ein Widerspruch ist. Kein von x verschiedenes Element kann also einer minimalen Gewinnkoalition angehören, somit sind alle Mitglieder außer $x$ machtlos. 5

Weitere Begriffsbildungen betreffen die Ausübung verschiedener Veto-Rechte sowie die Definition der Rolle eines (ggf. ausschlaggebenden) Vorsitzenden; sie sollen an dieser Stelle jedoch nicht weiter ausgeführt werden (man vergleiche hierzu u. a. Steiner 1966b, S. 75-78).

Der Zusammenhang zwischen rein theoretisch (in diesem Fall: mengentheoretisch) definierten Begriffen und damit verbundenen Alltagsvorstellungen kann zu weiterführenden Fragen und Diskussionen anregen, die wiederum in deduzierbare Sätze der Theorie der Abstimmungsmengen münden. Als Beispiele seien angeführt:

- In einer Abstimmungsmenge gibt es höchstens einen Diktator.

- Eine Blockkoalition kann nicht nur aus machtlosen Mitgliedern bestehen.

Eine rückblickende Betrachtung der aufgestellten Begriffe und Sätze sowie der zugehörigen Beweisideen zeigt, dass man zum Aufbau der gesamten Theorie als Ausgangspunkte

\footnotetext{
${ }^{4}$ Als Gegenbeispiel zu $D_{2} \Rightarrow D_{3}$ betrachten wir die Abstimmungsmenge $A=\{a, b, c, d\}$ mit zugehöriger Stimmenverteilung $(6,5,5,1)$ und dem Mehrheitsquotienten $q=\frac{3}{4}$ (vgl. Steiner 1966b, S. 68). Damit ist $\{a, b, c\}$ (einzige) minimale Gewinnkoalition und $D_{2}$ ist z. B. für $a$ erfüllt. Allerdings ist $\{a\}$ keine minimale Gewinnkoalition, d. h. $D_{3}$ ist nicht erfüllt.

${ }^{5}$ In diesem Beweis wird eine Aussage verwendet, die wiederum als Satz bewiesen werden muss, nämlich „Jede Teilmenge einer Verlustkoalition ist ebenfalls eine Verlustkoalition“. Der Beweis ist mit Hilfe von Beobachtung (i) (siehe oben) sowie der mengentheoretischen Regel $M \subseteq N \Leftrightarrow \bar{M} \supseteq \bar{N}$ leicht geführt. Zudem folgt aus dem Beweis direkt, dass, wenn $\{x\}$ eine minimale Gewinnkoalition ist, dies auch die einzige ist.
} 
nichts weiter benötigt als die in den Beobachtungen (i) und (ii) festgehaltenen Aussagen. Es gilt also, mathematisch sauber zu definieren, was man im Rahmen der Theorie unter einer Gewinn- bzw. Verlustkoalition verstehen möchte. Eine Möglichkeit besteht wie anfangs bereits angedeutet - in der Charakterisierung von Stimmenverteilungen und Mehrheitsquotienten:

Gegeben seien eine nicht-leere, endliche Menge $A$ und eine (von der konstanten 0-Funktion verschiedene) Abbildung $s: A \rightarrow \mathbb{N}_{0}$, welche wir Stimmenverteilung nennen. Ferner sei eine rationale Zahl $q$ mit $\frac{1}{2} \leq q \leq 1$, der sogenannte Mehrheitsquotient, gegeben. Durch $s$ ist ferner eine Abbildung $\sigma$ auf allen Teilmengen $K \subseteq A$ gegeben, nämlich

$$
\sigma(K):=\sum_{x \in K} s(x)
$$

Eine Teilmenge $K \subseteq A$ heißt Koalition. Man nennt $K$ eine Gewinnkoalition (bei gegebenen $s$ und $q$ ), falls

$$
\sigma(K)>\frac{1}{2} \cdot \sigma(A)\left(\text { falls } q=\frac{1}{2}\right) \quad \text { bzw. } \quad \sigma(K) \geq q \cdot \sigma(A)\left(\text { falls } q>\frac{1}{2}\right) .
$$

Das Tripel $(A, s, q)$ kann dann als Abstimmungsmenge bezeichnet werden. In der Terminologie der Strukturmathematik wird der Menge $A$ durch $s$ und $q$ eine Struktur aufgeprägt. Auffällig ist jedoch die Tatsache, dass die Stimmenverteilung $s$ und der Mehrheitsquotient $q$ beim Aufbau der Theorie größtenteils gar nicht benötigt wurden, sondern überwiegend mit Hilfe des Begriffs der Gewinnkoalition und seiner Eigenschaften gearbeitet wurde. Hinzu kommt die Kenntnis von Abstimmungssituationen wie beim UN-Sicherheitsrat, in denen eine konkrete Stimmenverteilung gar nicht angegeben wird. Dies wirft die Frage auf, ob man die Theorie der Abstimmungsmengen auch ohne die Konzepte der Stimmenverteilung und des Mehrheitsquotienten erstellen kann. Dies läuft auf eine „,axiomatische Fundierung" (Steiner 1966b, S. 79; Herv. im Orig.) hinaus, in der auf eine konkretinhaltliche Definition des Begriffs Gewinnkoalition verzichtet wird. Stattdessen wird dieser als implizit definierter Grundbegriff verwendet:

Eine nicht-leere Menge $A$, in der ein nicht-leeres System $\mathbb{G}$ von Teilmengen, genannt Gewinnkoalitionen, ausgezeichnet ist, heißt Abstimmungsmenge, wenn für alle $K \in \mathbb{G}$ gilt:

(I) $K \subseteq L(\subseteq A) \Rightarrow L \in \mathbb{G}$

(II) $K \in \mathbb{G} \Rightarrow \bar{K} \notin \mathbb{G}$

Die Axiome (I) und (II) entsprechen dabei genau den Beobachtungen (i) und (ii). Natürlich stellt sich nun die Frage, ob die beiden vorgeschlagenen Charakterisierungen von 
Abstimmungsmengen (wir wollen sie kurz ,s-gebunden“ und ,s-frei“ nennen) gleichwertig sind. Man prüft leicht nach, dass eine über Stimmenverteilung und Mehrheitsquotienten definierte Abstimmungsmenge die Axiome (I) und (II) erfüllt, d. h. jede $s$-gebundene Abstimmungsmenge kann als Konkretisierung (in moderner Terminologie: Modell) einer $s$-freien Abstimmungsmenge aufgefasst werden. Die umgekehrte Frage - „Kann man zu jeder $s$-freien Abstimmungsmenge eine Stimmenverteilung $s$ und einen passenden Mehrheitsquotienten $q$ angeben?" - lässt sich nicht so leicht beantworten. Es ist in der Regel nicht zu erwarten, dass Schülerinnen und Schüler diese Frage ohne Weiteres beantworten können. Es gibt jedoch ein verhältnismäßig einfaches Beispiel einer $s$-freien Abstimmungsmenge, von der man auch auf Schülerniveau einen Beweis vorführen kann, dass sich zu ihr keine passende Kombination aus Stimmenverteilung und Mehrheitsquotient finden lässt (siehe Steiner 1966b, S. 85-86): ${ }^{6}$

Als Abstimmungsmenge nehme man $A=\{a, b, c, d, e\}$ mit den minimalen Gewinnkoalitionen $G_{1}:=\{a, d, e\}$ und $G_{2}:=\{b, c, e\}$. Man kann leicht nachprüfen, dass die Axiome (I) und (II) erfüllt sind. Angenommen, es gäbe eine passende Stimmenverteilung $s$ mit $\sigma(A):=N$ und einen Mehrheitsquotienten $q>\frac{1}{2} \quad\left(\right.$ bzw. $\left.q=\frac{1}{2}\right)$.

Dann gilt, da $G_{1}$ und $G_{2}$ Gewinnkoalitionen sind, sowohl $\sigma\left(G_{1}\right) \geq q \cdot N$ (bzw. $>\frac{N}{2}$ ) als auch $\sigma\left(G_{2}\right) \geq q \cdot N$ (bzw. $>\frac{N}{2}$ ). Insgesamt erhält man

$$
s(a)+s(b)+s(c)+s(d)+2 s(e) \geq 2 q \cdot N(\text { bzw. }>N) .
$$

Andererseits gilt, da $B_{1}:=\{a, b, e\}$ und $B_{2}:=\{c, d, e\}$ Blockkoalitionen sind, sowohl $\sigma\left(B_{1}\right)<q \cdot N\left(\right.$ bzw. $\left.=\frac{N}{2}\right)$ als auch $\sigma\left(B_{2}\right)<q \cdot N\left(\right.$ bzw. $\left.=\frac{N}{2}\right)$. Insgesamt erhält man damit als Widerspruch zu obiger Gleichung

$$
s(a)+s(b)+s(c)+s(d)+2 s(e)<2 q \cdot N(\text { bzw. }=N) .
$$

Damit wird deutlich, dass in diesem Fall ,die begrifflich-axiomatische Methode weiter reicht als die konkret-numerische Methode“ (Steiner 1966b, S. 81), wenngleich letztere in der Praxis häufig gebraucht wird.

Abschließend kann den Schülerinnen und Schülern die Aufgabe gestellt werden, „eine vollständige Deduktion aller bisherigen Resultate aus dem Axiomensystem vorzunehmen und dabei die Definitionen und Sätze möglichst vorteilhaft anzuordnen, wobei nötigenfalls neue Beweise zu finden [sind]“ (Steiner 1966b, S. 81).

\footnotetext{
${ }^{6}$ Für das Beispiel des UN-Sicherheitsrates ist dies jedoch möglich (siehe Steiner 1966b, S. 82).
} 


\section{Didaktischer Kommentar}

Die geschilderte Unterrichtsreihe war nachweislich Steiners „Lieblingsbeispiel“, um Lernenden die Bedeutung des Mathematisierens und Axiomatisierens näher zu bringen, und „diente als Prototyp für sein didaktisches Denken“ (Biehler und Peter-Koop 2007, S. 4). Die verschiedenen Phasen der Unterrichtsreihe entsprechen dabei den zur damaligen Zeit wesentlichen methodischen Forderungen an eine sinnvolle Einbindung der Axiomatik in den Mathematikunterricht: „Entwickeln der Mathematik von Situationen aus, Mathematisieren, lokale[s] Ordnen, Axiomatisieren“ (Steiner 1966b, S. 66).

Wenngleich die Behandlung eines primär mengentheoretischen Themas aus heutiger Sicht etwas ,,angestaubt“ wirken mag, ist ein Einsatz von Steiners Ideen immer noch lohnenswert, da ohne weitreichende fachliche Kenntnisse sehr viel über Axiomatik gelernt werden kann. ${ }^{7}$ Im Folgenden möchte ich aufzeigen, welche Merkmale Steiners Unterrichtsreihe aus meiner Sicht auszeichnen und welche Erkenntnisse über die axiomatische Methode durch sie gefördert werden:

- Da das auf fachlicher Ebene benötigte Vorwissen der Schülerinnen und Schüler verhältnismäßig gering ist, ist die Einstiegshürde in die Thematik niedrig. Dies eröffnet die Möglichkeit, Teile der Unterrichtsreihe bereits in früheren Jahrgangsstufen einzusetzen (siehe auch Steiner 1966b, S. 66). Zudem sind das letztlich behandelte Axiomensystem und das darauf aufbauende Theoriegebäude der Abstimmungsmengen sehr überschaubar, so dass eine Konzentration auf und Diskussion über axiomatische Gesichtspunkte möglich sind, ohne den Überblick zu verlieren oder sich in fachlichen Spitzfindigkeiten zu verstricken.

- Im Zentrum von Steiners methodologischem Ansatz stehen die Alltagserfahrungen und Eigenaktivitäten der Schülerinnen und Schüler. Das Vorwissen, dass sie zu Abstimmungssituationen mitbringen, kann an verschiedenen Stellen der Unterrichtsreihe gewinnbringend eingebracht werden, unter anderem um die Adäquatheit und Vollständigkeit der entwickelten Konzepte zu überprüfen oder neue Begriffsbildungen anzuregen. Die mathematische Theorie inklusive ihrer axiomatischen Formulierung entwickelt sich damit genetisch aus den Schüleraktivitäten. Zudem können die Lernenden bei den unterrichtlichen Diskussionen die Rolle von „Experten“ einnehmen, wenn es beispielsweise darum geht, unter verschiedenen Möglichkeiten für eine Begriffsdefinition die „geeignetste“ auszuwählen. Dies hebt den Charak-

\footnotetext{
${ }^{7}$ Das auf Schülerseite benötigte Vorwissen beschränkt sich im Wesentlichen auf ein naives Verständnis mengentheoretischer Operatoren sowie rudimentäre aussagenlogische Kenntnisse (Implikation, Äquivalenz, Widerspruchsbeweis).
} 
ter mathematischer Wissensbildung als soziale Tätigkeit hervor (vgl. Steiner 1988, S. 199-200).

- Die Untersuchung des Zusammenhangs zwischen $s$-freien und s-gebundenen Abstimmungsmengen verdeutlicht, dass eine implizit-axiomatische Charakterisierung nicht die einzige Möglichkeit ist, eine mathematische Theorie aufzubauen, aber durchaus ihre Vorteile besitzt.

- Mit der Behandlung von Abstimmungsmengen liefert Steiner einen Strukturtyp von Mengen, welcher

a) von genügend vielen an sich wesentlichen Modellen aus erschlossen werden [kann] und damit eine strukturelle Betrachtungsweise in einer hinreichend reichen Welt von Erscheinungen [ermöglicht],

b) für deduktives Vorgehen auf elementarer Stufe in einem gewissen Ausmaß geeignet [ist], so daß auch ein Stück der zugehörigen allgemeinen Theorie entwickelt und nutzbar gemacht werden kann. (Steiner 1965, S. 17)

Die Kontrastierung der explorativen Phase (Beispiele für Abstimmungsmengen, Aufstellen von Beobachtungen und Definitionen, lokales Ordnen erster grundlegender Aussagen über Abstimmungsmengen) mit der finalen Erstellung einer streng deduktiven Darstellung auf der Basis eines Axiomensystems vermittelt fundamentale Kenntnisse über die Prozesse der mathematischen Theoriebildung. Dadurch wird dem Phänomen der antididaktischen Inversion entgegengewirkt: Der Unterschied zwischen den Schritten, die zur Herausbildung der mathematischen Theorie - ihrer Begriffe, Sätze, Beispiele und Beweismittel - geführt haben, und der fertigen axiomatisch-deduktiven Form wird expliziert und zum Gegenstand der unterrichtlichen Diskussion gemacht. „Neben die Mathematik als Fertigprodukt tritt damit gleichrangig die Mathematik als schöpferische Methode“" (Steiner 1965, S. 7; siehe auch Abschnitt 3.2). Damit wird klar, dass die Axiome keine willkürlichen Setzungen sind, sondern zu einem bestimmten Zweck in dieser Art und Weise formuliert werden. Gleichzeitig wird die Erkenntnis vermittelt, dass sowohl die Überlegungen, die zu diesem Axiomensystem geführt haben, als auch die außermathematischen Bedeutungen von Begriffen wie Diktator oder Veto-Recht für die finale deduktive Darstellung der Theorie nicht mehr benötigt werden. 


\subsubsection{Sätze aus dem Wüstensand (E. Cohors-Fresenborg et al.)}

Die in den 1980er Jahren am Institut für Kognitive Mathematik, Fachbereich Mathematik/Informatik der Universität Osnabrück entwickelte Unterrichtsreihe Sätze aus dem Wüstensand und ihre Interpretationen - Einführung in die axiomatische Auffassung von Mathematik ist für den Einsatz in der 8. Klasse konzipiert und verwendet die Metapher des „Vertragswerkes“, um Schülerinnen und Schülern die Funktion von Axiomensystemen und den darin vorkommenden implizit definierten Begriffen zu verdeutlichen. ${ }^{8}$ Diese Metapher bildet auch die Grundlage für weitere Unterrichtskonzepte, in denen axiomatische Aspekte bei der Behandlung von Rechengesetzen (negativer) rationaler Zahlen sowie bei der Lösung linearer Gleichungen und Ungleichungen bzw. Gleichungssysteme thematisiert werden (Cohors-Fresenborg, Kaune und Griep 1998a,b, 2015).

Im Folgenden soll die (auf eine Dauer von ca. 5 Wochen ausgelegte) Unterrichtsreihe Sätze aus dem Wüstensand genauer vorgestellt werden, in der die Vorstellungen zur axiomatischen Auffassung von Mathematik an zahlreichen Stellen von einer Meta-Perspektive aus betrachtet werden. Grundlage meiner Beschreibung ist das zugehörige Schülertextbuch (Cohors-Fresenborg et al. 2003b).

\section{Inhaltliche Beschreibung}

Die Unterrichtsreihe ist in eine archäologische Rahmengeschichte eingebettet:

Der Archäologe Friedrich Sand findet in der arabischen Wüste antike Schrifttafeln mit merkwürdigen Zeichen: I. $\underset{B b_{1} B b_{2}}{\bigvee} \neg b_{1}=b_{2}$

II. $\bigwedge_{B b_{1}} \bigwedge_{B b_{2}}\left(\neg b_{1}=b_{2} \rightarrow \bigvee_{K k}^{=1}\left(D b_{1} k \wedge D b_{2} k\right)\right)$

III. $\wedge \bigvee \neg D b k$ $K k B b$

Es handelt sich dabei um die Schriftsprache der (fiktiven) Maskonen. Beim Versuch einer Übersetzung fällt Herrn Sand auf, dass ihm nicht alle Schriftzeichen bekannt sind. Für diese wählt er zunächst Kunstworte (Bre, Ket, de-

\footnotetext{
${ }^{8}$ Die Unterrichtsmaterialien wurden im Rahmen des Modellversuchs Integration algorithmischer und axiomatischer Denkweisen in den gymnasialen Mathematikunterricht (Klasse 7/8) als Beitrag zur informations- und kommunikationstechnischen Bildung entwickelt, welcher durch das Niedersächsische Kultusministerium gefördert und in den Jahren 1987-1993 an zahlreichen Schulen erprobt und wissenschaftlich begleitet wurde. Die Zeitschrift Der Mathematikunterricht beinhaltet in Heft 3/1993 sechs Artikel, die verschiedene Aspekte dieses Schulversuchs genauer vorstellen.
} 
tieren) und kann damit immerhin eine vorläufige Übersetzung liefern:

I. Es gibt ein Bre $b_{1}$, es gibt ein Bre $b_{2}$, so dass gilt: $b_{1}$ ist verschieden von $b_{2}$.

II. Für alle Bre $b_{1}$, für alle Bre $b_{2}$ gilt: Wenn $b_{1}$ verschieden von $b_{2}$ ist, dann gibt es genau ein Ket $k$, so dass gilt: $b_{1}$ detiert $k$ und $b_{2}$ detiert $k$.

III. Für alle Ket $k$ gibt es ein Bre b, so dass gilt: $b$ detiert nicht $k^{9}{ }^{9}$

Wesentlicher Bestandteil der ersten Kapitel des Textbuches ist die Beschäftigung mit möglichen Interpretationen der Wüstensandsätze. So sind zwei vorgestellte Hypothesen, dass die Sätze I-III die Zusammensetzung antiker maskonischer Tempelorchester oder Durchführungsrichtlinien für Fechtturniere beschreiben; für die oben gewählten Kunstworte könnte sich dann - je nach Kontext - folgendes Wörterbuch ergeben:

\begin{tabular}{l|l|l} 
Kunstwort & Orchester-Interpretation & Turnier-Interpretation \\
\hline Bre & Musiker & Mannschaft \\
Ket & Instrument & Fechtkampf \\
detiert & spielt & nimmt teil an
\end{tabular}

Satz III beispielsweise lautet im Rahmen der Orchester-Interpretation wie folgt: Für alle Instrumente $k$ gibt es einen Musiker $b$, so dass gilt: $b$ spielt nicht $k$. Auf Basis der so interpretierten Aussagen können dann verschiedene Orchesterpläne daraufhin untersucht werden, welche der drei Sätze sie erfüllen und welche nicht. Die folgende Tabelle beschreibt einen möglichen Orchesterplan, der alle drei Sätze erfüllt (links), sowie einen, bei dem Satz II nicht erfüllt ist (rechts).

\begin{tabular}{|l|l|l|l|l|l|l|l|}
\hline Ali & Aladin & Assuman & Achmed & Ali & Aladin & Assuman & Achmed \\
\hline Harfe & Trommel & Horn & Rassel & Flöte & Flöte & Trommel & Geige \\
Flöte & Harfe & Trommel & Flöte & Geige & Trommel & Geige & Flöte \\
Pauke & Rassel & Pauke & Horn & Oboe & & & Trommel \\
\hline
\end{tabular}

Nach einer intensiven Behandlung der Orchester-Interpretation der Wüstensandsätze behandelt ein (der 5. Auflage des Schüler-Textbuches neu hinzugefügtes) Kapitel die Frage, wie man in der Symbolsprache der Maskonen grammatikalisch korrekte Sätze formulieren kann. Auf Grundlage eines Regelwerks, welches die „atomaren Satzbauteile“ (Cohors-Fresenborg et al. 2003a, S. 34) der Maskonensprache sowie Regeln für die Bildung neuer Sätze auflistet, können die Schülerinnen und Schüler mit Hilfe von Syntax-

\footnotetext{
${ }^{9}$ Die „Übersetzung“ von Satz III können Lernende auf Grundlage der beiden vorangegangenen Sätze bereits selbstständig formulieren.
} 
bäumen korrekte Sätze erstellen und vorgegebene Sätze auf Korrektheit überprüfen (siehe Abbildung 4.2).

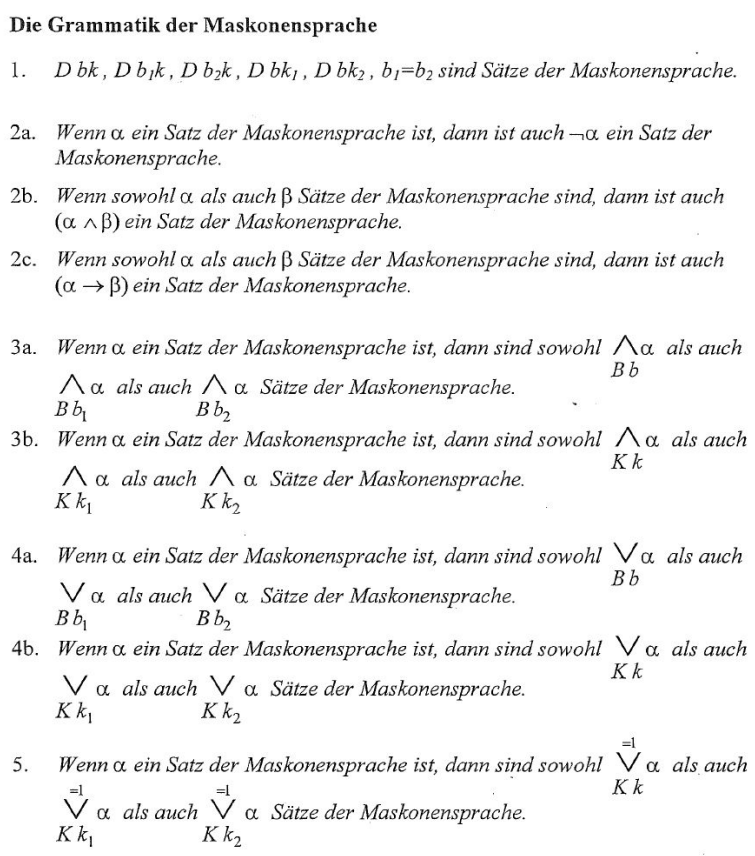

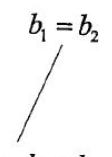

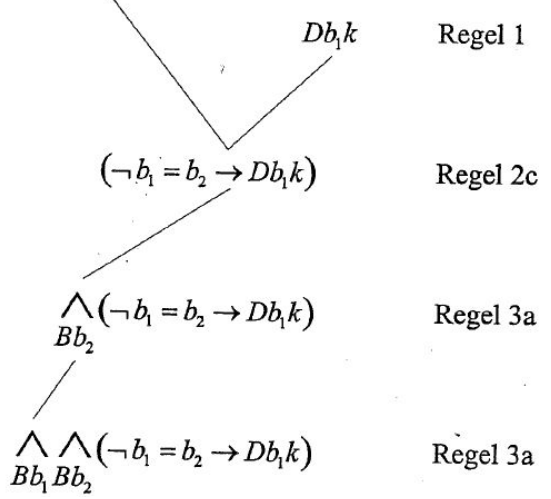

Abbildung 4.2: Grammatik der Maskonensprache - Regelwerk und Syntaxbaum ${ }^{10}$

Anschließend werden noch zahlreiche andere mögliche Interpretationen der Wüstensandsätze untersucht (siehe vor allem die Kapitel 3 und 4 in Cohors-Fresenborg et al. 2003b), was die intensive Diskussion über Fragen wie „Was bedeuten Worte wie Bre, Ket und detiert denn nun wirklich?“ oder „Liefern Interpretationen der Wüstensandsätze wahre Aussagen?“" ermöglicht. Dies führt schließlich auf das Konzept der impliziten Definition, dessen wichtigste Eigenschaften zusammenfassend und eingebettet in die archäologische Rahmengeschichte in dem Kapitel Friedrich Sands mathematische Märchenstunde (Cohors-Fresenborg et al. 2003b, S. 29-32) dargestellt werden:

Stellen wir uns vor, dass sich im Laufe der Geschichte der Maskonen eine Tradition ergeben hat, wie Turniere ausgetragen werden. Irgendwann einmal kam es zu einem ernsthaften Streitfall, der nicht für alle Beteiligten zufriedenstellend gelöst werden konnte. Daraufhin beschlossen die Maskonen, ein schriftliches Abkommen zu treffen, das für alle eindeutig festlegt, wie diese Turniere genau stattzufinden haben. Alle, die teilnehmen wollten, sollten sich daran halten müssen. Mit der Ausarbeitung des Textes wurden Priester beauftragt. [...]

Eine andere Gruppe von Priestern war etwa zur selben Zeit damit beschäftigt, Ordnung in die Besetzung der Tempelorchester zu bringen. Dieser Priester-

\footnotetext{
${ }^{10}$ Quelle: Grammatikregeln mit Erlaubnis der Autoren entnommen aus Cohors-Fresenborg et al. 2003b,
}

S. 13; Syntaxbaum mit Erlaubnis der Autoren entnommen aus Cohors-Fresenborg et al. 2003a, S. 40. 
gruppe gelang ebenfalls ein sehr kurzes Textstück zur Regelung ihrer Angelegenheiten.

Beide Gruppen brachten ihre Texte zu demselben Schreiber, der die Regeln für die Zukunft auf Papyrus festhalten sollte. Aber wie war der Schreiber erstaunt, als er bei einem Vergleich beider Texte feststellte, dass sich diese überaus ähnlich waren. Sollte es etwa überflüssig sein, zwei Texte zu erstellen? Doch sein Versuch, für beide Gruppen nur einen Text zu schreiben, scheiterte. Vier Wörter blieben übrig, um die ein heftiger Streit entbrannte. [...]

[D]er weise Oberpriester fand die Idee. Er dachte sich vier ${ }^{11}$ neue Wörter aus, die es bislang in der Sprache der Maskonen noch nicht gegeben hatte. Zwei klangen wie Substantive und zwei wie Wörter, die eine Beziehung ausdrücken. Mit diesen Wörtern formulierte er die Regeln und gab sie an seine Priester zurück. Diese waren glücklich mit der Lösung. Jeder sah die Freiheit, sich bei den Sätzen das denken zu können, was seinem Anliegen entsprach. [...]

Aufgrund der Bedeutung solcher Regelwerke sind im Laufe der Zeit einige Fachwörter zum Reden darüber eingeführt worden. Das Regelwerk selbst nennt man Axiomensystem. Die einzelnen Sätze daraus heißen Axiome. Die Kunstworte, die im Axiomensystem nicht weiter erklärt sind, wie „Bre“, „Ket“ und „,detiert“, bezeichnet man als Grundbegriffe des Axiomensystems. Interpretationen, die aus den Sätzen eines Axiomensystems wahre Aussagen machen, werden Modelle genannt. (Cohors-Fresenborg et al. 2003b, S. 29-30; Herv. im Orig.)

Damit ist klar, dass es die wahre Interpretation der Wüstensandsätze nicht geben kann. Die Grundbegriffe nehmen in dem abstrakt formulierten Regelwerk die Funktion von Leerstellen (Variablen) ein, die je nach Bedarf in unterschiedlicher Weise inhaltlich gefüllt (d. h. interpretiert) werden können.

Ein grundlegendes Verständnis für diesen abstrakten Charakter der implizit definierten Grundbegriffe wird durch das Übereinanderlegen geeignet vorbereiteter Folien gefördert (siehe Abbildung 4.3): Auf einer Folie ist der Grundtext der Wüstensandsätze ohne die Kunstwörter notiert, auf einer weiteren ausschließlich die Kunstwörter. Die zweite Folie kann dann beispielsweise durch eine Folie ausgetauscht werden, auf der die aus der

\footnotetext{
${ }^{11}$ Im Verlauf des Textbuches werden die Wüstensandsätze durch einen vierten ergänzt: Für alle Ket $k_{1}$, für alle Bre b gilt: Wenn $b k_{1}$ nicht detiert, dann gilt: Es gibt genau ein Ket $k_{2}$, so dass gilt: $b$ detiert $k_{2}$ und $k_{1}$ ist silukan zu $k_{2}$. Das neue Kunstwort silukan wird dabei explizit mit Hilfe der drei bereits vorhandenen Begriffe definiert: „, $k_{1}$ ist silukan zu $k_{2}$ “ bedeutet dasselbe wie „Es gibt kein Bre b, so dass gilt: $b$ detiert $k_{1}$ und $b$ detiert $k_{2}$ ". In Maskonensprache geschrieben erhält man
}

$$
\text { IV. } \bigwedge_{K k_{1}} \bigwedge_{B b}\left(\neg D b k_{1} \rightarrow \bigvee_{K k_{2}}^{=1}\left(D b k_{2} \wedge S k_{1} k_{2}\right)\right) \quad \text { mit } \quad S k_{1} k_{2}: \leftrightarrow \neg \bigvee_{B b}\left(D b k_{1} \wedge D b k_{2}\right)
$$


Orchester-Interpretation stammenden Begriffe Musiker, Instrument und spielt vermerkt sind. Eine reine „Kästchen-Folie“ kann von den Schülerinnen und Schülern dann selbstständig mit geeigneten Modell-Begriffen gefüllt werden.
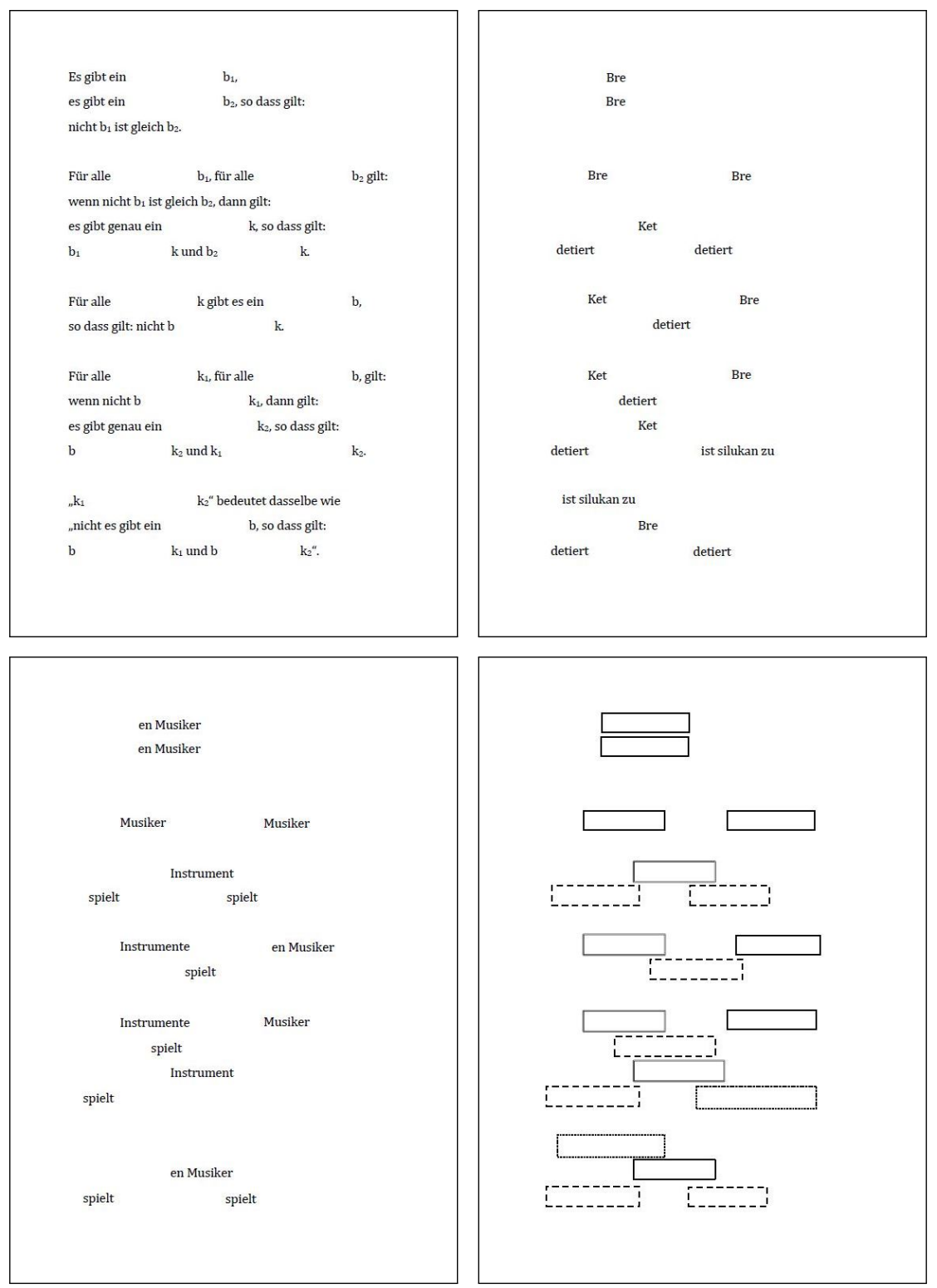

Abbildung 4.3: Foliensatz: Abstraktion bei den Wüstensandsätzen ${ }^{12}$

Ferner wird in dem oben zitierten Text eine für die Unterrichtsreihe zentrale Vorstellung zum Thema Axiomatik eingeführt: Axiomensysteme werden mit Regel-oder Vertragswerken verglichen, die zwischen verschiedenen Personen oder Parteien verabredet werden und an die man sich bei allen weiteren Aktivitäten zu halten hat. Diese Metapher wird anhand eines Vertragswerks zum Bau von Autobahnen verinnerlicht (Cohors-Fresenborg et al. 2003b, S. 33):

\footnotetext{
${ }^{12}$ Quelle: selbst erstellt nach Cohors-Fresenborg et al. 2003a, S. 78-79, 81-82.
} 
$\S 1$ Es gibt mindestens zwei verschiedene Autobahnkreuze.

$\$ 2 \mathrm{Zu}$ je zwei verschiedenen Autobahnkreuzen gibt es genau eine Autobahn, so dass beide Autobahnkreuze auf dieser Autobahn liegen.

$\S 3 \mathrm{Zu}$ jeder Autobahn gibt es mindestens ein Autobahnkreuz, das nicht auf dieser Autobahn liegt.

$\S 4 \mathrm{Zu}$ jeder Autobahn und zu jedem Autobahnkreuz, das nicht auf dieser Autobahn liegt, gibt es genau eine weitere Autobahn, so dass das Autobahnkreuz auf dieser weiteren Autobahn liegt und die beiden Autobahnen parallel sind.

Ausgehend von diesem Vertragswerk und einer Landkarte des fiktiven Emirats Dabou, auf der vier Orte eingezeichnet sind, lassen sich nun verschiedene Möglichkeiten finden und analysieren, ein (vertragsgemäßes) Netz aus Autobahnen und Autobahnkreuzen zu bauen.

Abschließend wird ausblickhaft der deduktive Aspekt der Axiomatik angesprochen, indem aus dem Autobahnbau-Vertrag streng logisch die Aussage „In jedem Autobahnmodell gibt es mindestens vier Autobahnkreuze " bewiesen wird.

\section{Didaktischer Kommentar}

Das herausstellendste Merkmal der Unterrichtsreihe ist ihr kognitionswissenschaftlich motivierter Zugang. Aufbauend auf der Metapherntheorie von Lakoff und Johnson (1980), nach der die zentrale Funktion einer Metapher darin besteht, einen relativ schwierig zu begreifenden Sachverhalt oder Vorgang durch bekanntere, leichter zugängliche Erfahrungen und Situationen verständlich zu machen, werden durch die Wüstensandsätze zwei mentale Modelle vermittelt, die zu einem vertieften Verständnis abstrakter Axiomensysteme und des Umgangs mit diesen beitragen (siehe auch Cohors-Fresenborg 2008):

1. Durch die archäologische Rahmengeschichte wird die Frage der Bedeutungsfindung für abstrakte Begriffe mit dem Übersetzen aus Fremdsprachen in Verbindung gesetzt. Zum einen wird der Prozess der Übertragung einer rein symbolischformal gegebenen Aussage (Maskonensprache) ins Deutsche (und umgekehrt) betrachtet. Zum anderen wird die Untersuchung verschiedener Modelle für ein gegebenes Axiomensystem mit der unterschiedlichen Interpretation der Kunstworte Bre, Ket, detiert und silukan verglichen.

2. Mit der Vertragswerk-Metapher wird eine für das Verständnis moderner Axiomatik grundlegende Vorstellung thematisiert. Beim Analysieren der Eigenschaften 
verschiedener Modelle der Wüstensandsätze (wie auch beim Prüfen formal notierter Aussagen auf syntaktische Korrektheit, siehe Abbildung 4.2) müssen die Schülerinnen und Schüler immer wieder durch genauen Verweis auf die verwendeten Paragraphen ihre Argumentationen belegen. Das vor diesem Hintergrund besonders intensiv diskutierte Vertragswerk zum Bau von Autobahnen eignet sich dabei, um die Auswirkungen zu untersuchen, die das Streichen oder Abändern bestimmter Regeln (Axiome) für das zu erstellende Autobahnnetz haben.

Beide mentalen Modelle zusammengenommen liefern eine tragbare Vorstellung von der modernen Auffassung axiomatischer Theorien als formale Denksysteme, wie sie von Hefendehl-Hebeker (2004, S. 144) beschrieben wird:

[D]as bedeutet, dass die auftretenden Begriffe nicht mehr den Charakter von Namen haben, sondern nur noch Variable (für Gegenstände, Funktionen und Prädikate) sind. Axiome beschreiben dann nicht mehr evidente Tatsachen, sondern stellen ein Vertragswerk für den Umgang mit den Begriffen dar, die für eine Interpretation der Variablen vorgesehen sind. (Herv. d. Verf.)

Im Gegensatz zu anderen Vorschlägen, die vornehmlich deduktive Aspekte oder den Prozess der Axiomatisierung thematisieren, stellt die Unterrichtsreihe also die Vermittlung geeigneter Metaphern zu abstrakten Axiomensystemen mit implizit definierten Grundbegriffen in den Vordergrund. Es werden nicht primär die Stufen der mathematischen Theorieentwicklung und Strukturierung behandelt, sondern ,eine erste Orientierung über Fragen der Fundierung mathematischer Begriffe, des Beweisens und der ontologischen Begründung der Mathematik“ (Cohors-Fresenborg et al. 2003a, S. 5) gegeben. Vor diesem Hintergrund ist es auch legitim, die Schülerinnen und Schüler direkt am Anfang der Unterrichtsreihe mit einem Axiomensystem (den Wüstensandsätzen) zu konfrontieren. Ein solches Vorgehen hat üblicherweise den Nachteil, dass die Rolle der Axiomatik in der Mathematik dadurch verfälscht dargestellt und der Eindruck vermittelt wird, Mathematik erschöpfe sich in der formal und logisch strengen Deduktion aus willkürlich gegebenen Ausgangssätzen (vgl. Cohors-Fresenborg et al. 2003a, S. 6; siehe auch Abschnitt 3.3). Da in der Unterrichtsreihe der deduktive Aspekt jedoch nur am Rande eine Rolle spielt und hinter die Frage der Bedeutungsfindung bei formalisierten mathematischen Texten zurücktritt, ist der gewählte Ansatz nachvollziehbar.

Viele der im Textbuch enthaltenen Übungsaufgaben bieten den Lernenden die Gelegenheit zur Metakognition (vgl. Cohors-Fresenborg et al. 2003a, S. 7). Insbesondere durch die Auseinandersetzung mit in das Schüler-Textbuch eingebauten fiktiven Äußerungen, die typische Fehler und Fehlvorstellungen beinhalten, wird den Schülerinnen und Schülern die Gelegenheit gegeben, sich eingehender mit dem Unterrichtsinhalt zu beschäftigen 
und so zu einem tieferen Verständnis zu gelangen. Außerdem steht den Lehrkräften damit eine Möglichkeit zur Verfügung, die Denkprozesse der Lernenden genauer zu beleuchten. Sowohl das Lehrerhandbuch zur Unterrichtsreihe (Cohors-Fresenborg et al. 2003a) als auch ein Artikel von Sjuts (1993) beinhalten zahlreiche Transkriptionen von Schüleräußerungen, die belegen, dass die durch die Wüstensandsätze vermittelten Vorstellungswelten nachhaltig sind.

Die wichtigsten Erkenntnisse bezüglich axiomatischer Denkweisen, die Schülerinnen und Schüler durch die Unterrichtsreihe gewinnen können, sind zusammengefasst die folgenden (siehe auch Sjuts 1993, S. 35-37):

- Eine Besonderheit der mathematischen Sprache ist, dass sie kontextfrei genutzt wird, d. h. die Bedeutung der in ihr vorkommenden Wörter - abgesehen von aussagenlogischen Begriffen - kann ausgeklammert werden. Dies stellt einen fundamentalen Unterschied zu anderen Sprachen dar, deren Verwendung wesentlich durch den Kontext mitbestimmt wird. Dies muss im Rahmen der für die Wüstensandsätze gewählten Übersetzungsmetaphorik thematisiert werden.

- Durch die vergleichende Betrachtung der Wörter Bre, Ket und detiert auf der einen und silukan auf der anderen Seite wird der Unterschied zwischen impliziten und expliziten Definitionen verdeutlicht. ${ }^{13}$

- Durch die Untersuchung zahlreicher verschiedener Interpretationen der Wüstensandsätze wird ein grundlegendes Verständnis davon vermittelt, was Abstraktion in der Mathematik bedeutet. Insbesondere die Folien-Aufgabe (siehe Abbildung 4.3) ist zur abschließenden ,Visualisierung und handgreiflichen Verankerung“ (CohorsFresenborg 2008, S. 18) gut geeignet. Was der abstrakte Charakter der Maskonensprache für den ontischen und epistemischen Status mathematischer Aussagen bedeutet, kann im Rahmen der Unterrichtsreihe nur angedeutet werden. Durch die Einsicht, dass es die wahre Interpretation der Wüstensandsätze nicht gibt, wird jedoch ein Voverständnis dieser Aspekte befördert.

- Die Oberpriester-Geschichte liefert eine Vorstellung davon, wie der Prozess der Axiomatisierung ablaufen kann: Man ordnet verschiedene, zunächst nicht zusammenhängende Theorien und erkennt strukturelle Gemeinsamkeiten zwischen ihnen, die man schließlich in einem abstrakt formulierten Regelwerk bündelt. Die in den Einzeltheorien inhaltlich interpretierten Begriffe werden dabei durch Begriffsvaria-

\footnotetext{
${ }^{13} \mathrm{Im}$ Gegensatz zu einer impliziten Definition, bei der die auftretenden Begriffe ausschließlich über die in den Axiomen formulierten Zusammenhänge charakterisiert sind, werden bei einer expliziten Definition bereits bekannte Begriffe zur Beschreibung eines unbekannten Begriffs verwendet.
} 
blen ersetzt, die semantisch nicht gefüllt sind. Zugleich werden anhand der Geschichte die für die mathematische Behandlung des Themas zentralen Begriffe eingeführt (Axiom, Axiomensystem, Grundbegriff, Modell, Abstraktion, implizite und explizite Definition).

Es ist in diesem Zusammenhang jedoch schade, dass den Schülerinnen und Schülern nicht mitgeteilt wird, dass die in der Unterrichtsreihe behandelten Sätze tatsächlich etwas mit Mathematik zu tun haben. Eine Behandlung der Inzidenzgeometrie, deren Axiome den Wüstensandsätzen bei geeigneter Interpretation entsprechen $($ Bre $=$ Punkt, Ket $=$ Gerade, detieren $=$ liegen auf, silukan $=$ parallel $)$, wäre hier durchaus wünschenswert und sinnvoll gewesen.

- Die Beschäftigung mit Vertragswerken schult den Umgang mit implizit definierten Grundbegriffen: Bei der Konstruktion von Modellen (z. B. im Kontext des Autobahnbau-Beispiels) darf ausschließlich auf das Vertragswerk Bezug genommen werden. Das gilt insbesondere für die verwendeten Grundbegriffe und die Beziehungen, in die sie durch das Vertragswerk zueinander gesetzt werden. Bei inhaltlich interpretierten Vertragswerken wie dem zum Bau von Autobahnen können dabei Interferenzen mit der alltäglichen Bedeutung von Begriffen wie „Autobahn“ oder „Autobahnkreuz“ entstehen. Bei den Überlegungen, welche Autobahnen durch welche Autobahnkreuze gemäß dem Regelwerk verbunden werden müssen, dürfen solche vertragsexternen Begriffsaspekte jedoch keine Rolle spielen.

- Ein Vertragswerk ist eine Übereinkunft, die zwei Parteien untereinander treffen. In unterschiedlichen Situationen können unterschiedliche Vertragstexte sinnvoll sein. Diese Vorstellung bildet den Grundstein dafür, die klassische Sicht mathematischer Aussagen als evidente Tatsachen zu erweitern und den hypothetisch-deduktiven Charakter der Mathematik zu betonen.

- Durch die Herleitung allgemeingültiger Modelleigenschaften unter streng logischer Berufung auf die Vertragsparagraphen sammeln die Lernenden erste Erfahrungen mit deduktivem Vorgehen und dem dabei erforderlichen Grad an Strenge. Auch die Untersuchung der Auswirkungen, die das Weglassen oder Hinzufügen eines Axioms (Paragraphen) auf die zulässigen Modelle haben, fällt unter diesen Aspekt. 


\subsubsection{Die Entdeckung der Axiomatik am Sechsstern (M. Wagenschein und M. Gerwig)}

Ein vergleichsweise aktueller Unterrichtsvorschlag stammt von Gerwig (2015) und greift inhaltlich wie methodisch die Ideen Martin Wagenscheins auf. Den didaktischen Hintergrund bildet die Lehrkunstdidaktik, die auf Wagenscheins Konzept des genetischen, sokratischen und exemplarischen Lehrens (siehe beispielsweise Wagenschein 1970, S. 6899), Wolfgang Klafkis Theorie der kategorialen Bildung sowie Gottfried Hausmanns Überlegungen zur dramaturgischen Inszenzierung von Unterrichtssequenzen (Hausmann 1959) aufbaut. Grundlegend für die lehrkunstdidaktische Unterrichtsgestaltung ist die Methodentrias exemplarisch - genetisch - dramaturgisch. ${ }^{14}$ Unterrichtseinheiten (auch Lehrstücke genannt) sind dabei

durchgestaltet wie ein Theaterstück, unterteilt in Akte und Szenen, die dem Aufgaben-, Handlungs- und Erkenntniszusammenhang stärker folgen als der Verteilung auf Wochenstunden und Lektionen. Diese Gliederung ermöglicht es, Denkhandlungen zu evozieren, Spannung aufzubauen zwischen alternativen Lösungsansätzen auf dem Weg zur Lösung, (historische) Lösungsansätze durchzuspielen und im Auffinden der Lösung ,Entspannung ' zu erzeugen. (Wildhirt und Gerwig 2013, S. 5)

Ausgangspunkt von Gerwigs Arbeit ist die ,paradoxe Situation“, dass das Beweisen zwar zweifelsohne „das Charakteristikum“ der Mathematik als wissenschaftlicher Disziplin sei, im Mathematikunterricht in aller Regel jedoch ein „Schattendasein“ führe (Gerwig 2015, S. 1; Herv. im Orig.). Gerwig entwickelt daraufhin drei Lehrstücke, die das Thema Beweisen aus unterschiedlichen Perspektiven beleuchten:

(1) Die Entdeckung der Axiomatik am Sechsstern (Gerwig 2015, S. 119-192)

(2) Beweisvielfalt erleben - der Satz des Pythagoras (Gerwig 2015, S. 193-258)

(3) Das Nichtabbrechen der Primzahlfolge (Gerwig 2015, S. 259-338)

Alle drei Lehrstücke behandeln zentrale Sätze aus Euklids Elementen, die auch von Wagenschein als Beispiele für genetische Lehrgänge thematisiert wurden. ${ }^{15}$

\footnotetext{
${ }^{14}$ Eine ausführliche Darstellung der historischen Entwicklung der Lehrkunstdidaktik sowie der Einflüsse Wagenscheins, Klafkis und Hausmanns inkl. Hinweisen auf weiterführende Literatur findet sich in Gerwig 2015, S. 53-116. Zudem ist 2013 ein Themenheft der Zeitschrift Der Mathematikunterricht (Heft 6) zur Lehrkunstdidaktik mit theoretischen Leitartikeln wie auch unterrichtspraktischen Beispielen erschienen. Die Internetseite http: / /www . lehrkunst . ch/ (letzter Zugriff: 29.09.17) bietet darüber hinaus zahlreiche weitere Informationen.

${ }^{15} \mathrm{Zu}$ (1) siehe Wagenschein 1970, S. 135-148, Wagenschein 1974 und Wagenschein 2008, S. 125-150; zu (2) siehe Wagenschein 1980, S. 251-267; zu (3) siehe Wagenschein 1980, S. 228-236.
} 
Die Inhalte des ersten Lehrstücks (in verschiedenen Vorstufen in den Jahrgangsstufen 7 und 8 in Deutschland und der Schweiz sowie in seiner vorläufigen Endform in einer 8. Klasse in der Schweiz erprobt) sollen im Folgenden dargestellt werden, da darin die Axiomatik eine explizite Rolle spielt. Wer sich insbesondere für die lehrkunstdidaktischen Details der Unterrichtsinszenierung interessiert, sei auf die Darstellung bei Gerwig (2015, S. 119-167) verwiesen, der sich zudem ein ausführliches Reflexionskapitel anschließt (S. 168-181).

\section{Inhaltliche Beschreibung}

Das Kernstück der Unterrichtseinheit bildet Wagenscheins Beweisvorschlag für die Beobachtung, dass sich der Radius eines Kreises genau sechs Mal auf dessen Rand abtragen lässt. Den klassischen Beweis ${ }^{16}$, der im Wesentlichen den Innenwinkelsatz für Dreiecke verwendet, lehnt Wagenschein ab, da er Zeugnis einer Unterrichtskultur sei, in der es überwiegend darum gehe, ,so viel Wissen von grundlegenden Sätzen [...] und von Fähigkeiten [...] anzusammeln, daß auftretende mathematische Probleme mit diesem , Handwerkszeug " sicher und schnell gelöst werden können“ (Wagenschein 1970, S. 137). Suggestiv fragt er: „Der Beweis bringt also zwar eine Entscheidung. Bringt er auch eine Klärung? Benutzt er nicht zu schweres Geschütz und verwendet unnötige Begriffe, verfährt er nicht unökonomisch und unelegant? Ein entbehrlich scheinender Aufwand verdeckt die Einsicht" (Wagenschein 1970, S. 138). Als Alternative zeigt Wagenschein auf, wie man den Satz durch Vereinfachungen und Umformulierungen der Problemstellung auf die - letztlich als evident zu betrachtende - Tatsache zurückführen kann, „, dass es in der Ebene in jeder Richtung immer möglich ist, eine Figur (z. B. ein Dreieck) so zu verschieben, dass seine [sic!] Punkte Spuren zurücklegen, die alle 1. gerade, 2. gleich lang, 3. parallel sind (Parallelverschiebung)“ (Gerwig 2015, S. 129). Abbildung 4.4 visualisiert die einzelnen Beweisschritte, welche im Folgenden erläutert werden:

Zunächst mache man sich klar, dass das Phänomen der sich exakt schließenden Zirkelrose, mit dem hier gestartet wird, äquivalent zu der Aussage über das exakt sechsmalige Abtragen des Kreisradius ist. Dies sieht man, wenn man anstatt sämtlicher Kreise nur deren Schnittpunkte mit dem Ursprungskreis betrachtet. Verbindet man diese Schnittpunkte und zeichnet zusätzlich die sechs zugehörigen Kreisradien in die Figur ein, erkennt man, dass man das Ausgangsproblem auch ohne Rückgriff auf die Kreisfigur wie folgt formulieren

\footnotetext{
${ }^{16}$ „Man muß die Radien zu den Eckpunkten zeichnen, dann sieht man: Das sind lauter gleichseitige, also auch gleichwinklige Dreiecke. Da die Winkelsumme in jedem beliebigen Dreieck (wie wir gelernt haben) ,einhundertundachtzig Grad beträgt', hat jeder Winkel in diesem besonderen Dreieck sechzig Grad. Und 6 mal 60 gibt eben 360!“ (Wagenschein 1970, S. 137).
} 


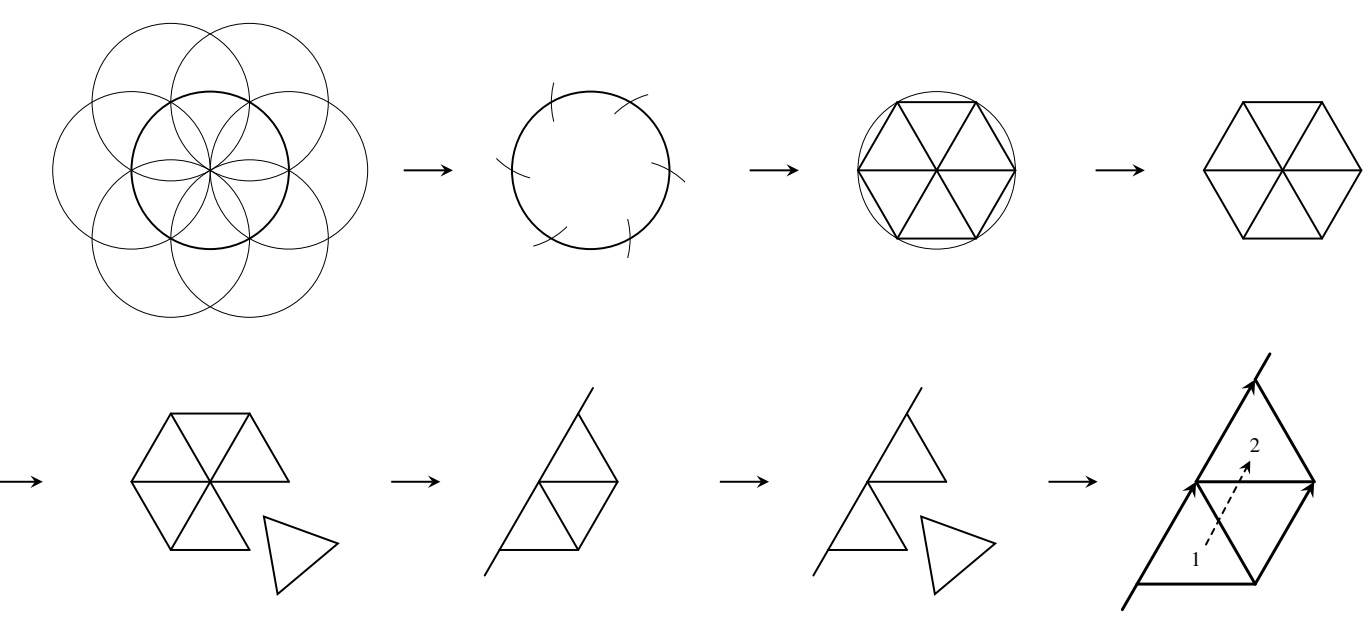

Abbildung 4.4: Visualisierung des Sechseck-Beweises ${ }^{17}$

kann: Lassen sich sechs gleichseitge Dreiecke lückenlos zusammenschieben? Ein Halbieren der Figur reduziert das Problem auf dieselbe Frage mit drei gleichseitigen Dreiecken: Liegen zwei Dreiecke an einer Geraden nebeneinander, lässt sich ein drittes Dreieck dann genau in die Lücke setzen? Nun kommt Wagenscheins Kniff: Man betrachtet die zwei nebeneinander liegenden Dreiecke dynamisch in dem Sinne, dass man sich das zweite als aus dem ersten Dreieck entstanden vorstellt, wenn man dieses entlang der Geraden um exakt eine Seitenlänge verschiebt. Alle Punkte des Dreiecks legen dabei dieselbe Spur zurück und alle diese Spuren sind gleich lang und parallel. Das bedeutet insbesondere, dass der Abstand zwischen den beiden Dreiecksspitzen exakt so lang wie eine Dreiecksseite ist. Deshalb passt zwischen die beiden Dreiecke exakt ein drittes „umgedrehtes“ gleichseitiges Dreieck.

Gerwig baut diesen Ansatz durch eigene Ergänzungen sowie die Ausarbeitung einiger bei Wagenschein nur angedeuteter Ideen zu einem Lehrstück mit einer Gesamtdauer von zwölf Unterrichtsstunden aus (vgl. Gerwig 2015, S. 143-144; eine komprimierte Darstellung des Lehrstücks findet man zudem in Gerwig 2013):

Ouvertüre (2 Stunden): Die Lernenden beschäftigen sich mit einer Vielzahl geometrischer Formen aus Natur und Kultur (z. B. Schneeflocken und Fensterrosen berühmter Bauwerke), deren prominenteste Gemeinsamkeit das regelmäßige Sechseck ist. Sie finden heraus, dass sich diese geometrische Figur mit Hilfe von Zirkel und Lineal durch eine sogenannte Zirkelrose (vgl. die Zeichnung oben links in Abbildung 4.4) konstruieren lässt. Im Anschluss wird eine gekürzte Version eines ,Sokratischen Dialogs“ von Alfréd Rényi gelesen (abgedruckt in Gerwig 2015, S. 382-383), welche die Idealität geometrischer Objekte thematisiert.

\footnotetext{
${ }^{17}$ Quelle: angelehnt an Gerwig 2015, S. 128.
} 
I. Akt (2 Stunden): Die Lehrkraft tritt verkleidet als Euklid von Alexandria auf, knüpft an den in der Ouvertüre behandelten Sokratischen Dialog an und diskutiert mit der Klasse die Frage, warum sich die (ideale) Zirkelrose genau schließt bzw. warum sich der Radius eines Kreises genau sechs Mal auf diesem abtragen lässt. Als Vorbereitung auf die Bearbeitung dieser Warum-Frage entwickeln die Lernenden allgemeine heuristische Strategien, die an der Tafel festgehalten werden, z. B.: „Nur benutzen, was bekannt oder selbstverständlich ist“ oder „Problem vereinfachen und umformulieren, ohne es jedoch im Kern zu verändern“.

II. Akt (2 Stunden): Das Ausgangsphänomen wird mit Hilfe der zuvor festgehaltenen Strategien nach Wagenscheins Vorbild (siehe oben) Schritt für Schritt analysiert und vereinfacht, bis man schließlich zur Lösung vordringt: die Möglichkeit der Parallelverschiebung in der Ebene. Als Denkimpuls kann die Lehrkraft den Schülerinnen und Schülern verschiedene „Ufer-Hilfen“ anbieten (siehe Gerwig 2015, S. 128).

III. Akt (2 Stunden): Die im II. Akt gefundene Begründung wird nochmals ,umgekehrt“ betrachtet, so dass verdeutlicht wird, wie bei einem mathematischen Beweis aus einer sofort einleuchtenden Tatsache deduktiv eine alles andere als triviale Aussage gefolgert wird. Anschließend wird der Original-Beweis aus Euklids Elementen analysiert. Dabei fällt auf, dass sich Euklid immer wieder auf andere, bereits bewiesene Aussagen beruft, für die er wiederum Beweise liefert und so weiter. Insgesamt beruht die von Euklid dargestellte Geometrie auf nur wenigen Definitionen, Postulaten und Axiomen. Dies wird von der Lehrkraft durch Vorstellen eines Schaubildes (ähnlich dem auf Seite 15 dieser Arbeit) verdeutlicht. Abschließend werden die Axiome und Postulate aus den Elementen gemeinsam betrachtet.

Epilog (4 Stunden): Analog zum Sechsstern-Beweis präsentiert Wagenschein auch eine alternative Begründung des Satzes von Thales, die dessen Gültigkeit ebenfalls auf die Möglichkeit der Parallelverschiebung zurückführt (siehe Wagenschein 1974, S. 65-67). Gerwig behandelt diesen Beweis als Abschluss seines Lehrstücks.

\section{Didaktischer Kommentar}

Insgesamt gelingt es Gerwig mit seinem Lehrstück, den Lernenden eine ,wirkliche Einsicht und ein echtes Erlebnis“ (Wittenberg 1963, S. 59) der (euklidischen) Axiomatik zu ermöglichen. Durch die intensiven heuristischen Phasen, in denen die Lernenden eigenständig nach einer Begründung für das Zirkelrosen-Phänomen suchen, und die anschließende Rückschau auf die fertige Argumentationskette ergibt sich die Notwendigkeit einer axiomatischen Basis genetisch aus den Schüleraktivitäten. 
Dennoch seien zwei kritische Anmerkungen, die auch von Hungerbühler (2015) erwähnt werden, an dieser Stelle erlaubt:

- Wagenschein formuliert die wesentliche Erkenntnis in Bezug auf die geometrische Axiomatik wie folgt:

[D]er Schüler ist nun reif für die Mitteilung der griechischen Entdeckung, daß und wie Axiomatik in der Geometrie möglich ist. Das heißt: daß ihre Wahrheiten nicht vereinzelt dastehen, sondern alle aus denselben einfachsten Selbstverständlichkeiten ableitbar sind und damit vollständig durchsichtig gemacht werden können. [...] Denn jeder hat ein Anrecht erfahren zu haben, was hier sich auftut: ein Gebiet, auf dem es Gewißheit gibt, Genauigkeit und Verstehen, und zwar ein restloses [...] ein Gebiet, in dem es möglich ist, alle Merkwürdigkeiten auf wenige evidente Grundwahrheiten zurückzuführen. (Wagenschein 1974, S. 68-69)

Auch Gerwig nimmt diese Sichtweise für sich in Anspruch (vgl. Gerwig 2015, S. 139-140), die als erste Heranführung an axiomatische Fragestellungen sicher auch ausreichend ist. Ein Mathematikunterricht, der sich dem Ziel verschreibt, ein möglichst authentisches Bild der Mathematik zu vermitteln, darf bei dieser Auffassung jedoch nicht stehen bleiben, sondern muss auch eine Konfrontation der Schülerinnen und Schüler mit der formalistischen Auffassung mathematischer Axiome als „abstrakte Spielregeln“ anstreben, aus denen sich Theorien deduzieren lassen, die auf verschiedene Modelle anwendbar sind. Es ist zwar nicht erforderlich, diesen Aspekt in das Lehrstück zu integrieren, allerdings sollten sich Lehrkräfte, die den von Wagenschein und Gerwig vorgezeichneten Weg gehen, bewusst sein, dass zumindest zu einem späteren Zeitpunkt eine Behandlung axiomatischer Themen aus Sicht der modernen Mathematik als Ergänzung sinnvoll wäre.

- Wagenscheins Kritik am „klassischen“ Beweis über die Winkelsumme im Dreieck ist nur bedingt nachvollziehbar, da man auch diesen so weit ,herunterbrechen“ kann, bis man bei evidenten Grundtatsachen angelangt. Inwiefern Wagenscheins Vorschlag diesem Beweis also überlegen ist hinsichtlich einer wirklichen Einsicht in den Begründungszusammenhang, ist zumindest fraglich. Hinzu kommt, dass in Wagenscheins Begründungskette stillschweigend ein Wechsel von einer objektorientierten zu einer abbildungsgeometrischen Sichtweise stattfindet. Hungerbühler (2015, S. 361) merkt dazu an, dass ,die griechische Geometrie [...] objektorientiert, nicht abbildungsorientiert“ war; der Begriff der „Translation [sei] ein moderner Fremdkörper im Beweis“. Vor diesem Hintergrund ist es lobenswert, dass Gerwig eine Behandlung des euklidischen Beweises in sein Lehrstück integriert. Dadurch wird den Schülerinnen und Schülern eine vertiefte Einsicht in die „Existenz 
eines durchdringenden Ordnungsgefüges“ (Gerwig 2015, S. 140) gewährt, welches letztendlich auf nur wenigen Axiomen beruht. Ein Problem, das sich daraus jedoch ergibt, ist die unterschiedliche axiomatische Basis, zu der beide Beweisversionen führen: zum einen die Parallelverschiebung, zum anderen das Parallelenpostulat. Diese beiden Aussagen sind jedoch aus fachlicher Sicht nicht äquivalent: „Es gibt Ebenen, in denen das Parallelenaxiom gilt, wo jedoch nicht zu allen Punktepaaren $P, Q$ eine Translation $\tau$ existiert mit $\tau(P)=Q^{“}$ (Hungerbühler 2015, S. 361).

\subsubsection{Hypothesen in der Mathematik (H. N. Jahnke)}

Hans Niels Jahnke stellt in seinen theoretischen und unterrichtspraktischen Arbeiten (siehe Jahnke 2007a,b, 2009, 2010 sowie Jahnke und Wambach 2013) den Gedanken in den Vordergrund, dass Beweise in der Mathematik nicht die (absolute) Wahrheit von Aussagen klären, sondern logisch-deduktive Zusammenhänge zwischen Sachverhalten in Form von ,wenn ..., dann ...-Aussagen“ aufzeigen. Hypothesen aufzustellen, mit ihnen zu experimentieren sowie ihre deduktiven Konsequenzen zu erforschen und dies als Kriterium für ihre Akzeptanz heranzuziehen, sei aus historischer und wissenschaftstheoretischer Sicht gerade auch für die Mathematik grundlegend. Als Beleg beruft Jahnke sich wesentlich auf die Arbeiten von Árpád Szabó zur Rolle der Axiome und Postulate in der vor- und nacheuklidischen Zeit (Szabó 1960 und Szabó 1978; siehe auch den Abschluss von Abschnitt 2.1.1) sowie auf das Konzept der extrinsischen Rechtfertigung (siehe auch die diesbezüglichen Ausführungen in Abschnitt 2.3).

Anhand zweier unterrichtspraktischer Vorschläge exemplifiziert Jahnke, wie bei Lernenden ein Bewusstsein für die einer Argumentation zugrunde liegenden Hypothesen geweckt werden kann. Zugleich betont er jedoch, dass solche Unterrichtsinterventionen nur dann nachhaltig sein können, wenn die Rolle von Annahmen und deren Konsequenzen und der Ursprung von Axiomen langfristig und über alle Jahrgangsstufen hinweg im Mathematikunterricht thematisiert werden (vgl. Jahnke und Wambach 2013, S. 482).

\section{Inhaltliche Beschreibung}

Der erste Unterrichtsvorschlag (Jahnke und Wambach 2013) ist auf etwa acht Schulstunden in einer 8. oder 9. Gymnasialklasse ausgelegt und behandelt die mathematische Darstellung eines antiken astronomischen Problems. Gemäß des geozentrischen Weltbildes ging man bekanntlich lange Zeit davon aus, dass die Erde der Mittelpunkt des Universums sei und sich alle übrigen Himmelskörper auf kreisförmigen Bahnen um sie beweg- 
ten. Ausgangspunkt der Unterrichtsreihe ist die sogenannte Anomalie der Sonne: Auf der einen Seite postulierte man, dass sich die Sonne mit konstanter Geschwindigkeit innerhalb eines Jahres auf einer Kreisbahn, deren Mittelpunkt mit dem Erdmittelpunkt übereinstimmt, exakt ein Mal um die Erde dreht. Als Konsequenz dieser Annahmen müssten die vier Jahreszeiten gleich lang sein. Auf der anderen Seite zeigen empirische Messungen, dass sie nicht von gleicher Länge sind. Auf Grundlage dieses Konflikts diskutieren die Schülerinnen und Schüler verschiedene Alternativen zu den Ausgangshypothesen über die Bewegungsbahn der Sonne und untersuchen, ob sich die aus diesen Modifikationen ergebenden Konsequenzen besser mit den empirischen Daten decken.

Der zweite Unterrichtsvorschlag für Schülerinnen und Schüler ab der 7. Klasse (Jahnke 2007b, 2009) thematisiert den Innenwinkelsatz für Dreiecke. Auch hier wählt Jahnke einen empirischen Zugang, in dem der Wechselwinkelsatz als eine aufgrund empirischer Messungen sinnvoll erscheinende Hypothese aufgefasst wird. Der Innenwinkelsatz lässt sich dann sowohl logisch-deduktiv daraus herleiten, als auch empirisch (approximativ) verifizieren. Dadurch wird wiederum der Wechselwinkelsatz als Ausgangshypothese gestärkt. Die Tatsache, dass aus dem Wechselwinkelsatz noch viele weitere Aussagen deduziert werden können, die einer Überprüfung durch Messungen standhalten, erhöht zugleich die Sicherheit des Innenwinkelsatzes. Hier ,fungiert die Geometrie als eine physikalische Theorie“ (Jahnke 2009, S. 26; Herv. im Orig.), an die wir glauben, weil ihre Aussagen mit unseren empirischen Erfahrungen übereinstimmen und nicht etwa, weil wir sie durch theoretische Beweise auf eine bestimmte Anzahl von Axiomen zurückführen können (vgl. Jahnke 2009, S. 26).

\section{Didaktischer Kommentar}

Jahnke spricht mit der Rechtfertigungsproblematik mathematischer Aussagen und der Betonung ihres hypothetischen Charakters einen wichtigen Aspekt der Theorieentwicklung und -sicherung an. Das Aufstellen von Vermutungen und das Einschätzen ihrer Glaubwürdigkeit (nicht zwangsläufig: Wahrheit) anhand der deduzierbaren und (quasi-)empirisch überprüfbaren Konsequenzen ist nah an der mathematischen Alltagspraxis. Zudem wirken die Unterrichtsideen auch der Gefahr der antididaktischen Inversion entgegen, die hier darin bestünde, die Ausgangssätze von vornherein als „offensichtlich wahr“ anzunehmen und dann lediglich wahrheitsübertragende deduktive Beweise für weiterführende Aussagen mit den Lernenden zu behandeln.

Beide Unterrichtsbeispiele zeigen deutlich, dass Jahnke den Prozess der Erkenntnisvalidierung in der Mathematik in enge Beziehung zum Vorgehen in anderen empirischen 
Wissenschaften setzen möchte. Im ersten Unterrichtsbeispiel ist dies ohnehin naheliegend, da hier kein immanent mathematisches Problem behandelt wird und man sich somit von Anfang an im empirisch-wissenschaftlichen Kontext bewegt. Bezogen auf den Unterrichtsvorschlag zur Geometrie drängt sich jedoch die Frage auf, worin genau laut Jahnke eigentlich der Unterschied zwischen Mathematik und empirischen Wissenschaften wie der Physik besteht. Eine wie in Jahnkes Unterrichtsreihe betriebene Geometrie

versteht sich vorerst als eine empirische Wissenschaft, deren Aussagen beobachtend und experimentell erschlossen und zeichnerisch wie rechnerisch verwertet werden und letztlich den Charakter von Hypothesen haben. Innerhalb des empirisch erworbenen Wissens lassen sich logische Stammbäume errichten und durch logisches Schließen weitere Aussagen gewinnen. (HefendehlHebeker und Hußmann 2003, S. 99)

Diese ontologisch verankerte Auffassung von Geometrie bzw. Mathematik im Allgemeinen ist nicht nur historisch bedeutsam, sondern auch der Erfahrungswelt der Lernenden sehr nah. Allerdings sei an dieser Stelle zumindest die Frage in den Raum gestellt, ob eine Abgrenzung mathematischer Argumentationsmuster von der Empirie nicht zumindest im Oberstufenbereich angestrebt werden sollte. Dies muss nicht zu einer Abkehr vom hypothetischen Charakter der Mathematik führen, denn eine Rechtfertigung mathematischer Aussagen bzw. der aus ihnen deduzierbaren Konsequenzen kann zusätzlich zu einer Überprüfung der Passung mit empirischen Messungen auch über die Untersuchung der innermathematischen Nützlichkeit geschehen.

\subsection{Zusammenfassung mit Blick auf die Lernziele}

Die vorgestellten Unterrichtsideen behandeln - teils mehr und teils weniger explizit - verschiedene Facetten axiomatischen Denkens und Arbeitens, von der euklidischen Auffassung über die Bedeutung von Abstraktion und impliziten Definitionen bis zur mathematischen Theorieentwicklung. Im Folgenden wird geschildert, inwiefern die in Abschnitt 4.1 aufgestellten übergeordneten Lernziele durch diese vier Unterrichtsreihen abgedeckt werden.

\section{Axiomatisierung von Abstimmungsmengen}

In Steiners Unterrichtskonzept steht der Prozess der mathematischen Theorieentwicklung im Vordergrund, insbesondere das lokale Ordnen verschiedener Aussagen über Abstimmungsmengen (LZ4). Wie bereits in Abschnitt 4.1 angedeutet, ist die Tätigkeit des lo- 
gischen Strukturierens eng verbunden mit deduktiven Aspekten, so dass die Schülerinnen und Schüler hier (vor allem zum Abschluss der Reihe) auch lernen, Aussagen aus dem aufgestellten Axiomensystem für Abstimmungsmengen zu deduzieren (LZ 5). Trotz der realweltlichen Verankerung des Themas wird durch den mengentheoretischen Formalismus die Hilbertsche Auffassung von Axiomen als impliziten Definitionen illustriert (LZ 3). Die Frage nach der Rechtfertigung von Axiomensystemen (LZ 6) wird bei Steiner zwar nicht explizit thematisiert, allerdings wird die „Nützlichkeit“ der impliziten Charakterisierung des Begriffs Gewinnkoalition für den Aufbau des Theoriegebäudes in der Unterrichtsreihe durchaus deutlich. Ähnliches gilt für LZ 1: Das Beweisen wird nicht bewusst auf der Meta-Ebene reflektiert, allerdings erfahren die Lernenden durch die Übungen zum lokalen Ordnen und Deduzieren, dass zur Begründung der behandelten Aussagen wiederum andere Aussagen über Abstimmungsmengen herangezogen werden (müssen).

\section{Sätze aus dem Wüstensand}

Die Materialien zur fiktiven Maskonensprache beschäftigen sich überwiegend mit der Bedeutung von Abstraktion. Durch die Behandlung verschiedener Modelle für die Wüstensandsätze setzen Lernende sich intensiv mit dem Wesen der impliziten Definiton auseinander (LZ3). Darüber hinaus fokussiert die Unterrichtsreihe verstärkt syntaktische Aspekte wie das korrekte Formulieren von Aussagen in der Maskonensprache. Es geht hier mehr um Formalisierung und weniger um axiomatische Aspekte. Zum Schluss der Unterrichtsreihe arbeiten die Schülerinnen und Schüler ansatzweise deduktiv auf Grundlage des Vertragswerks für den Autobahnbau (LZ5).

\section{Die Entdeckung der Axiomatik am Sechsstern}

Hintergrund von Gerwigs lehrkunstdidaktischen Ideen ist die euklidische Auffassung von Axiomen. Ausgehend vom Phänomen der Zirkelrose wird ein mathematisches Problem so lange rückwärts erschlossen, bis man zu einer nicht mehr beweisbedürftig erscheinenden Aussage gelangt. Dadurch wird sowohl die Natur mathematischen Beweisens - Rückführung einer zu zeigenden Aussage auf bereits bekannte Sachverhalte - deutlich (LZ1) als auch die evidenzbasierte Rechtfertigung für die Gültigkeit von Axiomen (LZ2).

\section{Hypothesen in der Mathematik}

Jahnkes Ideen zur Rolle von Hypothesen in der Mathematik sind die einzigen (mir bekannten) Unterrichtsvorschläge, die sich explizit und intensiv mit der Frage nach der 
Rechtfertigung axiomatischer Theorien beschäftigen (LZ 6). Zwar behandelt Jahnke nicht primär den Begriff Axiom und verwendet stattdessen überwiegend den Begriff Hypothese (vgl. Jahnke 2009, S. 26); sein Ansatz liefert aber eine wichtige Ergänzung zur rein euklidischen Rechtfertigung von Ausgangssätzen. Durch die Auffassung von Axiomen als Hypothesen wird neben einem geschärften Beweisverständnis (Beweise als Ketten von Wenn-dann-Aussagen, vgl. LZ 1) die Erkenntnis gefördert, dass mathematische Sätze nicht nur durch Selbstevidenz bzw. Deduzierbarkeit aus Selbstevidentem gerechtfertigt werden, sondern häufig auch durch die Nützlichkeit der aus ihnen ableitbaren Konsequenzen (bei Jahnke im Sinne von empirischer Überprüfbarkeit). Damit wird zumindest ein Teilaspekt der in LZ 6 angesprochenen Thematik adressiert. Eine Behandlung der Rechtfertigungsproblematik ohne empirische Argumentation findet hingegen nicht statt.

Die folgende Tabelle bietet eine Übersicht über die soeben beschriebene Abdeckung der in Abschnitt 4.1 formulierten Lernziele durch die vier Unterrichtsreihen:

\begin{tabular}{|l|c||c|c|c|c|c|c|}
\hline Unterrichtsreihe & Jgst. & LZ 1 & LZ 2 & LZ3 & LZ 4 & LZ 5 & LZ 6 \\
\hline \hline Abstimmungsmengen & $11 / 12$ & $(\checkmark)$ & & $\checkmark$ & $\checkmark$ & $\checkmark$ & $(\checkmark)$ \\
\hline Wüstensandsätze & 8 & & & $\checkmark$ & & $(\checkmark)$ & \\
\hline Sechsstern & $7 / 8$ & $\checkmark$ & $\checkmark$ & & & & \\
\hline Hypothesen in der M. & $8 / 9$ & $\checkmark$ & & & & & $(\checkmark)$ \\
\hline
\end{tabular}

Die Übersicht zeigt, dass alle in den Lernzielen formulierten Aspekte der axiomatischen Methode in mindestens einer der Unterrichtsreihen angesprochen werden. Daraus ließe sich folgern, dass eine Behandlung aller vier Unterrichtsreihen zu verschiedenen Zeitpunkten des Mathematikunterrichts ein abgerundetes Bild der Axiomatik fördert. Dieser Einschätzung soll nicht grundsätzlich widersprochen werden. Ich möchte jedoch einige Aspekte benennen, die die Entwicklung weiterer Unterrichtsmaterialien rechtfertigen:

Mit Ausnahme der Reihe zu Abstimmungsmengen sind alle Unterrichtsmaterialien für den Einsatz in der Sekundarstufe I konzipiert. Dies ist zum einen positiv zur Kenntnis zu nehmen, zeigt es doch, dass eine sinnvolle Thematisierung axiomatischer Aspekte durchaus auch mit jüngeren Lernenden gelingen kann. Allerdings sind der fachlichen und philosophischen Tiefe der Auseinandersetzung durch das Adressatenalter natürliche Grenzen gesetzt. Die Entwicklung von Konzepten für die Sekundarstufe II bietet das Potential, mit den Schülerinnen und Schülern noch stärker auf einer Meta-Ebene über Axiomatik zu sprechen und dabei zugleich ein Stück der Wissenschaftsgeschichte der Mathematik zu vermitteln. 
Die beiden umfassendsten der vier vorgestellten Unterrichtsreihen (siehe Abschnitte 4.2.1 und 4.2.2) behandeln zudem insofern keine ,realen“ Axiomensysteme, als den Schülerinnen und Schülern darin nicht vermittelt wird, in welchem mathematischen Themengebiet sie sich eigentlich bewegen. Der fachliche Hintergrund von Steiners Abstimmungsmengen ist die Spieltheorie, welche kein Thema der Schulmathematik ist und in diesem Sinne als „exotisch“ gelten muss (siehe auch Scholz 2007, S. 56). In diesem Fall dürfte es den Schülerinnen und Schülern schwer fallen, die Erkenntnisse aus der Unterrichtsreihe auf die übrigen ihnen bekannten mathematischen Inhalte zu übertragen. Ähnlich ist die Situation bei den Wüstensandsätzen: Die Axiome der Maskonen entsprechen zwar denen der Inzidenzgeometrie, allerdings wird diese mit den Schülerinnen und Schülern nicht diskutiert.

Nur die Ideen von Gerwig und Jahnke betreffen in einer den Lernenden transparenten Weise geometrische Axiomatik, beschränken sich dabei jedoch jeweils auf einen ganz bestimmten Aspekt der axiomatischen Methode. Es ist daher ein Desiderat, inhaltlich authentische Beispiele für axiomatische Betrachtungen zu entwickeln, d. h. solche Axiomensysteme zu behandeln, die im Laufe der Mathematikgeschichte tatsächlich Gegenstand der Diskussion gewesen sind, und den Lernenden diesen thematischen Bezug auch aufzuzeigen.

Ferner gibt es zur Rechtfertigungsproblematik von Axiomensystemen bisher kaum überzeugende Unterrichtsvorschläge. Jahnkes Arbeiten zur Bedeutung von Hypothesen in der Mathematik bilden hier eine Ausnahme, beschränken sich allerdings - wie oben bereits erwähnt - auf ein empiristisches Mathematikbild. Strebt man auch die Vermittlung einer formalen, ontologisch nicht mehr verankerten Auffassung von Axiomen an, sollte die Rechtfertigungsfrage auch in diesem Rahmen gestellt werden, d. h. man sollte Lernenden eine Antwort auf die Frage geben können, warum sich bestimmte abstrakte Axiomensysteme durchsetzen und andere nicht.

Abschließend sei erwähnt, dass die einzelnen Unterrichtsvorschläge zwar unterschiedliche epistemologische Auffassungen von Axiomen behandeln, jedoch kein Vergleich dieser Auffassungen bzw. keine Diskussion der Entwicklung von der euklidischen zur Hilbertschen Auffassung stattfindet. Am Beispiel der Geometrie kann man wissenschaftsgeschichtliche Aspekte zur Erläuterung dieses für die Mathematik maßgeblichen Auffassungswechsels gewinnbringend in unterrichtliche Arrangements integrieren. 



\section{Axiomatik mit Oberstufenschülern: Eigene Unterrichtsmaterialien}

Wie am Ende von Abschnitt 4.3 aufgezeigt wurde, besteht gerade im Oberstufenbereich noch Potential für die Entwicklung von Unterrichtskonzepten zur vertieften Auseinandersetzung mit axiomatischen Gesichtspunkten, vor allem im Hinblick auf eine explizite Thematisierung der unterschiedlichen epistemologischen Auffassungen sowie auf die Frage nach der Rechtfertigung von Axiomensystemen.

In diesem Kapitel werden drei von mir entwickelte Unterrichtsreihen vorgestellt, die in ihrer Gesamtheit die in Abschnitt 4.1 aufgestellten Lernziele abdecken und Schülerinnen und Schüler verstärkt zu einer Diskussion axiomatischer Denk- und Arbeitsweisen auf der Meta-Ebene anregen sollen.

In Abschnitt 5.1 werden die Grundsatzentscheidungen hinsichtlich des methodischen und inhaltlichen Formats der Unterrichtsideen erläutert. In den Abschnitten 5.2 bis 5.4 werden die drei Unterrichtsreihen behandelt, und zwar jeweils in drei Unterabschnitten: Eine Sachanalyse enthält in komprimierter Form den fachlichen Hintergrund des ausgewählten Themas; anschließend folgen allgemeine didaktische Anmerkungen in Bezug auf die Unterrichtsreihe (wesentliche Entscheidungen und didaktische Reduktionen), um schließlich die eigentliche Unterrichtsreihe zu präsentieren.

Die Beschreibung der Unterrichtsreihen ist dabei stets gleich strukturiert. Die folgenden Anmerkungen sollen diese Strukturierung transparent machen und den Leserinnen und Lesern zugleich eine Hilfestellung bieten, wie sie bei der Lektüre am besten vorgehen:

- Jede Unterrichtsreihe ist in mehrere Phasen unterteilt, zu denen jeweils bestimmte Arbeitsblätter und historische Textauszüge gehören, die in den Anhängen A.1 (Arbeitsblätter) und A.2 (Texte) zu finden sind. Der Verweis auf diese erfolgt mit Hilfe von Kürzeln, welche immer in der oberen rechten Ecke der Materialien zu finden sind. $\mathrm{Zu}$ den Arbeitsblättern existieren außerdem Musterlösungen (siehe Anhang A.4). 
- Zu Beginn jeder Beschreibung einer Unterrichtsreihe findet sich eine tabellarische Übersicht über die zugehörigen Phasen inkl. der in diesen Phasen verwendeten Materialien. Zudem werden in den Übersichtstabellen zu jeder Phase die wesentlichen Erkenntnisse mit Blick auf axiomatische Gesichtspunkte stichpunktartig festgehalten.

- Im Fließtext zu den Reihenbeschreibungen sind Zusammenfassungen und wesentliche Erkenntnisse in grauen Kästen hervorgehoben. Zusammengenommen fungieren sie als eine Art Wissensspeicher und bieten Lehrkräften beim Einsatz der Materialien eine Orientierung für mögliche ergebnissichernde Tafelanschriebe.

- Im Hinblick auf die Lektüre der Reihenbeschreibungen möchte ich folgende Leseempfehlung aussprechen: Pro Unterrichtsphase bietet es sich an, zunächst die im Anhang befindlichen, zugehörigen Arbeitsblätter und Textauszüge zu lesen und im Anschluss daran den entsprechenden Beschreibungstext. Bei Interesse - insbesondere, wenn man die Materialien konkret im Unterricht verwenden möchte - können zudem die ebenfalls im Anhang befindlichen Musterlösungen zu Rate gezogen werden, die jedoch für das grundlegende Nachvollziehen der Unterrichtsideen nicht notwendig sind.

\subsection{Didaktische Grundsatzentscheidungen}

\subsubsection{Themenauswahl}

Für die Entwicklung meiner Unterrichtsmaterialien habe ich drei Themengebiete (Geometrie, Wahrscheinlichkeitsrechnung, Zahlbereichserweiterungen) ausgewählt, deren Zusammenhang zu den in Abschnitt 4.1 aufgestellten Lernzielen im Folgenden erläutert wird. Die Materialien aus dem Bereich der Geometrie sind an der historischen Entwicklung axiomatischer Fragestellungen orientiert - mit den drei auch in Abschnitt 2.1 behandelten Stationen: Euklids Elemente, nichteuklidische Geometrie und Hilberts Grundlagen der Geometrie - und decken relativ umfassend die in den Lernzielen genannten Aspekte ab, wobei nicht jeder Aspekt tiefergehend behandelt wird. Die beiden anderen Unterrichtsreihen dienen der exemplarischen Vertiefung bestimmter Aspekte. Insgesamt wird die Abdeckung der Lernziele durch meine Unterrichtsreihen durch folgende Tabelle beschrieben. 


\begin{tabular}{|l||c|c|c|c|c|c|}
\hline Unterrichtsreihe & LZ 1 & LZ 2 & LZ3 & LZ 4 & LZ 5 & LZ 6 \\
\hline \hline Geometrie & $\checkmark$ & $\checkmark$ & $\checkmark$ & $\checkmark$ & $(\checkmark)$ & $(\checkmark)$ \\
\hline Wahrscheinlichkeitsrechnung & $(\checkmark)$ & & $\checkmark$ & $\checkmark$ & $\checkmark$ & \\
\hline Zahlbereichserweiterungen & & & $(\checkmark)$ & & & $\checkmark$ \\
\hline
\end{tabular}

\section{Geometrie}

Die ebene Geometrie ist und bleibt das Gebiet der Mathematik, in dem Schülerinnen und Schüler am häufigsten mit Beweisen konfrontiert werden (Innenwinkelsatz, Satz des Thales, Satzgruppe des Pythagoras u. a.). Die Sätze der Schulgeometrie bieten sich zum lokalen Ordnen mathematischer Theoreme an (LZ4; siehe auch Griesel 1963) und liefern damit eine Ausgangsbasis für die Beschäftigung mit der klassisch-inhaltlichen Sicht auf Axiomatik. Macht man die Annahmen deutlich, auf die sich ein geometrischer Beweis stützt (LZ 1), erkennen Lernende, dass man früher oder später bei Aussagen angelangt, die als nicht weiter beweisbedürftig erscheinen (LZ2).

Ein weiterführender Blick auf die nichteuklidische Geometrie (insbesondere ein Vergleich geometrischer Sachverhalte der ebenen und sphärischen Geometrie) fördert die Erkenntnis, dass Axiome nicht zwangsläufig aufgrund ihrer anschaulichen oder intuitiven Evidenz zu rechtfertigen sind, sondern stattdessen aufgrund der Nützlichkeit der sich aus ihnen ergebenden Theorie (beispielsweise im Sinne einer Passung mit empirischen Messungen; LZ 6).

Die Abkehr von der evidenzbasierten Sichtweise auf Axiome findet ihren Höhepunkt in Hilberts Grundlagen der Geometrie. Eine exemplarische Auseinandersetzung mit den Axiomen der Inzidenzgeometrie (inkl. erster kleiner Deduktionen; LZ 5) und verschiedenen inhaltlichen Belegungen der darin implizit definierten Grundbegriffe verdeutlicht die formale Sicht auf mathematische Theorien (LZ3). Ausschnitte aus dem Briefwechsel zwischen Hilbert und Frege zeigen zudem die epistemologischen Unterschiede zwischen dem klassischen (LZ 2) und dem modernen (LZ3) Standpunkt auf.

\section{Wahrscheinlichkeitsrechnung}

In der Wahrscheinlichkeitsrechnung können anhand der den Schülerinnen und Schülern bekannten Zugänge (Laplace- und frequentistischer Ansatz) gut die Grenzen inhaltlicher Begriffsbestimmungen aufgezeigt und mit der implizit-axiomatischen Begriffsdefinition verglichen werden (LZ3). Mit den Kolmogorov-Axiomen liegt zudem ein überschaubares und leicht verständliches Axiomensystem vor, ,,das zu dem Einfachsten gehört, das es in 
der Mathematik gibt“ (Freudenthal 1973b, S. 528), und das sich für lokales Ordnen und deduktive Übungen anbietet (LZ4 und 5).

\section{Zahlbereichserweiterungen}

Der Übergang von den reellen zu den komplexen Zahlen lässt Lernende erfahren, wie man auch mit Begriffen bzw. Aussagen, zu denen es anfangs keine anschauliche Repräsentation gibt, mathematisch operieren kann. Das Arbeiten mit ,Zahlen“ der Form $a+b \cdot i$ fand historisch gesehen zunächst auf einer rein syntaktisch-symbolischen Ebene statt, um Lösungsformeln für kubische und biquadratische Gleichungen zu finden. Man setzte zunächst voraus, dass es eine Zahl $i$ mit der Eigenschaft $i^{2}=-1$ gebe und dass man mit ihr und wie oben zusammengesetzten Zahlen wie gewohnt rechnen könne. Auch wenn nicht zuletzt die Visualisierung in der Zahlenebene durch Gauß und die Definition der komplexen Zahlen als Tupel des $\mathbb{R}^{2}$ zu deren Akzeptanz in der mathematischen Gemeinschaft geführt haben, zeigt dieses Beispiel, wie ertragreiche inner- und außermathematische Konsequenzen einer rein formalen Setzung Mathematiker zur weiteren Beschäftigung mit einem neuen Themengebiet anregen bzw. diese sogar erst ermöglichen können (LZ 6). Als kontrastierendes Beispiel für eine (zumindest aus Sicht der Schulmathematik) wenig ertragreiche Setzung dient die „Definition“ einer sogenannten bekloppten Zahl b mit der Eigenschaft $\frac{1}{0}=b$ bzw. $0 \cdot b=1$.

\subsubsection{Methodische und inhaltliche Leitideen}

Being aware of the role of assumptions, axioms and hypotheses is a key issue for understanding what a proof is. When students lack an awareness of the axiomatic basis of mathematical proof they will not be able to develop a habit of consciously and permanently asking for the assumptions underlying a valid reasoning. Thus, [...] at some points in the course of secondary education there should be classroom-based interventions addressing this difficulty and making the axiomatic organization of mathematics a theme. Students should be made aware that there are axioms in mathematics, what their role is and how mathematicians come to agree about which axioms should be accepted.

Mit dieser Aussage plädieren Jahnke und Wambach (2013, S. 469) ausdrücklich für eine Unterrichtskultur, in der die deduktive Ordnung mathematischer Theorien den Lernenden stärker bewusst gemacht wird. In der Tat ist es erstrebenswert, einige Aspekte der Axiomatik in den alltäglichen Mathematikunterricht zu integrieren. Dazu gehören aus meiner Sicht insbesondere ein Bewusstsein für den hypothetisch-deduktiven Charakter 
mathematischer Beweise und die damit verbundene Praxis, Axiome als Ausgangspunkte von Theoriegebäuden zu verwenden. Eine umfassende Behandlung, wie sie durch die Gesamtheit der Lernziele in Abschnitt 4.1 dargestellt wird, erscheint für den Regelunterricht allerdings unrealistisch.

Die hier vorgestellten Unterrichtskonzepte und -materialien sind - soweit mehr als einzelne Elemente gewählt werden - für den Einsatz jenseits des Regelunterrichts in der Oberstufe gedacht (etwa Workshops, AGs, Projektkurse und -wochen, etc.). Gleichwohl seien interessierte Lehrkräfte ausdrücklich dazu ermutigt, einzelne Bestandteile in den regulären Mathematikunterricht zu integrieren. Die notwendigen inhaltlichen Vorkenntnisse sind bewusst niedrig gehalten, so dass ein Einsatz in der Sekundarstufe II problemlos möglich sein sollte: Im Wesentlichen werden Kenntnisse der typischen Winkelsätze der ebenen Geometrie, Erfahrungen mit klassischen Zufallsexperimenten und der mengentheoretischen Modellierung stochastischer Aussagen sowie algebraische Grundfertigkeiten zum Lösen quadratischer und kubischer Gleichungen vorausgesetzt; stellenweise werden diese Aspekte im Rahmen der Unterrichtsreihen kurz wiederholt.

In den Beschreibungen der Unterrichtsreihen werden bewusst keine konkreten Angaben zum benötigten zeitlichen Umfang bei der Umsetzung gemacht. Eine mögliche zeitliche Aufteilung im Rahmen einer Workshop-Woche wird in Abschnitt 5.5 vorgestellt.

Bei der Entwicklung der Lernmaterialien habe ich mich an den folgenden Leitideen orientiert:

\section{Betrachtung inhaltlich authentischer Beispiele für Axiomatik}

Mit der Geometrie und der Wahrscheinlichkeitsrechnung stehen zwei Themen im Fokus meiner Materialien, die mathematikhistorisch eine wichtige Rolle gespielt haben und authentische Beispiele für axiomatisches Denken und Arbeiten darstellen. Gerade in der Oberstufe sollten die Schülerinnen und Schüler meines Erachtens mit Axiomensystemen konfrontiert werden, die sowohl Anknüpfungspunkte an die aus dem Regelunterricht bekannten Inhalte bieten als auch in ähnlicher Form in der Mathematik aufgetreten sind bzw. noch auftreten. Die Inhalte werden dabei didaktisch reduziert, jedoch nicht derart in ,Sachkontexte“ eingebettet, dass ihr mathematischer Ursprung für die Lernenden nicht mehr sichtbar ist.

Das Themengebiet Zahlbereichserweiterungen teilt die Eigenschaft der Authentizität mit den anderen beiden Themen, wenngleich der Bezug zur Axiomatik auf den ersten Blick nicht ersichtlich ist. Ich gehe darauf in Abschnitt 5.4.2 genauer ein. 


\section{Anknüpfung an das Schüler-Vorverständnis}

Dass man zu Beginn einer neuen Unterrichtsreihe auf die Vorerfahrungen und das Vorwissen der Lernenden zurückgreift, ist eine didaktische Selbstverständlichkeit. Im Hinblick auf axiomatische Aspekte ist allerdings damit zu rechnen, dass kaum solche Anknüpfungspunkte bestehen. Um den Schülerinnen und Schülern den Einstieg in das neue Thema dennoch zu erleichtern, beginnen die von mir entwickelten Unterrichtsreihen jeweils mit einem mathematischen Inhalt, der den Lernenden in der Sekundarstufe II bekannt sein sollte und anhand dessen erste axiomatische Fragestellungen entwickelt werden können. Im Falle der Geometrie sind dies die Winkelsätze, im Falle der Wahrscheinlichkeitsrechnung typische Zufallsexperimente zum Laplace'schen und frequentistischen Wahrscheinlichkeitsbegriff und im Falle der Zahlbereichserweiterungen das Lösen quadratischer Gleichungen mit Hilfe der $p$ - $q$-Formel.

\section{Diskussions- und Reflexionsphasen}

Wie bereits am Ende von Abschnitt 4.3 erwähnt, sollen sich meine Unterrichtsmaterialien unter anderem durch das verstärkte Einnehmen eines meta-mathematischen Standpunktes auszeichnen. Das bewusste Reflektieren über axiomatische Themen wirkt sich dabei auch auf die methodische Konzeption der Unterrichtsmaterialien aus:

Ursprünglich war angestrebt, diese in Form eines Schüler-Arbeitsheftes nach dem Vorbild des Aachener Schul-Hochschul-Projekts iMPACt (siehe Heitzer 2015) aufzubereiten, welches sich prinzipiell auch zum selbstständigen Durcharbeiten eignen sollte. Ich habe von dieser Idee jedoch schlussendlich wieder Abstand genommen, da sich gezeigt hat, dass dieses Format nur schwer mit dem angestrebten, erhöhten Ausmaß an diskursivem Denken vereinbar ist. Stattdessen wurden vor allem Arbeitsblätter entwickelt und historische Textauszüge verwendet (siehe auch nächster Punkt), die Anlässe zu vertieften Diskussions- und Reflexionsphasen über Axiomatik bieten sollen. Nicht alle angestrebten Ziele lassen sich dabei allein aus eigenen Aktivitäten der Lernenden motivieren, weshalb die Schülerinnen und Schüler anstelle eines konsequent genetischen Zugangs an einigen Stellen eher ,instruktiv“ mit bestimmten Sichtweisen und Problemstellungen konfrontiert werden und darüber diskutieren sollen. 


\section{Integration historischer Originalquellen}

Reading a source can be the trigger for establishing a dialogue with the ideas expressed. The source then becomes an interlocutor to be interpreted, to be questioned, to be answered and to be argued with. This applies especially to sources which discuss meta-mathematical issues such as the nature of the mathematical objects we handle, and the essence of mathematical activity. (Jahnke 2000, S. 296)

Die Sicht auf Axiome in der Mathematik hat sich im Laufe der Geschichte stark gewandelt. Dies wird nicht nur durch direkte Vergleiche axiomatischer Theoriedarstellungen etwa von Euklids Elementen und Hilberts Grundlagen der Geometrie - deutlich, sondern auch durch ein Studium meta-mathematischer Texte. Der Briefwechsel zwischen Frege und Hilbert über den Wahrheitsstatus geometrischer Axiome sei hier als ein eindrucksvolles Beispiel genannt (siehe auch Abschnitt 2.1.3). Die Verwendung historischer Textauszüge ist daher ein wesentlicher Bestandteil der Unterrichtsvorschläge, die sich hierdurch von den im vorigen Abschnitt präsentierten Konzepten unterscheiden. Die Herausforderung besteht dabei darin, solche Textpassagen auszuwählen, die als gehaltvolle Diskussions- und Reflexionsgrundlagen der zugehörigen axiomatischen Aspekte dienen können, die Lernenden jedoch in sprachlicher und inhaltlicher Hinsicht nicht überfordern.

\subsection{Unterrichtsreihe 1: Geometrie}

\subsubsection{Sachanalyse}

Die für die Unterrichtsreihe relevanten, fachlich-historischen Hintergründe sind in Abschnitt 2.1 dieser Arbeit bereits umfassend dargelegt worden. Es sollen an dieser Stelle daher lediglich die wesentlichen Aspekte in komprimierter Form wiederholt werden:

In Euklids Elementen wird mathematisches Wissen erstmals in einer axiomatisch-deduktiven Form präsentiert: Ausgehend von Definitionen, Axiomen und Postulaten wird das bis dato bestehende geometrische Wissen streng deduktiv hergeleitet und somit systematisch geordnet. Die Axiome und Postulate werden dabei (insbesondere in der Zeit nach Euklid) als absolut gültige Grundwahrheiten angesehen, die eines Beweises weder bedürfen noch überhaupt beweisbar sind, da sie dem menschlichen Geist als selbstevident einleuchten.

Versuche, die Unabhängigkeit des Parallelenpostulats von den übrigen Postulaten bzw. Axiomen zu beweisen, führten zu Beginn des 19. Jahrhunderts zur Entdeckung der nicht- 
euklidischen Geometrie, in welcher alle euklidischen Postulate/Axiome gelten bis auf das Parallelenpostulat, ohne dass sich jedoch ein logischer Widerspruch erzeugen lässt. Dies öffnete den Blick der Mathematiker für die Möglichkeit, dass der Geltungsanspruch der euklidischen Geometrie kein absoluter ist und die „Passung“ geometrischer Axiome mit der Realität keine Frage der reinen Anschauung (im Sinne Kants), sondern vielmehr eine (durch empirische Messungen gewonnene) Erfahrungstatsache ist. Diese Erkenntnis leitete die zunehmende theoretische Eigenständigkeit verschiedener (d. h. jeweils auf anderen Axiomen aufbauender) Geometrien ein, die sich damit zunehmend vom Anspruch der Realitätsbeschreibung lösten.

Seit Hilberts Grundlagen der Geometrie schließlich werden mathematische Theorien als formale Denksysteme aufgefasst, deren Grundbegriffe durch die zu Beginn aufgestellten Axiome implizit definiert werden, wodurch sich die Frage nach einer Wahrheit mathematischer Aussagen nicht mehr stellt.

\subsubsection{Didaktische Anmerkungen}

Grundlegende Idee bei der Konzeption dieser Unterrichtsreihe ist es, den Lernenden einen Überblick über die Entwicklung der Axiomatik in der Geometrie zu geben und sich dazu an den wesentlichen historischen Etappen (Euklids Elemente, Entdeckung nichteuklidischer Geometrie, Hilberts Grundlagen der Geometrie) zu orientieren. Daher hat die Einbindung historischer Quellen in dieser Reihe einen besonders hohen Stellenwert:

- Euklids Elemente: Die beispielhafte Auseinandersetzung mit Beweisen aus dem ersten Buch der Elemente gibt einen Einblick in den deduktiven Charakter der Mathematik und in die Praxis, bereits bewiesene Aussagen zur Begründung einer Behauptung heranzuziehen. Auch ausgewählte Definitionen sowie die Axiome und Postulate können anhand des „Originaltextes“ (d. h. anhand der deutschen Übersetzung) diskutiert werden.

- Hermann von Helmholtz' Über den Ursprung und die Bedeutung der geometrischen Axiome: Das bereits in Abschnitt 2.1.2 vorgestellte Gedankenexperiment über hypothetische zweidimensionale Wesen, die auf einer Kugeloberfläche leben, ist gut nachvollziehbar und vermittelt auf anschauliche Weise die sich wandelnde Auffassung von Axiomen hin zu einem empiristischen Standpunkt.

- Jean-Pierre Petits Geometrikon: Dieser mathematische Lern-Comic (siehe Anhang A.3) ist zwar kein historischer Text, passt jedoch sehr gut zur Diskussion über die Natur geometrischer Axiome: Der Protagonist des Comics nimmt geometri- 
sche Vermessungen vor ohne zu bemerken, dass er sich auf einer Kugel befindet. Die mit der Abkehr von evidenzbasierten Argumentationen verbundenen kognitiven Konflikte werden so in einem altersgerechten Format dargestellt.

- Henri Poincarés Wissenschaft und Hypothese: In diesem Textausschnitt wird für die theoretische Eigenständigkeit der verschiedenen denkbaren Geometrien argumentiert. Ganz explizit formuliert Poincaré hier die Auffassung, dass die Frage nach der Wahrheit geometrischer Axiome nicht zielführend sei. Vielmehr könnten unterschiedliche Geometrien mehr oder weniger bequem bzw. nützlich sein. Die in diesem Auszug geäußerten Gedanken eignen sich daher gut als Überleitung von realitätsgebundenen Auffassungen zu Hilberts abstrakter Sichtweise.

- Briefwechsel zwischen Frege und Hilbert: Zur kontrastierenden Diskussion der klassischen und modernen Sichtweise auf den Wahrheitsgehalt von Axiomen werden verschiedene Aussagen Gottlob Freges und David Hilberts verwendet, in denen die wesentlichen Unterschiede besonders pointiert dargelegt sind.

Der Fokus auf die historische Genese axiomatischer Aspekte bedingt, dass im Rahmen der Unterrichtsreihe nicht extensiv Geometrie betrieben werden kann - egal ob euklidisch oder nichteuklidisch. Insbesondere in Bezug auf die Behandlung nichteuklidischer Geometrie müssen daher zwei wesentliche Entscheidungen getroffen werden:

Zum einen ist es vom Umfang her nicht möglich, verschiedene Arten nichteuklidischer Geometrie ausführlich vorzustellen und zu behandeln. Ich habe mich für die sphärische Geometrie als Vertreter eines Modells der nichteuklidischen Geometrie entschieden, auch wenn dies fachlich nicht ganz sauber ist. Neben dem Parallelenpostulat sind auf der Sphäre nämlich noch weitere euklidische Axiome nicht erfüllt. ${ }^{1}$ Die Geometrie auf der Kugeloberfläche ist jedoch deutlich anschaulicher als die - in Anbetracht der Arbeiten rund um Gauß, Bolyai und Lobatschewski historisch gesehen eigentlich im Mittelpunkt stehende - hyperbolische Geometrie, deren zweidimensionale Modelle für die Zwecke dieser Unterrichtsreihe nicht praktikabel gewesen wären.

Zum anderen muss entschieden werden, wie man sich - über die bereits erwähnten historischen Texte hinaus - der sphärischen Geometrie nähern kann. Filler (1993) beschreibt fünf Möglichkeiten, diese aufzubauen. Neben zwei Varianten über Vektorgeometrie sowie einem differentialgeometrischen Ansatz, welche für diese Arbeit uninteressant sind, stehen zwei Zugänge zur Auswahl:

\footnotetext{
${ }^{1}$ Beispielsweise schneiden zwei sphärische Geraden (Großkreise) sich stets in zwei diametralen Punkten. Die Identifikation solcher Punktepaare als ein „sphärischer Punkt“ ergibt als Verallgemeinerung die elliptische Geometrie; allerdings erscheint dies im Hinblick auf die Behandlung mit Schülerinnen und Schülern als eine unnötige Komplikation, die wenig zum Verständnis der Axiomatik beiträgt.
} 
Bei der elementargeometrisch-euklidischen Behandlung werden die Eigenschaften und Sätze der sphärischen Geometrie sowie die Grundformeln der sphärischen Trigonometrie unter Zuhilfenahme der Zusammenhänge zwischen den geometrischen Objekten der Sphäre und denen des die Sphäre einbettenden euklidischen Raumes sowie der trigonometrischen Beziehungen in ebenen Dreiecken hergeleitet. Euklidische Behandlung heißt also nicht, daß darauf verzichtet wird, die sphärische Geometrie als eigenständige, nichteuklidische Geometrie aufzubauen, bedeutet aber, daß für den Aufbau die euklidische Geometrie genutzt wird. [...]

Bei einer nichteuklidischen synthetischen Behandlung wird auf die Verwendung der euklidischen Geometrie und der ebenen Trigonometrie verzichtet. Statt dessen wird die sphärische Geometrie synthetisch-axiomatisch aufgebaut. [...] Verkürzt ausgedrückt werden bei diesem Vorgehen Grundeigenschaften der sphärischen Geometrie [...] unbewiesen an den Anfang gestellt und auf Grundlage dieser Axiome die weiteren Eigenschaften der sphärischen Geometrie sowie die trigonometrischen Formeln hergeleitet. (Filler 1993, S. 45-46; Herv. im Orig.)

Ein rein axiomatischer Aufbau der sphärischen Geometrie im schulischen Kontext ist ebenso problematisch und ungeeignet wie ein rein axiomatischer Aufbau der euklidischen Geometrie. Er ist langwierig, überwiegend technisch und somit für die Lernenden wenig motivierend. Wenngleich die Einsicht in die Eigenständigkeit und Gleichwertigkeit alternativer Geometrien historisch gesehen aus dem (gescheiterten) Bestreben erwachsen ist, ohne Rückgriff auf die Anschauung und rein logisch aus der Verneinung des Parallelenpostulats einen Widerspruch herzuleiten, ist eine intensive Beschäftigung mit solchen Deduktionen mit Schülerinnen und Schülern ebenfalls nicht sinnvoll. Ich behandle daher die sphärische Geometrie von Anfang an unter Zuhilfename der euklidischen Geometrie. Das heißt allerdings nicht, dass diese in der Unterrichtsreihe komplett aufgebaut würde. Konkrete Resultate der euklidischen Geometrie spielen lediglich bei einem Arbeitsblatt zur Herleitung der Innenwinkelsumme für sphärische Dreiecke (GEOM3) eine Rolle. Darüber hinaus wird - auch unter Zuhilfenahme von Styroporkugeln - hauptsächlich mit der Modellvorstellung als Geometrie auf der Kugeloberfläche gearbeitet, um den Schülerinnen und Schülern einen enaktiv-visuellen Ankerpunkt zu bieten und somit möglichst zeiteffektiv eine grundlegende Vertrautheit mit dieser neuen Art von Geometrie zu schaffen. Darauf aufbauend können anhand der historischen Texte axiomatische Gesichtspunkte thematisiert werden.

Ich habe ferner bei der Ausgestaltung der Unterrichtsreihe auf einige bereits existierende Ideen zurückgegriffen, die sich aus meiner Sicht besonders gut in meine eigenen Konzepte integrieren lassen: 
- Die Beschäftigung mit der euklidischen Axiomatik erfolgt über das lokale Ordnen der aus dem Schulunterricht bekannten Winkelsätze. Die Idee, den deduktiven Charakter mathematischer Beweise anhand der Winkelsätze zu verdeutlichen, ist nicht neu und wurde beispielsweise von Griesel (1963) vorgeschlagen. Auch in dem Schulbuch von Kuypers, Lauter und Wuttke (1993, S. 116-131) dienen die Winkelsätze als Grundlage für eine Einführung in das mathematische Beweisen. Die graphische Darstellung der logischen Zusammenhänge in Pfeildiagrammen habe ich ebenfalls diesem Werk entnommen.

- Das Arbeitsblatt zur Innenwinkelsumme sphärischer Dreiecke (GEOM3) beruht wesentlich auf den Ideen in Filler 1993 (S. 13-19) und Filler 2010.

- In das Arbeitsblatt Geom4, welches sich mit der impliziten Definition geometrischer Grundbegriffe beschäftigt, habe ich Ideen aus der Wüstensand-Unterrichtsreihe einfließen lassen (siehe Abschnitt 4.2.2). 


\subsubsection{Beschreibung der Unterrichtsreihe}

Phase Thema (inkl. wesentlicher Tätigkeiten/Erkenntnisse) ABs / Texte

Einl. Die Sicherheit mathematischer Aussagen EINF1

- Richtigkeit mathematischer Aussagen einschätzen

- Möglichkeiten zur Begründung mathematischer

Aussagen sammeln und hinterfragen

1 Beweisen und Ordnen geometrischer Sätze

- Winkelsätze lokal ordnen

- deduktive Struktur der Geometrie erkennen und veranschaulichen

- grundlegende Begriffe zum Thema Beweisen kennen
GEOM1, Die Elemente

(Auszug)

\section{$2 \quad$ Sphärische Geometrie}

- Eigenschaften der sphärischen Geometrie erkunden und mit der euklidischen vergleichen

- Auffassung von geometrischen Axiomen als Erfahrungstatsachen (und damit die Abkehr von der evidenzbasierten Rechtfertigung ihrer Gültigkeit) nachvollziehen

- (theoretische) Gleichwertigkeit verschiedener Geometrien erkennen
GEOM2, GEOM3,

Hist 1, Hist2,

Geometrikon (Auszug)
$3 \quad$ Von Dimpfeln und Gronkeln

- Ersetzbarkeit der geometrischen Grundbegriffe durch Kunstworte erkennen und damit das Konzept der impliziten Definition nachvollziehen

- überschaubare Deduktionen aus abstrakt formulierten Axiomen durchführen

- euklidische und formalistische Sichtweise abschließend vergleichend diskutieren

\section{GEOM4, Hist3,}

HIST4

\section{Einleitung: Die Sicherheit mathematischer Aussagen (EINF1)}

Das Arbeitsblatt EINF1 dient als generelle Einführung in die Thematik und soll die Schülerinnen und Schüler für die Tatsache sensibilisieren, dass mathematische Aussagen den Ruf genießen, besonders sicher zu sein. Dazu werden sie zunächst mit sieben verschiede- 
nen Aussagen konfrontiert, deren Wahrheitsgehalt sie einschätzen sollen. Bei den meisten Aussagen sollte diese Einschätzung den Lernenden leicht fallen. Ab Aussage (5) geht es um Aussagen, bei denen sie sich eventuell nicht sicher sind bzw. erfahren können, dass sie sich geirrt haben. Gegebenenfalls vorhandenes Einzelvorwissen kann hier aufgegriffen und ansonsten der unten genannte Stand der Wissenschaft zur jeweiligen Aussage mitgeteilt werden.

- Aussage (1) ist „offensichtlich“"wahr.

- Die Aussagen (2) und (3) sind aus dem Unterricht bekannte, wahre Aussagen (1. binomische Formel bzw. Innenwinkelsatz).

- Aussage (4) ist falsch; als Gegenbeispiel kann ein gleichseitiges Dreieck genannt werden. Wird die Voraussetzung ergänzt, dass das Dreieck rechtwinklig ist, ist die Aussage wahr (Satz des Pythagoras).

- Bei Aussage (5) liegt eine Antwort zunächst nicht auf der Hand, sukzessives Einsetzen von Primzahlen liefert allerdings das Gegenbeispiel $2^{11}-1=2047=23 \cdot 89$.

- Aussage (6) ist der berühmte Große Satz von Fermat, welcher in den 1990er Jahren von Andrew Wiles und Richard Taylor bewiesen wurde. Es gibt allerdings nur eine Handvoll Mathematiker weltweit, die diesen Beweis komplett nachvollzogen haben.

- Bei Aussage (7) bietet sich ein induktives Vorgehen wie bei Aussage (5) an, allerdings wird man hier kein Gegenbeispiel finden können. Es handelt sich bei dieser Aussage um die Goldbach-Vermutung, ein bisher ungelöstes Problem der Mathematik. Die Gültigkeit der Aussage wurde mit Computer-Unterstützung bisher für alle geraden Zahlen bis $4 \cdot 10^{18}$ verifiziert (Stand: April 2012).

Im Anschluss an die Beschäftigung mit diesen sieben Aussagen werden mit den Lernenden Möglichkeiten gesammelt, wie man in der Mathematik den Wahrheitsgehalt einer Aussage überprüfen kann. Denkbare Antworten (unter Rückgriff auf die ggf. in Aufgabe 1 verwendeten Strategien) sind: als evident erkennen, Beweisen, Messen, an (vielen) Beispielen überprüfen, auf eine Autorität berufen (Beispiel: Großer Satz von Fermat), etc.

Das abschließende Zitat von Penelope Maddy in Aufgabe 3 dient dazu, diese Möglichkeiten kritisch zu reflektieren, empirische Vorgehensweisen wie das Überprüfen an vielen Beispielen zwar als erkenntnisfördernd, jedoch nicht absolute Sicherheit garantierend zu erkennen, und den Beweis als das Mittel zu identifizieren, mit dem Aussagen in der Mathematik „ein für allemal“ gesichert werden. 
Mit diesem Einsteig sind die Schülerinnen und Schüler auf die Grundproblematik eingestimmt. Daraufhin wird ihnen mitgeteilt, dass sie sich nun tiefergehend mit Beweisen in der Geometrie und dem daraus resultierenden Wahrheitsanspruch mathematischer Aussagen auseinandersetzen werden.

\section{Phase 1: Beweisen und Ordnen geometrischer Sätze (GEOM1)}

Das Arbeitsblatt GeOm1 beginnt mit einer geschlossenen Aufgabe zum Beweis des Satzes von Thales, bei der die Lernenden lediglich Lücken ausfüllen müssen. Dies dient dazu, das vorhandene Wissen zum schon länger zurückliegenden Thema „Beweisen in der Geometrie“ zu reaktivieren und Vertrautheit mit den geometrischen Inhalten herzustellen. Im Anschluss daran (Aufgabe 2) werden wesentliche Sätze der ebenen euklidischen Geometrie wiederholt und bewiesen. Hierzu werden die auf den Seiten 3 bis 5 des Arbeitsblattes abgedruckten Karten ausgeschnitten, um die schriftlich formulierten Aussagen den entsprechenden Skizzen zuzuordnen und anschließend Beweisideen zu den jeweiligen Sätzen zu sammeln. Je nach Zeitumfang und Vorkenntnisstand der Schülerinnen und Schüler kann auf die Behandlung einiger Sätze verzichtet werden (z. B. Außenwinkelsatz, Winkel im gleichseitigen Dreieck oder Innenwinkelsatz für Vierecke). Bei den Beweisen wird jeweils klar herausgestellt, welche anderen geometrischen Sätze in ihnen verwendet werden. Hierbei wird ebenfalls deutlich, dass sich für einige Sätze (auf Anhieb) keine Beweise finden lassen bzw. dass sie „offensichtlich wahr“ sind.

Aufgabe 3 ist das Kernstück dieser Unterrichtsphase. Die in den vorherigen beiden Aufgaben entdeckten logischen Zusammenhänge zwischen den verschiedenen Sätzen sollen nun graphisch in einem Pfeilschema dargestellt werden, indem jeweils Pfeile von denjenigen Sätzen auf einen zu beweisenden Satz zeigen, die für den Beweis direkt benötigt werden. Zur Bearbeitung dieser Aufgabe können die ausgeschnittenen Skizzen-Karten auf einem großen Blatt Papier (DIN A3 oder größer) verteilt und flexibel verschoben werden, bis ein geeignetes Pfeilschema gefunden worden ist. Anhand des aufgestellten Schaubildes wird die Notwendigkeit der Setzung von Axiomen nochmals graphisch fassbar. Natürlich handelt es sich bei den hier als Axiom zu bezeichnenden Ausgangssätzen (Nebenwinkelsatz, Parallelensatz, Kongruenzsatz SWS, Stufenwinkelsatz) mit Ausnahme des Parallelensatzes nicht um Axiome im Sinne Euklids, der diese Sätze bekanntlich auf noch grundlegendere Axiome zurückführen konnte. Im Sinne des lokalen Ordnens ist es hier jedoch didaktisch gerechtfertigt, diese Sätze als Axiome zu bezeichnen, da sie den Schülerinnen und Schülern in der Regel als evident und damit nicht beweisbedürftig erscheinen. 
Im Anschluss werden die wichtigsten Begriffe zum Thema „Beweisen“ eingeführt und die deduktive Struktur der Geometrie anhand einer Skizze visualisiert:

\section{Beweisen in der Mathematik - wichtige Begriffe}

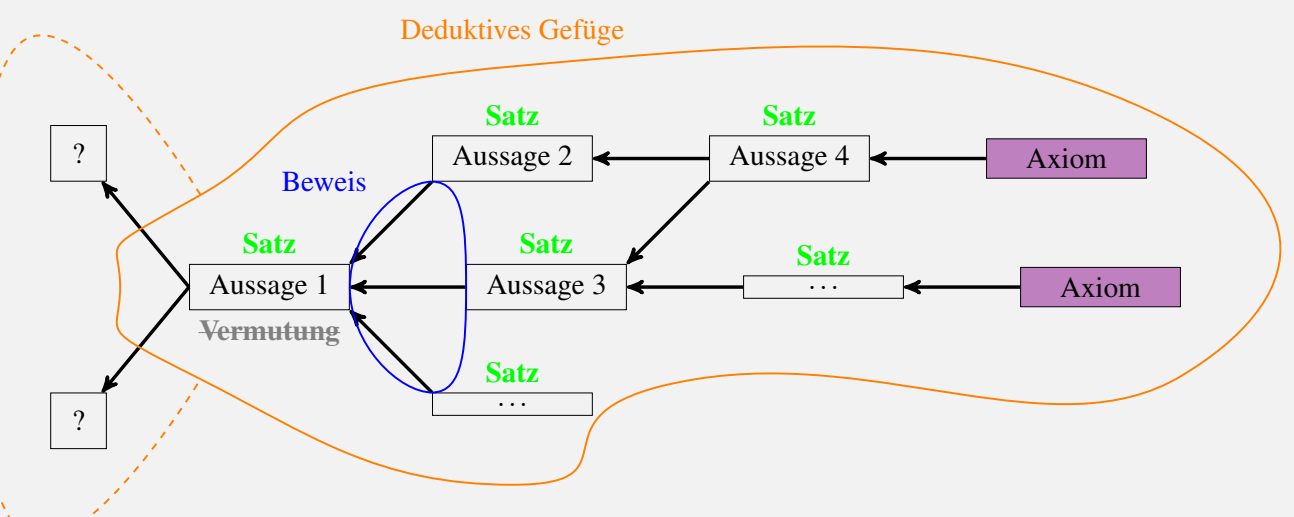

- Ein Beweis führt die Wahrheit einer Aussage auf die Wahrheit anderer Aussagen zurück, die entweder selbst schon bewiesen oder beweislos akzeptiert sind. Durch Beweise werden Wenn-dann-Beziehungen hergestellt: Wenn ... gilt, dann gilt auch ...!

- Eine Vermutung ist eine unbewiesene Aussage, von der man jedoch erwartet, dass sie wahr ist.

- Ein Satz ist eine bewiesene Aussage.

- Als Axiom bezeichnet man eine Aussage, die Mathematiker ohne Beweis akzeptieren und an den Anfang eines deduktiven Gefüges (s. u.) setzen.

- Die Gesamtheit aller bewiesenen oder ohne Beweis akzeptierten Aussagen eines Themengebiets (z. B. der Geometrie) nennt man deduktives Gefüge.

Der Begriff Wahrheit wird hier zunächst noch relativ unreflektiert bzw. in einem den Schülerinnen und Schülern vertrauten Sinne verwendet. Zum jetzigen Zeitpunkt der Unterrichtsreihe geht es noch nicht um die kritische Hinterfragung des Wahrheitsanspruchs mathematischer Aussagen.

Die Aufgaben 4 bis 6 vertiefen die Beschäftigung mit der deduktiven Ordnung der Geometrie, indem sie herausstellen, welche Auswirkungen der Verzicht auf bestimmte Aussagen sowie alternative Beweise auf das in Aufgabe 3 erstellte Schaubild haben. 
Im zweiten Teil dieser Phase setzen sich die Schülerinnen und Schüler mit Auszügen aus Euklids erstem Buch der Elemente auseinander. Dabei lesen sie zunächst die Definitionen, Postulate und Axiome (siehe auch Abschnitt 2.1.1) und diskutieren diese anhand einiger von der Lehrkraft gestellter Impulsfragen:

- Welche der Definitionen erklären einen Begriff wirklich? Welche sind weniger hilfreich?

Definitionen wie die eines stumpfen Winkels sind insofern hilfreich, als man durch sie weiß, was ein stumpfer Winkel ist, wenn man weiß, was ein rechter Winkel ist und was es für einen Winkel bedeutet, größer als ein anderer zu sein. Beim Definieren von Begriffen erhält man also ein ähnliches Schema wie beim Beweisen von Sätzen: Zur Erklärung eines Begriffes verwendet man andere Begriffe, deren Bedeutung man als bekannt voraussetzt. Dies führt dazu, dass man die Bedeutung bestimmter, grundlegender Begriffe als intuitiv gegeben annehmen muss.

$$
\text { Begriff } \Leftarrow \text { Begriffspaket } \mathrm{A} \Leftarrow \text { Begriffspaket } \mathrm{B} \Leftarrow \ldots \Leftarrow \text { Grundbegriffe }
$$

Der Begriff Punkt scheint ein solcher Grundbegriff zu sein. Zwar gibt Euklid auch hier eine Definition an, diese ist aber sehr vage und wenig hilfreich.

- Euklid unterscheidet zwischen Axiomen und Postulaten. Welche Unterschiede/Gemeinsamkeiten kannst du feststellen?

Naheliegende Aspekte sind hier die Unterscheidung in geometriespezifische (Postulate) und geometrieunabhängige Aussagen (Axiome) oder die Feststellung, dass die Postulate im Gegensatz zu den Axiomen geometrische Konstruktionen beschreiben. ${ }^{2}$ Eine Gemeinsamkeit (zumindest aus Schülersicht) sollte die intuitiv einleuchtende Wahrheit aller Aussagen sein.

- Wie kann man das fünfte Postulat durch eine Skizze veranschaulichen?

Da dieses Postulat das komplexeste von allen ist, hilft eine Visualisierung, um seine Aussage besser nachvollziehen zu können:

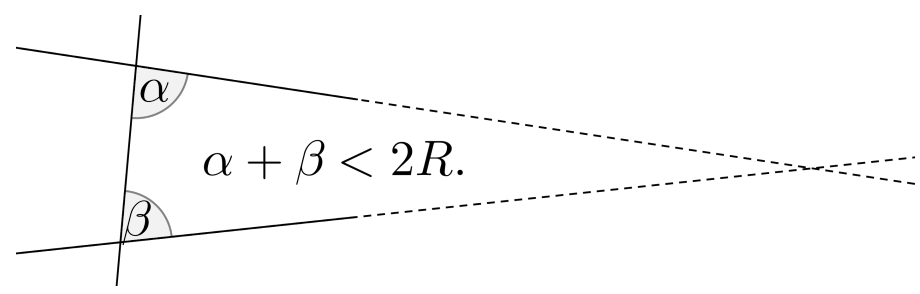

\footnotetext{
${ }^{2}$ Diese beiden Unterscheidungen treffen nicht in vollem Umfang zu, siehe dazu Abschnitt 2.1.1.
} 
Anschließend beschäftigen sich die Lernenden mit Euklids Beweis des Außen- und Innenwinkelsatzes, welche beide in Proposition 32 der Elemente enthalten sind. Dabei sollen sie diejenigen Aussagen identifizieren, die in dem Beweis verwendet werden und die daher entweder an einer früheren Stelle der Elemente bewiesen werden oder als Axiom bzw. Postulat festgehalten sein müssen.

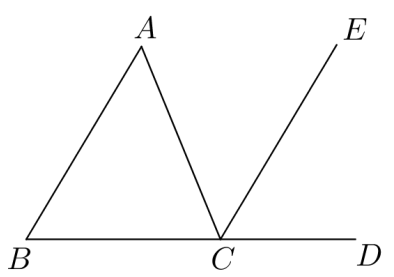

An jedem Dreieck ist der bei der Verlängerung einer Seite entstehende Außenwinkel den beiden gegenüberliegenden Innenwinkeln zusammen gleich, und die drei Winkel innerhalb des Dreiecks sind zusammen zwei Rechten gleich.

Das Dreieck sei $A B C$. Man verlängere eine Seite $B C$ nach $D$; ich behaupte, daß der Außenwinkel $A C D^{3}$ den beiden gegenüberliegenden Innnenwinkeln $C A B+A B C$ gleich ist und die drei Winkel innerhalb des Dreiecks $A B C+$ $B C A+C A B=2 R$.

Durch Punkt $C$ ziehe man $C E \|$ zur geraden Linie $A B$ (I, 31). Da $A B \| C E$ und sie von $A C$ geschnitten werden, sind die Wechselwinkel $B A C=A C E$ (I, 29). Ebenso ist, da $A B \| C E$ und sie von der geraden Linie $B D$ geschnitten werden, der äußere Winkel $E C D$ dem innen gegenüberliegenden $A B C$ gleich (I, 29). Wie oben bewiesen, ist auch $A C E=B A C$; also ist der ganze Winkel $A C D$ den beiden gegenüberliegenden Innenwinkeln $B A C+A B C$ gleich (Ax. 2).

Man füge $A C B$ beiderseits hinzu; dann sind $A C D+A C B=A B C+B C A+$ $C A B$. Aber $A C D+A C B=2 R$. (I, 13), also sind auch $A C B+C B A+C A B=2 R$.

In Euklids Beweis finden sich Verweise auf die Propositionen 13, 29 und 31 sowie das zweite Axiom; nicht von Euklid erwähnt wird die Verwendung des zweiten Postulats bei der Streckenverlängerung zu Beginn des Beweises. Die Beweisidee entspricht im Wesentlichen dem Vorgehen in Aufabe 6 des Arbeitsblattes GeOM1. Für den Fall, dass man den Kopieraufwand aufbringen kann und möchte, kann man den Schülerinnen und Schülern auch sämtliche Seiten der Elemente bis zu dieser Proposition austeilen ${ }^{4}$ und sie nun bitten, exemplarisch eine der erwähnten Propositionen samt Beweis anzuschauen und auch dort

\footnotetext{
${ }^{3}$ Berücksichtigt man bei der Notation von Winkeln auch die Orientierung, müsste hier eigentlich $D C A$ stehen. Euklid scheint bei seiner Notation jedoch prinzipiell den kleineren von den beiden Winkeln zu meinen, die durch die Angabe von drei Punkten festgelegt werden.

${ }^{4}$ In der von mir verwendeten Ausgabe (Euklid 2010) mit den Maßen $12 \mathrm{~cm} \times 3,5 \mathrm{~cm} \times 18,8 \mathrm{~cm}$ läuft das auf insgesamt zwölf DIN A4-Seiten hinaus, wobei auf eine DIN A4-Seite zwei Seiten der Elemente passen.
} 
die verwendeten Aussagen zu identifizieren und so weiter. Um die Komplexität des sich daraus ergebenden deduktiven Gefüges zu verdeutlichen, wird den Lernenden das zu Proposition 32 gehörende Pfeilschema präsentiert, welches aus Euklids Beweisen hervorgeht (siehe Abbildung 5.1).

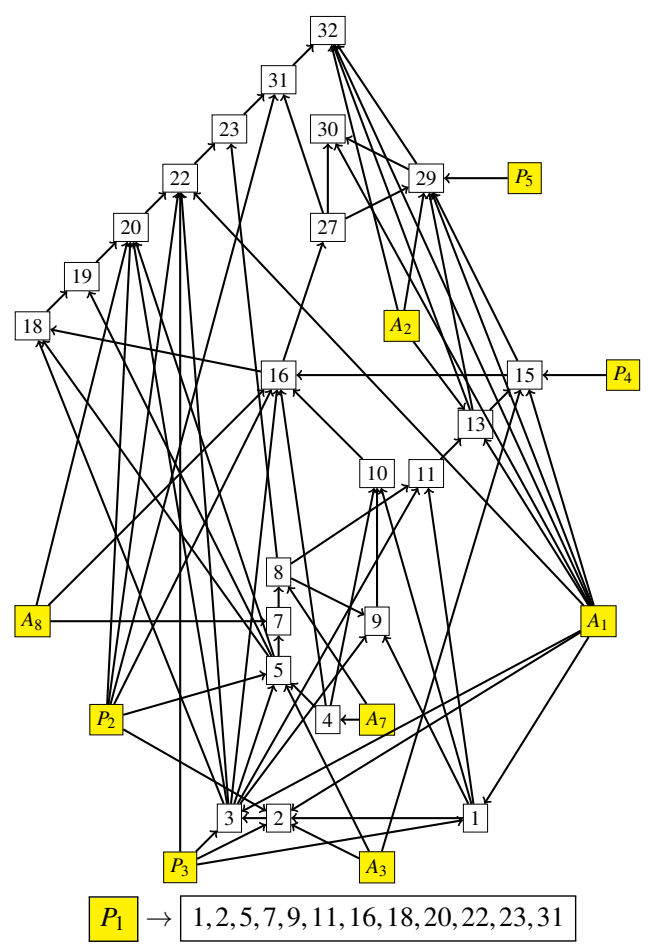

Abbildung 5.1: Pfeilschema zu Proposition 32

Den Abschluss dieser Phase und gleichzeitig den Übergang zur nächsten bildet ein Lehrervortrag zum Parallelenproblem, in dem zum einen die Äquivalenz von Euklids Fassung zur Playfair'schen Version (letztere ist den Lernenden - wenn auch nicht unter diesem Namen - vom Arbeitsblatt GEOM1 bereits bekannt) erwähnt und die Grundidee vorgestellt wird, die Unabhängigkeit des Parallelenpostulats durch einen Widerspruchsbeweis $\mathrm{zu}$ zeigen.

\section{Das Parallelenproblem}

EuKLIDs 5. Postulat $\left(P_{5}\right)$

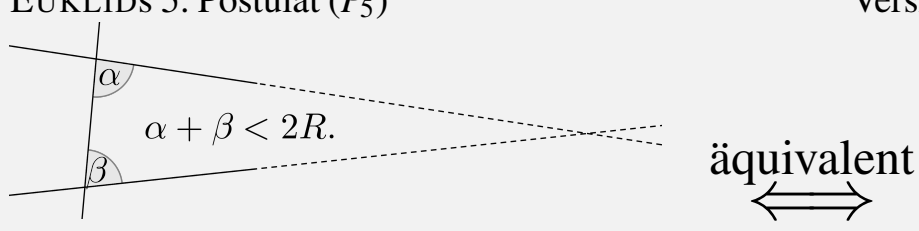

Version von JOHN PLAYFAIR $(P P)$

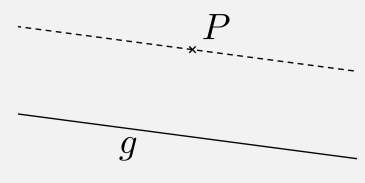

$P_{5}$ : Wenn eine gerade Linie zwei andere gerade Linien so schneidet, dass die innen auf derselben Seite entstehenden Winkel $\alpha$ und $\beta$ zusammen kleiner als zwei Rechte sind, dann 
schneiden diese beiden Linien sich auf der Seite, auf der die Winkel liegen, die zusammen kleiner als zwei Rechte sind.

$P P: Z u$ einer Geraden $g$ und einem Punkt $P$ außerhalb von $g$ gibt es genau eine zu $g$ parallele Gerade, die durch $P$ verläuft.

Frage: Lässt sich $P_{5}$ bzw. $P P$ aus den übrigen Axiomen/Postulaten herleiten?

Idee: Widerspruchsbeweis $\rightarrow$ Angenommen, die übrigen Axiome/Postulate gelten, $P_{5}$ bzw. $P P$ jedoch nicht. Lässt sich daraus ein logischer Widerspruch herleiten?

Auflösung: Die Antwort lautet nein, d. h. das Parallelenpostulat lässt sich nicht aus den übrigen Axiomen/Postulaten herleiten!

Den Lernenden wird mitgeteilt, dass die Auflösung des Parallelenproblems lange Zeit auf sich warten lassen hat. Um die Auflösung nachzuvollziehen, ist etwas Vorarbeit nötig, die in der zweiten Phase der Unterrichtsreihe geleistet wird.

\section{Phase 2: Sphärische Geometrie (GEOM2+3, HIST1+2)}

Als visuellen Einstieg lesen die Schülerinnen und Schüler die Seiten 2 bis 13 des mathematischen Comics Das Geometrikon (siehe Anhang A.3). Darin beschließt der Protagonist Anselm Wüßtegern, in einer nebligen Welt Geometrie zu betreiben, indem er Schnüre zwischen in den Boden gesteckten Stangen spannt. Die Stangen repräsentieren dabei Punkte und die Schnüre sind geodätische Linien, also kürzeste Verbindungen zwischen diesen Punkten. Bei seinen Messungen erhält Anselm jedoch seltsame Ergebnisse, die den bekannten Sätzen der euklidischen Geometrie widersprechen:

- Verlängert er eine Schnur von einem Punkt ausgehend kontinuierlich in eine Richtung, kommt er schlussendlich wieder am Ausgangspunkt an.

- Er spannt ein gleichseitiges Dreieck auf, dessen Innenwinkel nicht allesamt die Größe $60^{\circ}$ haben und in Summe größer als $180^{\circ}$ sind.

- Der Umfang und der Flächeninhalt eines aufgespannten Kreises sind kleiner als erwartet.

- Vergrößert Anselm den Radius des Kreises, wird die Kreislinie irgendwann zu einer Geraden; bei weiterer Vergrößerung ändert sich die Krümmung der Linie und der Umfang des Kreises nimmt sogar ab. 
All diese merkwürdigen Phänomene führen schließlich zu der Frage „Was ist passiert?“, welche die Lernenden nach aufmerksamer Lektüre des Comic-Ausschnittes ${ }^{5}$ ohne viel Mühe beantworten können sollten: Anselm befindet sich, ohne es zu wissen, auf einer Kugel und die Lehrsätze der euklidischen Geometrie sind damit nicht ohne Weiteres auf seine Situation (genauer: auf die Art der Oberfläche, auf der er sich befindet) anwendbar.

Den im Geometrikon-Ausschnitt beschriebenen Phänomenen gehen die Lernenden nun eigenständig und enaktiv auf den Grund. Sie verwenden Styroporkugeln, Stecknadeln und Wollfäden, um sphärische Geradenstücke und Dreiecke aufzuspannen und allgemeine Eigenschaften der Kugelgeometrie erkunden zu können (siehe Abbildung 5.2). Zur Unterstützung wird das Arbeitsblatt GEOM2 eingesetzt, welches einige Leitfragen für die eigenständigen Erkundungen enthält. Die Schülerergebnisse können anschließend im Plenum

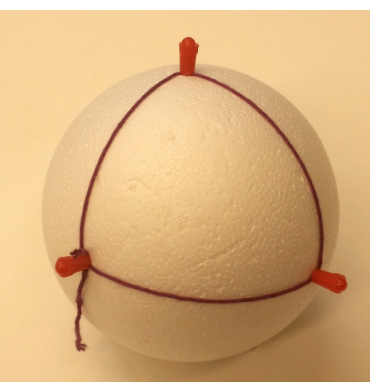

Abbildung 5.2: Styroporkugel diskutiert werden. Als Kontrolle bietet sich jedoch auch die fortgeführte Lektüre des Geometrikons an: Auf den Seiten 13 bis 21 werden die zuvor aufgeworfenen Fragen und Phänomene anschaulich geklärt.

Auf den Seiten 17 bis 19 des Mathe-Comics wird insbesondere dem Phänomen der nicht konstanten Innenwinkelsumme bei sphärischen Dreiecken genauer nachgegangen, unter anderem wird eine Formel erwähnt, welche die Innenwinkelsumme in Abhängigkeit zum Flächeninhalt eines sphärischen Dreiecks setzt. Da es sich bei diesem Lehrsatz der sphärischen Geometrie um einen starken Bruch mit der Erfahrungswelt der Lernenden handelt, wird er im Rahmen von GEOM3 unter Zuhilfenahme ausgewählter Aussagen der euklidischen Raumgeometrie (insb. der Formel für den Oberflächeninhalt einer Kugel) bewiesen.

Abschließend werden wesentliche Eigenschaften der euklidischen und der sphärischen Geometrie gegenübergestellt: ${ }^{6}$

\footnotetext{
${ }^{5}$ Um die Antwort der Frage nicht vorwegzunehmen, sollte die untere Hälfte von Seite 13 zunächst abgedeckt bzw. nicht an die Schülerinnen und Schüler verteilt werden.

${ }^{6}$ Methodisch bietet es sich hier an, die Eigenschaften der euklidischen Geometrie vorzugeben und die Schülerinnen und Schüler die zugehörigen Aussagen der sphärischen Geometrie selber formulieren zu lassen.
} 


\section{Vergleich zweier Geometrien}

\section{Euklidische Geometrie}

1. Zwei verschiedene Geraden haben höchstens einen Punkt gemeinsam.

2. Zwei Punkte legen eindeutig eine Gerade fest.

3. Jede Strecke kann beliebig verlängert werden.

4. $\mathrm{Zu}$ jeder Geraden $g$ und jedem Punkt $P$ außerhalb dieser Geraden gibt es genau eine Parallele zu $g$ durch $P$.

5. Die Innenwinkelsumme in jedem Dreieck beträgt $180^{\circ}$.

6. Ebene Dreiecke, die in allen drei Innenwinkeln übereinstimmen, sind im Allgemeinen nicht kongruent (sondern nur ähnlich).

7. $\mathrm{Zu}$ jedem vorgegebenen Mittelpunkt $M$ und Radius $r$ kann man einen Kreis zeichnen.

\section{Sphärische Geometrie}

1. Zwei verschiedene Großkreise schneiden sich stets in genau zwei (diametralen) Punkten.

2. Zwei nicht-diametrale Punkte legen eindeutig einen Großkreis fest.

3. Die maximale Länge einer sphärischen Strecke (Segment eines Großkreises) beträgt $2 \pi R$.

4. Es gibt keine parallelen Großkreise (siehe 1.).

5. Die Innenwinkelsumme ist in jedem sphärischen Dreieck größer als $180^{\circ}$ (und kleiner als $900^{\circ}$ ).

6. Sphärische Dreiecke, die in allen drei Innenwinkeln übereinstimmen, sind kongruent.

7. $\mathrm{Zu}$ jedem vorgegebenen Mittelpunkt $M$ und Radius $r<\pi R$ kann man einen sphärischen Kreis zeichnen ( $R$ ist der Kugelradius).

Um den Bogen zu axiomatischen Fragestellungen zu spannen, lesen die Lernenden nun einen Textauszug aus Hermann von Helmholtz' Vortrag Über den Ursprung und die Bedeutung der geometrischen Axiome, welcher in Abschnitt 2.1.2 bereits zitiert wurde (HIST1). Die Kernaussagen des Auszuges lassen sich wie folgt zusammenfassen: 
Ein neuer Blick auf geometrische Axiome

HeRmann VON Helmholtz: Ursprung und Bedeutung der geometrischen Axiome (1870)

\section{Gedankenexperiment:}

verstandbegabte, zweidimensionale Wesen betreiben Geometrie

LEBENSRAUM 1: unendliche Ebene

$\Downarrow$

Euklidische Geometrie
LEBENSRAUM 2:

Kugeloberfläche

$\Downarrow$

Sphärische Geometrie

anderer Lebensraum $\rightarrow$ andere empirische Erfahrungen

$\rightarrow$ ggf. andere geometrische Axiome

Das heißt: $\quad$ Axiome sind Erfahrungstatsachen!

Die in den Zeilen 27 bis 49 beschriebenen Eigenschaften der sphärischen Geometrie sind den Lernenden aufgrund der vorangestellten Erkundungsphasen bereits bekannt, so dass der Text zu weiten Teilen problemlos nachvollzogen werden kann. Besonders hinweisen sollte die Lehrkraft auf die Zeilen 9 bis 12, in denen erwähnt wird, dass sich die zweidimensionalen Wesen keine Vorstellung von einem höherdimensionalen Raum machen können. Dies hat insofern Konsequenzen für das hier beschriebene Verständnis der Natur geometrischer Axiome, als hieraus klar wird, dass diese Wesen insbesondere nicht wissen können bzw. sich nicht anschaulich vorstellen können, dass sie sich auf einer Kugeloberfläche befinden, da die Krümmung im dreidimensionalen Raum ein ihnen nicht bekanntes Konzept ist. Die naheliegende Schüleraussage, dass die Axiome der euklidischen wie auch der sphärischen Geometrie - wie bisher auch immer angenommen - anschaulich klar sind, greift hier also nicht, da die ,anschauliche Klarheit“ von einer dreidimensionalen Perspektive herrührt. Die Gültigkeit der Axiome lässt sich hingegen durch empirische Messungen überprüfen (entweder der Axiome selbst oder der aus ihnen deduzierbaren Konsequenzen).

Zur Verdeutlichung dieses neuen, empiristischen Standpunktes wird das Gedankenexperiment auf den dreidimensionalen Raum erweitert: Woher wissen wir eigentlich, dass die Sätze der (euklidischen) Raumgeometrie tatsächlich auch einer empirischen Überprüfung standhalten würden? Könnte es nicht sein, dass der uns umgebende Raum in einer vierten Dimension gekrümmt ist - was wir uns natürlich nicht anschaulich vorstellen könnenund sich beispielsweise Geraden oder Dreiecke in kosmisch großen Maßen ganz anders 
verhalten als bisher angenommen? Welche Auswirkungen eine solche Situation haben könnte, erfahren die Lernenden erneut durch die Lektüre eines Ausschnitts des Geometrikons (S. 34-39).

Ein Textauszug aus Henri Poincarés Wissenschaft und Hypothese (HIST2, siehe auch Abschnitt 2.1.2) greift von Helmholtz' Gedankenexperiment und die dadurch aufgeworfene Frage, welche Geometrie denn nun die Realität (d. h. den uns umgebenden physikalischen Raum) tatsächlich beschreibe, unmittelbar auf und bringt zum anderen die theoretische Eigenständigkeit der Geometrie als Vorstufe des formalistischen Standpunktes ${ }^{7}$ in die Diskussion mit ein. Die Kernaussagen sind im Folgenden zusammengefasst:

\section{Gleichzeitige Gültigkeit unterschiedlicher Geometrien}

HENRI POINCARÉ: Wissenschaft und Hypothese (1904)

- Geometrie ist eine exakte Wissenschaft, keine Experimentalwissenschaft.

- Verschiedene Geometrien können mehr oder weniger bequem oder nützlich zur Beschreibung der Realität sein, aber nicht per se richtig oder falsch.

- Axiome sind ,,auf Übereinkommen beruhende Festsetzungen“. Das bedeutet: die Wahl der Axiome wird von experimentellen Tatsachen geleitet, ist aber prinzipiell frei.

- Poincaré unterscheidet zwischen

a) der Geometrie als theoretischer (d.h. nicht an die Realität gebundener) Wissenschaft und

b) der Anwendung der Geometrie auf realweltliche Phänomene.

Während man auch bei von Helmholtz' Standpunkt noch von einer (von empirischen Erfahrungen abhängigen) Richtigkeit bzw. Wahrheit der Axiome reden kann, stellt Poincaré diesen Begriff explizit in Frage. Geometrische Axiome bzw. Aussagen sind nicht per se richtig/wahr, sondern mehr oder weniger bequem im Sinne einer möglichst einfachen Beschreibung der Realität. Auf theoretischer Ebene haben sie jedoch eher den Charakter von Konventionen bzw. Definitionen.

Poincarés Text ist inhaltlich sehr anspruchsvoll, so dass zu erwarten ist, dass die Lernenden durchaus Probleme haben, seine Ideen nachzuvollziehen. Dass eine Geometrie je

\footnotetext{
${ }^{7}$ Zeitlich erschien Poincarés Schrift zwar erst nach Hilberts Grundlagen der Geometrie, gedanklich ist ihr Einsatz als Übergang vom empiristischen zum formalistischen Standpunkt aufgrund des expliziten Bezugs auf das Helmholtz'sche Gedankenexperiment jedoch an dieser Stelle sinnvoll.
} 
nach den äußeren Gegebenheiten mehr oder weniger bequem ist, sollte jedoch aufgrund der Vorarbeiten im Kontext der sphärischen Geometrie nachvollziehbar sein.

Insbesondere der Gedanke der theoretischen Unabhängigkeit geometrischer Aussagen von anschaulichen Gegebenheiten dürfte für Schüler auf Grundlage des Textes jedoch zu vage bleiben. Er wird daher durch einen Lehrer-Impulsvortrag zur Auflösung des Parallelenproblems, welches am Ende der ersten Phase thematisiert wurde, vertiefend erklärt: Zum einen wird die hyperbolische Geometrie und ihre Eigenschaft, alle euklidischen Axiome bis auf das Parallelenpostulat zu erfüllen, kurz vorgestellt und zudem mit Hilfe von Poincarés Wörterbuch-Vorstellung (siehe auch Abschnitt 2.1.2) und die dadurch ermöglichte „Übersetzung“ von Sätzen der hyperbolischen Geometrie in solche der euklidischen und umgekehrt die Unabhängigkeit des Parallelenpostulats erläutert. Insbesondere das mentale Modell des Übersetzens - welches beispielsweise durch die Analogie zwischen Geraden und Großkreisen aus der sphärischen Geometrie bereits angebahnt wurde - ist für das Verständnis von besonderer Bedeutung und gleichzeitig ein wichtiger Grundstein für die nächste Unterrichtsphase.

\section{Auflösung des Parallelenproblems}

- Die hyperbolische Geometrie erfüllt alle Axiome der euklidischen Geometrie bis auf das Parallelenpostulat $P P$.

- Poincaré-Modell: Zu einer (hyperbolischen) Geraden gibt es mehrere Parallelen.

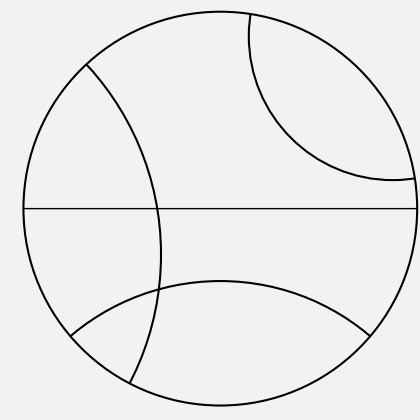

- Wörterbuch-Vorstellung:
Ebene
$\hat{=}$ das Innere einer Kreisscheibe
Punkte
$\hat{=}$ Punkte innerhalb der Kreisscheibe
Gerade
$\hat{=}$ Kreisbögen, die den Rand der Kreisscheibe senkrecht schneiden, sowie Durchmesserlinien


Satz der hyperbolischen $\stackrel{\text { übersetzen }}{\longleftrightarrow}$ Satz der euklidischen Geometrie Geometrie

- Widersprüche in der hyperbolischen Geometrie führen automatisch zu Widersprüchen in der euklidischen Geometrie (und umgekehrt).

- Ließe sich $P P$ in der euklidischen Geometrie aus den übrigen Axiomen beweisen, so ließe sich dieser Beweis mittels des Wörterbuchs auch in der hyperbolischen Geometrie führen. Das kann aber nicht sein!

\section{Phase 3: Von Dimpfeln und Gronkeln (GEOM4, HIST3+4)}

Im Zentrum dieser Unterrichtsphase steht die Auseinandersetzung mit dem Konzept der impliziten Definition und der damit verbundenen formalistischen Auffassung von Axiomen. Das Blatt GEOM4 greift die Idee der Kunstsprachenverwendung aus der in Abschnitt 4.2.2 vorgestellten Unterrichtsreihe auf und behält somit das mentale Modell des Übersetzens bei, welches auch aus dem vorangegangenen Impulsvortrag zur Auflösung des Parallelenproblems bekannt ist: Die Schülerinnen und Schüler werden mit drei Axiomen der Inzidenzgeometrie ${ }^{8}$ konfrontiert, allerdings sind die Begriffe Punkte, Geraden und liegen auf durch die Kunstworte Dimpfel, Gronkel und klubern ersetzt worden. Ohne die Bedeutung dieser Worte zu kennen, können die Lernenden dennoch allein aufgrund der festgelegten Axiome einige Aussagen über Dimpfel und Gronkel beweisen. Die zugehörigen Deduktionen sind weder kompliziert noch umfangreich (siehe Anhang A.4.1, S. 277), allerdings ist die geforderte stringente Form der Beweisführung unter ausschließlicher Berufung auf die Axiome in Kombination mit den Kunstworten für die Lernenden vermutlich derart ungewohnt, dass konkrete Hilfestellungen und Impulse durch die Lehrkraft gegeben werden müssen.

Im Anschluss werden die drei Axiome in zwei unterschiedlichen inhaltlichen Kontexten betrachtet. Im ersten Kontext werden die Kunstworte durch Begriffe aus dem musikalischen Umfeld ersetzt (Dimpfel $=$ Musiker, Gronkel $=$ Instrumente, klubern $=$ spielen $)$, so dass sich folgende Aussagen ergeben:

$A_{1}:$ Zu zwei verschiedenen Musikern $d_{1}$ und $d_{2}$ gibt es genau ein Instrument, das von $d_{1}$ und $d_{2}$ gespielt wird.

\footnotetext{
${ }^{8}$ Als Inzidenzgeometrie wird jener Teil der Geometrie bezeichnet, für den man lediglich die (durch entsprechende Axiome charakterisierte) Relation „liegen auf“ bzw. „gehen durch“ zwischen Punkten und Geraden benötigt.
} 
$A_{2}$ : Jedes Instrument wird von mindestens zwei Musikern gespielt.

$A_{3}$ : Es gibt mindestens drei Musiker, die nicht alle dasselbe Instrument spielen.

Im zweiten Kontext nimmt man die ,gewohnte“ inhaltliche Interpretation vor und ersetzt Dimpfel durch Punkte, Gronkel durch Geraden und klubern durch liegen auf/gehen durch: ${ }^{9}$

$A_{1}$ : Zu zwei verschiedenen Punkten $d_{1}$ und $d_{2}$ gibt es genau eine Gerade, die $d u r c h d_{1}$ und $d_{2}$ geht.

$A_{2}$ : Jede Gerade geht durch mindestens zwei Punkte.

$A_{3}$ : Es gibt mindestens drei Punkte, die nicht alle auf derselben Geraden liegen.

Neben der Tatsache, dass das Austauschen der Wörter und damit der inhaltlichen Interpretation die eigentliche Struktur (oder besser: Syntax) der Axiome nicht verändert, erkennen die Lernenden insbesondere, dass die zuvor geführten Beweise über Dimpfel und Gronkel nicht neu geführt werden müssen, da sie lediglich auf die in den Axiomen festgelegten Eigenschaften und Zusammenhänge zurückgreifen und somit völlig unabhängig von der inhaltlichen Bedeutung der Begriffe sind. Um Aussagen über Dimpfel und Gronkel beweisen zu können, muss man nicht wissen, was diese Dinge genau sind; relevant ist nur, was man in den Axiomen über Dimpfel und Gronkel erfährt. Das ist der Grundgedanke der impliziten Definition.

Als historisch bedeutsames Beispiel für diesen formalistischen Standpunkt werden ausgewählte Passagen aus David Hilberts Grundlagen der Geometrie gelesen (HIST3). Die Formulierungen des Blattes GEOM4 haben dabei Hilberts Sprachgebrauch bewusst antizipiert. Aussagen wie „Wir denken die Punkte, Geraden, Ebenen in gewissen gegenseitigen Beziehungen und bezeichnen diese Beziehungen durch Worte wie ,liegen ‘, ,zwischen“ [...]“ oder „Die Axiome dieser Gruppe stellen zwischen den oben eingeführten Dingen [...] eine Verknüpfung her“ sind für die Schülerinnen und Schüler somit leichter nachvollziehbar. Der Gedanke der Abstraktion, der hinter Hilberts Ausführungen steht (Punkt und Gerade sind nur Bezeichnungen für Dinge, deren Eigenschaften allein durch die Axiome definiert werden), wurde durch die Auseinandersetzung mit den Dimpfel-GronkelAussagen bereits eingeübt.

\footnotetext{
${ }^{9}$ Hierbei ist zu beachten, dass Passivkonstruktionen des Verbs klubern mit gehen durch übersetzt werden müssen, da es keine entsprechende Passiv-Variante der Verbs liegen auf gibt. „Ein Gronkel wird von einem Dimpfel geklubert" wird also beispielsweise übersetzt als „Eine Gerade geht durch einen Punkt“.
} 


\section{Abstrakte Sicht auf Axiome}

\section{DAVID HILBERT: Grundlagen der Geometrie (1899)}

- Man stellt sich unter Punkten, Geraden, etc. anschaulich nichts mehr vor.

- Relevant ist nur, in welchen Beziehungen die Begriffe zueinander stehen. Diese Beziehungen werden durch die Axiome beschrieben, z. B.:

Durch zwei Punkte geht genau eine Gerade.

Zwei Dimpfel klubern genau einen Gronkel.

$\rightarrow$ Prinzipiell kann man auch Kunstworte verwenden.

- Man sagt auch: Die Grundbegriffe (Punkt, Gerade, liegen auf / gehen durch) werden durch die Axiome implizit (= indirekt) definiert.

- Hilbert fragt nicht „Was ist ein Punkt / eine Gerade?“, sondern „Welche Bedingungen erfüllen / Merkmale haben Punkte und Geraden?“.

Die Auswirkungen, die diese Sichtweise auf den Wahrheitsanspruch mathematischer Aussagen hat, kommen in dem Briefwechsel zwischen Gottlob Frege und David Hilbert sehr gut zur Geltung (siehe auch Abschnitt 2.1.3). Als Abschluss dieser Unterrichtsreihe sollen die Schülerinnen und Schüler deshalb besonders markante und aussagekräftige Passagen daraus lesen und die darin auftretenden unterschiedlichen Sichtweisen herausarbeiten (HIsT4, bestehend aus einem Blatt mit Aussagen Freges und einem weiteren mit darauf eingehenden Aussagen Hilberts). Methodisch bietet sich hier (nach einer ersten Lektüre beider Textausschnitte) eine Aufteilung in zwei Gruppen an, wobei sich eine Gruppe intensiv mit Freges Argumenten und Sichtweisen beschäftigt und die andere mit Hilbert. Darauf aufbauend wird eine Plenumsdiskussion über die Frage geführt, ob Axiome nun selbstevidente Wahrheiten oder inhaltsleere Aussagen sind (bzw. welche Argumente jeweils für und gegen diese beiden Auffassungen sprechen).

\section{Zwei verschiedene Sichtweisen auf Axiome}

\section{Freges Position:}

- Axiome können nicht die Bedeutung eines Begriffs klären, das ist Sache der Definitionen.

- Definitionen geben einem (bisher bedeutungslosen) Begriff eine Bedeutung.

- Mathematische Aussagen (Sätze, Axiome) dürfen nur Begriffe enthalten, de- 
ren Bedeutung bereits geklärt ist. Nur so kann über die Wahrheit dieser Aussagen entschieden werden.

- Axiome sind Sätze, die offensichtlich wahr sind und daher keiner Begründung bedürfen.

- Aus der Wahrheit der Axiome folgt ihre Widerspruchsfreiheit.

\section{Hilberts Position:}

- Axiome beschreiben die Merkmale bestimmter Begriffe (wie Punkt, Gerade) und definieren diese somit.

- Bestimmte Begriffe (wie Punkt, Gerade) kann man nicht anders als durch Axiome definieren, da alles andere zu wirr und vage wäre.

- Die Wahrheitsfrage von Axiomen ist bei Hilbert von relativem Charakter: Wenn sich die (prinzipiell willkürlich wählbaren) Axiome eines deduktiven Gefüges nicht widersprechen, dann nennt man sie ,wahr“ (bezüglich des zugehörigen deduktiven Gefüges).

Die abschließende Diskussion von Freges und Hilberts stark divergierenden Ansichten bildet einen geeigneten Abschluss dieser Unterrichtsreihe, da durch Freges Aussagen nochmals der Bogen zum Beginn der Unterrichtsreihe mit dem Fokus auf die euklidische Auffassung gespannt wird. Das Resultat dieser Diskussion muss nicht (und sollte auch nicht) die Entscheidung für eine der beiden Sichtweisen sein. Vielmehr geht es darum, zu erkennen, dass man Axiome ohne inhaltliche Interpretation auffassen und dennoch damit arbeiten kann und dass diese Tatsache durch die behandelten historischen Entwicklungen kontinuierlich angebahnt wurde. Dennoch ist beispielsweise die Frage berechtigt, ob man nicht in Wahrheit doch realitätsbezogene geometrische Sachverhalte beschreiben möchte, auch wenn man Dimpfel und Gronkel statt Punkte und Gerade sagen kann.

\subsection{Unterrichtsreihe 2: Wahrscheinlichkeitsrechnung}

\subsubsection{Sachanalyse}

Wenige Begriffe in der Mathematik sind so kontrovers diskutiert worden wie der Wahrscheinlichkeitsbegriff. Die historische Betrachtung weist zahlreiche Versuche aus, eine 
zufriedenstellende Definition für dieses Konzept zu liefern. Zu den (auch für die Schulmathematik) bedeutendsten Beiträgen gehören die Ansätze von Pierre-Simon de Laplace (1749-1827) und Richard von Mises (1883-1953). Laplace fasst Wahrscheinlichkeit als Verhältnis der günstigen Ergebnisse eines Zufallsexperiments zu allen möglichen Ergebnissen auf unter der Voraussetzung, dass nichts dagegen spricht, letztere als ,gleichmöglich“ anzusehen (Prinzip vom unzureichenden Grunde):

Die Theorie des Zufalls [...] besteht darin, alle Ereignisse derselben Art auf eine gewisse Anzahl gleich möglicher Fälle zurückzuführen, d. h. auf solche, über deren Existenz wir in gleicher Weise im Unklaren sind, und dann die Zahl der Fälle zu bestimmen, die dem Ereignis, dessen Wahrscheinlichkeit man sucht, günstig sind. Das Verhältnis dieser Zahl zu der aller möglichen Fälle ist das Maass [sic!] dieser Wahrscheinlichkeit, die also nur ein Bruch ist, dessen Zähler die Zahl der günstigen Fälle, und dessen Nenner die Zahl aller möglichen Fälle ist. (de Laplace 1886, S. 3)

Abgesehen davon, dass der Begriff der Gleichmöglichkeit wieder den Begriff der Wahrscheinlichkeit beinhaltet und diese Definition somit zirkulär ist, führte insbesondere das Bedürfnis, eine größere Anzahl von Phänomenen mit stochastischen Methoden zu behandeln (und nicht nur solche, bei denen man von vorher bekannten, gleichmöglichen Versuchsausgängen ausgehen kann), zur Weiterentwicklung des Wahrscheinlichkeitsbegriffs (vgl. Steinbring 1980, S. 126-127). ${ }^{10}$

Der österreichische Mathematiker Richard von Mises prägte den frequentistischen Ansatz, für den die Konzepte des Kollektivs und der Unmöglichkeit eines Spielsystems zentral sind: Wahrscheinlichkeitstheoretische Untersuchungen können auf prinzipiell unbegrenzt reproduzierbare gleichartige Massenerscheinungen angewendet werden, bei denen die relative Häufigkeit einer bestimmten Merkmalsausprägung gegen einen Grenzwert konvergiert, der bei willkürlicher Wahl einer Teilfolge unverändert bleibt (siehe von Mises 1964). Diesem Zugang liegt eine objektivistische Vorstellung von Wahrscheinlichkeit zugrunde, die hier ähnlich einer physikalischen Größe durch relative Häufigkeiten approximativ bestimmt wird. Insbesondere Einzelereignisse (beispielsweise „Wie wahrscheinlich ist es, dass es morgen regnet?"), die nicht unter den Kollektiv-Begriff fallen, lassen sich mit der frequentistischen Theorie jedoch nicht fassen und benötigen daher einen subjektivistischen Ansatz. Dieser betrachtet Wahrscheinlichkeit als Grad der subjektiven Überzeugung bzgl. des Eintretens eines Ereignisses unter Einbeziehung aller relevanten (auch informeller) Informationen (vgl. Wolpers und Götz 2002, S. 84-86).

${ }^{10} \mathrm{Zu}$ Grenzen und Beschränkungen des Laplace'schen Wahrscheinlichkeitsbegriffs siehe auch Wolpers und Götz 2002, S. 81-82. 
Der Frequentismus ist für die Praxis von großer Bedeutung. Allerdings ist die Ableitung des Wahrscheinlichkeitsbegriffs aus der (empirischen) Beobachtung der Stabilisierung relativer Häufigkeiten unter anderem deshalb problematisch, weil der damit zusammenhängende analytische Konvergenzbegriff mathematisch nicht haltbar ist. Es existiert nämlich nicht zu jedem $\varepsilon>0$ ein $n_{\varepsilon}$, so dass die relativen Häufigkeiten $h_{n}$ für $n>n_{\varepsilon}$ höchstens um $\varepsilon$ von einem (als tatsächliche Wahrscheinlichkeit angenommenen) Wert $p$ abweichen. Es handelt sich vielmehr um eine stochastische Konvergenz, d. h. die Wahrscheinlichkeit, dass sich die relativen Häufigkeiten bei zunehmender Versuchszahl von der tatsächlichen Wahrscheinlichkeit $p$ unterscheiden, wird immer kleiner. Diese sprachliche Explikation des stochastischen Konvergenzbegriffs macht deutlich, dass auch hier wieder der eigentlich zu definierende Begriff der Wahrscheinlichkeit ins Spiel kommt (vgl. Schreiber 1979, S. 239-241, Steinbring 1980 sowie Wolpers und Götz 2002, S. 85-86).

Eine mathematisch zufriedenstellende Lösung gelang schließlich dem russischen Mathematiker Andrei Kolmogorov (1903-1987). In Grundbegriffe der Wahrscheinlichkeitsrechnung (Kolmogorov 1933) definierte er Wahrscheinlichkeit implizit als normierte, $\sigma$ additive Maßfunktion auf geeigneten Mengenalgebren. Die folgende axiomatische Beschreibung entspricht im Wesentlichen Kolmogorovs Charakterisierung und dient - mitunter in leicht abgewandelter Form - in den meisten einführenden Lehrbüchern zur Stochastik auch heute noch als Definition eines Wahrscheinlichkeitsmaßes:

Sei $\Omega$ eine nichtleere Menge und $\mathcal{A} \subseteq \operatorname{Pot}(\Omega)$ eine $\sigma$-Algebra, d. h.:

(A1) $\Omega \in \mathcal{A}$;

(A2) Aus $A \in \mathcal{A}$ folgt $\Omega \backslash A:=\bar{A} \in \mathcal{A}$;

(A3) Für höchstens abzählbar viele Teilmengen $A_{1}, A_{2}, A_{3}, \ldots$ mit $A_{i} \in \mathcal{A}$ für alle $i$ gilt

$$
\bigcup_{i=1}^{\infty} A_{i} \in \mathcal{A} \text {. }
$$

Eine Abbildung $P: \mathcal{A} \rightarrow \mathbb{R}$ heißt Wahrscheinlichkeitsmaß, wenn die folgenden Axiome erfüllt sind:

(K1) $P(A) \geq 0$ für alle $A \in \mathcal{A} \quad$ (Nichtnegativität),

(K2) $P(\Omega)=1 \quad$ (Normiertheit),

(K3) Für höchstens abzählbar viele, paarweise disjunkte Teilmengen $A_{1}, A_{2}, A_{3}, \ldots$ mit $A_{i} \in \mathcal{A}$ für alle $i$ gilt

$$
P\left(\bigcup_{i=1}^{\infty} A_{i}\right)=\sum_{i=1}^{\infty} P\left(A_{i}\right) \quad(\sigma \text {-Additivität })
$$


Dieser Ansatz war eine logische und notwendige Konsequenz aus den bisher gescheiterten Definitionsversuchen; ,die implizite Definition wird zum Ausdruck der theoretischen Eigenständigkeit des Wahrscheinlichkeitsbegriffs“ (Steinbring 1980, S. 136). Dabei entfalten sich Tragweite und Bedeutung von Kolmogorovs maß- und mengentheoretischer Axiomatik insbesondere im Falle unendlicher Ergebnismengen. Die Forderung der $\sigma$ Additivität für Wahrscheinlichkeitsmaße, deren Folgerungen wie beispielsweise die 0-1Gesetze $^{11}$ durchaus nicht von allen akzeptiert wurden und werden, nimmt im theoretischen Aufbau eine zentrale Stellung ein. Kolmogorov selbst nimmt diesbezüglich einen pragmatischen Standpunkt ein: „Unendliche Wahrscheinlichkeitsfelder erscheinen nur als idealisierte Schemata reeller zufälliger Prozesse. Wir beschränken uns dabei willkürlich auf solche Schemata, welche dem Stetigkeitsaxiom VI genügen. ${ }^{12}$ Diese Beschränkung erwies sich bis jetzt bei den verschiedensten Untersuchungen als zweckmäßig“ (Kolmogorov 1933, S. 14). Der letzte Satz zeigt dabei, dass die Verwendung des Stetigkeitsaxioms nicht so ,willkürlich“ ist, wie es sich in dem vorangestellten Satz zunächst anhört.

Bei aller Abstraktheit der maßtheoretischen Sichtweise darf nicht vergessen werden, dass die Probleme einer inhaltlichen Interpretation des Wahrscheinlichkeitsbegriffs mit der axiomatischen Grundlegung nicht aus der Welt geschafft sind: „The old questions eliminated by Kolmogorov's axiomatics have not died out. They come back, not in their ontological form, but as questions as to how to apply probability“ (Freudenthal, zit. n. Steinbring 1980, S. 137). Vielmehr werden besagte Probleme zum Zwecke der mathematischlogischen Darstellung der Theorie vorübergehend ausgeblendet.

${ }^{11} \mathrm{Zu}$ den 0-1-Gesetzen gehört unter anderem das Lemma von Borel-Cantelli (siehe auch Klenke 2006, S. 51): Seien $A_{1}, A_{2}, \ldots$ Ereignisse und $A=\limsup _{n \rightarrow \infty} A_{n}$. Dann gilt:

(i) Ist $\sum_{n=1}^{\infty} P\left(A_{n}\right)<\infty$, so ist $P(A)=0$.

(ii) Sind die $A_{n}$ paarweise unabhängig und $\sum_{n=1}^{\infty} P\left(A_{n}\right)=\infty$, so ist $P(A)=1$.

Eine informelle Variante des zweiten Teils des Lemmas ist das Infinite-Monkey-Theorem (siehe auch https://de.wikipedia.org/wiki/Infinite-Monkey-Theorem; letzter Zugriff: 13.10.2017), welches besagt, dass ein endlos und zufällig auf einer Schreibmaschine tippender Affe fast sicher irgendwann sämtliche Werke Shakespeares schreiben wird.

${ }^{12}$ Kolmogorov verwendet ein zur $\sigma$-Additivität äquivalentes Axiom (siehe Kolmogorov 1933, S. 13): Für eine abnehmende Folge $A_{1} \supset A_{2} \supset A_{3} \supset \ldots$ von höchstens abzählbar vielen $A_{i} \in \mathcal{A}$ mit $\bigcap_{i=1}^{\infty} A_{i}=\emptyset$ gilt

$$
\lim _{n \rightarrow \infty} P\left(A_{n}\right)=0
$$




\subsubsection{Didaktische Anmerkungen}

Die vorgestellte Unterrichtsreihe soll die durch Kolmogorov begründete implizit-axiomatische Charakterisierung des Wahrscheinlichkeitsbegriffs mit den beiden inhaltlich orientierten Zugängen von Laplace und von Mises vergleichen und erste deduktive Übungen auf Basis der Kolmogorov-Axiome bereitstellen. Sie kann und soll die Entwicklung der Wahrscheinlichkeitsrechnung einschließlich der fachlichen Hintergründe in all ihrer Komplexität jedoch nur eingeschränkt wiedergeben. Zudem müssen einige für die Herausbildung stochastischen Denkens und adäquater Grundvorstellungen relevante Aspekte dem Regelunterricht überlassen werden. Beispielsweise erscheint eine schülergerechte Thematisierung der Kontroverse zwischen subjektivistischen und objektivistischen Ansätzen vor dem Hintergrund einer angemessenen Einschätzung der mit dem Wahrscheinlichkeitsbegriff verbundenen Vorstellungen für den Stochastikunterricht sinnvoll, auch wenn sie in aktuellen Schulbüchern in der Regel nicht erfolgt. Sie ist für die im Rahmen dieser Arbeit entwickelte Unterrichtsreihe allerdings nicht relevant, da sie nicht mit axiomatischen Fragestellungen zusammenhängt.

Von Mises' Zugang wird in dieser Unterrichtsreihe (wie auch in der Schule) nur vereinfacht behandelt, da eine intensive Auseinandersetzung mit dem Kollektivbegriff und dem Prinzip der Unmöglichkeit eines Spielsystems nicht durchführbar ist. Dass eine Definition des Wahrscheinlichkeitsbegriffs über eine Konvergenzaussage relativer Häufigkeiten zirkulär ist und der frequentistische Ansatz - trotz seiner im Vergleich zum Laplace-Ansatz breiteren Anwendbarkeit - nur auf bestimmte Arten von Zufallsphänomenen anwendbar ist, kann man Schülerinnen und Schülern aber dennoch verständlich machen.

Auch die eigentliche fachliche Rolle von Kolmogorovs Axiomensystem (siehe oben) wird man selbst in Zusatzkursen der Sekundarstufe II kaum vermitteln können. Ziel der Unterrichtsreihe ist es vielmehr, Kolmogorovs abstrakt-mengentheoretischen Zugang zum Wahrscheinlichkeitsbegriff zu verdeutlichen und einige einfache Deduktionen zu behandeln. Für diese Zwecke erscheint eine Fokussierung auf endliche Wahrscheinlichkeitsräume ausreichend. In diesem Fall kann eine Thematisierung der Axiome für $\sigma$-Algebren entfallen, da die Potenzmenge als Definitionsbereich von Wahrscheinlichkeitsmaßen gewählt werden kann. Auch die $\sigma$-Additivität reduziert sich auf endliche Additivität für disjunkte Mengen, so dass letztlich eine einfache und überschaubare axiomatische Basis übrig bleibt, die auch in vielen Schulbüchern im Rahmen kurzer Exkurse erwähnt wird (siehe z. B.: Brandt, Riemer und Wollmann 2012, S. 16; Griesel et al. 2011, S. 398-399; Lergenmüller, Schmidt und Krüger 2012, S. 55). ${ }^{13}$

\footnotetext{
${ }^{13} \mathrm{Im}$ endlichen Fall ist das Axiomensystem von Kolmogorov äquivalent zur Konstruktion eines Wahr-
} 
Neben der Kontrastierung verschiedener Zugänge zum Wahrscheinlichkeitsbegriff bieten die Kolmogorov-Axiome und die aus ihnen unmittelbar deduzierbaren Aussagen über Wahrscheinlichkeitsmaße eine gute Basis, um das lokale Ordnen zu üben. Ein wesentlicher Bestandteil der Unterrichtsreihe ist daher die eigenständige Entdeckung und Systematisierung logischer Zusammenhänge zwischen elementaren wahrscheinlichkeitstheoretischen Aussagen. Der Vorteil ist dabei, dass die zugehörigen Beweise zumeist sehr einfach und kurz sind und es für viele Aussagen verschiedene Beweismöglichkeiten und damit auch verschiedene Möglichkeiten gibt, eine axiomatische Basis aufzustellen.

Bezüglich des für die Formulierung der Kolmogorov-Axiome nötigen mengentheoretischen Zugangs zum Ereignisbegriff merkt Freudenthal an, dass spätestens mit der Einführung von Zufallsvariablen die Mengensysteme zugunsten aussagenlogischer Propositionen als Argumente von Wahrscheinlichkeitsmaßen in den Hintergrund treten. Dies sei angesichts des Sprachgebrauchs in der Stochastik - wo man schließlich über Wahrscheinlichkeiten von Ereignissen rede - ohnehin intuitiver zugänglich (vgl. Freudenthal 1974, S. 261). Der Mengenlehre kommt seinem Eindruck nach die Rolle eines Feigenblatts zu: Man verwende sie, weil es „modisch“ (fashionable, S. 262) sei; der eigentliche Kern der Stochastik werde damit aber verdeckt. Freudenthals Aussagen stammen natürlich aus einer Zeit, als die Auswirkungen der Neuen Mathematik noch deutlich spürbar waren, was man vor allem seinen Beschreibungen ,gängiger Schulbücher“ anmerkt, die heutzutage kaum noch zutreffen. Dennoch ist es richtig, dass das Ausmaß an mengentheoretischem Formalismus im Schulunterricht wohlüberlegt sein will. Ein eher informeller Umgang mit rudimentären mengentheoretischen Schreibweisen und Operationen ist in vielen aktuellen Schulbüchern jedoch üblich (siehe z. B.: Brandt, Riemer und Wollmann 2012; Griesel et al. 2011; Lergenmüller, Schmidt und Krüger 2012) und für eine Einführung der Kolmogorov-Axiome ausreichend.

Obwohl die Auseinandersetzung mit Kolmogorovs Axiomensystem im Vordergrund der Unterrichtsreihe steht, darf der Bezug der Theorie zur Anwendung natürlich nicht fehlen. Die Wahl der Axiome ist nicht willkürlich und auch die Sätze, die man im Rahmen einer formalisierten Theorie aus diesen deduziert, sind im Vorfeld meist durch Anwendungs-

scheinlichkeitsmaßes über Zähldichten (das gilt auch noch für den abzählbar unendlichen Fall, wenn die endliche Additivität durch $\sigma$-Additivität ersetzt wird), so dass man Wahrscheinlichkeit auch in dieser Form definieren könnte (siehe z. B. Cramer und Kamps 2008, S. 155). Die Gleichwertigkeit beider Definitionen können Schülerinnen und Schüler leicht nachprüfen. Eine ausführliche Thematisierung der für überabzählbare Ergebnismengen auftretenden Probleme (die ja unter anderem auch eine Diskussion des Begriffs der Ereignisalgebra beinhaltet) würde aber den Rahmen der Unterrichtsreihe sprengen. Es ist denkbar, den Lernenden zumindest von der Existenz solcher Probleme zu berichten und dadurch weitere Gründe für die Kolmogorov-Axiome als Ausgangspunkt auch im endlichen Fall zu nennen. Mit elementaren Kenntnissen der Integralrechnung kann man auch zeigen, dass für $\Omega=\mathbb{R}$ und über Riemann-Dichten definierte Wahrscheinlichkeitsmaße stets $P(\{\omega\})=0$ gelten muss. Bei der Darstellung der Unterrichtsreihe im folgenden Abschnitt wird dieser Aspekt jedoch nicht weiter behandelt. 
probleme motiviert und in informeller Form bekannt. Nur vor dem Hintergrund der Bemühungen einer inhaltlichen Definition des Wahrscheinlichkeitsbegriffs und in der Auseinandersetzung mit anwendungsorientierten Aufgaben können Schülerinnen und Schüler einen Eindruck davon gewinnen, was Kolmogorovs Zugang leistet und was nicht.

\subsubsection{Beschreibung der Unterrichtsreihe}

Phase Thema (inkl. wesentlicher Tätigkeiten/Erkenntnisse) $\quad$ ABs / Texte

\begin{tabular}{|c|c|c|}
\hline 1 & $\begin{array}{l}\text { Reflexion der klassischen Zugänge zum } \\
\text { Wahrscheinlichkeitsbegriff } \\
\text { - den Laplace'schen und frequentistischen Zugang zum } \\
\text { Wahrscheinlichkeitsbegriff anhand typischer } \\
\text { Zufallsexperimente wiederholen } \\
\text { - Probleme und Grenzen der beiden klassischen } \\
\text { Zugänge benennen }\end{array}$ & $\begin{array}{l}\text { WKEIT1, Hist5, } \\
\text { Hist6 }\end{array}$ \\
\hline 2 & $\begin{array}{l}\text { Mengentheoretische Modellierung stochastischer } \\
\text { Situationen } \\
\text { - stochastische Situationen mengentheoretisch } \\
\text { modellieren }\end{array}$ & W KEIT2 \\
\hline 3 & $\begin{array}{l}\text { Logisches Ordnen wahrscheinlichkeitstheoretischer } \\
\text { Aussagen } \\
\text { - logische Zusammenhänge zwischen einfachen } \\
\text { wahrscheinlichkeitstheoretischen Aussagen entdecken } \\
\text { - kleinere Deduktionen durchführen } \\
\text { - verschiedene Möglichkeiten zum Aufstellen eines } \\
\text { Axiomensystems erkunden }\end{array}$ & W KEIT3 \\
\hline 4 & $\begin{array}{l}\text { Deduktives Arbeiten mit den Kolmogorov-Axiomen } \\
\text { - Kolmogorovs abstrakt-axiomatischen Zugang zum } \\
\text { Wahrscheinlichkeitsbegriff mit den beiden } \\
\text { klassisch-inhaltlichen Zugängen vergleichen } \\
\text { - auf Grundlage der Kolmogorov-Axiome ein Stück } \\
\text { Wahrscheinlichkeitstheorie deduktiv entwickeln }\end{array}$ & $\begin{array}{l}\text { HIST7, WKEIT4, } \\
\text { WKEIT5 }\end{array}$ \\
\hline
\end{tabular}




\section{Phase 1: Reflexion der klassischen Zugänge zum Wahrscheinlichkeitsbegriff (WKEIT1, HIST5+6)}

Ausgangspunkt und roter Faden der Unterrichtsreihe ist die Frage Was ist Wahrscheinlichkeit?, welche auch als Haupt-Überschrift auf sämtlichen entwickelten Arbeitsblättern vermerkt ist.

Das erste Arbeitsblatt WKEIT1 beschäftigt sich anhand typischer Zufallsexperimente mit den klassischen Zugängen zum Wahrscheinlichkeitsbegriff. Aufgabe 1 legt dabei den Laplace'schen Zugang zugrunde und Aufgabe 2 erfordert die Schätzung einer Wahrscheinlichkeitsverteilung aufgrund der sich ergebenden relativen Häufigkeiten bei einem mehrfach ausgeführten Zufallsexperiment. Zusammen mit den beiden historischen Textauszügen Hist5 (insb. Z. 21-35) und Hist6 (insb. Z. 19-42) versuchen die Schülerinnen und Schüler, mögliche Definitionen für den Begriff Wahrscheinlichkeit aufzustellen.

\section{Der Wahrscheinlichkeitsbegriff: Klassische Definitionen}

\section{Laplace'scher Ansatz:}

Sind bei einem Zufallsexperiment alle möglichen Ausgänge gleichwahrscheinlich, so ist die Wahrscheinlichkeit für ein Ereignis gleich dem Quotienten

$$
\frac{\text { Anzahl der günstigen Ausgänge }}{\text { Anzahl aller möglichen Ausgänge }} \text {. }
$$

\section{Frequentistischer Ansatz:}

Führt man eine große Anzahl von Wiederholungen desselben Zufallsexperiments durch, nähert sich die relative Häufigkeit, mit der ein beobachtetes Ereignis auftritt, immer mehr der tatsächlichen Wahrscheinlichkeit für dieses Ereignis. Wenn also bei $n$ Durchführungen eines Zufallsexperiments ein Ereignis $E$ mit der absoluten Häufigkeit $m$ auftritt, so kann man annähernd $P(E)=h_{n}(E)=\frac{m}{n}$ setzen.

Im Anschluss an diese Definitionsversuche werden mit Hilfe des Arbeitsblattes und der beiden Textauszüge problematische Aspekte thematisiert. Insbesondere von Mises erwähnt in HIST6 einige Grenzen des Laplace'schen Wahrscheinlichkeitsbegriffs und die Aufgaben 3 und 4 von WKEIT1 stoßen Diskussionen über den frequentistischen Zugang an. 


\section{Schwierigkeiten mit den klassischen Ansätzen}

\section{Probleme des Laplace-Ansatzes:}

- „Wahrscheinlickeit“ wird mit Hilfe des Begriffs „gleichwahrscheinlich“ erklärt. $\Rightarrow$ Zirkelschluss

- Die Gleichwahrscheinlichkeit der Versuchsausgänge ist eine Annahme, die sich nur im Nachhinein als richtig oder falsch herausstellen kann.

- Die Annahme der Gleichwahrscheinlichkeit ist in vielen Situationen nicht gerechtfertigt bzw. sinnvoll möglich.

\section{Probleme des frequentistischen Ansatzes:}

- Die Annäherung der relativen Häufigkeiten an die (tatsächliche) Wahrscheinlichkeit ist nicht sicher. Es wird lediglich die Wahrscheinlichkeit immer geringer, dass die relativen Häufigkeiten von der tatsächlichen Wahrscheinlichkeit abweichen. $\Rightarrow$ Zirkelschluss

- Häufig möchte man die Wahrscheinlichkeit von nicht wiederholbaren Ereignissen bestimmen (z. B. Regenwahrscheinlichkeit für das kommende Wochenende); dies ist mit dem frequentistischen Ansatz nicht möglich.

Die bewusste Reflexion der beiden klassischen Zugänge liefert einen wichtigen Grundstein, um die später eingeführte abstrakte Auffassung zu verstehen.

\section{Phase 2: Mengentheoretische Modellierung stochastischer Situationen (WKEIT2)}

In der zweiten Unterrichtsphase wird, abhängig davon, in welchem Ausmaß sie bereits im Regelunterricht behandelt wurde, die mengentheoretische Modellierung von Ereignissen und Zufallsexperimenten besprochen, um eine effektive Schreibweise zur Formulierung einfacher wahrscheinlichkeitstheoretischer Sätze zur Verfügung zu haben. Die Schülerinnen und Schüler sollen am Ende dieser Phase die Modellierbarkeit stochastischer Situationen auf mengentheoretischer Basis verstehen und Begriffe wie Ergebnismenge, (Elementar-)Ereignis und Gegenereignis mit dieser Sichtweise in Verbindung bringen.

Grundsätzlich kann zu Beginn dieser Phase ein Impulsvortrag der Lehrkraft stehen, in dem die wesentlichen Begriffe erläutert und an einem einfachen Beispiel illustriert werden. Die folgende Tabelle ist angelehnt an Büchter und Henn (2005, S. 137) und enthält die wichtigsten mengentheoretischen Begriffe: 
Mengentheoretische Grundbegriffe

\begin{tabular}{c|c} 
Begriff & Erklärung \\
\hline \hline Ergebnismenge & $\begin{array}{c}\text { Menge } \Omega=\left\{\omega_{1}, \omega_{2}, \ldots\right\} \text { aller möglichen } \\
\text { Ergebnisse eines Zufallsexperiments }\end{array}$ \\
\hline Elementarereignis & $\begin{array}{c}\text { einelementige Teilmenge }\{\omega\} \\
\text { der Ergebnismenge } \Omega\end{array}$ \\
\hline Ereignis & Teilmenge $A$ der Ergebnismenge $\Omega$ \\
\hline Sicheres Ereignis & $A=\Omega$ \\
\hline Unmögliches Ereignis & $A=\emptyset$ \\
\hline Unvereinbare Ereignisse & $\begin{array}{c}\text { Schnittmenge } \\
\text { Gegenereignis des Ereignisses } A\end{array}$ \\
\hline Und-Ereignis & $A \cap B=\{\omega \in \Omega \mid \omega \in A$ und $\omega \in B\}$ \\
\hline zweier Ereignisse $A$ und $B$ & Vereinigungsmenge $A \cap B=\emptyset$ \\
\hline Oder-Ereignis & $A \cup B=\{\omega \in \Omega \mid \omega \in A$ oder $\omega \in B\}$ \\
\hline zweier Ereignisse $A$ und $B$ & \\
\hline
\end{tabular}

Diese Begrifflichkeiten werden anhand des folgenden Roulette-Beispiels ${ }^{14}$ veranschaulicht:

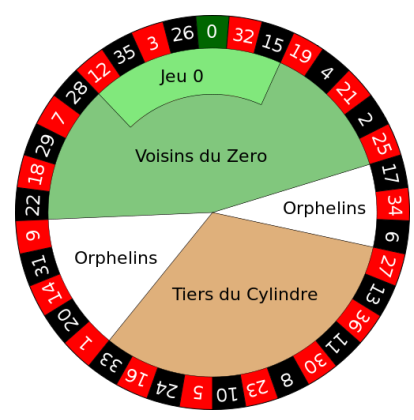

Es sei

$$
\Omega=\{0,1,2,3, \ldots, 36\}
$$

- $A$ : Die Kugel fällt auf ein rotes Feld.

$A=\{1,3,5,7,9,12,14,16,18,19,21,23,25,27,30,32,34,36\}$

- $B$ : Die Kugel fällt auf eine ungerade Zahl.

$B=\{1,3,5,7, \ldots, 35\}$

- $C$ : Die Kugel fällt auf eine Primzahl.

$C=\{2,3,5,7,11,13,17,19,23,29,31\}$

\footnotetext{
${ }^{14}$ Bildquelle: https://de.wikipedia.org/wiki/Roulette (letzter Zugriff: 05.05.2018), Autor: Betzaar (CC BY-SA 3.0).
} 
- Sicheres Ereignis:

$D$ : Die Kugel fällt auf eine Zahl zwischen 0 und 36 (inklusive).

$D=\Omega$

- Gegenereignis zu A:

$E$ : Die Kugel fällt nicht auf ein rotes Feld.

$E=\Omega \backslash A=\{0,2,4,6,8,10,11,13,15,17,20,22,24,26,28,29,31,33,35\}$

- Und-Ereignis von A und B:

$F$ : Die Kugel fällt auf eine rote und ungerade Zahl.

$F=\{1,3,5,7,9,19,21,23,25,27\}$

- Oder-Ereignis von A und B:

$G$ : Die Kugel fällt auf eine rote oder eine ungerade Zahl. $G=\{1,3,5,7,9,11,12,13, \ldots, 19,21,23,25,27,29,30,31, \ldots, 36\}$

- Die Ereignisse $E$ und $F$ sind unvereinbar, denn $E \cap F=\emptyset$

Das Arbeitsblatt WKEIT2 dient zur Einübung des mengentheoretischen Formalismus und enthält schulbuchtypische Aufgaben. ${ }^{15}$

\section{Phase 3: Logisches Ordnen wahrscheinlichkeitstheoretischer Aussagen (WKEIT3)}

In dieser Phase werden die logischen Zusammenhänge von Aussagen untersucht, die im Rahmen einer Theorie der Wahrscheinlichkeitsrechnung Gültigkeit besitzen sollten. Für die bloße Einführung der Kolmogorov-Axiome ist diese Unterrichtsphase zwar nicht notwendig, allerdings bietet sich an dieser Stelle eine sehr gute Gelegenheit, lokales Ordnen zu üben.

Das Arbeitsblatt W KEIT3 listet zu Beginn neun elementare wahrscheinlichkeitstheoretische Aussagen auf, die Lernende mit einem schulüblichen Erfahrungsschatz stochastischer Aufgaben nachvollziehen und inhaltlich interpretieren können. Man kann dazu unter anderem auf Aufgabe 1 des Arbeitsblattes WKEIT 1 rekurrieren. Beispielsweise erfordert die Beantwotung der Frage Wie wahrscheinlich ist es, beim einfachen Wurf eines idealen Würfels eine gerade Zahl oder ein Primzahl zu werfen? die Verwendung der Regel (3) $P(A \cup B)=P(A)+P(B)-P(A \cap B)$.

Anschließend wird nach Zusammenhängen zwischen den Regeln gefragt und exemplarisch gezeigt, wie eine Aussage aus anderen herleitbar ist. Dabei sind die Deduktionen nicht kompliziert, die Art der Beweisführung - neben der Verwendung von ein oder zwei der übrigen Regeln benötigt man stellenweise einfache „,mengentheoretische Tricks“ wie

\footnotetext{
${ }^{15}$ Aufgabe 4 soll dabei aufzeigen, dass es auch stochastische Situationen gibt, in denen die Ergebnismenge theoretisch unendlich sein kann. Dieser Aspekt ist für den weiteren Verlauf der Unterrichtsreihe jedoch nicht relevant.
} 
die geschickte disjunkte Zerlegung einer Menge - jedoch ungewohnt, so dass gemeinsame Beispiele vorausgestellt und ggf. weitere Hilfsimpulse gegeben werden sollten. Die Ergebnisse dieser Erkundungsphase ${ }^{16}$ werden in einer Tabelle festgehalten:

\section{Logische Abhängigkeiten zwischen den neun Regeln}

\begin{tabular}{cc||c|c|c|c|c|c|c|c|c} 
& $\downarrow$ Regel ... kann man folgern aus $\rightarrow$ & 1 & 2 & 3 & 4 & 5 & 6 & 7 & 8 & 9 \\
\hline \hline 1 & $P(\Omega)=1$ & $\mathbf{0}$ & $\times$ & & & & & $\times$ & & \\
\hline 2 & $P(\emptyset)=0$ & $\times$ & $\mathbf{0}$ & & & & & $\times$ & & \\
\hline 3 & $P(A \cup B)=P(A)+P(B)-P(A \cap B)$ & & & $\mathbf{0}$ & $\times$ & & & & & \\
\hline 4 & $P(A \uplus B)=P(A)+P(B)$ & & $\times$ & $\times$ & $\mathbf{\square}$ & & & & & \\
\hline 5 & $P(A) \geq 0$ & & & & & $\mathbf{0}$ & $\times$ & $\times$ & & \\
\hline 6 & $P(A) \leq 1$ & & & & & $\times$ & $\mathbf{0}$ & $\times$ & & \\
\hline 7 & $P(\bar{A})=1-P(A)$ & $\times$ & & & $\times$ & & & $\mathbf{0}$ & & \\
\hline 8 & $A \subseteq B \Rightarrow P(B \backslash A)=P(B)-P(A)$ & & & & $\times$ & & & & $\mathbf{\square}$ & \\
\hline 9 & $A \subseteq B \Rightarrow P(A) \leq P(B)$ & & & & $\times$ & $\times$ & & & & $\mathbf{0}$ \\
\hline
\end{tabular}

In der Tabelle finden sich beispielsweise in der zu Regel (7) gehörenden Zeile Kreuze in den Spalten (1) und (4), was bedeutet, dass diese Regel aus den Regeln (1) und (4) hergeleitet werden kann. Die Endform der Tabelle ist jedoch nicht eindeutig, es ist Spielraum für unterschiedliche Beweiswege gegeben (siehe Seite $280 \mathrm{f}$. für eine Übersicht möglicher Herleitungen). Obige Tabelle zeigt das Gemeinschaftsergebnis einer Gruppe von Q1-Schülern, mit der eine frühere Version der Unterrichtsreihe erprobt wurde.

Anhand der fertig ausgefüllten Tabelle wird das Augenmerk der Lernenden darauf gelenkt, dass es nicht möglich ist, alle Aussagen zu beweisen, ohne einen Zirkelschluss zu erzeugen. Zur Lösung dieses Problems können einige Aussagen ohne Beweis an den Anfang gestellt und die restlichen aus diesen zugrunde gelegten Anfangsaussagen (= Axiomen) gefolgert werden. Es gibt verschiedene Überlegungen, an denen man sich bei einer solchen Auswahl orientieren kann:

- Die Regeln (4) und (7) werden besonders häufig zur Herleitung anderer Regeln verwendet und scheinen somit „,besonders grundlegend“ zu sein.

- Es gibt besonders eng verbundene Regeln, die wechselseitig zur Herleitung der jeweils anderen herangezogen werden (Regeln (1) und (2), (3) und (4) sowie (5) und (6)). Je eine dieser Regeln könnte als Axiom gewählt werden.

\footnotetext{
${ }^{16}$ Es bietet sich an, die Herleitungen in Partner- oder Gruppenarbeit finden zu lassen, so dass sich jedes Schülerteam mit ein oder zwei der neun Regeln befassen kann.
} 
- Es ist naheliegend, eher „einfache“ Regeln wie (4) als Axiome zu nehmen und daraus ,kompliziertere“ wie (3) herzuleiten.

- Umgekehrt könnte man jedoch argumentieren, dass Regel (4) ein Spezialfall von Regel (3) ist und somit in einer Folgerungskette erst später vorkommen sollte.

- Die Regeln (8) und (9) werden zur Herleitung anderer Regeln überhaupt nicht verwendet. Sie kommen daher als Axiom nicht in Frage.

Die Schülerinnen und Schüler stellen mit Hilfe solcher Überlegungen und der Tabelle verschiedene mögliche Axiomensysteme auf und deduzieren die jeweils restlichen Aussagen. Es fällt auf, dass mindestens drei Axiome benötigt werden, um jeweils alle übrigen Aussagen ableiten, also ein vollständiges ${ }^{17}$ Axiomensystem aufstellen zu können. Dabei gibt es verschiedene (äquivalente) Möglichkeiten, z. B. (1,4,5), (1,4,6) oder (4,5,7). Abbildung 5.3 zeigt, wie man diese Möglichkeiten mit Hilfe der bereits aus der Unterrichtsreihe zur Geometrie (siehe Abschnitt 5.2) bekannten Pfeilschemata entdecken kann. Das linke Schaubild basiert auf der Strategie, aus den Regelpaaren (1)/(2), (3)/(4) und (5)/(6) jeweils eine Regel als Axiom auszuwählen; das rechte Schema basiert auf der Strategie, die Regeln (4) und (7) aufgrund ihrer häufigen Verwendung bei den Deduktionen als Axiome zu wählen. Bei letzterem Vorgehen zeigt sich jedoch, dass nicht alle übrigen Regeln auf diese Weise hergeleitet werden können (die gestrichelte Linie deutet an, bis zu welcher Stelle man gelangt), so dass man eine weitere Regel als Axiom hinzunehmen muss.
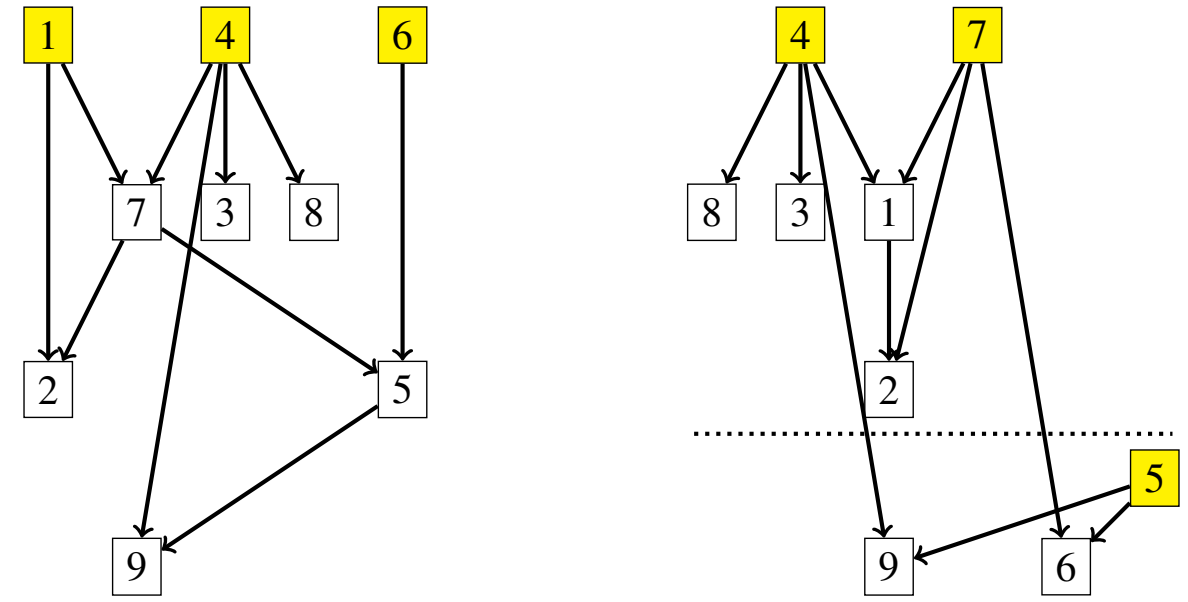

Abbildung 5.3: Mögliche Axiomensysteme mit zugehörigen deduktiven Gefügen

Auch Axiomensysteme von vier (oder mehr) Regeln sind möglich, jedoch ist man bestrebt, minimale Systeme zu finden, denn eine Aussage als Axiom zu setzen, obwohl man sie aus den übrigen Axiomen folgern könnte, ist unbefriedigend und unnötig.

\footnotetext{
${ }^{17}$ Vollständigkeit ist hier natürlich nur sehr eingeschränkt auf die neun behandelten Aussagen zu beziehen.
} 
Die Schülerinnen und Schüler erfahren so, dass eine gewisse Flexibilität (dieser Begriff beschreibt die Situation deutlich besser als „Willkür“) bei der Auswahl einer geeigneten axiomatischen Basis besteht. Das mathematische Resultat, nämlich die Herleitung aller übrigen Regeln, ist jedoch das gleiche.

\section{Phase 4: Deduktives Arbeiten mit den Kolmogorov-Axiomen (HIST7, WKEIT4+5)}

Nach der Auseinandersetzung mit selbst aufgestellten Axiomensystemen wird den Lernenden das Kolmogorov'sche Axiomensystem präsentiert, welches der Auswahl der Regeln (1), (4) und (5) entspricht:

\section{Die Kolmogorov-Axiome der Wahrscheinlichkeitsrechnung}

Es sei $\Omega$ eine endliche Menge. Eine Funktion $P$ bezeichnet man als Wahrscheinlichkeitsmaß auf $\Omega$, wenn sie jeder Teilmenge $A \subseteq \Omega$ eine reelle Zahl $P(A)$ zuordnet, die auch Wahrscheinlichkeit von $A$ genannt wird, und folgende Eigenschaften besitzt:

(K1) Es gilt $P(A) \geq 0$ für alle $A \subseteq \Omega$.

(K2) Es gilt $P(\Omega)=1$.

(K3) Sind zwei Teilmengen $A$ und $B$ aus $\Omega$ disjunkt (d.h. $A \cap B=\emptyset$ ), dann gilt

$$
P(A \cup B)=P(A)+P(B) .
$$

(K1) bis (K3) nennt man Kolmogorov-Axiome.

Dieser Zugang wird mit den beiden bekannten Definitionen aus Phase 1 verglichen: Während die Laplace-Definition und der frequentistische Ansatz zu klären versuchen, was Wahrscheinlichkeit (in bestimmten Anwendungssituationen) genau ist und zugleich Möglichkeiten anbieten, Wahrscheinlichkeiten zu berechnen, verzichtet Kolmogorov auf eine inhaltliche Interpretation des Begriffs und gibt „nur“ charakteristische Eigenschaften an, die Wahrscheinlichkeiten erfüllen sollen. Dabei ist die Wahl der Axiome natürlich durch die Eigenschaften der anwendungsorientierten Wahrscheinlichkeitsbegriffe geleitet. Zur Kontrastierung der inhaltlichen Definitionsversuche (insbesondere des frequentistischen Ansatzes) mit Kolmogorovs Zugang bietet sich die Lektüre des Textausschnittes HIST7 an, in dem Kolmogorov nicht nur seine Axiome vorstellt, sondern sich auch zum Verhältnis dieser zur Erfahrungswelt äußert und dabei explizit zum einen auf Hilberts Grundla- 
gen der Geometrie als Vorbild für seinen eigenen Zugang verweist als auch auf Richard von Mises hinsichtlich einer realweltlichen Interpretation der Axiome:

- In seiner Einleitung (Z.1-12) verweist Kolmogorov explizit auf Hilberts Grundlagen der Geometrie, so dass an dieser Stelle gut ein Rückbezug zur vorangegangenen Unterrichtsreihe hergestellt werden kann: Genau wie Hilbert die Geometrie (zunächst) losgelöst von der Anschauung aufbaut, möchte Kolmogorov die Wahrscheinlichkeitstheorie ohne konkrete inhaltliche Interpretation der relevanten Begriffe (Ereignis, Wahrscheinlichkeit) formulieren.

- Kolmogorovs Beschreibung der Axiome (Z. 13-24) stimmt nicht exakt mit der weiter oben präsentierten überein, da er auch die Axiome für das Mengensystem, welches als Definitonsbereich des Wahrscheinlichkeitsmaßes $P$ dient, angibt. Den Lernenden kann man an dieser Stelle mitteilen, das ein genaues Nachvollziehen von Kolmogorovs Konzept eines Mengenkörpers für diese Unterrichtsreihe nicht notwendig ist.

- Im zweiten Abschnitt des Textes (Z. 25-46) beschreibt Kolmogorov, wie man die abstrakt formulierten Axiome inhaltlich interpretieren könnte. Er verfolgt dabei den frequentistischen Ansatz und verdeutlicht so auch, welchen anwendungsorientierten Ursprung die Formulierung seiner Axiome hatte. Interessant ist jedoch vor allem sein Hinweis in Fußnote 3, dass die inhaltlichen Ausführungen zum Verhältnis der Axiome zur Erfahrungswelt für die weitere mathematische Darstellung der Wahrscheinlichkeitstheorie nicht relevant seien und daher von Lesern, die ausschließlich an letzterem interessiert sind, ausgelassen werden könnten.

Zur Verdeutlichung der Tatsache, dass es in der Mathematik eine typische Vorgehensweise ist, den Bezug zur Realität zum Zwecke der mathematischen Darstellung einer Theorie vorübergehend auszuklammern, bearbeiten die Lernenden nun die Arbeitsblätter W KEIT4 und WKEIT5 (oder zumindest ausgewählte Aufgaben), auf denen mit den KolmogorovAxiomen deduktiv gearbeitet wird. WKEIT4 konzentriert sich dabei auf relativ einfache Deduktionen und die Überprüfung der Wahrscheinlichkeitsmaß-Eigenschaft durch Überprüfung der Axiome (K1) bis (K3). Auf WKEIT5 werden die Begriffe der stochastischen Unabhängigkeit und der bedingten Wahrscheinlichkeit formal definiert und die nötigen Beweisschritte für den Satz von der totalen Wahrscheinlichkeit und den Satz von Bayes behandelt. $^{18}$

\footnotetext{
${ }^{18}$ Der Begriff der bedingten Wahrscheinlichkeit wird hier wie gewohnt über die Definition $P_{B}(A)=\frac{P(A \cap B)}{P(B)}$ für $P(B)>0$ eingeführt; die stochastische Unabhängigkeit wird (anders als im Schulunterricht üblich) über die Multiplikationsformel $P(A \cap B)=P(A) \cdot P(B)$ definiert. Der in Aufgabe 2b) von WKEIT5 beschriebene Zusammenhang zwischen stochastischer Unabhängigkeit und bedingter Wahrscheinlich-
} 
Die Schülerinnen und Schüler können anhand der entwickelten Deduktionen nachvollziehen, dass der Bezug zu realen Anwendungssituationen (etwa die Interpretation von Mengen als Ereignisse eines Zufallsexperiments) für die Beweisführung keine Rolle spielt, wenngleich Begriffe wie die der bedingten Wahrscheinlichkeit oder der stochastischen Unabhängigkeit ebenso wie die bewiesenen Sätze durch Anwendungsprobleme motiviert sind. Während sie im Rahmen des axiomatischen Ansatzes als inhaltsleere, rein formal definierte Begriffe bzw. Aussagen erscheinen, gewinnen sie erst in der Anwendung an Bedeutung. Um dies zu verdeutlichen, sollten die auf W KEIT5 behandelten Begriffe und Sätze auch im Rahmen einer typischen Anwendungsaufgabe aus dem Stochastikunterricht diskutiert werden (vor allem, falls die Lernenden diese Vorkenntnisse aus dem Regelunterricht noch nicht mitbringen).

Wichtig ist, mit den Lernenden zum Schluss noch einmal den Bogen zur Ausgangsfrage Was ist Wahrscheinlichkeit? zu schlagen und die behandelten Zugänge zum Wahrscheinlichkeitsbegriff vor diesem Hintergrund erneut zu diskutieren:

- Auch Kolmogorovs axiomatischer Zugang klärt letztlich nicht die Frage, was Wahrscheinlichkeit genau ist. Dies ist jedoch gar nicht sein Ziel. Mathematiker ,,vergessen“ manchmal bewusst den Bezug eines Begriffs zu realen Anwendungen, um sich ausschließlich auf die mathematische Beschreibung der logischen Zusammenhänge zwischen den einzelnen Aussagen eines Themengebiets konzentrieren zu können.

- Die Probleme, welche die inhaltlichen Definitionen des Wahrscheinlichkeitsbegriffs bereiten (siehe Phase 1), werden durch die abstrakt-axiomatische Darstellung nicht gelöst. Sie tauchen wieder auf, sobald man die Theorie wieder anwenden möchte. Schon eine einfache Frage wie Mit welcher Wahrscheinlichkeit erscheint beim zweifachen Würfelwurf ein Pasch? kann nicht allein auf Grundlage des axiomatischen Wahrscheinlichkeitsbegriffs beantwortet werden. In diesem Fall müssen zunächst Wahrscheinlichkeiten für die Elementarereignisse festgelegt werden, was jedoch

keit tritt im Rahmen des hier gewählten axiomatischen Zugangs als deduzierbare Folgerung auf. Im regulären Stochastikunterricht dient er in der Regel als Motivation und Definition des Unabhängigkeitsbegriffs, aus der wiederum die Multiplikationsformel folgt. Beide Definitionsansätze sind im Prinzip gleichwertig. Den Unterschied in der Reihenfolge kann man mit den Schülerinnen und Schülern kurz diskutieren und erörtern, welche Vorzüge die Definition über die Multiplikationsformel - trotz der geringeren intuitiven Klarheit - im Rahmen des deduktiven Aufbaus hat. So umfasst sie beispielsweise auch die Fälle $P(A)=0$ und $P(B)=0$, ist als ,Rechenregel“ einfacher handhabbar und die Symmetrie des Unabhängigkeitsbegriffs tritt klarer zutage (vgl. Büchter und Henn 2005, S. 170-171). Darüber hinaus birgt eine zu starke Betonung der Eigenschaft $P_{B}(A)=P(A)$ bzw. $P_{A}(B)=P(B)$ auch die Gefahr von Fehlvorstellungen. Stochastische Unabhängigkeit ist zu einem gewissen Grad ein theoretischer Begriff, der nicht mit kausaler oder realer Unabhängigkeit von Ereignissen gleichgesetzt werden darf. Es lässt sich bereits an sehr einfachen Zufallsexperimenten verdeutlichen, ,dass nicht zu jeder formal ermittelbaren Unabhängigkeit von Ereignissen auch eine entsprechende intuitive Vorstellung von der Unabhängigkeit dieser Ereignisse entwickelbar ist“" (Wolpers und Götz 2002, S. 163). 
von der jeweiligen Situation abhängig ist und daher eine inhaltliche Interpretation des Wahrscheinlichkeitsbegriffs erfordert.

- Man mag die Verwendung einer axiomatischen Begriffsbestimmung als Kapitulation der Mathematik vor dem Anspruch der Wirklichkeitsbeschreibung ansehen. Bestimmte inhaltliche Aspekte eines Begriffs lassen sich in einer mathematischen Definition einfach nicht fassen. Andererseits kann man es auch als fruchtbare Methode ansehen, inhaltliche Vorstellungen bei der mathematischen Darstellung einer Theorie für eine Weile auszuklammern. Im Falle des Wahrscheinlichkeitsbegriffs hat das den Vorteil, dass die in den Kolmogorov-Axiomen festgehaltenen Eigenschaften von Vertretern verschiedener Auffassungen von Wahrscheinlichkeit als formaler Rahmen akzeptiert und genutzt werden können. Die mathematische Theorie wird so gewissermaßen auf festen Grund gestellt.

Die beiden grundlegenden Sichtweisen auf den Wahrscheinlichkeitsbegriff lassen sich wie folgt in komprimierter Form beschreiben:

\section{Was ist Wahrscheinlichkeit? - Zwei Ansätze}

\section{Kolmogorovs Ansatz:}

- Kolmogorov fragt nicht „Was ist Wahrscheinlichkeit?“, sondern „Welche Eigenschaften (Axiome) sollen Wahrscheinlichkeiten erfüllen?“.

- Inhaltliche Vorstellungen zu den Begriffen sind für die mathematische Darstellung nicht nötig; die Begriffe sind rein formal definiert.

- Die Sätze der Wahrscheinlichkeitstheorie lassen sich allein aus den Axiomen beweisen.

\section{Inhaltlicher Ansatz (Laplace, von Mises):}

- Die Begriffe sind bereits in den Definitionen eng mit Anwendungssituationen verknüpft.

- Möchte man konkrete Anwendungsaufgaben lösen, muss man den Wahrscheinlichkeitsbegriff inhaltlich interpretieren. 


\subsection{Unterrichtsreihe 3: Zahlbereichserweiterungen}

\subsubsection{Sachanalyse}

Zahlbereichserweiterungen haben in der Geschichte der Mathematik stets eine herausragende Rolle gespielt. Für die Mathematik ,als eine deduktiv geordnete Welt eigener Art“ (Winter 1995, S. 37) sind diese Erweiterungen „,[v]on fundamentaler intellektueller aber auch sehr praktischer Bedeutung“ (Winter 1995, S. 40). Die Prozesse zur Anerkennung neuer Zahlbereiche innerhalb der mathematischen Gemeinschaft waren dabei jedoch immer langwierig und und stießen auf viel Widerstand, waren sie doch mit grundlegenden Vorstellungsumbrüchen hinsichtlich bis dato gewohnter Anschauungen und bekannter Rechenregeln verbunden (siehe auch Malle 2007c).

Die vorliegende Unterrichtsreihe beschäftigt sich mit der Entstehung der komplexen Zahlen. ${ }^{19}$ Diese tauchen erstmals im 16. Jahrhundert $n$. Chr. bei Versuchen auf, kubische und biquadratische Gleichungen zu lösen. Bei den damals entwickelten Lösungsformeln entstehen als Zwischenergebnisse Terme, die Wurzeln aus negativen Zahlen enthalten. Beispielsweise erhält man mit den von dem Italiener Girolamo Cardano (1501-1576) veröffentlichten Lösungsformeln für kubische Gleichungen der Form $x^{3}+p x+q=0$ solche „seltsamen“ Ergebnisse, die man unter der Annahme der Gültigkeit bestimmter Rechenregeln aber zu einem sinnvollen reellwertigen Ergebnis weiter umformen kann. Diese zunächst auf rein formal-arithmetischer Ebene bestehende Nützlichkeit von Wurzeln aus negativen Zahlen führte zumindest dazu, dass sich Mathematiker weiter mit ihnen beschäftigten und ihre Existenz durch eine geometrische Veranschaulichung zu rechtfertigen versuchten, wenngleich die Anerkennung als eigenständige Zahlen durch die mathematische Gemeinschaft noch lange auf sich warten ließ. Bezeichnungen wie „sophistische“, „unmögliche“, „falsche“, „eingebildete“ oder ,imaginäre“ Zahlen (Malle 2007c, S. 10) zeugen von dem Unbehagen, das viele Mathematiker bei der Auseinandersetzung mit diesen neuen mathematischen Objekten verspürt haben müssen.

Neben der offensichtlichen Nützlichkeit imaginärer Zahlen beim Rechnen sowie bei der möglichst einfachen Darstellung von Gleichungslösungen ${ }^{20}$ trug vor allem ihre Visualisierung in der Zahlenebene durch Gauß erheblich zu ihrer Akzeptanz bei (siehe auch Alten et al. 2014, S. 331-332).

\footnotetext{
${ }^{19}$ Ein Abriss der wesentlichen historischen Entwicklungen findet sich in Alten et al. 2014, S. 270-283.

${ }^{20}$ Nicht nur kann im Falle quadratischer Gleichungen auf die Positivität der Diskriminante verzichtet werden. Die Verwendung imaginärer Größen gipfelte schließlich im Fundamentalsatz der Algebra: Jede Gleichung der Form $z^{n}+a_{n-1} \cdot z^{n-1}+\ldots+a_{1} \cdot z+a_{0}=0$ hat (inkl. Vielfachheiten) genau $n$ Lösungen (siehe auch Alten et al. 2014, S. 303-309, 355-359).
} 
Eine „logisch einwandfreie arithmetische Begründung der komplexen Zahlen“ (Alten et al. 2014, S. 333) lieferte schließlich William Rowan Hamilton (1805-1865). Er definierte eine komplexe Zahl als Zahlentupel $(a, b)$ mit reellen Komponenten $a$ und $b .{ }^{21}$ Addition und Multiplikation lassen sich dann wie folgt definieren:

$$
\begin{gathered}
(a, b)+(c, d):=(a+c, b+d) \\
(a, b) \cdot(c, d):=(a c-b d, a d+c b)
\end{gathered}
$$

Mit der Notation $a+b \cdot i:=(a, b)$, welche die Identifikation einer reellen Zahl $a$ mit dem Zahlenpaar $(a, 0)$ sowie der imaginären Zahl $i$ mit dem Zahlenpaar $(0,1)$ impliziert, lässt sich dies intuitiv schreiben als

$$
\begin{gathered}
(a+b \cdot i)+(c+d \cdot i)=(a+c)+(b+d) \cdot i \\
(a+b \cdot i) \cdot(c+d \cdot i)=(a c-b d)+(a d+c b) \cdot i
\end{gathered}
$$

wobei $i^{2}=-1$ ausgenutzt wird, was sich wiederum in der Form $(0,1) \cdot(0,1)=(-1,0)$ leicht aus der Definition der Multiplikation herleiten lässt. Hamiltons Trick bestand also darin, die Rechenverknüpfungen für die Zahlenpaare so zu wählen, dass sie dem intuitiven Rechnen in der Form $a+b \cdot i$ entsprechen.

\subsubsection{Didaktische Anmerkungen}

Zunächst ist anzumerken, dass das Thema Zahlbereichserweiterungen in der Regel nicht im Kontext der Axiomatik behandelt wird (wenn man von der Charakterisierung der natürlichen Zahlen durch die Dedekind-Peano-Axiome absieht; siehe hierzu beispielsweise Hischer 2012, S. 207-238).

Bei der Einführung neuer Zahlbereiche sprechen Mathematiker von Definitionen oder Konstruktionen: Die ganzen Zahlen werden als Menge von Äquivalenzklassen auf $\mathbb{N} \times \mathbb{N}$ bezüglich der Äquivalenzrelation $(a, b) \sim(c, d): \Leftrightarrow a+d=c+b$ definiert, wie auch die komplexen Zahlen als Zahlentupel $(a, b) \in \mathbb{R}^{2}$ definiert werden. Das hängt damit zusammen, dass die jeweiligen Zahlbereiche unter Rückgriff auf bereits bekannte Zahlbereiche charakterisiert werden, wohingegen bei ,echten“ axiomatischen Zugängen die Grundbegriffe durch die Axiome implizit definiert werden.

\footnotetext{
${ }^{21}$ Die anderen gängigen Charakterisierungen der komplexen Zahlen als Faktorring $\mathbb{R}[X] /\left(X^{2}+1\right)$ bzw. über Matrizen der Form $\left(\begin{array}{cc}a & -b \\ b & a\end{array}\right) \in \mathbb{R}^{2 \times 2}$ spielen im Rahmen dieser Arbeit keine Rolle.
} 
Ich habe mich dennoch dazu entschieden, eine Unterrichtsreihe aus diesem Themengebiet zu konzipieren, da das Vorgehen bei der Anerkennung neuer Zahlbereiche gerade in den Anfangsphasen viel mit axiomatischen Aspekten zu tun hat. Freudenthal spricht vom algebraischen Prinzip:

Gewisse Symbolde [...] deuten ein Objekt nicht explizit sondern implizit an, ihre Bedeutung rührt, wenigstens anfangs, ganz daher, daß man in bestimmter Weise mit ihnen rechnet. [...] Man nenne die unbekannte Größe $x$, stelle die Bedingungen fest, denen sie genügt, und rechne mit $x$ nach den üblichen Rechengesetzen und so, als ob es die Bedingungen erfüllt [...].

Es ist das eine typische algebraische Art des Inter- und Extrapolierens, ich möchte sagen, das formale oder axiomatische Inter- und Extrapolieren; man hat bei gewissen Operationen gewisse Gesetze konstatiert und verlangt, daß diese Gesetze bei der Erweiterung erhalten bleiben. Die „Permanenz der Rechengesetze" ist eine natürliche Forderung, und historisch war es auch so, daß über die Art der Fortsetzung mit Permanenzargumenten entschieden wurde, und daß die Fortsetzung ins Komplexe sich dadurch verzögerte, daß da gewisse Gesetze (die Ordnungseigenschaften) ihre Gültigkeit verlieren müssen. (Freudenthal 1973a, S. 210-212)

Das hier beschriebene Vorgehen weist Ähnlichkeiten mit dem Prinzip der extrinsischen Rechtfertigung auf. Es werden Forderungen aufgestellt, die (bezogen auf den neuen Zahlbereich) nicht den Charakter evidenter Tatsachen haben, sondern als hypothetische Setzungen aufgefasst werden können, und über deren Akzeptanz auf Grundlage der aus den Forderungen folgenden Konsequenzen entschieden wird (z. B.: Weist der neue Zahlbereich eine Körper-Struktur auf? Lässt er sich auf andere mathematische Gebiete anwenden?). Das Themenfeld Zahlbereichswerweiterungen bietet sich also besonders gut zur Verdeutlichung innermathematischer Rechtfertigungsprozesse an. Die Einführung der komplexen Zahlen bietet dabei den zusätzlichen Vorteil, dass Schülerinnen und Schüler in der Regel noch keine anschauliche Vorstellung von diesen Zahlen haben und die erste Begegnung damit ganz natürlich auf einer rein formalen Ebene stattfindet. Damit steht eine evidenzbasierte Begründung der Ausgangsforderungen nicht zur Verfügung und eine Rechtfertigung kann zunächst nur über die algebraische Nützlichkeit der neuen Zahlen erfolgen.

Um die Diskussion der Sinnhaftigkeit einer Setzung wie $i^{2}=-1$ zusätzlich zu fördern, wird - nach einer kurzen Einstiegsphase anhand zweier fiktiver Lösungen einer quadratischen Gleichung - ein historischer Ansatz für die Beschäftigung mit den komplexen Zahlen gewählt (siehe auch Malle 2007b): Das Hauptaugenmerk liegt auf dem Versuch, kubische Gleichungen mit Hilfe der Cardanischen Formel zu lösen. Der Vorteil gegenüber einer Motivation über quadratische Gleichungen besteht darin, dass die Lernenden leicht 
einsehen können, dass kubische Gleichungen immer eine reelle Lösung haben müssen und man (bei geeigneter Wahl der Parameter der Gleichung) mit überschaubarem algebraischen Aufwand nachweisen kann, dass die Lösungsterme der Cardanischen Formel, in denen mitunter Wurzeln aus negativen Zahlen vorkommen, einer reellwertigen Lösung entsprechen. Dadurch lassen sich stärkere Argumente für die Beschäftigung mit imaginären Zahlen formulieren, während bei quadratischen Gleichungen leichter der Eindruck entstehen kann, es handele sich dabei nur um eine algebraische Spielerei.

Die Visualisierung in der Gauß'schen Zahlenebene sowie Hamiltons Definition über geordnete Zahlenpaare werden in der Unterrichtsreihe in Form eines Lehrervortrags behandelt, um die letzten Schritte zur Akzeptanz der komplexen Zahlen aufzuzeigen, ansonsten jedoch nicht intensiver behandelt. Stattdessen soll das kalkülhafte Verwenden von bekannten Rechenregeln anhand weiterer Zahlbereichserweiterungen vertieft werden:

Eine wesentliche gedankliche Hürde beim Übergang von den natürlichen (oder den positiven rationalen) zu den negativen Zahlen ist die Begründung, warum die Multiplikation zweier negativer Zahlen eine positive Zahl ergibt. In der didaktischen Literatur werden zahlreiche Möglichkeiten diskutiert, die Einführung der negativen Zahlen und der zugehörigen Rechenregeln in der Sekundarstufe I anschaulich und kontextgebunden zu behandeln (siehe vom Hofe und Hattermann 2014, Hußmann und Schindler 2014, Pallack 2014 oder Malle 2007a). Ich möchte das Für und Wider der einzelnen Ansätze hier nicht diskutieren. Aus fachlicher Sicht und auch im Zusammenhang mit dem Thema Axiomatik ist der formal-abstrakte Ansatz über das Permanenzprinzip aber grundlegend (siehe auch Walcher und Wittmann 2012). Daher soll in der konzipierten Unterrichtsreihe der logisch-deduktive Zusammenhang zwischen der Regel minus mal minus ergibt plus und der Forderung der Gültigkeit des Distributivgesetzes herausgestellt werden.

Abschließend soll der Versuch, die Durchführbarkeit der - sonst häufig und fälschlicherweise als ,verboten“ deklarierten - Division durch Null für einen (hypothetischen) neuen Zahlbereich zu fordern, ein Beispiel liefern, bei dem zumindest aus Sicht der Schulmathematik eine Rechtfertigung der Ausgangsforderungen sehr problematisch wird. 


\subsubsection{Beschreibung der Unterrichtsreihe}

\begin{tabular}{ccc} 
Phase & Thema (inkl. wesentlicher Tätigkeiten/Erkenntnisse) & ABs / Texte \\
\hline \hline 1 & Einstieg & ZBE1 \\
- & anhand zweier fiktiver Schülerlösungen einer \\
& quadratischen Gleichung erste Argumente für und \\
& \\
& gegen das Rechnen mit Wurzeln aus negativen Zahlen \\
& sammeln
\end{tabular}

2 Lösung kubischer Gleichungen

ZBE2, ZBE3, ZBE4

- anhand der Auseinandersetzung mit kubischen

Gleichungen weitere Argumente für und gegen das

Rechnen mit Wurzeln aus negativen Zahlen sammeln

- rückblickend die (innermathematische) Nützlichkeit der komplexen Zahlen als Rechtfertigung für Setzungen wie $i^{2}=-1$ nachvollziehen

$3 \quad$ Negative und bekloppte Zahlen

ZBE5

- den Zusammenhang zwischen der Regel minus mal minus ergibt plus und der Gültigkeit des

Distributivgesetzes erkennen

- auf Grundlage des Versuches, mit einer bekloppten Zahl $b$ mit der Eigenschaft $0 \cdot b=1$,wie gewohnt“ zu rechnen, zusammenfassend die Möglichkeiten und Grenzen von rein formalen Setzungen in der Mathematik diskutieren

\section{Phase 1: Einstieg (ZBE1)}

Zu Beginn der Unterrichtsreihe werden die Lernenden auf ZBE1 mit zwei unterschiedlichen (fiktiven) Schülerlösungen der quadratischen Gleichung $x^{2}+x+3=0$ konfrontiert. Während die erste Bearbeitung die Gleichung als unlösbar deklariert, da die Diskriminante kleiner als 0 ist, wird in der zweiten Bearbeitung mit den mittels $p$ - $q$-Formel erhaltenen Ausdrücken $-\frac{1}{2}+\sqrt{-\frac{11}{4}}$ und $-\frac{1}{2}-\sqrt{-\frac{11}{4}}$,einfach die Probe gemacht" und es stellt sich heraus, dass sie tatsächlich die Gleichung erfüllen. Anhand dieser Situation werden in einer ersten Diskussionsphase Argumente für und gegen die Verwendung von Wurzeln aus negativen Zahlen gesammelt: 
- Für die Verwendung spricht, dass bisher unlösbare Gleichungen lösbar werden, wenn man mit Wurzeln aus negativen Zahlen ,wie gewohnt“ rechnet und die Wurzel beim Quadrieren ,einfach wegfallen lässt““.

- Gegen die Verwendung spricht, dass die gefundenen Lösungen sich geometrisch nicht als Nullstellen des Graphen der zugehörigen Parabel visualisieren lassen und dass bisher aus dem Mathematikunterricht die Regel bekannt ist, dass für eine Zahl $x$ stets $x^{2} \geq 0$ gilt.

Zu diesem Zeitpunkt sollte die Sinnhaftigkeit der Verwendung zumindest noch stark angezweifelt werden, da über die bloße arithmetische Möglichkeit des kalkülhaften Rechnens hinaus keine erkennbaren Vorteile bestehen.

\section{Phase 2: Lösung kubischer Gleichungen (ZBE2+3(+4))}

Als historische Einführung in die Thematik dieser Phase lesen die Schülerinnen und Schüler den Text auf ZBE2. Es handelt sich dabei um einen mathematikhistorischen Exkurs, der die Bemühungen einiger italienischer Mathematiker um die Lösung kubischer Gleichungen schildert. Die historischen Fakten sind größtenteils den Ausführungen von Alten et al. (2014, S. 270-278) entnommen.

Anschließend führt das Arbeitsblatt ZBE3 die Lernenden schrittweise an die Erkenntnis heran, dass man mit Wurzeln aus negativen Zahlen nicht nur „wie gewohnt“ rechnen kann, sondern dabei in bestimmten Fällen sogar sinnvolle, reellwertige Lösungen entstehen. Aufgabe 1 dient dabei vorwiegend als Wiederholung: Durch systematisches Probieren und/oder Raten bzw. genaues Hinschauen sollen Lösungen ausgewählter kubischer Gleichungen ermittelt werden (Teil a). Ferner werden zu den auf ZBE2 erwähnten historischen Textaufgaben die zugehörigen Gleichungen aufgestellt (Teil b). In Aufgabenteil c beschäftigen sich die Lernenden mit der Tatsache, dass man bei den in Teil $b$ aufgestellten kubischen Gleichungen durch Ausprobieren einzelner Werte oder Raten keine Lösung findet. Man kann aus dem Schulwissen über das Unendlichkeitsverhalten von Polynomfuntkionen dritten Grades jedoch anschaulich schließen, dass kubische Gleichungen immer mindestens eine reelle Lösung haben müssen. Dies ist ein wesentlicher Unterschied zu quadratischen Gleichungen, bei denen die Frage nach einer Lösung unter bestimmten Rahmenbedingungen (Diskriminante $<0$ ) anschaulich überhaupt keinen Sinn ergibt.

Die Cardanische Lösungsformel wird auf diesem Arbeitsblatt zunächst einfach präsentiert und in Aufgabe 2 auf eine Reihe von kubischen Gleichungen angewendet. ${ }^{22}$ Die

\footnotetext{
${ }^{22}$ Das Aufgabenblatt ZBE4 behandelt schrittweise den Beweis der Cardanischen Formel und ist als Zusatz
} 
restlichen Aufgaben behandeln ausgewählte kubische Gleichungen, bei denen sich die zunächst kompliziert aussehenden Lösungsterme, die sich aus der Cardanischen Formel ergeben, mit überschaubarem algebraischen Aufwand vereinfachen lassen. Jede Aufgabe soll dabei eine bestimmte Erkenntnis fördern, die im Anschluss an die Bearbeitung im Plenum formuliert und diskutiert wird:

- Aufgabe 3: Die Cardanische Formel liefert korrekte reellwertige Ergebnisse, auch wenn man dies der Darstellung auf den ersten Blick nicht ansieht (Hinweis: In dieser Aufgabe - wie auch in den bisherigen Aufgaben - treten in den Lösungstermen noch keine Quadratwurzeln aus negativen Zahlen auf).

- Aufgabe 4: In bestimmten Fällen treten in dem Lösungsterm der Cardanischen Formel Quadratwurzeln aus negativen Zahlen auf (und zwar bei $\left(\frac{q}{2}\right)^{2}<\left(\frac{p}{3}\right)^{2}$ ), obwohl das Gesamtergebnis offensichtlich eine reelle Zahl sein muss.

- Aufgaben 5+6: Wenn man in solchen Fällen mit den Quadratwurzeln ,wie gewohnt" rechnet und dabei $\sqrt{x}^{2}=x$ unabhängig vom Vorzeichen von $x$ ausnutzt, erhält man sinnvolle, reelle Ergebnisse.

Diese Erkenntnisse werden die Zweifel an der Existenz von Wurzeln aus negativen Zahlen bei den Schülerinnen und Schülern nicht komplett ausräumen, doch sollte nach Bearbeitung dieser Aufgaben ein geschärftes Bewusstsein für deren Nützlichkeit entstanden sein: Wenn man Zahlen der Form $\sqrt{-a}$ mit $a>0$ (zumindest vorübergehend) akzeptiert und die gewohnten Rechenregeln auf sie anwendet, erzielt man deutliche Fortschritte beim Lösen von Gleichungen.

Uneingeschränkt kann man bekannte Rechenregeln dabei allerdings nicht anwenden, wie die folgenden beiden Beispiele verdeutlichen:

(i) $-1=\sqrt{-1}^{2}=\sqrt{-1} \cdot \sqrt{-1}=\sqrt{(-1) \cdot(-1)}=\sqrt{1}=1$

(ii) Ist $\sqrt{-1}$ eigentlich größer oder kleiner als 0 ?

- Angenommen, $\sqrt{-1}>0$.

Dann folgt $-1=\sqrt{-1} \cdot \sqrt{-1}>0 \cdot \sqrt{-1}=0$.

- Angenommen, $\sqrt{-1}<0$, also $-\sqrt{-1}>0$.

Dann folgt: $-1=(-\sqrt{-1}) \cdot(-\sqrt{-1})>0 \cdot(-\sqrt{-1})=0$.

für Schülerinnen und Schüler gedacht, die an einer Herleitung der Formel interessiert sind. Es hat für die Ziele der Unterrichtsreihe jedoch keine Relevanz und kann somit bei der Durchführung weggelassen werden. 
Im ersten Fall wird deutlich, dass die Regel $\sqrt{a} \cdot \sqrt{b}=\sqrt{a \cdot b}$ für negative $a$ und $b$ nicht wie gewohnt gültig ist, im zweiten Fall werden grundlegende Eigenschaften der Ordnungsrelation < verletzt. Neben die offensichtliche Nützlichkeit beim Lösen kubischer Gleichungen treten also recht grundlegende Probleme in anderen Bereichen. Die bisherigen Vor- und Nachteile des Umgangs mit Wurzeln aus negativen Zahlen werden in folgender Übersicht zusammengefasst:

\section{Rechnen mit Wurzeln aus negativen Zahlen: Sinnvoll?}

\section{Pro:}

- Wenn man „wie gewohnt“ rechnet, lassen sich bisher unlösbare Gleichungen lösen.

- Mit der Cardanischen Formel erhält man korrekte reelle Ergebnisse, auch wenn man zwischenzeitlich mit Wurzeln aus negativen Zahlen rechnet.

- Im Falle kubischer Gleichungen kann man die - zunächst kompliziert aussehenden - Lösungsterme nach Vereinfachung sogar geometrisch als Nullstellen eines Polynoms dritten Grades deuten.

\section{Contra:}

- Bisher galt: Quadrate von Zahlen sind immer positiv.

- Eine „Lösung“ quadratischer Gleichungen mit negativer Diskriminante ergibt anschaulich keinen Sinn.

- Es können beim Rechnen Widersprüche auftreten, z. B.:

$$
-1=(\sqrt{-1})^{2}=\sqrt{-1} \cdot \sqrt{-1}=\sqrt{(-1) \cdot(-1)}=\sqrt{1}=1
$$

- Wurzeln aus negativen Zahlen können nicht angeordnet werden, d. h. es gilt weder $\sqrt{-1}<0$ noch $\sqrt{-1}>0$.

Um weitere, wichtige Gründe für die Tatsache zu nennen, dass die komplexen Zahlen sich letztlich als Erweiterung der reellen Zahlen durchgesetzt haben, werden den Lernenden in einem Impulsvortrag die abschließenden historischen Etappen vorgestellt: 


\section{Kurze Geschichte der komplexen Zahlen}

Als Kurzschreibweise für eine Zahl, deren Quadrat - 1 ergibt, hat man sich anstelle von $\sqrt{-1}$ auf den Buchstaben $i$ (für imaginär) geeinigt. Zahlen der Form $a+b \cdot i$ mit $a, b \in \mathbb{R}$ nennt man komplexe Zahlen.

CARL FRIEDRICH GAUSS (1777-1855) fand eine geometrische Deutung für komplexe Zahlen als Punkte bzw. Pfeile in der Zahlenebene:

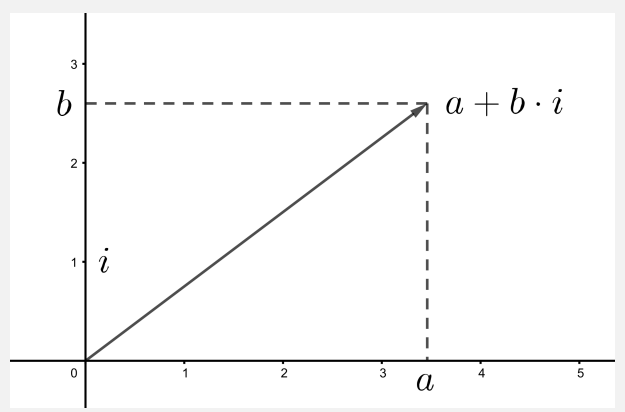

Damit lässt sich beispielsweise die Addition komplexer Zahlen geometrisch deuten:

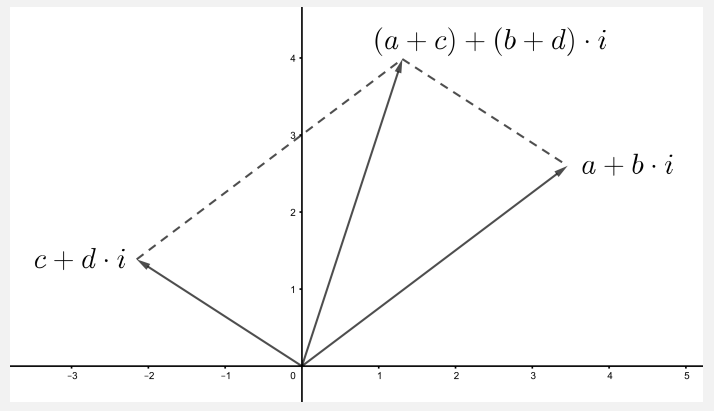

WILLIAM ROWAN HAMILTON (1805-1865) definierte komplexe Zahlen schließlich als geordnete Zahlenpaare $(a, b)$, auf denen Addition und Multiplikation wie folgt definiert sind:

$$
\begin{gathered}
(a, b)+(c, d)=(a+c, b+d) \\
(a, b) \cdot(c, d)=(a c-b d, a d+c b)
\end{gathered}
$$

Mit der Identifikation $(a, b):=a+b \cdot i$ (insb. also $(a, 0):=a$ und $(0,1):=i$ ) entspricht dies dem gewohnten Rechnen:

$$
\begin{gathered}
(a+b \cdot i)+(c+d \cdot i)=(a+c)+(b+d) \cdot i \\
(a+b \cdot i) \cdot(c+d \cdot i)=(a c-b d)+(a d+c b) \cdot i
\end{gathered}
$$


Das Vorgehen bei der Begründung der komplexen Zahlen kann rückblickend wie folgt beschrieben werden:

\section{Rechtfertigung der komplexen Zahlen}

- Komplexe Zahlen tauchen zunächst auf rein formaler Ebene als Wurzeln aus negativen Zahlen beim Lösen bestimmter Gleichungen auf. Eine anschauliche Vorstellung davon existiert zunächst nicht.

- Man postuliert vorübergehend, dass Zahlen, deren Quadrat negativ ist, existieren und man mit ihnen ,wie gewohnt“ rechnen kann.

- Man überprüft, welche Konsequenzen sich aus der mathematischen Arbeit mit komplexen Zahlen ergeben.

- Die Nützlichkeit (beispielsweise beim Lösen von Gleichungen oder der Verknüpfung von geometrischen und algebraischen Aspekten) überwiegt schließlich und die komplexen Zahlen werden als eigenständige mathematische Objekte anerkannt.

Wenngleich für die finale Akzeptanz durch die mathematische Gemeinschaft die geometrische Visualisierung wie auch die Definition mittels geordneter Zahlenpaare ausschlaggebend waren, so stellt das anfängliche kalkülhafte Rechnen mit formalen Symbolen, an die man aus ganz praktischen Gründen gewisse Forderungen stellt, doch eine wesentliche mathematische Tätigkeit dar.

\section{Phase 3: Negative und bekloppte Zahlen (ZBE5)}

Das in der vorherigen Phase kennengelernte Vorgehen zur Rechtfertigung rein formal eingeführter Zahlsymbole soll in der dritten und letzten Phase vertieft werden, indem es auf andere Zahlbereichserweiterungen angewendet und in diesem Zusammenhang diskutiert wird. Auf ZBE5 wird zunächst die den Schülerinnen und Schülern bekannte Regel beleuchtet, dass minus mal minus gleich plus ist. Dazu wird zum einen eine schulbuchtypische Begründung über Permanenzreihen vorgestellt (siehe z. B. Lergenmüller und Schmidt 2007, S. 27 oder Pallack 2013, S. 199) und anschließend mit einer Begründung ähnlich dem Vorgehen bei komplexen Zahlen verglichen (siehe auch Winter 1983a, S. 192). Ziel ist es, in beiden Fällen die impliziten Forderungen herauszuarbeiten, die in die jeweiligen Begründungen einfließen. Beide Begründungen sind nämlich eng mit der Forderung der fortwährenden Gültigkeit des Distributivgesetzes verknüpft. 
Während diese Forderung im zweiten Beispiel mehr oder weniger offensichtlich im Rechenschritt $g^{2}+g+1=g \cdot(g+1)$ zu Tage tritt, ist sie im Permanenzreihen-Argument nur versteckt enthalten. Sie wird jedoch deutlich, wenn man danach fragt, warum die gewählte Fortsetzung der Permanenzreihe - abgesehen von einem rein strukturell-ästhetischen Empfinden - logisch und sinnvoll ist. Betrachten wir hierzu die beiden Gleichungen $(-3) \cdot 1=-3$ und $(-3) \cdot(-1)=+3$, aus denen folgt:

$$
(-3)+3=(-3) \cdot 1+(-3) \cdot(-1) \stackrel{!}{=}(-3) \cdot(1+(-1))=(-3) \cdot 0=0
$$

Es muss das Distributivgesetz gelten, damit die Gleichungskette korrekt ist. Es gibt hier logisch betrachtet zwei Argumentationsrichtungen:

\section{Rechtfertigung der Regel minus mal minus ergibt plus}

(i) Wenn $(-) \cdot(-)=(+)$ festgelegt wird, so ist die Gültigkeit des Distributivgesetzes eine logische Konsequenz dieser Forderung.

(ii) Wenn umgekehrt die Gültigkeit des Distributivgesetzes gefordert wird, so ist $(-) \cdot(-)=(+)$ eine logische Konsequenz dieser Forderung.

In beiden Fällen kann man den Lernenden verdeutlichen, dass bei der Begründung neuer Zahlbereiche Forderungen an diese aufgestellt werden, die wiederum zu ganz bestimmten Konsequenzen führen, deren Nützlichkeit bzw. Erwünschtheit man zur Bewertung und schließlich Rechtfertigung der ursprünglichen Forderungen heranzieht.

Es wurden bisher zwei Beispiele behandelt, in denen neue Zahlen durch die rein formale Forderung der Gültigkeit bestimmter Eigenschaften begründet wurden. Die Frage liegt nahe, ob man in der Mathematik nach Belieben Eigenschaften für potentielle neue Zahlen einfach definieren kann. In Aufgabe 3 werden die Schülerinnen und Schüler mit der Forderung konfrontiert, es solle eine sogenannte bekloppte Zahl $b$ geben, welche $\frac{1}{0}=b$ bzw. $0 \cdot b=1$ erfüllt. $^{23}$ Was geschieht, wenn man mit $b$ versucht, ,,wie gewohnt“ zu rechnen? Einige Beispiele genügen, um zu verdeutlichen, dass die auftretenden Probleme ungleich schwerwiegender sind als in den bisher behandelten Fällen:

\footnotetext{
${ }^{23}$ Auf diese Idee hat mich ein MathePlus-Zusatzkurs des Couven-Gymnasiums gebracht, in dem ich frühere Versionen meiner Unterrichtsideen erprobt habe. Auch die Bezeichnung bekloppte Zahl für $b$ stammt von den Schülerinnen und Schülern.
} 
Konsequenzen der Zulassung einer bekloppten Zahl b

(i) $b^{2}=b \cdot b=\frac{1}{0} \cdot \frac{1}{0}=\frac{1 \cdot 1}{0 \cdot 0}=\frac{1}{0}=b$

(ii) $1=0 \cdot b=(0+0) \cdot b=0 \cdot b+0 \cdot b=1+1=2$

(iii) $1=0 \cdot b=(0 \cdot 0) \cdot b=0 \cdot(0 \cdot b)=0 \cdot 1=0$

Während man bei (i) noch sagen kann, dass die hier durchgeführte Rechnung eben eine Eigenschaft der bekloppten Zahl $b$ beschreibt, zeigen die Rechnungen in (ii) und (iii), dass sowohl das Distributivgesetz als auch das Assoziativgesetz für bekloppte Zahlen nicht gelten können, da man sonst Widersprüche erhält. Obwohl es in der Mathematik natürlich Bereiche gibt, in denen mit der Gleichung $\frac{1}{0}=\infty$ (nicht aber $0 \cdot \infty=1$ ) gearbeitet wird, liegt aus Schülersicht an dieser Stelle doch zumindest die Vermutung nahe, dass man mit der bekloppten Zahl $b$ eine formale Setzung hat, die man rein mathematisch zwar tätigen kann, die aber aufgrund mangelnder Nützlichkeit wenig Sinn ergibt.

\subsection{Erfahrungen aus der Umsetzung mit Schülergruppen}

Die konzipierten Unterrichtsmaterialien wurden in unterschiedlichen Entwicklungsstadien in Oberstufen-Zusatzkursen des Couven-Gymnasiums Aachen pilotiert und final im Rahmen einer Workshop-Woche an der RWTH erprobt. Über die Pilotierungen soll an dieser Stelle nur kurz berichtet werden. Durch sie hat sich die grundsätzliche inhaltliche Ausrichtung der Unterrichtsreihen nicht geändert; es wurden lediglich einzelne ThemenBausteine ergänzt und das konzeptionelle Format leicht angepasst.

\subsubsection{Pilotierung}

Im Rahmen einer Kooperation mit dem Couven-Gymnasium in Aachen bestand die Möglichkeit, Vorformen der in den vorherigen drei Abschnitten beschriebenen Unterrichtsreihen in Projektkursen der Jahrgangsstufe 11 (Q 1) zu erproben. Es handelte sich überwiegend um in überdurchschnittlichem Maße an Mathematik interessierte Schülerinnen und Schülern, die sich freiwillig für die Teilnahme an dem jeweiligen Projektkurs entschieden hatten. Folgende Tabelle gibt einen Überblick über den zeitlichen Rahmen der Erprobungen. 


\begin{tabular}{|l|l|l|}
\hline Unterrichtsreihe & Dauer & Schülerzahl \\
\hline \hline Wahrscheinlichkeitsrechnung & $3 \times 90 \mathrm{~min}$ & $13 / 15$ \\
Geometrie & $4 \mathrm{~h}$ (Workshoptag) & 12 \\
Komplexe und „bekloppte“ Zahlen & $2 \times 90 \mathrm{~min}$ & 14 \\
\hline
\end{tabular}

Die Unterrichtsreihe zur Wahrscheinlichkeitsrechnung wurde im Sommer 2013 in zwei Projektkursen erprobt, die anderen beiden Unterrichtsreihen wurden unmittelbar hintereinander im Sommer 2014 in einem Projektkurs durchgeführt, wobei der Geometrie-Teil als Workshoptag an der RWTH Aachen stattfand. Die behandelten Inhalte sowie die auf Grundlage der Erprobungen vorgenommen Änderungen und Ergänzungen werden im Folgenden kurz erläutert.

Wahrscheinlichkeitsrechnung: Die im Sommer 2013 erprobte Unterrichtsreihe umfasste mit Ausnahme der historischen Texte die in Abschnitt 5.3 beschriebenen Phasen 1 bis 3. Die Unterrichtsreihe endete aus Zeitgründen (es handelte sich um die letzten Doppelstunden vor den Sommerferien) mit dem Ausfüllen der Tabelle über die logischen Zusammenhänge zwischen den wahrscheinlichkeitstheoretischen Aussagen und einer abschließenden Diskussion über Möglichkeiten, aus diesen ein geeignetes Axiomensystem aufzustellen.

Geometrie: Fokus dieser Erprobung war die Auseinandersetzung mit der sphärischen Geometrie. Ausgangspunkt war - wie auch bei der in Abschnitt 5.2 dargestellten Endform - das lokale Ordnen ausgewählter Sätze der euklidischen Geometrie (z. B. Satz des Thales). Allerdings wurde diese Phase in der Pilot-Erprobung zeitlich straffer und mit weniger Eigenarbeitsphasen der Schülerinnen und Schüler durchgeführt. Auch Euklids Elemente wurden in einem Impulsvortrag nur als Exkurs erwähnt. Die Aktivitäten im Zusammenhang mit der sphärischen Geometrie (Comic-Ausschnitte aus dem Geometrikon, Arbeiten mit den Styroporkugeln, Auseinandersetzung mit dem auf von Helmholtz beruhenden Gedankenexperiment) entsprachen im Wesentlichen der in dieser Arbeit vorgestellten Form, wobei das Gedankenexperiment als Lehrervortrag vorgestellt und kein historischer Textauszug dazu gelesen wurde. Hilberts Grundlagen der Geometrie und das Konzept der impliziten Definition wurden nur in einem kurzen Exkurs behandelt.

Komplexe und „bekloppte“ Zahlen: Da die Teilnehmerinnen und Teilnehmer des Projektkurses das Thema Komplexe Zahlen bereits im Unterricht behandelt hatten, bot sich die Gelegenheit, rückblickend das Vorgehen zu diskutieren, eine Zahl $i$ mit der Eigenschaft $i^{2}=-1$ zu postulieren und damit ,wie gewohnt“ zu rechnen. Dabei entfielen wesentliche Teile der in Abschnitt 5.4 beschriebenen Phasen 1 und 2, denn auch die Definition über geordnete Zahlenpaare war den Schülerinnen und Schülern bereits bekannt. 
Die Diskussion über die Nützlichkeit der Setzung $i^{2}=-1$ war demnach geprägt von dem bereits vorhandenen Wissen, dass komplexe Zahlen in der Mathematik verbreitet sind und genutzt werden, so dass gerade mögliche Contra-Argumente nicht intensiv diskutiert wurden. Die mögliche Definition einer bekloppten Zahl $b$ mit der Eigenschaft $0 \cdot b=1 \mathrm{bzw}$. $\frac{1}{0}=b$ wurde im Rahmen der Erprobung ebenfalls diskutiert. Die Idee dazu stammte sogar aus der Lerngruppe, die auch die Bezeichnung bekloppte Zahl vorschlug. Die Tatsache, dass die Diskussion teilweise von den Schülerinnen und Schülern initiiert wurde, zeigte, dass die Möglichkeit der Zahlbereichserweiterung und die damit zusammenhängenden Rechtfertigungsfragen für Lernende kognitiv sehr anregend sind.

\section{Aus der Pilotierung resultierende Veränderungen}

Grundsätzlich erwiesen sich die verwendeten Unterrichtsmaterialien als geeignet zur Vermittlung axiomatischer Inhalte und stießen bei den Schülerinnen und Schülern auf großes Interesse. Als besonders motivierend und ergiebig zeichneten sich in den Pilotierungen das lokale Ordnen der wahrscheinlichkeitstheoretischen Aussagen sowie die Diskussion der bekloppten Zahl $b$ ab.

Insgesamt war ein wesentlicher Unterschied der in den Pilotierungen erprobten Unterrichtsreihen im Vergleich zu den in dieser Arbeit beschriebenen finalen Versionen, dass sie separat voneinander durchgeführt wurden und noch nicht in ein umfassendes LernzielKonzept eingebettet waren, wie es in den Abschnitten 4.1 und 5.1 ausgeführt wird. So wurden die Lernziele 3 (moderne Auffassung von Axiomen, implizite Definition), 5 (deduktives Arbeiten) und 6 (Rechtfertigung von Axiomensystemen) nur ansatzweise verfolgt, da die entsprechenden Unterrichtsphasen bei den Erprobungen zeitlich zu knapp angesetzt waren bzw. teilweise noch nicht vollständig entwickelt waren (beispielsweise im Falle der Wahrscheinlichkeitsrechnung und der Zahlbereichserweiterungen).

Hinsichtlich der in Abschnitt 5.1.2 geschilderten inhaltlichen und methodischen Leitideen wurden die Betrachtung inhaltlich authentischer Beispiele für Axiomatik und die Anknüpfung an das Schüler-Vorverständnis bereits umgesetzt und auch die Bedeutung von Diskussions- und Reflexionsphasen berücksichtigt. Bei der Überarbeitung wurde auf die zusätzliche Integration historischer Originalquellen zur Stärkung der diskursiven Phasen geachtet. Dies sollte vor allem der intensiveren Auseinandersetzung mit über die evidenzbasierte Sicht hinausgehenden Aspekten von Axiomatik dienen.

Bezogen auf die einzelnen Unterrichtsreihen wurden die folgenden wesentlichen Änderungen vorgenommen: 
- Alle Reihen wurden inhaltlich ausgeweitet. Das gilt insbesondere für die Geometrie, bei der bestimmte in der Pilotierung nur ausblickhaft behandelte Aspekte nun intensiver thematisiert werden. Dazu gehört eine gründlichere Auseinandersetzung mit der euklidischen Geometrie anhand der Winkelsätze und des Studiums ausgewählter Passagen aus den Elementen, die Vermittlung des Konzepts der impliziten Definition durch das Dimpfel-Gronkel-Axiomensystem sowie die vergleichende Auseinandersetzung mit der klassischen und modernen Sicht auf Axiome anhand des Briefwechsels zwischen Frege und Hilbert.

- Die Unterrichtsreihe zu den Kolmogorov-Axiomen wurde hauptsächlich durch die in Abschnitt 5.3 beschriebene Phase 4 ergänzt, um zum einen erste Aussagen aus den Kolmogorov-Axiomen zu deduzieren (und damit LZ 5 stärker zu berücksichtigen) und zum anderen Kolmogorovs Zugang intensiver mit den inhaltlich orientierten Zugängen von Laplace und von Mises zu vergleichen und somit einen Rückbezug zum Beginn der Unterrichtsreihe herzustellen.

- Die Ideen zum Themenfeld Zahlbereichserweiterungen wurden in der Pilotierung nur stark verkürzt erprobt, da - wie oben erwähnt - die komplexen Zahlen in dem Projektkurs bereits eingeführt worden waren. Für die finale Erprobung wurden daher vor allem die Phasen 1 und 2 ausgearbeitet. Dabei wurde besonderer Wert auf eine schrittweise Diskussion der Nützlichkeit imaginärer Zahlen gelegt, die sich an bestimmte Erkenntnisse beim Lösen kubischer Gleichungen orientiert. Zudem wurde als Vertiefung der Rechtfertigungsproblematik die Auseinandersetzung mit der Regel minus mal minus ergibt plus ergänzt.

\subsubsection{Erprobung im Rahmen einer Workshop-Woche}

Nach den oben geschilderten Piloterprobungen und daraus folgenden Überarbeitungen wurden die in dieser Arbeit vorgestellten Versionen der Unterrichtsmaterialien in der ersten Sommerferienwoche 2016 im Rahmen einer Workshop-Woche an der RWTH Aachen erprobt. Damit sollte zum einen die reine Funktionalität der entwickelten Unterrichtsmaterialien (Verstehen die Schülerinnen und Schüler die Aufgabenstellungen? Eignen sich die historischen Textauszüge als Ausgangspunkt für Diskussionen? usw.) getestet und zum anderen untersucht werden, inwiefern die sechs übergeordneten Lernziele aus Abschnitt 4.1 von den Teilnehmerinnen und Teilnehmern erreicht würden.

Zur besseren nachträglichen Reflexion wurde die Workshop-Woche auf Video aufgezeichnet. Zudem wurden die Schülerinnen und Schüler an einigen Stellen aufgefordert, ih- 
re Gedanken zu bestimmten Frage- und Problemstellungen schriftlich in Form von Kurzaufsätzen zu fixieren. Die sechs Aufgabenstellungen ${ }^{24}$ und zugehörigen Schülerantworten sind in Anhang B zu finden.

Im Folgenden werden zunächst die organisatorischen Rahmenbedingungen der WorkshopWoche und deren Konsequenzen für die Aussagekraft der Erprobung beschrieben. Anschließend werden der genauere Workshopverlauf und Erfahrungen bezüglich der Funktionalität der verwendeten Materialien vorgestellt.

\section{Zeitlicher Rahmen}

An den ersten vier Tagen der Workshop-Woche wurden je drei „Schul-Doppelstunden“ von jeweils 90 Minuten mit dazwischen liegenden Pausen durchgeführt. Am letzten Workshoptag erfolgten ein letzter 90-Minuten-Block sowie eine ca. 45-minütige Abschlussphase. Grob entfielen dabei die folgenden Zeiten auf die jeweiligen Bestandteile:
Einstieg:
ca. 45 Minuten
Unterrichtsreihe 1 (Geometrie):
ca. $6 \times 90$ Minuten
Unterrichtsreihe 2 (Wahrscheinlichkeitsrechnung):
ca. 4,5 $\times 90$ Minuten
Unterrichtsreihe 3 (Zahlbereichserweiterungen):
ca. $2 \times 90$ Minuten
Abschluss:
ca. 45 Minuten

Eine detailliertere Übersicht über den zeitlichen Ablauf und die in den einzelnen Zeitslots behandelten Inhalte findet sich auf den Seiten 186-190. Die zeitliche Gestaltung hat sowohl Vor- als auch Nachteile:

- Eine zusammenhängende Workshop-Woche bietet im Gegensatz zu wöchentlich stattfindenden schulischen Zusatzkursen die Möglichkeit, konzentriert und ohne unnötigen Wiederholungsbedarf an einem Thema zu arbeiten. Zudem kann die Phasierung des Unterrichts offener gestaltet werden, da nicht nach jeweils exakt 90 Minunten ein gesichertes Lernergebnis vorliegen muss, sondern mit Pausenzeitpunkt und -länge flexibel auf den lerngruppenspezifischen Verlauf reagiert werden kann.

- Andererseits werden die Schülerinnen und Schüler innerhalb von einer Woche mit sehr vielen neuen Inhalten konfrontiert, die zudem einen über weite Strecken anderen Denkmodus und Zugang zur Mathematik erfordern, als dies aus dem Regelunterricht vertraut ist. Obwohl die Unterrichtsreihen intensive Diskussions- und

\footnotetext{
${ }^{24}$ Die Anzahl der Kurzaufsätze stimmt nur zufällig mit der Anzahl der übergeordneten Lernziele überein; es besteht keine 1:1-Passung zwischen Lernzielen und Kurzaufsatz-Themen.
} 
Reflexionsphasen vorsehen, ist davon auszugehen, dass die vorgestellten axiomatischen Gesichtspunkte dadurch weniger nachhaltig vermittelt werden können.

- Die Erprobung hat zudem gezeigt, dass der hier gewählte zeitliche Rahmen die Untergrenze für eine sinnvolle Erprobung sämtlicher Materialien darstellt. Einzelne Aufgaben der Arbeitsblätter wurden aus Zeitgründen nicht bearbeitet (im tabellarischen Verlaufsplan weiter unten in grauen Kommentaren angemerkt).

\section{Teilnehmerzahl- und zusammensetzung}

An der Workshop-Woche nahmen 2 Schülerinnen und 2 Schüler von insgesamt 3 unterschiedlichen Schulen teil, die kurz zuvor die zehnte Jahrgangsstufe (EF) abgeschlossen hatten. Die Teilnehmerzahl war damit leider äußerst niedrig. ${ }^{25}$ Dies hatte zwar den Vorteil, dass man sich als Lehrkraft sehr viel intensiver mit der einzelnen Schülerin und dem einzelnen Schüler auseinandersetzen konnte; gerade in den Diskussionsphasen wäre eine höhere Teilnehmerzahl der Intention des Workshops allerdings zuträglich gewesen.

Zusätzlich zur geringen Teilnehmerzahl schränkt auch die Freiwilligkeit des Workshopangebots die Übertragbarkeit der Erprobungsergebnisse auf andere Unterrichtsformen ein. Es ist davon auszugehen, dass an der Workshop-Woche nur besonders interessierte und ohnehin mathematikaffine Schülerinnen und Schüler teilgenommen haben. Grundsätzlich entspricht dies aber der Haupt-Zielgruppe der Unterrichtsreihen, da der Anteil intrinsisch motivierter Lernender in Oberstufen-Zusatzkursen in der Regel ebenfalls überdurchschnittlich ist.

\section{Verlaufsplan}

Im Folgenden wird ein tabellarischer Überblick über den Verlauf der Workshop-Woche mit leicht gerundeten Zeitangaben gegeben. Die Inhalte werden dabei in Stichpunkten und mit Verweis auf die im Anhang befindlichen Aufgabenblätter und Textauszüge benannt, da sie in den Abschnitten 5.2 bis 5.4 ausführlich beschrieben wurden.

\footnotetext{
${ }^{25}$ Hierzu ist anzumerken, dass die organisatorischen Rahmenbedingungen für die Akquise ausreichend vieler Teilnehmer nicht optimal waren, da gerade in der Ferienzeit die RWTH Aachen eine große Zahl von konkurrierenden Angeboten wie Schülerunis stellt.
} 


\section{Tag}

\section{Dauer Inhalt/Aktivitäten}

\begin{tabular}{rc}
\hline \hline $15 \mathrm{~min}$ & Begrüßung, Vorstellungsrunde, Organisatorisches \\
\hline $30 \mathrm{~min}$ & Einführung: Die Sicherheit mathematischer Aussagen [EINF1] \\
& - Aufg. 1+2 im PlENUM \\
& - KURZAUFSATZ 1 (= Aufg. 3): Sicherheit mathematischer Aussagen \\
&
\end{tabular}

80 min Beweisen und Ordnen geometrischer Sätze [GEOM1]

- Aufg. 1+2 in PA* (nicht alle Beweise wurden im Rahmen von Aufg. 2b in PA entwickelt, einige wurden kurz frontal vorgestellt), Aufg. 3 im PLENUM,

Aufg. 5+6 in PA (Aufg. 4 ausgelassen)

- IMPUlsvortRAG: Beweisen in der Mathematik

\section{0 min Euklids Elemente}

- Definitionen, Postulate und Axiome lesen und diskutieren

- Beweise von Prop. 32 und 13: verwendete Sätze identifizieren

- IMPULSVORTRAG: Parallelenproblem

- KurZaufsatz 2: Beweise „offensichtlicher“ Aussagen

60 min Sphärische Geometrie Teil I [Geometrikon, GEOM2]

- SuS lesen S. 2-13(oben) des Geometrikons $\rightarrow$ Was ist passiert?

- GEOM2 in PA

*Anmerkung: Die Abkürzung PA steht für „Partnerarbeit“. Wird im Verlaufsplan angegeben, dass eine Partnerarbeitsphase stattgefunden hat, so ist damit auch die anschließende Besprechung im Plenum impliziert. 


\section{Tag}

\begin{tabular}{|c|c|}
\hline Dauer & Inhalt/Aktivitäten \\
\hline \multirow[t]{2}{*}{$30 \mathrm{~min}$} & Von Dimpfeln und Gronkeln [GEOM4] ${ }^{*}$ \\
\hline & - 1. Seite von GEOM4 in PA \\
\hline \multirow[t]{4}{*}{$60 \mathrm{~min}$} & Sphärische Geometrie Teil II [Auszug Geometrikon, GEOM3] \\
\hline & - SuS lesen S. 13-21 des Geometrikons \\
\hline & - GEOM3 in PA \\
\hline & $\begin{array}{l}\text { - PLENUM: Gegenüberstellung wesentlicher Eigenschaften der euklidischen } \\
\text { und sphärischen Geometrie }\end{array}$ \\
\hline \multirow[t]{5}{*}{$90 \mathrm{~min}$} & Die Natur geometrischer Axiome [HIST1, HIST2] \\
\hline & - SuS lesen HIST 1; anschließend Diskussion im Plenum \\
\hline & - KuRZAUfSATZ 3: Natur geometrischer Axiome \\
\hline & - SuS lesen Hist2; anschließend Diskussion im Plenum \\
\hline & - IMPULSVORTRAG: Auflösung des Parallelenproblems \\
\hline \multirow[t]{4}{*}{$80 \mathrm{~min}$} & Hilbert-Frege-Kontroverse [GEOM4, Hist3, Hist4] \\
\hline & - Rest von GEOM4: Interpretationen von Dimpfel, Gronkel, klubern \\
\hline & - SuS lesen Hist3; anschließend Diskussion im Plenum, Bezug zu GeOM4 \\
\hline & - SuS lesen HisT4 und arbeiten die unterschiedlichen Standpunkte heraus \\
\hline
\end{tabular}

\footnotetext{
*Anmerkung: Der erste Teil von GeOM4 wurde bereits am Anfang des zweiten Workshoptages behandelt und bewusst als Exkurs präsentiert. Damit sollte verhindert werden, dass die Schülerinnen und Schüler allein schon aufgrund der Tatsache, dass sie sich bisher ausschließlich mit Geometrie beschäftigt haben, auf die Idee kommen, dass die Dimpfel-Gronkel-Axiome etwas mit Geometrie zu tun haben.
} 


\section{Tag}

\begin{tabular}{|c|c|}
\hline Dauer & Inhalt/Aktivitäten \\
\hline \multirow[t]{3}{*}{$45 \mathrm{~min}$} & Abschluss Geometrie \\
\hline & - KURZAUfsatz 4: Zwei verschiedene Sichtweisen auf Axiome \\
\hline & - Abschlussdiskussion \\
\hline \multirow[t]{4}{*}{$100 \min$} & $\begin{array}{l}\text { Klassische Zugänge zum Wahrscheinlichkeitsbegriff [WKEIT1, } \\
\text { HIsT5, HIST6] }\end{array}$ \\
\hline & - Aufg. $1+2$ in PA, Aufg. $3+4$ im PlenUm \\
\hline & - Hist5 und Hist6 lesen und mögliche Definitionen für den Begriff \\
\hline & Wahrscheinlichkeit aufstellen; Grenzen der klassischen Zugänge diskutieren \\
\hline \multirow[t]{3}{*}{$45 \mathrm{~min}$} & Mengentheoretische Grundlagen [W KEIT2] \\
\hline & - IMPULSVORTRAG: Mengentheoretische Modellierung \\
\hline & - WKeIT2 in PA \\
\hline \multirow[t]{4}{*}{$90 \mathrm{~min}$} & Elementare Regeln Teil I [WKEIT3] \\
\hline & - Logische Zusammenhänge zwischen den Regeln erkunden, Beweise im \\
\hline & Plenum vorstellen \\
\hline & - Tabelle gemeinsam ausfüllen, dabei verschiedene Möglichkeiten diskutieren \\
\hline
\end{tabular}




\section{Tag}

\section{Dauer Inhalt/Aktivitäten}

45 min Elementare Regeln Teil II [WKEIT3]

- verschiedene Möglichkeiten diskutieren, auf Grundlage der Tabelle ein Axiomensystem aufzustellen

$120 \mathrm{~min}$ Kolmogorov-Axiome [HIST7, W KEIT4, W KeIT5]

- SuS lesen Hist7, Vergleich mit Hilbert

- Aufg. 1 von WKeIT4 in PA (Aufg. 2 ausgelassen)

- Aufg. 2+3 von WKeIT5 in PA (Aufg. 1 ausgelassen)

- Behandlung einer „typischen“ Schulbuchaufgabe zum Satz von Bayes*

- KuRZAufsatz 5: Ansätze zum Wahrscheinlichkeitsbegriff

- Abschließende Zusammenfassung zu Kolmogorovs Ansatz

\section{0 min Kubische Gleichungen [ZBE1, ZBE2, ZBE3]}

- ZBE1 als Einführung im Plenum diskutieren

- ZBE2 lesen

- ZBE3 in PA bearbeiten, Sammlung von Pro- und Contra-Argumenten bzgl. der Verwendung imaginärer Zahlen (Aufg. 6 ausgelassen)

*Anmerkung: Als ,,typische“ Schulbuchaufgabe wurde behandelt:

In einer Schulklasse befinden sich 30\% Mädchen und 70\% Jungen. Von den Mädchen ernähren sich $80 \%$ vegetarisch; unter den Jungen gibt es 30\% Vegetarier. Es wird eine Person zufällig gewählt. Sie ist Vegetarier(in). Mit welcher Wahrscheinlichkeit handelt es sich um einen Jungen?

Lösung: Wir betrachten folgende Ereignisse:

$A$ : Die Person ist ein Junge. $\quad \bar{A}: \quad$ Die Person ist ein Mädchen.

$B$ : Die Person ist Vegetarier(in). $\quad \bar{B}: \quad$ Die Person ist kein(e) Vegetarier(in).

Gesucht ist also die bedingte Wahrscheinlichkeit $P_{B}(A)$. Aus der Aufgabenstellung kann man die folgenden Wahrscheinlichkeiten direkt ablesen: $P(A)=\frac{7}{10}, \quad P(\bar{A})=\frac{3}{10}, \quad P_{A}(B)=\frac{3}{10}, \quad P_{\bar{A}}(B)=\frac{8}{10}$

Dann erhält man mit dem Satz von der totalen Wahrscheinlichkeit: $P(B)=\frac{3}{10} \cdot \frac{7}{10}+\frac{8}{10} \cdot \frac{3}{10}=\frac{45}{100}$

Der Satz von Bayes schließlich liefert:

$$
P_{B}(A)=\frac{P_{A}(B) \cdot P(A)}{P(B)}=\frac{\frac{3}{10} \cdot \frac{7}{10}}{\frac{45}{100}}=\frac{21}{45}=46, \overline{6} \%
$$




\section{Tag}

\section{Dauer Inhalt/Aktivitäten}

90 min Komplexe Zahlen, weitere Zahlbereichserweiterungen [ZBE3, ZBE5]

- scheinbare Widersprüche beim Rechnen mit $\sqrt{-1}$ diskutieren

- IMPULSVORTRAG: Symbol $i$, Gauß-Visualisierung, Hamilton-Def.

- ZBE5: Regel $(-) \cdot(-)=(+)$ und Def. einer „bekloppten“Zahl $b$ diskutieren

45 min $\quad$ Abschluss
- KuRZAufs Atz 6: Mathematikbilder
- Abschlussdiskussion
- Evaluation der Workshop-Woche*

*Anmerkung: Zum Abschluss wurden die Schülerinnen und Schüler gebeten, einen Evaluationsbogen über die Workshop-Woche (auf dem Bogen als Seminar bezeichnet) auszufüllen. Die Ergebnisse sind in Anhang $\mathrm{C}$ zu finden. Aufgrund der geringen Teilnehmerzahl lassen sich die Rückmeldungen weder in positiver (was besonders gut gefallen hat) noch in negativer (was nicht so gut gefallen hat) Sicht verallgemeinern, so dass über den Anhang hinaus nicht näher auf die Evaluation eingegangen wird. 


\section{Zur Funktionalität der Unterrichtsmaterialien}

Die Aufgabenstellungen der Arbeitsblätter haben sich durchweg als hinreichend verständlich erwiesen. An einigen wenigen Stellen wurden leichte sprachliche Anpassungen vorgenommen, welche in den im Anhang dieser Arbeit zur Verfügung gestellten Versionen bereits eingearbeitet sind.

Auch der Einsatz der historischen Texte hat sich weitgehend bewährt, wenngleich die darauf aufbauenden Diskussionsphasen aufgrund der Teilnehmerzahl nicht immer so ergiebig waren wie erwartet. Insbesondere die Texte von Poincaré (HIST2) sowie Frege und Hilbert (HIST4) erwiesen sich als sehr herausfordernd. Bei künftigen Durchführungen wäre zu überlegen, ob HIST2 ersatzlos entfallen kann. Ein Übergang von der Beschäftigung mit der sphärischen Geometrie zu Hilberts Grundlagen der Geometrie ist durch den Impulsvortrag zur Auflösung des Parallelenproblems und die darin behandelte WörterbuchVorstellung dennoch möglich. Der Wegfall von HIST2 würde zudem die verhältnismäßig große Anzahl historischer Texte gegen Ende der Geometrie-Reihe reduzieren.

Die Unterrichtsreihe zur Geometrie ist rückblickend im vorderen Teil ein wenig zu lang. Insbesondere die intensive Beschäftigung mit den Winkelsätzen erschien den Schülerinnen und Schülern möglicherweise unterfordernd. Unterrichtsvorschläge, die sich mit derartigen geometrischen Aspekten beschäftigen, sind in der Regel für die Sekundarstufe I konzipiert und auch auf der Meta-Ebene werden an dieser Stelle noch keine komplexen axiomatischen Themen diskutiert. Eine denkbare Lösung wäre, die Behandlung des Arbeitsblattes GEOM1 deutlich zu straffen und die deduktive Struktur der Geometrie relativ unmittelbar anhand der Auszüge aus den Elementen zu thematisieren.

Um die Effektivität der Ergebnissicherung zu steigern, wurde ein sogenanntes Wissensspeicher-Heft angefertigt, welches eine ähnliche Funktion wie die aus dem Mathematikunterricht bekannten Regelhefte erfüllt: Zu Beginn der Workshop-Woche wurde den Teilnehmerinnen und Teilnehmern ein schmales Heft mit einigen leeren DIN A4-Seiten ausgehändigt, welches im Verlauf der Woche zunehmend mit ausgewählten Tafelanschrieben gefüllt wurde. Als Orientierung für die Auswahl geeigneter Inhalte können die grauen Kästen in den Abschnitten 5.2 bis 5.4 dienen.

\subsubsection{Lernzielerreichung}

Die folgenden Ausführungen betreffen die Frage, inwieweit die übergeordneten Lernziele aus Abschnitt 4.1 von den Schülerinnen und Schülern erreicht wurden. In die Auswertung 
sind dabei die Aktivitäten und Äußerungen sowie die schriftlichen Textproduktionen der Schülerinnen und Schüler innerhalb der Kurzaufsätze ${ }^{26}$ eingeflossen. Bezüglich der Aktivitäten und mündlichen Äußerungen dienen die eigenen Beobachtungen - unmittelbar während der Workshopdurchführung sowie aufgrund der Videoaufnahmen - als Basis.

Grundsätzlich ist dabei anzumerken, dass die beschriebene Erprobung lediglich „Existenzbeweise" für bestimmte Lernzielerreichungen liefern kann. Insbesondere ist mit der hier vorgenommenen Auswertung nicht der Anspruch einer empirisch-wissenschaftlichen Beforschung der Unterrichtsreihen verbunden. Dies ist nicht nur aufgrund der geringen Teilnehmerzahl unmöglich, sondern auch, weil Entwicklung, Durchführung und Evaluation der Unterrichtsmaterialien von derselben Person vorgenommen wurden.

Lernziel 1: Die Schülerinnen und Schüler erklären, dass beim mathematischen Beweisen die Gültigkeit einer Aussage auf die Gültigkeit bereits akzeptierter Aussagen zurückgeführt wird, und begründen damit die Notwendigkeit, als Ausgangspunkte mathematischer Beweisführungen Axiome zu setzen.

Dieses Lernziel wurde bereits zu Beginn der Workshop-Woche mit dem lokalen Ordnen der Winkelsätze verfolgt. Die schematische Darstellung des deduktiven Gefüges über Pfeildiagramme erwies sich dabei als besonders ergiebig. Den hypothetisch-deduktiven Charakter mathematischer Aussagen fasste ein Schüler wie folgt zusammen: Auf die Frage, wie sicher mathematische Sätze seien, antwortete er: ,[...] genauso sicher wie die Axiome, von denen sie ausgehen“. Eine andere Schülerin antwortete: „Wenn die Axiome, von denen sie ausgehen, sicher sind, sind auch die mathematischen Sätze sicher“. Eine ähnliche Antwort gab ein Schüler auf die Frage, was genau Beweise eigentlich beweisen: „Sie beweisen, dass, wenn die Regeln / Sätze, von denen sie ausgehen, wahr sind, auch der bewiesene Satz stimmt".

Auch das Ordnen der wahrscheinlichkeitstheoretischen Aussagen mit Hilfe der Tabelle hat sich als förderlich für das Erreichen des Lernziels erwiesen. Insbesondere die Notwendigkeit der Setzung von Axiomen trat hier nochmals in den Vordergrund, da das sukzessive Beweisen der Aussagen ansonsten zu Zirkelschlüssen führen würde (was hier wegen der begrenzten Anzahl und formalen Schlichtheit der zur Verfügung stehenden Aussagen offensichtlicher ist als in der Geometrie).

Dass die Schülerinnen und Schüler bezüglich des hypothetisch-deduktiven Charakters der Mathematik durchaus schon Vorwissen aus der Schule mitbringen, zeigt der folgende Satz

${ }^{26}$ Sämtliche Kurzaufsätze finden sich in Anhang B. In den Texten zur Lernzielerreichung erfolgen Zitate aus den Kurzaufsätzen mit Verweis auf den Verfasser bzw. die Verfasserin des Textes (Sm1 / Sm2 für die beiden männlichen Schüler und Sw1 / Sw2 für die beiden weiblichen Schülerinnen) sowie auf den Kurzaufsatz, aus dem die zitierte Passage stammt. 
eines Schülers, welcher ganz zu Beginn der Workshop-Woche verfasst wurde: „Wenn also diese Grundlagen [der Mathematik, T.H.], die dem Menschen fast selbstverständlich erscheinen, wahr sind, dann muss auch die gesamte Mathematik wahr sein“"(Sm1, Kurzaufsatz1; Herv. d. Verf.).

Lernziel 2: Die Schülerinnen und Schüler erläutern die Deutung von Axiomen als Tatsachen, die aufgrund ihrer Evidenz ohne Beweis akzeptierbar sind.

Erwartungsgemäß konnten die Schülerinnen und Schüler diese Sichtweise ohne große Probleme nachvollziehen, wie etwa die folgende Aussage zeigt: „Außerdem sollen Axiome und Postulate wirklich sofort einleuchten“ (Sm1, Kurzaufsatz 2). Dies hängt ohne Zweifel mit den starken inhaltlichen Vorstellungen etwa zu den geometrischen Grundbegriffen oder auch zum Wahrscheinlichkeitsbegriff zusammen, welche die zugehörigen Axiome als klar und nicht beweisbedürftig erscheinen lassen. Die Nähe der evidenzbasierten Sicht auf Axiome zur Erfahrungswelt der Schülerinnen und Schüler spiegelt sich auch in der von diesen immer wieder geäußerten Auffassung wider, der Realitätsbezug der Mathematik (und damit auch die Wahrheit der Axiome) sei maßgeblich für deren Sinnhaftigkeit. Man vergleiche hier auch die Ausführungen zu LZ 6.

Eine Variante der evidenzbasierten Sichtweise findet sich in folgender Schüleräußerung: „Allgemein kann man auch noch sagen, die einfache Mathematik erscheint sicher, da sie durch allgegenwärtige Beispiele erkennbar/beweisbar ist [...]“ (Sm2, Kurzaufsatz 1). Hier tritt eine empiristische Auffassung zum Vorschein, welche die Mathematik deshalb als gesichert betrachtet, weil ihre grundlegenden Axiome über Jahrtausende hinweg empirisch nicht widerlegt werden konnten.

Lernziel 3: Die Schülerinnen und Schüler erläutern die Deutung von Axiomen als Aussagen, welche inhaltlich nicht interpretiert werden und die Grundbegriffe eines mathematischen Gebiets implizit definieren.

Besonders ergiebig zum Erreichen dieses Lernziels war das Arbeitsblatt GEOM4, auf dem die geometrischen Grundbegriffe durch Kunstworte ersetzt werden. Die Wörterbuch-Metapher, welche auch im Impulsvortrag zur Auflösung des Parallelenproblems angesprochen wurde, erwies sich dabei als tragfähige mentale Vorstellung zur abstrakten Formulierung von Axiomen. Die Schülerinnen und Schüler erkannten, dass ein für das DimpfelGronkel-System geführter Beweis nicht erneut geführt werden muss, wenn man die Axiome geometrisch deutet, sondern dass lediglich die Wörter ausgetauscht werden müssen.

In diesem Zusammenhang entstand zudem eine Diskussion darüber, ob es den Lernenden leichter falle, Beweise unter Verwendung der Kunstworte zu führen oder in der „üblichen“ geometrischen Interpretation der Axiome. Man war sich zwar einig, dass das Nachdenken 
über die Axiome und die Beweise leichter falle, wenn die Begriffe ,nicht so abwegig sind wie Dimpfel und Gronkel“", es auf der anderen Seite bei unmittelbar mit einer Vorstellung verbundenen Begriffen aber schwieriger wäre, sich bei der Beweisführung ,nur auf die Axiome zu beschränken“. Wenn man an Punkte und Geraden denke, passiere es schneller, dass man wie Euklid zusätzliche anschauliche Gegebenheiten in den Beweisschritten verwende, die zuvor nicht explizit benannt wurden.

Die beiden mit den Lernzielen 2 und 3 verbundenen Auffassungen von Axiomen wurden von den Schülerinnen und Schülern vor allem im Zusammenhang mit der Frage der Sinnhaftigkeit der Mathematik kritisch beleuchtet. Näheres dazu findet sich in den Ausführungen zu LZ 6.

Lernziel 4: Die Schülerinnen und Schüler untersuchen die logischen Zusammenhänge ausgewählter mathematischer Aussagen sowie verschiedene Möglichkeiten, aus diesen Aussagen ein (minimales) Axiomensystem auszuwählen.

Das lokale Ordnen einer überschaubaren Anzahl mathematischer Aussagen stand an zwei Stellen der Workshop-Woche im Vordergrund: zum einen bei der Untersuchung der Winkelsätze der ebenen Geometrie (GEOM1) und zum anderen im Rahmen der Wahrscheinlichkeitsrechnung (WKEIT3). Insbesondere die Phase der Beweisfindung und anschließenden Ordnung der wahrscheinlichkeitstheoretischen Aussagen stieß bei den Schülerinnen und Schülern auf reges Interesse und großes Engagement. Es wurden verschiedene Beweiswege und unterschiedliche Möglichkeiten vorgestellt und diskutiert, auf Grundlage der entdeckten logischen Zusammenhänge drei passende Axiome auszuwählen. Die auf den Seiten 163 und 164 beschriebenen Strategien zur Auswahl geeigneter Axiome wurden dabei von den Lernenden größtenteils selbstständig entdeckt. Dies zeigt zum einen das hohe Potential dieser Phase für kreative Aktivitäten und zum anderen, dass auch abstrakt-formale Themen intrinsisch motivierend wirken können.

Lernziel 5: Die Schülerinnen und Schüler leiten exemplarisch aus einem gegebenen Axiomensystem deduktiv weitere Aussagen her.

Deduktive Übungen waren wesentlicher Bestandteil der Unterrichtsreihe zur Wahrscheinlichkeitsrechnung (W KEIT3+4+5) wie auch in kleinerem Rahmen der Unterrichtsreihe zur Geometrie (GEOM4). Es wurde besonderer Wert darauf gelegt, die einzelnen Beweisschritte durch Verweis auf das jeweils verwendete Axiom bzw. den jeweils verwendeten Satz zu rechtfertigen. Obwohl das streng deduktive Vorgehen im Rahmen der Erprobungen nur angedeutet werden konnte und für die Schülerinnen und Schüler sehr ungewohnt war, so dass ich während der Arbeitsphasen mehrmals Hilfestellung durch Hinweise auf mögliche Beweisideen geben musste, stieß es generell auf großes Interesse. Überhaupt 
war die Motivation gerade in den eher formal-abstrakt geprägten Phasen 3 und 4 der Unterrichtsreihe zur Wahrscheinlichkeitsrechnung besonders hoch.

Lernziel 6: Die Schülerinnen und Schüler beschreiben Kriterien zur Rechtfertigung einer auf Axiomen aufbauenden Theorie.

Die Rechtfertigung einer auf Axiomen aufbauenden Theorie ist für die Schülerinnen und Schüler stark mit deren Sinnhaftigkeit verbunden. Insbesondere in den Kurzaufsätzen 4 und 6, in denen sie mit zwei unterschiedlichen Sichtweisen auf Axiome bzw. Mathematik im Allgemeinen konfrontiert sind, wird dieser Aspekt immer wieder betont. Die Rechtfertigungsproblematik eignet sich damit besonders gut zur kritischen Reflexion der klassischen und modernen Sicht auf Axiome (LZ2 und LZ3). Einige Beispiele aus den Kurzaufsätzen:

- „Weil Axiome der Startpunkt von Beweisen, Sätzen und ganzen Systemen sind, können sie eigentlich jeder Art sein und willkürlich festgesetzt werden. Andererseits macht es aber auch nur relativ wenig Sinn, von absolut realitätsfernen Axiomen auszugehen, da die Ergebnisse, zu denen man kommen könnte, nicht in der Realität gelten.“ (Sm1, Kurzaufsatz 4)

- „Zum einen brauchen wir realitätsnahe Axiome, denn welchen Sinn hat die Mathematik, wenn man sich alles nur ausdenkt und es keiner Realität entspricht [...].“ (Sw2, Kurzaufsatz 4)

- „Ich kann die Position A [Mathematik als formales Spiel mit den Axiomen als Spielregeln, T.H.] nachvollziehen und finde die Idee interessant. Jedoch wüsste ich nicht, was einem diese Sicht auf Axiome und die Mathematik nutzen würde, da sie keinerlei Aussage über die Relevanz der Mathematik macht.“ (Sm2, Kurzaufsatz 6)

- „Willkürlichen nicht-interpretierten Axiomen kann man auch schwer einen Sinn geben, was dazu führt, dass der Nutzen des Axioms infrage gestellt wird.“ (Sw1, Kurzaufsatz 6)

Die Textbeispiele zeigen, dass der Realitätsbezug von Axiomen und mathematischen Aussagen aus Schülersicht ein wesentliches Kriterium für deren Sinnhaftigkeit ist. Zwar zeigen sie grundsätzlich Verständnis für die abstrakte Sichtweise bzw. können diese nachvollziehen (siehe auch LZ3); sie scheint jedoch keinen wesentlichen Beitrag dazu zu leisten, der Mathematik einen konkreten Sinn zu geben. ${ }^{27}$ Diese Ansicht erinnert an ei-

\footnotetext{
${ }^{27}$ Es ist auffällig, dass sich die Schülerinnen und Schüler bei ihrer Auseinandersetzung mit der formalistischen Sicht auf Mathematik nicht zumindest in Kurzaufsatz 6 auf ihre Erfahrungen aus dem Themenfeld Zahlbereichserweiterungen beziehen, zumal sie die Vorteile eines zunächst völlig abstrakten Vorgehens anhand der Cardanischen Formel meinem Eindruck nach nachvollzogen hatten. Meine Vermutung ist,
} 
ne ähnliche Äußerung von Courant und Robbins (2010, S. xxi), die ich daher nochmals zitieren möchte:

Der Lebensnerv der mathematischen Wissenschaft ist bedroht durch die Behauptung, Mathematik sei nichts anderes als ein System von Schlüssen aus Definitionen und Annahmen, die zwar in sich widerspruchsfrei sein müssen, sonst aber von der Willkür des Mathematikers geschaffen werden. Wäre das wahr, dann würde die Mathematik keinen intelligenten Menschen anziehen. Sie wäre eine Spielerei mit Definitionen, Regeln und Syllogismen ohne Ziel und Sinn. (Herv. d. Verf.)

In ähnlicher Weise stellt auch Kolmogorov eine Verbindung zwischen Sinnhaftigkeit und Realitätsbezug her, wenn er mit folgenden Worten auf die mögliche inhaltliche Interpretation seiner Axiome verweist: „Ein Leser, der den folgenden Axiomen sofort einen konkreten Sinn geben will, soll sogleich $§ 2$ [Das Verhältnis zur Erfahrungswelt, T.H.] lesen“ (Kolmogorov 1933, S. 2; Herv. d. Verf.).

Teilweise erkennen die Lernenden jedoch auch in der formalistischen Auffassung ein Sinn: „Ich sehe die Ansicht, dass Axiome [inhaltlich nicht interpretierte, T.H.] Bedingungen sind, als sinnvoller an. Diese Art der Axiome lässt zum einen Widersprüche verschiedener Axiome aus verschiedenen Axiomen zu, da der Anspruch der Axiome nicht ist allgemeingültig und richtig zu sein. Zusätzlich schließt diese Ansicht der Axiome nicht aus, dass Axiome, wahre offensichtliche Tatsachen“ sein können, jedoch bietet diese Axiomen-Ansicht die Möglichkeit, Annahmen als Axiome zu benutzen und lässt dadurch jegliches Gedankenexperiment wie auch die Annahme einer Hypothese und deren Folgen zu“ (Sm2, Kurzaufsatz 4).

Der Schüler Sm2 bezieht sich im zweiten Satz auf die verschiedenen möglichen Geometrien, deren Aussagen sich aus einem inhaltlichen Blickwinkel zu widersprechen scheinen, aus theoretischer Sicht jedoch gleichberechtigt nebeneinander stehen können. ${ }^{28}$ Dass Axiome dabei keinen Anspruch auf Allgemeingültigkeit erheben können, ist eine zutreffende und wichtige Facette eines formalistischen Standpunktes. Interessant ist auch die beschriebene Verknüpfung von inhaltlicher und abstrakter Sichtweise: Axiome kann man immer noch interpretieren, so dass sie zu wahren Aussagen (im ontischen Sinne) werden; die klassische und moderne Sicht auf Axiome widersprechen sich also nicht, sondern letztere ist eher als Ergänzung der ersteren zu sehen. Die Formulierung, dass abstrakt for-

dass sie die dritte Unterrichtsreihe nicht in dem Ausmaß mit der Axiomatik in Verbindung gebracht haben, wie dies ursprünglich intendiert war, da der Begriff Axiom in dieser Unterrichtsreihe kaum auftaucht.

${ }^{28}$ Man vergleiche dies etwa mit Freges Position, der das noch für unmöglich hielt: „Wenn die euklidische Geometrie wahr ist, so ist die nichteuklidische Geometrie falsch, und wenn die nichteuklidische wahr ist, so ist die euklidische Geometrie falsch“ (zit. n. Frege 1969, S. 183). 
mulierte Axiome, ,jegliches Gedankenexperiment wie auch die Annahme einer Hypothese und deren Folgen" zulassen, betont wiederum den hypothetisch-deduktiven Charakter der Mathematik, dem zufolge man nicht notwendigerweise von unbezweifelbaren Tatsachen ausgehen muss, um Mathematik zu treiben.

\subsubsection{Zusammenfassung und Konsequenzen}

An dieser Stelle werden die wichtigsten Erkenntnisse aus den Erprobungen zusammengefasst und Empfehlungen für zukünftige Umsetzungen ausgesprochen:

Die Schülerinnen und Schüler hatten großes Interesse an den behandelten Themen und zeigten sich in der Lage, die axiomatische Struktur der Mathematik nachzuvollziehen und auf der Meta-Ebene zu diskutieren. Insbesondere bei der Diskussion über den Sinn und Zweck der Mathematik nahmen sie reflektierte Haltungen ein und setzten sich kritisch mit den unterschiedlichen Sichtweisen auf Axiome auseinander. Dabei stellten sie einen engen Zusammenhang zwischen der Sinnhaftigkeit der Mathematik und ihrem Realitätsbezug her, wie er auch von bekannten Mathematikern postuliert wird.

Insgesamt lässt sich festhalten, dass die sechs übergeordneten Lernziele zu weiten Teilen erreicht wurden. Insbesondere realitätsgebundene Auffassungen von Axiomen konnten die Schülerinnen und Schüler leicht in ihr Mathematikbild integrieren und als Beleg für die Sinnhaftigkeit der Mathematik heranziehen. Die abstrakte Sicht erforderte erwartungsgemäß ein höheres $\mathrm{Ma} ß$ an bewusster Vermittlung. Die Lernenden konnten sie mit Hilfe der Wörterbuch-Vorstellung im Rahmen des Dimpfel-Gronkel-Beispiels sowie der historischen Textquellen jedoch gut nachvollziehen und sie zeigten sich gerade bei Phasen, in denen auf formaler Ebene gearbeitet wurde (beispielsweise beim lokalen Ordnen der wahrscheinlichkeitstheoretischen Aussagen), besonders engagiert und motiviert.

Eine Einsicht in die Nützlichkeit des formalistischen Standpunkts konnte hingegen nur teilweise vermittelt werden. Zwar wurde als Vorteil erkannt, dass unterschiedliche, abstrakt formulierte Theorien jeweils von Axiomen ausgehen können, die zunächst nicht offensichtlich erscheinen oder sich zusammengenommen widersprechen würden, und damit Raum für (hypothetisch-deduktive) Gedankenexperimente lassen. Andererseits ließen sich die - wie Wittenberg (1963, S. 55) schreibt - ,organischen Notwendigkeiten“ der modernen Mathematik nur sehr eingeschränkt in der Workshop-Woche verdeutlichen. Dies erscheint allerdings in Anbetracht des - gemessen am Umfang der Lernziele - knappen zeitlichen Rahmens und der wenigen Vorkenntnisse der Lernenden zum Thema Axiomatik nicht verwunderlich (man vergleiche auch den letzten Punkt unten). 
Um das diskursive Potential der Unterrichtsmaterialien besser nutzen zu können, wäre eine höhere Teilnehmerzahl wünschenswert (man vergleiche auch den freien Schülerkommentar in Anhang C). Dadurch könnten die eigenständigen Arbeitsphasen in Partnerbzw. Gruppenarbeit effektiver gestaltet werden und die Diskussions- und Reflexionsphasen erhielten mehr Dynamik.

Das erprobte Format der einmaligen, gebündelten Durchführung der Unterrichtsreihen im Rahmen einer Workshop-Woche hatte mit Blick auf die konzentrierte Auseinandersetzung mit einem Thema sicher Vorteile. Gleichzeitig sind damit der Nachhaltigkeit der erarbeiteten Inhalte und Erkenntnisse aber Grenzen gesetzt. Es ist daher aus meiner Sicht empfehlenswert, axiomatische Gesichtspunkte über einen längeren Zeitraum und an verschiedenen Stellen der Schullaufbahn (auch in der Sekundarstufe I) mit Schülerinnen und Schülern zu thematisieren. 


\section{Fazit}

Mit dieser Arbeit wurden drei Hauptziele verfolgt: einen Gesamtüberblick über die Bedeutung der axiomatischen Methode in der Mathematik zu geben, interessierten Lehrkräften die Grundlage für eine didaktisch fundierte eigene Entscheidung über die Behandlung axiomatischer Themen mit Lernenden zur Verfügung zu stellen und konkrete Möglichkeiten aufzuzeigen, axiomatische Denk- und Arbeitsweisen zum Gegenstand von Lernumgebungen zu machen.

Basierend auf mathematikhistorischen und -philosophischen Betrachtungen haben sich drei grundlegende Typen von Axiomatik herauskristallisiert. Diese sind mit jeweils unterschiedlichen Zielsetzungen in Bezug auf die Darstellung mathematischer Theorien wie auch divergierenden Auffassungen über den ontischen Status mathematischer Objekte und den epistemischen Status mathematischer Aussagen verbunden:

- Die Euklidische Axiomatik strebt an, ein bestimmtes mathematisches Gebiet (beispielsweise die Geometrie) zu beschreiben, und stellt dabei Aussagen an den Anfang ihrer Theorien, die als absolut wahr (bzgl. einer wie auch immer gearteteten Realität) betrachtet werden. Aus diesen Axiomen deduzierte Aussagen sind damit ebenfalls unumstößlich wahr.

- Die Hilbertsche Axiomatik hat ebenfalls zum Ziel, ein bestimmtes Themengebiet vollständig zu charakterisieren, verzichtet aber auf die ontologische Verankerung der mathematischen Grundbegriffe, welche völlig abstrakt aufgefasst und durch die Axiome implizit definiert werden. Die Frage nach der Wahrheit von Axiomen stellt sich dann nicht mehr, von mathematischem Interesse ist nur noch deren Widerspruchsfreiheit.

- Die Bourbakische Axiomatik teilt den formalen Charakter mit der Hilbertschen Axiomatik, behandelt jedoch Axiomensysteme, die explizit verschiedene, nichtisomorphe Modelle besitzen. Dadurch können inhaltlich verschiedene, aber strukturell ähnliche Themengebiete in abstrakt formulierten Theorien zusammengefasst werden. 
Durch Gödels Unvollständigkeitssätze stellte sich in der ersten Hälfte des 20. Jahrhunderts heraus, dass es nicht gelingen kann, die gesamte Mathematik formal auf ein widerspruchsfreies System von Axiomen aufzubauen und damit ihre absolute Sicherheit zu garantieren. Dennoch hat die axiomatische Methode das Selbstverständnis der Mathematik wesentlich geprägt und bleibt eine unverzichtbare Grundlage für die systematische Darstellung mathematischer Theorien.

Im Fokus der didaktischen Betrachtungen standen die Reformbestrebungen der Neuen Mathematik und die in diesem Zusammenhang intensiv geführte Debatte über die Sinnhaftigkeit, Axiomatik zum Gegenstand des Mathematikunterrichts zu machen. Obwohl die didaktischen Argumente gegen eine Axiomatikbehandlung schwer wiegen und bei neuen Investitionen berücksichtigt werden müssen, kann aus der Rücknahme der Reform nicht grundsätzlich auf die Ungeeignetheit axiomatischer Denk- und Arbeitsweisen als Unterrichtsthema geschlossen werden. Dies liegt unter anderem daran, dass eine Vielzahl institutioneller und organisatorischer Rahmenbedingungen erheblich zum Scheitern der Neuen Mathematik beigetragen und dabei die inhaltlichen Aspekte überlagert haben.

Eine Haupterkenntnis bleibt aber, dass ein axiomatischer Aufbau ganzer Schulstoffgebiete aus methodischen, lernpsychologischen und zeitlich-organisatorischen Gründen nicht zielführend ist. Axiomatik aus diesen Gründen komplett aus dem Mathematikunterricht zu verbannen, wie es im Anschluss an die Neue Mathematik zum Teil getan wurde und bis heute nachzuwirken scheint, ist jedoch aus meiner Sicht ebenfalls kaum vertretbar: Einblicke in den axiomatischen Aufbau der Mathematik und die unterschiedlichen Sichtweisen auf Axiome gehören zu einem abgerundeten Gesamtbild der Mathematik, wie es in der Schule angebahnt werden sollte. Zu berücksichtigen sind dabei sowohl der Produktals auch der Prozesscharakter der Mathematik. Nn Bezug auf Axiomatik impliziert dies, dass Schülerinnen und Schüler nicht nur lernen sollten, mit vorgegebenen Axiomensystemen deduktiv zu arbeiten, sondern auch Einsicht in das Zustandekommen verschiedener Axiomensysteme gewinnen sollten.

Als Orientierung für die Behandlung axiomatischer Denk- und Arbeitsweisen mit Schülerinnen und Schülern habe ich sechs übergeordnete Lernziele formuliert, welche in Summe ein relativ abgerundetes Bild dieser Thematik beschreiben. Eine Analyse bestehender Unterrichtsideen hat ergeben, dass es gerade für die Sekundarstufe I bereits überzeugende Materialien gibt, die einen Großteil dieser Lernziele abdecken. Es wurde jedoch auch deutlich, dass gerade hinsichtlich der fachlich-philosophischen Tiefe wie auch der Authentizität der behandelten Themenbereiche noch Potential für die Entwicklung geeigneter Konzepte vorhanden ist, welche insbesondere in der Sekundarstufe II anzusiedeln sind und dort (je nach eigener Entscheidung) überwiegend im fakultativen Bereich. 
Im Rahmen dieser Arbeit wurden drei Unterrichtsreihen entwickelt und erprobt, die authentische Beispiele von Axiomatik behandeln und Lernenden die Gelegenheit bieten, auf einer Meta-Ebene axiomatische Charakteristika der Mathematik zu diskutieren:

- Anhand der Geometrie wird die historische Entwicklung der axiomatischen Methode - insbesondere mit Blick auf den Wahrheitsanspruch mathematischer Aussagen - nachgezeichnet, von Euklids Elementen über die Entdeckung nichteuklidischer Geometrien bis zu Hilberts Grundlagen der Geometrie.

- Am Beispiel der Wahrscheinlichkeitsrechnung werden inhaltliche Ansätze zur Begriffsbestimmung mit dem abstrakt-axiomatischen Zugang verglichen. Anhand einer überschaubaren Anzahl von Aussagen wird die Eigentätigkeit des Axiomatisierens in den Vordergrund gestellt; anschließend werden wahrscheinlichkeitstheoretische Aussagen aus dem Kolmogorov-Axiomensystem deduziert.

- Das Thema Zahlbereichserweiterungen bietet die Gelegenheit, formale Setzungen wie $i^{2}=-1$ oder $0 \cdot b=1$ kritisch zu hinterfragen und Möglichkeiten ihrer Rechtfertigung zu diskutieren.

Bei den Unterrichtsreihen habe ich mich bewusst dafür entschieden, die behandelten Inhalte nicht derart in Sachkontexte oder Geschichten einzubetten, dass ihr mathematischer Ursprung verdeckt würde. Axiomatische Fragestellungen werden - beispielsweise durch den Gebrauch historischer Originalquellen - in einer Form behandelt, die den mathematikhistorischen Gegebenheiten zumindest ähnlich ist.

Die Erfahrungen aus der Erprobung der Unterrichtsreihen haben gezeigt, dass axiomatische Themen bei Schülerinnen und Schülern auf Interesse stoßen und die entwickelten Materialien zu einer reflektierteren Haltung bezüglich der Bedeutung der Axiomatik in der Mathematik beitragen. Die übergeordneten Lernziele wurden dabei zu weiten Teilen erreicht; in den Diskussions- und Reflexionsphasen wie auch in den Textproduktionen der Lernenden spielte vor allem das Thema der Sinnhaftigkeit der Mathematik eine große Rolle, was zu einer intensiven Auseinandersetzung mit der klassischen und modernen Sicht auf Axiomatik und der Rechtfertigungsproblematik führte.

Zur Nachhaltigkeit der vermittelten Inhalte lässt sich auf Basis der Investitionen im Rahmen dieser Dissertation nichts sagen. Es ist davon auszugehen, dass ihr unter anderem aufgrund der komprimierten, einmaligen Auseinandersetzung Grenzen gesetzt sind. Es wäre definitiv zu begrüßen, wenn Schülerinnen und Schüler über einen längeren Zeitraum an verschiedenen Stellen ihres Bildungsweges (auch in der Sekundarstufe I) exemplarische und altersgemäße Einblicke in Aspekte der Axiomatik erhielten, und so zu einer vertieften Reflexion auf der Meta-Ebene befähigt würden. 
Ich hoffe, mit dieser Arbeit die Axiomatik nach einer gewissen „Über-Eliminierung“ im schulischen Umfeld wieder mehr interessierten Menschen zugänglich zu machen. Axiomatisches Denken und Arbeiten ist ein zu wichtiger Bestandteil der Mathematik, um in der Schule komplett ausgeblendet zu werden. 


\section{A Unterrichtsmaterialien}

\section{A.1 Arbeitsblätter}

\section{Übersicht}

\begin{tabular}{|c|c|c|}
\hline Arbeitsblatt & Thema & Seiten \\
\hline EINF1 & Die Sicherheit mathematischer Aussagen & 204 \\
\hline GEOM 1 & Beweisen und Ordnen geometrischer Sätze & 205-209 \\
\hline GEOM2 & $\begin{array}{l}\text { Sphärische Geometrie - Erste Erkundungen auf der } \\
\text { Kugeloberfläche }\end{array}$ & $210-211$ \\
\hline GEOM3 & $\begin{array}{l}\text { Flächeninhalt und Innenwinkelsumme sphärischer } \\
\text { Dreiecke }\end{array}$ & 212 \\
\hline GEOM4 & Von Dimpfeln und Gronkeln & $213-214$ \\
\hline W KEIT1 & $\begin{array}{l}\text { Was ist Wahrscheinlichkeit? - Klassische } \\
\text { Zufallsexperimente }\end{array}$ & $215-216$ \\
\hline W KEIT2 & $\begin{array}{l}\text { Was ist Wahrscheinlichkeit? - Mengentheoretische } \\
\text { Grundbegriffe }\end{array}$ & 217 \\
\hline WKEIT3 & Was ist Wahrscheinlichkeit? - Elementare Regeln & $218-220$ \\
\hline W KEIT4 & Was ist Wahrscheinlichkeit? - Die Kolmogorov-Axiome & $221-222$ \\
\hline W KEIT5 & $\begin{array}{l}\text { Was ist Wahrscheinlichkeit? - Bedingte Wahrscheinlichkeit } \\
\text { und stochastische Unabhängigkeit }\end{array}$ & 223 \\
\hline ZBE1 & Quadratische Gleichungen - Zwei Schülerlösungen & 224 \\
\hline ZBE2 & Kubische Gleichungen - Mathematikhistorischer Exkurs & 225 \\
\hline ZBE3 & Kubische Gleichungen - Die Cardanische Formel & $226-227$ \\
\hline ZBE4 & $\begin{array}{l}\text { Herleitung der Cardanischen Formel - Für } \\
\text { Algebra-Experten }\end{array}$ & $228-229$ \\
\hline ZBE5 & Weitere Zahlbereichserweiterungen & 230 \\
\hline
\end{tabular}




\section{Die Sicherheit mathematischer Aussagen}

1. Betrachten Sie die folgenden mathematischen Aussagen. Welche sind Ihrer Meinung nach wahr und welche nicht? Bei welchen sind Sie sich nicht sicher?

(1) $2+2=4$

(2) $(a+b)^{2}=a^{2}+2 a b+b^{2}$ für $a, b \in \mathbb{R}$

(3) Die Summe der Innenwinkel in einem Dreieck beträgt stets $180^{\circ}$.

(4) In einem Dreieck mit den Seiten $a, b$ und $c$, wobei $c$ die längste der drei Seiten sei, gilt stets $a^{2}+b^{2}=c^{2}$.

(5) Wenn $p$ eine Primzahl ist, so ist $2^{p}-1$ ebenfalls eine Primzahl.

(6) Die Gleichung $x^{n}+y^{n}=z^{n}$ (wobei $x, y, z \in \mathbb{N}$ ) hat für $n \geq 3$ keine Lösung.

(7) Jede gerade natürliche Zahl größer als 2 kann als Summe zweier Primzahlen dargestellt werden.

2. Welche Möglichkeiten kennen Sie, um herauszufinden, ob eine mathematische Aussage richtig oder falsch ist? (Nennen Sie mindestens drei verschiedene Aspekte.)

1)

2)

3)

4)

5)

3. Die amerikanische Wissenschaftsphilosophin Penelope Maddy (geb. 1950) meint:

Von all den merkwürdigen und unterschiedlichen Dingen, die wir glauben, gibt es nur wenige, denen wir mehr Glauben schenken, als den Wahrheiten einfacher Mathematik. Nach einem Beispiel für eine durch und durch zuverlässige Tatsache gefragt, werden sich viele abwenden vom gesunden Menschenverstand - "früher dachte man auch, Menschen könnten nicht fliegen" von Naturwissenschaft - "die Sonne ist bislang jeden Tag aufgegangen, aber das muss morgen nicht so sein" - hin zu der Sicherheit der Arithmetik - „aber 2 plus 2 ist sicher 4".

Nehmen Sie Stellung zu dieser Aussage. Ist der hier beschriebene Ruf der Mathematik gerechtfertigt? Was macht mathematische Aussagen in den Augen vieler Leute so sicher? 


\section{Beweisen und Ordnen geometrischer Sätze}

1. Vervollständigen Sie den Beweis des Satzes des Thales, indem Sie die Lücken ausfüllen:

Liegt der Punkt $C$ eines Dreiecks $A B C$ auf dem Halbkreis über der Seite $A B$, dann hat das Dreieck bei $C$ einen rechten Winkel.

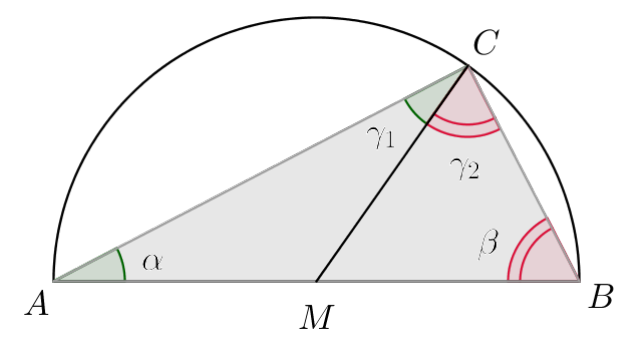

Beweis: Wir betrachten das Dreieck $A B C$, wobei $A$ und $B$ die Endpunkte des Kreisdurchmessers sind und $C$ ein beliebiger weiterer Punkt auf dem Kreisbogen ist. Wir zeichnen nun die Strecke $C M$, wobei $M$ der (Halb-)Kreismittelpunkt ist.

- Die Winkel und sowie und sind jeweils Basiswinkel der grund des Dreiecke $A M C$ bzw. $M B C$ und somit auf-

- Aufgrund des identisch. gilt ferner:

$$
\alpha+\beta+\gamma_{1}+\gamma_{2}=
$$

- Es folgt $2 \cdot\left(\gamma_{1}+\gamma_{2}\right)=$ , denn

- Daraus folgt direkt

$$
\left(\gamma_{1}+\gamma_{2}=\right) \gamma=
$$

2. a) Ordnen Sie den Skizzen auf den Karteikarten die passende Formulierung des dargestellten geometrischen Sachverhaltes zu.

b) Formulieren Sie Beweise für möglichst viele der Aussagen und markieren Sie explizit, welche Sätze in den verschiedenen Beweisschritten verwendet werden. 
3. Ordnen Sie die Karteikarten so, dass die logischen Zusammenhänge zwischen den einzelnen Aussagen erkennbar werden. Übertragen Sie diese Ordnung nach folgendem Schema in Ihr Heft.

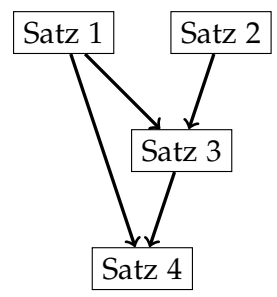

[Die Pfeile sollen angeben, welche Sätze beim Beweis eines anderen Satzes verwendet wurden. Z. B.: Im Beweis von Satz 4 wurden die Sätze 1 und 3 verwendet.]

4. Welche Auswirkungen auf das in Aufgabe 3 erstellte Schema hat es, wenn man auf die folgende Aussage verzichten wollte?

a) Innenwinkelsatz für Dreiecke

b) Parallelensatz

c) Kongruenzsatz SWS

d) Wechselwinkelsatz

e) Nebenwinkelsatz

5. a) Beweisen Sie den Stufenwinkelsatz mit Hilfe des Wechsel- und Scheitelwinkelsatzes.

b) Wie müsste das in 3. erstellte Schaubild aufgrund dieses Beweises geändert werden?

6. a) Beweisen Sie den Außenwinkelsatz mit Hilfe der folgenden Skizze (dabei soll die Gerade $g$ parallel zur Strecke $A B$ sein):

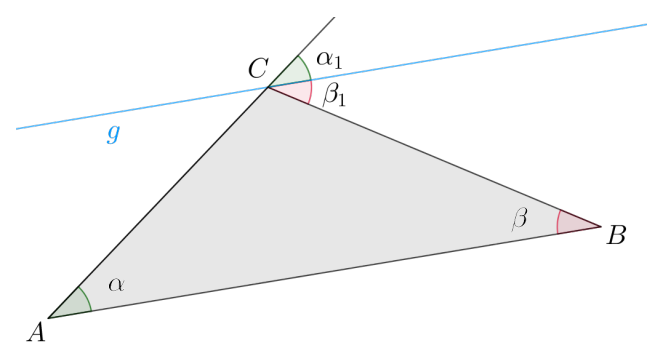

Folgern Sie anschließend den Innenwinkelsatz aus dem Außenwinkelsatz.

b) Wie müsste das in 3. erstellte Schaubild aufgrund von Teil a) geändert werden? 

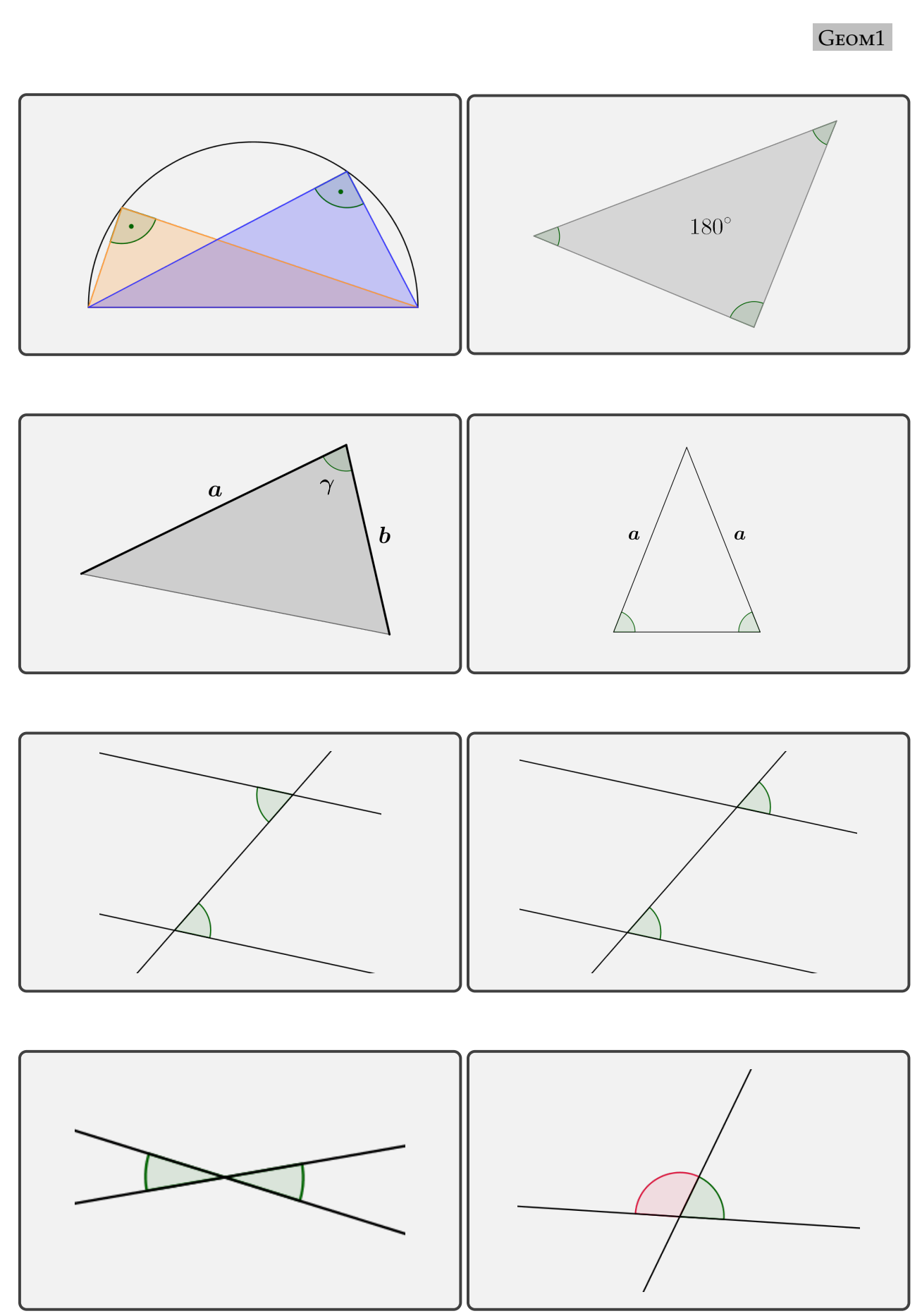
GEом1
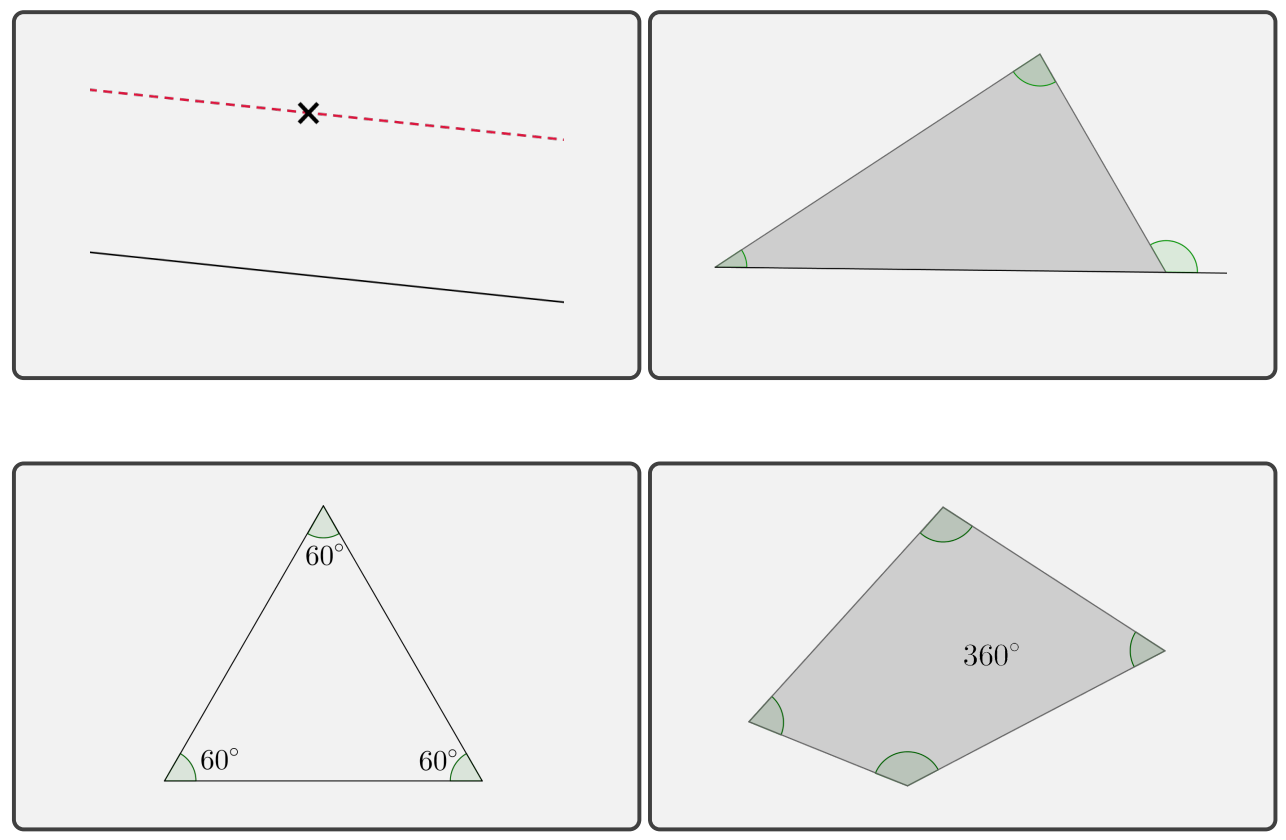


\section{Gеом1}

WINKEL IM GLEICHSEITIGEN DREIECK

In einem gleichseitigen Dreieck hat jeder der Innenwinkel die Größe $60^{\circ}$.

INNENWINKELSATZ FÜR VIERECKE

Die Summe der Winkel in jedem Viereck beträgt $360^{\circ}$.

\section{PARAllelensatz}

$\mathrm{Zu}$ einer gegebenen Geraden $g$ und einem Punkt außerhalb von $g$ gibt es genau eine $\mathrm{zu} g$ parallele Gerade, die durch diesen Punkt verläuft.

NeBENWINKELSATZ

Nebenwinkel ergänzen sich zu $180^{\circ}$.

WECHSELWINKELSATZ

Wechselwinkel an parallelen Geraden sind gleich groß.

KongruenZsATZ SWS

Zwei Dreiecke, die in zwei Seitenlängen und in dem von diesen Seiten eingeschlossenen Winkel übereinstimmen, sind kongruent.

\section{AussenWINKELSATZ}

In jedem Dreieck ist die Summe zweier Innenwinkel gleich dem nicht anliegenden Außenwinkel.

INNENWINKELSATZ FÜR DREIECKE

Die Summe der Winkel in einem Dreieck beträgt $180^{\circ}$.

\section{BASISWINKELSATZ}

In jedem gleichschenkligen Dreieck sind die Basiswinkel gleich groß.

\section{SCHEITELWINKELSATZ}

Scheitelwinkel an sich schneidenden Geraden haben dieselbe Größe.

STUFENWINKELSATZ

Stufenwinkel an parallelen Geraden sind gleich groß. 


\section{Sphärische Geometrie}

Erste Erkundungen auf der Kugeloberfläche

Die kürzeste Verbindung zwischen zwei Punkten auf der Kugeloberfläche erhält man, wenn man einen Faden zwischen diesen spannt. Welches Gebilde - welches wir ",sphärische Gerade“ nennen wollen - erhält man, wenn man diesen Faden über die Punkte hinaus verlängert und weiter spannt?

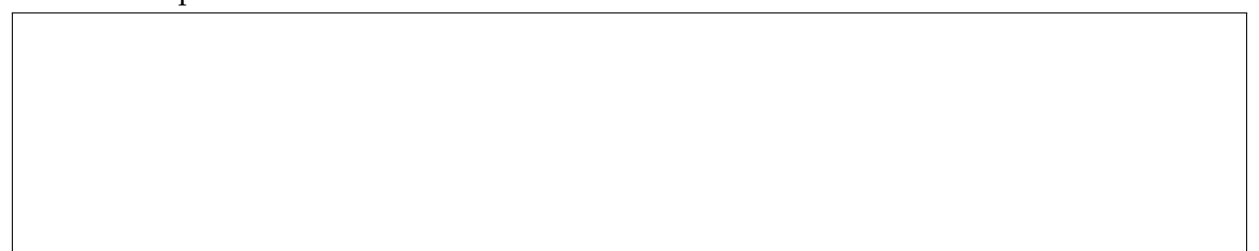

In der Euklidischen Geometrie schneiden sich zwei Geraden in genau einem Punkt oder sie sind parallel. Wie verhalten sich zwei sphärische Geraden?

Wenn man in der Euklidischen Geometrie zu einer gegebenen Geraden $g$ alle Punkte betrachtet, die von dieser den gleichen Abstand haben und auf derselben Seite von $g$ liegen, erhält man eine zu $g$ parallele Gerade. Was erhält man, wenn man alle Punkte betrachtet, die zu einer sphärischen Geraden den gleichen (sphärischen) Abstand haben und auf derselben Seite liegen? 
Ein „sphärischer Kreis“ ist die Menge aller Punkte auf einer Kugeloberfläche, die von einem fest gewählten Punkt $M$ denselben (sphärischen) Abstand (= Radius) haben. Was passiert, wenn man den Radius vergrößert bzw. verkleinert?

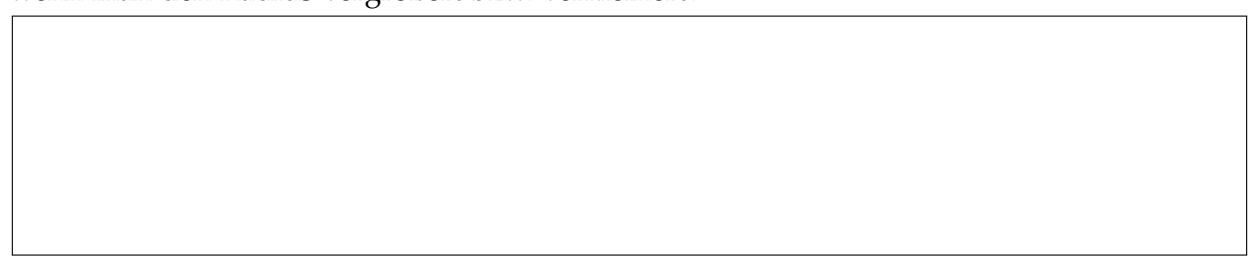

Wie sieht ein sphärisches Dreieck aus,

a) das genau drei rechte Winkel hat?

b) das genau zwei rechte Winkel hat?

c) dessen Innenwinkelsumme möglichst groß ist?

d) dessen Innenwinkelsumme möglichst nah an $180^{\circ}$ liegt?

In der Euklidischen Geometrie gilt:

Unter drei verschiedenen Punkten einer Geraden gibt es stets genau einen Punkt, der zwischen den beiden anderen liegt.

Was kann man über drei Punkte auf einer sphärischen Geraden aussagen? 


\section{Flächeninhalt und Innenwinkelsumme sphärischer Dreiecke}

Auf der Ihnen vorliegenden Styroporkugel sind die drei Großkreise $A B, A C$ und $B C$ eingezeichnet, wodurch das Kugeldreieck $\triangle A B C$ entsteht. Die Punkte $A^{d}, B^{d}$ und $C^{d}$ bezeichnen die zu $A, B$ und $C$ diametralen (d.h. in Bezug auf den Kugelmittelpunkt gegenüberliegenden) Punkte.

1. Nummerieren Sie alle auf der Kugel sichtbaren Kugeldreiecke und listen Sie sie auf:

$\triangle A B C$,

2. Finden Sie Kugelzweiecke, die $\triangle A B C$ beinhalten und schraffieren Sie sie in unterschiedlichen Farben.

3. Welche Kugelzweiecke bleiben übrig? Vergleichen Sie diese mit den bereits schraffierten Kugelzweiecken. Welchen Zusammenhang kann man erkennen?

4. Schraffieren Sie die weißen Kugelzweiecke nun ebenfalls (geschickt!) farbig.

5. Wir geben zunächst eine allgemeine Formel zur Berechnung des Flächeninhalts eines Kugelzweiecks an: In der nebenstehenden Abbildung lässt sich erkennen, dass das Verhältnis zwischen dem Winkelmaß $\alpha$ eines Kugelzweiecks und dem eines Vollwinkels $\left(360^{\circ}=\right.$ $2 \pi$ ) gleich dem Verhältnis des Flächeninhalts $F_{Z}$ des Zweiecks zum Oberflächeninhalt $O$ der gesamten Kugel ist.

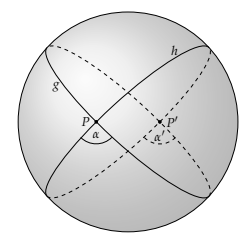

Welche Formel für den Flächeninhalt $F_{Z}$ ergibt sich aus dieser Überlegung?

$$
F_{Z}=
$$

(Erinnerung: $O=4 \cdot \pi \cdot R^{2}$, wobei $R$ der Kugelradius ist.)

6. Berechnen Sie (bei gegebenem Kugelradius $R$ ) anhand Ihrer Vorüberlegungen den Flächeninhalt des Kugeldreiecks $\triangle A B C$.

(Hinweis: Überlegen Sie, wie sich die Kugeloberfläche geeignet aus Teilzweiecken zusammensetzen lässt.)

7. Welcher Zusammenhang zwischen der Innenwinkelsumme eines Kugeldreiecks und seinem Flächeninhalt ergibt sich aus der in 6. berechneten Formel? 


\section{Von Dimpfeln und Gronkeln}

Gegeben sei eine nicht-leere Menge D von Dingen, die wir Dimpfel nennen, sowie eine nicht-leere Menge $G$ von Dingen, die wir Gronkel nennen.

Zwischen Dimpfeln und Gronkeln besteht eine Beziehung, die wir mit dem Wort klubern bezeichnen. Wir sagen etwa: „Ein Dimpfel klubert einen Gronkel“ bzw. „Ein Gronkel wird von einem Dimpfel geklubert".

Für Dimpfel und Gronkel sollen die folgenden Axıome gelten:

$A_{1}: \mathrm{Zu}$ zwei verschiedenen Dimpfeln $d_{1}$ und $d_{2}$ gibt es genau einen Gronkel, der von $d_{1}$ und $d_{2}$ geklubert wird.

$A_{2}$ : Jeder Gronkel wird von mindestens zwei Dimpfeln geklubert.

$A_{3}$ : Es gibt mindestens drei Dimpfel, die nicht alle denselben Gronkel klubern.

\section{Aufgabe:}

Leiten Sie aus den Axiomen folgende Aussagen über Dimpfel und Gronkel her:

a) Zwei verschiedene Gronkel werden von höchstens einem Dimpfel gleichzeitig geklubert.

b) Es gibt mindestens drei verschiedene Gronkel.

c) Wenn $d_{1}$ und $d_{2}$ Dimpfel sind, dann findet man stets einen dritten Dimpfel $d_{3}$, so dass $d_{1}, d_{2}$ und $d_{3}$ nicht denselben Gronkel klubern. 


\section{Von Musikern und Instrumenten}

Gegeben sei eine nicht-leere Menge $D$ von Personen, die wir Musiker nennen, sowie eine nicht-leere Menge $G$ von Dingen, die wir Instrumente nennen.

Zwischen Musikern und Instrumenten besteht eine Beziehung, die wir mit dem Wort spielen bezeichnen. Wir sagen etwa: „Ein Musiker spielt ein Instrument“ bzw. „Ein Instrument wird von einem Musiker gespielt".

Für Musiker und Instrumente sollen die folgenden Axıome gelten:

$A_{1}: \mathrm{Zu}$ zwei verschiedenen wird von mindestens zwei

$A_{2}$ : Jedes

$A_{3}:$ Es gibt mindestens drei $a$ und $b$ gibt es genau wird.

\section{Von Punkten und Geraden}

Gegeben sei eine nicht-leere Menge $\mathbb{P}$ von Dingen, die wir Punkte nennen, sowie eine nichtleere Menge $G$ von Dingen, die wir Geraden nennen.

Zwischen Punkten und Geraden besteht eine Beziehung, die wir mit dem Wort bezeichnen. Wir sagen etwa: bzw. “.

Für Punkte und Geraden sollen die folgenden Axıome gelten:

$$
\begin{aligned}
& A_{1}: \\
& A_{2}: \\
& A_{3}:
\end{aligned}
$$




\section{Was ist Wahrscheinlichkeit?}

Klassische Zufallsexperimente
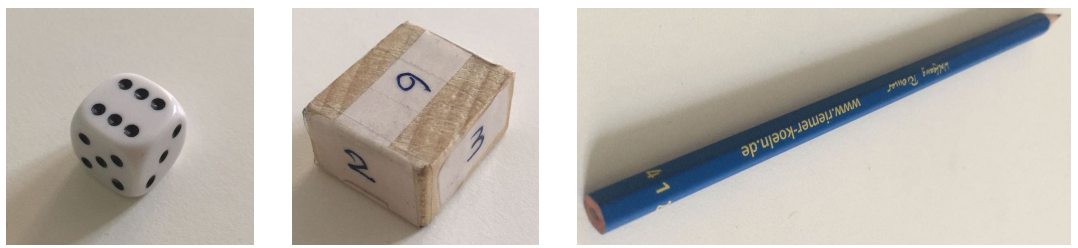

1. In den obigen Abbildungen sehen Sie drei verschiedene Zufallsgeneratoren, die alle mit den Ziffern 1 bis 6 beschriftet sind. Schätzen Sie für alle drei Objekte vor der Durchführung eines Zufallsexperiments die Wahrscheinlichkeiten für die sechs möglichen Augenzahlen und ermitteln Sie auf dieser Grundlage, wie groß die Wahrscheinlichkeit für die folgenden Ereignisse jeweils wäre. Begründen Sie Ihre Schätzungen.
A: Es liegt eine gerade Zahl oben.
B: Es liegt eine Primzahl oben.
C: Es liegt eine gerade Zahl oder eine Primzahl oben.
D: Es liegt eine gerade Zahl und eine Primzahl oben.
E: Es liegt weder eine gerade Zahl noch eine Primzahl oben.

2. a) Werfen Sie die Zufallsgeneratoren jeweils $120 \mathrm{Mal}(60 \mathrm{Mal})$ und füllen Sie für jedes Objekt folgende Tabelle aus:

\begin{tabular}{c|c|c|c} 
Ergebnis & Strichliste & abs. H. & rel. H. \\
\hline \hline 1 & & & \\
\hline 2 & & & \\
\hline 3 & & & \\
\hline 4 & & & \\
\hline 5 & & & \\
\hline 6 & & & \\
\hline
\end{tabular}

b) Stellen Sie auf Grundlage der ausgefüllten Tabelle eine im Vergleich zu Aufgabe 1 ggf. revidierte Wahrscheinlichkeitsverteilung für jeden Zufallsgenerator auf:

\begin{tabular}{|c|r|r|r|r|r|r|}
\hline Ergebnis & \multicolumn{1}{|c|}{1} & \multicolumn{1}{c|}{2} & \multicolumn{1}{c|}{3} & \multicolumn{1}{c|}{4} & \multicolumn{1}{c|}{5} & \multicolumn{1}{c|}{6} \\
\hline Wahrscheinlichkeit & $\%$ & $\%$ & $\%$ & $\%$ & $\%$ & $\%$ \\
\hline
\end{tabular}




\section{WKEIT1}

3. Tom: "Ich habe 6 Mal gewürfelt, es ist aber kein Mal eine Sechs gekommen. Kann es dann trotzdem sein, dass die Wahrscheinlichkeit, eine Sechs zu würfeln, bei meinem Würfel ein Sechstel beträgt?"

Anja: „Ich habe 600 Mal gewürfelt und es ist 200 Mal eine Sechs gekommen. Beträgt die Wahrscheinlichkeit, eine Sechs zu würfeln, bei meinem Würfel nun ein Sechstel oder nicht doch eher ein Drittel?"

Was würden Sie den beiden antworten?

4. Der oben abgebildete Quader wurde 1000 Mal geworfen. Dabei wurden zwischendurch die relativen Häufigkeiten der Augenzahl 3 notiert:

\begin{tabular}{|c|c|}
\hline Anzahl Würfe & rel. H. \\
\hline \hline 200 & $35 \%$ \\
\hline 400 & $31,5 \%$ \\
\hline 600 & $30,8 \%$ \\
\hline 800 & $31,7 \%$ \\
\hline 1000 & $30,4 \%$ \\
\hline
\end{tabular}

Welche Wahrscheinlichkeit würden Sie für das Ereignis „Beim Würfeln des Quaders erscheint die Augenzahl 3“ angeben? 


\section{Was ist Wahrscheinlichkeit?}

Mengentheoretische Grundbegriffe

1. Eine ideale Münze wird dreimal hintereinander geworfen. Geben Sie eine passende Ergebnismenge $\Omega$ an und stellen Sie die folgenden Ereignisse als Teilmengen von $\Omega$ dar. Welche Wahrscheinlichkeiten kann man den Ereignissen zuordnen?

A: „Es fällt genau zweimal Zahl."

B: "Es fällt mindestens zweimal Wappen."

C: "Es fällt höchstens zweimal Wappen."

D: „Beim ersten Wurf fällt Zahl und insgesamt fällt mindestens einmal Wappen."

2. Eine Schale enthält 20 Kugeln mit den Zahlen 0 bis 19. Es wird eine Kugel verdeckt gezogen. Es sei E : "Die Zahl auf der Kugel ist eine Primzahl“ und F : "Die Zahl auf der Kugel ist durch 5 teilbar". Geben Sie die folgenden Ereignisse in Mengenschreibweise und in Worten an:

$$
E \cap F, \quad E \cup F, \quad \bar{E}, \quad \overline{E \cup F}
$$

3. Wir betrachten das Zufallsexperiment des zweifachen Würfelwurfs. Geben Sie für die folgenden Situationen jeweils eine geeignete Ergebnismenge $\Omega$ an und bestimmen Sie die Wahrscheinlichkeiten für die Elementarereignisse:

(i) Die beiden Würfel werden hintereinander geworfen.

(ii) Die beiden Würfel werden gleichzeitig geworfen und die Würfel sind nicht unterscheidbar.

(iii) Man interessiert sich ausschließlich für die Augensumme der beiden Würfe.

4. Beim Spiel „Mensch ärgere dich nicht" darf man seine Spielfigur erst dann aus der Startposition heraus auf sein Startfeld des Spielfeldes stellen, wenn man eine Sechs gewürfelt hat. Geben Sie eine passende Ergebnismenge $\Omega$ für diese Situation an und ermitteln Sie die Wahrscheinlichkeit, dass beim ersten (zweiten, dritten, ..., $k$-ten) Wurf zum ersten Mal eine Sechs gewürfelt wird. 
WKEIT3

\section{Was ist Wahrscheinlichkeit?}

Elementare Regeln

Folgern Sie die folgenden Regeln für Wahrscheinlichkeiten jeweils aus den anderen. Notieren Sie anschließend explizit, welche der anderen Regeln Sie zur Herleitung benötigt haben.

(1) $P(\Omega)=1$

(2) $P(\emptyset)=0$

(3) $P(A \cup B)=P(A)+P(B)-P(A \cap B)$

(4) $P(A \uplus B)=P(A)+P(B)^{*}$

(5) $P(A) \geq 0$

(6) $P(A) \leq 1$

(7) $P(\bar{A})=1-P(A)$

(8) $A \subseteq B \Rightarrow P(B \backslash A)=P(B)-P(A)$

(9) $A \subseteq B \Rightarrow P(A) \leq P(B)$

\begin{tabular}{rr||r|r|r|r|r|r|r|l|l} 
& $\downarrow$ Regel ... kann man folgern aus $\rightarrow$ & 1 & 2 & 3 & 4 & 5 & 6 & 7 & 8 & 9 \\
\hline \hline 1 & $P(\Omega)=1$ & & & & & & & & & \\
\hline 2 & $P(\emptyset)=0$ & & & & & & & & & \\
\hline $3 \quad P(A \cup B)=P(A)+P(B)-P(A \cap B)$ & & & & & & & & & \\
\hline $4 \quad P(A \uplus B)=P(A)+P(B)$ & & & & & & & & & \\
\hline 5 & $P(A) \geq 0$ & & & & & & & & & \\
\hline 6 & $P(A) \leq 1$ & & & & & & & & & \\
\hline 7 & $P(\bar{A})=1-P(A)$ & & & & & & & & & \\
\hline 8 & $A \subseteq B \Rightarrow P(B \backslash A)=P(B)-P(A)$ & & & & & & & & & \\
\hline 9 & $A \subseteq B \Rightarrow P(A) \leq P(B)$ & & & & & & & & & \\
\hline
\end{tabular}

${ }^{*}$ Hinweis zu Regel (4): Die Schreibweise $A \uplus B$ soll kennzeichnen, dass es sich um die Vereinigung von disjunkten Mengen handelt, d. h. $A \cap B=\emptyset$. 
WKEIT3

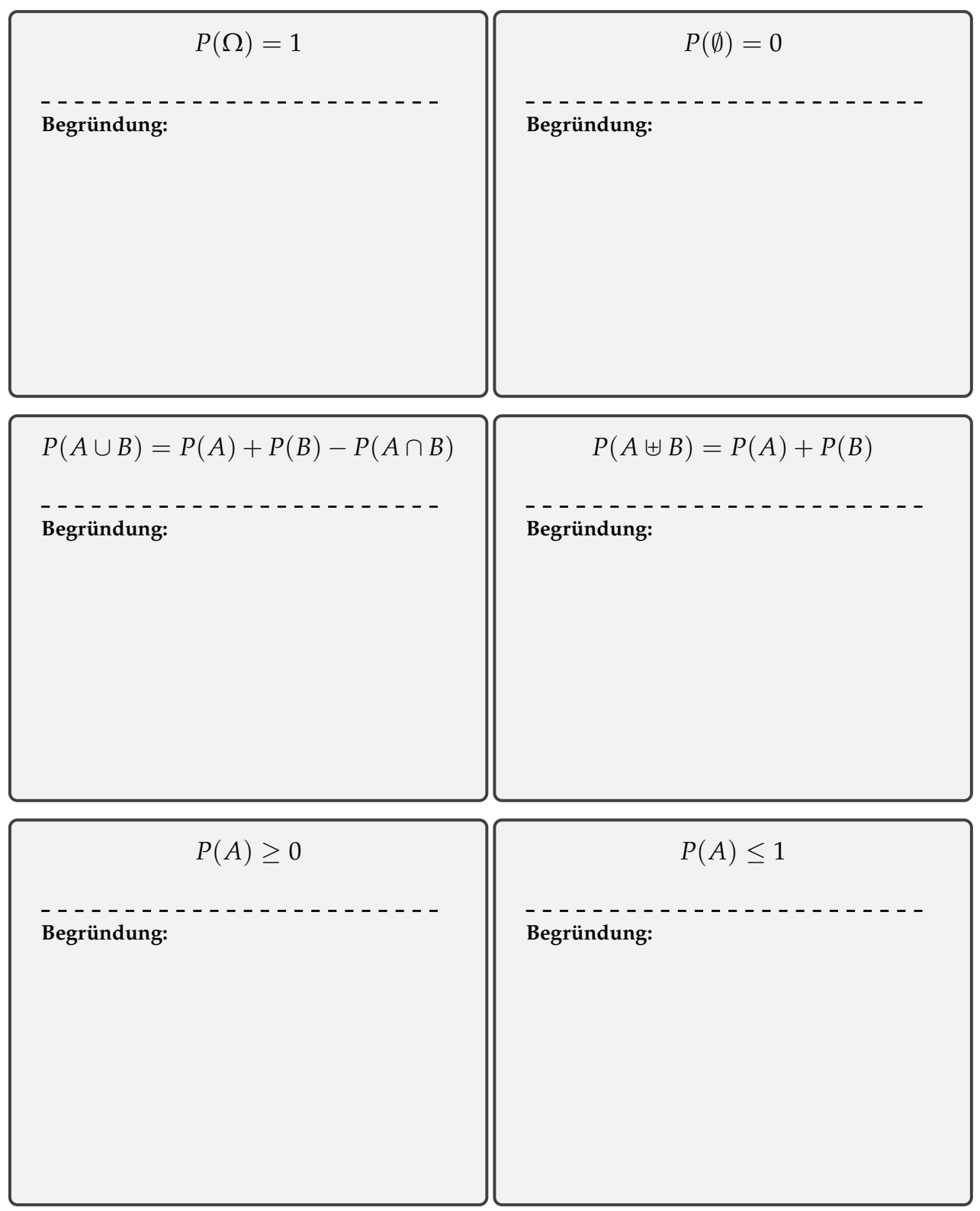


WKeIT3

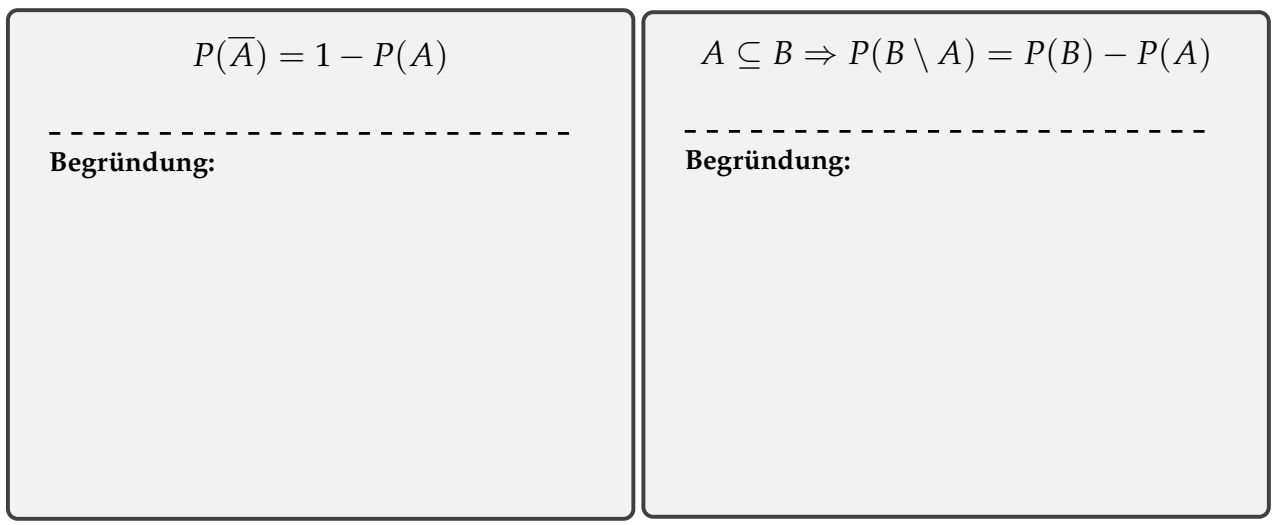

$A \subseteq B \Rightarrow P(A) \leq P(B)$

Begründung: 


\section{Was ist Wahrscheinlichkeit?}

Die Kolmogorov-Axiome

Es sei $\Omega$ eine endliche Menge. Eine Funktion $P$ bezeichnet man als Wahrscheinlichkeitsmaß auf $\Omega$, wenn sie jeder Teilmenge $A \subseteq \Omega$ eine reelle Zahl $P(A)$ zuordnet, die auch Wahrscheinlichkeit von $A$ genannt wird, und folgende Eigenschaften besitzt:

(K1) Es gilt $P(A) \geq 0$ für alle $A \subseteq \Omega$.

(K2) Es gilt $P(\Omega)=1$.

(K3) Sind zwei Teilmengen $A$ und $B$ aus $\Omega$ disjunkt (d.h. $A \cap B=\emptyset$ ), dann gilt

$$
P(A \cup B)=P(A)+P(B) .
$$

(K1) bis (K3) nennt man die Kolmogorov-Axiome der Wahrscheinlichkeitsrechnung.

1. a) Leiten Sie aus den Kolmogorov-Axiomen die folgenden Eigenschaften von Wahrscheinlichkeitsmaßen in einer "sinnvollen" Reihenfolge her:
i) $P(A) \leq 1$
iv) $P(\emptyset)=0$
ii) $P(A \cup B)=P(A)+P(B)-P(A \cap B)$
v) $P(\bar{A})=1-P(A)$
iii) $A \subseteq B \Rightarrow P(B \backslash A)=P(B)-P(A)$
vi) $A \subseteq B \Rightarrow P(A) \leq P(B)$

b) Es seien $A_{1}, \ldots, A_{n} \subseteq \Omega$ paarweise disjunkt. Zeigen Sie:

$$
P\left(A_{1} \cup \ldots \cup A_{n}\right)=P\left(A_{1}\right)+\ldots+P\left(A_{n}\right)
$$

c) Zeigen Sie für beliebige Ereignisse $A, B, C$ :

$$
P(A \cup B \cup C)=P(A)+P(B)+P(C)-P(A \cap B)-P(B \cap C)-P(C \cap A)+P(A \cap B \cap C)
$$

2. a) Gegeben sei eine Menge $\Omega$ mit $n$ Elementen und eine Funktion $L$, die durch

$$
L(A)=\frac{|A|}{n} \quad \text { für } A \subseteq \Omega
$$

(wobei $|A|$ die Anzahl der Elemente der Menge $A$ angibt) definiert ist. Weisen Sie nach, dass $L$ ein Wahrscheinlichkeitsmaß ist.

b) Gegeben seien eine Menge $\Omega$ und ein Element $\omega \in \Omega$. Wir definieren eine Funktion $\delta_{\omega}$, die jeder Teilmenge $A$ aus $\Omega$ den Wert 1 zuordnet, wenn $\omega \in A$, und allen übrigen Teilmengen den Wert 0 zuordnet.

$$
\delta_{\omega}(A):= \begin{cases}1, & \text { falls } \omega \in A, \\ 0, & \text { falls } \omega \notin A\end{cases}
$$

Zeigen Sie, dass $\delta_{\omega}$ ein Wahrscheinlichkeitsmaß ist. 


\section{WKEIT4}

c) Gegeben sei die Menge $\Omega=\left\{\omega_{1}, \omega_{2}, \omega_{3}, \omega_{4}, \omega_{5}\right\}$. Durch welche der folgenden Bedingungen wird ein Wahrscheinlichkeitsmaß auf $\Omega$ festgelegt? (Hinweis: Statt $P\left(\left\{\omega_{i}\right\}\right)$ schreiben wir kurz $P\left(\omega_{i}\right)$.)
(i) $P\left(\omega_{1}\right)=P\left(\omega_{2}\right)=\ldots=P\left(\omega_{5}\right)=\frac{1}{2}$
(ii) $P\left(\omega_{1}\right)=P\left(\omega_{2}\right)=\ldots=P\left(\omega_{5}\right)=\frac{1}{5}$
(iii) $P\left(\omega_{1}\right)=1, P\left(\omega_{2}\right)=P\left(\omega_{3}\right)=\ldots=P\left(\omega_{5}\right)=0$
(iv) $P\left(\omega_{1}\right)=\frac{1}{2}, P\left(\omega_{2}\right)=\frac{1}{4}, P\left(\omega_{3}\right)=\frac{1}{5}, P\left(\omega_{4}\right)=\frac{1}{25}, P\left(\omega_{5}\right)=\frac{1}{100}$
(v) $P\left(\omega_{1}\right)=\frac{3}{4}, P\left(\omega_{2}\right)=\frac{1}{2}, P\left(\omega_{3}\right)=P\left(\omega_{4}\right)=0, P\left(\omega_{5}\right)=-\frac{1}{4}$

d) Formulieren Sie eine Methode, mit der man auf einer beliebigen (nicht-leeren) endlichen Menge $\Omega=\left\{\omega_{1}, \ldots, \omega_{n}\right\}$ ein Wahrscheinlichkeitsmaß definieren kann. 


\section{Was ist Wahrscheinlichkeit?}

Bedingte Wahrscheinlichkeit und stochastische Unabhängigkeit

Gegeben seien eine Menge $\Omega$ und ein Wahrscheinlichkeitsmaß $P$.

- Wenn $P(B)>0$ ist, dann nennt man

$$
P_{B}(A):=\frac{P(A \cap B)}{P(B)}
$$

die bedingte Wahrscheinlichkeit von $A$ unter der Bedingung $B$.

- Zwei Ereignisse $A$ und $B$ heißen stochastisch unabhängig, wenn gilt:

$$
P(A \cap B)=P(A) \cdot P(B) .
$$

Andernfalls nennt man $A$ und B stochastisch abhängig

1. Gegeben sei ein Wahrscheinlichkeitsmaß $P$ auf der Menge $\Omega$. Weisen Sie nach, dass die Funktion $P_{B}$ (für fest gewähltes $B \subseteq \Omega$ mit $P(B)>0$ ) ebenfalls ein Wahrscheinlichkeitsmaß ist.

2. Beweisen Sie folgende Aussagen:

a) $P(A \cap B)=P_{B}(A) \cdot P(B)=P_{A}(B) \cdot P(A)$

b) Wenn $A$ und $B$ stochastisch unabhängig sind und $P(A), P(B)>0$ gilt, so ist $P_{B}(A)=P(A)$ bzw. $P_{A}(B)=P(B)$

c) Die Ereignisse $A$ und $B$ seien stochastisch unabhängig. Dann sind auch die Ereignisse
(i) $\bar{A}$ und $B$
(ii) $A$ und $\bar{B}$ sowie
(iii) $\bar{A}$ und $\bar{B}$

stochastisch unabhängig.

3. Beweisen Sie:

a) $P(B)=P_{A}(B) \cdot P(A)+P_{\bar{A}}(B) \cdot P(\bar{A})$ (Satz von der totalen Wahrscheinlichkeit)

b) $P_{B}(A)=\frac{P_{A}(B) \cdot P(A)}{P(B)}$ (Satz von Bayes) 


\section{Quadratische Gleichungen}

Zwei Schülerlösungen

Ivo und Tobias sollen die quadratische Gleichung

$$
x^{2}+x+3=0
$$

lösen.

Lösung Ivo

$$
\begin{gathered}
p-q-\text { Formel } x^{2}+x+3=0 \\
\Rightarrow \quad x=-\frac{1}{2} \pm \sqrt{\underbrace{\frac{1}{4}-3}} \\
\Rightarrow L=\{\}
\end{gathered}
$$

Lösung Tobias

$$
\begin{aligned}
& x^{2}+x+3=0 \\
\Rightarrow \quad & x=-\frac{1}{2} \pm \sqrt{\frac{1}{4}-3} \\
\Rightarrow & \mathbb{L}=\left\{-\frac{1}{2}+\sqrt{-\frac{11}{4}},-\frac{1}{2}-\sqrt{-\frac{11}{4}}\right\}
\end{aligned}
$$

Probe:

$$
\begin{aligned}
& \left(-\frac{1}{2}+\sqrt{-\frac{11}{4}}\right)^{2}+\left(-\frac{1}{2}+\sqrt{-\frac{11}{4}}\right)+3 \\
& =\frac{1}{4}-2 \cdot \frac{1}{2} \sqrt{-\frac{11}{4}}-\frac{11}{4}-\frac{1}{2}+\sqrt{-\frac{11}{4}}+3 \\
& =\frac{1}{4}-\frac{11}{4}-\frac{1}{2}+3=-\frac{12}{4}+3=0 \\
& \text { Funktioniert genauso fur }-\frac{1}{2}-\sqrt{-\frac{11}{4}} \\
& \rightarrow \text { Diè beiden Lösungen stimunen. }
\end{aligned}
$$

Ivo erhält für seine Lösung die volle Punktzahl; Tobias erhält 0 Punkte, woraufhin er sich bei seinem Mathe-Lehrer beschweren möchte.

Welche Argumente kann Tobias anbringen? Wie kann wiederum der Lehrer die unterschiedliche Punktevergabe rechtfertigen? 


\section{Kubische Gleichungen}

Mathematikhistorischer Exkurs

Im Italien des 16. Jahrhunderts beschäftigten sich die Mathematiker unter anderem mit der Suche nach geeigneten Lösungsverfahren für verschiedene Typen von Gleichungen. Während sie sich mit linearen und quadratischen Gleichungen (d.h. Gleichungen der Form $a \cdot x+b=0$ bzw. $\left.a \cdot x^{2}+b \cdot x+c=0\right)$ schon recht gut auskannten, bereitete ihnen die Lösung sogenannter kubischer Gleichungen $\left(a \cdot x^{3}+b \cdot x^{2}+c \cdot x+d=0\right)$ noch erhebliche Probleme.

1515 soll es dem Mathematikprofessor SCIPIONE DEL FERRo gelungen sein, Lösungsterme der allgemeinen kubischen Gleichung $x^{3}+a \cdot x=b$ zu bestimmen. Er veröffentlichte seine Ergebnisse jedoch nie.

Zwanzig Jahre später forderte Del FerRos Schüler Antonio MARIA FIORE den in Venedig lebenden Mathematiker Niccolò TARTAGLIA zu einem öffentlichen Wettstreit heraus: Beide sollten dem jeweils anderen 30 Aufgaben stellen, die es innerhalb von 30 Tagen zu lösen galt. Bei den Aufgaben von Fiore an Tartaglia handelte es sich ausschließlich um solche, die auf die Lösung kubischer Gleichungen hinausliefen. Hier sind einige Beispiele:

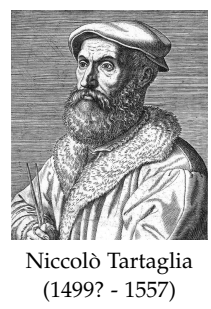

1. Finde mir eine Zahl derart, daß, wenn ihr Kubus addiert wird, das Resultat sechs ist.

2. Finde mir zwei Zahlen in doppelter Proportion derart, wenn das Quadrat der größeren Zahl multipliziert wird mit der kleineren, und wenn dieses Produkt zu den zwei ursprünglichen Zahlen addiert wird, das Ergebnis vierzig sein wird.

9. Finde mir eine Zahl derart, daß, vier ihrer Kubikwurzeln hinzugefügt, siebzehn herauskommt.

15. Ein Mann verkauft einen Saphir für 500 Dukaten und macht dabei einen Gewinn in der dritten Potenz seines Kapitals. Wie groß ist dieser Profit?

Buchstäblich im letzten Moment (in der Nacht vom 29. auf den 30. Tag) gelang es TARTAGLIA, die Lösungen mit Hilfe einer allgemeinen Formel $\mathrm{zu}$ finden und somit den Wettstreit $\mathrm{zu}$ gewinnen.

TARTAGLIAs Erfolg sprach sich schnell herum und so wurde der Arzt Girolamo Cardano auf ihn aufmerksam. Er flehte TartaGLIA förmlich an, ihm seine Lösungsmethode mitzuteilen und versprach hoch und heilig, diese nicht an Dritte weiterzugeben. Schließlich gab TARTAGLIA nach und erläuterte CARDANo den Rechenweg, um die kubischen Gleichungen zu lösen (interessanter Weise in Gedichtform!).

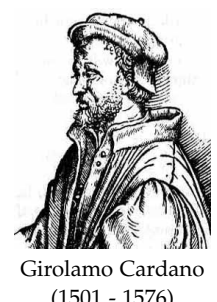

1545 veröffentlichte CARDANO die Lösungsmethode entgegen seines Versprechens in seinem Werk Ars Magna sive de regulis algebraicis (Die große Kunst oder über die Regeln der Algebra), was TARTAGLIA verständlicherweise sehr erzürnte. 


\section{Kubische Gleichungen}

Die Cardanische Formel

1. a) Geben Sie jeweils eine Lösung der folgenden kubischen Gleichungen an:

(i) $x^{3}-6 x^{2}+11 x=6$

(ii) $x^{3}+x+10=0$

(iii) $x^{3}+6 x=20$

b) Welche kubischen Gleichungen verbergen sich jeweils hinter den vier exemplarisch aufgeführten Aufgaben von Fiore an TARTAGLiA?

c) Es wird Ihnen (vermutlich) nicht gelingen, zu den kubischen Gleichungen aus Aufgabenteil b) eine Lösung zu finden. Warum kann man dennoch überzeugt sein, dass es eine Lösung geben muss?

Eine 1545 von GIROLAmo CARDANO veröffentlichte Lösungsformel für kubische Gleichungen lautet wie folgt:

Eine Lösung der reduzierten kubischen Gleichung $x^{3}=p \cdot x+q$ ist gegeben durch

$$
x=\sqrt[3]{\frac{q}{2}+\sqrt{\left(\frac{q}{2}\right)^{2}-\left(\frac{p}{3}\right)^{3}}}+\sqrt[3]{\frac{q}{2}-\sqrt{\left(\frac{q}{2}\right)^{2}-\left(\frac{p}{3}\right)^{3}}} .
$$

(Wie man diese Formel beweisen kann, wird auf einem separaten Übungsblatt behandelt, welches Sie freiwillig bearbeiten können.)

2. Geben Sie mit Hilfe der Cardanischen Formel eine Lösung zu den in Aufgabe 1b) aufgestellten kubischen Gleichungen an. Sie müssen den Lösungsterm nicht so weit wie möglich vereinfachen.

3. a) Zeigen Sie mit Hilfe der Cardanischen Formel, dass die kubische Gleichung

$$
x^{3}+6 x=20
$$

aus Aufgabenteil 1a)(iii) die Lösung $\sqrt[3]{10+\sqrt{108}}+\sqrt[3]{10-\sqrt{108}}$ hat.

b) Andererseits ist 2 offensichtlich eine Lösung der Gleichung. Zeigen Sie:

$$
\sqrt[3]{10+\sqrt{108}}+\sqrt[3]{10-\sqrt{108}}=2
$$

(Hinweis: Benutzen Sie die Faktorisierung $x^{3}+6 x-20=(x-2) \cdot\left(x^{2}+2 x+10\right)$.)

4. Die Gleichung $x^{3}=x$ besitzt offensichtlich die drei Lösungen $-1,1$ und 0 . Welches Ergebnis liefert die Cardanische Formel? Wie interpretieren Sie dieses Ergebnis? 
5. Betrachten Sie die Gleichung $x^{3}=15 x+4$. Diese hat 4 als reelle Lösung. Die Cardanische Formel liefert als Lösung

$$
\sqrt[3]{2+\sqrt{-121}}+\sqrt[3]{2-\sqrt{-121}}
$$

a) Weisen Sie die Identität

$$
(2+\sqrt{-1})^{3}=2+\sqrt{-121}=2+11 \sqrt{-1}
$$

nach. Zeigen Sie analog $(2-\sqrt{-1})^{3}=2-11 \sqrt{-1}$.

b) Verwenden Sie Teil a), um

$$
\sqrt[3]{2+\sqrt{-121}}+\sqrt[3]{2-\sqrt{-121}}=4
$$

zu folgern.

6. Raten Sie eine Lösung der Gleichung

$$
x^{3}-6 x+4=0
$$

und bestimmen Sie andererseits den Lösungsterm mit der Cardanischen Formel. Weisen Sie auch hier die Übereinstimmung nach.

(Hinweis: Berechnen Sie $(1+\sqrt{-1})^{3}$.) 


\section{Herleitung der Cardanischen Formel}

Für Algebra-Experten

1. a) Machen Sie sich klar, dass man das Lösen einer kubischen Gleichung der Form

$$
a \cdot x^{3}+b \cdot x^{2}+c \cdot x+d=0
$$

(wobei $a \neq 0$ ) stets auf das Lösen einer kubischen Gleichung der Form

$$
x^{3}+A \cdot x^{2}+B \cdot x+C=0
$$

zurückführen kann, indem Sie die Koeffizienten $A, B, C$ in Abhängigkeit von $a, b, c, d$ angeben.

b) Zeigen Sie mit Hilfe der Substitution $x=y-\frac{A}{3}$, dass man die Lösung jeder Gleichung der Form

$$
x^{3}+A \cdot x^{2}+B \cdot x+C=0
$$

auf die Lösung einer Gleichung der Form

$$
(*) \quad y^{3}=p \cdot y+q
$$

zurückführen kann. Geben Sie dazu die Koeffizienten $p$ und $q$ in Abhängigkeit von $A, B, C$ an.

Man nennt $(*)$ auch die reduzierte Form einer kubischen Gleichung.

2. Wir denken uns die Lösung von $(*)$ aus zwei Zahlen $u$ und $v$ (wobei $u, v \neq 0$ ) zusammengesetzt, d.h. $y=u+v$.

a) Zeigen Sie, dass die Gleichung $(*)$ genau dann erfüllt ist, wenn

$$
\text { (1) } p=3 u v \quad \text { und } \quad \text { (2) } q=u^{3}+v^{3} \text {. }
$$

b) Löst man Gleichung (1) nach $v$ auf und setzt dies entsprechend in Gleichung (2) ein, erhält man

$$
u^{3}+\left(\frac{p}{3 u}\right)^{3}=q .
$$

Zeigen Sie mit Hilfe der Substitution $z=u^{3}$, dass diese Gleichung äquivalent zu der quadratischen Gleichung

$$
(* *) \quad z^{2}-q z+\left(\frac{p}{3}\right)^{3}=0
$$

ist. 
3. a) Zeigen Sie durch Lösen von $(* *)$ :

$$
u=\sqrt[3]{\frac{q}{2}+\sqrt{\left(\frac{q}{2}\right)^{2}-\left(\frac{p}{3}\right)^{3}}} \text { oder } u=\sqrt[3]{\frac{q}{2}-\sqrt{\left(\frac{q}{2}\right)^{2}-\left(\frac{p}{3}\right)^{3}}}
$$

b) Wie kann man ohne Rechnen direkt erkennen, dass auch

$$
v=\sqrt[3]{\frac{q}{2}+\sqrt{\left(\frac{q}{2}\right)^{2}-\left(\frac{p}{3}\right)^{3}}} \text { oder } v=\sqrt[3]{\frac{q}{2}-\sqrt{\left(\frac{q}{2}\right)^{2}-\left(\frac{p}{3}\right)^{3}}}
$$

gilt?

c) Folgern Sie die Cardanische Formel:

Eine Lösung der reduzierten kubischen Gleichung $y^{3}=p \cdot y+q$ ist gegeben durch

$$
y=u+v=\sqrt[3]{\frac{q}{2}+\sqrt{\left(\frac{q}{2}\right)^{2}-\left(\frac{p}{3}\right)^{3}}}+\sqrt[3]{\frac{q}{2}-\sqrt{\left(\frac{q}{2}\right)^{2}-\left(\frac{p}{3}\right)^{3}}} .
$$




\section{Weitere Zahlbereichserweiterungen}

1. Im Schulunterricht wird die Regel „minus mal minus gleich plus“ häufig über sog. Permanenzreihen begründet. Diskutieren Sie folgende Argumentation:

Wir wissen bereits, dass das Produkt aus einer positiven und einer negativen Zahl ein negatives Vorzeichen hat, z.B. $5 \cdot(-3)=-15$ oder $(-2) \cdot 3=-6$.

Wir betrachten nun die folgende Zahlenreihe:

$$
\begin{aligned}
& (-3) \cdot 3=-9 \\
& (-3) \cdot 2=-6 \\
& (-3) \cdot 1=-3 \\
& (-3) \cdot 0=0 \\
& (-3) \cdot(-1)=+3 \\
& (-3) \cdot(-2)=+6
\end{aligned}
$$

In jeder Zeile verringert sich der zweite Faktor um 1 und das Ergebnis erhöht sich stets um 3. Die Regel „minus mal minus gleich plus“ ist also eine logische Ergänzung der Zahlenreihe.

2. Vergleichen Sie die Begründung aus 1. mit folgender Argumentation (Ausgangssituation: Wir kennen nur die Menge der natürlichen Zahlen $\mathbb{N}_{0}=\{0,1,2,3, \ldots\}$ ):

Die Gleichung $x+1=0$ ist in $\mathbb{N}_{0}$ nicht lösbar. Wir definieren einfach eine "Zahl“ $g$ mit der Eigenschaft $g+1=0$.

Diese neue "Zahl" $g$ ist offensichtlich keine der bisher bekannten natürlichen Zahlen. Wenn wir aber davon ausgehen, dass wir mit $g$ "wie gewohnt“ rechnen können, so erhalten wir:

$$
g^{2}=g^{2}+0=g^{2}+g+1=g \cdot(\underbrace{g+1}_{=0})+1=1
$$

Die Zahl $g$ bezeichnen wir nun mit -1 und haben somit gezeigt:

$$
(-1) \cdot(-1)=+1
$$

3. Herr R. Umbastler hat eine Idee:

"Es heißt ja immer, man darf nicht durch 0 dividieren. Was ist aber, wenn ich mir einfach eine neue Zahl b (eine sog. "bekloppte“ Zahl) definiere, die genau die Eigenschaft

$$
\frac{1}{0}=: b \quad b z w . \quad 0 \cdot b=1
$$

hat?"

Was halten Sie von diesem Vorschlag? Welche Probleme ergeben sich, wenn man auch mit dieser neuen Zahl $b$ „wie gewohnt" rechnen möchte? 


\section{A.2 Historische Textauszüge}

Übersicht

\begin{tabular}{cll} 
Text & Auszug aus & Quelle \\
\hline \hline Hist1 & $\begin{array}{l}\text { Über den Ursprung und die } \\
\text { Bedeutung der geometrischen Axiome }\end{array}$ & von Helmholtz 1883, S. 8-10 \\
\hline Hist2 & Wissenschaft und Hypothese & Poincaré 1904, S. 49-52 \\
\hline Hist3 & Grundlagen der Geometrie & Hilbert 1962, S. 2-5 \\
\hline Hist4 & Wissenschaftlicher Briefwechsel & Frege 1976, S. 61-63, 65-66, 79 \\
\hline Hist5 & Philosophischer Versuch über die & de Laplace 1886, S. 2-3 \\
& Wahrscheinlichkeiten & \\
\hline Hist6 & Grundlagen der & von Mises 1964, S. 57-58, 65-66, \\
& Wahrscheinlichkeitsrechnung & 69-70 \\
\hline Hist7 & Grundbegriffe der & Kolmogorov 1933, S. 1-4 \\
& Wahrscheinlichkeitsrechnung &
\end{tabular}


HermanN von Helmholtz (1821-1894)

Auszug aus

"Über den Ursprung und die Bedeutung der geometrischen Axiome“ (1870)

Denken wir uns - darin liegt keine logische Unmöglichkeit - verstandbegabte Wesen von nur zwei Dimensionen, die an der Oberfläche irgend eines unserer festen Körper leben und sich 3 bewegen. Wir nehmen an, dass sie nicht die Fähigkeit haben, irgend etwas außerhalb dieser Oberfläche wahrzunehmen, wohl aber Wahrnehmungen zu machen, ähnlich den unsrigen, innerhalb der Ausdehnung der Fläche, in der sie sich bewegen. Wenn sich solche Wesen ihre Geometrie ausbilden, so würden sie ihrem Raume natürlich nur zwei Dimensionen zuschreiben. Sie würden ermitteln, dass ein Punkt, der sich bewegt, eine Linie beschreibt, und eine Linie, die sich bewegt, eine Fläche, was für sie das vollständigste Raumgebilde wäre, das sie kennen. Aber sie würden sich ebenso wenig eine Vorstellung machen können von einem weiteren räumlichen Gebilde, das entstände, wenn eine Fläche sich aus ihrem flächenhaften Raume herausbewegte, als wir es können von einem Gebilde, das durch Herausbewegung eines Körpers aus dem uns bekannten Raume entstände. [...]

Jene Flächenwesen würden ferner auch kürzeste Linien in ihrem flächenhaften Raume ziehen können. Das wären nicht nothwendig gerade Linien in unserem Sinne, sondern was wir nach geometrischer Terminologie geodätische Linien der Fläche, auf der jene leben, nennen würden, Linien, wie sie ein gespannter Faden beschreibt, den man an die Fläche anlegt, und der ungehindert an ihr gleiten kann. Ich will mir erlauben, im Folgenden dergleichen Linien als die geradesten Linien der bezeichneten Fläche (beziehlich eines gegebenen Raumes) zu bezeichnen, um dadurch ihre Analogie mit der geraden Linie in der Ebene hervorzuheben. [...]

Wenn nun Wesen dieser Art auf einer unendlichen Ebene lebten, so würden sie genau dieselbe Geometrie aufstellen, welche in unserer Planimetrie ${ }^{1}$ enthalten ist. Sie würden behaupten, dass zwischen zwei Punkten nur eine gerade Linie möglich ist, dass durch einen dritten, außerhalb derselben liegenden Punkt nur eine Parallele mit der ersten geführt werden kann, dass übrigens gerade Linien in das Unendliche verlängert werden können, ohne dass ihre Enden sich wieder begegnen, und so weiter. [...]

${ }_{27}$ Nun könnten aber intelligente Wesen dieser Art auch an der Oberfläche einer Kugel leben. 28 Ihre kürzeste oder geradeste Linie zwischen zwei Punkten würde dann ein Bogen des größten Kreises sein, der durch die betreffenden Punkte zu legen ist. Jeder größte Kreis, der durch 30 zwei gegebene Punkte geht, zerfällt dabei in zwei Theile. Wenn beide ungleich lang sind, ist

${ }^{1}$ Planimetrie $=$ Geometrie der Ebene 
31 der kleinere Theil allerdings die einzige kürzeste Linie auf der Kugel, die zwischen diesen

32 beiden Punkten besteht. Aber auch der andere größere Bogen desselben größten Kreises ist

33 eine geodätische oder geradeste Linie, $d$. h. jedes kleinere Stück desselben ist eine kürzeste

34 Linie zwischen seinen beiden Endpunkten. Wegen dieses Umstandes können wir den Be-

35 griff der geodätischen oder geradesten Linie nicht kurzweg mit dem der kürzesten Linie

36 identifizieren. Wenn nun die beiden gegebenen Punkte Endpunkte desselben Durchmessers

${ }_{37}$ der Kugel sind, so schneiden alle durch diesen Durchmesser gelegten Ebenen Halbkreise

38 aus der Kugelfläche, welche alle kürzesten Linien zwischen den beiden Endpunkten sind. In

39 einem solchen Falle gibt es also unendlich viele unter einander gleiche kürzeste Linien zwi-

40 schen den beiden gegebenen Punkten. Somit würde das Axiom, dass nur eine kürzeste Linie

41 zwischen zwei Punkten bestehe, für die Kugelbewohner nicht ohne eine gewisse Ausnahme

42 gültig sein.

43 Parallele Linien würden die Bewohner der Kugel gar nicht kennen. Sie würden behaupten,

44 dass beliebige zwei geradeste Linien, gehörig verlängert, sich schließlich nicht nur in einem

45 sondern in zwei Punkten schneiden müssten. Die Summe der Winkel in einem Dreieck wür-

46 de immer größer sein als zwei Rechte, und umso größer, je größer die Fläche des Dreiecks.

47 Eben deshalb würde ihnen auch der Begriff der geometrischen Ähnlichkeit der Form zwi-

48 schen größeren und kleineren Figuren derselben Art fehlen. Denn ein größeres Dreieck muss

49 nothwendig andere Winkel haben als ein kleineres. [...]

50 Es ist klar, dass die Wesen auf der Kugel bei denselben logischen Fähigkeiten, doch ein ganz

51 anderes System geometrischer Axiome aufstellen müssten, als die Wesen auf der Ebene,

52 und als wir selbst in unserem Raume von drei Dimensionen. Diese Beispiele zeigen uns

53 schon, dass, je nach der Art des Wohnraumes, verschiedene geometrische Axiome aufgestellt

54 werden müssten von Wesen, deren Verstandeskräfte den unsrigen ganz entsprechend sein

55 könnten. 
HENRI POINCARÉ (1854-1912)

Auszug aus

"Wissenschaft und Hypothese" (1904)

Ist es gewiß, daß unsere Geometrie die rechte ist, denn es sind doch mehrere Geometrien möglich? Die Erfahrung lehrt uns ohne Zweifel, daß die Summe der Winkel eines Dreiecks gleich zwei Rechten ist; aber das ist nur der Fall, wenn wir mit zu kleinen Dreiecken operieren; nach Lobatschewsky ist der Unterschied von zwei Rechten der Oberfläche des Dreiecks proportional: kann diese Differenz nicht wahrnehmbar werden, wenn wir mit größeren Dreiecken operieren und wenn unsere Messungen genauer werden? Die Euklidische Geometrie würde für uns damit nur eine vorläufig richtige Geometrie sein.

Um über diese Meinung zu disputieren, müssen wir uns vor allem fragen: Welches ist die Natur der geometrischen Axiome? [...]

- Wir wollen [...] die Vorstellung von Lebewesen ohne Dicke (resp. Höhe) wieder aufnehmen [...]; wir können wohl kaum annehmen, daß sich diese Wesen, wenn sie einen Verstand gleich dem unsrigen hätten, die Euklidische Geometrie aneignen würden, welche allen ihren Erfahrungen widerspräche.

14 Sollen wir nun daraus schließen, daß die geometrischen Axiome erfahrungsmäßige Wahr15 heiten sind? Man experimentiert doch nicht mit idealen geraden Linien oder Kreisen; man 6ann dazu nur wirkliche Gegenstände brauchen. [...]

17 Wenn die Geometrie eine Experimental-Wissenschaft wäre, so würde sie aufhören, eine ex18 akte Wissenschaft zu sein, sie würde also einer beständigen Revision zu unterwerfen sein. $[\ldots]$

20 [Die geometrischen Axiome] sind auf Übereinkommen beruhende Festsetzungen; unter allen 21 möglichen Festsetzungen wird unsere Wahl von experimentellen Tatsachen geleitet; aber sie bleibt frei und ist nur durch die Notwendigkeit begrenzt, jeden Widerspruch zu vermeiden. 3 In dieser Weise können auch die Postulate streng richtig bleiben, selbst wenn die erfahrungs24 mäßigen Gesetze, welche ihre Annahme bewirkt haben, nur annähernd richtig sein sollten.

25 Mit anderen Worten: die geometrischen Axiome [...] sind nur verkleidete Definitionen.

26 Was soll man dann aber von der folgenden Frage denken: Ist die Euklidische Geometrie 27 richtig?

28 Die Frage hat keinen Sinn.

29 Ebenso könnte man fragen, ob das metrische System richtig ist und die älteren Maß-Systeme

30 falsch sind [...]. Eine Geometrie kann nicht richtiger sein wie eine andere; sie kann nur

31 bequemer sein. 
DAvid Hilbert (1862-1943)

Auszug aus

"Grundlagen der Geometrie“ (1899/1962)

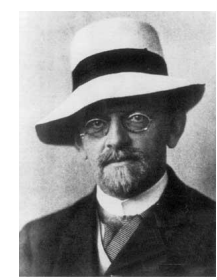

Wir denken drei verschiedene Systeme von Dingen: die Dinge des ersten Systems nennen wir Punkte und bezeichnen sie mit $A, B, C, \ldots$; die Dinge des zweiten Systems nennen wir Geraden und bezeichnen sie mit $a, b, c, \ldots$; die Dinge des dritten Systems nennen wir Ebenen und bezeichnen sie mit $\alpha, \beta, \gamma, \ldots[\ldots]$.

Wir denken die Punkte, Geraden, Ebenen in gewissen gegenseitigen Beziehungen und bezeichnen diese Beziehungen durch Worte wie "liegen“, ,zwischen“, ,kongruent", ,parallel“, "stetig“; die genaue und für mathematische Zwecke vollständige Beschreibung dieser Beziehungen erfolgt durch die Axiome der Geometrie. [...]

\section{§2. Die Axiomgruppe I: Axiome der Verknüpfung.}

$22[\ldots]$

\section{§3. Die Axiomgruppe II: Axiome der Anordnung.}

24 Die Axiome dieser Gruppe definieren den Begriff „zwischen“ und ermöglichen auf Grund 25 dieses Begriffes die Anordnung der Punkte auf einer Geraden, in einer Ebene oder im Raume.

26 Erklärung. Die Punkte einer Geraden stehen in gewissen Beziehungen zueinander, zu deren

27 Beschreibung uns insbesondere das Wort "zwischen“ dient. 


\section{Hist3}

28 II 1. Wenn ein Punkt B zwischen einem Punkt A und einem Punkt C liegt, so sind $A, B, C$ drei verschiedene Punkte einer Geraden, und B liegt dann auch zwischen $C$ und $A$.

3о II 2. Zu zwei Punkten A und $C$ gibt es stets wenigstens einen Punkt B auf der Geraden AC, so daß $C$ zwischen $A$ und $B$ liegt.

II 3. Unter irgend drei Punkten einer Geraden gibt es nicht mehr als einen, der zwischen den beiden anderen liegt. 


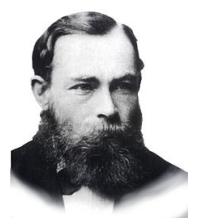

Gottlob Frege (1848-1925) / DAvid Hilbert (1862-1943)

Auszug aus

„Wissenschaftlicher Briefwechsel"

FRege an Hilbert:

1 Ganz anderer Art sind wohl die Erklärungen der $\S \S 1$ und 3, bei denen die Bedeutungen der Wörter "Punkt", "Gerade“, ,zwischen“ nicht angegeben, sondern als bekannt vorausgesetzt werden. Wenigstens scheint es so. Man ist aber auch im Unklaren darüber, was Sie Punkt nennen. [...] Bedenklich sind mir die Sätze (§1), dass die genaue und vollständige Beschreibung von Beziehungen durch die Axiome der Geometrie erfolge, und dass (§3) Axiome den Begriff „zwischen“ definieren. Damit wird etwas den Axiomen aufgebürdet, was Sache der Definitionen ist. Dadurch scheinen mir die Grenzen zwischen Definitionen und Axiomen in bedenklicher Weise verwischt zu werden [...].

Jede Definition enthält ein Zeichen (einen Ausdruck, ein Wort), das vorher noch keine Bedeutung hatte, dem erst durch die Definition eine Bedeutung gegeben wird. [...] Die anderen Sätze (Axiome, Grundgesetze, Lehrsätze) dürfen kein Wort enthalten und kein Zeichen, dessen Sinn und Bedeutung oder dessen Beitrag zum Gedankenausdruck nicht bereits völlig feststände, sodass über den Sinn des Satzes, den darin ausgedrückten Gedanken kein Zwei-

4 fel ist. Es kann sich dann nur darum handeln, ob dieser Gedanke wahr sei, und worauf dann

5 etwa seine Wahrheit beruhe. Axiome und Lehrsätze können also nie die Bedeutung eines

16 in ihnen vorkommenden Zeichens oder Wortes erst festsetzen wollen, die vielmehr schon

17 feststehen muss. [...]

18 Axiome nenne ich Sätze, die wahr sind, die aber nicht bewiesen werden, weil ihre Erkenntnis 9 aus einer von der logischen ganz verschiedenen Erkenntnisquelle fliesst, die man Raumanschauung nennen kann. Aus der Wahrheit der Axiome folgt, dass sie einander nicht wider1 sprechen. [...]

22 Wenn ich Ihr Axiom II.1 als solches aufstellte, so setzte ich dabei die Bedeutungen der Aus23 drücke „etwas ist Punkt einer Geraden“ und „B liegt zwischen $A$ und $C$ “ als vollständig 24 und unzweideutig bekannt voraus [...]. Dann kann das Axiom nicht dazu dienen, etwa das

25 Wort „,Zwischen“ genauer zu erklären [...]. Es scheint kaum etwas Anderes übrig zu bleiben 26 als die Annahme, das Wort „zwischen“ habe in II.1 übrhaupt noch keine Bedeutung. Dann

27 kann aber II.1 nicht wahr, also kein Axiom sein in meinem Sinne des Wortes, der, wie ich 28 meine, der allgemein angenommene ist. 


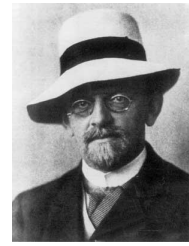

\section{DAvid Hilbert (1862-1943) / Gottlob Frege (1848-1925) \\ Auszug aus \\ „Wissenschaftlicher Briefwechsel"}

Hilbert an Frege:

29 Sie sagen, meine Erklärung in $\S 3$ sei keine Definition des Begriffes „zwischen“. Denn es

so fehlen die Merkmale. Ja diese Merkmale sind ja ausführlich in den Axiomen II.1 - [II.4]

31 angegeben. Wenn man aber das Wort Definition genau im hergebrachten Sinne nehmen

32 will, so hat man zu sagen: "Zwischen“ ist eine Beziehung für die Punkte einer Geraden, die

зз folgende Merkmale hat: II.1 ... [II.4].

34 Sie sagen weiter: „Ganz anders sind wohl die Erklärungen in §1, wo die Bedeutungen Punkt,

35 Gerade, ... nicht angegeben, sondern als bekannt vorausgesetzt werden." Hier liegt wohl der

36 Cardinalpunkt des Missverständnisses. Ich will nichts als bekannt voraussetzen [...]. Wenn

37 man nach andern Definitionen für "Punkt“, etwa durch Umschreibungen wie ausdehnungs-

38 los etc. sucht, so muss ich solchem Beginnen allerdings aufs entschiedenste widersprechen;

39 man sucht da etwas, was man nie finden kann, weil nichts da ist, und alles verliert sich

40 und wird wirr und vage und artet in Versteckspiel aus. Wollen Sie meine Axiome lieber

41 Merkmale der [...] Begriffe nennen, so würde ich dagegen gar nichts einzuwenden haben

$42 \quad[\ldots]$.

43 Sie schreiben: „Axiome nenne ich Sätze ... Aus der Wahrheit der Axiome folgt, dass sie einan-

44 der nicht widersprechen." Es hat mich sehr interessirt, gerade diesen Satz bei Ihnen zu lesen,

45 da ich nämlich, solange ich über solche Dinge denke, schreibe und vortrage, immer gerade

46 umgekehrt sage: Wenn sich die willkürlich gesetzten Axiome nicht einander widersprechen

47 mit sämtlichen Folgen, so sind sie wahr, so existieren die durch die Axiome definirten Dinge.

48 Das ist für mich das Criterium der Wahrheit und der Existenz. [...]

49 Also, um die Hauptsache noch einmal zu sagen: Die Umnennung „Merkmale“ statt „Axio-

50 me" etc. ist doch nur eine Aeusserlichkeit und überdies Geschmackssache - ist aber jeden-

51 falls leicht zu bewerkstelligen. Dagegen in 3 Zeilen eine Definition des Punktes geben zu

52 wollen, ist meines Erachtens eine Unmöglichkeit, da vielmehr erst der ganze Aufbau der

53 Axiome die vollständige Definition giebt. [...]

54 Meine Meinung ist eben die, dass ein Begriff nur durch seine Beziehungen zu anderen Begrif-

55 fen logisch festgelegt werden kann. Diese Beziehungen, in bestimmten Aussagen formulirt,

56 nenne ich Axiome und komme so dazu, dass die Axiome [...] die Definitionen der Begriffe

57 sind. 
PierRe-Simon De LAPlace (1749-1827)

Auszug aus

„Philosophischer Versuch über die Wahrscheinlichkeiten" (1814)

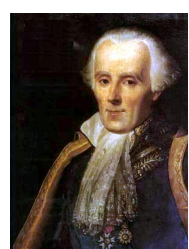

Wir müssen also den gegenwärtigen Zustand des Weltalls als die Wirkung seines früheren Zustandes und andererseits als die Ursache dessen, der folgen wird, betrachten. Eine Intelligenz, welche für einen gegebenen Augenblick alle Kräfte, von denen die Natur belebt ist, sowie die gegenseitige Lage der Wesen, die sie zusammen setzen, kennen würde, und überdies umfassend genug wäre, um diese gegebenen Grössen einer Analyse zu unterwerfen, würde in derselben Formel die Bewegungen der grössten Weltkörper wie die des leichtesten Atoms ausdrücken: nichts würde für sie ungewiss sein und Zukunft wie Vergangenheit ihr offen vor Augen liegen. Der menschliche Geist bietet in der Vollendung, die er der Astronomie zu geben gewusst hat, ein schwaches Bild dieser Intelligenz. Seine Entdeckungen auf dem Gebiete der Mechanik und Geometrie verbunden mit der Entdeckung der allgemeinen Schwere, haben es ihm möglich gemacht, durch dieselben analytischen Ausdrücke die vergangenen wie zukünftigen Zustände zu umfassen. Durch Anwendung derselben Methode auf einige andere Gegenstände seiner Kenntnisse ist er dahin gelangt, die beobachteten Phänomene auf allgemeine Gesetze zurückzuführen und diejenigen vorauszusehen, die gegebene Umstände herbeiführen müssen. Alle diese Bemühungen beim Aufsuchen der Wahrheit zielen dahin $a b$, ihn unablässig der eben angenommenen Intelligenz näher zu bringen, der er aber immer unendlich ferne bleiben wird. Dieses Streben, welches dem menschlichen Geschlechte eigenthümlich ist, erhebt eben den Menschen über das Thier, und seine Fortschritte auf diesem Gebiete unterscheiden die Nationen und Jahrhunderte und machen ihren wahren Ruhm aus. [...]

21 Die Wahrscheinlichkeit bezieht sich zum Theil auf diese Unwissenheit, zum Theil auf unsere 22 Kenntnisse. Wir wissen, dass von drei oder einer grösseren Zahl von Ereignissen eines sich 23 ereignen muss; aber nichts veranlasst uns zu glauben, dass das eine von ihnen sich eher als die anderen ereignen wird. In diesem Zustande der Ungewissheit ist es uns unmöglich, 25 über ihr Eintreffen mit Sicherheit etwas auszusagen. Es ist jedoch wahrscheinlich, dass eines 26 dieser Ereignisse, das aufs gerathewohl herausgerissen wird, nicht eintreffen wird, weil wir 27 mehrere gleich mögliche Fälle sehen, welche seine Existenz ausschliessen, während nur ein einziger ihr günstig ist.

29 Die Theorie des Zufalls (des hasards) besteht darin, alle Ereignisse derselben Art auf eine gewisse Anzahl gleich möglicher Fälle zurückzuführen, d. h. auf solche, über deren Existenz wir in gleicher Weise im Unklaren sind, und dann die Zahl der Fälle zu bestimmen, die dem Ereigniss, dessen Wahrscheinlichkeit man sucht, günstig sind. Das Verhältniss dieser Zahl zu der aller möglichen Fälle ist das Maass dieser Wahrscheinlichkeit, die also nur ein Bruch 34 ist, dessen Zähler die Zahl der günstigen Fälle, und dessen Nenner die Zahl aller möglichen 5 Fälle ist. 


\section{RichaRd von Mises (1883-1953) \\ Auszug aus \\ „Grundlagen der Wahrscheinlichkeitsrechnung" (1919)}

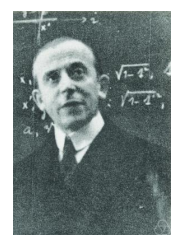

1 Dem Bedürfnis nach einer exakten Grundlegung der Wahrscheinlichkeitsrechnung wird sich kein Mathematiker verschliessen, der eines der bestehenden Lehrbücher zur Hand nimmt: in der Tat kann man den gegenwärtigen Zustand kaum anders als dahin kennzeichnen, dass die Wahrscheinlichkeitsrechnung heute eine mathematische Disziplin nicht ist. Kein Autor erhebt sich wesentlich über die Auffassung der von Laplace herrührenden "Definition", die Wahrscheinlichkeit eines Ereignisses sei der Quotient aus der Anzahl der "dem Ereignis günstigen Fälle“ durch die aller "gleichmöglichen Fälle“. Ganz abgesehen von der hier eingeschlossenen petitio principii [lat.: Zirkelschluss] - denn "gleichmöglich“ kann wohl nichts anderes heissen als "gleich wahrscheinlich" - ist die übliche Darstellung weit davon entfernt, diesen Satz, soweit ihm überhaupt ein exakter Sinn zukommt, als Begriffsbestimmung für das im weiteren Verlaufe „Wahrscheinlichkeit" Genannte aufrechtzuerhalten. Wie soll man auch etwa die Wahrscheinlichkeit, mit einem falschen Würfel sechs zu werfen, wie die Lebens- oder Sterbens-Wahrscheinlichkeit dieser „Definition“ unterordnen [...]? [...]

4 Als erstes Beispiel [...] betrachten wir den folgenden Fall. Es sei ein Körper, der mehr oder weniger genau die Gestalt eines kleinen Würfels besitzt, an seinen sechs Ebenen Seitenflächen mit den Zahlen 1 bis 6 beschrieben. Der Körper wird in einen Becher gelegt und aus diesem auf den Tisch geworfen; sobald er zur Ruhe gelangt, erscheint eine der sechs Zahlen auf der Oberseite. [...]

9 Die Erfahrung lehrt erstens, dass, sobald nur die Zahl der Würfe hinreichend gross ist, die relative Häufigkeit jedes der sechs Merkmale annähernd konstant bleibt. Man macht die Beobachtung gewöhnlich an einem sog. „richtigen“ Würfel, d. h. bei einem, der möglichst genau, nach Gestalt und Massenverteilung, die Würfelsymmetrie besitzt, und bei dem sich daher aus Symmetriegründen jede der sechs Konstanten gleich, also vom Betrage $\frac{1}{6}$, herausstellen muss. Aber auch mit Würfeln, die dieser Bedingung nicht entsprechen, sind Versuche angestellt worden, die unsere Behauptung bestätigen. Wie gross die annähernd konstant bleibenden relativen Häufigkeiten sind, ist für uns hier ganz gleichgültig; es genügt, dass man in dem Verlauf der Häufigkeitszahlen bei wachsender Zahl der Würfe immer ein Verhalten erkennt, das in der Annahme der Existenz eines Grenzwertes für eine ins Unendliche ausgedehnte Wurfreihe einen angemessenen Ausdruck findet. [...]

3o Die meisten Schwierigkeiten hat man in dem ältesten und ursprünglichsten Anwendungsge31 biet der Wahrscheinlichkeitsrechnung, in der Theorie der Glücksspiele, gefunden. Man hat hier 32 gemeint, für die "Gleichmöglichkeit" der sechs Seiten eines Würfels apriorische Erkenntnis3 quellen heranziehen zu müssen und danach auch versucht, diese Anschauungsweise auf alle anderen Gebiete der Wahrscheinlichkeitsrechnung auszudehnen. Allein es ist klar, dass die

35 Gleichheit der sechs Wahrscheinlichkeiten $W_{1}$ bis $W_{6}$ nichts anderes als eine Forderung ist, 
36 durch die ein ",richtiger“ Würfel definiert wird. Ob die Forderung in einem bestimmten Fall

37 erfüllt ist, also von der Richtigkeit eines Würfels, kann man sich nicht anders überzeugen,

38 [...] als indem man eine grosse Zahl $N$ von Würfen ausführt und die Quotienten $N_{A}: N$

39 untersucht. Denn selbst wenn die völlige Homogenität des Materials gesichert erscheint,

40 bleibe es immer ungewiss, welchen Einfluss die kleinsten Abweichungen von der Gestalts-

41 symmetrie, die sich eben der Kleinheit wegen der unmittelbaren Beobachtung entziehen, bei

42 fortgesetzter Steigerung von $N$ auf das Ergebnis der Würfe ausüben. 
ANDREI Kolmogorov (1903-1987)

Auszug aus

"Grundbegriffe der Wahrscheinlichkeitsrechnung" (1933)

Die Wahrscheinlichkeitstheorie als mathematische Disziplin soll und kann genau in demselben Sinne axiomatisiert werden wie die Geometrie oder die Algebra. Das bedeutet, daß, nachdem die Namen der zu untersuchenden Gegenstände und ihrer Grundbeziehungen sowie die Axiome, denen diese Grundbeziehungen zu gehorchen haben, angegeben sind, die ganze weitere Darstellung sich ausschließlich auf diese Axiome gründen soll und keine Rücksicht auf die jeweilige konkrete Bedeutung dieser Gegenstände und Beziehungen nehmen darf.

Dementsprechend wird in $\S 1$ der Begriff eines Wahrscheinlichkeitsfeldes als eines gewissen Bedingungen genügenden Mengensystems definiert. Was die Elemente dieser Mengen sind, ist dabei für die rein mathematische Entwicklung der Wahrscheinlichkeitsrechnung völ1 lig gleichgültig (man vgl. die Einführung der geometrischen Grundbegriffe in HiLberTs 12 "Grundlagen der Geometrie“ [...]).

14 Es sei $\Omega$ eine Menge von Elementen $\omega_{1}, \omega_{2}, \omega_{3}, \ldots$, welche man elementare Ereignisse nennt, 15 und $\mathcal{F}$ eine Menge von Teilmengen aus $\Omega$; die Elemente der Menge $\mathcal{F}$ werden weiter zufällige 16 Ereignisse genannt.

$17 \quad$ I. $\mathcal{F}$ ist ein Mengenkörper ${ }^{2}$.

II. $\mathcal{F}$ enthält die Menge $\Omega$.

III. Jeder Menge $A$ aus $\mathcal{F}$ ist eine nichtnegative reelle Zahl $P(A)$ zugeordnet. Diese Zahl $P(A)$ nennt man die Wahrscheinlichkeit des Ereignisses $A$.

$21 \quad$ IV. $P(\Omega)=1$.

V. Wenn $A$ und $B$ disjunkt sind, so gilt

$$
P(A \cup B)=P(A)+P(B) .
$$
men I-V genügt, nennt man ein Wahrscheinlichkeitsfeld.

${ }^{1}$ Ein Leser, der den folgenden Axiomen sofort einen konkreten Sinn geben will, soll sogleich den §2 lesen.

${ }^{2}$ Vgl. Hausdorff: Mengenlehre 1927 S. 78. Ein Mengensystem heißt ein Körper, wenn Summe, Durchschnitt und Differenz von zwei Mengen des Systems wieder dem System angehören. 
26 Die Anwendung der Wahrscheinlichkeitsrechnung auf die reelle Erfahrungswelt geschieht

27 nach dem folgenden Schema:

28 1. Es wird ein gewisser Komplex $\mathfrak{S}$ von Bedingungen vorausgesetzt, welcher unbeschränkter

29 Wiederholung fähig ist. [...]

30 A. Man kann praktisch sicher sein, daß, wenn man den Komplex der Bedingungen $\mathfrak{S}$ eine 31 große Anzahl von $n$ Malen wiederholt und dabei durch $m$ die Anzahl der Fälle bezeichnet,

32 bei denen das Ereignis $A$ stattgefunden hat, das Verhältnis $m / n$ sich von $P(A)$ nur wenig 33 unterscheidet.

34 B. Ist $P(A)$ sehr klein, so kann man praktisch sicher sein, daß bei einer einmaligen Realisation 35 der Bedingungen $\mathfrak{S}$ das Ereignis $A$ nicht stattfindet.

36 Empirische Deduktion der Axiome. Gewöhnlich kann man voraussetzen, daß das System $\mathcal{F}$ 37 der in Betracht kommenden Ereignisse $A, B, C, \ldots$, denen gewisse Wahrscheinlichkeiten zu38 geschrieben sind, einen Mengenkörper bildet, welcher $\Omega$ als Element enthält (Axiome I und

39 II sowie die erste Hälfte des Axiomes III - die Existenz der Wahrscheinlichkeiten). Es ist

40 ferner evident, daß stets $0 \leq \frac{m}{n} \leq 1$ ist, so daß die zweite Hälfte des Axioms III durchaus

41 als natürlich erscheint. Für das Ereignis $\Omega$ gilt immer $m=n$, weshalb man natürlicherweise

$42 \quad P(\Omega)=1$ setzt (Axiom IV). Sind schließlich $A$ und $B$ miteinander unverträglich $(\mathrm{d}$. $\mathrm{h}$. sind

43 die Mengen $A$ und $B$ disjunkt), so ist $m=m_{1}+m_{2}$, wobei $m, m_{1}, m_{2}$ der Reihe nach die

44 Anzahl der Versuche bezeichnet, in denen das Ereignis $A \cup B$ bzw. $A$ bzw. $B$ auftritt. Daraus

45 folgt

$$
\frac{m}{n}=\frac{m_{1}}{n}+\frac{m_{2}}{n}
$$

46 Es erscheint also als angebracht, $P(A \cup B)=P(A)+P(B)$ zu setzen.

\footnotetext{
${ }^{3}$ Ein Leser, der sich nur für die rein mathematische Entwicklung der Theorie interessiert, braucht diesen Paragraphen nicht zu lesen - die weitere Darstellung beruht auf den Axiomen des $\$ 1$ und benutzt nicht die Überlegungen des gegenwärtigen Paragraphen. In diesem wollen wir uns mit dem bloßen Hinweis auf die empirische Entstehung der Axiome der Wahrscheinlichkeitsrechnung begnügen und lassen deshalb eine eingehende philosophische Untersuchung des Wahrscheinlichkeitsbegriffes in der Erfahrungswelt bewußt beiseite. In der Darstellung der notwendigen Voraussetzungen für die Anwendbarkeit der Wahrscheinlichkeitsrechnung auf die Welt der reellen Geschehnisse folgt der Verfasser im hohen Maße den Ausführungen von Herrn von Mises [...].
} 


\section{A.3 Auszüge aus dem Geometrikon}

Das Geometrikon ist ein von dem französischen Astrophysiker Jean-Pierre Petit (geb. 1937) erstellter, populärwissenschaftlicher mathematischer Comic, der in die Grundlagen der nichteuklidischen Geometrie einführt. Er steht unter der URL

\section{http://www.savoir-sans-frontieres.com/JPP/telechargeables/ \\ Deutch/das_geometrikon.htm}

zum freien Download zur Verfügung. Da im Vorwort explizit die Erlaubnis erteilt wird, „die vorliegende Datei zu vervielfältigen, entweder in digitaler Form oder in Form gedruckter Kopien und sie in Bibliotheken oder im Rahmen von Schule, Universität oder Vereinen zu verbreiten“, werden in diesem Anhang die für meine Unterrichtskonzepte relevanten Seiten zur Verfügung gestellt.

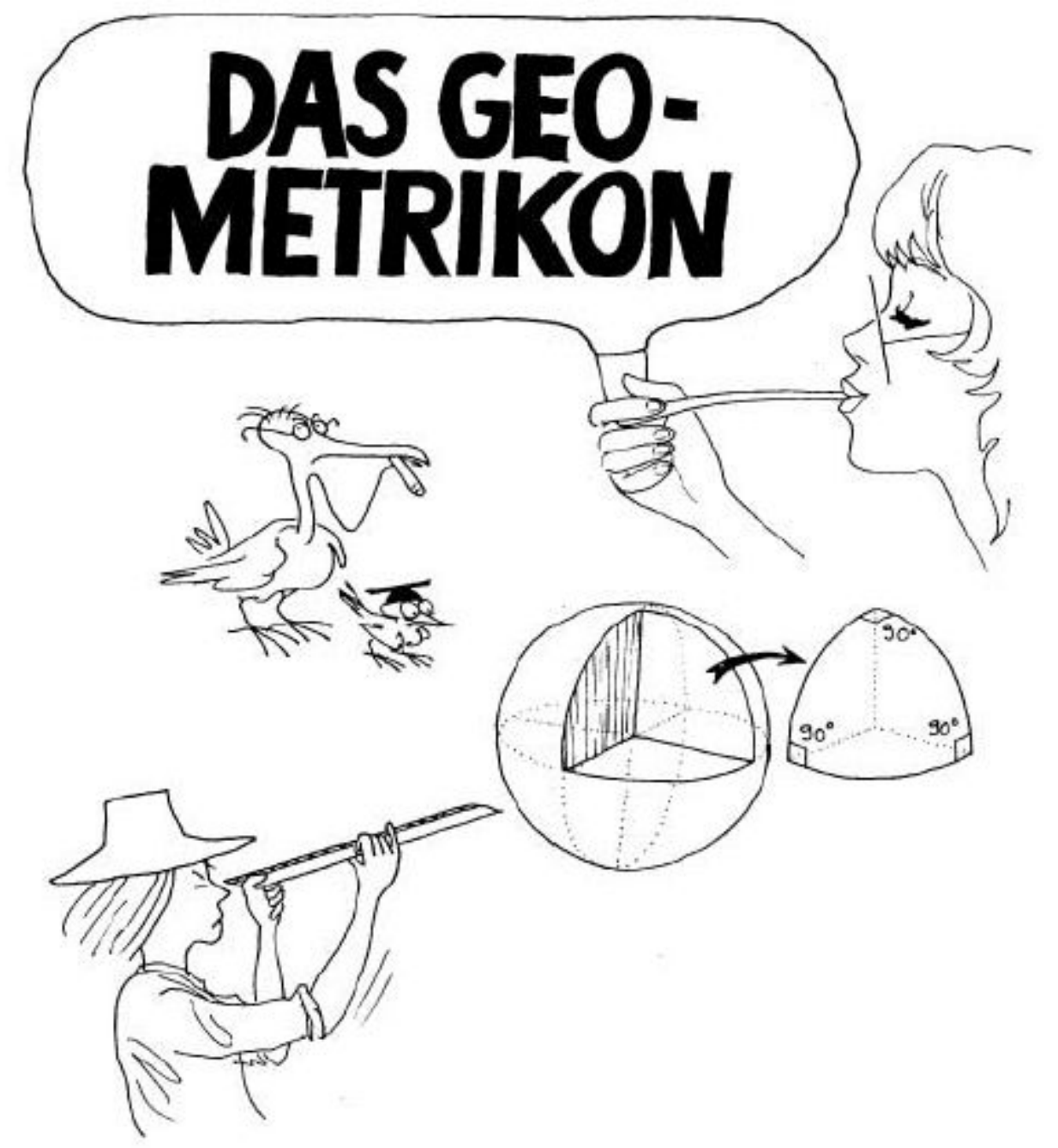


Die Gesellschaft Euklid\& Co wurde im dritten Jahrhundert vor Christus in Alexandrien gegriundet. Zweitansendzweihundert Jahre lang florierte das Gesdic̈ft. Die Produkte werden überall geschätet, und die Kundschaft war zufrieden und treen.

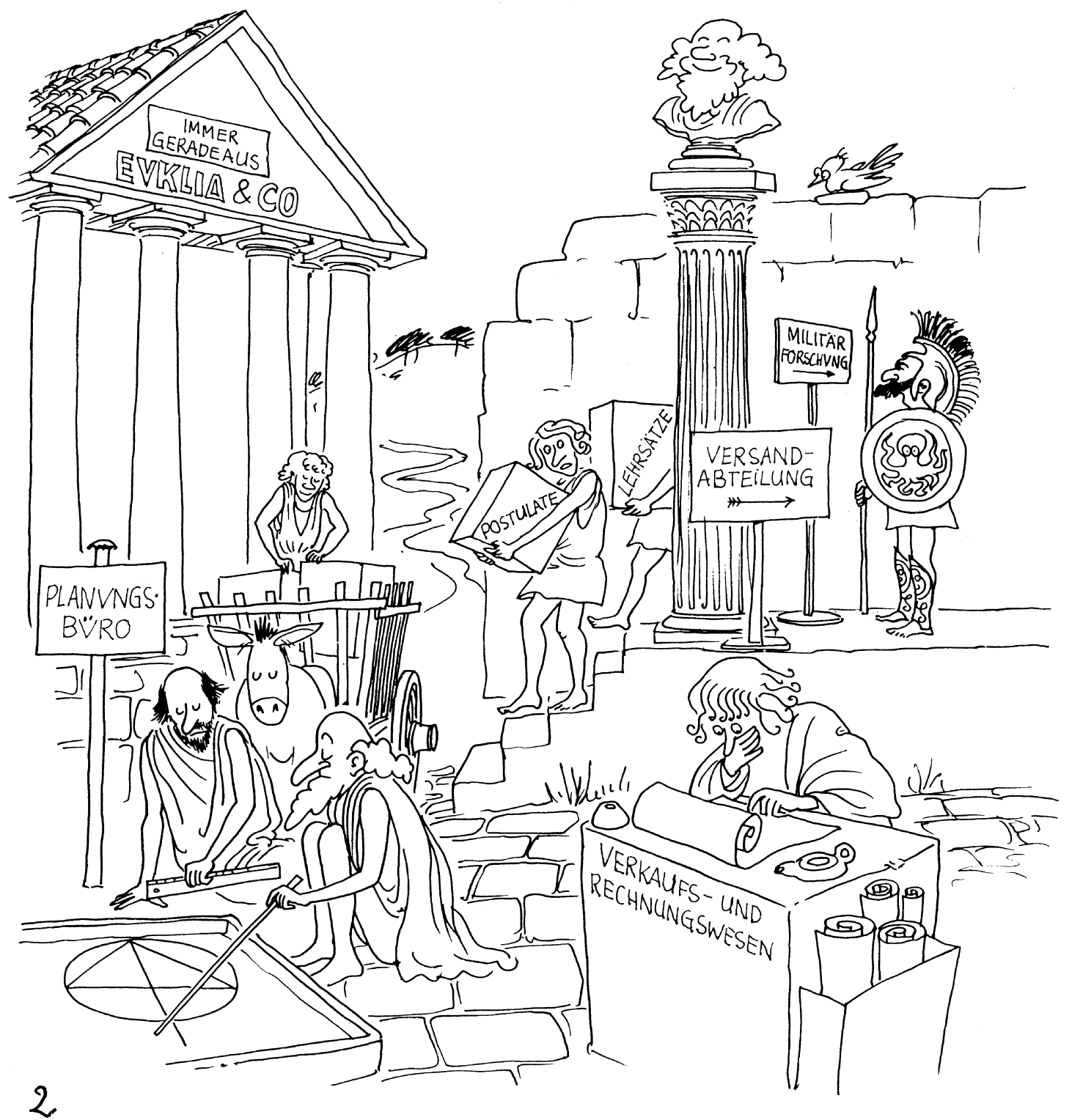


Dann aber änderte sich des Geschmack der Kunden. Aufgrened seltsamer Experimente fragten sich zunächst wenige cud domn inmer mehr, of die Produkte von Euklid\& 6 wirklich überall und für alles die besten seien.

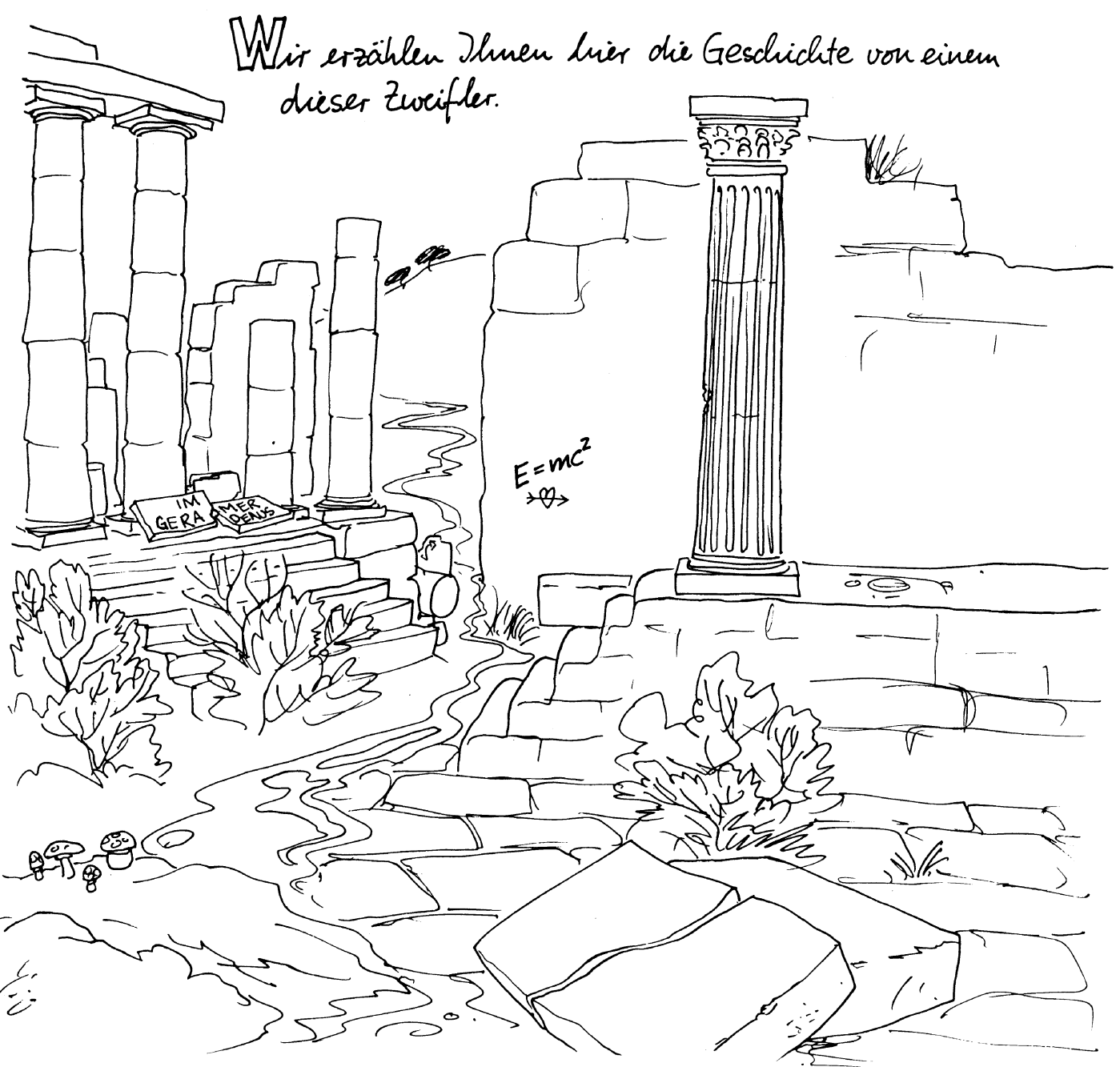




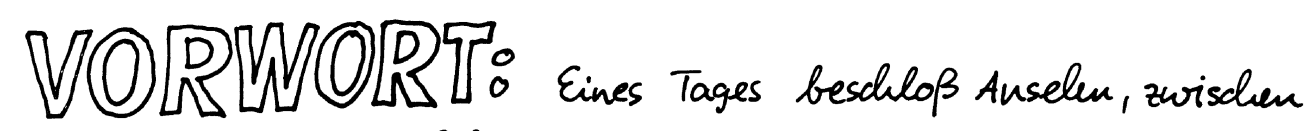
zwei Stangen eine Schnur zu spannen.

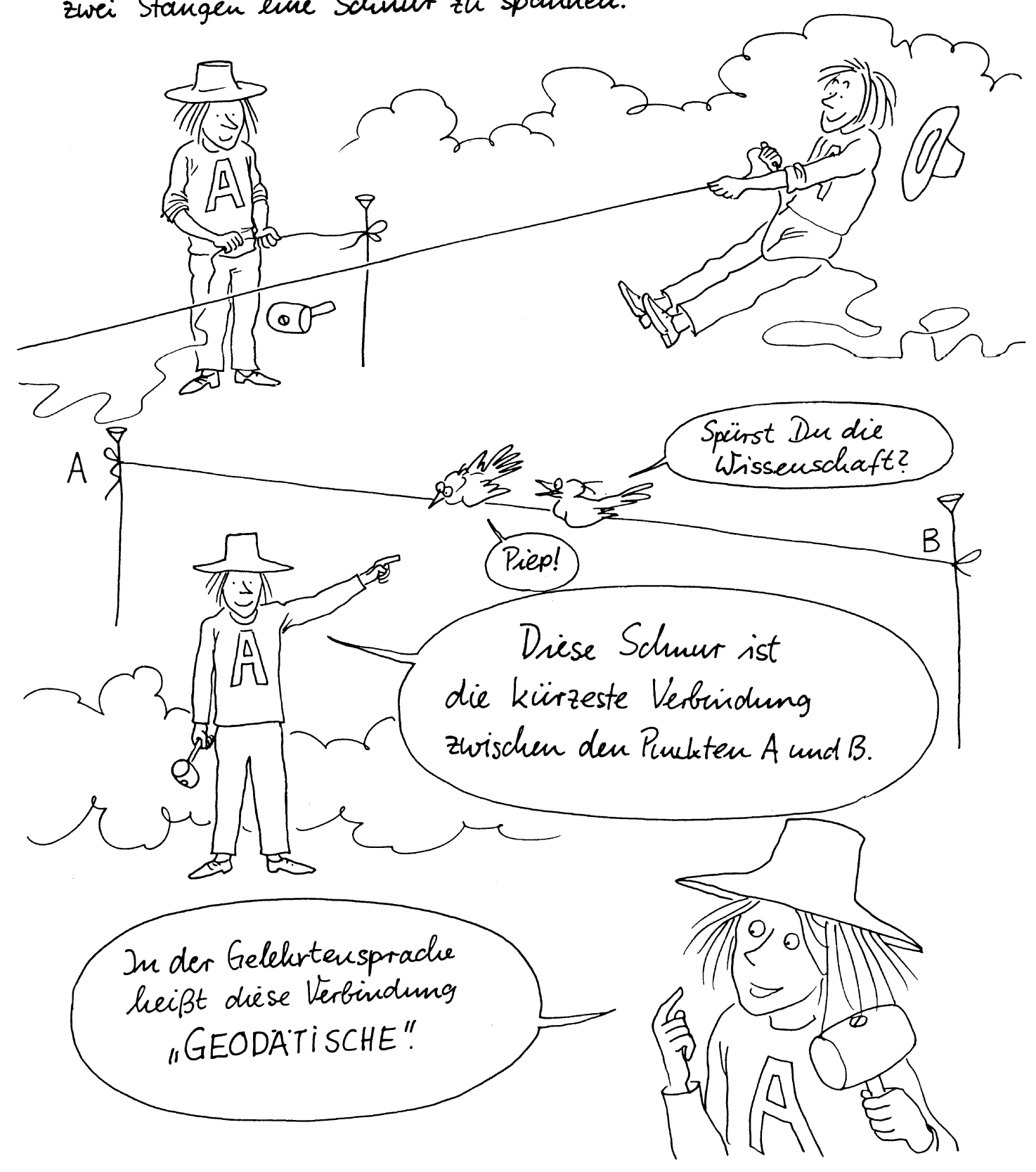

4. 
Mit drei gespannten Fäden, das heißt uit drei Geodätischen...

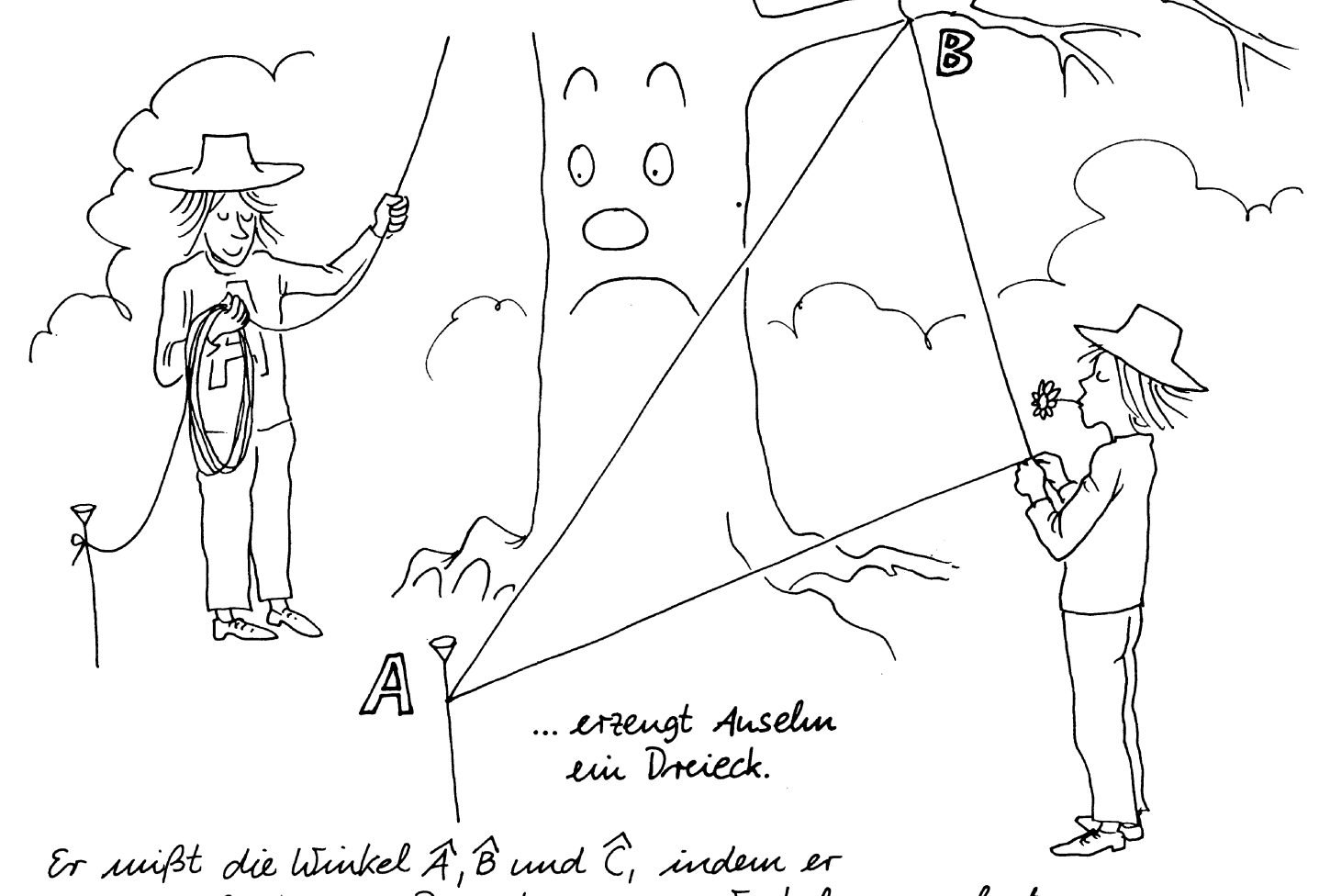
an jede Spritze des Dreiecks seinen Winkelmesser legt,

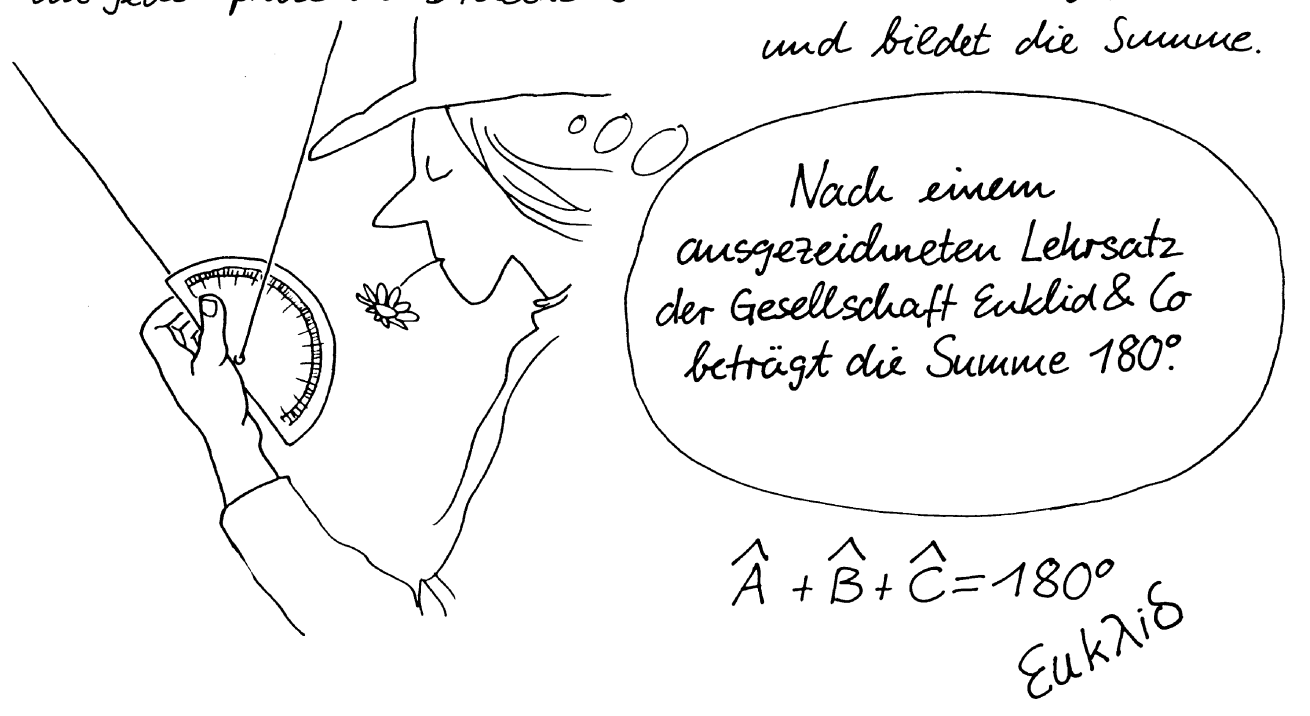


Die Welt, in der Anselun lebte, war nebelig und trïb.

Man sah manchual die Hand vor den Augen indet.
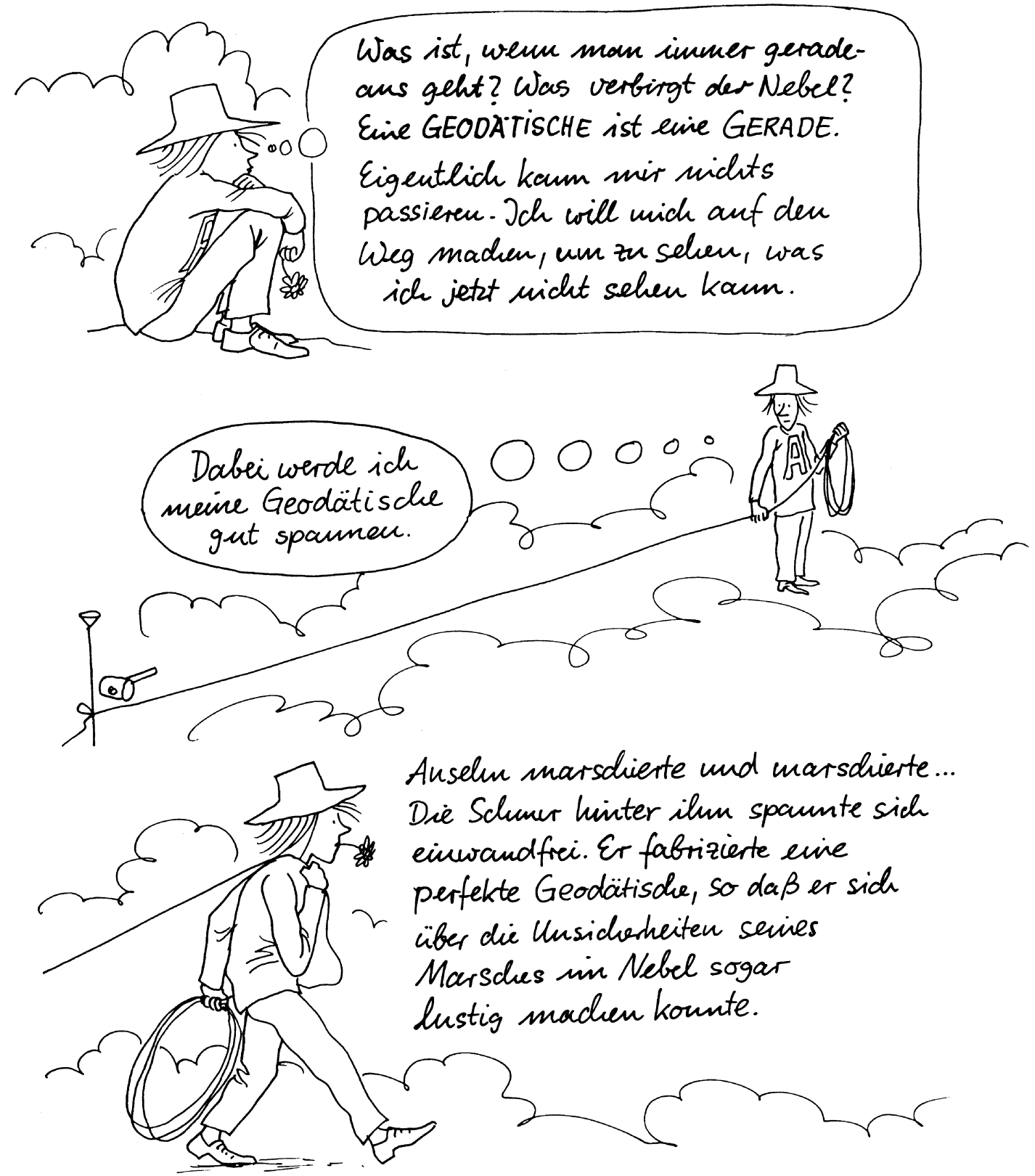

6 


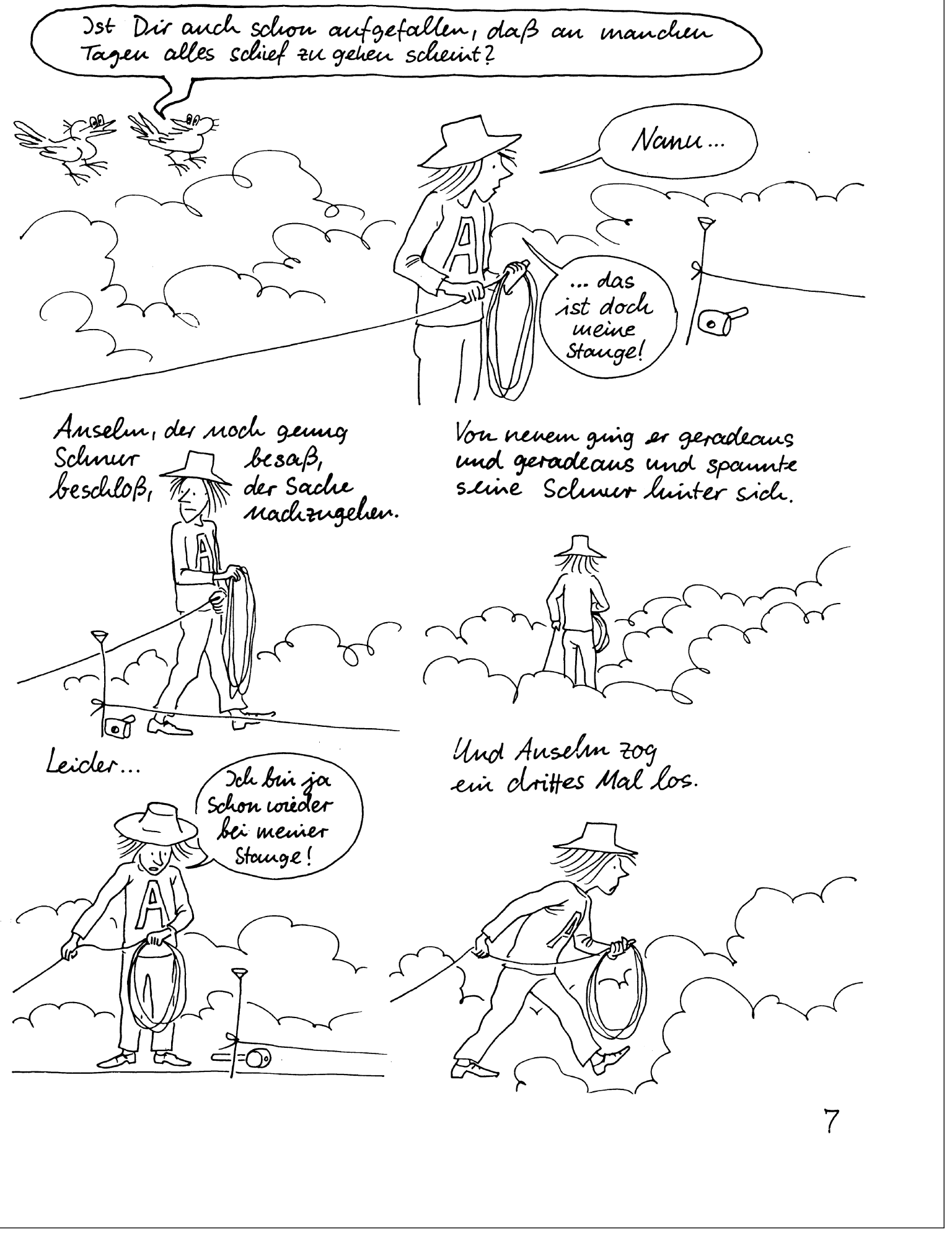




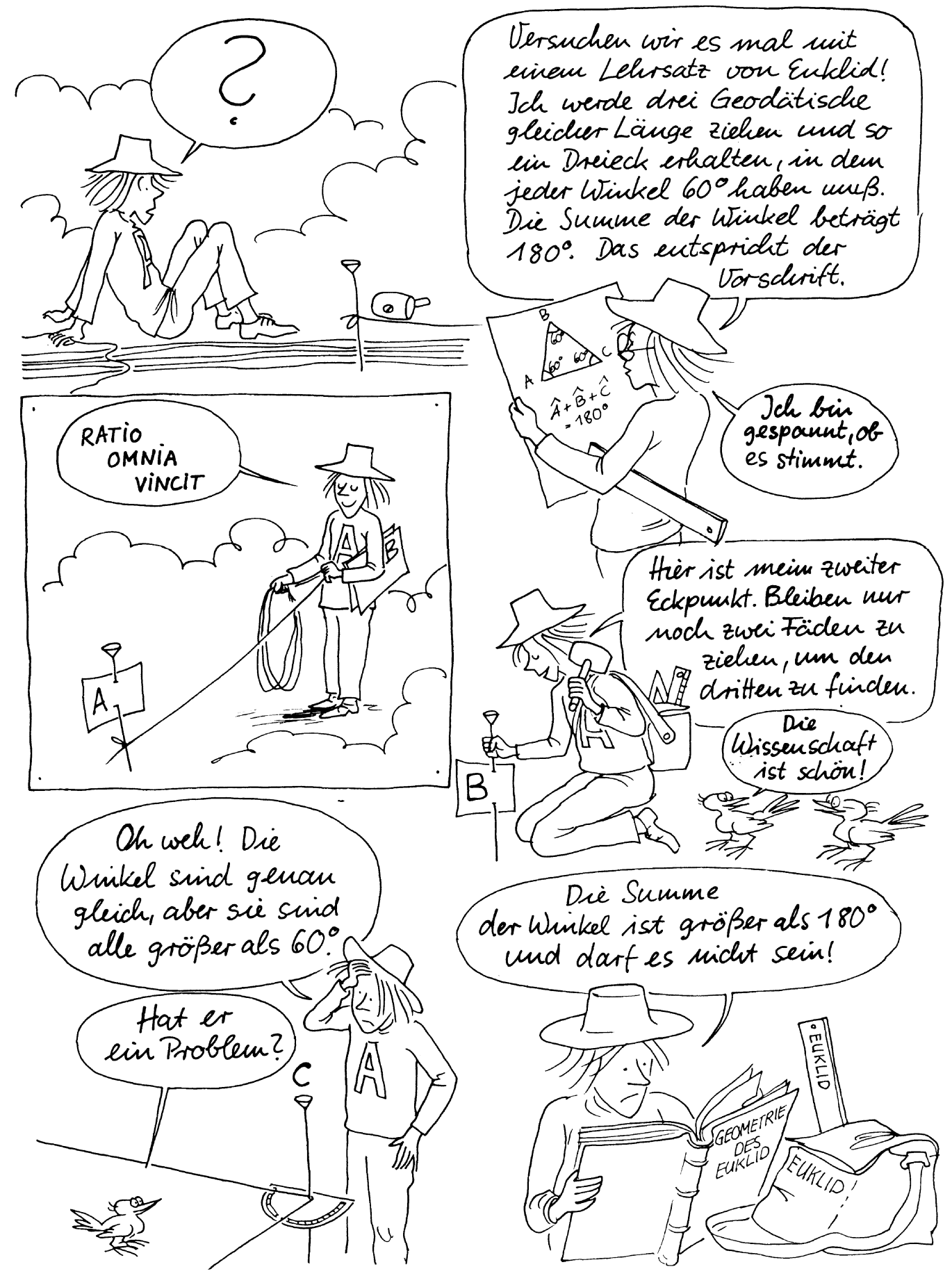

8 


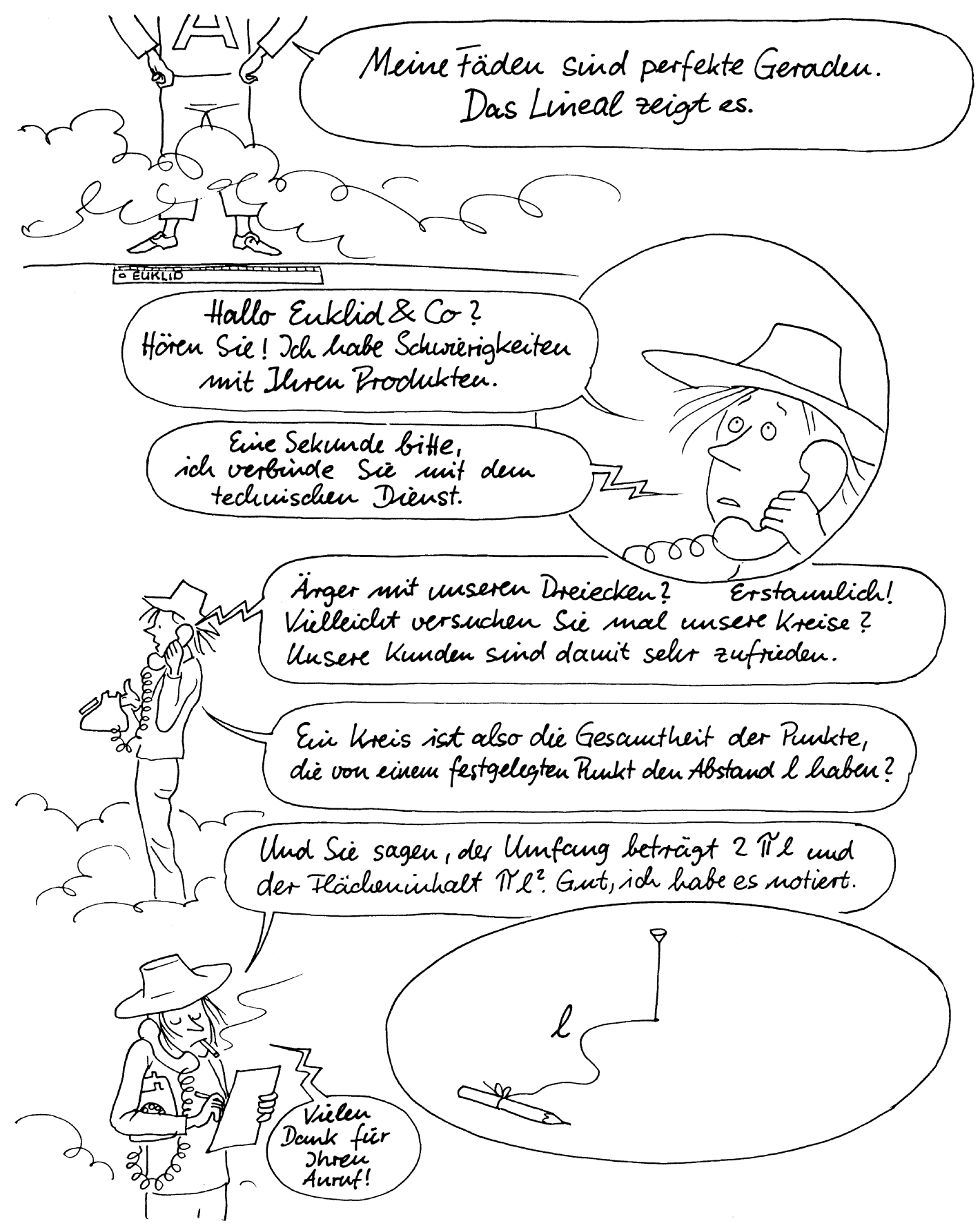




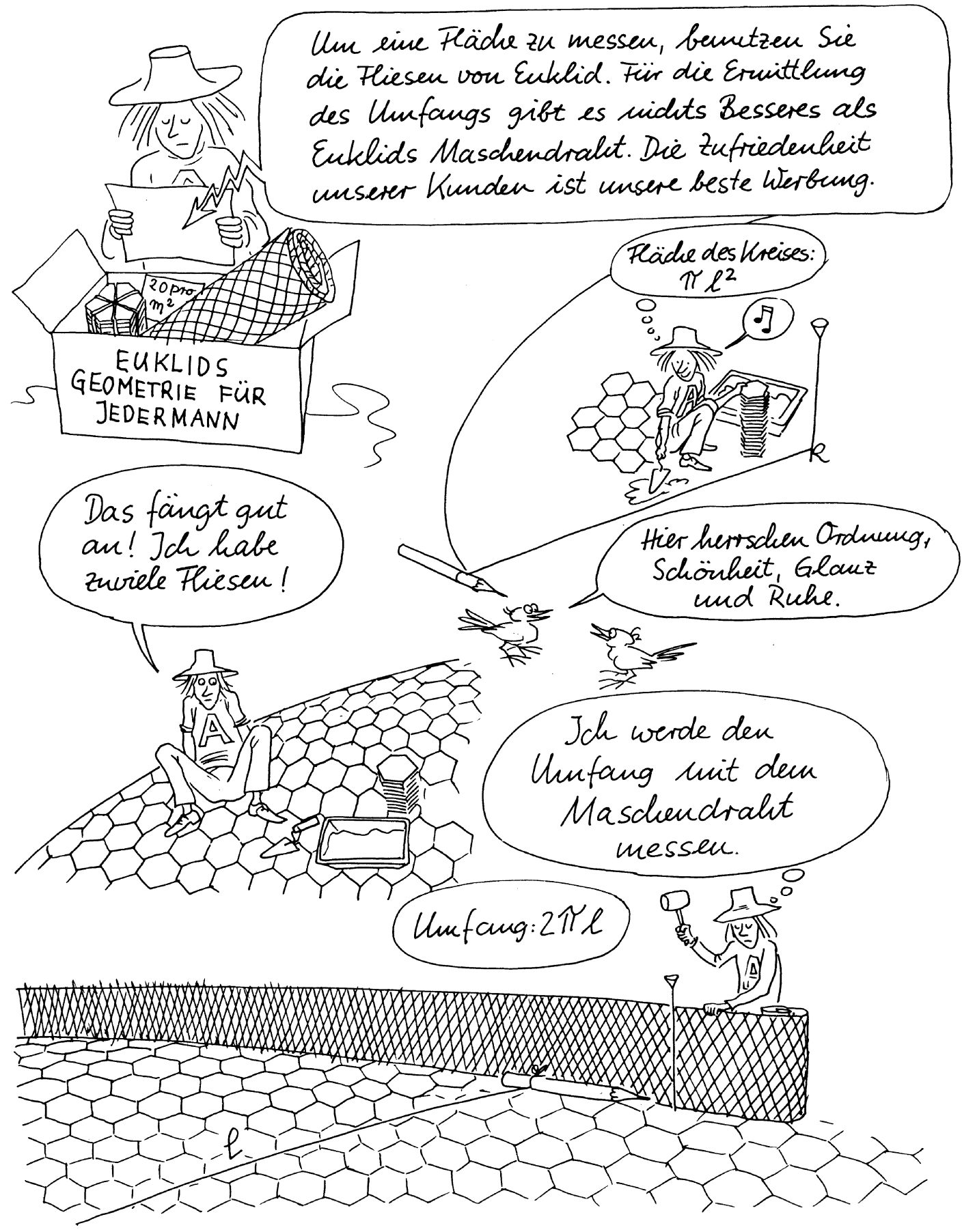

10 

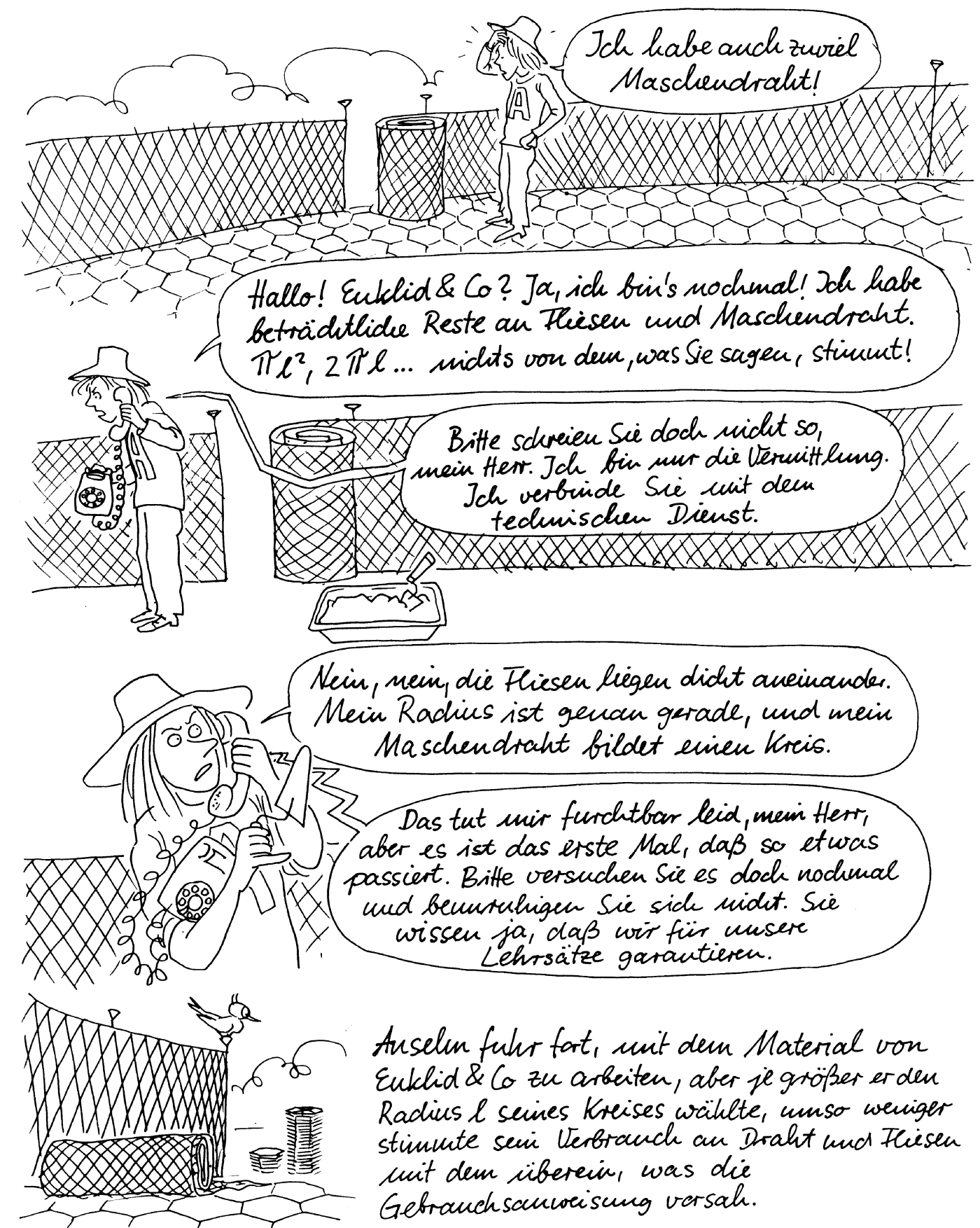

Auselun fulur fart, unit dem Material von Euklid \& Co zu arbeiten, aber je größer er den Radius $l$ seines Kreises wählte, unso weriger stimmente sein Verbranch an Draht nud Fliesen uit dem ïberein, was die Gebranch sanwoisung versah. 


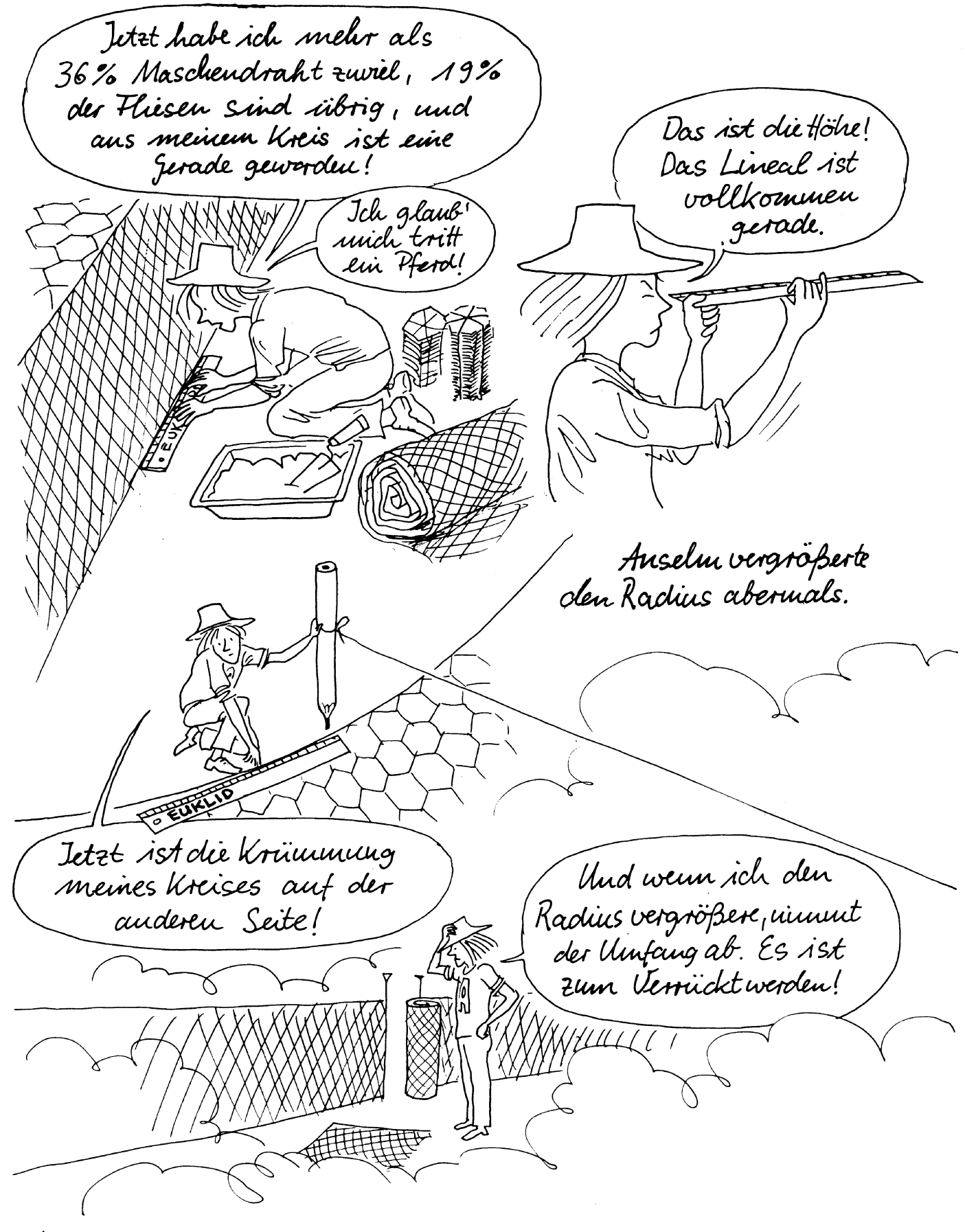

12 
Nach einigen weiteren Versuchen:

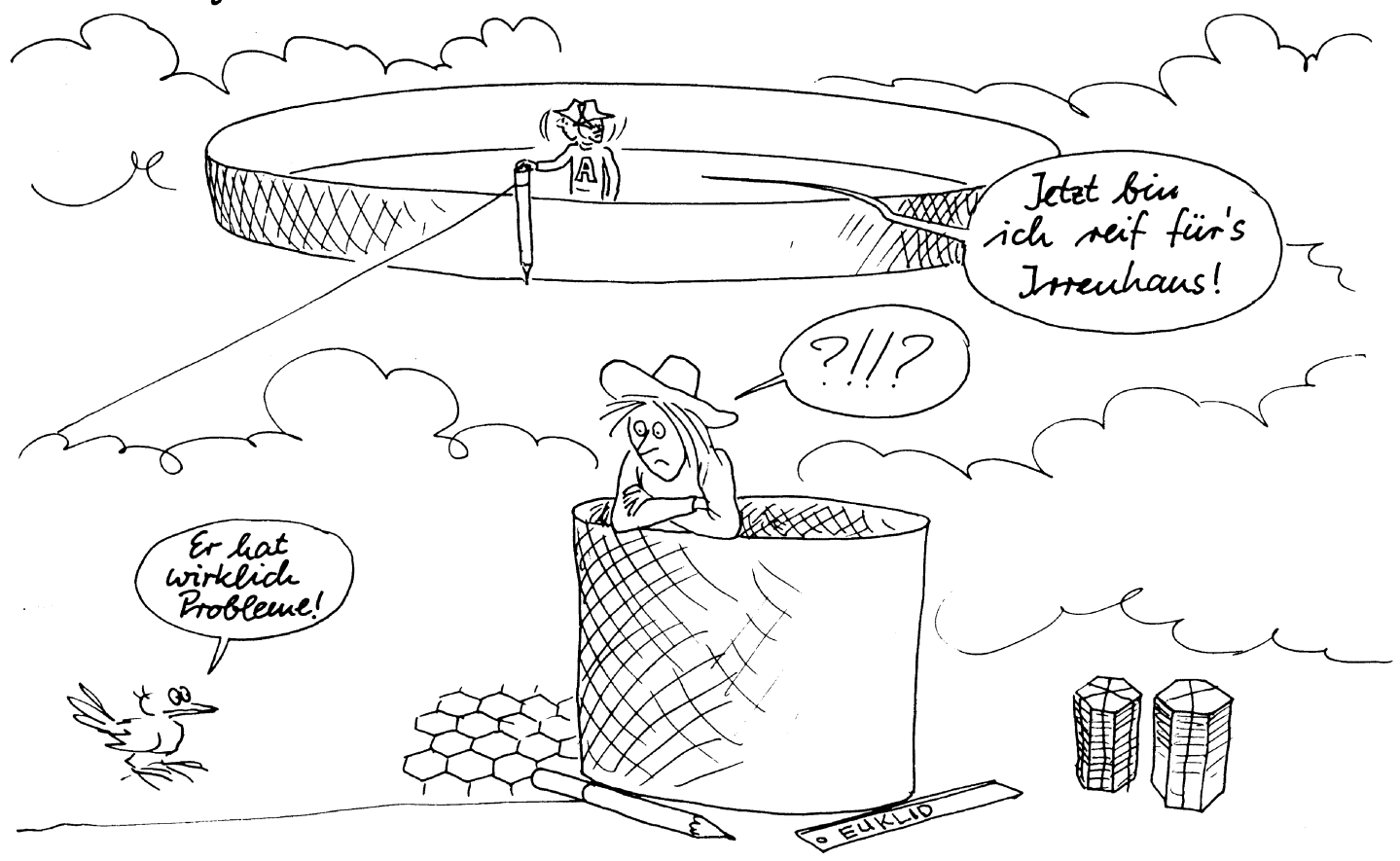

\section{WIAS ISE RASSIFRT?}

Un die Trage zu beantworten, wollen wir den Nebel vertreiben.

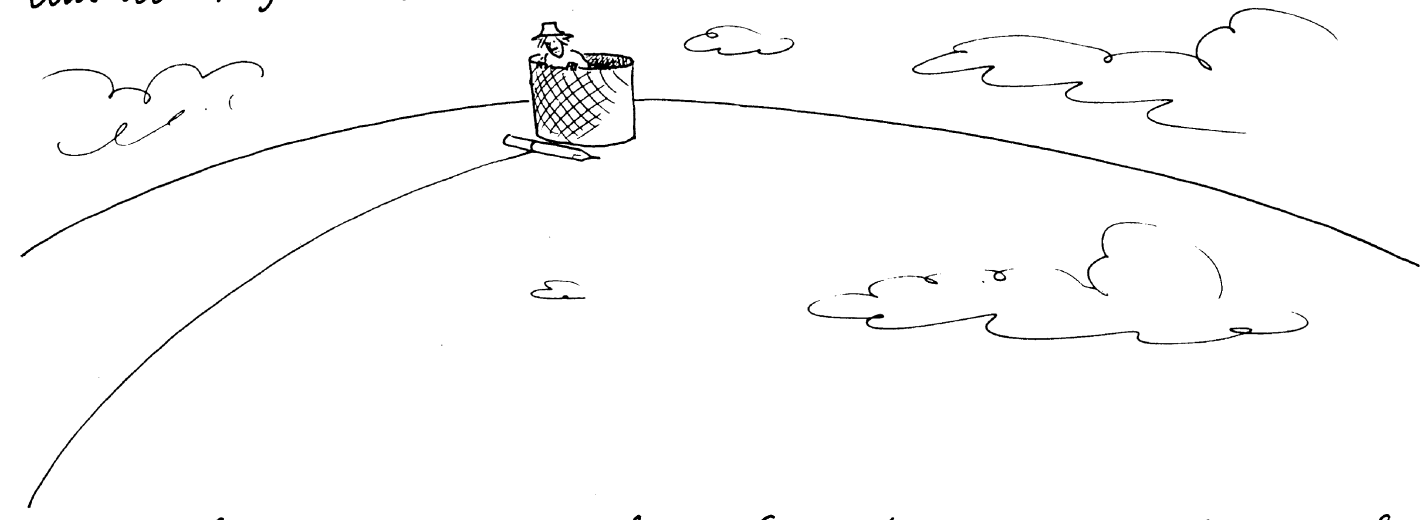

Anselm hat die Regelu der ebenen Geometrie angewendet mud sieht num plöthlich, daß er sich auf einer Kugel befindet. 


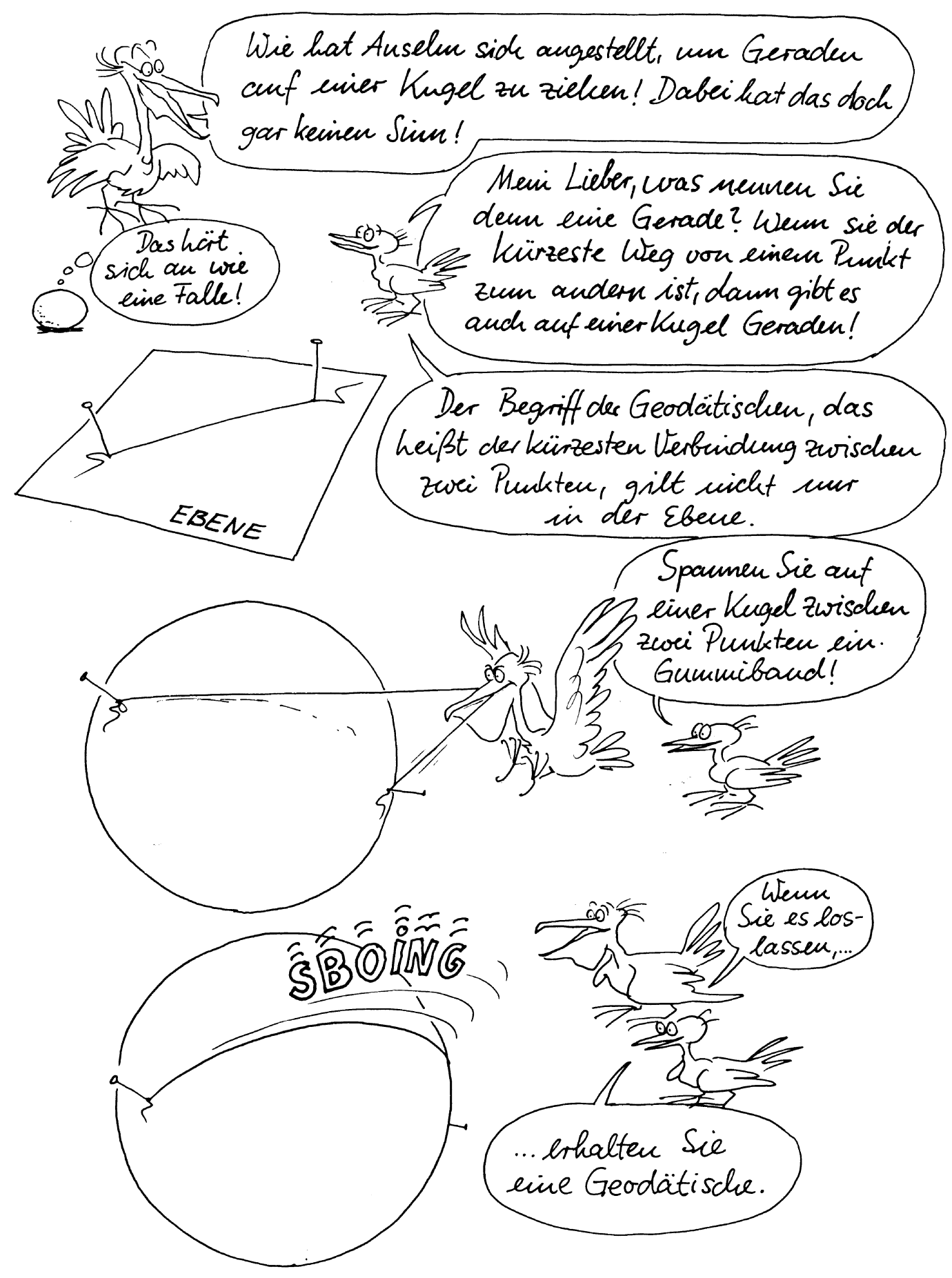

14 


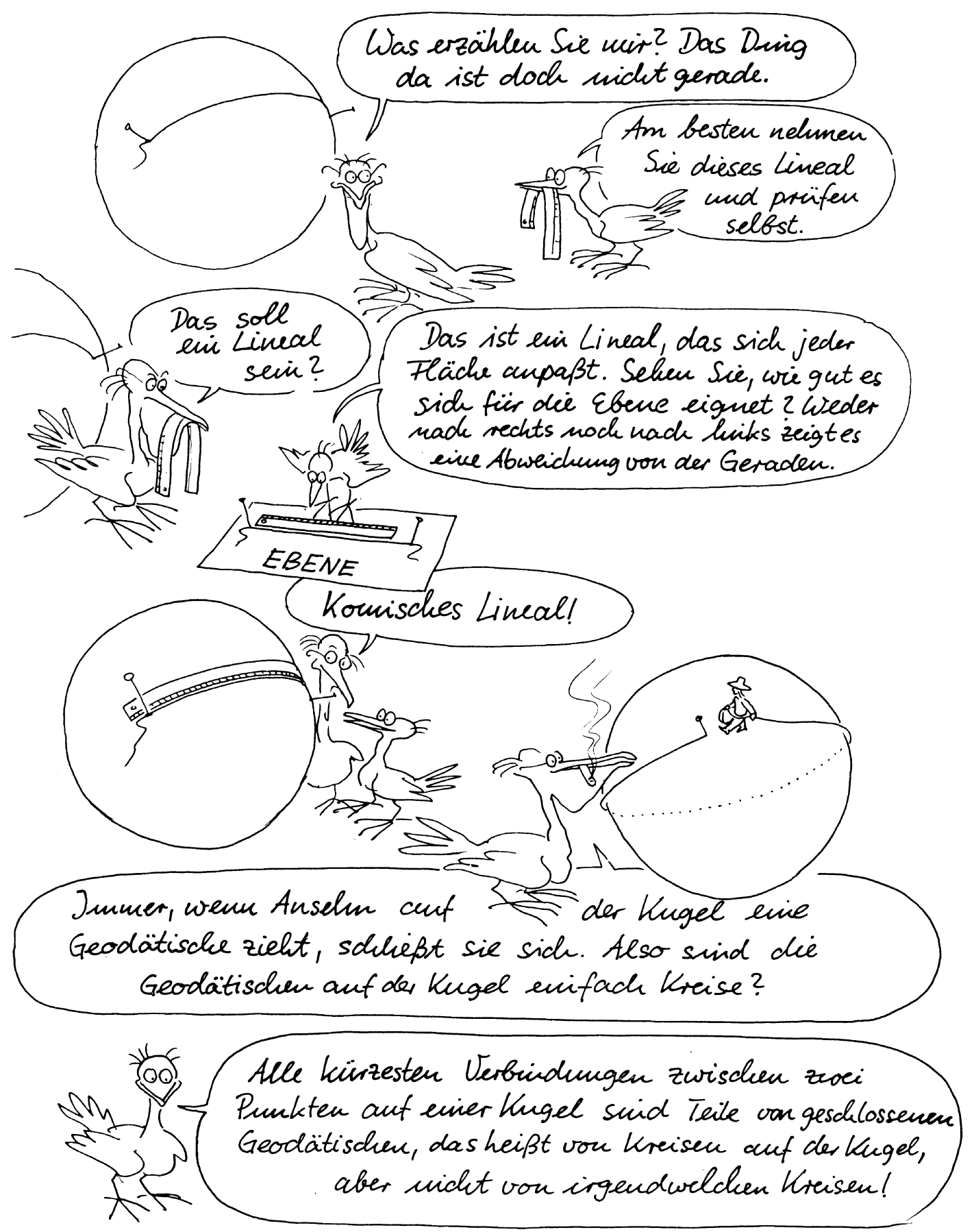




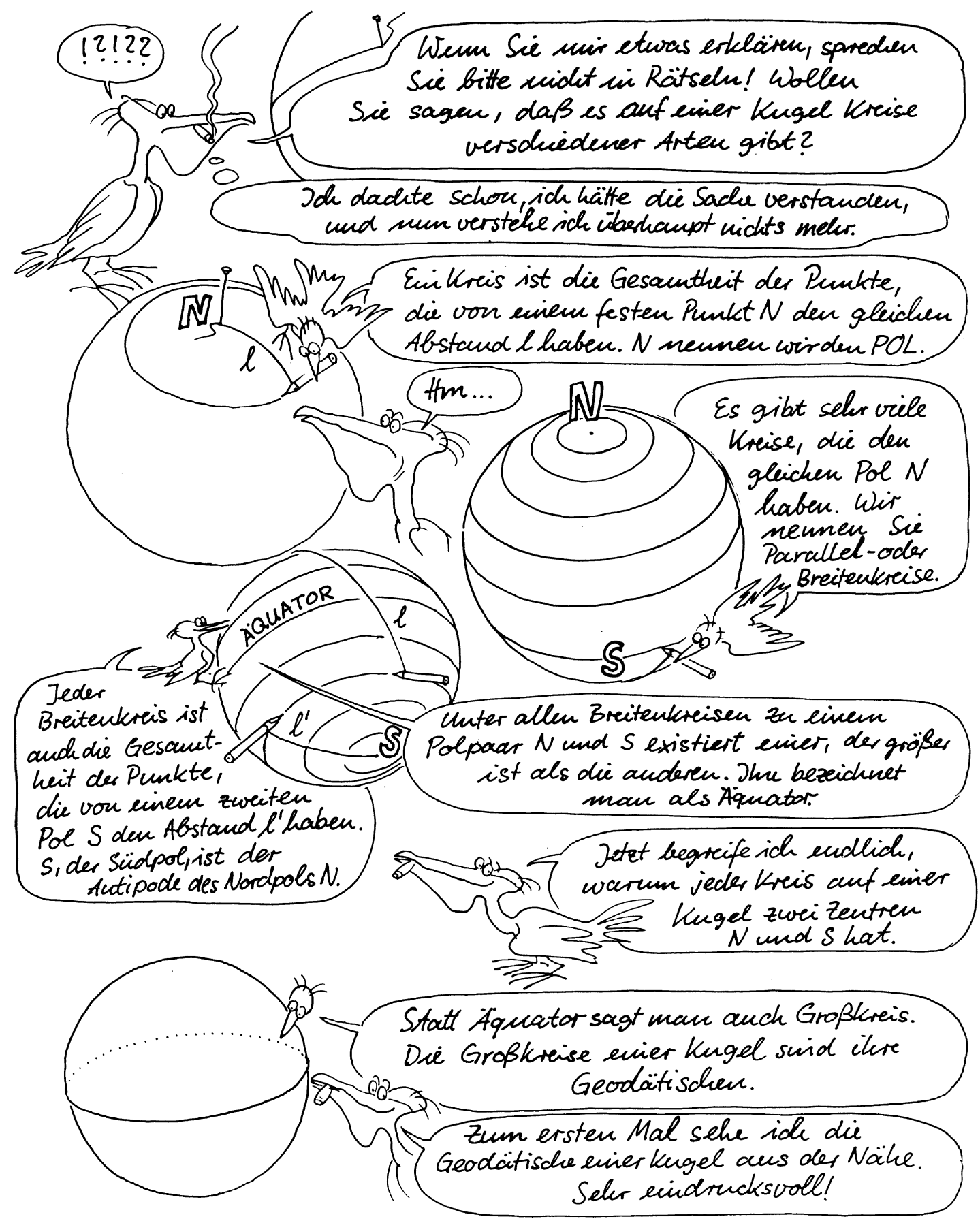




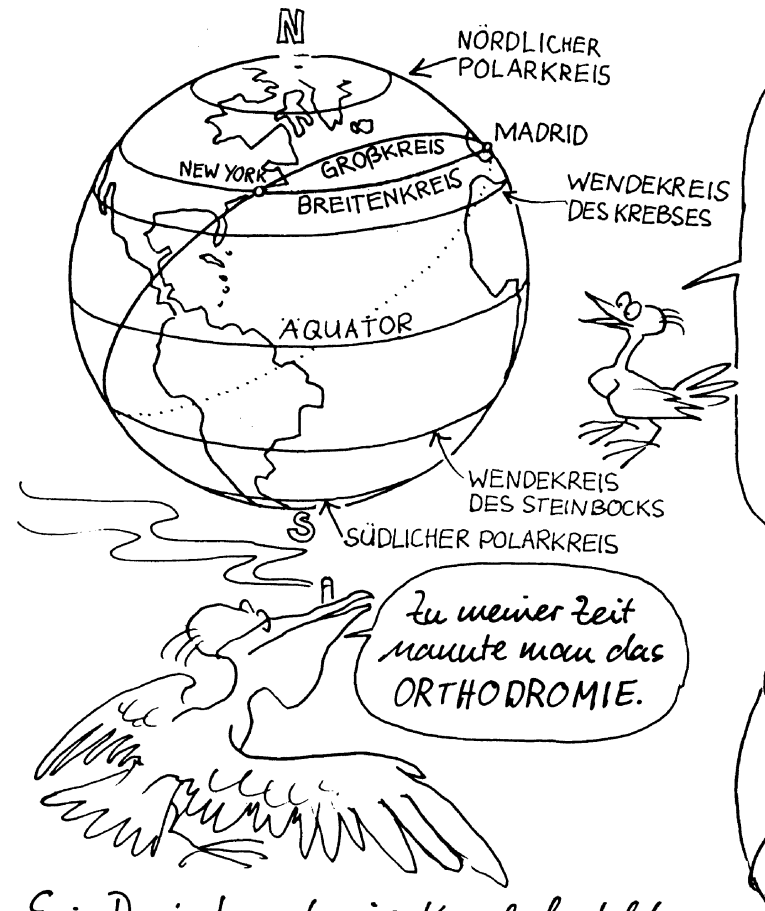

Ein Dreieck ant einerkugel besteht aus drei Bögen, die Teile vou Großkreisen sind.

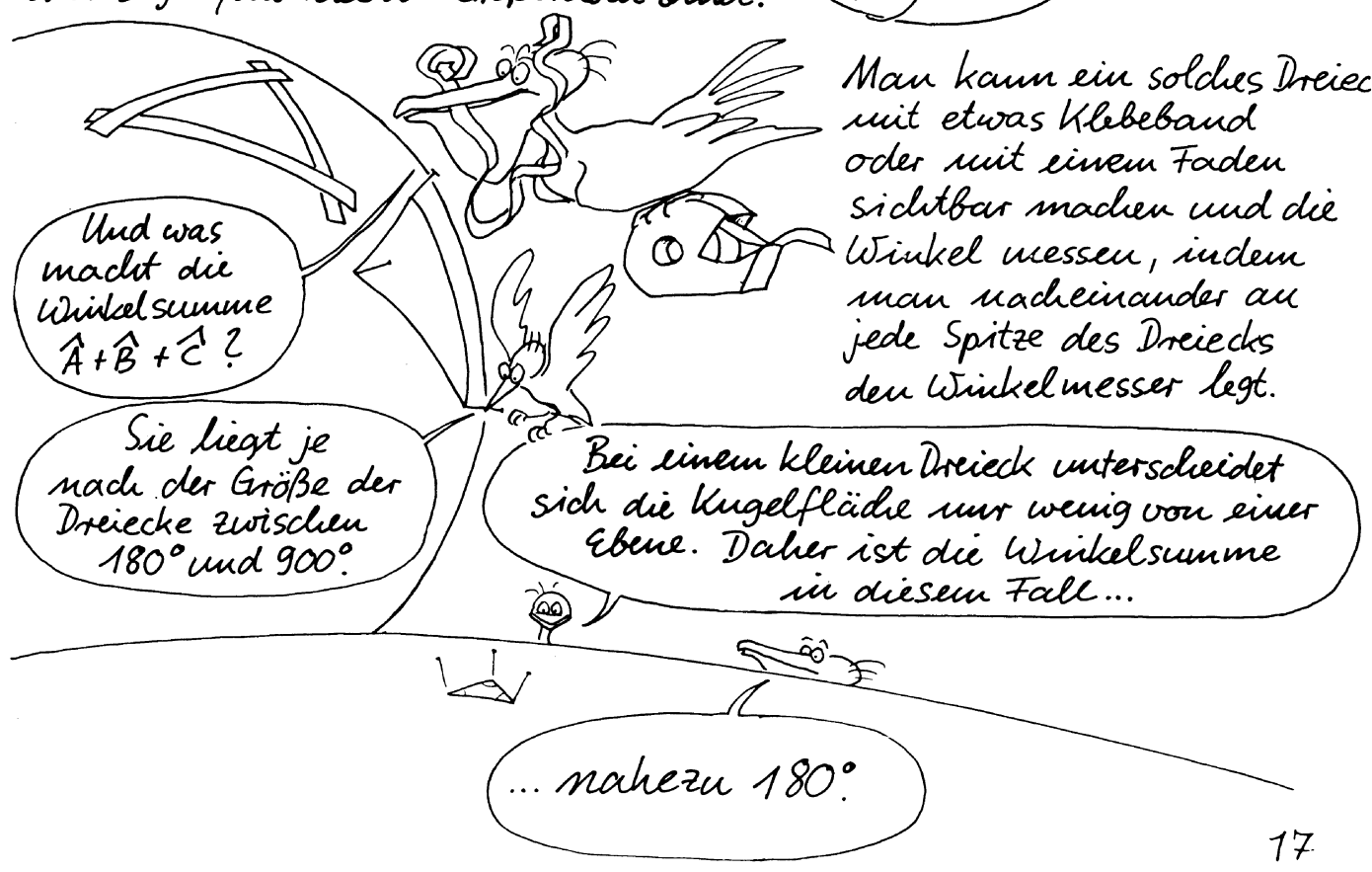

Auf derErde sind die Polarkreise und die Weadelereise Breitenkreise. Madrid und New York hiegen aut deunselben Breitentireis. Aber dieser ist vidit die kürzeste Verbiudung zwischen ilneu. Diese ergibt sich vielmehr erst, weun man den Großkreis durch die beiden Städte zeicluet.

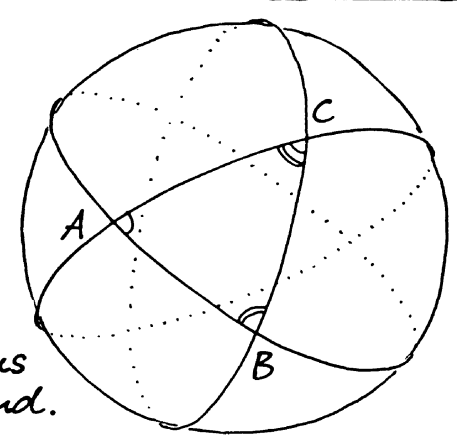

rieck uit etwas Klebeband oder uit einem Faden sichtbar machen und die Winkel messen, indem nan uacheinander an jede Spitze des Dreiecks den Wrickel messer legt. 


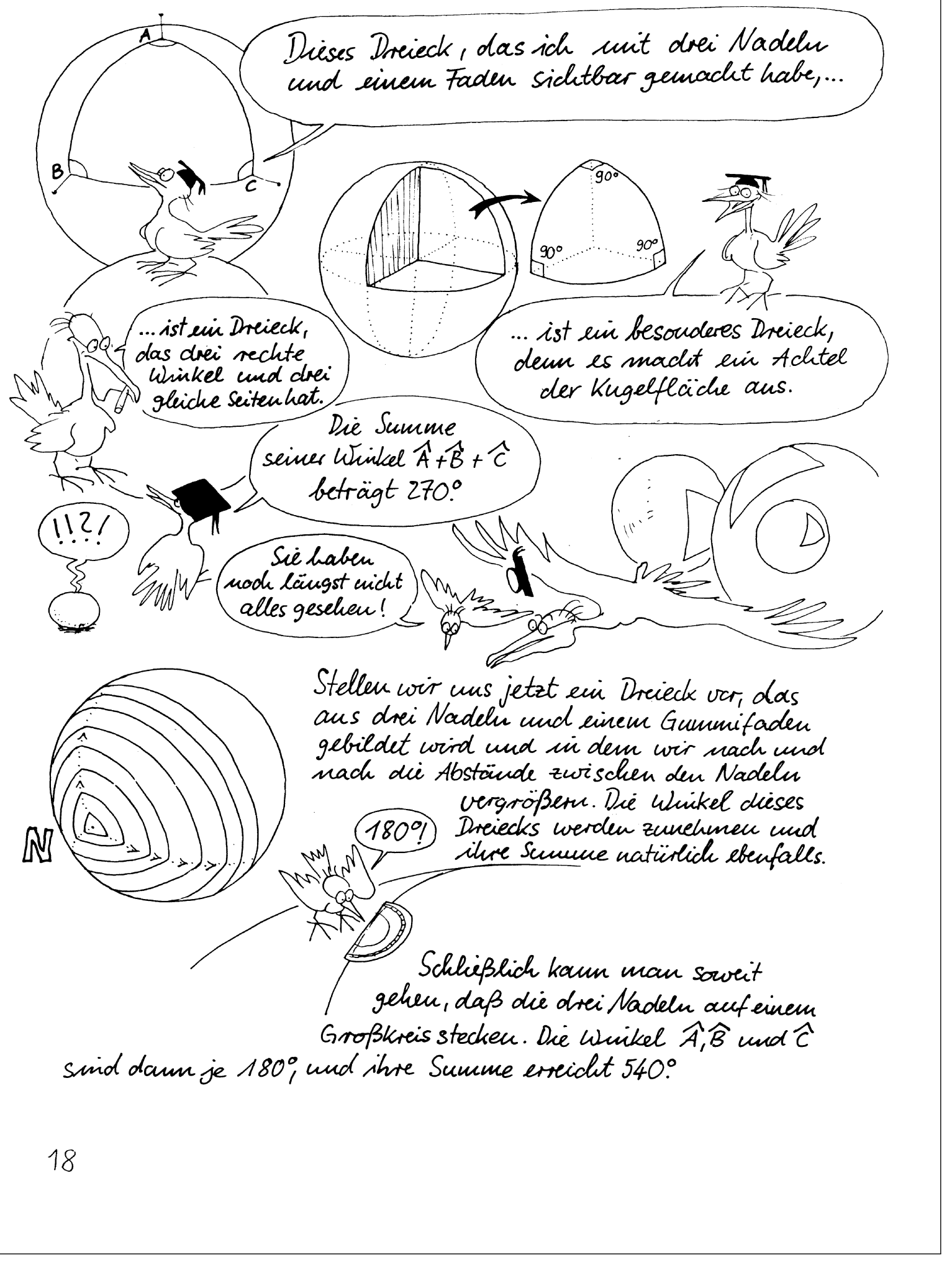



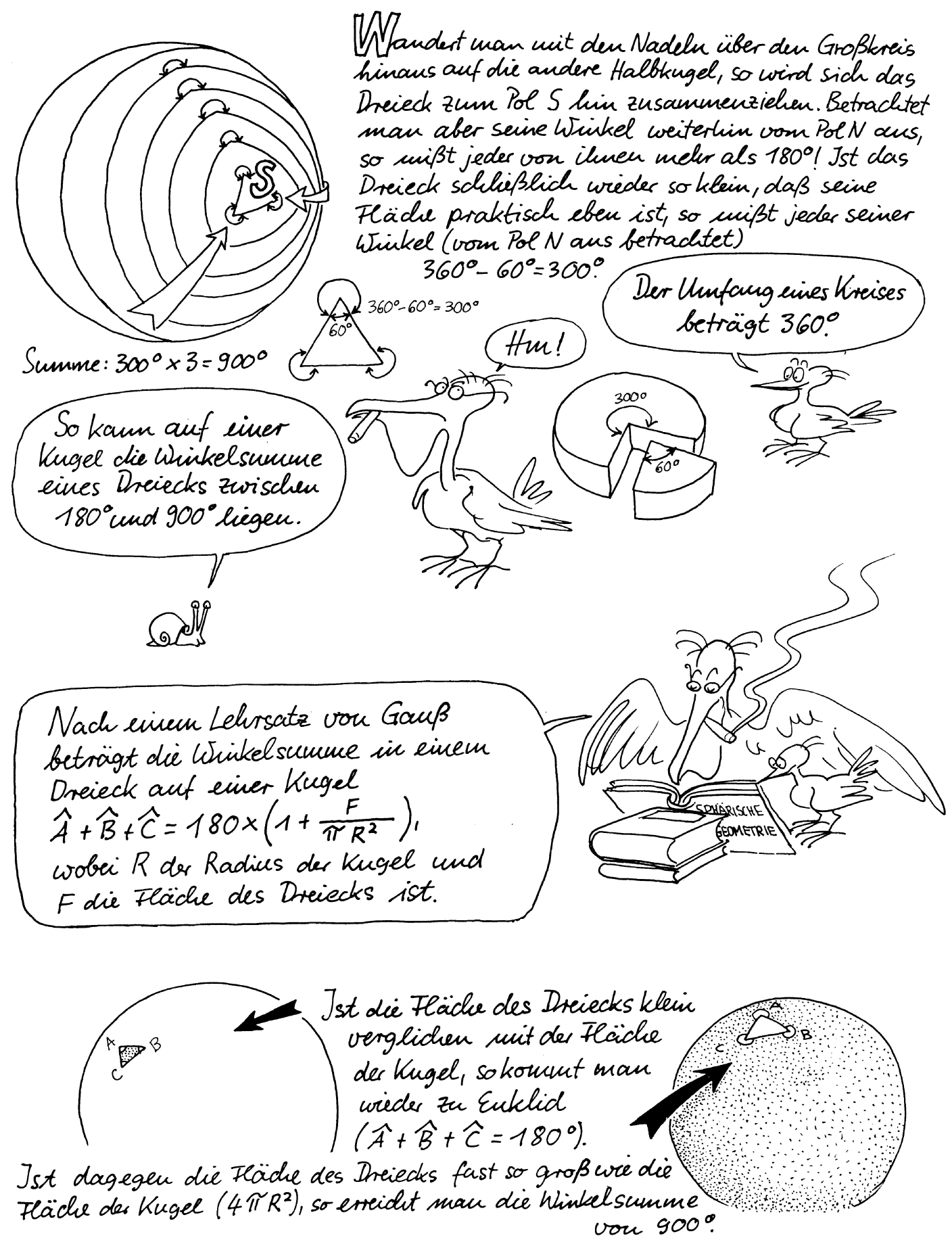


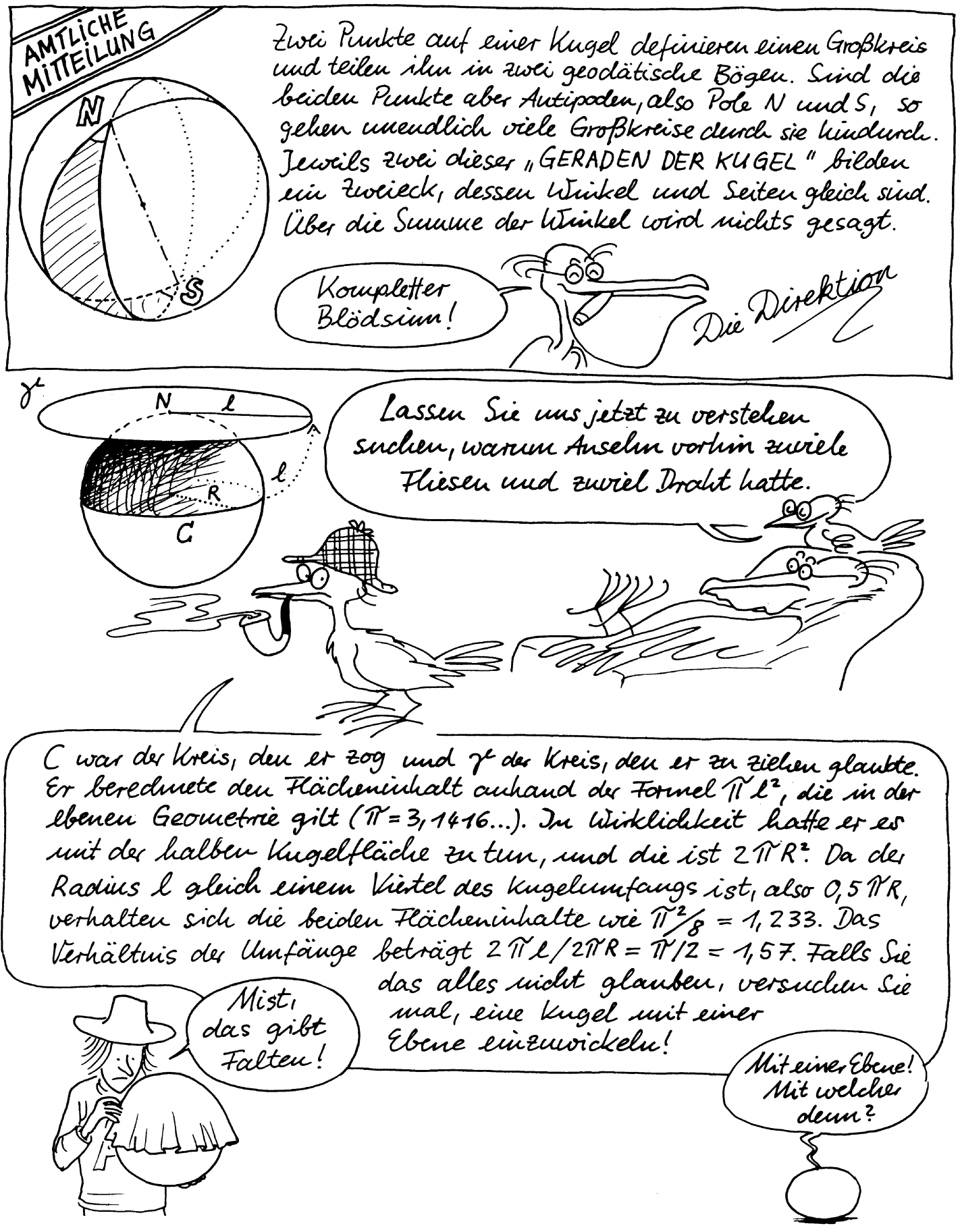

20 

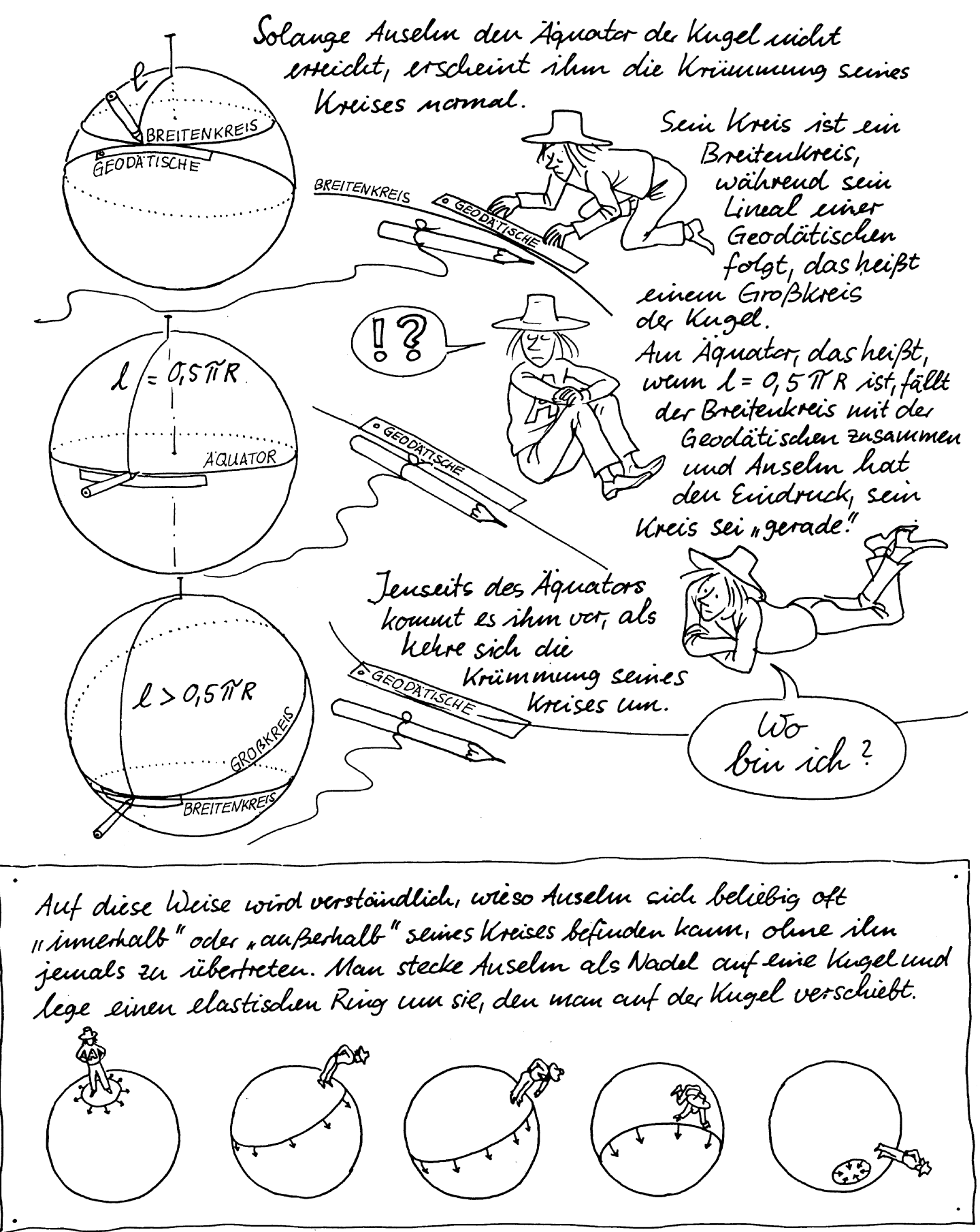


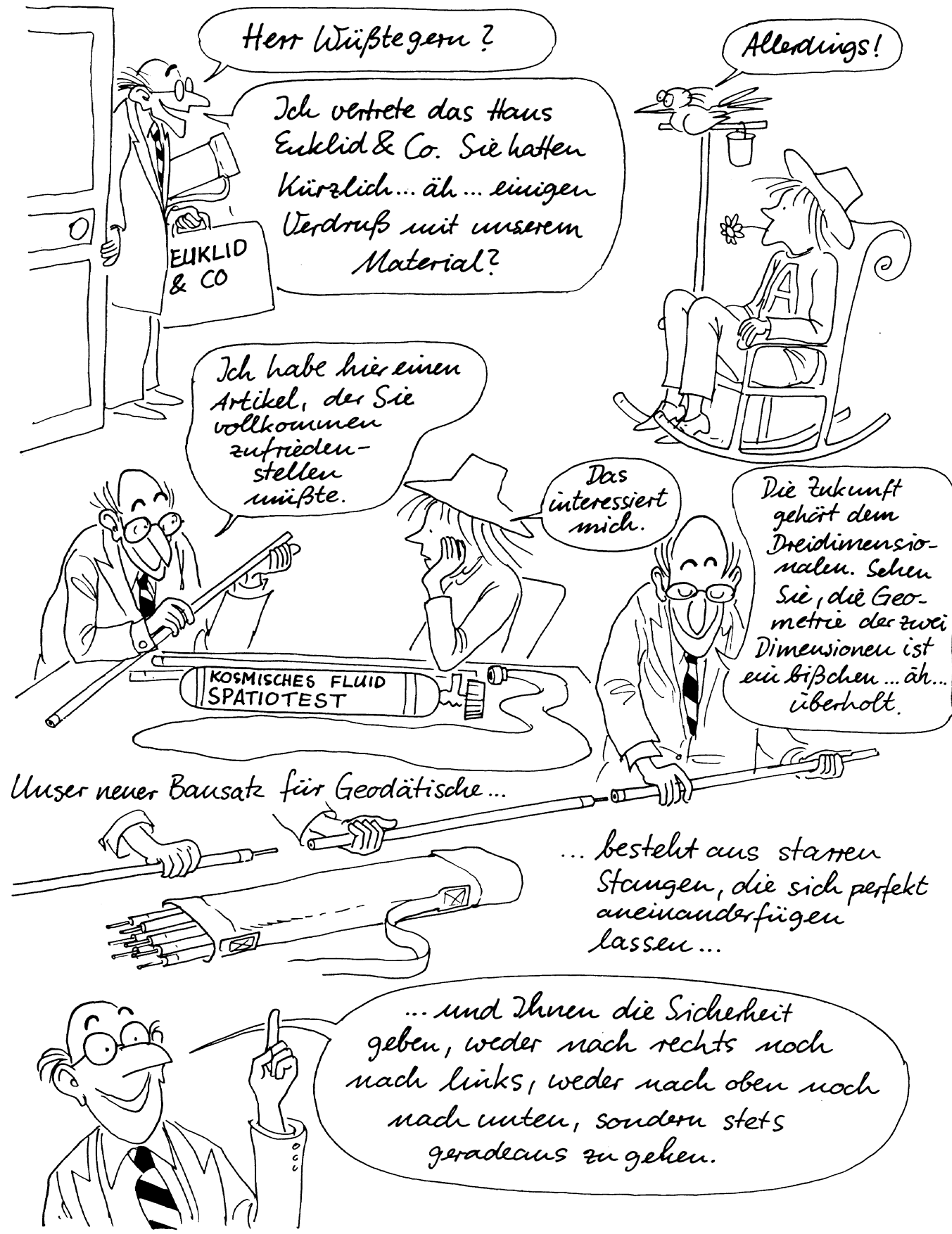




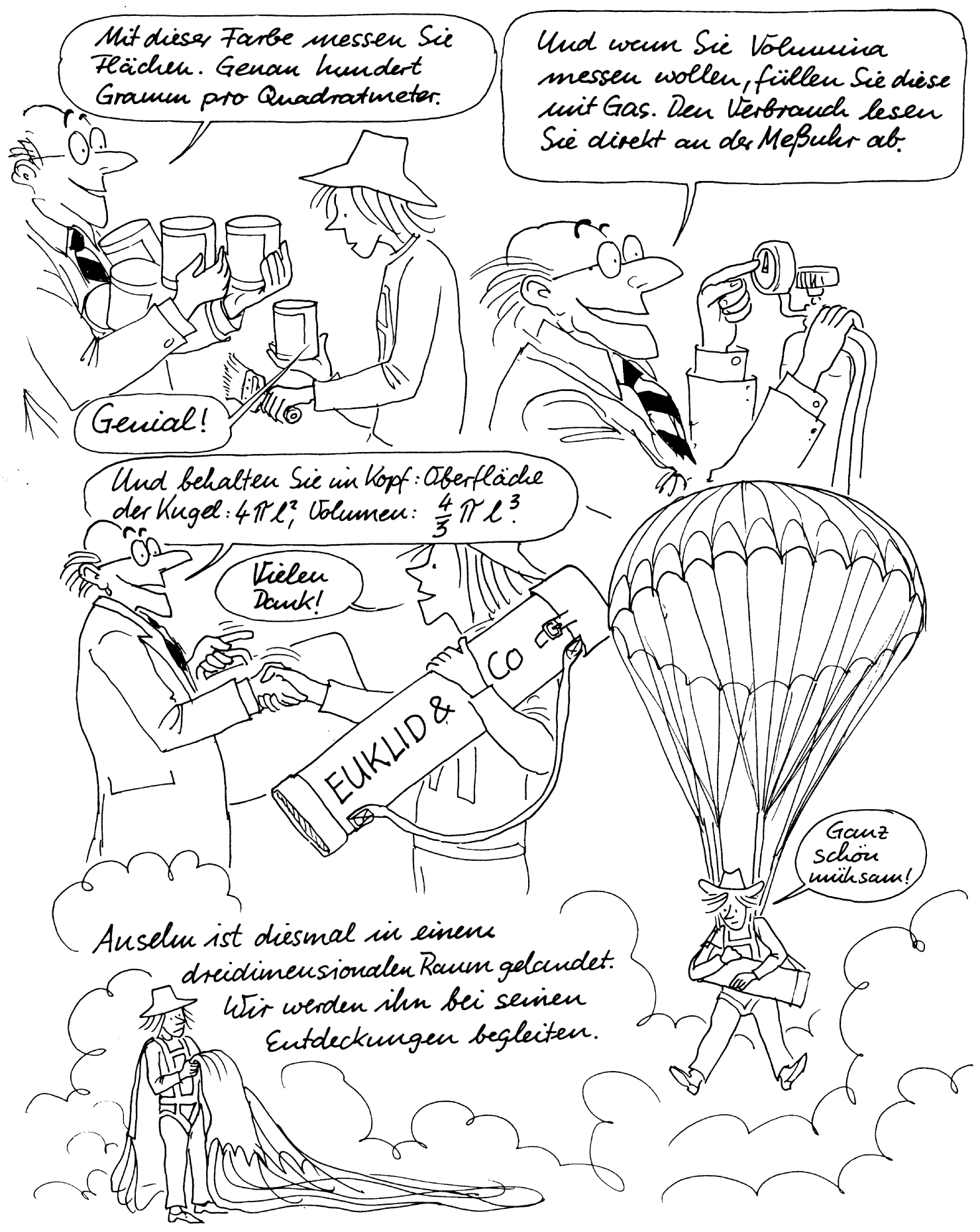




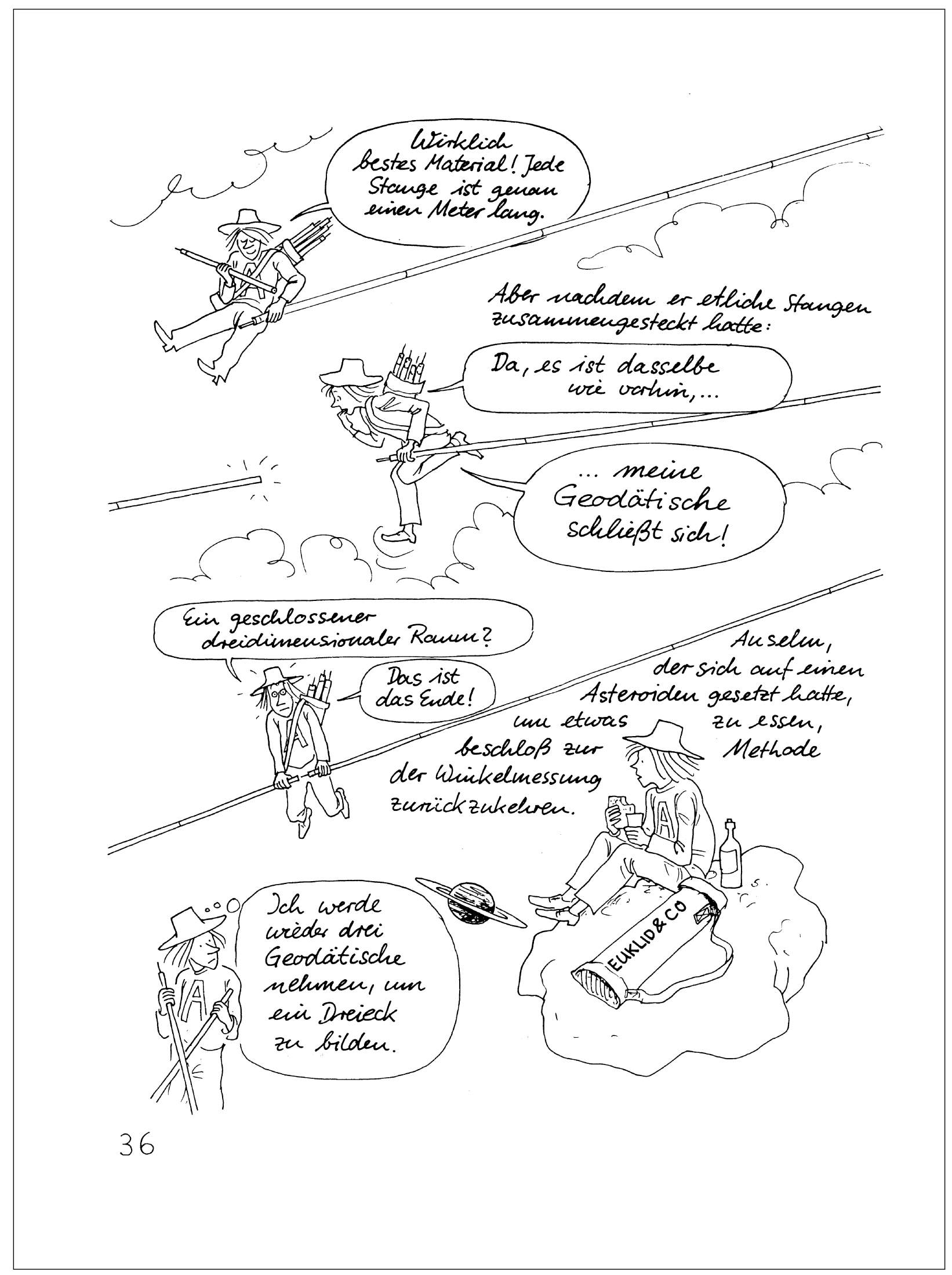




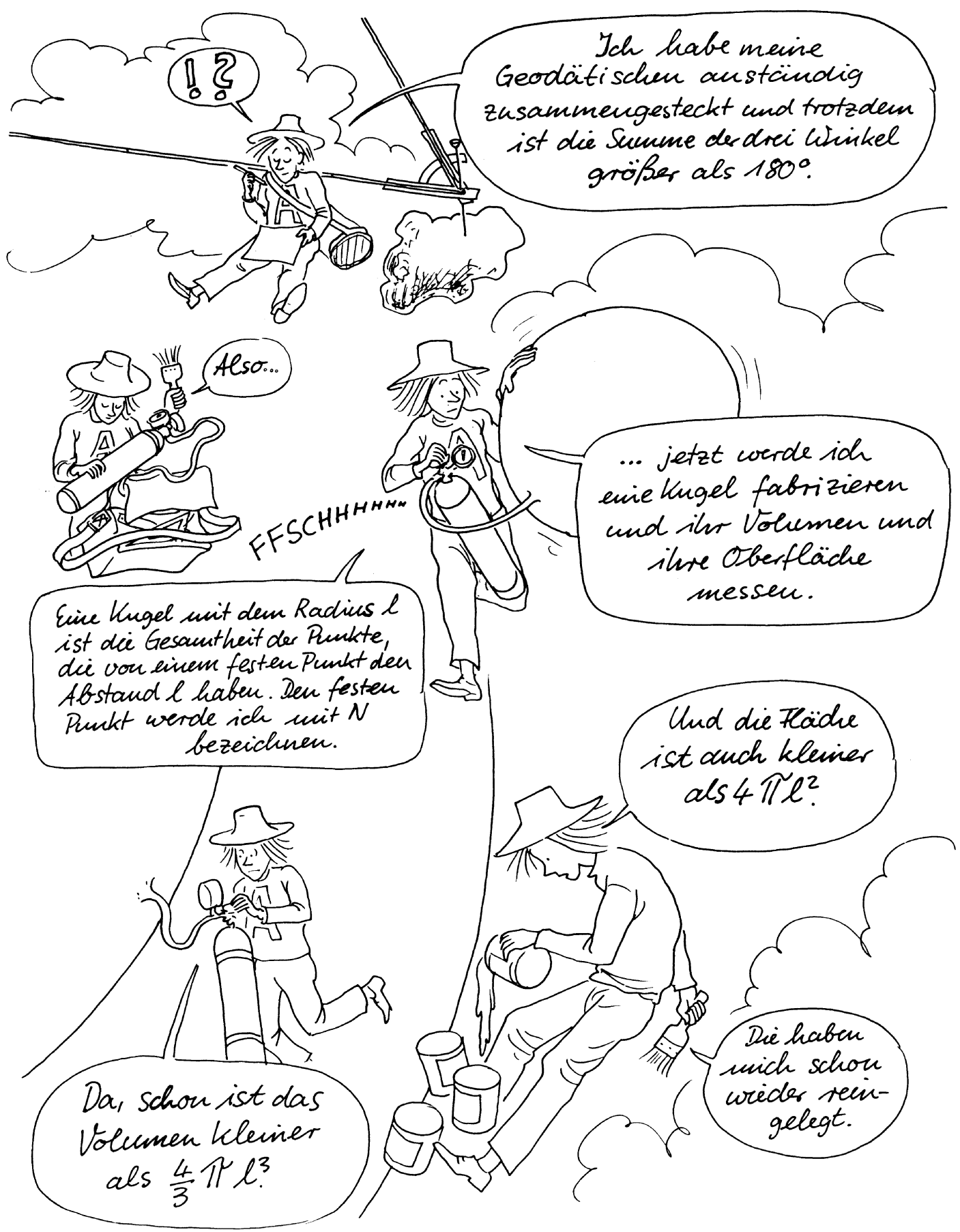


Anselm vergrößest deu Radius der kugel.

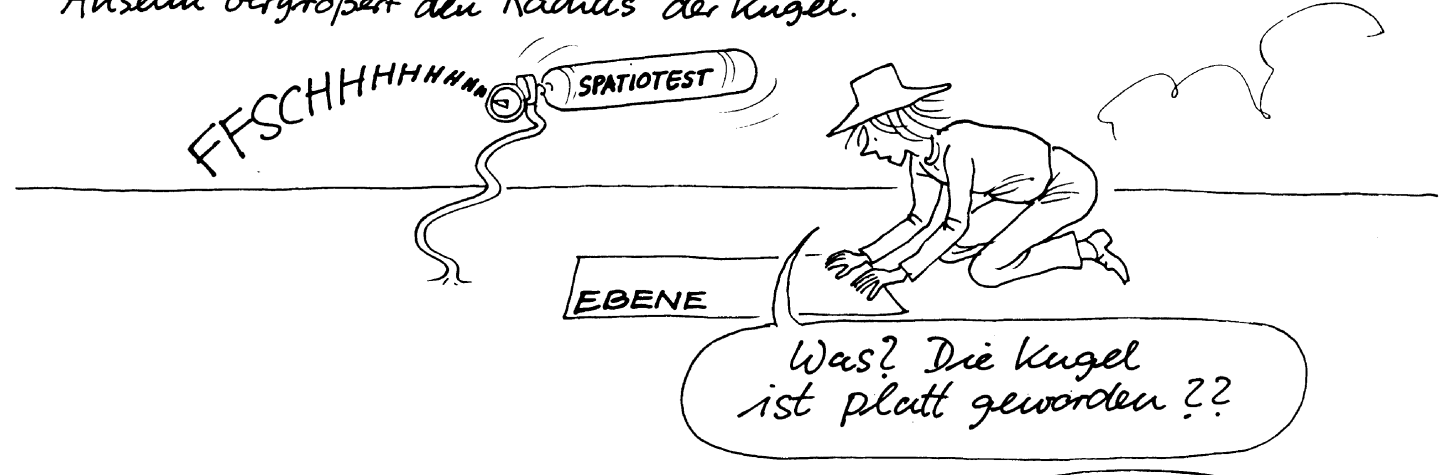

Auselun vergrößert die Kugel weiter.
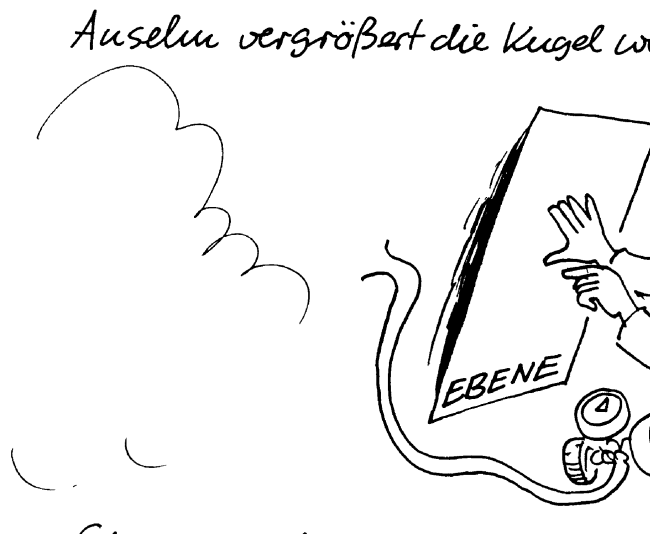

Etwas später:

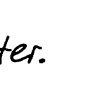

Jetzt kelert
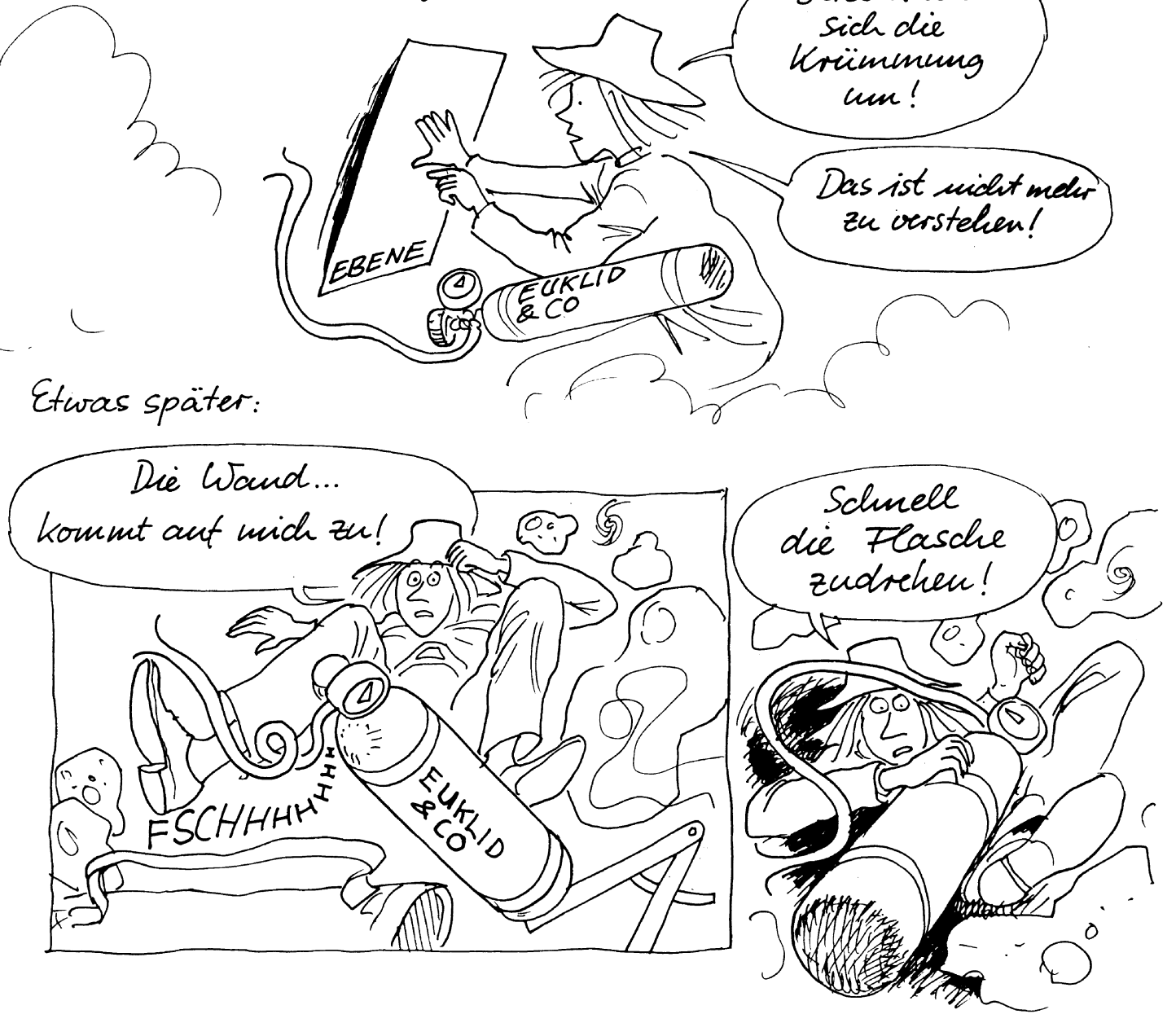

38 


\section{A.4 Lösungen und Kommentare}

\section{A.4.1 Geometrie}

\section{Geом1: Beweisen und Ordnen geometrischer Sätze}

1. Wir betrachten das Dreieck $A B C$, wobei $A$ und $B$ die Endpunkte des Kreisdurchmessers sind und $C$ ein beliebiger weiterer Punkt auf dem Kreisbogen ist. Wir zeichnen nun die Strecke $C M$, wobei $M$ der (Halb-)Kreismittelpunkt ist.

- Die Winkel $\alpha$ und $\gamma_{1}$ sowie $\beta$ und $\gamma_{2}$ sind jeweils Basiswinkel der gleichschenkligen Dreiecke $A M C$ bzw. $M B C$ und somit aufgrund des Basiswinkelsatzes identisch.

- Aufgrund des Innenwinkelsatzes gilt ferner $\alpha+\beta+\gamma_{1}+\gamma_{2}=180^{\circ}$.

- Es folgt $2 \cdot\left(\gamma_{1}+\gamma_{2}\right)=180^{\circ}$, denn $\alpha+\beta+\gamma_{1}+\gamma_{2}=(\overbrace{\alpha}^{=\gamma_{1}}+\gamma_{1})+(\overbrace{\beta}^{=\gamma_{2}}+\gamma_{2})$.

- Daraus folgt direkt $\left(\gamma_{1}+\gamma_{2}=\right) \gamma=90^{\circ}$.

2. a)
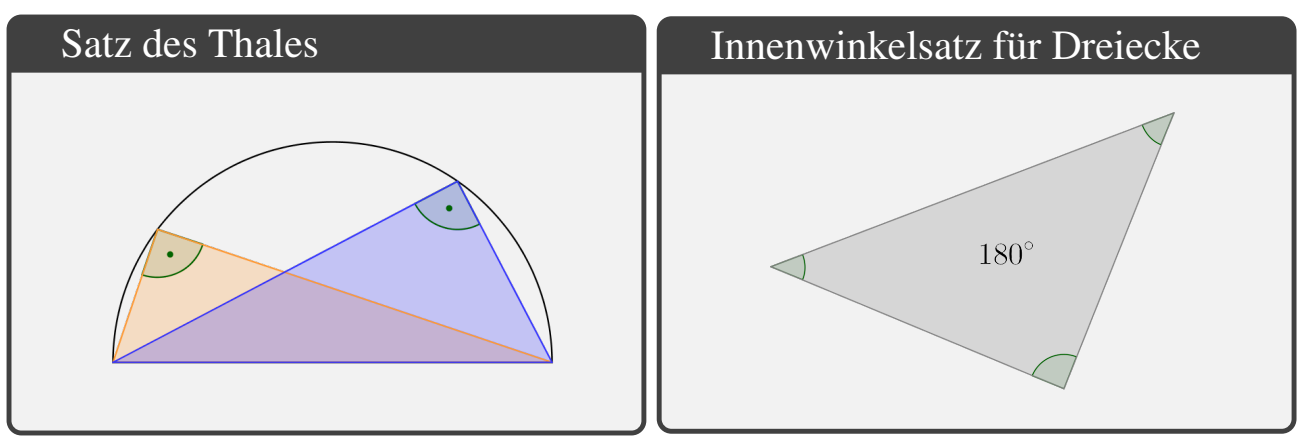

\section{Kongruenzsatz SWS}

Basiswinkelsatz
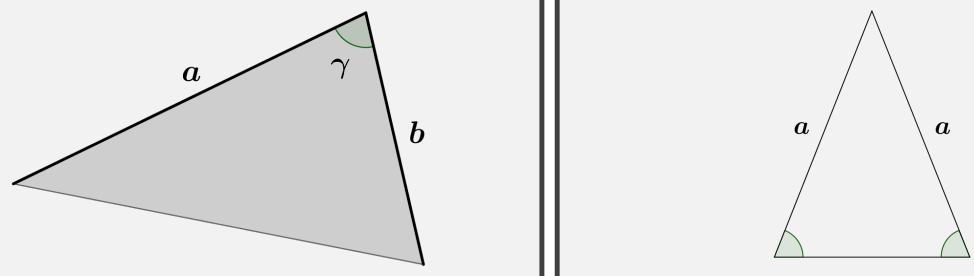


\section{Wechselwinkelsatz}

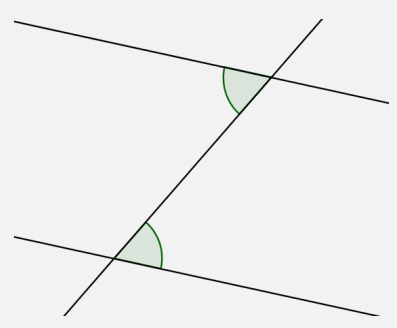

\section{Scheitelwinkelsatz}

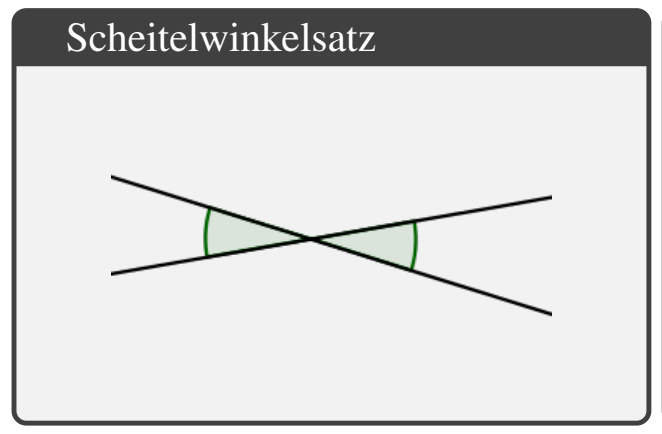

\section{Stufenwinkelsatz}

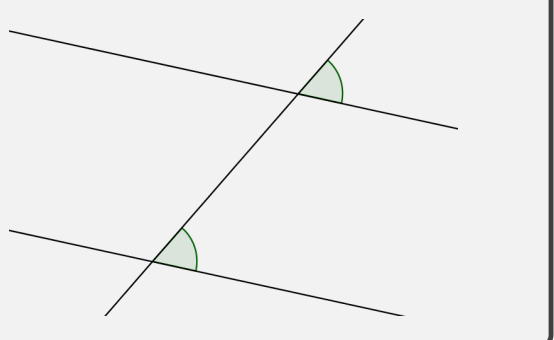

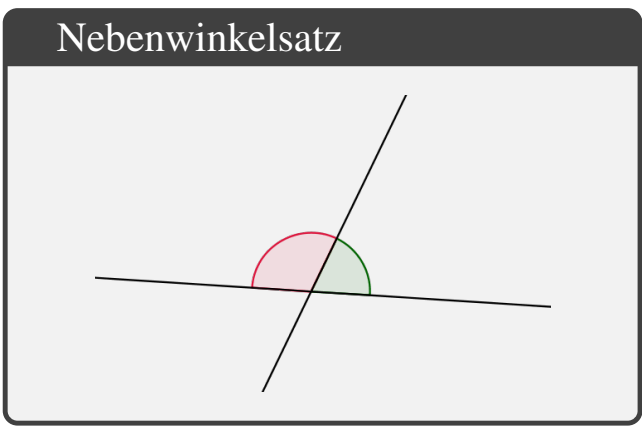

\section{Parallelensatz}

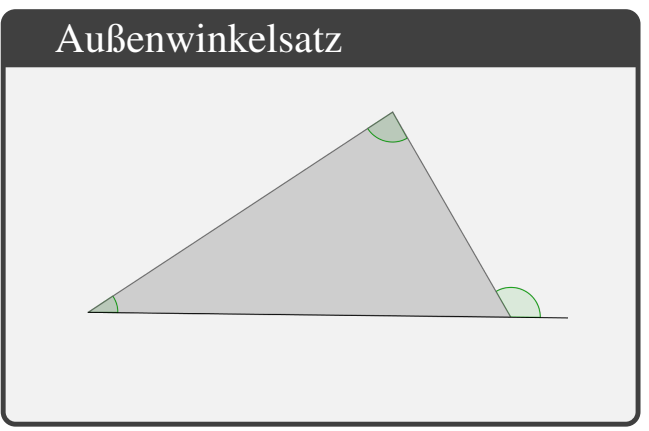

\section{Winkel im gleichs. Dreieck}

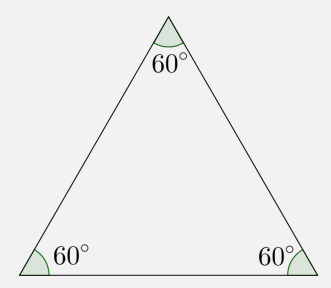

Innenwinkelsatz für Vierecke

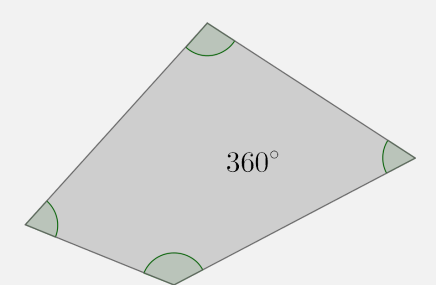


b) Satz des Thales: siehe Aufgabe 1 .

\section{Innenwinkelsatz für Dreiecke:}

- Man ziehe die Parallele zur Strecke $\overline{A B}$ durch $C$ (Parallelensatz).

- Es gilt $\alpha=\tilde{\alpha}$ und $\beta=\tilde{\beta}$ (Wechselwinkelsatz).

- Es gilt $\alpha+\beta+\gamma=\tilde{\alpha}+\tilde{\beta}+\gamma=180^{\circ}$ (Nebenwinkelsatz).

\section{Innenwinkelsatz für Vierecke:}

- Man ziehe eine Diagonale (z. B. von $B$ nach $D$ ).

- Nach dem Innenwinkelsatz für Dreiecke gilt $\alpha+\beta_{1}+\delta_{1}=180^{\circ}$ sowie $\alpha+\beta_{2}+\delta_{2}=180^{\circ}$.

- Insgesamt: $\alpha+\left(\beta_{1}+\beta_{2}\right)+\gamma+\left(\delta_{1}+\delta_{2}\right)=$ $360^{\circ}$

\section{Außenwinkelsatz:}

- Es gilt $\beta+\delta=180^{\circ}$ (Nebenwinkelsatz).

- Es gilt $\beta+(\gamma+\alpha)=180^{\circ}$ (Innenwinkelsatz für Dreiecke).

- Daraus folgt $\alpha+\gamma=\delta$.
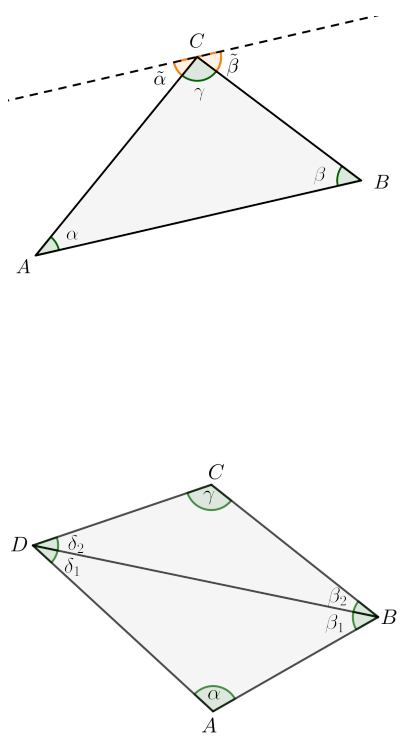

\section{Winkel im gleichseitigen Dreieck:}

- Nach dem Basiswinkelsatz gilt $\alpha=\beta=\gamma$.

- Ferner gilt $\alpha+\beta+\gamma=3 \cdot \alpha=180^{\circ}$ (Innenwinkelsatz für Dreiecke).

- Insgesamt folgt $\alpha=\beta=\gamma=60^{\circ}$.

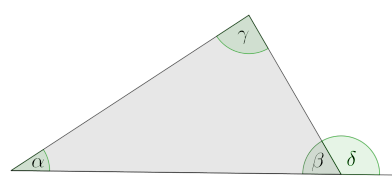

\section{Scheitelwinkelsatz:}

- Es gilt $\alpha+\gamma=\beta+\gamma=180^{\circ}$ (Nebenwinkelsatz).

- Also gilt auch $\alpha=\beta$.

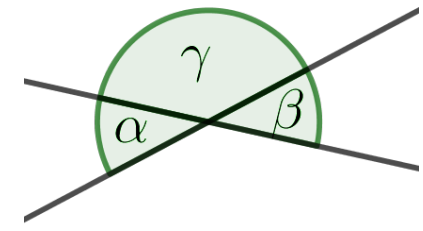




\section{Basiswinkelsatz:}

- Man ziehe die Winkelhalbierende durch $C$.

- Die Dreiecke $A M C$ und $M B C$ sind kongruent (Kongruenzsatz SWS).

- Insbesondere gilt also $\alpha=\beta$.

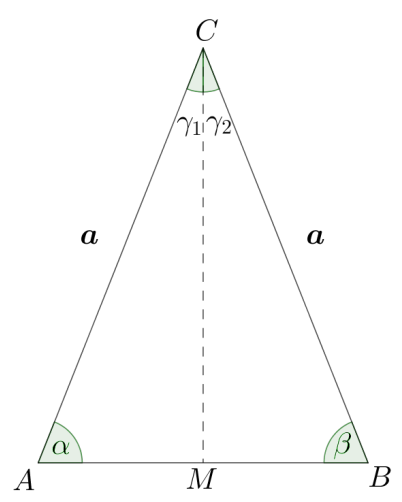

\section{Wechselwinkelsatz:}

- Es gilt $\alpha=\tilde{\alpha}$ (Stufenwinkelsatz).

- Es gilt $\tilde{\alpha}=\beta$ (Scheitelwinkelsatz).

- Insgesamt gilt also $\alpha=\beta$.

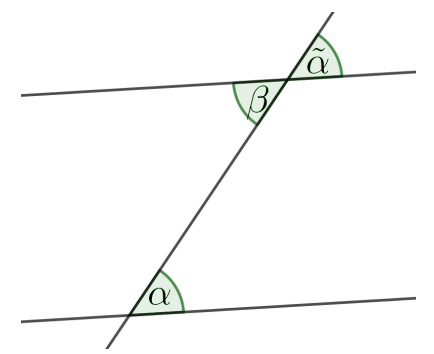

3.

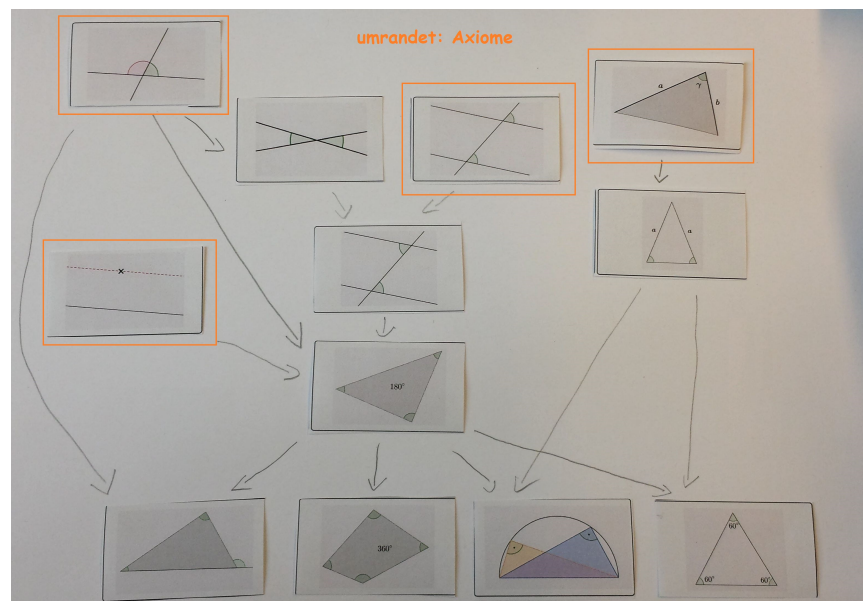

4. Der Verzicht auf die Gültigkeit einer bestimmten Aussage hat zur Folge, dass alle Aussagen, für deren Herleitung man die ,gestrichene“ Aussage benötigt, ebenfalls ihre Gültigkeit innerhalb des deduktiven Gefüges verlieren (es sei denn, man findet alternative Beweise, die die „gestrichenen“ Aussagen nicht verwenden). In Bezug auf die in dieser Aufgabe genannten Sätze verlieren also folgende weitere Aussagen ihre Gültigkeit:

a) Innenwinkelsatz für Dreiecke $\rightarrow$ Außenwinkelsatz, Innenwinkelsatz für Vierecke, Satz des Thales, Winkel im gleichseitigen Dreieck

b) Parallelensatz $\rightarrow$ Innenwinkelsatz für Dreiecke, Außenwinkelsatz, Innenwinkelsatz für Vierecke, Satz des Thales, Winkel im gleichseitigen Dreieck 
c) Kongruenzsatz SWS $\rightarrow$ Basiswinkelsatz, Satz des Thales, Winkel im gleichseitigen Dreieck

d) Wechselwinkelsatz $\rightarrow$ Innenwinkelsatz für Dreiecke, Außenwinkelsatz, Innenwinkelsatz für Vierecke, Satz des Thales, Winkel im gleichseitigen Dreieck

e) Nebenwinkelsatz $\rightarrow$ Scheitelwinkelsatz, Wechselwinkelsatz, Innenwinkelsatz für Dreiecke, Außenwinkelsatz, Innenwinkelsatz für Vierecke, Satz des Thales, Winkel im gleichseitigen Dreieck

5. a)

- Es gilt $\alpha=\gamma$ (Wechselwinkelsatz).

- Es gilt $\gamma=\beta$ (Scheitelwinkelsatz).

- Insgesamt gilt also $\alpha=\beta$.

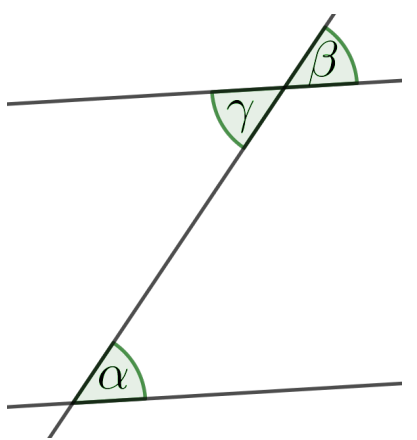

b) Die Pfeile, die von Scheitel- und Stufen- zum Wechselwinkelsatz führen, entfallen; dafür führen Pfeile vom Scheitel- und Wechsel- zum Stufenwinkelsatz. In diesem Schaubild muss dann der Wechselwinkelsatz anstelle des Stufenwinkelsatzes als Axiom umrandet werden.

\section{6. a) Außenwinkelsatz:}

- Die Gerade $g$ existiert nach dem Parallelensatz.

- Es gilt $\alpha=\alpha_{1}$ (Stufenwinkelsatz) sowie $\beta=\beta_{1}$ (Wechselwinkelsatz).

- Insgesamt folgt $\alpha+\beta=\alpha_{1}+\beta_{1}$.

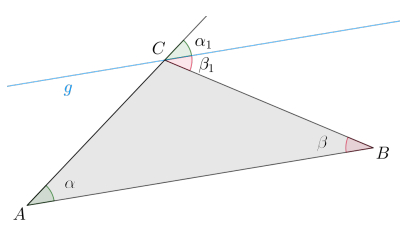

\section{Innenwinkelsatz für Dreiecke:}

- Es gilt $\beta+\delta=180^{\circ}$ (Nebenwinkelsatz).

- Es gilt $\delta=\alpha+\gamma($ Außenwinkelsatz).

- Daraus folgt $\alpha+\beta+\gamma=180^{\circ}$.

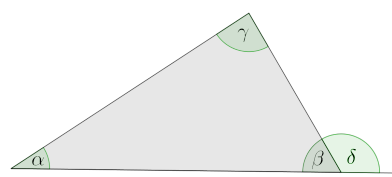


b)

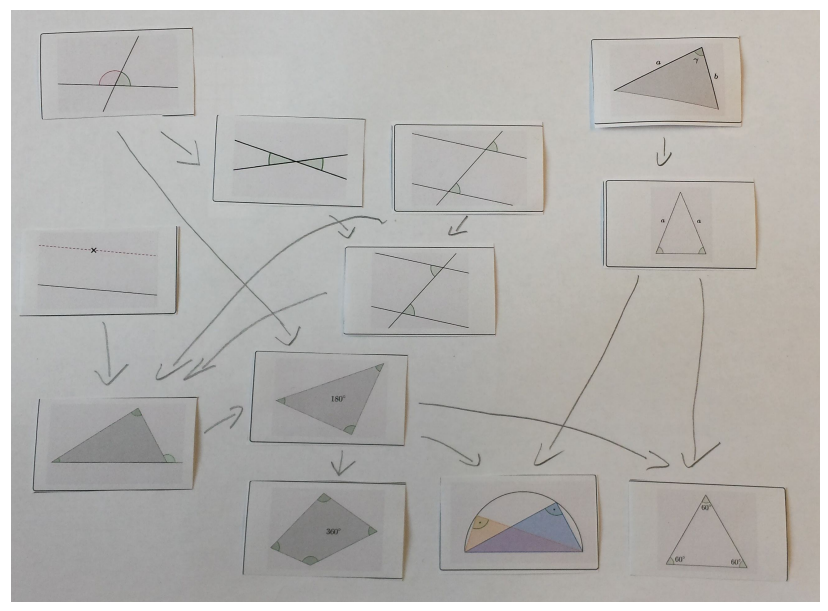




\section{GEOM3: Flächeninhalt und Innenwinkelsumme sphärischer Dreiecke}

1. $\triangle A B C, \triangle A^{d} B C, \triangle A B^{d} C, \triangle A B C^{d}, \triangle A^{d} B^{d} C, \triangle A^{d} B C^{d}, \triangle A B^{d} C^{d}, \triangle A^{d} B^{d} C^{d}$

2. - Die Dreiecke $\triangle A B C$ und $\triangle A^{d} B C$ bilden zusammen ein sphärisches Zweieck mit dem Winkel $\alpha=\angle B A C$.

- Die Dreiecke $\triangle A B C$ und $\triangle A B^{d} C$ bilden zusammen ein sphärisches Zweieck mit dem Winkel $\beta=\angle C B A$.

- Die Dreiecke $\triangle A B C$ und $\triangle A B C^{d}$ bilden zusammen ein sphärisches Zweieck mit dem Winkel $\gamma=\angle A C B$.

3./4. Die übrigen Zweiecke sind diejenigen, die jeweils das (zum Dreieck $\triangle A B C$ kongruente) Dreieck $\triangle A^{d} B^{d} C^{d}$ beinhalten. Es lassen sich Paare von Zweiecken finden (und farbgleich schraffieren, welche jeweils die gleichen diametralen Eckpunkte $\left(A A^{d}, B B^{d}\right.$ und $\left.C C^{d}\right)$ und damit auch die gleichen Winkel haben. Diese Zweiecke sind jeweils kongruent. Ebenso sind die Dreiecke $\triangle A^{d} B C$ und $\triangle A B^{d} C^{d}, \triangle A B^{d} C$ und $\triangle A^{d} B C^{d}$ sowie $\triangle A B C^{d}$ und $A^{d} B^{d} C$ kongruent.

5. Aus $\frac{\alpha}{2 \pi}=\frac{F_{Z}}{4 \pi R^{2}}$ folgt $F_{Z}=2 \alpha R^{2}$.

6. Wir bezeichnen den Flächeninhalt des Dreiecks $\triangle A B C$ (bzw. $\triangle A^{d} B^{d} C^{d}$ ) mit $F$, den des Dreiecks $\triangle A^{d} B C$ (bzw. $\triangle A B^{d} C^{d}$ ) mit $F_{1}$, den des Dreiecks $\triangle A B^{d} C$ (bzw. $\triangle A^{d} B C^{d}$ ) mit $F_{2}$ und den des Dreiecks $\triangle A B C^{d}$ (bzw. $A^{d} B^{d} C$ ) mit $F_{3}$. Die Dreicke $\triangle A B C, \triangle A^{d} B C, \triangle A^{d} B^{d} C$ und $\triangle A B^{d} C$ bilden eine Halbsphäre. Es gilt also

$$
(*) \quad F+F_{1}+F_{2}+F_{3}=2 \pi R^{2} .
$$

Ferner gilt nach der Formel aus 5.:

$$
F+F_{1}=2 \alpha R^{2}, \quad F+F_{2}=2 \beta R^{2}, \quad F+F_{3}=2 \gamma R^{2}
$$

Addieren dieser drei Gleichungen liefert: $3 F+F_{1}+F_{2}+F_{3}=2 R^{2} \cdot(\alpha+\beta+\gamma)$ Zusammen mit $(*)$ erhält man:

$$
2 R^{2} \cdot(\alpha+\beta+\gamma)=2 \pi R^{2}+2 F \Leftrightarrow F=R^{2} \cdot(\alpha+\beta+\gamma-\pi)
$$

7. Je größer der Flächeninhalt eines sphärischen Dreiecks ist, desto größer ist auch dessen Winkelsumme. 


\section{GEOM4: Von Dimpfeln und Gronkeln}

a) Angenommen, zwei verschiedene Gronkel würden beide von (mindestens) zwei verschiedenen Dimpfeln $d_{1}$ und $d_{2}$ geklubert werden. Dann gäbe es zu zwei verschiedenen Dimpfeln mehr als einen Gronkel, der von beiden geklubert wird. Das steht im Widerspruch zu $A_{1}$.

b) 1) Nach $A_{3}$ gibt es drei verschiedene Dimpfel $d_{1}, d_{2}, d_{3}$, die nicht alle denselben Gronkel klubern.

2) Zu je zwei dieser Dimpfel gibt es nach $A_{1}$ genau einen Gronkel, der von beiden Dimpfeln geklubert wird. Nennen wir diese $G_{12}, G_{23}$ und $G_{13}$.

3) Dieser Gronkel sind paarweise verschieden, denn wäre z.B. $G_{12}=G_{13}$, würden die drei Dimpfel $D_{1}, D_{2}, D_{3}$ denselben Gronkel klubern. Widerspruch zu $1)$.

c) 1) Die Dimpfel $d_{1}$ und $d_{2}$ klubern nach $A_{1}$ genau einen Gronkel $G_{1}$.

2) Es sei $G_{2}$ ein von $G_{1}$ verschiedener Gronkel (welcher nach Teil b) auch existiert).

3) $G_{2}$ wird nach $A_{2}$ von mindestens zwei Dimpfeln geklubert.

4) Würden beide Dimpfel auch $G_{1}$ klubern, hätte man einen Widerspruch zu $A_{1}$. Mindestens ein Dimpfel (nennen wir ihn $d_{3}$ ) klubert also nicht $G_{1}$.

\section{Von Musikern und Instrumenten:}

$A_{1}$ : Zu zwei verschiedenen Musikern $d_{1}$ und $d_{2}$ gibt es genau ein Instrument, das von $d_{1}$ und $d_{2}$ gespielt wird.

$A_{2}$ : Jedes Instrument wird von mindestens zwei Musikern gespielt.

$A_{3}$ : Es gibt mindestens drei Musiker, die nicht alle dasselbe Instrument spielen.

\section{Von Punkten und Geraden:}

$A_{1}:$ Zu zwei verschiedenen Punkten $d_{1}$ und $d_{2}$ gibt es genau eine Gerade, die durch $d_{1}$ und $d_{2}$ geht.

$A_{2}$ : Jede Gerade geht durch mindestens zwei Punkte.

$A_{3}$ : Es gibt mindestens drei Punkte, die nicht alle auf derselben Geraden liegen. 


\section{A.4.2 Wahrscheinlichkeitsrechnung}

\section{WKEIT1: Was ist Wahrscheinlichkeit? Klassische Zufallsexperimente}

1. Zu erwarten ist hier, dass die Schülerinnen und Schüler für den Laplace-Würfel und die Bleistifte a priori eine Wahrscheinlichkeit von 1/6 für alle Augenzahlen schätzen und sich bei einer Schätzung für die Augenzahlen des Quaders an den unterschiedlich großen Flächen orientieren. Beispielhaft wird in dieser Musterlösung mit den Schätzungen $P(3)=P(4)=30 \%, P(1)=P(6)=15 \%$ und $P(2)=P(5)=5 \%$ gerechnet. Die anzustellenden Rechnungen sind dann:

$$
\begin{aligned}
& \text { - } P(A)=P(2)+P(4)+P(6) \\
& \text { - } P(B)=P(2)+P(3)+P(5) \\
& \text { - } P(C)=P(2)+P(3)+P(4)+P(5)+P(6)=P(A)+P(B)-P(2) \\
& \text { - } P(D)=P(2) \\
& \text { - } P(E)=1-P(C)=P(1)
\end{aligned}
$$

2. Individuelle Schülerlösungen

3. Zu Tom: Die Anzahl der durchgeführten Würfe ist sehr niedrig, so dass das Nichtauftreten einer bestimmten Augenzahl durchaus üblich ist und noch keine hohe Ausagekraft bzgl. der Auftretenswahrscheinlichkeit hat.

$\mathrm{Zu}$ Anja: Aufgrund der hohen Versuchszahl beträgt die Wahrscheinlichkeit, eine Sechs zu würfeln, vermutlich tatsächlich 200/600 = 1/3 und nicht 1/6, wobei das geschilderte Ergebnis rein theoretisch auch bei einem „,normalen“ Würfel auftreten könnte (was allerdings sehr unwahrscheinlich, aber eben nicht unmöglich ist).

4. Individuelle Schülerlösungen. [Da die relativen Häufigkeiten immer zumindest minimal schwanken werden, ist es nicht möglich, einen exakten Wert anzugeben. Vielmehr dienen die relativen Häufigkeiten als Schätzung für die tatsächliche Wahrscheinlichkeit, die mit zunehmender Wurfzahl immer genauer wird.] 


\section{WKEIT2: Was ist Wahrscheinlichkeit? Mengentheoretische Grundbegriffe}

1. $\Omega=\{W W W, Z W W, W Z W, W W Z, Z Z W, Z W Z, W Z Z, Z Z Z\}$

$$
\begin{aligned}
& A=\{Z Z W, Z W Z, W Z Z\} \Rightarrow P(A)=\frac{3}{8} \\
& B=\{Z W W, W Z W, W W Z, W W W\} \Rightarrow P(B)=\frac{4}{8}=\frac{1}{2} \\
& C=\{W W Z, W Z W, Z W W, W Z Z, Z W Z, Z Z W, Z Z Z\} \Rightarrow P(C)=\frac{7}{8} \\
& D=\{Z Z W, Z W Z, Z W W\} \Rightarrow P(D)=\frac{3}{8}
\end{aligned}
$$

\section{2. in Mengenschreibweise:}

$E \cap F=\{5\}$

$E \cup F=\{0,2,3,5,7,10,11,13,15,17,19\}$

$\bar{E}=\{0,1,4,6,8,9,10,12,14,15,16,18\}$

$\overline{E \cup F}=\{1,4,6,8,9,12,14,16,18\}$

\section{in Worten:}

$E \cap F$ : Die Zahl ist eine Primzahl und durch 5 teilbar.

$E \cup F$ : Die Zahl ist eine Primzahl oder durch 5 teilbar.

$\bar{E}$ : Die Zahl ist keine Primzahl.

$\overline{E \cup F}$ : Die Zahl ist weder eine Primzahl noch durch 5 teilbar.

3. (i) $\Omega=\{(1,1),(1,2), \ldots,(6,6)\}$ mit $P(\{\omega\})=\frac{1}{36}$

(ii) $\Omega=\{(1 \mid 1),(1 \mid 2), \ldots,(6 \mid 6)\}$, wobei $(k \mid j)$ kein geordnetes Paar ist, d.h. insb. $(k \mid j)=(j \mid k)$. Es gilt dann: $P(\{(k \mid k)\})=\frac{1}{36}$ und $P(\{(k \mid j)\})=\frac{2}{36}(k \neq j)$

(iii) $\Omega=\{2,3, \ldots, 12\}$ mit

$$
\begin{aligned}
& P(\{2\})=P(\{12\})=\frac{1}{36}, P(\{3\})=P(\{11\})=\frac{2}{36}, P(\{4\})=P(\{10\})=\frac{3}{36}, \\
& P(\{5\})=P(\{9\})=\frac{4}{36}, P(\{6\})=P(\{8\})=\frac{5}{36}, P(\{7\})=\frac{6}{36} .
\end{aligned}
$$

4. $\Omega=\mathbb{N}=\{1,2,3, \ldots\}$. Die Wahrscheinlichkeit, dass beim $k$-ten Wurf zum ersten Mal eine Sechs gewürfelt wird, beträgt $\left(\frac{5}{6}\right)^{k-1} \cdot \frac{1}{6}$. 


\section{WKEIT3: Was ist Wahrscheinlichkeit? Elementare Regeln}

Im Folgenden werden zu jeder der neun Regeln - falls möglich - verschiedene Möglichkeiten aufgezeigt, diese aus den jeweils übrigen herzuleiten:

(1) $P(\Omega)=1$

- aus (4) und (7):

$P(\Omega)=P(A \uplus \bar{A})=P(A)+P(\bar{A})=P(A)+1-P(A)=1$

- aus (2) und (7):

$P(\Omega)=P(\bar{\emptyset})=1-P(\emptyset)=1-0=1$

(2) $P(\emptyset)=0$

- $\operatorname{aus}(1)$ und (4):

$1=P(\Omega)=P(\Omega \uplus \emptyset)=P(\Omega)+P(\emptyset)=1+P(\emptyset) \Rightarrow P(\emptyset)=0$

- aus (1) und (7):

$P(\emptyset)=P(\bar{\Omega})=1-P(\Omega)=1-1=0$

(3) $P(A \cup B)=P(A)+P(B)-P(A \cap B)$

- aus (4) und (8):

$$
\begin{aligned}
P(A \cup B) & =P((A \backslash(A \cap B)) \uplus(B \backslash(A \cap B)) \uplus(A \cap B)) \\
& =P(A \backslash(A \cap B))+P(B \backslash(A \cap B))+P(A \cap B) \\
& =P(A)-P(A \cap B)+P(B)-P(A \cap B)+P(A \cap B) \\
& =P(A)+P(B)-P(A \cap B)
\end{aligned}
$$

- nur aus (4):

$$
\begin{aligned}
P(A \cup B) & =P((A \backslash B) \uplus(B \backslash A) \uplus(A \cap B)) \\
& =P(A \backslash B)+P(A \cap B)+P(B \backslash A)+P(A \cap B)-P(A \cap B) \\
& =P((A \backslash B) \uplus(A \cap B))+P((B \backslash A) \uplus(A \cap B))-P(A \cap B) \\
& =P(A)+P(B)-P(A \cap B)
\end{aligned}
$$

(4) $P(A \uplus B)=P(A)+P(B)$

- aus (2) und (3):

$$
P(A \uplus B)=P(A)+P(B)-P(\underbrace{A \cap B}_{=\emptyset})=P(A)+P(B)
$$


(5) $P(A) \geq 0$

- aus (6) und (7):

$1=P(A)+P(\bar{A})$. Da $P(A), P(\bar{A}) \leq 1$ nach (6), muss $P(A) \geq 0$ gelten.

- aus (2) und (9):

$\emptyset \subseteq A \Rightarrow 0=P(\emptyset) \leq P(A)$

(6) $P(A) \leq 1$

- aus (5) und (7): $1=P(A)+P(\bar{A})$. Da $P(A), P(\bar{A}) \geq 0$ nach (5), muss $P(A) \leq 1$ gelten.

- $\operatorname{aus~(1)~und~(9):~}$

$A \subseteq \Omega \Rightarrow P(A) \leq P(\Omega)=1$

(7) $P(\bar{A})=1-P(A)$

- aus (1) und (4):

$1=P(\Omega)=P(A \uplus \bar{A})=P(A)+P(\bar{A}) \Rightarrow P(\bar{A})=1-P(A)$

- aus (1) und (8):

$P(\bar{A})=P(\Omega \backslash A)=P(\Omega)-P(A)=1-P(A)$

(8) $A \subseteq B \Rightarrow P(B \backslash A)=P(B)-P(A)$

- nur aus (4):

$P(B)=P((B \backslash A) \uplus A)=P(B \backslash A)+P(A) \Rightarrow P(B \backslash A)=P(B)-P(A)$

(9) $A \subseteq B \Rightarrow P(A) \leq P(B)$

- $\operatorname{aus~(4)~und~(5):~}$

$$
P(B)=P((B \backslash A) \uplus A)=\underbrace{P(B \backslash A)}_{\geq 0}+P(A) \geq P(A)
$$

- aus (5) und (8):

$$
P(B)-P(A)=P(B \backslash A) \geq 0 \Rightarrow P(A) \leq P(B)
$$




\section{WKEIT4: Was ist Wahrscheinlichkeit? Die Kolmogorov-Axiome}

1. a) Individuelle Schülerlösungen auf Grundlage der Bearbeitung von WKEIT3.

b) Streng genommen müsste hier die Verallgemeinerung von (K3) mittels vollständiger Induktion bewiesen werden. Es reicht aber eine ,,informelle“ Argumentation, die das mehrmalige Anwenden von (K3) beinhaltet, z. B.:

$P\left(A_{1} \cup \ldots \cup A_{n}\right) \stackrel{(K 3)}{=} P\left(A_{1}\right)+P\left(A_{2} \cup \ldots \cup A_{n}\right) \stackrel{(K 3)}{=} \ldots \stackrel{(K 3)}{=} P\left(A_{1}\right)+\ldots+P\left(A_{n}\right)$

c) Es gilt:

$$
\begin{array}{ll}
1 a)(i i) & P(A \cup B \cup C) \\
= & P(A)+P(B \cup C)-P(A \cap(B \cup C)) \\
\stackrel{1 a)(i i)}{=} & P(A)+P(B)+P(C)-P(B \cap C)-\overbrace{P(A \cap(B \cup C))} \\
\stackrel{1 a)(i i)}{=} & P(A)+P(B)+P(C)-P(B \cap C)-(P(A \cap B)+P(A \cap C)-P(A \cap B \cap C)) \\
= & P(A)+P(B)+P(C)-P(A \cap B)-P(B \cap C)-P(C \cap A)+P(A \cap B \cap C)
\end{array}
$$

2. a) Wir überprüfen, ob $L$ die Kolmogorov-Axiome erfüllt:

(K1) Es ist $L(A)=\frac{|A|}{n} \geq 0$ für alle Teilmengen $A$, da stets $|A| \geq 0$ ist.

(K2) Es ist $|\Omega|=n$, also $L(\Omega)=\frac{n}{n}=1$.

(K3) Seien $A$ und $B$ disjunkt. Dann gilt für die Anzahl der Elemente der Vereinigung $A \cup B:|A \cup B|=|A|+|B|$. Dann gilt:

$$
L(A \cup B)=\frac{|A \cup B|}{n}=\frac{|A|+|B|}{n}=\frac{|A|}{n}+\frac{|B|}{n}=L(A)+L(B)
$$

b) Wir überprüfen, ob $\delta_{\omega}$ die Kolmogorov-Axiome erfüllt:

(K1) ist klar aufgrund der Definition von $\delta_{\omega}$.

(K2) Es ist $\omega \in \Omega$ und damit $\delta_{\omega}(\Omega)=1$ nach Definition von $\delta_{\omega}$.

(K3) Seien $A$ und $B$ disjunkt und $\omega \in \Omega$. Dann gibt es drei sich gegenseitig ausschließende Möglichkeiten:

(i) $\omega \notin A \cup B \Rightarrow \delta_{\omega}(A \cup B)=0=\overbrace{\delta_{\omega}(A)}^{=0}+\overbrace{\delta_{\omega}(B)}^{=0}$

(ii) $\omega \in A \Rightarrow \delta_{\omega}(A \cup B)=1=\overbrace{\delta_{\omega}(A)}^{=1}+\overbrace{\delta_{\omega}(B)}^{=0}$ 
(iii) $\omega \in B \Rightarrow \delta_{\omega}(A \cup B)=1=\overbrace{\delta_{\omega}(A)}^{=0}+\overbrace{\delta_{\omega}(B)}^{=1}$

Insgesamt gilt also $\delta_{\omega}(A \cup B)=\delta_{\omega}(A)+\delta_{\omega}(B)$ für disjunkte Mengen $A, B$.

c) Durch (ii), (iii) und (iv) werden Wahrscheinlichkeitsmaße festgelegt, durch (i) und (v) nicht (bei (i) wäre die Gesamtwahrscheinlichkeit $P(\Omega)=2,5>1$ und bei (v) ist $P\left(\omega_{5}\right)$ negativ.

d) Man legt nicht-negative Werte für die Elementarwahrscheinlichkeiten $P\left(\omega_{i}\right)$ fest, so dass $\sum_{i=1}^{n} P\left(\omega_{i}\right)=1$. Die Wahrscheinlichkeit für ein Ereignis $A$ ergibt sich dann aus der Summe der zu $A$ gehörigen $\omega_{i}$, d. h.

$$
P(A):=\sum_{\omega_{i} \in A} P\left(\omega_{i}\right)
$$




\section{WKEIT5: Was ist Wahrscheinlichkeit? Bedingte Wahrscheinlichkeit und stochastische Unabhängigkeit}

1. Wir überprüfen, ob $P_{B}$ die Kolmogorov-Axiome erfüllt:

(K1) Es ist $P_{B}(A)=\frac{P(A \cap B)}{P(B)} \geq 0$ für alle Teilmengen $A$, da $P$ nach Voraussetzung (K1) erfüllt.

(K2) $P_{B}(\Omega)=\frac{P(\Omega \cap B)}{P(B)}=\frac{P(B)}{P(B)}=1$

(K3) Seien $A_{1}$ und $A_{2}$ disjunkt. Dann gilt

$$
\begin{aligned}
P_{B}\left(A_{1} \cup A_{2}\right) & =\frac{P\left(\left(A_{1} \cup A_{2}\right) \cap B\right)}{P(B)}=\frac{P\left(\left(A_{1} \cap B\right) \cup\left(A_{2} \cap B\right)\right)}{P(B)} \\
& =\frac{P\left(A_{1} \cap B\right)+P\left(A_{2} \cap B\right)}{P(B)}=P_{B}\left(A_{1}\right)+P_{B}\left(A_{2}\right),
\end{aligned}
$$

denn $A_{1} \cap B$ und $A_{2} \cap B$ sind ebenfalls disjunkt und $P$ erfüllt nach Voraussetzung (K3).

2. a) $\quad P(A \cap B)=\frac{P(A \cap B)}{P(B)} \cdot P(B)=P_{B}(A) \cdot P(B)$

- $P(A \cap B)=P(B \cap A)=\frac{P(B \cap A)}{P(A)} \cdot P(A)=P_{A}(B) \cdot P(A)$

b) $P_{B}(A)=\frac{P(A \cap B)}{P(B)}=\frac{P(A) \cdot P(B)}{P(B)}=P(A)$

- $P_{A}(B)=\frac{P(B \cap A)}{P(A)}=\frac{P(B) \cdot P(A)}{P(A)}=P(B)$

c) (i) Es ist $B=(\bar{A} \cap B) \cup(A \cap B)$ eine disjunkte Vereinigung. Deshalb gilt:

$$
P(B) \stackrel{(K 3)}{=} P(\bar{A} \cap B)+P(A \cap B) \Leftrightarrow P(\bar{A} \cap B)=P(B)-P(A \cap B)
$$

Wegen der stochastischen Unabhängigkeit von $A$ und $B$ folgt

$$
P(\bar{A} \cap B)=P(B)-P(A \cap B)=P(B)-P(A) \cdot P(B)=\overbrace{(1-P(A))}^{=P(\bar{A})} \cdot P(B) .
$$

(ii) Die stochastische Unabhängigkeit von $A$ und $\bar{B}$ folgt analog zu (i) mit der disjunkten Vereinigung $A=(A \cap B) \cup(A \cap \bar{B})$.

(iii) Nach (ii) sind $A$ und $\bar{B}$ stochastisch unabhängig. Nach (i) sind dann auch $\bar{A}$ und $\bar{B}$ stochastisch unabhängig. 
3. a) Mit der selben disjunkten Vereinigung aus Aufgabe 2c) hat man:

$$
P(B)=P(A \cap B)+P(\bar{A} \cap B) \stackrel{2 a)}{=} P_{A}(B) \cdot P(A)+P_{\bar{A}}(B) \cdot P(\bar{A})
$$

b) $P_{B}(A)=\frac{P(A \cap B)}{P(B)} \stackrel{2 a)}{=} \frac{P_{A}(B) \cdot P(A)}{P(B)}$ 


\section{A.4.3 Zahlbereichserweiterungen}

\section{ZBE3: Kubische Gleichungen. Die Cardanische Formel}

1. a) (i) „Raten“ oder systematisches Probieren liefert $x=1$ als mögliche Lösung.

(ii) $x^{3}+x+10=0 \Leftrightarrow x \cdot\left(x^{2}+1\right)=-10$. Also muss $x<0$ sein. Systematisches Probieren oder genaues Hinsehen liefert $x=-2$ als mögliche Lösung.

(iii) $x^{3}+6 x=20 \Leftrightarrow x \cdot\left(x^{2}+6\right)=20$. Systematisches Probieren oder genaues Hinsehen liefert $x=2$ als mögliche Lösung.

b) 1. $x^{3}+x=6$

2. Die „zwei Zahlen in doppelter Proportion“ sein $x$ und $2 x$. Dann erhält man gemäß der Aufgabe die kubische Gleichung $(2 x)^{2} \cdot x+2 x+x=$ $4 x^{3}+3 x=40$.

9. Die gesuchte Zahl sei $x$. Dann liefert die Aufgabe zunächst $x+4 \cdot \sqrt[3]{x}=$ 17. Mit $y:=\sqrt[3]{x}$ lässt sich dies als die kubische Gleichung $y^{3}+4 y=17$ darstellen.

15. $x^{3}+x=500$

c) Visualisiert man die kubischen Gleichungen als Graphen von Polynomfunktionen dritten Gerades, so erkennt man wegen $\lim _{x \rightarrow-\infty} f(x)= \pm \infty$ und $\lim _{x \rightarrow \infty} f(x)=$ $\mp \infty$, dass jedes $y \in \mathbb{R}$ mindestens einmal als Funktionswert auftreten muss, die kubische Gleichung $f(x)=y$ also mindestens eine reelle Lösung haben muss.

2. - $x^{3}+x=6 \Leftrightarrow x^{3}=\underbrace{(-1)}_{=p} \cdot x+\underbrace{6}_{=q}$. Damit erhält man:

$$
x=\sqrt[3]{3+\sqrt{9+\frac{1}{27}}}+\sqrt[3]{3-\sqrt{9+\frac{1}{27}}}
$$

- $4 x^{3}+3 x=40 \Leftrightarrow x^{3}=\left(-\frac{3}{4}\right)+10$. Damit erhält man:

$$
x=\sqrt[3]{5+\sqrt{25+\frac{1}{64}}}+\sqrt[3]{5-\sqrt{25+\frac{1}{64}}}
$$


- $y^{3}+4 y=17 \Leftrightarrow y^{3}=(-4) \cdot y+17$. Damit erhält man:

$$
y=\sqrt[3]{\frac{17}{2}+\sqrt{\frac{289}{4}+\frac{64}{27}}}+\sqrt[3]{\frac{17}{2}-\sqrt{\frac{289}{4}+\frac{64}{27}}}
$$

- $x^{3}+x=500 \Leftrightarrow x^{3}=(-1) \cdot x+500$. Damit erhält man:

$$
x=\sqrt[3]{250+\sqrt{250^{2}+\frac{1}{27}}}+\sqrt[3]{250-\sqrt{250^{2}+\frac{1}{27}}}
$$

3. a) Die Aussage folgt direkt durch Einsetzen mit $p=-6$ und $q=20$, also

$$
\frac{q}{2}=10, \quad\left(\frac{q}{2}\right)^{2}=100 \quad \text { und } \quad\left(\frac{p}{3}\right)^{3}=(-2)^{3}=-8 .
$$

b) Es ist $x^{3}+6 x-20=(x-2) \cdot\left(x^{2}+2 x+10\right)=(x-2) \cdot \underbrace{\left((x+1)^{2}+9\right)}_{\geq 0}$. Nach a) ist $\hat{x}=\sqrt[3]{10+\sqrt{108}}+\sqrt[3]{10-\sqrt{108}}$ eine Lösung der Gleichung, also muss $\hat{x}-2=0$ oder $(\hat{x}+1)^{2}+9=0$ sein. Letzteres ist aber nicht möglich, so dass $\hat{x}=2$ gelten muss.

Hinweis: Genau genommen muss hier die Reellwertigkeit von $\sqrt[3]{10+\sqrt{108}}+$ $\sqrt[3]{10-\sqrt{108}}$ als Begründung angeführt werden; zu diesem Zeitpunkt der Unterrichtsreihe sind Wurzeln aus negativen Zahlen in den Lösungstermen der Cardanischen Formel allerdings noch nicht aufgetreten, so dass die hier vorgestellte Begründung ausreichend ist.

4. Mit der Cardanischen Formel erhält man als Lösung

$$
x=\sqrt[3]{0+\sqrt{0-\frac{1}{27}}}+\sqrt[3]{0-\sqrt{0-\frac{1}{27}}}=\sqrt[3]{\frac{1}{3} \cdot \sqrt{-\frac{1}{3}}}+\sqrt[3]{-\frac{1}{3} \cdot \sqrt{-\frac{1}{3}}}
$$

Da die Gleichung nur 3 Lösungen hat und diese bekannt sind, müsste dieser Ausdruck also entweder -1, 1 oder 0 entsprechen. Ignoriert man erstmal die Tatsache, dass in dem Ausdruck $\sqrt{-\frac{1}{3}}$ auftaucht, könnte man einfach wie gewohnt folgendermaßen weiterrechnen:

$$
\sqrt[3]{\frac{1}{3} \cdot \sqrt{-\frac{1}{3}}}+\sqrt[3]{-\frac{1}{3} \cdot \sqrt{-\frac{1}{3}}}=\sqrt[3]{\frac{1}{3} \cdot \sqrt{-\frac{1}{3}}}-\sqrt[3]{\frac{1}{3} \cdot \sqrt{-\frac{1}{3}}}=0
$$


5. a) Es gilt:

$$
\begin{aligned}
(2+\sqrt{-1})^{3} & =2^{3}+3 \cdot 2^{2} \cdot \sqrt{-1}+3 \cdot 2 \cdot \sqrt{-1}^{2}+\sqrt{-1}^{3} \\
& =8+12 \sqrt{-1}+6 \cdot(-1)-1 \sqrt{-1} \\
& =2+11 \sqrt{-1}=2+\sqrt{-121}
\end{aligned}
$$

Analog zeigt man $(2-\sqrt{-1})^{3}=2-11 \sqrt{-1}$.

b) Es gilt:

$$
\sqrt[3]{2+\sqrt{-121}}+\sqrt[3]{2-\sqrt{-121}} \stackrel{\text { a) }}{=} 2+\sqrt{-1}+2-\sqrt{-1}=2+2=4
$$

6. Mit dem Hinweis ist ein vorheriges „Erraten“ einer reellwertigen Lösung nicht notwendig. Die Cardanische Formel liefert

$$
x=\sqrt[3]{-2+\sqrt{-4}}+\sqrt[3]{-2-\sqrt{-4}}
$$

Wie in Aufgabe 5 zeigt man nun $(1 \pm \sqrt{-1})^{3}=-2 \pm \sqrt{-4}$ und erhält damit

$$
x=1+\sqrt{-1}+1-\sqrt{-1}=2 .
$$




\section{ZBE4: Herleitung der Cardanischen Formel}

1. a) Dividieren beider Seiten der Gleichung durch $a \neq 0$ liefert

$$
A=\frac{b}{a}, \quad B=\frac{c}{a}, \quad C=\frac{d}{a}
$$

b) Zur Bearbeitung wird die Formel $(a-b)^{3}=a^{3}-3 a^{2} b+3 a b^{3}-b^{3}$ benötigt.

$$
\begin{array}{lll} 
& x^{3}+A x^{2}+B x+C & =0 \\
\stackrel{x=y-\frac{A}{3}}{\Leftrightarrow}\left(y-\frac{A}{3}\right)^{3}+A\left(y-\frac{A}{3}\right)^{2}+B\left(y-\frac{A}{3}\right)+C & =0 \\
\Leftrightarrow & y^{3}-A y^{2}+\frac{1}{3} A^{2} y-\frac{1}{27} A^{3}+A y^{2}-\frac{2}{3} A^{2} y+\frac{1}{9} A^{3}+B y-\frac{1}{3} A B+C & =0 \\
\Leftrightarrow & y^{3}+y\left(-\frac{1}{3} A^{2}+B\right)+\left(\frac{2}{27} A^{3}-\frac{1}{3} A B+C\right) &
\end{array}
$$

Man setze nun $p:=\frac{1}{3} A^{2}-B$ und $q:=-\frac{2}{27} A^{3}+\frac{1}{3} A B-C$. Damit hat die Gleichung die Form $y^{3}=p \cdot y+q$.

2. a) Es gilt:

$$
\overbrace{(u+v)^{3}}^{=y^{3}}=u^{3}+3 u^{2} v+3 u v^{2}+v^{3}=\underbrace{3 u v}_{=p} \cdot \overbrace{(u+v)}^{=y}+\underbrace{u^{3}+v^{3}}_{=q}
$$

b) Es gilt:

$$
\begin{aligned}
& u^{3}+\left(\frac{p}{3 u}\right)^{3}=q \\
& z=\mu^{3} \quad z+\left(\frac{p}{3}\right)^{3} \cdot \frac{1}{z}=q \\
& \stackrel{z \neq 0}{\Leftrightarrow} z^{2}+\left(\frac{p}{3}\right)^{3}=q z \\
& \Leftrightarrow \quad z^{2}+q z+\left(\frac{p}{3}\right)^{3}=0
\end{aligned}
$$

3. a) Die $p$ - $q$-Formel liefert:

$$
\left(u^{3}=\right) z=\frac{q}{2} \pm \sqrt{\left(\frac{q}{2}\right)^{2}-\left(\frac{p}{3}\right)^{3}}
$$


Daraus folgt:

$$
u=\sqrt[3]{z}=\sqrt[3]{\frac{q}{2} \pm \sqrt{\left(\frac{q}{2}\right)^{2}-\left(\frac{p}{3}\right)^{3}}}
$$

b) Auflösen der Gleichung (1) nach $u$ anstatt $v$ und Einsetzen in die (bzgl. $u$ und $v$ symmetrische) Gleichung (2) liefert $v^{3}+\left(\frac{p}{3 v}\right)^{3}=q$.

Man kann also exakt die selben Rechnungen wie in 2b) und 3a) durchführen und erhält für $v$ das gleiche Ergebnis.

c) Wählt man beispielsweise

$$
u^{3}=\frac{q}{2}+\sqrt{\left(\frac{q}{2}\right)^{2}-\left(\frac{p}{3}\right)^{3}}
$$

so muss wegen $u^{3}+v^{3}=q$

$$
v^{3}=\frac{q}{2}-\sqrt{\left(\frac{q}{2}\right)^{2}-\left(\frac{p}{3}\right)^{3}}
$$

gelten. Analog gilt: Wählt man $u^{3}=\frac{q}{2}-\ldots$, so muss $v^{3}=\frac{q}{2}+\ldots$ gelten. 


\section{A.5 Hinweise zu Bildquellen und -nutzungsrechten}

- Kugelzweieck (S. 212):

selbst erstellt nach Filler 1993, S. 13.

- Niccolò Tartaglia (S. 225):

https://commons.wikimedia.org/wiki/File:Niccolò_Tartaglia.jpg

Letzter Zugriff: 05.05.2018 (CC 0).

- Girolamo Cardano (S. 225):

https://commons.wikimedia.org/wiki/File:Cardano.jpg

Letzter Zugriff: 05.05.2018 (CC 0).

- Hermann von Helmholtz (S. 232):

https://commons.wikimedia.org/wiki/File:Hermann_von_Helmholtz.jpg Letzter Zugriff: 05.05.2018 (CC 0).

- Henri Poincaré (S. 234):

https://commons.wikimedia.org/wiki/File:Young_Poincare.jpg

Letzter Zugriff: 05.05.2018 (CC 0).

- David Hilbert (S. 235, 237-238):

https://www.flickr.com/

Letzter Zugriff: 05.05.2018, Autor: Aldo Cavini Benedetti (CC BY-NC-SA 2.0).

- Gottlob Frege (S. 237-238):

https://commons.wikimedia.org/wiki/File:Young_frege.jpg

Letzter Zugriff: 05.05.2018 (CC 0).

- Pierre-Simon de Laplace (S. 239):

https://commons.wikimedia.org/wiki/File:Pierre-Simon_Laplace.jpg Letzter Zugriff: 05.05.2018 (CC 0).

- Richard von Mises (S. 240):

https://opc.mfo.de/detail?photo_id=2896

Letzter Zugriff: 05.05.2018, Autor: Konrad Jacobs (CC BY-SA 2.0).

- Andrei Kolmogorov (S. 242):

https: / /opc.mfo.de/detail?photoID=7493

Letzter Zugriff: 05.05.2018, Autor: Konrad Jacobs (CC BY-SA 2.0). 



\section{B Schüler-Kurzaufsätze}

In diesem Anhang befinden sich alle Kurzaufsatz-Themen und Schülerantworten aus der in Abschnitt 5.5.2 beschriebenen Erprobung. Insgesamt liegen (mit Ausnahme von Aufsatz 1) für jedes Aufsatz-Thema vier Schülerantworten von je zwei weiblichen Schülerinnen (Sw1 und Sw2) und zwei männlichen Schülern (Sm1 und Sm2) vor.

\section{Übersicht}

\begin{tabular}{cll} 
Kurzaufsatz & Thema & Seiten \\
\hline \hline 1 & Sicherheit mathematischer Aussagen & $294-295$ \\
2 & Beweise „offensichtlicher“ Aussagen & $296-297$ \\
3 & Natur geometrischer Axiome & $298-299$ \\
4 & Zwei verschiedene Sichtweisen auf Axiome & $300-301$ \\
5 & Ansätze zum Wahrscheinlichkeitsbegriff & $302-303$ \\
6 & Mathematikbilder & $304-306$
\end{tabular}




\section{Kurzaufsatz 1: Sicherheit mathematischer Aussagen}

Die amerikanische Wissenschaftsphilosophin PENELOPE MADDY (geb. 1950) meint:

Von all den merkwürdigen und unterschiedlichen Dingen, die wir glauben, gibt es nur wenige, denen wir mehr Glauben schenken, als den Wahrheiten einfacher Mathematik. Nach einem Beispiel für eine durch und durch zuverlässige Tatsache gefragt, werden sich viele abwenden vom gesunden Menschenverstand - „früher dachte man auch, Menschen könnten nicht fliegen “ - von Naturwissenschaft - ,, die Sonne ist bislang jeden Tag aufgegangen, aber das muss morgen nicht so sein" - hin zu der Sicherheit der Arithmetik - ,aber 2 plus 2 ist sicher 4“.

Nehmen Sie Stellung zu dieser Aussage. Ist der hier beschriebene Ruf der Mathematik gerechtfertigt? Was macht mathematische Aussagen in den Augen vieler Leute so sicher?

Antwort Sw1

[hat diesen Kurzaufsatz nicht mitgeschrieben.]

Antwort Sw2

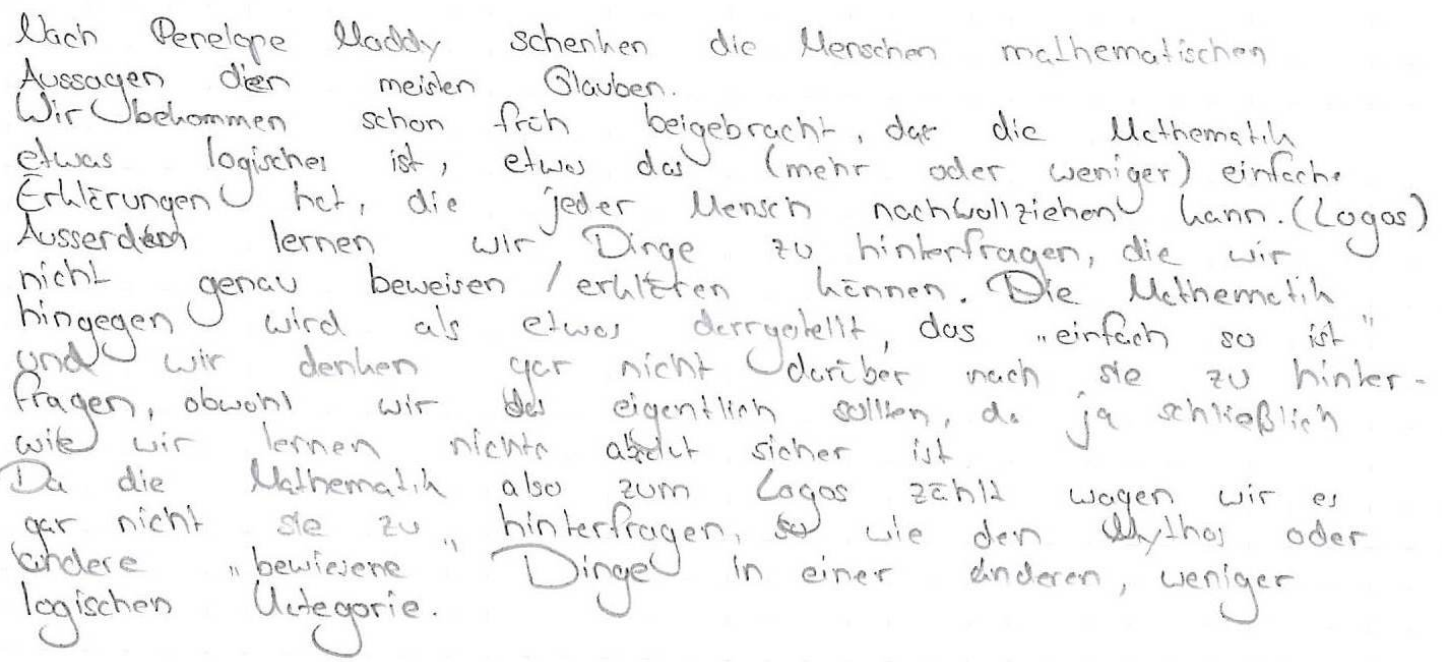


295

Antwort Sm1

Viele Menschen haller die Mathervatish fir sicherer als alles andero, Geraode da sie sie

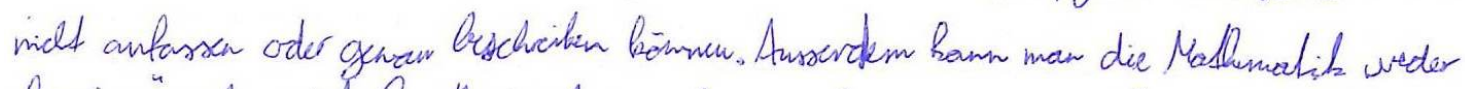

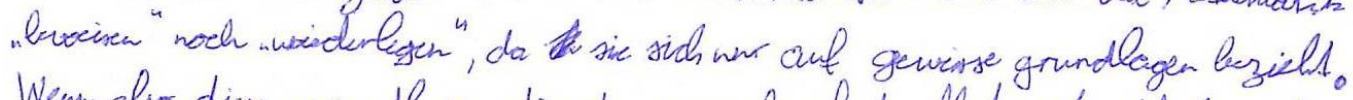

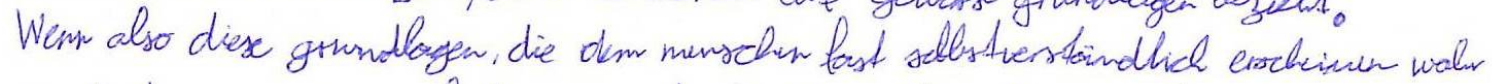
sind, dann muss auch die gesamte Mathmostib wahr ssin. Aesserden ist die

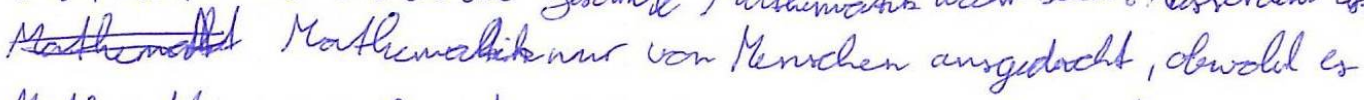
Mathmatibern to vorbomnt, als wirdm sie sie nur nentdechea."

Antwort Sm2

lel wirde die Mathematih sclon als sidweralsalle anderen Notwwissen schalfen bezseichen, da sir der Grundbanstein alb ist. Die Problum der Mathematik werden hierbat ant die ourderen Wissenschafter inbertrayer, jedod entsfetion dench beantwortete Fragen neve bew. Wistere Fragm und somit enstatum burch eie Ewieritery der Mathematic in

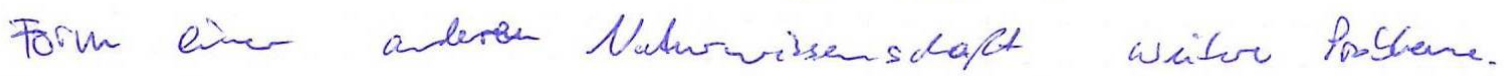
Die Mitheratiin ist olso wisht so sider, will sil selsst

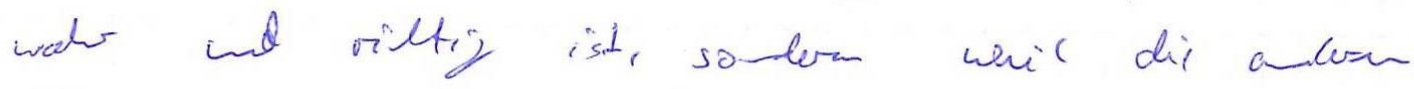

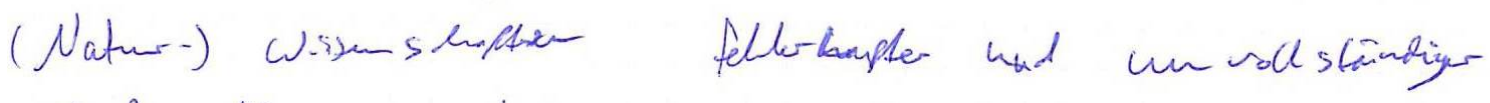

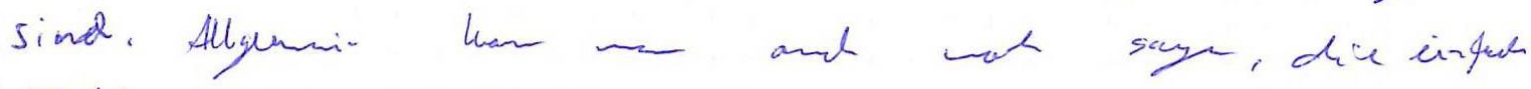
Mathe mation ershint sicher. du sie durd

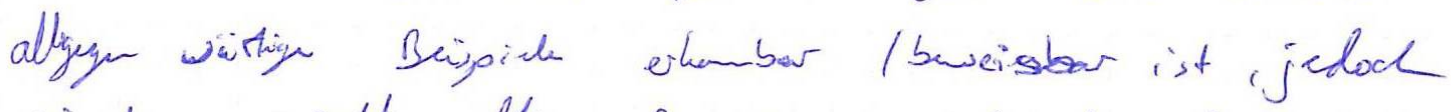
sise milt olle Beispick un bedingt unt imour walu. 


\section{Kurzaufsatz 2: Beweise „offensichtlicher“ Aussagen}

Euklid führt in den Elementen teilweise sehr ausführliche Beweise für Aussagen, die man auch für „anschaulich klar“ oder „offensichtlich“ halten kann. Ist das aus Ihrer Sicht notwendig? Warum tut Euklid das wohl?

\section{Antwort Sw1}

Ich finde diese Beweise notwendig, da dadurch jeder einzelner Schritt erklät wird und nachvollzogen werden kann. Somit können die Aussagen sehr genau wiederlegt werden und so Wachleichen sich auch nicht so leicht Fehler ein. Durch diese ausfïhrliche Beweise werden Aussagen, die zunächst als woffentsichtlich on gehalten werden, doch nicht so offentsichtlich, wie man vermutet.

\section{Antwort Sw2}

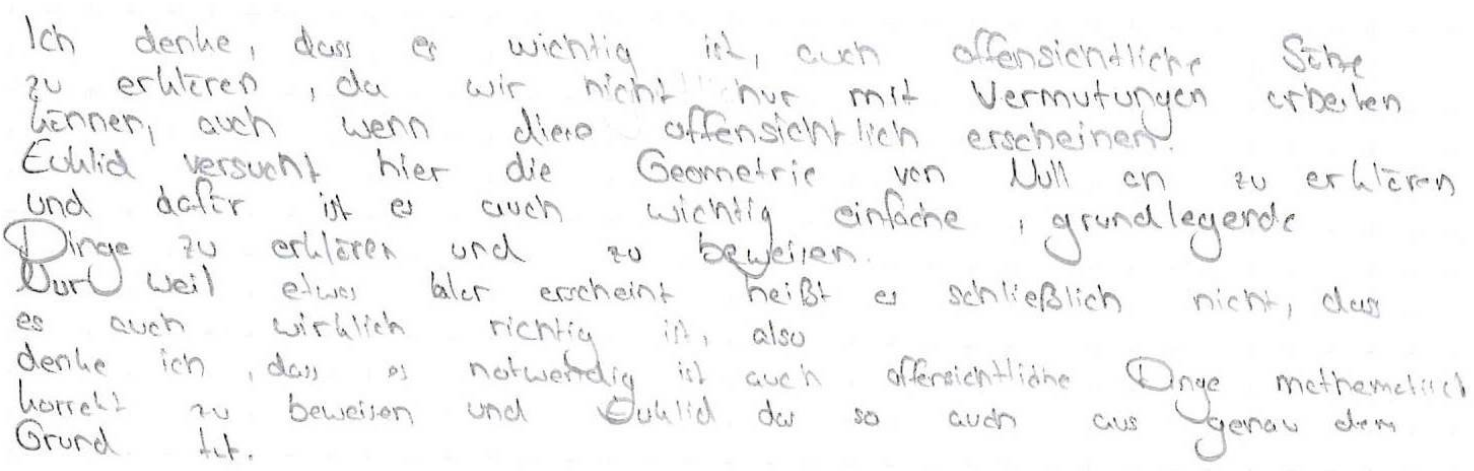


297

Antwort Sm1

Er versmcht, so wenig Axiome und Postulate zunutzen, wen so viel wie wöglich zu bewevisen. Ausserdem sollen Asiome und Postalate wirblich sofert

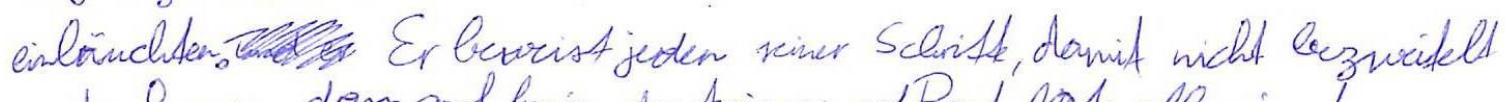
werder hawn, dass anf baris der Axioneund Patulate allexins Anssagen vallstewdig richtig sind. Idr vernute, die Ansagen, de schor sehr nah an Axiomen lisgew, warch an schwierigstess zn bewciscen, da

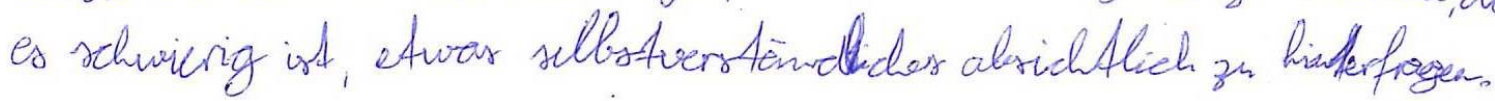

Antwort Sm2

Ich whide diese Bewabe als sinuroll ansehen, da Echlid dadurch dic homplexeren Alssugen infacher beweisen beann. Zusätalich ist der Beweis einer befinition nidat zu fern. Also als Definition ist de Beweis micht moghich, dor er Z.B. ant

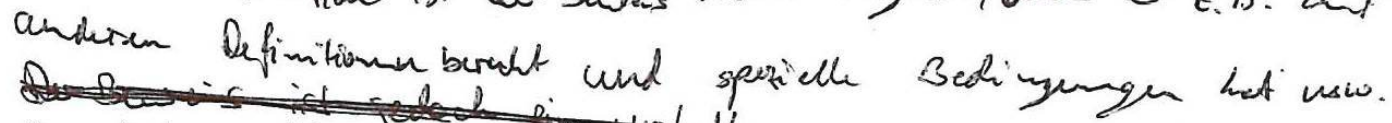

Bsp: Nibenwinhl sat:

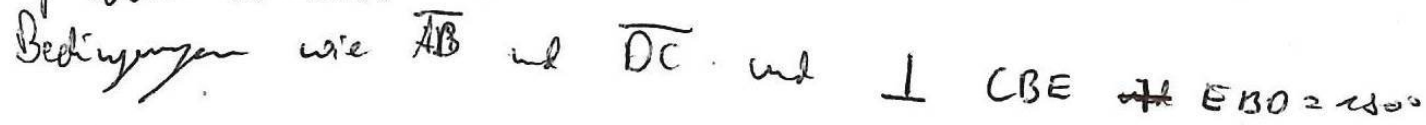

$$
\begin{aligned}
& \rightarrow \text { CBE }=90^{\circ} \\
& \rightarrow \text { ESP } 290^{\circ}
\end{aligned}
$$

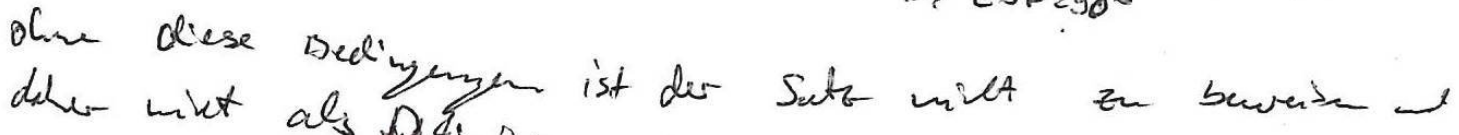

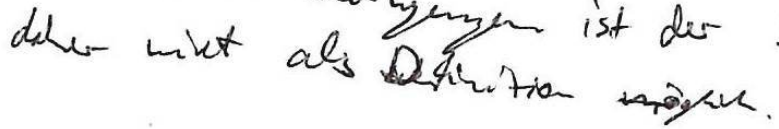




\section{Kurzaufsatz 3: Natur geometrischer Axiome}

Lesen Sie die Seiten 34-38 aus dem Geometrikon.

Was ist hier passiert? Welche Auswirkungen haben Anselms Erfahrungen auf die geometrischen Axiome für den dreidimensionalen Raum?

\section{Antwort Sw1}

Auf einer Ebene widrde sich die Fläche eines Kreises vergrößern, wenn der Radius vergroßßert wird. Jedoch verhleinert bew. zieht sich ein Körper in dreidimensionalem Raum zusammen, wenn der Ragivs vergrößhert wird. Das Volumen breitet sich aus und die Struktur des Korpers wird veräandert. Bildet man Dreieche in einem dreidimensionalem Rawm, betragt vertiatt verhalten sich die Dreieche wie spharische Dreiecke. Die Innenwinhelsumme kann ebentalls größer als $180^{\circ}$ sein.

\section{Antwort Sw2}

Dic "2-dimersionalen" Messungen im 3-dimensionalen Raum zeigen Durch die Raumhirmmung hal er die selben Ergebnisie wie Als er zB. den Radius seiner Uugel vergrißßert wird dieve plati, da wenn der Rrd $\pi R$ ist che Uugel in die entgegen Iph 3-dimensionlalen Raum gelten schließsich andere Axiome al im 2. ". "also brauch! er andere an bre Axiome, die an den 3-d. Raum angepast sind.

\section{Antwort Sm1}

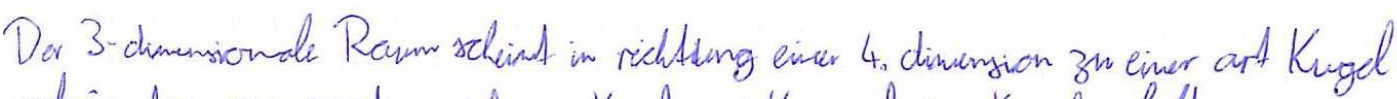

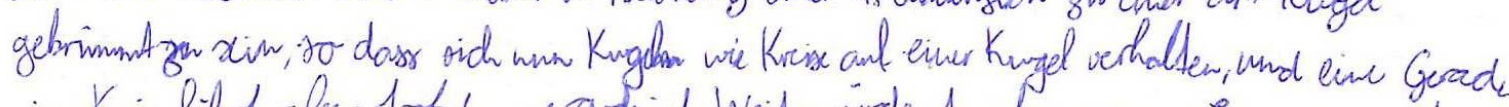

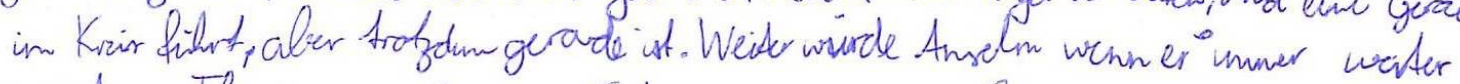
gerodeang Fliegt auch ohne sime Stanger wieder ane Startpunbt anbonmon, was beduntet, days Eublids. Axione curch ine veränderter form und in 3-dimensionalen 
299

Antwort Sm2

Wiefermal maet die krümangny Anselm eimen Strich durch die Rechneng.

Dieses mal ist jedoch die Krimmeny des draidinusonam

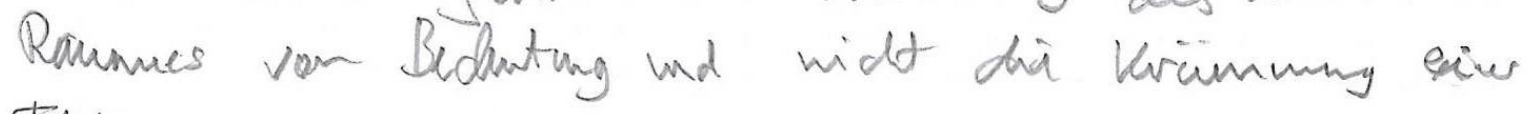
Fliche.

Balurd andern sich die tusbritungs ridtung oder kruimmung des hiptballows bew der kugel und die vermuidhich feste gerade stange eirdet in itrem Anfaing.

Auch die winhal, das volumen und die oberfaia Von vörpern ist nilt wir bei der angenommo Axiomen fis den dieidimensibualen Paum, 


\section{Kurzaufsatz 4: Zwei verschiedene Sichtweisen auf Axiome}

Sind Axiome

a) offensichtlich wahre Aussagen oder

b) inhaltlich nicht interpretierte und willkürliche Setzungen/Bedingungen?

\section{Antwort Sw1}

Memer Meinung nach sind Axiome offentsichtlich wahre Aussagen. Axiome stellen Bedingungen von den Begriffen dar, die immer erfüllt sein müssen, um auch daraws Folgerungen zu erschließen und um weitere Axiome darauf aufzubaven. Diese Begriffe sind definiert. Denn so kionnem die Atiome realitatsnah aufgestellt werden und arch innerhalb eines Axiom kann man die Begriffe logisch zueinanderstellen. Außerdem werden a Axiom tem tid Axiome nicht wiederlegt, tweld was auch realitätsnah und logisch heraustellt.

\section{Antwort Sw2}

Ich denhe empdxiome sollten sowchl Eredie Reallith als auch for Gedunhenexperimente mutheth sein, je nochdent in welcher Umgebung bzw. Raum Zum genuht werden. hat die Mathematih, wenn man sich alles nur ousdenkt und es heiner Realitel entopricht, zum andoren hat curn die Lillkier of einen groben Uuten, da man nicht alles realitetsnah betrachlen hann.

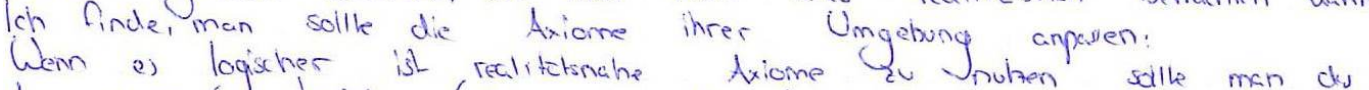
Wean e) logischer ist realitetsnahe taiome zu nutzen salle man des tun, wenn os logischer (oder aurh einfocher) ist willhirliche Aviome zu nuthen 
301

Antwort Sm1

Weil Axiome der Startpmult won Bewrizen, Sätzen und ganzen Systheme sind,

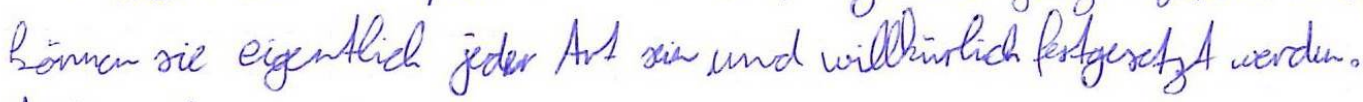
Awderacits wacht es aber awch mur relativ wenig Sinn, van absolust realitaitsfermen Axioneen Auszngher, da die Ergebnisse, zh

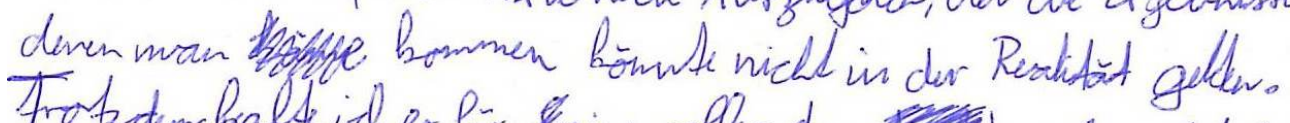

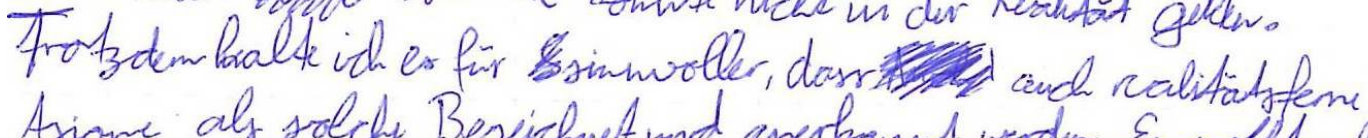
Asiome als solche Bezichnet und averhannt werdew. Es sollte allevelings Blor sim, ob sich eine Grypu von Axionem ouf die Realifids oder nicht bozicht.

Antwort Sm2

Ich selu die tusicht, duss Axiome Bedingannan sind, als sinaroder an.

Biese fit der Axiome tösst zum bine Widersprinche veschicdemen Axiome aus voschiedewer fystemen our, da des troponer der

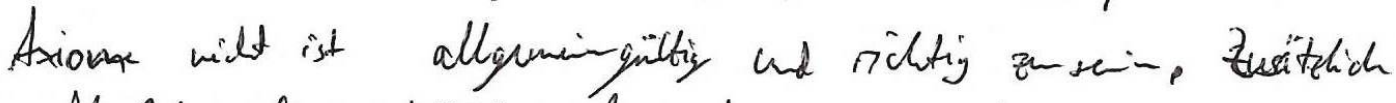

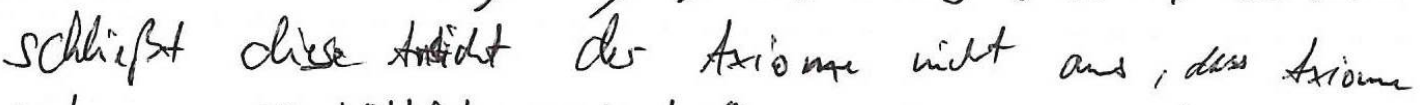

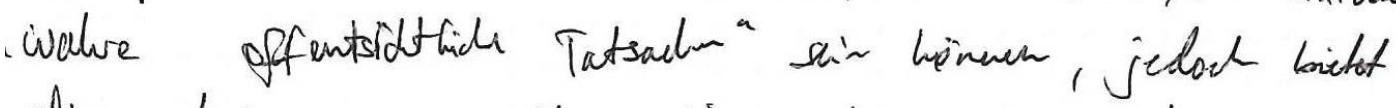

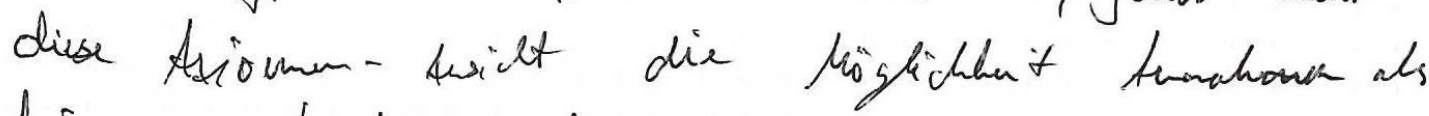

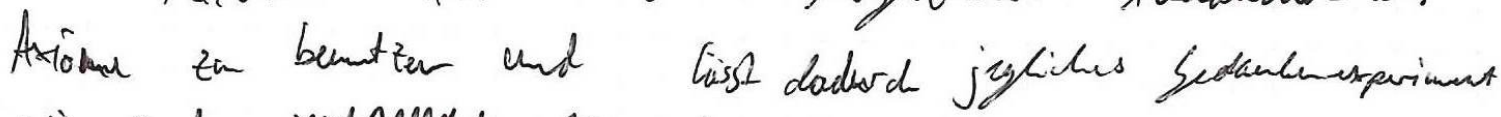
wie and abie Amatine cives bypostere and dom folgen zetu.

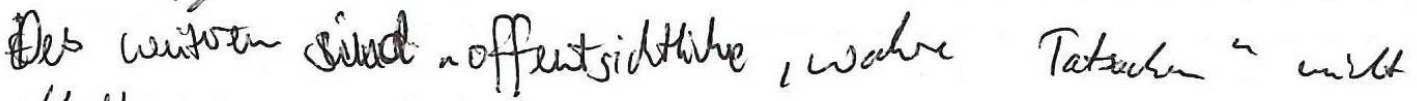
lotbthriand

und sind semit nibt gevwagummapen wothr.

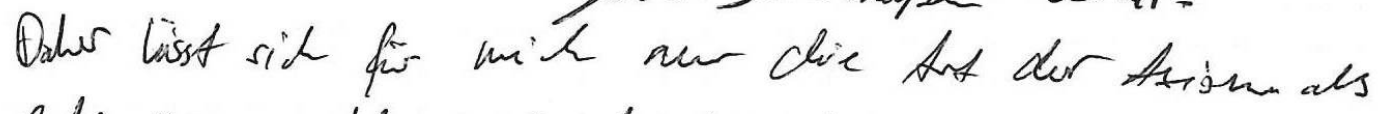
Redhingergen verstelum and als simuded anschen. 


\section{Kurzaufsatz 5: Ansätze zum Wahrscheinlichkeitsbegriff}

Miriam meint:

Wenn ich typische Stochastik-Aufgaben lösen möchte, komme ich alleine mit Kolmogorovs abstraktem Ansatz nicht weiter, sondern brauche auch den Laplace-Ansatz oder den frequentistischen Ansatz. Was bringen mir die Kolmogorov-Axiome dann überhaupt?

Was würden Sie ihr antworten?

\section{Antwort Sw1}

Ich wiurde sagen bei typischen Stochastih-Aufgaben, wie 2.B. eine Autgabe in der man die bedingte Wahrscheinlichneit berechnet werden soll, $x$ wïrden die Axiome von Kolmogorov ausreichen, da solche Berechnungen mit Definitionen festgelegt sind. Jedoch benotigt man den Laplace- Ansatz oder den frequentistis chen Ansatz bei Aufgabern, wo man 2. B. die Wahrscheinlicheit feststellen möch te.

\section{Antwort Sw2}

Die Axiome ven Ulmogerov sind dazu da, um theth de theorie

des Laplare - und frequentistuchen - Ansches besser nochuoliziehen zu liennen

und die Gesamke Theurke der Wchrscheinlichleiten bester "v verstehen.

Der abstraite trisatr beschreibt des grundlegende, das hinter der Anwenduyg
ven Laplare und Milis stecht. 
303

Antwort Sm1

Erst Mittilfe der Axiome Kolmogorow bönnen reglw bewiesen werder, mithille

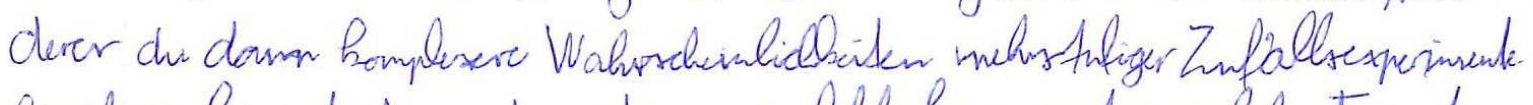
berchnen bounst. Ansserdin istes zurn felhkrfrien untzer solder tormaln

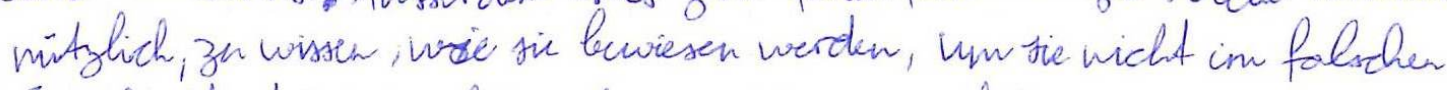
sevehbontext zn gebrancher und yo zor falschen evgebnissen zu benmer. Deshailb ist es Siruboll, die Axiome Kolmogorovos, die

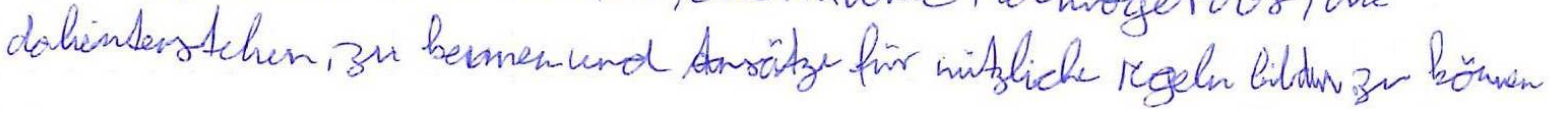

Antwort Sm2

Mit Hilfe do Kolmogorto Asiome lasen sich Säze und Regeler der

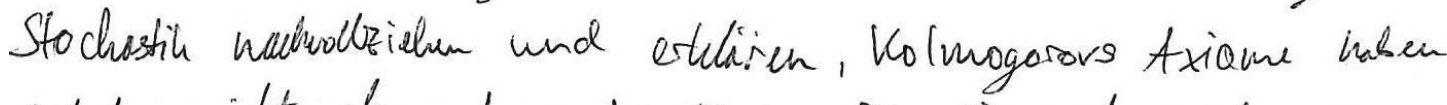
jedach midt den Anspruch hösung fïr line Aluwendeng zer sein.

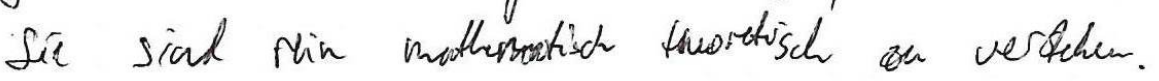

Sobald man line Anwendeng ciber die Therre hinaus

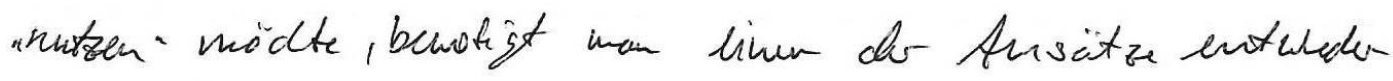
von Mises ader huplace.

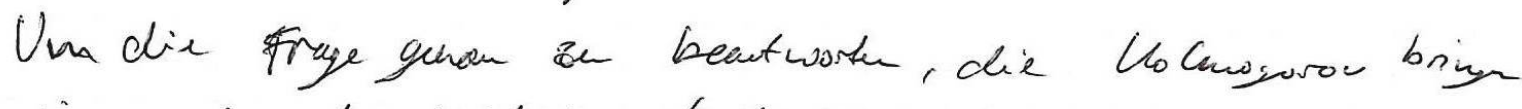

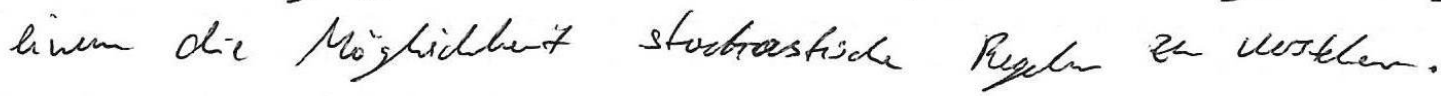




\section{Kurzaufsatz 6: Mathematikbilder}

A) Mathematik ist wie ein formales Spiel: Die Begriffe sind die Spielfiguren und die Axiome sind die Spielregeln.

B) Axiomensysteme sind kein Selbstzweck. Eine Theorie, die sich aus einem Axiomensystem aufbauen lässt, ist nur dann gerechtfertigt, wenn sie die Lösung eines bis dahin fremden Problems herbeiführt.

Was sagen Sie zu diesen beiden Meinungen? Wie würden Sie sich positionieren?

\section{Antwort Sw1}

Ich wurde mich zu der zweiten Meinung positionieren. Denn man kann nicht willkirlich Axiomensysteme aufstellen. Die Begriffe missen definiert sein and in dem Axiom Logisch aufgestellt werden, um reale Probleme lösen is hönnen. Dean Wenn man Axiome irgendwie zusammenstellt, wie dies in der Zuer ersten Meinung vertreten wird, dann wird es schwivenger Probleme zu lösem. Willkürlichen nicht-interpretienten Atiomen Kann man aych schwer einen Sinn geben, was dazo führt, den Nutzen des Axioms infrage
zo ste gestellt wird. 


\section{Antwort Sw2}

A) Mathematih ist wie ein formales Spiel:

Zum einen braucht man Regein, dio der Realitet entsprechen, um damit reale Probleme tüsen zu Lünnen, denn wir nutzen die Mathematih schließlich um probleme zu toser.

B) Axiomensysteme sind hein Selbstzwech:

Zum anderen ist es rein mathematisch trohdem mëglich Axiomensysteme cufustellon, die heiner Reclitzt ehtsprechen und auch nicht dazu da sind um reake Probleme zu lüsen, sondern nur cuf der Theorie beruhen.

Ich denhe, dws es zwor sinnuall sein hann sich Axiomensysteme vorzustellen, die heiner Realrtet entsprechen, aber im Endefeht doch unnetig sind, doe mon auch mit dirton ior am Ende af ein redles Problem schließen will, es. De Mathematilh machl ouch bringt biouch nur wirllich einen When, wenn wir sie af die Realitil onwender.

\section{Antwort Sm1}

Miver Minis

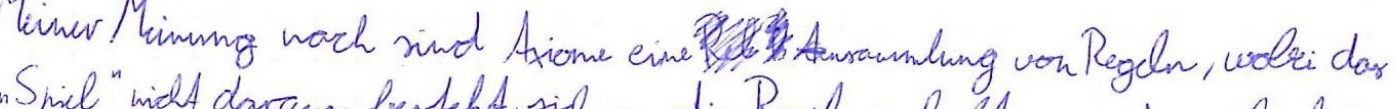

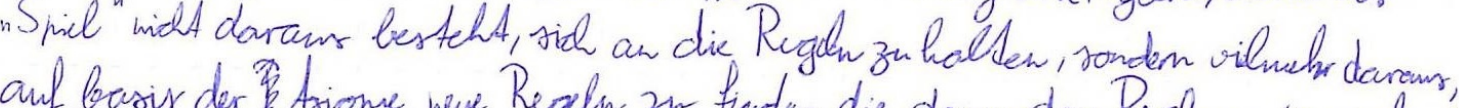

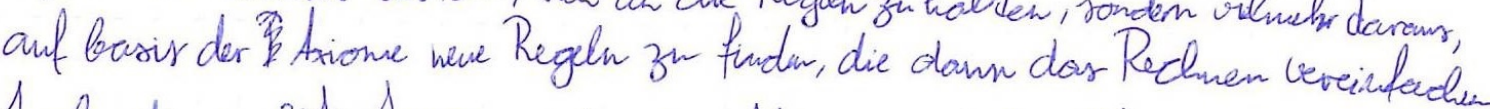

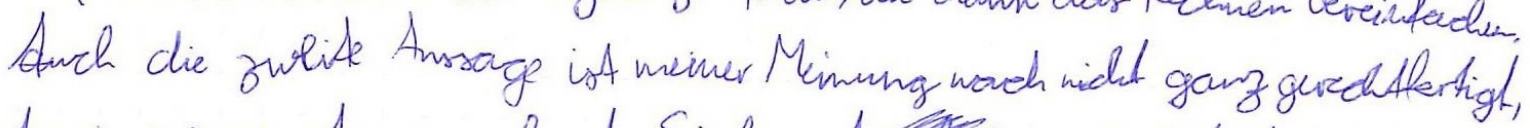
da sie mir zu stotng vorkond. Sie lasaght nambich, dass niar nur dann "spielen" dast, wenn man om enste auch wirlslich line richtige, abor

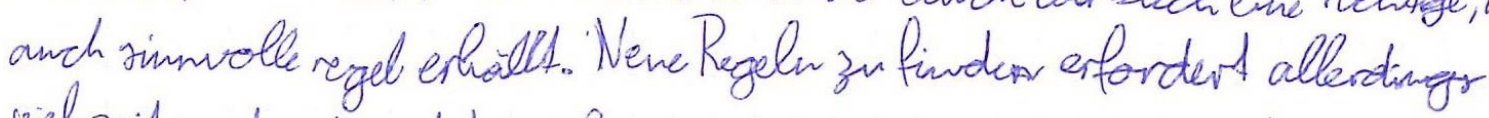

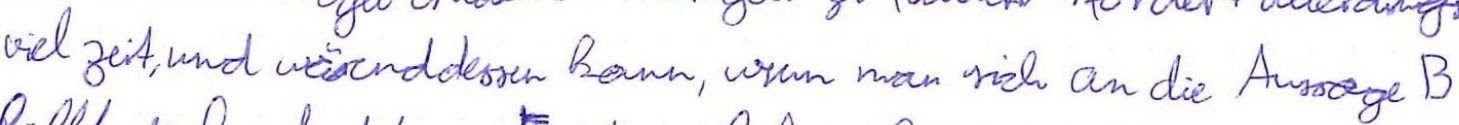
Paollt, die breativitat ans toust werlobrengeher. 
306

B Schüler-Kurzaufsätze

Antwort Sm2

Ich haun die Position A wach wodkieher und Finde die lclee interessant.

Jedoch wïste ich nidt, was inem diese Sicht ant Axiome and die Mathenatic mutzen wirde, da sie hierbi tussay iberdie Reluang der Mathematic hat.

Pasition B wirde ich eingesdrainht zestimanen. Nationite ist das Estulle ines Honomensystems

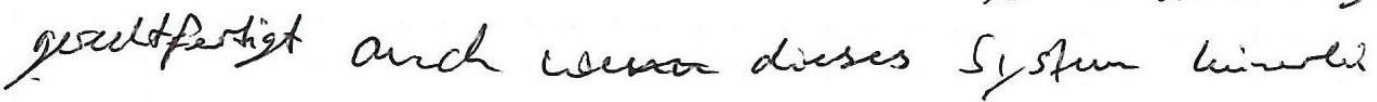
wele Erhentinis zuciost oder bewisht. Id wiirde jedoch sagen, doss in Axromensysten micht simuroch ist, wan es gar him Motzen hats Somit ist jechs Axiommsysera geraltfolgt, aber wictt unbedingt simmerd obr mitzhich. 


\section{Evaluationsergebnisse der Workshop-Woche}

Zum Abschluss der Workshop-Woche (hier Seminar genannt) füllten die Schülerinnen und Schüler einen Evaluationsbogen aus. Die Ergebnisse sind hier zusammengefasst.

\section{Freie Kommentarfelder}

Das hat mir besonders gut gefallen:

- Das Thema Stochastik fand ich gut und zweckmäßig sinnvoll.

- der Inhalt

- Durch Beweise etc. hat man verstanden, wozu dies gemacht wird.

- die Erklärungen der Themen, teilweise auf der Basis von historischen Originaltexten (z. B. Briefwechsel)

Das könnte man besser machen:

- mehr Animierung zum Mitarbeiten (öfter Zweiergespräch oder Stille, weil keiner mitgearbeitet hat)

- zu nah an normalem Unterricht für die erste Ferienwoche

- der Aufbau (nicht immer Bezug zum eigentlichen Thema)

Sonstige Anmerkungen:

- Die Teilnehmer-Anzahl war etwas zu niedrig (würde so ca. 8 vorschlagen), um flüssig zu arbeiten. 


\begin{tabular}{|c|c|c|c|c|c|}
\hline Zum Inhalt insgesamt: & $\begin{array}{c}\text { trifft voll } \\
\mathrm{zu}\end{array}$ & & & & $\begin{array}{l}\text { trifft gar } \\
\text { nicht zu }\end{array}$ \\
\hline Das Thema des Seminars fand ich interessant. & 3 & 1 & & & \\
\hline Das Seminar bot einen guten Einblick ins Thema. & 3 & 1 & & & \\
\hline Ich habe viel gelernt. & 3 & 1 & & & \\
\hline Ich hatte schon Vorerfahrungen mit dem Thema. & & 1 & 3 & & \\
\hline Besonders gefallen hat/haben mir: & $\begin{array}{c}\text { trifft voll } \\
\mathrm{zu}\end{array}$ & & & & $\begin{array}{l}\text { trifft gar } \\
\text { nicht zu }\end{array}$ \\
\hline - die Beschäftigung mit den Winkelsätzen der euklidischen Geometrie & & 1 & 2 & 1 & \\
\hline - die Erkundungen der Eigenschaften der Kugelgeometrie & 1 & 2 & & 1 & \\
\hline - die Auszüge aus dem Mathe-Comic & & 2 & 1 & 1 & \\
\hline - die Diskussion historischer Textauszüge (Helmholtz, Poincaré, Hilbert, Frege) & 1 & & 3 & & \\
\hline - das Dimpfel-Gronkel-Beispiel & & 3 & 1 & & \\
\hline - die Zufallsexperimente mit den Würfeln, Quadern und Stiften & 2 & & 2 & & \\
\hline - das Herleiten der stochastischen Regeln und Ausfüllen der Tabelle & 3 & & 1 & & \\
\hline $\begin{array}{l}\text { - die Diskussion der Möglichkeiten, aufgrund der Tabelle ein Axiomensystem } \\
\text { aufzustellen }\end{array}$ & 1 & 3 & & & \\
\hline - das rein formale Beweisen stochastischer Aussagen & 2 & 2 & & & \\
\hline - die Diskussion über die Sinnhaftigkeit, Wurzeln aus negativen Zahlen zu ziehen & 2 & 1 & 1 & & \\
\hline - das Lösen kubischer Gleichungen mit der Cardano-Formel & 1 & 1 & 2 & & \\
\hline
\end{tabular}




\begin{tabular}{|c|c|c|c|c|c|}
\hline Zur Methodik: Was hat dir gut gefallen? & $\begin{array}{c}\text { trifft voll } \\
\mathrm{zu}\end{array}$ & & & & $\begin{array}{l}\text { trifft gar } \\
\text { nicht zu }\end{array}$ \\
\hline - die gemeinsame Diskussion über axiomatische Aspekte & 1 & 3 & & & \\
\hline - die Verwendung historischer Quellen im Seminar & 2 & & 2 & & \\
\hline - die „Experimentier-Phasen“ (Styroporkugeln, Zufallsexperimente, etc.) & 1 & 2 & 1 & & \\
\hline - die Impulsvorträge des Seminarleiters & 2 & 2 & & & \\
\hline - das Verwendung von Arbeitsblättern & 2 & & 1 & 1 & \\
\hline Abwechslung und Schwierigkeitsgrad & zu hoch & & & & zu niedrig \\
\hline Der Anteil an reinen Vortragsphasen war ... & & & 3 & 1 & \\
\hline Der Anteil an gemeinsamen Diskussionsphasen war ... & & 2 & 2 & & \\
\hline Der Anteil an eigenständigem Arbeiten war ... & & 2 & 1 & 1 & \\
\hline Der Schwierigkeitsgrad war insgesamt ... & & 2 & 2 & & \\
\hline Gesamtbewertung & sehr gut & & & & mangelhaft \\
\hline Ich gebe dem Seminar die Gesamtnote: & & 4 & & & \\
\hline
\end{tabular}





\section{Literatur}

Alten, Heinz-Wilhelm, Alireza Djafari Naini, Bettina Eick, Heiko Wesemüller-Kock, Menso Folkerts, Hartmut Schlosser, Karl-Heinz Schlote und Hans Wußing (2014). 4000 Jahre Algebra: Geschichte, Kulturen, Menschen. 2. Aufl. Berlin: Springer.

Andersson, Gunnar (1989). Wahr und falsch; Wahrheit. In: Handlexikon zur Wissenschaftstheorie. Hrsg. von Helmut Seiffert und Gerard Radnitzky. München: Ehrenwirth, S. 369-375.

Artmann, Benno und Hubert Weller (1981). Eine gestufte Hinführung zur Axiomatik in der Linearen Algebra. In: Der Mathematikunterricht 27(2), S. 48-58.

Arzarello, Ferdinando, Maria Giuseppina Bartolini Bussi, Allen Yuk Lun Leung, Maria Alessandra Mariotti und Ian Stevenson (2012). Experimental Approaches to Theoretical Thinking: Artefacts and Proofs. In: Proof and Proving in Mathematics Education. The 19th ICMI Study. Hrsg. von Gila Hanna und Michael de Villiers. Dordrecht: Springer, S. 97-137.

Athen, Hermann (1966). Die Modernisierungstendenzen im Nürnberger Rahmenplan für Mathematik. In: Der Mathematikunterricht 12(3), S. 87-106.

Bedürftig, Thomas und Roman Murawski (2012). Philosophie der Mathematik. 2. Aufl. Berlin: De Gruyter.

Behnke, Heinrich und Hans-Georg Steiner (1956a). Der Begriff des Vektors in der wissenschaftlichen Literatur. In: Der Mathematikunterricht 2(1), S. 5-23.

- (1956b). Der Begriff des Vektors in der wissenschaftlichen Literatur (Zweiter Teil). In: Der Mathematikunterricht 2(4), S. 65-92.

Berghammer, Rudolf (2013). Ordnungen und Verbände. Grundlagen, Vorgehensweisen und Anwendungen. Wiesbaden: Springer Vieweg.

Bernays, Paul (1950/1976). Mathematische Existenz und Widerspruchsfreiheit. In: Ders. Abhandlungen zur Philosophie der Mathematik. Darmstadt: Wissenschaftliche Buchgesellschaft, S. 92-106.

Biehler, Rolf und Andrea Peter-Koop (2007). Hans-Georg Steiner: a life dedicated to the development of didactics of mathematics as a scientific discipline. In: ZDM. Mathematics Education 39, S. 3-30. 
Blank, Albert A. (1966). The Use and Abuse of the Axiomatic Method. In: The Role of Axiomatics and Problem Solving in Mathematics. Hrsg. von The Conference Board of the Mathematical Sciences. Washington, D.C.: Ginn and Company, S. 13-19.

Bloor, David (1983). Wittgenstein. A Social Theory of Knowledge. London: Macmillan Press.

Blum, Werner und Arnold Kirsch (1991). Preformal proving: Examples and reflections. In: Educational Studies in Mathematics 22(2), S. 183-203.

Bogensperger, Brigitte (2014). Die Neue Mathematik und was von ihr übrig blieb. Diplomarbeit. Universität Wien.

Bourbaki, Nicolas (1950). The Architecture of Mathematics. In: The American Mathematical Monthly 57, S. 221-232.

- (1961a). Die Architektur der Mathematik I. In: Physikalische Blätter 17(4), S. 161166.

- (1961b). Die Architektur der Mathematik II. In: Physikalische Blätter 17(5), S. 212218.

Brandt, Dieter, Wolfgang Riemer und Alexander Wollmann, Hrsg. (2012). Lambacher Schweizer. Mathematik für Gymnasien - Stochastik. Stuttgart: Ernst Klett.

Bruner, Jerome S. (1973). Der Prozeß der Erziehung. 3. Aufl. Düsseldorf: Schwann.

- (1974). Entwurf einer Unterrichtstheorie. Berlin: Cornelsen.

Brunner, Esther (2014). Mathematisches Argumentieren, Begründen und Beweisen. Grundlagen, Befunde und Konzepte. Berlin: Springer Spektrum.

Büchter, Andreas und Hans-Wolfgang Henn (2005). Elementare Stochastik - Eine Einführung in die Mathematik der Daten und des Zufalls. Berlin: Springer.

Buck, Robert C. (1966). The Role of a Naive Axiomatics. In: The Role of Axiomatics and Problem Solving in Mathematics. Hrsg. von The Conference Board of the Mathematical Sciences. Washington, D.C.: Ginn and Company, S. 20-26.

Bürger, Heinrich (1979). Beweisen im Mathematikunterricht - Möglichkeiten der Gestaltung in der Sekundarstufe I und II. In: Beweisen im Mathematikunterricht. Hrsg. von Willibald Dörfler und Roland Fischer. Wien: Höder-Pichler-Tempsky, S. 103-134.

Cabassut, Richard, AnnaMarie Conner, Filyet Aslı İşçimen, Fulvia Furinghetti, Hans Niels Jahnke und Francesca Morselli (2012). Conceptions of Proof - In Research and Teaching. In: Proof and Proving in Mathematics Education. The 19th ICMI Study. Hrsg. von Gila Hanna und Michael de Villiers. Dordrecht: Springer, S. 169-190.

Cantor, Georg (1895). Beiträge zur Begründung der transfiniten Mengenlehre. In: Mathematische Annalen 46, S. 481-512.

Cohors-Fresenborg, Elmar (2008). Kognition beim Lernen von Mathematik. Erkenntnisgewinn durch Bildung von mentalen Modellen und den Gebrauch von Metaphern. In: 
Vom Sinn und von der Schwierigkeit des Erinnerns. Hrsg. von Claus Urban und Joachim Engelhardt. Berlin: LIT, S. 246-279.

Cohors-Fresenborg, Elmar, Christa Kaune und Mathilde Griep (1998a). Vertragswerke über den Umgang mit Zahlen - Handbuch für Lehrerinnen und Lehrer. 3. Aufl. Schriftenreihe des Forschungsinstituts für Mathematikdidaktik 17. Osnabrück.

- (1998b). Vertragswerke über den Umgang mit Zahlen - Textbuch für Schülerinnen und Schüler. 3. Aufl. Schriftenreihe des Forschungsinstituts für Mathematikdidaktik 16. Osnabrück.

- (2015). Vertragsgemäßes Rechnen - Arbeitsbuch für Schülerinnen und Schüler in Klasse 6. 3. Aufl. Schriftenreihe des Forschungsinstituts für Mathematikdidaktik 45. Osnabrück.

Cohors-Fresenborg, Elmar, Mathilde Griep, Christa Kaune und Inge Schwank (2003a). Sätze aus dem Wüstensand und ihre Interpretationen - Handbuch für Lehrerinnen und Lehrer. 3. Aufl. Schriftenreihe des Forschungsinstituts für Mathematikdidaktik 15. Osnabrück.

- (2003b). Sätze aus dem Wüstensand und ihre Interpretationen - Textbuch für Schülerinnen und Schüler zur Einführung in die axiomatische Auffassung von Mathematik. 5. Aufl. Schriftenreihe des Forschungsinstituts für Mathematikdidaktik 2. Osnabrück.

Corry, Leo (1989). Linearity and Reflexivity in the Growth of Mathematical Knowledge. In: Science in Context 3(2), S. 409-440.

- (1997). David Hilbert and the Axiomatization of Physics (1894-1905). In: Archive for History of Exact Sciences 51, S. 83-198.

Courant, Richard und Herbert Robbins (2010). Was ist Mathematik? 5. Aufl. Übers. von Iris Runge. Berlin: Springer.

Cramer, Erhard und Udo Kamps (2008). Grundlagen der Wahrscheinlichkeitsrechnung und Statistik. Ein Skript für Studierende der Informatik, der Ingenieur- und Wirtschaftswissenschaften. 2. Aufl. Berlin: Springer.

Curry, Haskell B. (1951). Outlines of a Formalist Philosophy of Mathematics. Amsterdam und London: North-Holland Publishing Company.

Damerow, Peter (1977). Die Reform des Mathematikunterrichts in der Sekundarstufe I. Eine Fallstudie zum Einflu $\beta$ gesellschaftlicher Rahmenbedingungen auf den Prozeß der Curriculum-Reform (Band 1: Reformziele, Reform der Lehrpläne). Stuttgart: Ernst Klett.

Davis, Philip J. und Reuben Hersh (1996). Erfahrung Mathematik. Übers. von Jeanette Zehnder. Basel: Birkhäuser Verlag. 
de Laplace, Pierre-Simon (1886). Philosophischer Versuch über die Wahrscheinlichkeiten. Nach der sechsten Auflage des Originals übersetzt von Norbert Schwaiger. Leipzig: Duncker \& Humblot.

de Villiers, Michael (1990). The role and function of proof in mathematics. In: Pythagoras 24, S. 17-24.

- (2010). Experimentation and Proof in Mathematics. In: Explanation and Proof in Mathematics. Philosophical and Educational Perspectives. Hrsg. von Gila Hanna, Hans N. Jahnke und Helmut Pulte. New York: Springer, S. 205-222.

Deiser, Oliver (2010). Einführung in die Mengenlehre. Die Mengenlehre Georg Cantors und ihre Axiomatisierung durch Ernst Zermelo. 3. Aufl. Berlin: Springer.

Dieudonné, Jean (1961). New Thinking in School Mathematics. In: New Thinking in School Mathematics. Hrsg. von Organisation for European Economic Co-operation, S. 31-49.

Duval, Raymond (1991). Structure du raisonnement déductive et apprentissage de la démonstration. In: Educational Studies in Mathematics 22(3), S. 233-261.

Ebbinghaus, Heinz-Dieter (1974). Zur mengentheoretischen Begründung der natürlichen Zahlen. In: Der Mathematikunterricht 20(5), S. 27-42.

Euklid (2010). Die Elemente. 4. Aufl. Ostwalds Klassiker der exakten Wissenschaften Band 235. Hrsg. und übers. von Clemens Thaer. Frankfurt am Main: Harri Deutsch.

Feynman, Richard P. (1965). New Textbooks for the "New" Mathematics. In: Engineering and Science 28(6), S. 9-15.

Filler, Andreas (1993). Euklidische und nichteuklidische Geometrie. Mannheim: BI Wissenschaftsverlag.

- (2010). Geometrie auf der Kugeloberfläche. In: Der Mathematikunterricht 56(6), S. 1627.

Fischer, Roland und Günther Malle (2004). Mensch und Mathematik. Eine Einführung in didaktisches Denken und Handeln. 2. Aufl. München: Profil-Verlag.

Frege, Gottlob (1969). Nachgelassene Schriften. Hrsg. von Hans Hermes, Friedrich Kambartel und Friedrich Kaulbach. Hamburg: Felix Meiner.

- (1976). Wissenschaftlicher Briefwechsel. Hrsg. von Gottfried Gabriel, Hans Hermes, Friedrich Kambartel, Christian Thiel und Albert Veraart. Hamburg: Felix Meiner.

Freudenthal, Hans (1963). Was ist Axiomatik, und welchen Bildungswert kann sie haben? In: Der Mathematikunterricht 9(4), S. 5-29.

- (1973a). Mathematik als pädagogische Aufgabe. Bd. 1. Stuttgart: Ernst Klett.

- (1973b). Mathematik als pädagogische Aufgabe. Bd. 2. Stuttgart: Ernst Klett.

- (1974). The Crux of Course Design in Probability. In: Educational Studies in Mathematics 5, S. 261-277. 
Gentzen, Gerhard (1936). Die Widerspruchsfreiheit der reinen Zahlentheorie. In: Mathematische Annalen 112, S. 493-565.

Gerwig, Mario (2013). Lehrstückportrait: Euklids Sechsstern - die Entdeckung des Beweisens. In: Der Mathematikunterricht 59(6), S. 24-25.

- (2015). Beweisen verstehen im Mathematikunterricht. Axiomatik, Pythagoras und Primzahlen als Exempel der Lehrkunstdidaktik. Wiesbaden: Springer Spektrum.

Gödel, Kurt (1931a). Über formal unentscheidbare Sätze der Principia mathematica und verwandter Systeme I. In: Monatshefte für Mathematik und Physik 38, S. 173-198.

- (1931b). Diskussion zur Grundlegung der Mathematik. In: Erkenntnis 2(1), S. 135151.

- (1947/1983). What is Cantor's continuum problem? In: Philosophy of mathematics: Selected readings. Hrsg. von Paul Benacerraf und Hilary Putnam. 2. Aufl. Cambridge: Cambridge University Press, S. 470-485.

Griesel, Heinz (1963). Lokales Ordnen und Aufstellen einer Ausgangsbasis, ein Weg zur Behandlung der Geometrie der Unter- und Mittelstufe. In: Der Mathematikunterricht 9(4), S. 55-65.

- (1965). Die Leitlinie Menge-Struktur im gegenwärtigen Mathematikunterricht. In: Der Mathematikunterricht 11(1), S. 40-53.

Griesel, Heinz, Andreas Gundlach, Helmut Postel und Friedrich Suhr, Hrsg. (2011). Elemente der Mathematik. Qualifikationsphase Grund-und Leistungskurs. Braunschweig: Schroedel.

Grotemeyer, Karl P. (1965). Zum strukturellen Aufbau der Mathematik. In: Der Mathematikunterricht 11(4), S. 73-93.

Halmos, Paul R. (1957). Nicolas Bourbaki. In: Scientific American 196(5), S. 88-99.

Hamann, Tanja (2011). „Macht Mengenlehre krank?“ - Die Neue Mathematik in der Schule. In: Beiträge zum Mathematikunterricht 2011. Vorträge auf der 45. Tagung für Didaktik der Mathematik vom 21.2. bis 25.2.2011. Hrsg. von Reinhold Haug und Lars Holzäpfel. Freiburg: WTM-Verlag, S. 347-350.

- (2013). „Macht Mengenlehre krank?“ - Kritik an der Neuen Mathematik in der Grundschule. In: Beiträge zum Mathematikunterricht 2013. Vorträge auf der 47. Tagung für Didaktik der Mathematik vom 4.3.2013 bis 8.3.2013. Hrsg. von Gilbert Greefrath, Friedhelm Käpnick und Martin Stein. Münster: WTM-Verlag, S. 412-415.

- (2017). Die „Mengenlehre“ im Anfangsunterricht - historische Darstellung einer gescheiterten Unterrichtsreform in der Bundesrepublik Deutschland. Dissertation. Universität Hildesheim.

Hanna, Gila (2000). Proof, explanation and exploration: An overview. In: Educational Studies in Mathematics 44, S. 5-23. 
Hartshorne, Robin (2010). Geometry: Euclid and Beyond. New York: Springer.

Hausmann, Gottfried (1959). Didaktik als Dramaturgie des Unterrichts. Heidelberg: Quelle und Meyer.

Heath, Thomas L. (2013). The Thirteen Books of Euclid's Elements. 2. Aufl. Bd. 1. Translated with introduction and commentary. New York: Dover Publications.

Hefendehl-Hebeker, Lisa (2004). Perspektiven für einen künftigen Mathematikunterricht. In: Konsequenzen aus PISA: Perspektiven der Fachdidaktiken. Hrsg. von Horst Bayrhuber, Bernd Ralle, Kristina Reiss, Lutz-Helmut Schön und Helmut Johannes Vollmer. Innsbruck: StudienVerlag, S. 141-190.

Hefendehl-Hebeker, Lisa und Stephan Hußmann (2003). Beweisen - Argumentieren. In: Mathematik-Didaktik. Praxishandbuch für die Sekundarstufe I und II. Hrsg. von Timo Leuders. Berlin: Cornelsen Scriptor, S. 93-106.

Heintz, Bettina (2000). Die Innenwelt der Mathematik. Zur Kultur und Praxis einer beweisenden Disziplin. Wien: Springer.

Heitzer, Johanna (2015). Das Aachener Schul-Hochschul-Projekt iMPACt. In: Übergänge konstruktiv gestalten. Ansätze für eine zielgruppenspezifische Hochschuldidaktik Mathematik. Hrsg. von Jürgen Roth, Thomas Bauer, Herbert Koch und Susanne Prediger. Wiesbaden: Springer Spektrum, S. 3-18.

Hemmi, Kirsti (2008). Students' encounter with proof: the condition of transparency. In: ZDM. Mathematics Education 40, S. 413-426.

Hempel, Carl G. (1945). Geometry and Empirical Science. In: The American Mathematical Monthly 52, S. 7-17.

- (1945/1983). On the nature of mathematical truth. In: Philosophy of mathematics: Selected readings. Hrsg. von Paul Benacerraf und Hilary Putnam. 2. Aufl. Cambridge: Cambridge University Press, S. 377-393.

Heymann, Hans W. (1996). Allgemeinbildung und Mathematik. Weinheim: Beltz.

Hilbert, David (1900). Mathematische Probleme. In: Nachrichten von der Königl. Gesellschaft der Wissenschaften zu Göttingen. Mathematisch-physikalische Klasse aus dem Jahre 1900. S. 253-297.

- (1962). Grundlagen der Geometrie. 9. Aufl. Stuttgart: Teubner.

- (1964). Neubegründung der Mathematik. In: Ders. Hilbertiana. Fünf Aufsätze. Darmstadt: Wissenschaftliche Buchgesellschaft, S. 12-32.

- (2015). Grundlagen der Geometrie (Festschrift 1899). Hrsg. und komm. von Klaus Volkert. Berlin: Springer Spektrum.

Hilbert, David und Paul Bernays (1934). Grundlagen der Mathematik. Bd. 1. Berlin: Springer. 
Hischer, Horst (2012). Grundlegende Begriffe der Mathematik: Entstehung und Entwicklung. Struktur - Funktion - Zahl. Wiesbaden: Springer Spektrum.

- (2016). (Mathematisches) Modellieren als Axiomatisieren - eine ungewohnte Sicht? In: Mitteilungen der Gesellschaft für Didaktik der Mathematik 100, S. 51-56.

Hock, Tobias, Johanna Heitzer und Inge Schwank (2016). Axiomatisches Denken und Arbeiten im Mathematikunterricht. In: Journal für Mathematik-Didaktik 37(1), S. 181208.

Höffe, Otfried (2009). Aristoteles: Die Hauptwerke. Ein Lesebuch. Tübingen: francke.

Hoffmann, Dirk W. (2013a). Die Gödel'schen Unvollständigkeitssätze. Eine geführte Reise durch Kurt Gödels historischen Beweis. Berlin: Springer Spektrum.

- (2013b). Grenzen der Mathematik. Eine Reise durch die Kerngebiete der mathematischen Logik. 2. Aufl. Berlin: Springer Spektrum.

Hungerbühler, Norbert (2015). Nachwort: Ein Paradigma für die lehrkunstdidaktische Erarbeitung unterrichtstauglicher und bildungsförderlicher didaktischer Werke (Teil II). In: Gerwig, Mario. Beweisen verstehen im Mathematikunterricht. Axiomatik, Pythagoras und Primzahlen als Exempel der Lehrkunstdidaktik. Wiesbaden: Springer Spektrum, S. 359-363.

Hußmann, Stephan und Maike Schindler (2014). Ein Kontext für negative Zahlen - auch für die Multiplikation. In: Mathematik lehren 183, S. 28-32.

Irvine, Andrew D. (1990). Nominalism, Realism \& Physicalism in Mathematics: An Introduction to the Issues. In: Physicalism in Mathematics. Hrsg. von Andrew D. Irvine. Dordrecht: Kluwer, S. ix-xxvi.

Jahnke, Hans N. (2000). The use of original sources in the mathematics classroom. In: History in Mathematics Education. The ICMI Study. Hrsg. von John Fauvel und Jan van Maanen. Dordrecht: Kluwer, S. 291-328.

- (2007a). Beweisen und hypothetisch-deduktives Denken. In: Der Mathematikunterricht 53(5), S. 10-21.

- (2007b). Proofs and hypotheses. In: ZDM. Mathematics Education 39, S. 79-86.

- (2008). Theorems that admit exceptions, including a remark on Toulmin. In: ZDM. Mathematics Education 40, S. 363-371.

- (2009). Hypothesen und ihre Konsequenzen. In: Praxis der Mathematik in der Schule 30, S. 26-30.

- (2010). The Conjoint Origin of Proof and Theoretical Physics. In: Explanation and Proof in Mathematics. Philosophical and Educational Perspectives. Hrsg. von Gila Hanna, Hans N. Jahnke und Helmut Pulte. New York: Springer, S. 17-32. 
Jahnke, Hans N. und Stefan Ufer (2015). Argumentieren und Beweisen. In: Handbuch der Mathematikdidaktik. Hrsg. von Regina Bruder, Lisa Hefendehl-Hebeker, Barbara Schmidt-Thieme und Hans-Georg Weigand. Berlin: Springer Spektrum, S. 331-356.

Jahnke, Hans N. und Ralf Wambach (2013). Understanding what a proof is: a classroombased approach. In: ZDM. Mathematics Education 45, S. 469-482.

Jänisch, Klaus (2008). Topologie. 8. Aufl. Berlin: Springer.

Kahle, Reinhard (2007). Die Gödelschen Unvollständigkeitssätze. In: Mathematische Semesterberichte 54, S. 1-12.

Kalmár, László (1967). Foundations of Mathematics - Whither Now? In: Problems in the Philosophy of Mathematics. Hrsg. von Imre Lakatos. North-Holland Publishing Company, S. 187-194.

Kant, Immanuel (1966). Kritik der reinen Vernunft. Stuttgart: Reclam.

Kapur, Jagat N. (1977). New mathematics movement - is it over? In: International Journal of Mathematical Education in Science and Technology 8(3), S. 259-267.

Kilpatrick, Jeremy (2012). The new math as an international phenomenon. In: ZDM. Mathematics Education 44, S. 563-571.

Kirsch, Arnold (1967). Gehören die Peano-Axiome in den Schulunterricht? In: Der Mathematikunterricht 13(3), S. 11-24.

- (1976). Eine ,intellektuell ehrliche“ Einführung des Integralbegriffs in Grundkursen. In: Didaktik der Mathematik 4, S. 87-105.

- (1979). Beispiele für prämathematische Beweise. In: Beweisen im Mathematikunterricht. Hrsg. von Willibald Dörfler und Roland Fischer. Klagenfurt: Hölder-PichlerTempsky, S. 261-274.

Klenke, Achim (2006). Wahrscheinlichkeitstheorie. Berlin: Springer.

Kline, Morris (1966). Mathematics and Axiomatics. In: The Role of Axiomatics and Problem Solving in Mathematics. Hrsg. von The Conference Board of the Mathematical Sciences. Washington, D.C.: Ginn and Company, S. 57-62.

- (1972). Mathematical Thought from Ancient to Modern Times. New York: Oxford University Press.

- (1973). Why Johnny Can't Add: The Failure of the New Math. New York: St. Martin's Press.

Klotzek, Benno (2001). Euklidische und nichteuklidische Elementargeometrien. Frankfurt am Main: Harri Deutsch.

Koch, Helmut (2004). Einführung in die Mathematik. 2. Aufl. Berlin: Springer.

Kolmogorov, Andrei N. (1933). Grundbegriffe der Wahrscheinlichkeitsrechnung. Berlin: Springer. 
Kultusministerkonferenz, Hrsg. (2015). Bildungsstandards im Fach Mathematik für die Allgemeine Hochschulreife (Beschluss der Kultusministerkonferenz vom 18.10.2012). Köln: Wolters Kluwer.

Kuntze, Sebastian (2009). Beweisen - was ist das? Gesprächs-,,Rahmen“ und Reflexionsanlässe schaffen. In: Mathematik lehren 155, S. 12-17.

Kuypers, Wilhelm, Josef Lauter und Hans Wuttke, Hrsg. (1993). Mathematik. 7. Schuljahr. Berlin: Cornelsen.

Lakatos, Imre (1979). Beweise und Widerlegungen. Die Logik mathematischer Entdeckungen. Hrsg. von John Worrall und Elie Zahar. Braunschweig: Vieweg \& Sohn.

- (1982a). Renaissance des Empirismus in der neueren Philosophie der Mathematik? In: Ders. Philosophische Schriften: Mathematik, empirische Wissenschaft und Erkenntnistheorie. Braunschweig: Vieweg, S. 23-41.

- (1982b). Unendlicher Regreß und Grundlagen der Mathematik. In: Ders. Philosophische Schriften: Mathematik, empirische Wissenschaft und Erkenntnistheorie. Braunschweig: Vieweg, S. 3-22.

Lakoff, George und Mark Johnson (1980). Metaphors We Live By. Chicago: The University of Chicago Press.

Laugwitz, Detlef (1965). Der Streit um die Methode in der modernen Mathematik. In: Neue Sammlung. Göttinger Blätter für Kultur und Erziehung 5, S. 9-23.

- (1966). Sinn und Grenzen der axiomatischen Methode. In: Der Mathematikunterricht 12(3), S. 16-39.

Laures, Gerd und Markus Szymik (2015). Grundkurs Topologie. 2. Aufl. Heidelberg: Springer Spektrum.

Leng, Mary (2010). Preaxiomatic Mathematical Reasoning: An Algebraic Approach. In: Explanation and Proof in Mathematics. Philosophical and Educational Perspectives. Hrsg. von Gila Hanna, Hans N. Jahnke und Helmut Pulte. New York: Springer, S. 4758.

Lenné, Helge (1969). Analyse der Mathematikidaktik in Deutschland. Stuttgart: Ernst Klett.

Lergenmüller, Arno und Günter Schmidt, Hrsg. (2007). Mathematik Neue Wege. Arbeitsbuch für Gymnasien - 6. Schuljahr. Braunschweig: Schroedel.

Lergenmüller, Arno, Günter Schmidt und Katja Krüger, Hrsg. (2012). Mathematik Neue Wege. Arbeitsbuch für Gymnasien - Stochastik. Braunschweig: Schroedel.

Leuders, Timo (2011). Perspektiven von Mathematikunterricht. In: Mathematik-Didaktik. Praxishandbuch für die Sekundarstufe I und II. Hrsg. von Timo Leuders. 6. Aufl. Berlin: Cornelsen, S. 15-58. 
Leuders, Timo und Kathleen Philipp (2014). Mit Beispielen zum Erkenntnisgewinn. Experiment und Induktion in der Mathematik. In: Mathematica didactica 37, S. 163-190.

Maddy, Penelope (1988a). Believing the Axioms. I. In: The Journal of Symbolic Logic 53(2), S. 481-511.

- (1988b). Believing the Axioms. II. In: The Journal of Symbolic Logic 53(3), S. 736764.

- (1990). Realism in Mathematics. Oxford: Clarendon Press.

- (2011). Defending the Axioms: On the Philosophical Foundations of Set Theory. New York: Oxford University Press.

Malle, Günther (2007a). Die Entstehung negativer Zahlen. Der Weg vom ersten Kennenlernen bis zu eigenständigen Denkobjekten. In: Mathematik lehren 142, S. 52-57.

- (2007b). Die spannende Suche nach dem $i$. Wie sich Zahlvorstellungen entwickeln. In: Mathematik lehren 142, S. 60-63.

- (2007c). Zahlen fallen nicht vom Himmel. Ein Blick in die Geschichte der Mathematik. In: Mathematik lehren 142, S. 4-11.

Mengenlehre: 3+5=5+3 (1974). In: Der Spiegel 13, S. 62-79.

Meschkowski, Herbert (1964). Einführung in die moderne Mathematik. 2. Aufl. Mannheim: Bibliographisches Institut.

- (1965). Mathematik als Bildungsgrundlage. Braunschweig: Vieweg.

- (1990). Denkweisen großer Mathematiker. Ein Weg zur Geschichte der Mathematik. Braunschweig: Vieweg.

Meyer, Michael (2006). Entdecken und Begründen im Mathematikunterricht. Von der Abduktion zum Argument. Dissertation. Hildesheim: Franzbecker.

- (2007). Entdecken und Begründen im Mathematikunterricht - Zur Rolle der Abduktion und des Arguments. In: Journal für Mathematik-Didaktik 28, S. 286-310.

Moon, Bob (1986). The 'New Maths' Curriculum Controversy. An International Story. London: The Falmer Press.

Ossa, Erich (2009). Topologie. Eine anschauliche Einführung in die geometrischen und algebraischen Grundlagen. 2. Aufl. Wiesbaden: Vieweg + Teubner.

Pallack, Andreas, Hrsg. (2013). Fundamente der Mathematik. Nordrhein-Westfalen. Gymnasium - Klasse 6. Berlin: Cornelsen.

- (2014). Die Multiplikation ganzer Zahlen - mit oder ohne Kontext? In: Mathematik lehren 183, S. 25-27.

Pasch, Moritz (1882). Vorlesungen über neuere Geometrie. Leipzig: Teubner.

Pedemonte, Bettina (2007). How can the relationship between argumentation and proof be analysed? In: Educational Studies in Mathematics 66(1), S. 23-41. 
Picht, Georg (1964). Die deutsche Bildungskatastrophe. Analyse und Dokumentation. Olten: Walter-Verlag.

Platon (2010). Der Staat. Köln: Anaconda.

Poincaré, Henri (1904). Wissenschaft und Hypothese. Übers. von F. und L. Lindemann. Leipzig: Teubner.

Polya, George (1949/1995). Schule des Denkens. Vom Lösen mathematischer Probleme. 4. Aufl. Tübingen: Francke.

Proklos (1970). A Commentary on the First Book of Euclid's Elements. Translated with Introduction and Notes by Glenn R. Morrow. Princeton, NJ: Princeton University Press.

Radnitzky, Gerard (1989). Definition. In: Handlexikon zur Wissenschaftstheorie. Hrsg. von Helmut Seiffert und Gerard Radnitzky. München: Ehrenwirth, S. 27-33.

Riemann, Bernhard (2013). Über die Hypothesen, welche der Geometrie zu Grunde liegen. Historisch und mathematisch kommentiert von Jürgen Jost. Berlin: Springer Spektrum.

Rosser, John B. (1936). Extensions of some theorems of Gödel and Church. In: Journal of Symbolic Logic 1(3), S. 87-91.

Schichl, Hermann und Roland Steinbauer (2009). Einführung in das mathematische Arbeiten. Berlin: Springer.

Schoenfeld, Alan H. (1985). Mathematical Problem Solving. Orlando: Academic Press.

Scholz, Roland W. (2007). Game and decision theory in mathematics education: epistemological, cognitive and didactical perspectives. In: ZDM. Mathematics Education 39, S. 51-61.

Schönbeck, Jürgen (2003). Euklid. Basel: Birkhäuser Verlag.

Schreiber, Alfred (1979). Probleme bei der Einführung des Wahrscheinlichkeitsbegriffs. In: Mathematica didactica 2(4), S. 235-246.

Schreiber, Peter (2010a). Einführung: Euklid und die Geschichte seines Werkes. In: Euklid. Die Elemente. 4. Aufl. Ostwalds Klassiker der exakten Wissenschaften Band 235. Hrsg. und übers. von Clemens Thaer. Frankfurt am Main: Harri Deutsch, S. iii-xvi.

- (2010b). Zur Geschichte der Parallelenproblems und der nichteuklidischen Geometrien. In: Der Mathematikunterricht 56(6), S. 3-15.

Scriba, Christoph J. und Peter Schreiber (2010). 5000 Jahre Geometrie: Geschichte, Kulturen, Menschen. 3. Aufl. Berlin: Springer.

Shapiro, Stewart (2000). Thinking about Mathematics: The Philosophy of Mathematics. New York: Oxford University Press.

Sjuts, Johann (1993). Zur didaktisch-methodischen Reorganisation des SI-Mathematikunterrichts. In: Der Mathematikunterricht 39(3), S. 27-43. 
Steinbring, Heinz (1980). Zur Entwicklung des Wahrscheinlichkeitsbegriffs - Bernoullis Theorem aus didaktischer Sicht. In: Journal für Mathematik-Didaktik 1(3), S. 115-141. Steiner, Hans-Georg (1959). Das moderne mathematische Denken und die Schulmathematik. In: Der Mathematikunterricht 5(4), S. 5-79.

- (1965). Menge, Struktur, Abbildung als Leitbegriffe für den mordernen mathematischen Unterricht. In: Der Mathematikunterricht 11(1), S. 5-19.

- (1966a). Einfache Verknüpfungsgebilde als Vorfeld der Gruppentheorie. In: Der Mathematikunterricht 12(2), S. 5-18.

- (1966b). Mathematisierung und Axiomatisierung einer politischen Struktur. Ein Beispiel zur axiomatischen Methode im mathematischen Unterricht. In: Der Mathematikunterricht 12(3), S. 66-86.

- (1966c). Verschiedene Aspekte der axiomatischen Methode im Unterricht. In: Les Repercussions de la Recherche Mathématique sur l'Enseignement. Luxemburg, S. 31-70.

- (1968). Examples of exercises in mathematization on the secondary school level. In: Educational Studies in Mathematics 1(1/2), S. 181-201.

- (1969a). Eine mathematische Theorie der Abstimmungsgremien. Dissertation. Darmstadt: TU Darmstadt.

- (1969b). Examples of exercises in mathematization. An extension of the theory of voting bodies. In: Educational Studies in Mathematics 1(3), S. 289-299.

- (1975). Nichtmeßbare Abstimmungsgremien. In: Mathematisch-Physikalische Semesterberichte 22(1), S. 53-67.

- (1976a). An example of the axiomatic method in instruction: The mathematization of a political structure. In: The Mathematics Teacher 55, S. 520-528.

- (1976b). Finite geometries and non-measurable voting bodies. In: Educational Studies in Mathematics 7(1/2), S. 139-146.

- (1988). Mathematization of voting systems: some classroom experiences. In: International Journal of Mathematical Education in Science and Technology 19(2), S. 199213.

Steiner, Mark (1973). Platonism and the Causal Theory of Knowledge. In: The Journal of Philosophy 70(3), S. 57-66.

Stone, Marshall H. (1961). Reform in School Mathematics. In: New Thinking in Shool Mathematics. Hrsg. von Organisation for European Economic Co-operation, S. 14-29. Szabó, Árpád (1960). Anfänge des Euklidischen Axiomensystems. In: Archive for History of Exact Sciences 1, S. 37-106.

- (1978). The Beginnings of Greek Mathematics. Dordrecht: D. Reidel Publishing Company. 
Tait, William W. (1986). Truth and Proof: The Platonism of Mathematics. In: Synthese 69, S. 341-370.

Tall, David (2013). How Humans Learn to Think Mathematically - Exploring the Three Worlds of Mathematics. New York: Cambridge University Press.

Tapp, Christian (2013). An den Grenzen des Endlichen. Das Hilbertprogramm im Kontext von Formalismus und Finitismus. Berlin: Springer Spektrum.

Tarski, Alfred (1936). Der Wahrheitsbegriff in den formalisierten Sprachen. In: Studia Philosophica 1, S. 261-405.

Tietze, Uwe-Peter und Frank Förster (2000). Fachdidaktische Grundfragen des Mathematikunterrichts in der Sekundarstufe II. In: Mathematikunterricht in der Sekundarstufe II. Band 1: Fachdidaktische Grundfragen - Didaktik der Analysis. Hrsg. von Uwe-Peter Tietze, Manfred Klika und Hans Wolpers. 2. Aufl. Braunschweig: Vieweg, S. 1-177. van der Waerden, Bartel L. (1967). Klassische und moderne Axiomatik. In: Elemente der Mathematik 22(1), S. 1-4.

van Hiele, Pierre M. (1959). A Child's Thought and Geometry. In: English translation of selected writings of Dina van Hiele-Geldorft and Pierre M. van Hiele. Hrsg. von David Fuys, Dorothy Geddes und Rosamond Tischler. Brooklyn: Brooklyn College, School of Education.

Verhulst, Ferdinand (2010). Eine kühle Sicht der Wahrheit - Beweisen und Begründen in der Mathematik. In: Das Zebra-Buch zur Geometrie. Hrsg. von Ferdinand Verhulst und Sebastian Walcher. Berlin: Springer, S. 199-248.

Volkert, Klaus (2013). Das Undenkbare denken. Die Rezeption der nichteuklidischen Geometrie im deutschsprachigen Raum (1860-1900). Berlin: Springer Spektrum.

Vollrath, Hans-Joachim (1994). Strukturelles Denken im Algebraunterricht. In: Der Mathematikunterricht 40(4), S. 5-25.

Vollrath, Hans-Joachim und Jürgen Roth (2012). Grundlagen des Mathematikunterrichts in der Sekundarstufe. 2. Aufl. Heidelberg: Spektrum Akademischer Verlag.

vom Hofe, Rudolf und Mathias Hattermann (2014). Zugänge zu negativen Zahlen. In: Mathematik lehren 183, S. 2-7.

von Helmholtz, Hermann (1883). Über den Ursprung und die Bedeutung der geometrischen Axiome. In: Ders. Vorträge und Reden. Bd. 2. Braunschweig: Vieweg, S. 1-31. von Mises, Richard (1964). Grundlagen der Wahrscheinlichkeitsrechnung. In: Selected Papers of Richard von Mises: Probability and Statistics, General. Hrsg. von Philipp Frank et al. Nachdruck des Originals von 1919. Providence, RI: American Mathematical Society, S. 57-105.

von Neumann, John und Oskar Morgenstern (1944). Theory of Games and Economic Behavior. Princeton: Princeton University Press. 
Wagenschein, Martin (1970). Ursprüngliches Verstehen und exaktes Denken. Bd. 2. Stuttgart: Ernst Klett.

- (1974). Entdeckung der Axiomatik. In: Der Mathematikunterricht 20(1), S. 52-70.

- (1980). Naturphänomene sehen und verstehen. Genetische Lehrgänge. Hrsg. von Hans Ch. Berg. Stuttgart: Ernst Klett.

- (2008). Verstehen lehren. Genetisch - Sokratisch - Exemplarisch. 4. Aufl. Weinheim: Beltz.

Walcher, Sebastian und Erich Ch. Wittmann (2012). Minus mal minus. Zum Fundament der COACTIV-Studie. In: Der mathematische und naturwissenschaftliche Unterricht 65(6), S. 371-377.

Weber, Heinrich (1893). Die allgemeinen Grundlagen der Galois'schen Gleichungstheorie. In: Mathematische Annalen 43, S. 521-549.

Wigner, Eugene (1960). The Unreasonable Effectiveness of Mathematics in the Natural Sciences. In: Communications in Pure and Applied Mathematics 13(1), S. 1-14.

Wildhirt, Susanne und Mario Gerwig (2013). Das Konzept der Lehrkunstdidaktik verdeutlicht am Thema Wahrscheinlichkeitsrechnung. In: Der Mathematikunterricht 59(6), S. 4-10.

Wille, Matthias (2015). Die Metaphysik der Mathematik. Mathematische Modellbildung und metaphysische Spekulation. In: Der Mathematikunterricht 61(6), S. 10-17.

Winter, Heinrich (1975). Allgemeine Lernziele für den Mathematikunterricht? In: ZDM. Mathematics Education 7, S. 106-116.

- (1983a). Über die Entfaltung begrifflichen Denkens im Mathematikunterricht. In: Journal für Mathematik-Didaktik 4(3), S. 175-204.

- (1983b). Zur Problematik des Beweisbedürfnisses. In: Journal für Mathematik-Didaktik 4(1), S. 59-95.

- (1995). Mathematikunterricht und Allgemeinbildung. In: Mitteilungen der Gesellschaft für Didaktik der Mathematik 61, S. 37-46.

Wittenberg, Alexander I. (1963). Bildung und Mathematik. Stuttgart: Ernst Klett.

Wittmann, Erich Ch. (1981). Grundfragen des Mathematikunterrichts. 6. Aufl. Braunschweig: Vieweg.

Wittmann, Erich Ch. und Gerhard Müller (1988). Wann ist ein Beweis ein Beweis? In: Mathematikdidaktik: Theorie und Praxis. Festschrift für Heinrich Winter. Hrsg. von Peter Bender. Berlin: Cornelsen, S. 237-257.

Witzke, Ingo (2011). Zur Theorieentwicklung in der Mathematik. In: Beiträge zum Mathematikunterricht 2011. Vorträge auf der 45. Tagung für Didaktik der Mathematik vom 21.2. bis 25.2.2011. Hrsg. von Reinhold Haug und Lars Holzäpfel. Freiburg: WTMVerlag, S. 911-915. 
- (2012). Mathematik - eine (naive) Naturwissenschaft im Schulunterricht? In: Beiträge zum Mathematikunterricht 2012. Vorträge auf der 46. Tagung für Didaktik der Mathematik vom 5.3.2012 bis 9.3.2012. Hrsg. von Matthias Ludwig und Michael Kleine. Weingarten: WTM-Verlag, S. 949-952.

- (2013). Unterschiedliche Auffassungen von Mathematik - ein Ansatzpunkt zur Klärung der Übergangsproblematik im Fach Mathematik? In: Mathematik im Übergang Schule/Hochschule und im ersten Studienjahr. Extended Abstracts zur 2. khdm-Arbeitstagung, S. 172-174.

Wolpers, Hans und Stefan Götz (2002). Mathematikunterricht in der Sekundarstufe II. Band 3: Didaktik der Stochastik. Hrsg. von Uwe-Peter Tietze, Manfred Klika und Hans Wolpers. Braunschweig: Vieweg.

Worrall, John (1979). A Reply to David Bloor. In: The British Journal for the History of Science 12(1), S. 71-81.

Wußing, Hans (2009). 6000 Jahre Mathematik. Eine kulturgeschichtliche Zeitreise - 2. Von Euler bis zur Gegenwart. Berlin: Springer.

Zech, Friedrich (2002). Grundkurs Mathematikidaktik. 10. Aufl. Weinheim: Beltz. 



\section{Index}

Ableitung (in formalen Systemen), 45

Alphabet (eines formalen Systems), 43

Argumentationsbasis, 84-85

Argumentieren, 83-85

ARISTOTELES, 10

Aussage, 45

Aussageform, 45

Axiom

Archimedisches Axiom, 18, 30

Auswahlaxiom, 59

Axiom vs. Postulat, 10-11

Axiome als Vertragswerke, 108-112

Axiome der Peano-Arithmetik, 44

Extensionalitätsaxiom, 58

Stetigkeitsaxiom, 155

Unendlichkeitsaxiom, 58

Vollständigkeitsaxiom, 29, 31

Axiomatik

abstrahierende, 63

Bourbakische, 63

charakterisierende, 62

euklidische, 61

formale, 63

formalistische, 64

Hilbertsche, 62

inhaltliche, 63

klassische, 63

moderne, 63
Axiomensystem

monomorphes, 62

polymorphes, 63

Begründen, 83-85

Beweis(en), 10, 16, 56-57, 60, 83-87, 139

formaler Beweis, 45

Funktionen des Beweisens, 86

inhaltlich-anschauliche Beweise, 84

präformale Beweise, 84

prämathematische Beweise, 84

VON BOLYAI, JOHANN, 20

VON BOLYAI, WOLFGANG, 18

Bourbaki-Gruppe, 38

BRUNER, JEROME, 82

CANTOR, GeOrg, 37

CARDANO, Girolamo, 169

CAYley, ARTHUR, 36

Clavius, Christoph, 18

Curry, Haskell B., 57

Definition, 10

explizite, 111

implizite, 31-33

Dialektik, 14

DIEUDONNÉ, JEAN, 68

Elemente, 8-16

Axiome, 9 
Definitionen, 9

Postulate, 9

Entscheidbarkeit, 43

Epistemologie, 7

EUKLID, 8

Finitismus, 42

Formalismus, 31, 55-56

Hilbert als Formalist, 34-35

Spielformalismus, 35

Formel (eines formalen Systems), 43

FrAENKEL, ABRAHAM, 37

Frege, GotTlob, 32

FREUDENTHAL, HANS, 76

GAUSS, CARL FrIEDRICH, 20

Gefüge, deduktives, 139

GentZen, Gerhard, 49

Geometrie

elliptische, 20, 133

hyperbolische, 23-27

Inzidenzgeometrie, 149

nichteuklidische, 18-28

sphärische, 20

GÖDEL, KURT, 42, 54, 57

Gödelscher Unvollständigkeitssatz

erster, 46

zweiter, 48

Gruppentheorie, 36-37

HAMiLTON, WiLliam ROWAN, 170

HAUSMANN, GotTFRIED, 113

von Helmholtz, Hermann, 21

HILBERT, DAVID, 29

Hilbertprogramm, 42

Intuition, mathematische, 54

Intuitionismus, 53

Inversion, antididaktische, 76
KIRSCH, ARNOLD, 82

KLAFKI, WOLFGANG, 113

KLEIN, Felix, 24

Kolmogorov, AndReI, 154

Kolmogorov-Axiome, 154

Konstruktivismus, 51

Korrektheit, 46

Korrespondenztheorie, 31

LAKATOS, IMRE, 59

LAMBERT, JOHANN HEINRICH, 19

DE LAPLACE, PIERRE-SiMON, 153

LEGENDRE, AdRIEN-MARIE, 18

Lehrkunstdidaktik, 113-114

LOBATSCHEWSKI, NiKOLAI I., 20

Menge

naiver Mengenbegriff, 37

strukturierte Menge, 38

Metamathematik, 42

VON MISES, RICHARD, 153

Modell, 23

Klein-Modell, 24

Poincaré-Modell, 24-25

Modellieren, 24

Negationsvollständigkeit, 46

Neue Mathematik, 65-74

Nominalismus, 53

Nürnberger Rahmenplan, 69-72

Ontologie, 7

Ordnen, lokales, 77

PASCH, MORITZ, 29

PeAno, Giuserpe, 29

Peano-Arithmetik, 43-45

Physikalismus, 54

PLATON, 13

Platonismus, 53-54 
PLAYFAIR, JOHN, 18

POINCARÉ, HENRI, 24

Postulat, 8-16

Parallelenpostulat, 17-18

Postulat vs. Axiom, 10-11

Prädikatenlogik, 44

Presburger-Arithmetik, 49

Proposition, 12

Quasi-Empirismus, 59-60

Realismus, 53

Rechtfertigung

extrinsische, 58

intrinsische, 58

Relation, 39

ROSSER, JOHN BARKLEY, 46

Royaumont-Konferenz, 67-68

Russelsche Antinomie, 37

SACCHERI, GIROLAMO, 18

Satz (siehe auch Theorem), 139

Sprache (eines formalen Systems), 43

STEINER, HANS-GEORG, 77, 96

Stone, Marshall Harvey, 67

Struktur

algebraische Struktur, 38

Ordnungsstruktur, 39

Halbordnung, 40

lineare Ordnung, 40

Quasiordnung, 40

Striktordnung, 40

Totalordnung, 40

topologische Struktur, 40

Strukturmathematik, 36-41

System, formales, 43

TARSKI, Alfred, 57

Term (eines formalen Systems), 43
Theorem, 45

Unabhängigkeit, 30

Unvollständigkeitssätze, siehe Gödelscher Unvollständigkeitssatz

Variable

freie, 44

gebundene, 44

Verknüpfung, 38

Vermutung, 139

Vollständigkeit, 30

semantische, 46

syntaktische, siehe Negationsvollständigkeit

WAGenschein, MARTIN, 113

Wahrheit, 16-17, 27, 31-34, 45, 56-58

Wahrscheinlichkeitsbegriff

frequentistischer, 153

Laplace'scher, 153

objektivistischer, 153

subjektivistischer, 153

Wahrscheinlichkeitsmaß, siehe KolmogorovAxiome

WALLIS, JOHN, 18

WEBER HEINRICH, 36

Widerspruchsfreiheit, 30, 46

relative, 26

WIENER, HERMANN, 29

WittenberG, AleXAnder I., 81-83

Wohlordnungssatz, 59

Zahlen

bekloppte, 179

komplexe, 170

ZERMELO, ERNST, 37 